Comparative Effectiveness Review

Number 199

\title{
Treatments for Basal Cell and Squamous Cell Carcinoma of the Skin
}
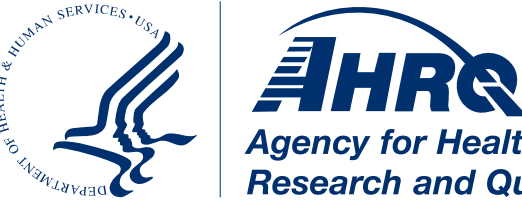

Agency for Healthcare

Research and Quality
Effective Health

Care Program 


\section{Comparative Effectiveness Review}

Number 199

\section{Treatments for Basal Cell and Squamous Cell Carcinoma of the Skin}

(with addendum)

Prepared for:

Agency for Healthcare Research and Quality

U.S. Department of Health and Human Services

5600 Fishers Lane

Rockville, MD 20857

www.ahrq.gov

Contract No. 290-2015-00002-I

Prepared by:

Brown Evidence-based Practice Center

Providence, RI

Investigators:

Aaron Drucker, M.D.

Gaelen P. Adam, M.L.I.S.

Valerie Langberg, M.S.

Abhilash Gazula, M.P.H.

Bryant Smith, M.P.H.

Farah Moustafa, M.D.

Martin A. Weinstock, M.D., Ph. D.

Thomas A. Trikalinos, M.D.

AHRQ Publication No. 17(18)-EHC033-EF

December 2017 


\section{Key Messages}

\section{Purpose of Review}

Assess comparative effectiveness and safety of treatments for basal cell carcinoma (BCC) and squamous cell carcinoma (SCC).

\section{Key Messages}

- Comparative evidence on treatment of BCC and SCC is limited. Many comparisons were evaluated in one or two randomized controlled trials only.

- Surgery and radiotherapy have lower recurrence rates for BCC than interventions that destroy lesions with heat or cold, photodynamic therapy (PDT), or curettage.

- There is moderate confidence that PDT for BCC is associated with better cosmetic outcomes than surgery.

- Serious adverse events, events leading to treatment discontinuation, and treatment site infections were uncommon with all treatments for BCC.

- Recurrence rates for SCC in situ were lower with PDT and cryotherapy than with drugs. Evidence was insufficient to draw conclusions for other treatments. 
This report is based on research conducted by the Brown Evidence-based Practice Center (EPC) under contract to the Agency for Healthcare Research and Quality (AHRQ), Rockville, MD (Contract No. 290-2015-00002-I). The findings and conclusions in this document are those of the authors, who are responsible for its contents; the findings and conclusions do not necessarily represent the views of AHRQ. Therefore, no statement in this report should be construed as an official position of AHRQ or of the U.S. Department of Health and Human Services.

\section{None of the investigators have any affiliations or financial involvement that conflicts with the material presented in this report.}

The information in this report is intended to help health care decisionmakers-patients and clinicians, health system leaders, and policymakers, among others-make well-informed decisions and thereby improve the quality of health care services. This report is not intended to be a substitute for the application of clinical judgment. Anyone who makes decisions concerning the provision of clinical care should consider this report in the same way as any medical reference and in conjunction with all other pertinent information, i.e., in the context of available resources and circumstances presented by individual patients.

This report is made available to the public under the terms of a licensing agreement between the author and the Agency for Healthcare Research and Quality. This report may be used and reprinted without permission except those copyrighted materials that are clearly noted in the report. Further reproduction of those copyrighted materials is prohibited without the express permission of copyright holders.

AHRQ or U.S. Department of Health and Human Services endorsement of any derivative products that may be developed from this report, such as clinical practice guidelines, other quality enhancement tools, or reimbursement or coverage policies, may not be stated or implied. This report may periodically be assessed for the currency of conclusions. If an assessment is done, the resulting surveillance report describing the methodology and findings will be found on the Effective Health Care Program Web site at www.effectivehealthcare.ahrq.gov. Search on the title of the report.

Persons using assistive technology may not be able to fully access information in this report. For assistance contact EffectiveHealthCare@ahrq.hhs.gov.

Suggested citation: Drucker A, Adam GP, Langberg V, Gazula A, Smith B, Moustafa F, Weinstock MA, Trikalinos TA. Treatments for Basal Cell and Squamous Cell Carcinoma of the Skin. Comparative Effectiveness Review No. 199. (Prepared by the Brown Evidence-based Practice Center under Contract No. 290-2015-00002-I.) AHRQ Publication No. 17(18)EHC033-EF. Rockville, MD: Agency for Healthcare Research and Quality; December 2017. www.effectivehealthcare.ahrq.gov/reports/final.cfm. DOI: https://doi.org/10.23970/AHRQEPCCER199 


\section{Preface}

The Agency for Healthcare Research and Quality (AHRQ), through its Evidence-based Practice Centers (EPCs), sponsors the development of systematic reviews to assist public- and private-sector organizations in their efforts to improve the quality of health care in the United States. These reviews provide comprehensive, science-based information on common, costly medical conditions, and new health care technologies and strategies.

Systematic reviews are the building blocks underlying evidence-based practice; they focus attention on the strength and limits of evidence from research studies about the effectiveness and safety of a clinical intervention. In the context of developing recommendations for practice, systematic reviews can help clarify whether assertions about the value of the intervention are based on strong evidence from clinical studies. For more information about AHRQ EPC systematic reviews, see www.effectivehealthcare.ahrq.gov/reference/purpose.cfm.

AHRQ expects that these systematic reviews will be helpful to health plans, providers, purchasers, government programs, and the health care system as a whole. Transparency and stakeholder input are essential to the Effective Health Care Program. Please visit the Web site (www.effectivehealthcare.ahrq.gov) to see draft research questions and reports or to join an email list to learn about new program products and opportunities for input.

If you have comments on this systematic review, they may be sent by mail to the Task Order Officer named below at: Agency for Healthcare Research and Quality, 5600 Fishers Lane, Rockville, MD 20857, or by email to epc@ahrq.hhs.gov.

Gopal Khanna, M.B.A.

Director

Agency for Healthcare Research and Quality

Stephanie Chang, M.D., M.P.H.

Director

Evidence-based Practice Center Program

Center for Evidence and Practice Improvement

Agency for Healthcare Research and Quality
Arlene S. Bierman, M.D., M.S.

Director

Center for Evidence and Practice Improvement Agency for Healthcare Research and Quality

Lionel Bañez, M.D.

Task Order Officer

Center for Evidence and Practice Improvement Agency for Healthcare Research and Quality 


\section{Key Informants}

In designing the study questions, the EPC consulted several Key Informants who represent the end-users of research. The EPC sought the Key Informant input on the priority areas for research and synthesis. Key Informants are not involved in the analysis of the evidence or the writing of the report. Therefore, in the end, study questions, design, methodological approaches, and/or conclusions do not necessarily represent the views of individual Key Informants.

Key Informants must disclose any financial conflicts of interest greater than $\$ 10,000$ and any other relevant business or professional conflicts of interest. Because of their role as end-users, individuals with potential conflicts may be retained. The TOO and the EPC work to balance, manage, or mitigate any conflicts of interest.

The list of Key Informants who provided input to this report follows:

Sarah Arron, M.D.

Dermatologist/Associate Professor

University of California

San Francisco, CA

Melody J. Eide, M.D. M.P.H.

Dermatologist/Assistant Professor of

Medicine

University of South Dakota Sanford School

of Medicine

Rapid City Medical Center

Rapid City, SD

Charles Hayley

Medical Director/Medicaid,

Noridian

Lawrence, KS
William Higgins, M.D., M.Be.

Assistant Professor of Dermatology

Brown University

Providence, RI

Eleni Linos, M.D.

Dermatologist/Health Services Researcher

University of California

San Francisco, CA

Dr. Yakub Puthawala, M.D.

Radiation Oncologist

Chief of Brachytherapy Services

Rhode Island Hospital

Providence, RI

Patients (2)

Rhode Island

Names withheld for privacy protection 


\section{Technical Expert Panel}

In designing the study questions and methodology at the outset of this report, the EPC consulted several technical and content experts. Broad expertise and perspectives were sought. Divergent and conflicting opinions are common and perceived as healthy scientific discourse that results in a thoughtful, relevant systematic review. Therefore, in the end, study questions, design, methodologic approaches, and/or conclusions do not necessarily represent the views of individual technical and content experts.

Technical Experts must disclose any financial conflicts of interest greater than $\$ 10,000$ and any other relevant business or professional conflicts of interest. Because of their unique clinical or content expertise, individuals with potential conflicts may be retained. The TOO and the EPC work to balance, manage, or mitigate any potential conflicts of interest identified.

The list of Technical Experts who provided input to this report follows.

Sarah Arron, M.D.*

Dermatologist/Associate Professor

University of California

San Francisco, CA

Elizabeth Toni Barnes, M.D.*

Department of Radiation Oncology

University of Toronto

Toronto, ON, Canada

An-Wen Chan, M.D.

Associate Professor

University of Toronto

Toronto, ON, Canada

Melody Eide, M.D., M.P.H.*

Dermatologist/Assistant Professor of

Medicine

University of South Dakota Sanford School

of Medicine

Rapid City Medical Center

* Provided input on Draft Report.
Rapid City, SD

Charles Hayley

Medical Director/Medicaid, Noridian

Lawrence, KS

William Higgins, M.D., M.Be. Assistant Professor of Dermatology

Brown University

Providence, RI

Eleni Linos, M.D.

Dermatologist/Health Services Researcher University of California

San Francisco, CA 


\section{Peer Reviewers}

Prior to publication of the final evidence report, EPCs sought input from independent Peer reviewers without financial conflicts of interest. However, the conclusions and synthesis of the scientific literature presented in this report do not necessarily represent the views of individual reviewers.

Peer Reviewers must disclose any financial conflicts of interest greater than $\$ 10,000$ and any other relevant business or professional conflicts of interest. Because of their unique clinical or content expertise, individuals with potential nonfinancial conflicts may be retained. The TOO and the EPC work to balance, manage, or mitigate any potential nonfinancial conflicts of interest identified.

The list of Peer Reviewers follows:

Mary-Margaret Chren, M.D. Department of Dermatology

University of California at San Francisco

San Francisco VA Medical Center

San Francisco, CA

Graham Colditz, M.D.

Department of Surgery

Washington University School of Medicine

St. Louis, MO

Robert P. Dellavalle, M.D., Ph.D., M.S.P.H. Professor of Dermatology and Public Health University of Colorado School of Medicine
Colorado School of Public Health Chief, Dermatology Service

U.S. Department of Veterans Affairs Eastern Colorado Health Care System Denver, CO

Naveed Nosrati, M.D.

Division of Plastic Surgery

Department of Surgery

Indiana University

Indianapolis, IN 


\section{Treatments for Basal Cell and Squamous Cell Carcinoma of the Skin}

\section{Structured Abstract}

Introduction. Basal cell carcinoma (BCC) and squamous cell carcinoma (SCC) are among the most common malignancies in the United States. There are many potential management strategies for BCCs and SCCs, and the choice of management strategy for an individual patient is not straightforward. We aimed to comprehensively collect information on the comparative effectiveness and safety of each currently used therapeutic strategy for both BCC and SCC.

Data sources. We conducted literature searches in MEDLINE ${ }^{\circledR}$, the Cochrane Central Trials Registry and Cochrane Database of Systematic Reviews, and Embase ${ }^{\circledR}$ up to March 2017. We also perused the reference lists of published relevant clinical practice guidelines and systematic reviews. We recorded information on recurrence, histologic clearance, clinical clearance, patientor observer-rated cosmetic outcomes, adverse effects, quality of life, costs and resources, mental health, patient satisfaction, and mortality. We estimated intervention effects (differences in outcomes between treatments) and the mean frequency of the outcome with each treatment using network meta-analyses.

Results We identified 58 randomized controlled trials and 51 nonrandomized comparative studies comparing 21 interventions in 9 categories. Nearly all reported results for recurrence or cure rate outcomes and adverse events, and many reported results for cosmetic outcomes. Few studies reported results using validated instruments for quality of life, mental health, or patient satisfaction with treatment. Data were sparse, especially for analyses at the individualintervention level. For BCCs, surgical interventions and radiation were associated with lower recurrence rates than interventions that destroy lesions with heat or cold and photodynamic therapy (PDT), and may have lower recurrence rates than curettage. Recurrence rates did not differ significantly between imiquimod and excision. The data were not sufficient to draw conclusions about the comparison of curettage with interventions that destroy lesions with heat or cold, or PDT versus other intervention categories. For SCC in situ, interventions that destroy the lesions with heat or cold and PDT were associated with lower recurrence rates than 5fluorouracil. Data on the relative effect of thermal interventions versus PDT were not precise enough to draw conclusions.

Conclusions. Based on sparse evidence, surgical and radiation treatments have lower recurrence rates than other modalities for the treatment of low-risk BCC, and PDT appears to have superior cosmetic outcomes. Large gaps remain in the literature regarding the comparison of individual interventions and SCC in situ, with very little or no information on immunocompromised patients, patients with limited life expectancy, and patients with specific lesion categories, including high-risk BCCs and invasive SCCs.

May 2019 update: an addendum is located at the end of the main report, before the appendixes. 


\section{Contents}

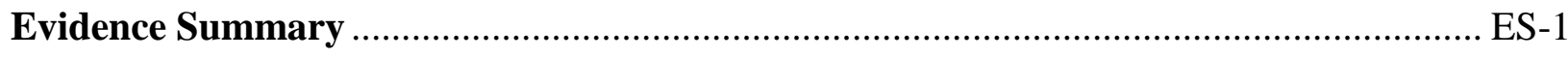

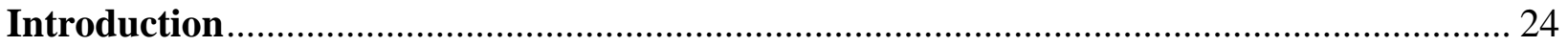

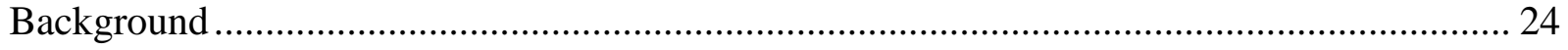

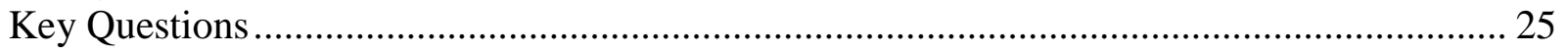

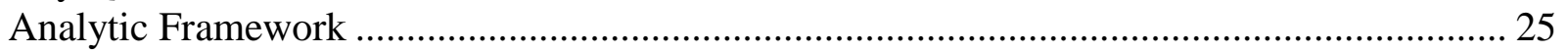

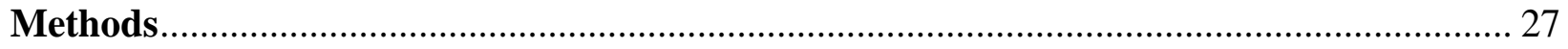

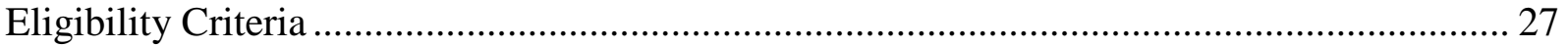

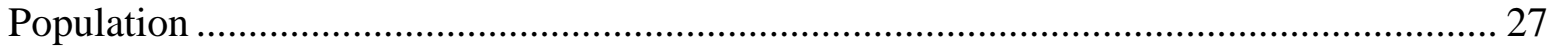

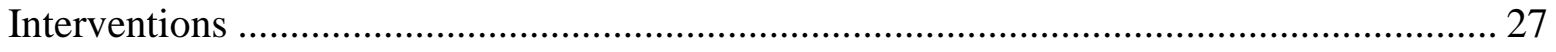

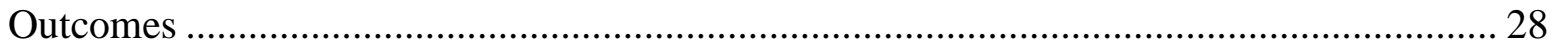

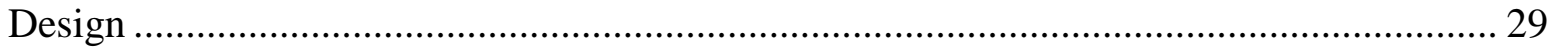

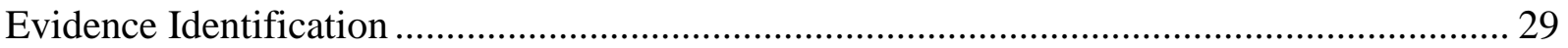

Data Extraction and Data Management ................................................................................... 29

Assessment of Methodological Risk of Bias of Individual Studies...................................... 30

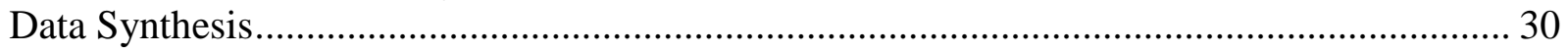

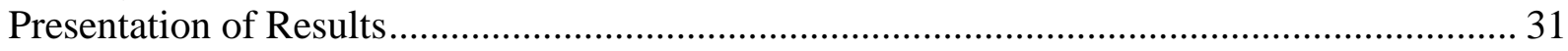

Evidence Graphs ............................................................................................... 31

Relative Effects Tables ................................................................................................ 33

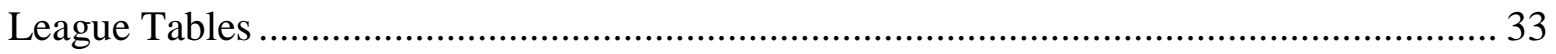

Grading the Strength of Evidence (SOE) for Major Comparisons and Outcomes .................... 34

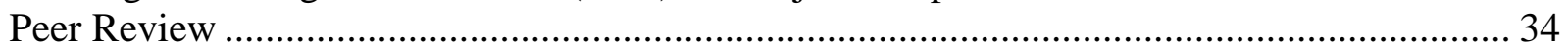

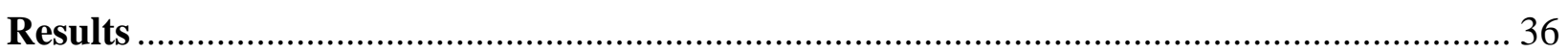

Summary of Studies ............................................................................................................ 36

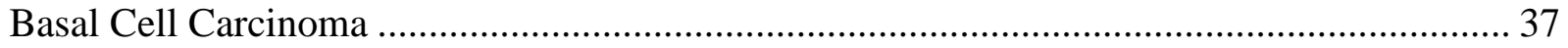

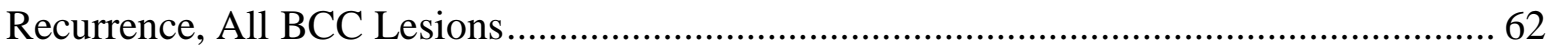

Lack of Histologic Clearance (All BCC Lesions) ………………………........................... 73

Lack of Clinical Clearance, All BCC Lesions ..................................................................... 86

Patient-Reported Cosmetic Outcomes, All BCC Lesions...................................................... 97

Quality of Life, All BCC Lesions .................................................................................. 102

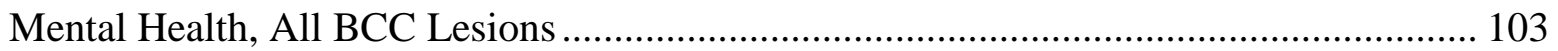

Patient Satisfaction, All BCC Lesions ........................................................................ 103

Mortality, All BCC Lesions ............................................................................................. 103

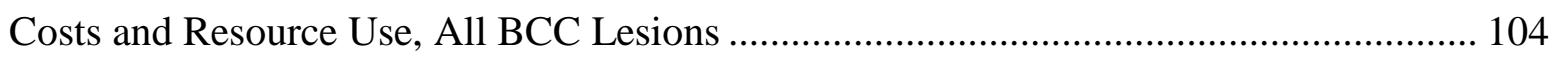

Adverse Events, All BCC Lesions............................................................................... 105

Dose Response Analyses for Drugs, All BCC Lesions ...................................................... 110

Special Populations ..................................................................................................... 110 


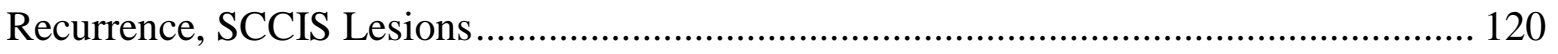

Lack of Histological Clearance, SCCIS Lesions ............................................................. 124

Lack of Clinical Clearance, SCCIS Lesions .................................................................. 126

Patient-Reported Cosmetic Outcomes, SCCIS Lesions...................................................... 133

Observer-Reported Cosmetic Outcomes, SCCIS Lesions .................................................. 133

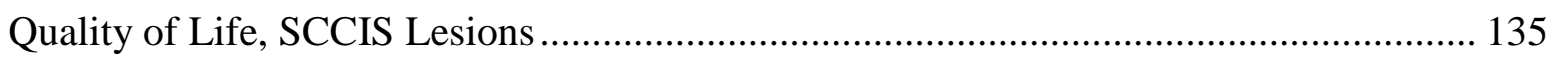

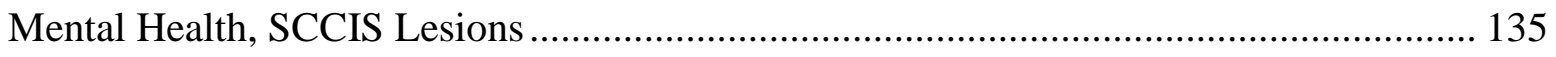

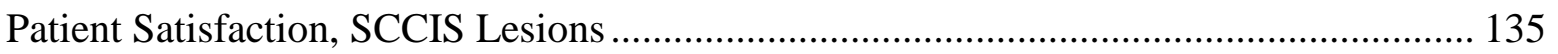

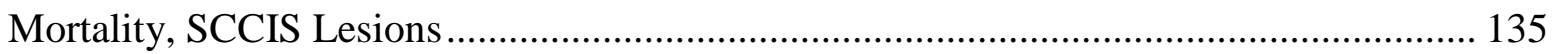

Costs and Resource Use, SCCIS Lesions ..................................................................... 135

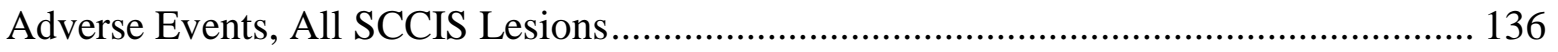

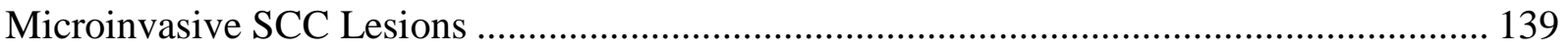

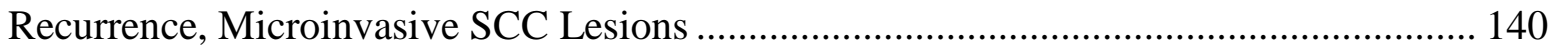

Lack of Histological Clearance, Microinvasive SCC Lesions.............................................. 140

Observer-Reported Cosmetic Outcomes, Microinvasive SCC Lesions ............................... 140

Adverse Events, Microinvasive SCC Lesions .................................................................... 140

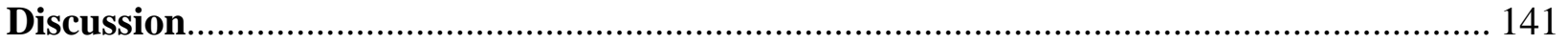

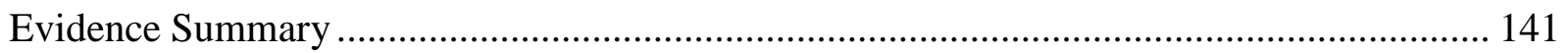

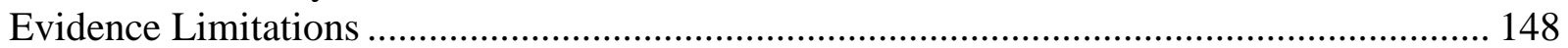

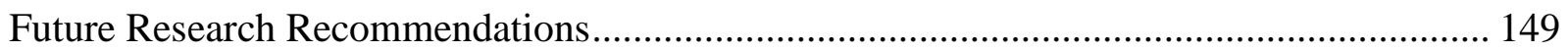

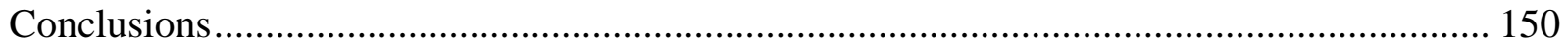

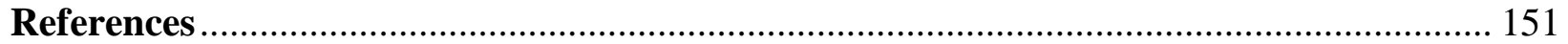

\section{Tables}

Table A. Population, interventions, outcomes, timing, and setting .......................................... ES-2 Table B. Mean frequency (percent) of outcomes per intervention category based on direct and indirect data (all BCCs) .................................................................................................. ES-9 Table C. Mean frequency of outcomes per intervention category based on direct and indirect data (SCCIS). ES-13

Table D. Summary conclusions for BCC lesions and strength of the relevant evidence ........ ES-17 Table E. Summary conclusions for SCCIS lesions and strength of the relevant evidence..... ES-20 Table 1. Relative odds ratios for an outcome between intervention categories (Figure 3) ........... 33

Table 2. Mean and forecasted event fractions by intervention category ........................................ 34

Table 3. Characteristics of RCTs of superficial BCCs ……………………………………........ 42

Table 4. Characteristics of RCTs of nodular BCC …………................................................... 46

Table 5. Characteristics of RCTs of high-risk BCC lesions ......................................................... 54

Table 6. Characteristics of RCTs of mixed types of BCC lesions............................................ 54

Table 7. Sample information, recurrence (all BCC lesions, intervention categories) .................... 63 
Table 8. Relative odds ratios for recurrence between intervention categories (all BCC lesions,

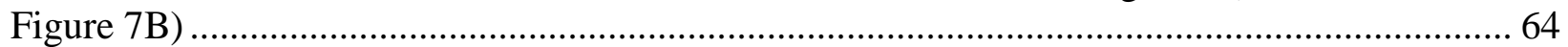

Table 9. Mean and forecasted recurrence rates by intervention category (all BCC lesions)......... 64

Table 10. Sample information, recurrence (all BCC lesions, individual interventions)................. 65

Table 11. Relative odds ratios for recurrence between individual interventions (all BCC lesions,

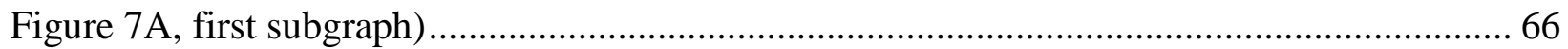

Table 12. Relative odds ratios for recurrence between individual interventions (all BCC lesions,

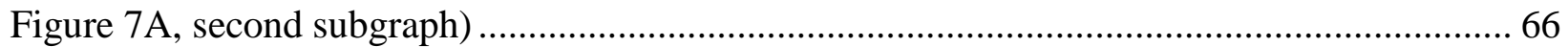

Table 13. Mean recurrence rates by intervention (all BCC lesions) ................................................ 68

Table 14. Relative odds ratios for recurrence between interventions (predominantly superficial

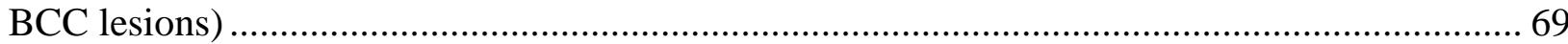

Table 15. Mean recurrence rates by intervention category (predominantly superficial BCC

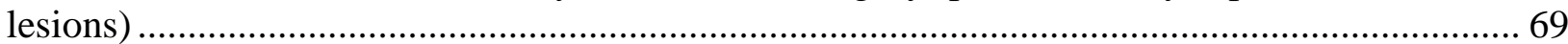

Table 16. Sample information (predominantly nodular lesions) ……………………................ 70

Table 17. Relative odds ratios for recurrence between interventions (predominantly nodular BCC

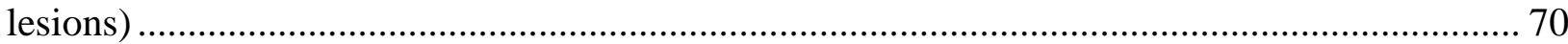

Table 18. Mean and forecasted recurrence rates by intervention category (predominantly nodular

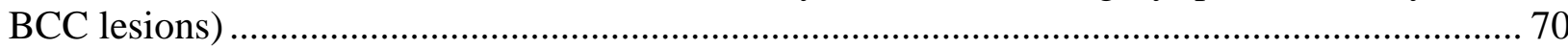

Table 19. Subgroup results by lesion size and location for recurrence (BCC lesions).................. 71

Table 20. Sample information, lack of histological clearance (all BCC lesions, intervention

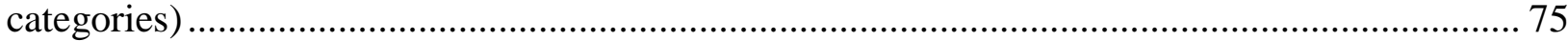

Table 21. Relative odds ratios for lack of histologic clearance between intervention categories

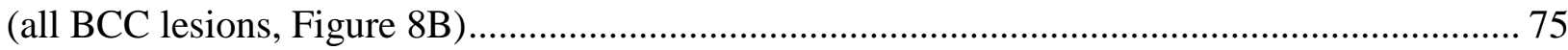

Table 22. Mean and forecasted lack of histologic clearance fractions by intervention category (all BCC lesions) .................................................................................................................... 76

Table 23. Sample information, lack of histological clearance (all BCC lesions, individual

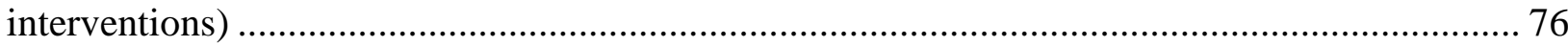
Table 24. Relative odds ratios for lack of histological clearance between individual interventions

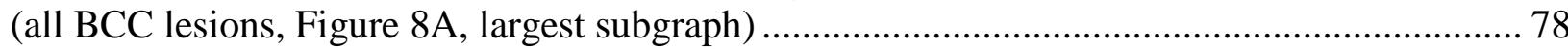
Table 25. Relative odds ratios for lack of histological clearance between individual interventions (all BCC lesions, Figure 8, other subgraphs) ............................................................................. 78 Table 26. Relative odds ratios for lack of histological clearance between individual interventions (all BCC lesions, Figure 8A, 2 more subgraphs) ……………....................................................... 80 Table 27. Mean and forecasted lack of histological clearance fractions by intervention category

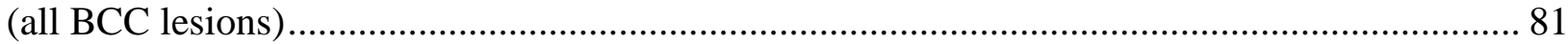

Table 28. Sample information, lack of histological clearance (superficial lesions) ...................... 82 Table 29. Relative odds ratios for lack of histological clearance between intervention categories (predominantly superficial BCC lesions)..................................................................................... 83

Table 30. Mean fraction of lesions without histological clearance by intervention category (predominantly superficial BCC lesions)..................................................................................... 83

Table 31. Sample information, lack of histological clearance (nodular lesions) ............................ 83 Table 32. Relative odds ratios for lack of histological clearance between intervention categories (nodular BCC lesions)........................................................................................................... 84 
Table 33. Mean and forecasted lack of histological clearance fractions by intervention category

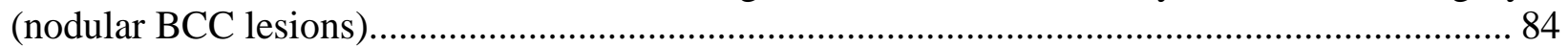

Table 34. Subgroup results for lack of histological clearance in superficial BCCs ....................... 85

Table 35. Sample information, lack of clinical clearance (all BCC lesions, intervention

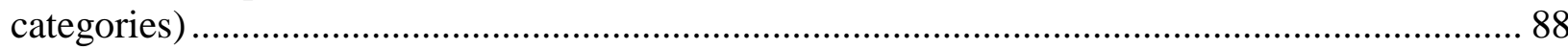
Table 36. Relative odds ratios for lack of clinical clearance between intervention categories (all

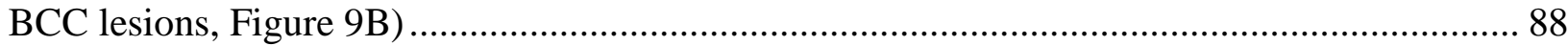
Table 37. Mean and forecasted lack of clinical clearance fractions by intervention category (all

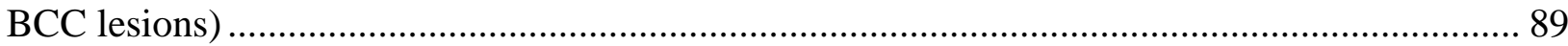

Table 38. Sample information, lack of clinical clearance (all BCC lesions, individual interventions).....

Table 39. Relative odds ratios for lack of clinical clearance between individual interventions (all BCC lesions, Figure 9A, largest subgraph) 91

Table 40. Relative odds ratios for lack of clinical clearance between individual interventions (all BCC lesions, Figure 9A, remaining subgraphs).

Table 41. Mean and forecasted lack of clinical clearance fractions by individual intervention (all

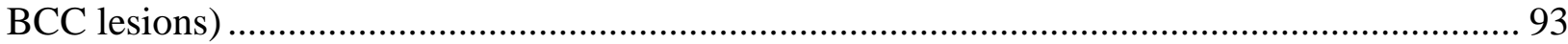

Table 42. Sample information, lack of clinical clearance (superficial BCC lesions) .................... 93

Table 43. Relative odds ratios for lack of clinical clearance between intervention categories (superficial BCC lesions) ...................................................................................................... 94

Table 44. Mean and forecasted lack of clinical clearance fractions by intervention category (superficial BCC lesions) .................................................................................................... 94

Table 45. Sample information, lack of clinical clearance (superficial BCC lesions) .................... 94

Table 46. Relative odds ratios between intervention categories for lack of clinical clearance (nodular BCC lesions)............................................................................................................ 95 Table 47. Mean fractions of lesions with no clinical clearance by intervention category (nodular

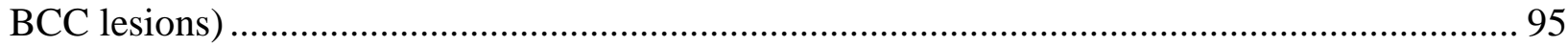

Table 48. Subgroup results for lack of clinical clearance in BCC lesions..................................... 96 Table 49. Sample information, patient-reported cosmetic outcomes (all BCC lesions, intervention categories) ..................................................................................................................... 97

Table 50. Relative odds ratios between intervention categories for at least good cosmetic outcome as assessed by patients (all BCC lesions, Figure 10) ..................................................... 98 Table 51. Mean and forecasted fractions of lesions with at least good cosmetic outcome as assessed by patients (all BCC lesions)....................................................................................... 99 Table 52. Sample information, observer-reported cosmetic outcomes (all BCC lesions, intervention categories) ....................................................................................................... 99

Table 53. Relative odds ratios between intervention categories for at least good cosmetic outcome as assessed by an observer (all BCC lesions, Figure 11) .............................................. 100 Table 54. Mean fractions of lesions with at least good cosmetic outcome as assessed by an

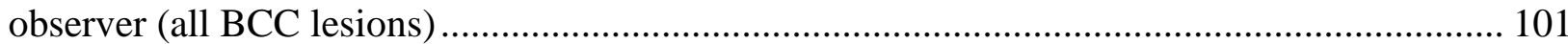

Table 55. Quality of life measured with Skindex ……………............................................... 103

Table 56. Sample information, adverse events (all BCC lesions, intervention categories)......... 108 Table 57. Mean fractions of adverse events, using each RCT's definitions (all BCC lesions).. 109 
Table 58. Summary of phase II or II/III trials comparing different doses or intensities of

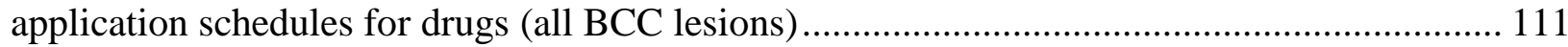

Table 59. Characteristics of studies of SCC populations................................................... 118

Table 60. Sample information, recurrence (SCCIS, intervention categories) .......................... 121

Table 61. Relative odds ratios for recurrence between intervention categories (SCCIS lesions,

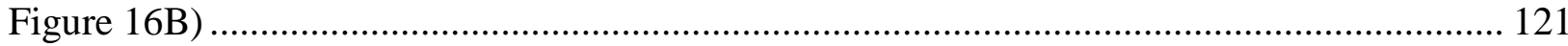

Table 62. Mean recurrence rates by intervention category (SCCIS lesions) ............................ 122

Table 63. Sample information, recurrence (SCCIS, interventions) ....................................... 122

Table 64. Relative odds ratios for recurrence between individual interventions (SCCIS lesions,

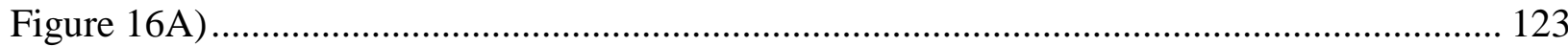

Table 65. Mean and forecasted recurrence rates by intervention category (SCCIS lesions)...... 123

Table 66. Subgroup analyses by lesion location and size: results for recurrence (SCCIS lesions)

Table 67. Sample information, lack of histological clearance (SCCIS) ................................. 125

Table 68. Relative odds ratios for lack of histological clearance between individual interventions

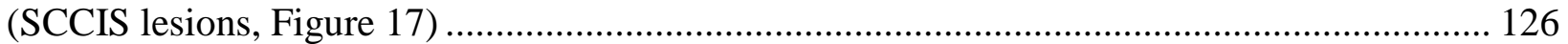

Table 69. Mean lack of histological clearance (all SCCIS lesions)...................................... 126

Table 70. Sample information, lack of clinical clearance (SCCIS, intervention categories) ..... 128

Table 71. Relative odds ratios for lack of clinical clearance between intervention categories

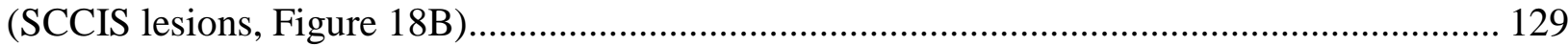

Table 72. Mean and forecasted lack of clinical clearance fractions by intervention category

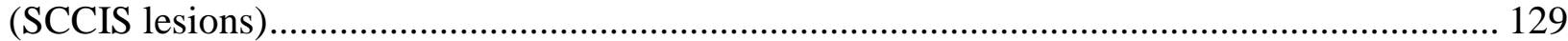

Table 73. Sample information, lack of clinical clearance (SCCIS, interventions)................... 129

Table 74. Relative odds ratios for lack of clinical clearance between individual interventions

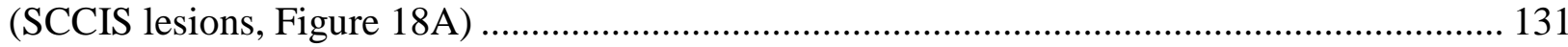

Table 75. Mean and forecasted lack of clinical clearance fractions by intervention (SCCIS lesions)

Table 76. Subgroup analyses by lesion location and size: results for lack of clinical clearance

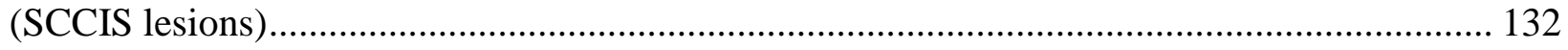

Table 77. Sample information, observer-reported cosmetic outcomes (SCCIS)...................... 133

Table 78. Relative odds ratios between interventions for at least good cosmetic outcome, as assessed by an observer (SCCIS lesions, Figure 19)

Table 79. Mean fractions of lesions with at least good cosmetic outcome, as assessed by an

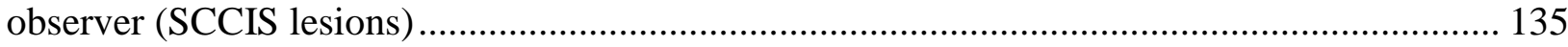

Table 80. Sample information, adverse events (SCCIS) …................................................... 138

Table 81. Mean fractions of adverse events, using each RCT's definitions (SCCIS lesions) .... 139

Table 82. Summary conclusions for BCC lesions and strength of the relevant evidence .......... 143

Table 83. Summary conclusions for SCCIS lesions and strength of the relevant evidence ....... 146

\section{Figures}

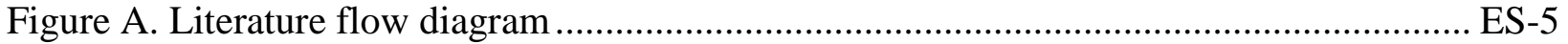

Figure B. Evidence graph depicting compared treatments in RCTs of BCC lesions .............. ES-6

Figure C. Evidence graphs for recurrence, histologic clearance, and clinical clearance from RCTs of BCC lesions 
Figure D. Evidence graph depicting compared interventions in RCTs of SCC lesions ES-11

Figure E. Evidence graphs for recurrence, histologic clearance, and clinical clearance for RCTs of SCC lesions. ES-12

Figure 1. Analytic framework for treatments for basal cell and squamous cell carcinoma of the skin 26

Figure 2. Example evidence graph depicting comparisons between individual interventions ..... 32

Figure 3. Evidence graph depicting comparisons between intervention categories ................... 32

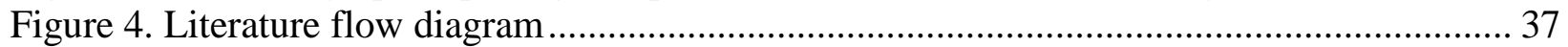

Figure 5. Evidence graph depicting compared treatments in RCTs of BCC lesions ................... 38

Figure 6. Evidence graphs for recurrence, histologic clearance, and clinical clearance from RCTs

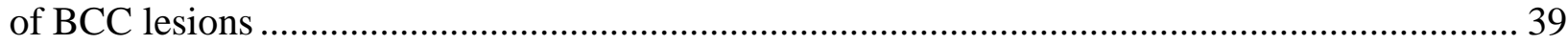

Figure 7. Evidence graph of RCTs evaluating recurrence in BCCs across (A) individual interventions and (B) types of interventions

Figure 8. Evidence graph of RCTs evaluating lack of histological clearance in BCCs across (A)

individual interventions and (B) types of interventions..................................................... 74

Figure 9. Evidence graph of RCTs evaluating lack of clinical clearance in BCCs across (A)

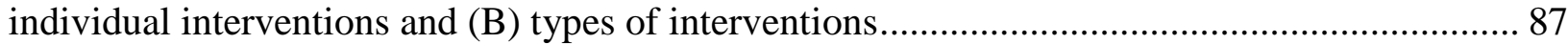

Figure 10. Evidence graph of RCTs comparing patient-assessed cosmetic outcomes (all BCC lesions)

Figure 11. Evidence graph of RCTs comparing observer-assessed cosmetic outcomes (all BCC

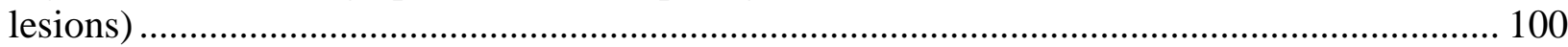

Figure 12. Evidence graph of RCTs comparing frequency of adverse events (all BCC lesions)105

Figure 13. Evidence graph depicting compared treatments in RCTs of SCC lesions ............... 114

Figure 14. Evidence graph depicting compared treatment types in RCTs of SCC lesions ........ 115

Figure 15. Evidence graphs for recurrence, histologic clearance, and clinical clearance for RCTs

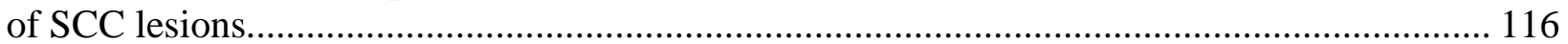

Figure 16. Evidence graph of RCTs evaluating recurrence in SCCIS across (A) individual

interventions and (B) types of interventions .............................................................. 120

Figure 17. Evidence graph of RCTs evaluating lack of histological clearance in SCCIS lesions

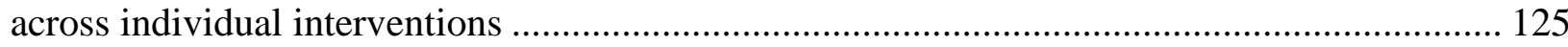

Figure 18. Evidence graph of RCTs evaluating lack of clinical clearance in SCCIS lesions across

(A) individual interventions and (B) types of interventions

Figure 19. Evidence graph of RCTs comparing observer-assessed cosmetic outcomes (all SCC

lesions)

Figure 20. Evidence graph of RCTs comparing frequency of adverse events (SCCIS lesions). 136

\section{Appendixes}

Appendix A. Search Strategy

Appendix B. Excluded Studies

Appendix C. Design Details

Appendix D. Baselines

Appendix E. Arm Details

Appendix F. Risk of Bias 
Appendix G. Summary Results From Unadjusted NRCS

Appendix H. Adverse Events Reported

Appendix I. Study-Level Results 


\section{Evidence Summary}

\section{Introduction}

Skin cancers, including basal cell carcinoma (BCC) and squamous cell carcinoma (SCC), are the most common malignancies in the United States. ${ }^{1}$ BCC and SCC, the 2 most common skin cancers, are collectively referred to as keratinocyte carcinomas. Over 5.4 million of these lesions are diagnosed in 3.3 million people in the United States annually, ${ }^{2,3}$ and the global burden of disease from keratinocyte carcinomas is estimated at 12.9 disability-adjusted life years per 100,000 persons. ${ }^{4}$ Generally keratinocyte carcinomas are not aggressive and do not metastasize or kill as often as melanoma, which is the third most common skin cancer. ${ }^{5}$ However, SCC can metastasize and is estimated to kill between 3900 and 8800 people in the United States each year. ${ }^{6}$ A more common problem is that BCC and SCC and their treatment may result in disfigurement or disability, which can adversely impact quality of life. ${ }^{3}$ The recent Surgeon General's call to action to prevent skin cancer at the population level emphasizes the public health importance of dealing with these cancers. ${ }^{7}$

There are many potential management strategies for BCC and SCC, including surgical excision without intraoperative evaluation of the margins, surgical excision with intraoperative evaluation of the margins, destruction via temperature gradients, ionizing radiation, photodynamic interventions, medical therapies, various combinations of the aforementioned therapies, and watchful waiting.

The choice of management strategy for an individual patient with a specific keratinocyte carcinoma is complex, and it is not clear how various therapeutic options perform relative to each other. In addition, interventions for treating skin cancers differ substantially in cost. ${ }^{3,8-10}$

The objective of this systematic review is to comprehensively synthesize information on the comparative effectiveness and safety of each of the above-mentioned therapeutic strategies for both BCC and SCC.

\section{Key Questions}

The review addresses two Key Questions for adult patients with BCC or SCC of the skin. Each Key Question will be answered separately for BCC and SCC:

Key Question 1: What is the comparative effectiveness of various interventions, overall and in subgroups of interest?

Key Question 2: How do the adverse events associated with the various interventions compare overall and in subgroups of interest?

\section{Methods}

The Brown Evidence-based Practice Center (EPC) conducted this review based on a systematic review of the published scientific literature, using established methodologies as outlined in the Agency for Healthcare Research and Quality (AHRQ) Methods Guide for Effectiveness and Comparative Effectiveness Reviews. ${ }^{11}$ The Prospero registration number is CRD42016043353. Below is a summary of the methods; details are provided in the methods section of the full report. 


\section{Eligibility Criteria}

We use the population, intervention, comparator, outcomes, timing, and setting (PICOTS formalism to define the characteristics of the eligible studies for this review. Details are in Table A.

Table A. Population, interventions, outcomes, timing, and setting

PICOTS and Description

Population

Primary basal cell carcinoma (BCC) and squamous cell carcinoma (SCC)

Subpopulations of interest

People who are immunocompromised

People with a limited life expectancy

We excluded subpopulations based on rare genetic factors

Subgroups as defined by location or grade of lesion

Interventions (organized into categories A through $\mathbf{J}$ )

A. Surgical excision without intraoperative evaluation of the margins

B. Surgical excision with intraoperative evaluation of the margins

Mohs micrographically controlled surgery

Surgery with examination of frozen sections

C. Interventions that destroy the lesion via temperature gradients

(C1) Cryotherapy

(C2) Diathermy/electrodesiccation

(C3) Curettage of the lesion plus diathermy (cauterization) of margins

(C4) Curettage of the lesion plus cryotherapy

(C5) $\mathrm{CO}_{2}$ laser therapy

D. Interventions that destroy the lesion with ionizing radiation

(D1) External beam radiation with photons (X or gamma rays), electrons (beta rays), or positively charged particles (e.g., protons, helium nuclei/alpha rays), at orthovoltage or megavoltage energies, or using in-office radiation machines

(D2) Brachytherapy with superficial application or interstitial application (pleisiotherapy) of radiation sources (usually emitting beta or alpha rays)

E. Photodynamic interventions

(E1) 5-aminolevulinic acid (ALA) + blue light

(E2) Methyl aminolevulinate (MAL) + red light

(E3) Other forms of PDT

F. Medical interventions

(F1) 5-fluorouracil (5-FU)

(F2) Imiquimod

(F3) Interferon (IFN alpha-2a/2b or INF beta)

(F4) Ingenol mebutate

(F5) Other medical interventions, including BEC-5 cream, Bleomycin, Methotrexate, Diclofenac, and Hedgehog inhibitors (Vismodegib, Sonidegib)

G. Shave excision

H. Curettage without diathermy

I. Placebo 


\begin{tabular}{|l|}
\hline \multicolumn{2}{|l|}{ PICOTS and Description } \\
\hline J. No treatment \\
\hline Outcomes \\
\hline Recurrence \\
\hline Histological clearance \\
\hline Clinical clearance \\
\hline Cosmetic outcomes \\
\hline Quality of life \\
\hline Mental health \\
\hline Patient satisfaction with treatment \\
\hline Mortality \\
\hline Adverse events \\
\hline Timing: any \\
\hline Setting: any \\
\hline
\end{tabular}

\section{Design}

We evaluated all randomized controlled trials (RCTs) and all comparative nonrandomized controlled studies (NRCSs) that took steps to control for patient- or lesion-level confounders such as medical history, age, education, lesion type, size, location and stage. NRCSs that reported only crude results were identified and tabulated but were excluded from the report. Those results are in Appendix G.

\section{Evidence Identification, Data Extraction, and Assessment of Methodological Risk of Bias of Individual Studies}

We conducted literature searches of studies in PubMed, the Cochrane Central Trials Registry, the Cochrane Database of Systematic Reviews, and EMBASE up to March 8, 2017 to identify primary research studies meeting our criteria. All citations found through literature searches and other sources were independently screened by two researchers.

Each study was extracted by one member of the review team and reviewed and confirmed by at least one other experienced methodologist. Disagreements were resolved by discussion among the team. Data was extracted into a customized form in Systematic Review Data Repository (SRDR) online system (http://srdr.ahrq.gov).

We assessed elements of the design of each study based on predefined criteria. For RCTs, we used the Cochrane risk of bias tool, ${ }^{12}$ which asks about risk of selection bias, performance bias, detection bias, attrition bias, reporting bias, and other potential biases. For observational studies, we used relevant questions from the Newcastle Ottawa Scale. ${ }^{13}$ We obtained a minimum bound for the number of unpublished studies through a clinicaltrials.gov search.

\section{Data Synthesis and Grading the Strength of Evidence (SOE)}

All included studies were summarized in narrative form and in summary tables that include the important features of the study populations. Lesions were divided by subtype (superficial, nodular, or high-risk BCC, SCC, or mixed populations) for analysis to ensure that the treatments would be most comparable. Where possible, lesions were also evaluated by size and location. Trial arms with fewer than five lesions were not included in the analysis, because they contribute 
minimal information, and in some instances, necessitated adding model parameters that were difficult to estimate.

We conducted pairwise and network meta-analyses with mixed effects (random intercepts and fixed intervention slopes) or full-random effects (random intercepts and random slopes) multilevel models within the generalized linear and latent mixed models. To aid the interpretation of these analyses we also present model-based estimates for the mean frequency of an outcome in the examined interventions, as well as forecasts of the frequency of the outcome in a new setting (e.g., a new study, or in a new population) that is similar to the studies in the meta-analysis.

For each major conclusion, we graded the strength of the body of evidence as per the AHRQ Methods Guide on assessing the strength of evidence. ${ }^{11}$ We judged the applicability within and across studies with reference to demographics of enrolled participants, the location and severity of the lesions, and the availability of treatments.

\section{Peer Review}

A draft version of this report was reviewed by invited and public reviewers. Revisions of the draft were made, where appropriate, based on their comments. The draft and final reports have also been reviewed by the Task Order Officer and an Associate Editor from another EPC. However, the findings and conclusions are those of the authors, who are responsible for the contents of the report.

\section{Results}

The literature searches yielded 15813 citations (Figure A), of which 15278 were excluded in abstract screening. A search of the reference lists of relevant systematic reviews yielded another 85 studies, which brought the total number screened in full text to 534. The 109 included studies (described in 125 papers) report 58 RCTs and 51 NRCSs. 
Figure A. Literature flow diagram

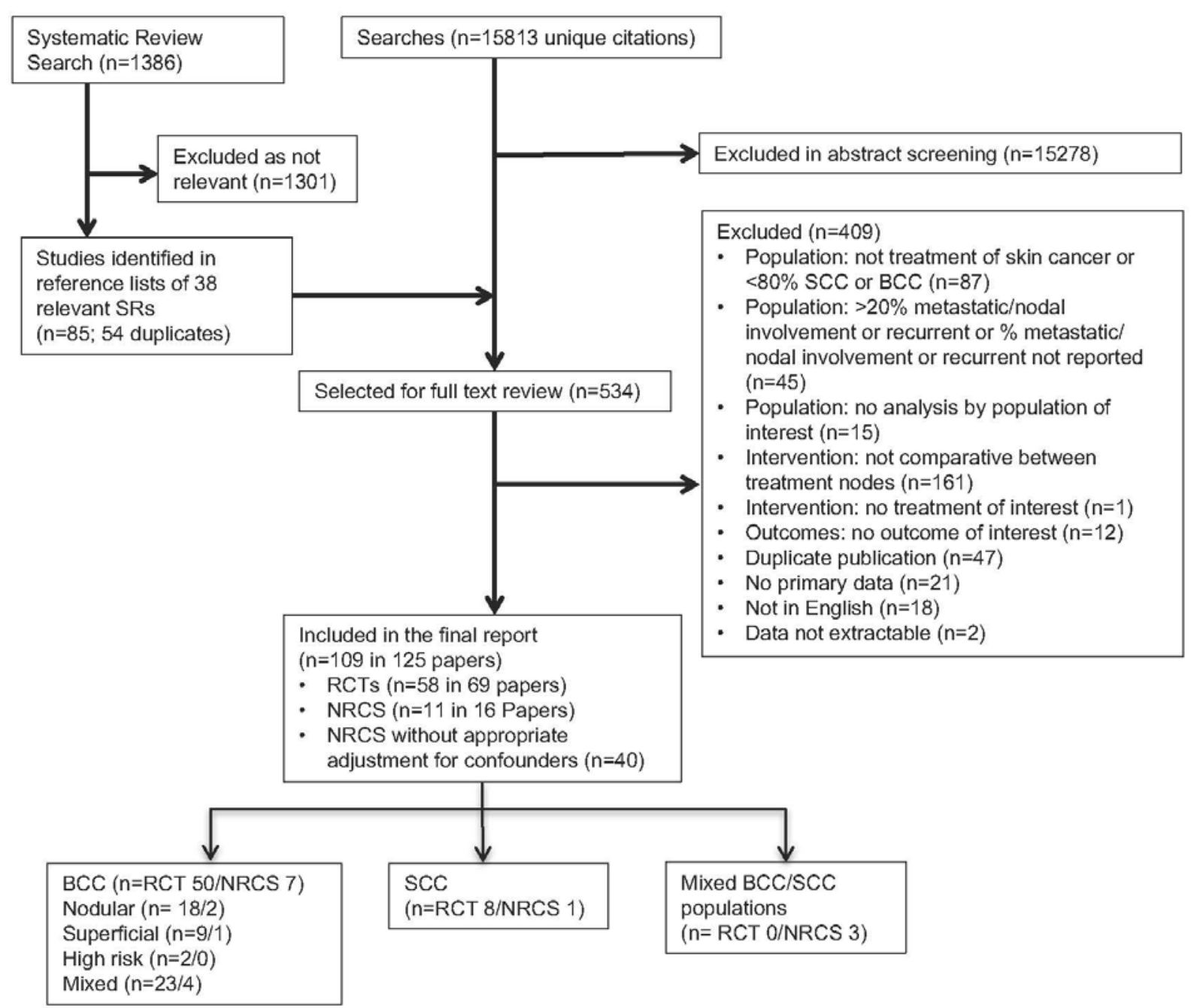

$\mathrm{SR}$ = systematic review; SCC = squamous cell carcinoma; BCC = basal cell carcinoma; RCT = randomized controlled trial; NRCS = nonrandomized comparative study

The studies primarily reported on BCC, with a minority reporting results for SCC. Nearly all reported results for recurrence or cure rate outcomes and adverse events, and many reported results for cosmetic outcomes. Few studies reported results using validated instruments for quality of life, mental health, or patient satisfaction with treatment. Because there was insufficient evidence for these outcomes, these results are presented in the full report only, as are results for specific types of BCC and other subgroups.

Details on how to read the graphs and tables are provided in the methods section of the full report. Analyses by specific intervention and results of studies that could not be included in the meta-analysis are given in the results section of the full report.

\section{Basal Cell Carcinoma}

The evidence graph in Figure B suggests that limited conclusions can be drawn about which individual intervention is best (with respect to each outcome) for two reasons: (1) some 
interventions have never been compared with other interventions, directly or indirectly, and (2) There are few studies for any given comparison.

The evidence is even more sparse when one considers the information that is actually available for specific outcomes. Figure $\mathrm{C}$ shows the evidence graphs for the outcomes for which we have the most data, namely recurrence, lack of histologic clearance, and lack of clinical clearance. Results are given in Table B.

The RCTs included patients and lesions that are typically encountered in clinical practice, but the lack of information on treatment effect heterogeneity with respect to patient-level factors limits extrapolation to individual patients. No RCT focused on patients who were immunocompromised or had substantially limited life expectancy.

\section{Figure B. Evidence graph depicting compared treatments in RCTs of BCC lesions}

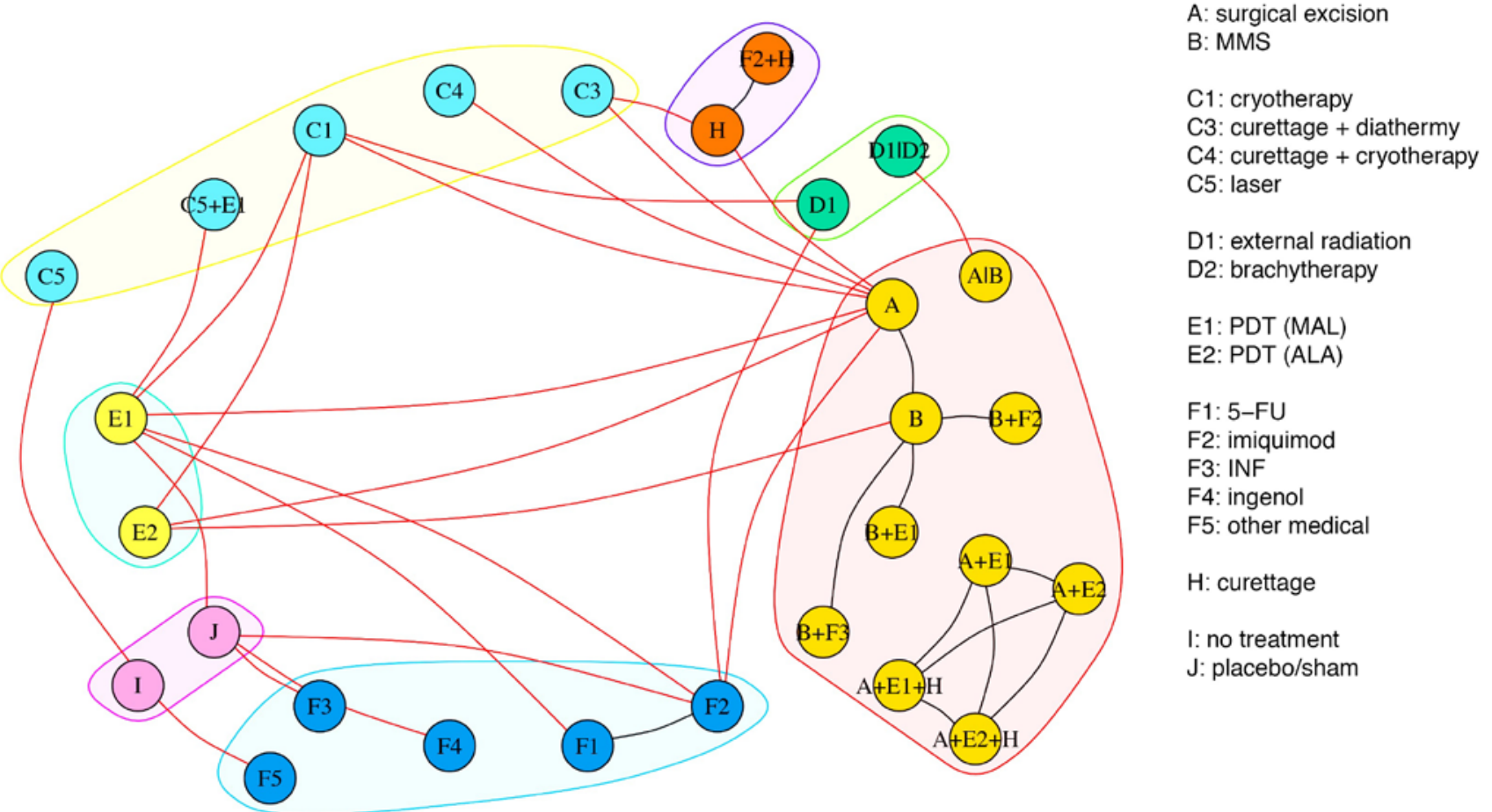

MMS = Mohs micrographic surgery; PDT = photodynamic therapy; ALA = 5-aminolevulinic acid; $\mathrm{MAL}=$ methyl aminolevulinate; FU = fluorouracil, INF = interferon 
Figure C. Evidence graphs for recurrence, histologic clearance, and clinical clearance from RCTs of BCC lesions

(A) Recurrence

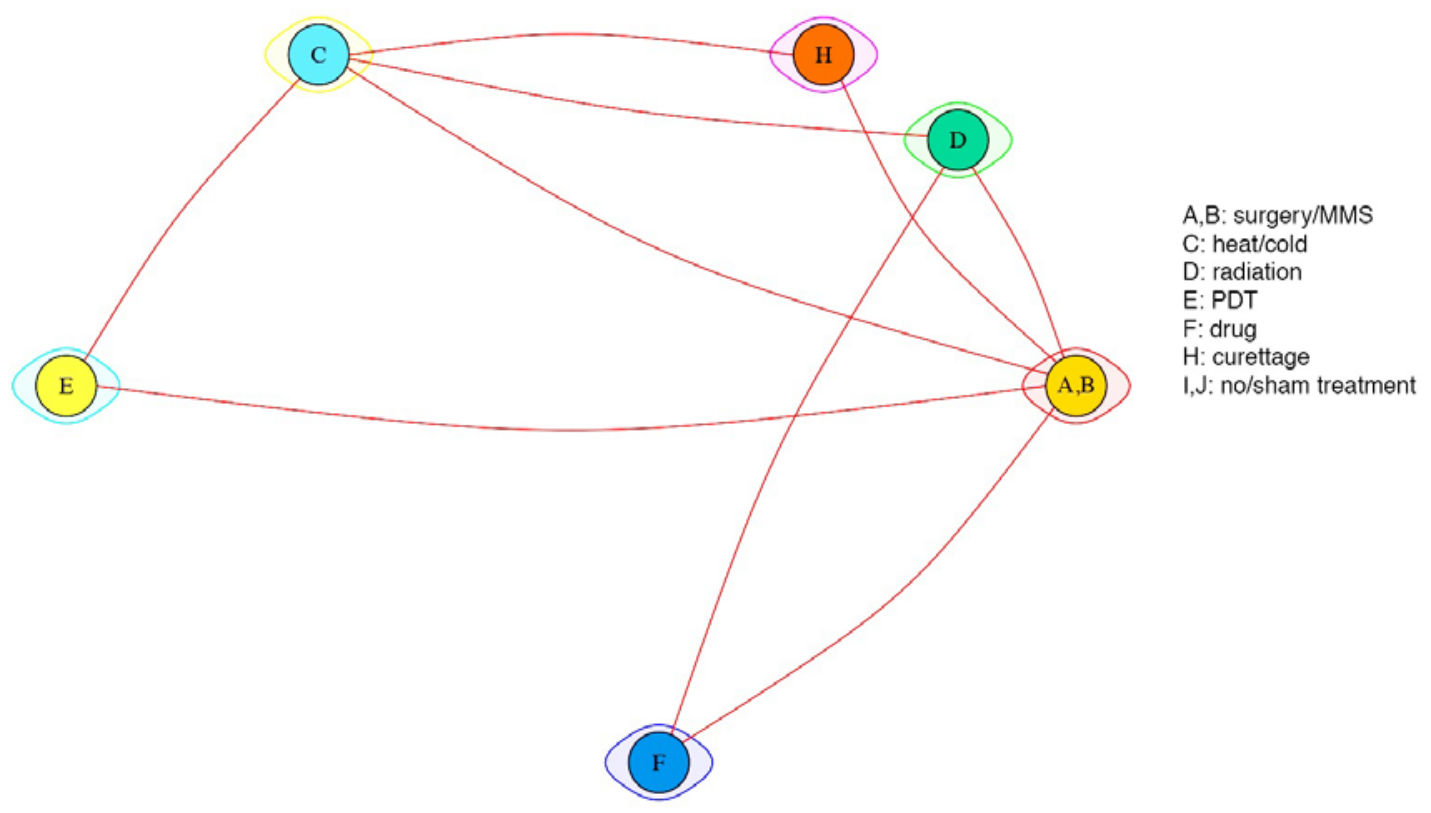

(B) Lack of histologic clearance

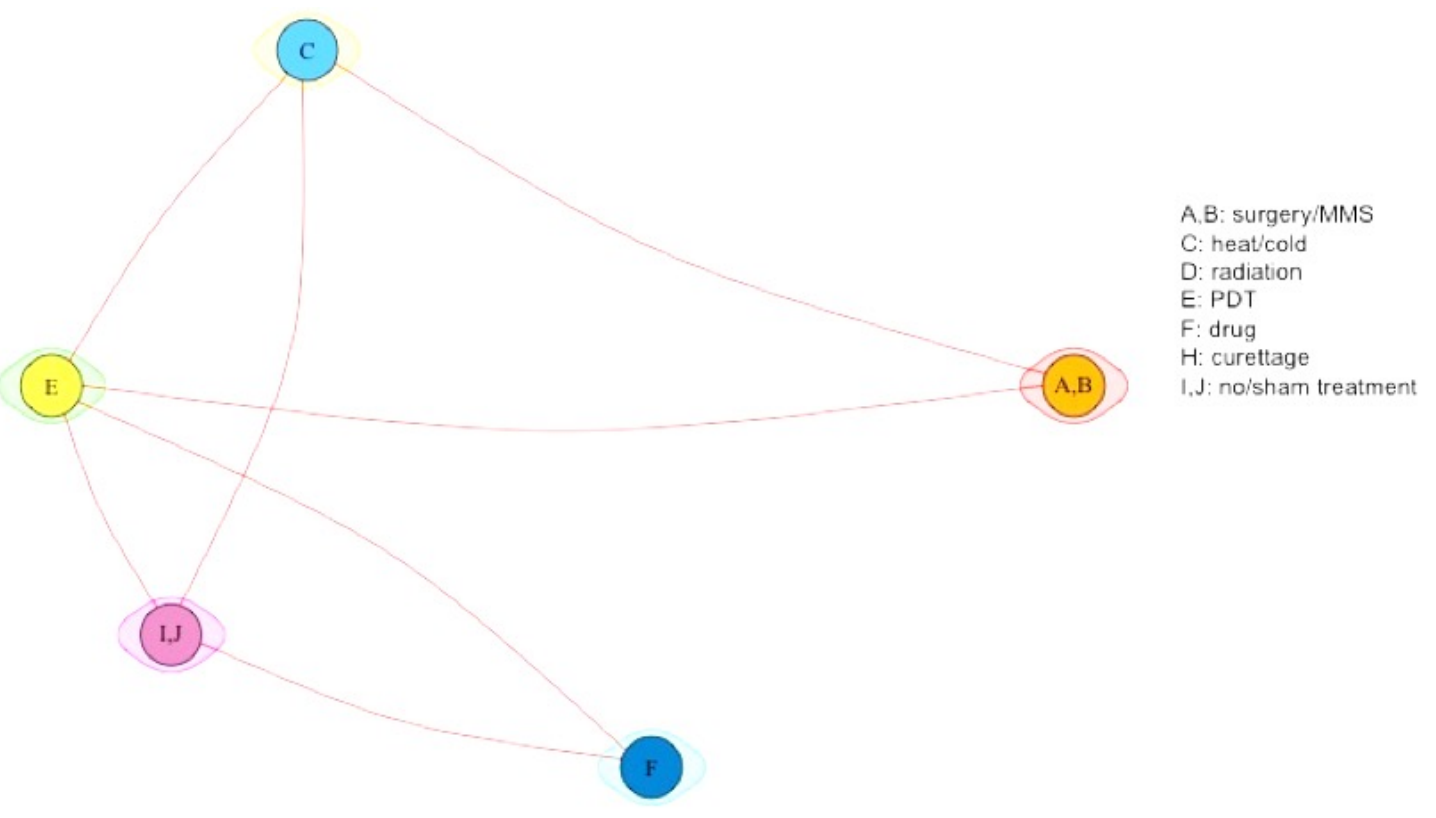




\section{(C) Lack of clinical clearance}

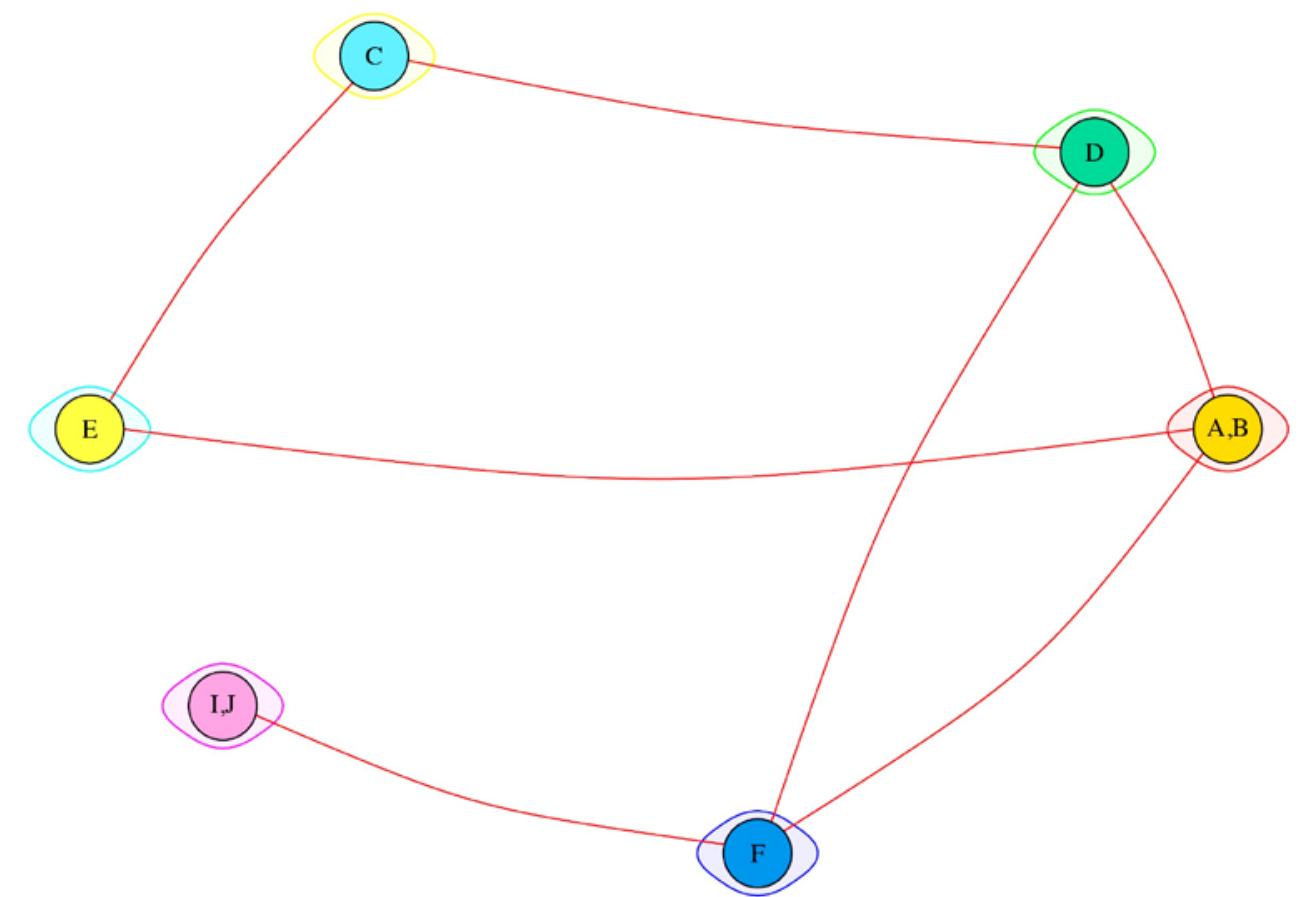

A,B: surgery/MMS

C: heat/cold

D: radiation

E: PDT

$\mathrm{F}$ : drug

$\mathrm{H}$ : curettage

$\mathrm{I}, \mathrm{J}:$ no/sham treatment

MMS = Mohs micrographic surgery; PDT = photodynamic therapy 
Table B. Mean frequency (percent) of outcomes per intervention category based on direct and indirect data (all BCCs)

\begin{tabular}{|c|c|c|c|c|c|c|c|c|c|}
\hline $\begin{array}{l}\text { Interve } \\
\text { ntion } \\
\text { Type }\end{array}$ & $\begin{array}{l}\text { Recurre } \\
\text { nce } \\
(95 \% \mathrm{Cl})\end{array}$ & $\begin{array}{l}\text { Lack of } \\
\text { Histologic } \\
\text { Clearance } \\
(95 \% \mathrm{Cl})\end{array}$ & $\begin{array}{l}\text { Lack of } \\
\text { Clinical } \\
\text { Clearance } \\
(95 \% \mathrm{Cl})\end{array}$ & $\begin{array}{l}\text { Cosmetic } \\
\text { Outcomes: } \\
\text { Patient } \\
\text { Reported } \\
(95 \% \mathrm{Cl})\end{array}$ & $\begin{array}{l}\text { Cosmetic } \\
\text { Outcomes: } \\
\text { Observer } \\
\text { Reported } \\
(95 \% \mathrm{Cl})\end{array}$ & $\begin{array}{l}\text { AEs } \\
\text { Leading } \\
\text { to } \\
\text { Disconti } \\
\text { nuation } \\
(95 \% \mathrm{Cl})\end{array}$ & $\begin{array}{l}\text { Serious } \\
\text { AEs } \\
(95 \% \\
\mathrm{Cl})\end{array}$ & $\begin{array}{l}\text { AEs: } \\
\text { Pain } \\
\text { (95\% } \\
\text { Cl) }\end{array}$ & $\begin{array}{l}\text { AEs: } \\
\text { Infectio } \\
\text { n (95\% } \\
\text { Cl) }\end{array}$ \\
\hline $\begin{array}{l}\text { Surgery/ } \\
\text { MMS } \\
(A, B)\end{array}$ & $\begin{array}{l}3.4(1.5 \\
7.6)\end{array}$ & $\begin{array}{l}1.2(0.1 \\
15.9)\end{array}$ & $\begin{array}{l}3.0(0.8 \\
10.7)\end{array}$ & $\begin{array}{l}88.8(73.7 \\
95.7)\end{array}$ & $\begin{array}{l}55.0(34.7 \\
73.8)\end{array}$ & $\begin{array}{l}\text { Not } \\
\text { defined* }\end{array}$ & $\begin{array}{l}0.6(0.2 \\
2.4)\end{array}$ & $\begin{array}{l}21.5 \\
(8.1, \\
46.2)\end{array}$ & $\begin{array}{l}5.5(2.8, \\
10.7)\end{array}$ \\
\hline $\begin{array}{l}\text { Heat/col } \\
d(C)\end{array}$ & $\begin{array}{l}21.2 \\
(14.0 \\
30.7)\end{array}$ & $\begin{array}{l}24.9(8.2 \\
55.0)\end{array}$ & $\begin{array}{l}11.9(4.2 \\
29.1)\end{array}$ & $\begin{array}{l}60.5(32.4 \\
83.0)\end{array}$ & $\begin{array}{l}74.3(51.5, \\
88.8)\end{array}$ & $\begin{array}{l}0.9(0.0 \\
20.1)\end{array}$ & $\begin{array}{l}2.6(0.2 \\
31.0)\end{array}$ & $\begin{array}{l}12.9 \\
(0.8, \\
73.1)\end{array}$ & NA \\
\hline $\begin{array}{l}\text { Radiatio } \\
\mathrm{n}(\mathrm{D})\end{array}$ & $\begin{array}{l}4.4(1.7, \\
10.5) \\
\end{array}$ & & $\begin{array}{l}4.7(0.8, \\
23.4) \\
\end{array}$ & $\begin{array}{l}79.1 \text { (55.2, } \\
92.1)\end{array}$ & $\begin{array}{l}25.5(7.1, \\
60.7) \\
\end{array}$ & & & & \\
\hline PDT (E) & $\begin{array}{l}21.1 \\
(14.0 \\
31.3)\end{array}$ & $\begin{array}{l}19.5(6.4 \\
46.4)\end{array}$ & $\begin{array}{l}14.7(6.1 \\
31.3)\end{array}$ & $\begin{array}{l}97.9(93.1 \\
99.4)\end{array}$ & $\begin{array}{l}88.7(78.9, \\
94.2)\end{array}$ & $\begin{array}{l}\text { Not } \\
\text { defined* }\end{array}$ & $\begin{array}{l}0.7(0.2 \\
2.7)\end{array}$ & $\begin{array}{l}20.7 \\
(8.2, \\
43.3)\end{array}$ & $\begin{array}{l}0.5(0.1, \\
2.4)\end{array}$ \\
\hline $\begin{array}{l}\text { Drugs } \\
\text { (F) }\end{array}$ & $\begin{array}{l}3.1(0.2 \\
39.0)\end{array}$ & $\begin{array}{l}35.6(16.5 \\
60.8)\end{array}$ & $\begin{array}{l}16.6(5.3 \\
41.6)\end{array}$ & $\begin{array}{l}94.2(37.5 \\
99.8)\end{array}$ & $\begin{array}{l}76.3(52.8 \\
90.2)\end{array}$ & $\begin{array}{l}4.9(2.0 \\
11.6)\end{array}$ & $\begin{array}{l}3.6(2.0 \\
6.5)\end{array}$ & $\begin{array}{l}9.9 \\
(4.4 \\
20.9)\end{array}$ & $\begin{array}{l}0.5(0.1, \\
3.7)\end{array}$ \\
\hline $\begin{array}{l}\text { Curetta } \\
\text { ge }(\mathrm{H})\end{array}$ & $\begin{array}{l}20.0(5.4, \\
51.9)\end{array}$ & & & & & & & & \\
\hline $\begin{array}{l}\text { No/sha } \\
\mathrm{m} \\
\text { treatme } \\
\mathrm{nt}(\mathrm{I}, \mathrm{J})\end{array}$ & & $\begin{array}{l}83.5(65.5 \\
93.1)\end{array}$ & $\begin{array}{l}84.2(50.6, \\
96.5)\end{array}$ & & $\begin{array}{l}89.8(40.1, \\
99.1)\end{array}$ & $\begin{array}{l}1.0(0.2 \\
4.4)\end{array}$ & $\begin{array}{l}2.4(0.3 \\
15.2)\end{array}$ & $\begin{array}{l}2.9 \\
(0.9 \\
9.4)\end{array}$ & NA \\
\hline
\end{tabular}

Note: Black cells indicate interventions that have no data for that outcome.

$\mathrm{AE}=$ adverse event; $\mathrm{MMS}=$ Mohs micrographic surgery; $\mathrm{PDT}=$ photodynamic therapy; $\mathrm{BCC}=$ basal cell carcinoma; $\mathrm{NA}=$ not applicable; $\mathrm{CI}=$ confidence interval

* Surgical interventions and PDT are one-time therapies that cannot be “discontinued”. For parsimony of exposition, however, in the descriptive analyses in the Table we assigned 0 discontinuation to these interventions.

\section{Recurrence}

In total, 13 RCTs (1664 lesions) were included in this analysis, and cumulative sample sizes per comparison ranged from 27 to 355.

For parsimony of exposition, we only list predicted mean frequencies of events with each intervention category across the included RCTs, based on their estimated relative effects in network meta-analysis (Table B). (For more results, including by specific intervention and for subgroups, refer to the full report.)

\section{Lack of Histological Clearance}

In total, 15 RCTs (1940 lesions) were included in this analysis, and cumulative sample sizes per comparison ranged from 44 to 1196 . Table B shows the mean fraction of lesions without histologic clearance across the included RCTs. (For more results, refer to the full report.)

\section{Lack of Clinical Clearance}

In total, 14 RCTs (1734 lesions) were included in this analysis, and cumulative sample sizes per comparison ranged from 27 to 420 . For each intervention category, Table B shows the mean fraction of lesions without clinical clearance across the included RCTs. (For more results, refer to the full report.) In general, the mean fractions for lack of histologic clearance for individual 
interventions are in congruence with the corresponding fractions estimated for intervention categories.

\section{Patient-Reported Cosmetic Outcomes, All BCC Lesions}

In total, seven RCTs (752 lesions) were included in this analysis. In Table B drugs and photodynamic therapy (PDT) are associated with highest percentages of good cosmetic outcomes, followed by surgical treatments, radiation, and interventions that use heat or cold to destroy the lesion. (For detailed results, refer to the full report.)

\section{Observer-Reported Cosmetic Outcomes, All BCC Lesions}

In total, 10 RCTs (1460 lesions) were included in this analysis. Table B shows that the percentage of lesions with good or better cosmetic outcomes ranged between 74.3 and 89.8 percent for interventions that destroy the lesion with heat or cold (C), drugs (F), PDT (E) and no or sham treatment $(\mathrm{I}, \mathrm{J})$, and was 55.0 percent for surgical treatments (A,B). Radiation (D) had the smallest percentage of good or better cosmetic outcome. However, the confidence intervals for these proportions are wide, so we could not draw any strong conclusions.

\section{Adverse Events, All BCC Lesions}

In Table B drugs were most likely to have adverse events leading to discontinuation (4.9\%; 95\% CI, 2.0 to 20.1); other interventions types had a much smaller percentage (1.2\%). The number of adverse events characterized as "serious" by the investigators was smaller than 3.6 percent for all intervention categories. Pain after treatment was most commonly encountered for surgical interventions (21.5\%) and for PDT (20.7\%). Infections at the treatment site were described in 5.5 percent of lesions with surgical treatments (95\% CI 28 to 10.7) and were reported in less than 1 percent for PDT and drugs. No information on infections was available for treatments that destroy lesions with heat or cold or for no (or sham) treatment.

\section{Squamous Cell Carcinoma}

The evidence graphs in Figures D and E depict eight comparisons between 10 interventions organized in four intervention categories, none of which are in the surgical or radiation category. Most RCTs included only participants with SCC in situ (SCCIS); one included participants with microinvasive SCC. It is not included in this analysis, but is summarized in the full report. Information on each comparison is provided by at most three RCTs, and for most comparisons, by a single RCT.

Figure E shows the corresponding evidence graphs for the outcomes for which we have the most data, namely recurrence and lack of clinical clearance. Evidence on other outcomes (quality of life, cosmetic outcomes, costs or resource use) is even sparser and is given in the full report. Results are given in Table C. 
Figure D. Evidence graph depicting compared interventions in RCTs of SCC lesions

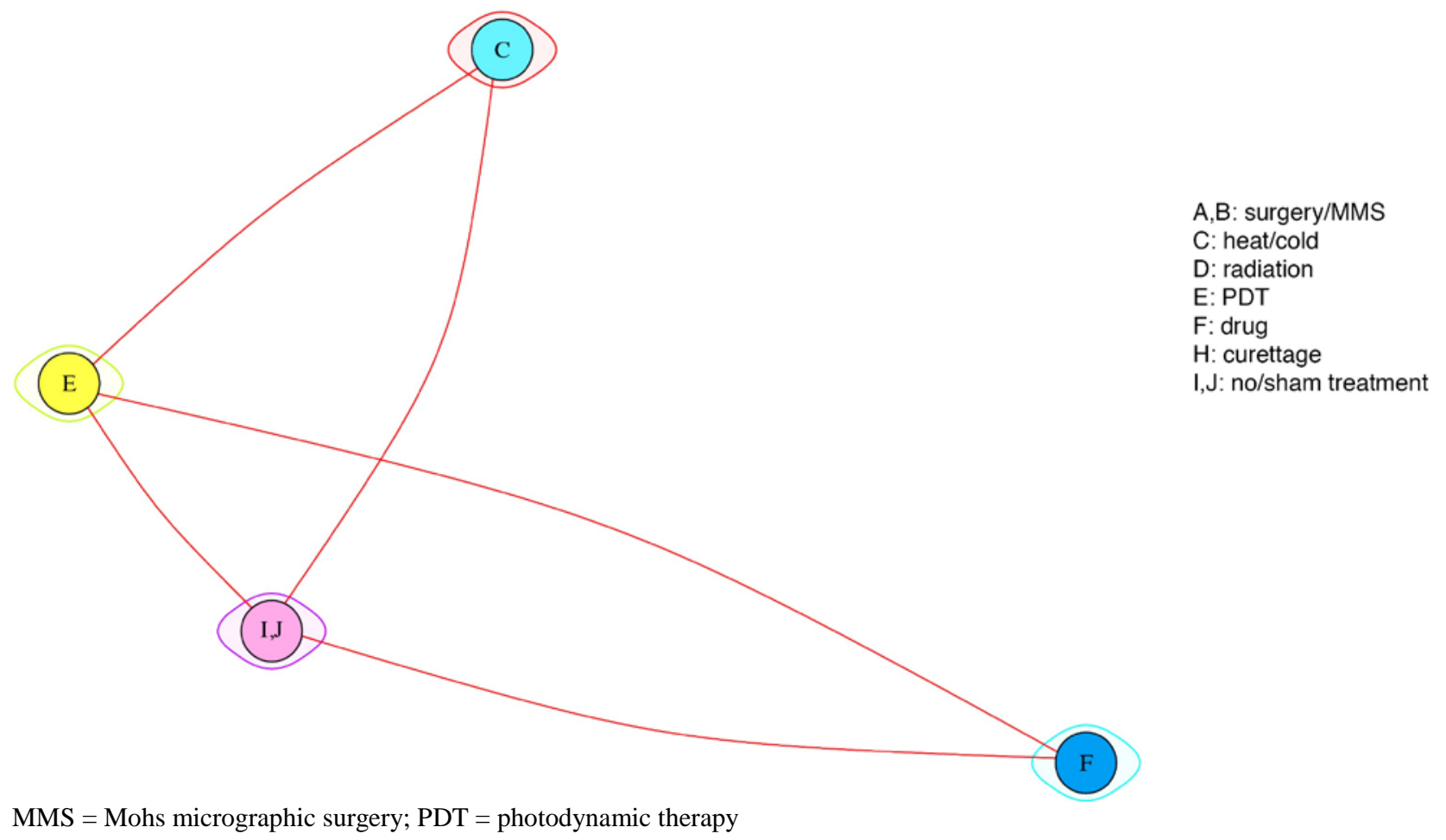


Figure E. Evidence graphs for recurrence, histologic clearance, and clinical clearance for RCTs of SCC lesions

\section{(A) Recurrence}

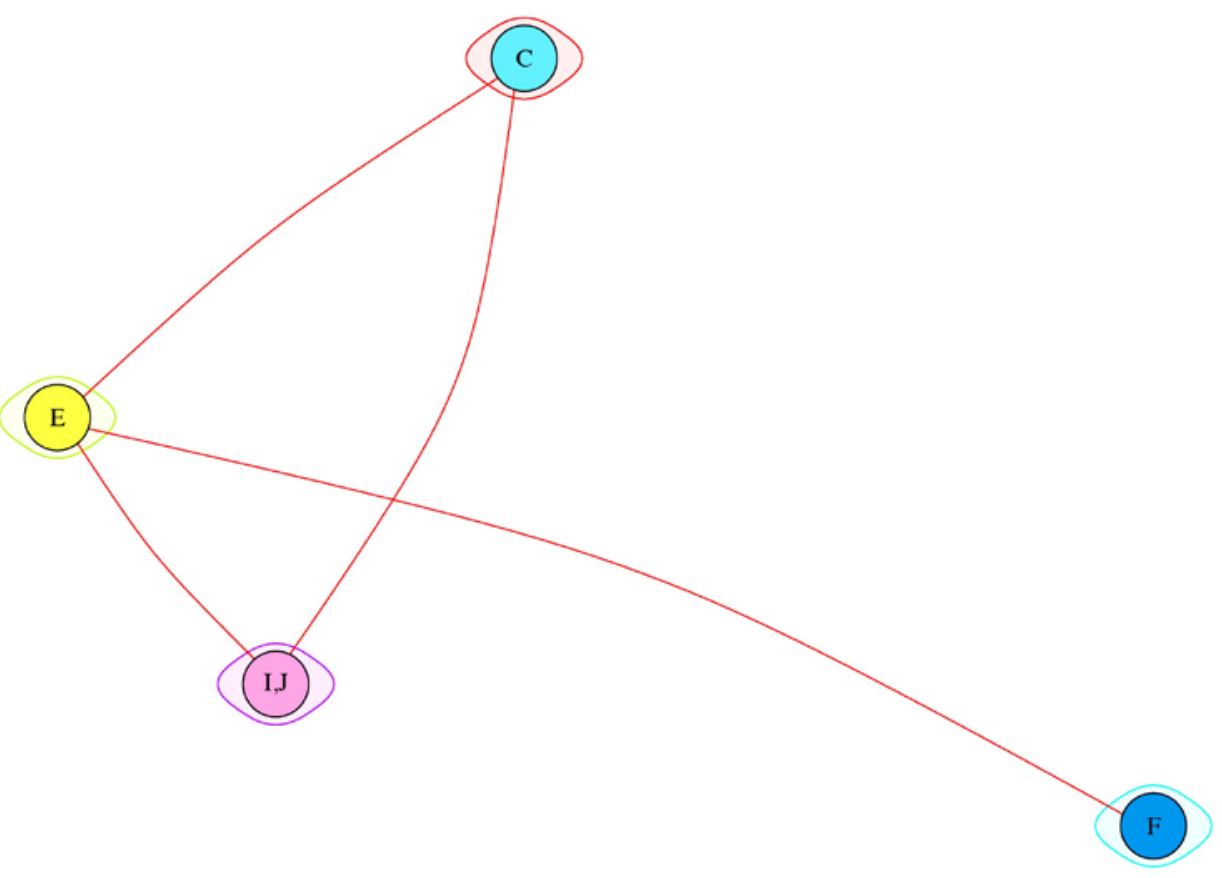

A,B: surgery/MMS
C: heat/cold
D: radiation
E: PDT
F: drug
H: curettage
I,J: no/sham treatment

(B) Lack of clinical clearance

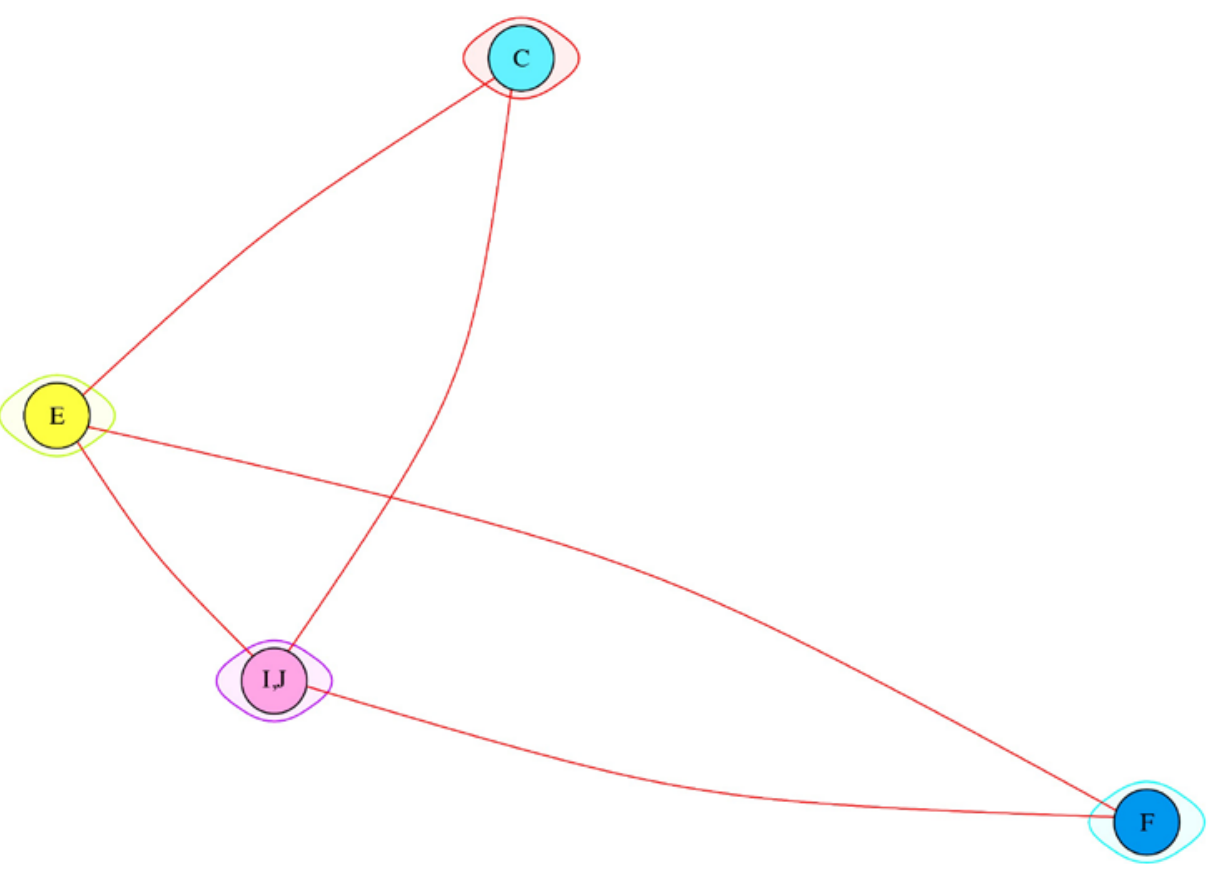

A,B: surgery/MMS

C: heat/cold

D: radiation

E: PDT

F: drug

$\mathrm{H}$ : curettage

$\mathrm{I}, \mathrm{J}$ : no/sham treatment

MMS = Mohs micrographic surgery; PDT = photodynamic therapy 
Table C. Mean frequency of outcomes per intervention category based on direct and indirect data (SCCIS)

\begin{tabular}{|c|c|c|c|c|c|c|}
\hline $\begin{array}{l}\text { Treatment } \\
\text { type }\end{array}$ & $\begin{array}{l}\text { Recurrence } \\
\text { Rates (95\% } \\
\text { Cl) }\end{array}$ & $\begin{array}{l}\text { Lack of } \\
\text { Clinical } \\
\text { Clearance } \\
(95 \% \mathrm{Cl})\end{array}$ & $\begin{array}{l}\text { Adverse Events } \\
\text { Leading to } \\
\text { Discontinuation } \\
(95 \% \mathrm{Cl})\end{array}$ & $\begin{array}{l}\text { Serious } \\
\text { Adverse } \\
\text { Events } \\
(95 \% \mathrm{Cl})\end{array}$ & $\begin{array}{l}\text { Adverse } \\
\text { Events: Pain } \\
\text { After } \\
\text { Treatment } \\
(95 \% \mathrm{Cl})\end{array}$ & $\begin{array}{l}\text { Adverse } \\
\text { Events: } \\
\text { Infection } \\
(95 \% \mathrm{Cl})\end{array}$ \\
\hline $\begin{array}{l}\text { Heat/ } \\
\text { cold }(\mathrm{C})\end{array}$ & $15.1(8.1,26.5)$ & $10.8(3.1,31.3)$ & $1.9(0.6,6.4)$ & $0.9(0.1,6.1)$ & $34.1(20.0,51.6)$ & $0(0,31)$ \\
\hline PDT (E) & $17.7(10.8,27.8)$ & $14.9(5.4,34.9)$ & Not defined* & $0.5(0.0,7.7)$ & $23.4(12.4,39.5)$ & $0(0,31)$ \\
\hline Drugs (F) & $51.5(28.9,73.5)$ & $29.2(8.4,65.1)$ & $13.3(3.4,40.5)$ & NA & NA & NA \\
\hline $\begin{array}{l}\mathrm{No} / \\
\text { sham } \\
\text { treatment } \\
(\mathrm{I}, \mathrm{J})\end{array}$ & $50.0(11.2,88.8)$ & $88.0(54.2,97.8)$ & $4.7(0.9,20.1)$ & $0(0,32.2)$ & $28.4(9.7,59.3)$ & NA \\
\hline
\end{tabular}
interval.

*PDT is a one time interventions that cannot be "discontinued"; for parsimony of exposition, however, in the descriptive analyses in the Table we assigned 0 discontinuation events to PDT.

\section{Recurrence}

In Table C interventions that destroy the lesion with heat or cold (C) and PDT (E) had on average lower recurrence rates (15.1 and 17.7 percent, respectively) compared to drugs or no/sham treatment. Of note, the average recurrence rate with drugs is 51.5 percent (95\% CI 28.9 to 73.5), reflecting the high recurrence rates observed in the single RCT comparing 5-FU with PDT (ALA).

\section{Lack of Histological Clearance}

Data were very sparse (2 RCTs, 50 lesions), and results are not summarized here. Refer to the full report.

\section{Lack of Clinical Clearance}

In Table $C$ the fraction of lesions without clinical clearance was between 10.8 and 29.2 percent in the active treatments and 88 percent with placebo, which is similar to the results by individual comparisons. However, the confidence intervals for each estimate are wide.

\section{Patient-Reported Cosmetic Outcomes, All SCC Lesions}

We did not identify any studies with results for this outcome in this population.

\section{Observer-Reported Cosmetic Outcomes, All SCC Lesions}

Data were very sparse (2 RCTs, 204 lesions), and results are not summarized here. Refer to the full report.

\section{Adverse Events, All SCCIS Lesions}

In Table $\mathrm{C}$ the highest mean frequency of adverse events leading to treatment discontinuation (3 RCTs; 292 participants) was 13.3 percent (95\% CI, 3.4 to 40.5) for drugs (F); it was less than 1.2 percent for other intervention categories. The frequency of adverse events characterized as "serious" by the investigators (1 RCT; 225 participants) was smaller than 1 percent for all intervention categories. In the two RCTs that reported pain after treatment, between 23.4 and 
34.1 percent reported pain regardless of treatment (including sham treatments). The outcome of infection at the treatment site was reported in a single RCT (36 participants) at 0 percent.

\section{Discussion}

Within the existing evidence, with respect to BCC recurrence, surgical treatments and radiation therapy appear to be (statistically significantly) better than interventions that destroy lesions with heat or cold, PDT, or curettage. However, PDT was associated with improved cosmetic outcomes. With regards to drugs for the treatment of BCC, recurrence rates with imiquimod were not significantly different than with surgical excision in a single large RCT. Given that lack of recurrence is, essentially, cure from disease, these results support the effectiveness of surgical and radiation treatment for low-risk BCC. Full details in Tables D and E.

We acknowledge that the clinical applicability of some of these results is limited. The comparisons between intervention categories are not as informative as comparisons between individual interventions. We have provided analyses at the individual intervention level, but opt not to draw conclusions based on them, because most are based on indirect data and small numbers. In addition, the analyses cannot adequately account for heterogeneity of the populations in included studies, particularly for low-risk BCCs, because, although the RCTs had comparable populations (see Tables 3-6 of the full report), many did not stratify their results by histologic subtype (superficial or nodular) or location. Thus, we were unable to incorporate these important factors into the analyses. For example, radiation (because of its expense and poor cosmetic outcomes) is rarely used in routine clinical practice to treat low-risk BCC; its use is generally limited to patients with high risk or recurrent disease or for patients with contraindications to surgery. However, the four RCTs that included radiation arms did not differ significantly in population from the other studies included in the low-risk BCC network, with the exception that they included a larger percentage of lesions in high-risk (face, eyelids) areas.

Conversely, use of topical drugs is generally limited to primary, superficial tumors. Therefore, comparisons of the efficacy of radiation and drugs for the low-risk BCCs included in our study may not be relevant in the clinical decision making for most patients and clinicians. That said, the analysis contains an RCT that looks at the direct comparison of radiation and imiquimod in a high-risk location (eyelids), so it might be that they are more relevant for low-risk lesions in high-risk locations. $^{14}$

For SCCIS, the use of cryotherapy and PDT is supported over topical 5-fluorouracil with regards to recurrence. However, how these treatments perform for SCCIS compared with surgical treatments, which are commonly used in clinical practice, is not ascertainable based on the currently available evidence.

For patients and clinicians, though, cure is not the only important endpoint. All of the treatments under study are associated with benefits and drawbacks that patients and clinicians consider routinely. For example, while external beam radiation therapy is effective, its remote sequelae, such as skin atrophy and the development of secondary tumors, make it less advisable for younger patients. For patients for whom cosmesis is a primary concern, treatment with PDT may be preferable despite its higher recurrence rates. Despite sparse evidence on their ability to cure BCC and SCCIS, some patients may prefer the convenience provided by topical medical treatments such as 5-fluorouracil and imiquimod, which can be applied by the patient at home; this contrasts with the multiple visits to hospitals or specialty clinics required for radiation therapy which are not be practical for some patients. Access to treatments will also impact 
clinical decisionmaking. Specialty care is not available in all communities; while primary care physicians can perform basic surgical procedures and prescribe topical medications, they do not have access to specialized treatments, such as Mohs micrographic surgery (MMS), radiotherapy, and PDT.

Perhaps the most striking observation is the dearth of information that is available comparing interventions for these very common cancers. For example, only 13 RCTs (1664 lesions) examining BCC recurrence were included, of which 20 lesions were treated with curettage. Further, the amount of evidence in the 10 comparisons with head to head data was limited: the number of RCTs per comparison ranged between 1 and 3 , and the cumulative number of lesions ranged between 27 and 347. The small sample sizes of these RCTs adds to concerns about the generalizability of our results to the treatment of all cutaneous BCC and SCC.

For SCC, data on recurrence are even sparser. For SCCIS, only 4 RCTs (348 lesions) compared 4 types of interventions, namely a drug (imiquimod), interventions that destroy lesions with heat or cold, PDT, and sham treatments. Surgical interventions and curettage, therapies commonly used for SCCIS in clinical practice, were not examined.

Only one RCT evaluated treatments for invasive SCC, the subgroup of SCC that are most likely to recur or metastasize, and thus most important to evaluate. ${ }^{15}$ In clinical practice, these lesions are routinely treated with surgical excision with or without intraoperative margin evaluation, and in most cases are considered appropriate for Mohs surgery in the American Academy of Dermatology appropriate use criteria. ${ }^{16}$ Radiation is also used for invasive SCC. The lack of evidence comparing efficacy among these commonly used treatments is striking.

Adjuvant radiotherapy and new drugs (including epidermal growth factor receptor inhibitors, such as cetuximab and erlotinib) that may be used as adjuvant treatment in the case of positive margins postexcision or in the case of advanced disease were not within the scope of this review but also have utility in treating BCC and SCC lesions.

With few exceptions and for most outcomes, individual studies were deemed to have at most moderate risk of confounding, selection, or measurement biases. The risk of bias of individual studies was not a major determinant for the conclusions in the tables. By far the major concern is that the evidence is sparse when one considers the richness of the clinical questions that can be posed, including questions that may have important health and cost implications for insurers and patients. For example, there are no studies on the effectiveness of external radiation therapy delivered with portable machines in the office setting versus radiation therapy delivered in specialized facilities or other interventions. Empirical data on this radiation therapy modality would be useful because there are only limited data on radiation therapy to extrapolate from.

Other large gaps remain in the knowledge base: There is no information on subgroups of patients who have limited life expectancy, are frail, or who are immunocompromised (e.g., have chronic lymphocytic leukemia and other malignancies, immunodeficiency disorders, or who receive immunomodulating or immunosuppressive treatments). There is limited or no information on high risk BCC lesions, and on invasive SCCs. There is limited data on patientand lesion-specific modifiers of intervention effects.

Finally, outcomes such as histological clearance and clinical clearance are surrogates for lesion recurrence. In particular, clinical clearance may help physicians choose among PDT, medical, and radiation-based therapies, but is not an informative outcome for surgical interventions: any surgical treatment, regardless of margin control, removes all clinically visible tumor. Therefore, our conclusion in Table D that surgical interventions are better than all other interventions with respect to clinical clearance, while very likely to be true, is almost 
meaningless. Adverse events were inconsistently reported. For analysis, they were grouped based on study author's definitions, which may have led to some misclassification.

\section{Evidence Gaps}

We have identified a number of important gaps in the medical literature on the topic of treating BCC and SCC. First, more trials are needed comparing commonly used treatment modalities such as simple excision, Mohs surgery, PDT and topical medical therapy. Further, in order to justify routine use of various forms of radiotherapy for these patients, more trials comparing radiotherapy with other modalities are needed in select populations for whom radiotherapy may be appropriate.

Second, all trials for BCC and SCC should, where possible, use recurrent disease as a primary or secondary outcome, as in our opinion it is the most clinically important outcome. Trials should also attempt to incorporate measures of health care resource utilization, which were lacking in our review of the existing evidence save for one RCT and one NRCS. ${ }^{17,18}$ Future trials would also benefit from standardization and consistent definition of all outcomes, particularly adverse events and patient-reported outcomes such as cosmesis. To this end, we encourage the development of a core outcome set as is being done for other skin diseases such as psoriasis (The International Dermatology Outcome Measures) ${ }^{19}$ and atopic dermatitis (Harmonizing Outcome Measures for Eczema). ${ }^{20}$

Third, while more evidence is needed overall, future research should also focus on specific subgroups that have minimal evidence to date. Aggressive histologic subtypes of BCC, including infiltrative and sclerosing patterns, account for very little of the evidence found in our review. No comparative evidence was found on keratinocyte carcinomas in high-risk groups such as organ transplant recipients and patients with other altered immune states. Patients with limited life-expectancy are another subgroup of interest.

Fourth, better monitoring of population trends in BCCs and SCCs can help focus research on the most consequential subtypes. Such monitoring can be performed by the Surveillance, Epidemiology, and End Results (SEER) Program (which currently ignores these cancers), the Centers for Disease Control and Prevention (CDC), or large health organizations. While the volume of these tumors makes surveillance logistically difficult and costly, advances in health information technology and big data analytic techniques should make it more feasible. ${ }^{21}$

Given how common these tumors are and their burden on the health care system, research funding directed to determine the most effective and cost-effective measures for these tumors is needed. It is incumbent on funding agencies and health care payers to fund research examining important questions in this field. Patients, clinicians, payers, and research funders would benefit from a decision analysis of the management of BCC and SCC lesions.

\section{Conclusions}

Based on sparse evidence, surgical, radiation and topical drug treatments have lower recurrence rates than other modalities for the treatment of low-risk BCC, and PDT appears to have superior cosmetic outcomes. Large gaps remain in the literature regarding the comparison of individual interventions, and very little or no information on immunocompromised patients, patients with limited life expectancy, and on patients with specific lesion categories, including high risk BCCs and invasive SCCs. In order for clinicians, patients and payers to make informed decisions regarding the treatment of these lesions, new RCT or high-quality NRCS evidence is needed. 
Table D. Summary conclusions for BCC lesions and strength of the relevant evidence

\begin{tabular}{|c|c|c|c|c|}
\hline Conclusion statement & $\begin{array}{l}\text { RoB } \\
\text { (evidence } \\
\text {-base) }\end{array}$ & Consistency & Precision & Directness \\
\hline
\end{tabular}

(1) Surgical interventions $(A, B)$ and radiation Moderate Possibly

(D) were associated with lower

recurrence rates than interventions that

destroy lesions with heat or cold (C), and

PDT (E)

(moderate to high strength of evidence)

(2) Curettage $(H)$ may have higher recurrence rates than surgical interventions $(A, B)$ or radiation $(D)$

(3) Imiquimod (F) was associated with recurrence rates that were not significantly different than that of surgical interventions $(A, B)$

(4) [Imprecise data on the comparison on curettage and interventions that destroy lesions with heat or cold (C) or PDT (E)]

Histologic clearance, all BCC

(1) Surgical interventions $(A, B)$ were associated with better histological clearance outcomes and were statistically significantly better than interventions that destroy lesions with heat or cold (C), PDT (E), drugs (F), and placebo $(\mathrm{I}, \mathrm{J})$.

(2) Interventions that destroy lesions with heat or cold (C), PDT (E), and drugs (F) have better histological outcomes than placebo $(\mathrm{I}, \mathrm{J})$

(3) [imprecise data on the relative comparisons of nonsurgical active interventions]

Clinical clearance, all BCC

(1) Surgical interventions $(A, B)$ were associated with better clinical clearance outcomes than PDT (E), drugs (F) and placebo $(\mathrm{I}, \mathrm{J})$

(2) All active treatments were associated with better clinical clearance outcomes than placebo

(3) [Imprecise data on relative comparisons between nonsurgical active treatments]

\section{Moderate Possibly}

Varies by Mix of direct

comparison from and indirect

consistent

(No robust

indications of

inconsistency)

precise to

data

imprecise.

and indi

High
(2) Low
(3) Low
(4) [Insufficient]

consistent

(No robust

indications of

inconsistency)

Varies by

Mix of direct

comparison from and indirect

precise to

data

ata

(1) High

2) Moderate to

high

(3) [Insufficient]

(No robust

indications of

inconsistency)
(1) High

(2) Moderate to

high

Varies by Mix of direct comparison from and indirect precise to data imprecise.

(3) [Insufficient]

Comments

- Surgery/MMS $(A, B)$ had significantly fewer recurrences than heat/cold, PDT, and curettage; not significantly fewer than radiation; and not significantly more than drugs (7 RCTs; 2 NRCSs)

- Heat/cold (C) interventions had significantly more recurrences than surgery and radiation; not significantly more than drugs and curettage, and not significantly fewer than PDT (7 RCTs)

- Radiation (D) had significantly fewer recurrences than thermal interventions and PDT, not significantly fewer than curettage, and not significantly more than surgery and drugs (3 RCTs)

- PDT (E) had significantly more recurrences than radiation and surgery, and not significantly more than heat/cold, drugs, and curettage (6 RCTs, 1 NRCS)

- Imiquimod (F) had more recurrences than surgery, but not significantly so (1 $\mathrm{RCT})$

- Curettage $(\mathrm{H})$ had significantly more recurrences than surgery, not significantly more recurrences than drugs and radiation, and not significantly fewer recurrences than PDT and heat/cold (2 RCTs)

- Surgery (A,B) performed significantly better than heat/cold, drugs, and placebo, and nonsignificantly better than PDT (2 RCTs)

- Thermal interventions (C) performed significantly better than placebo, nonsignificantly better than drugs, nonsignificantly worse than PDT, and significantly worse than surgery (2 RCTs)

- PDT (E) performed significantly better than placebo, nonsignificantly better than drugs and heat/cold, and nonsignificantly worse than surgery (7 RCTs, 1 NRCS)

- Drugs (F) performed significantly better than placebo, nonsignificantly worse than PDT and heat/cold, and significantly worse than surgery (8 RCTs, 2 (NRCSs)

- Surgery (A,B) performed statistically significantly better than drugs and placebo, and nonsignificantly better than heat/cold and PDT (4 RCTs); this comparison is less relevant as surgery ought to achieve $100 \%$ clinical clearance

- Thermal interventions (C)performed statistically significantly better than plecebo, nonsignificantly better than drugs and PDT, and nonsignificantly worse than surgery (3 RCTs)

- PDT (E) performed statistically significantly better than placebo, nonsignificantly better than drugs, and nonsignificantly worse than surgery and heat/cold (7 RCTs)

- Drugs (F) performed statistically significantly better than placebo, 


\begin{tabular}{|c|c|c|c|c|c|c|}
\hline Conclusion statement & $\begin{array}{l}\text { RoB } \\
\text { (evidence } \\
\text {-base) }\end{array}$ & Consistency & Precision & Directness & $\begin{array}{l}\text { Overall } \\
\text { Rating }\end{array}$ & Comments \\
\hline
\end{tabular}

nonsignificantly worse than PDT and heat/cold, and significantly worse than surgery (5 RCTs)

Patient-reported cosmetic outcomes, all

$B C C$

(1) PDT is associated with better cosmetic outcomes than other intervention categories

(2) [Imprecise data on relative comparisons between nonsurgical active intervention categories]

Observer-reported cosmetic outcomes, all $B C C$

(1) PDT is associated with significantly better cosmetic outcomes than surgery $(A, B)$

(2) [PDT may be associated with better cosmetic outcomes compared to nonsurgical active intervention categories]

(3) [Imprecise data on relative comparisons between heat/cold $(C)$, radiation, and drugs (D)]

\section{Adverse effects, all BCC}

(1) Serious adverse events, adverse events leading to discontinuation and infections of the treated site are uncommon with surgical interventions $(A, B)$, heat or cold (C), PDT (E) and drugs (F)

(2) For the interventions above, on average 1 in 10 to 1 in 5 patients report experiencing pain after treatment
Moderate Possibly consistent

(No robust

indications of inconsistency)

\section{Varies by} and indirect

precise to

imprecise.

Imprecise for

most

comparisons

comparisons

based on

indirect data)
(1) Low

(2) Insufficient

- (A,B) Surgery had significantly better outcomes than heat/cold and radiation, significantly worse outcomes than PDT, and nonsignificantly worse outcomes than drugs (4 RCTs)

- Thermal interventions (C)had significantly worse outcomes than surgery and PDT and nonsignificantly worse than radiation and drugs (2 RCTs)

- Radiation (D) had nonsignificantly better outcomes than heat/cold, nonsignificantly worse outcomes than drugs, and significantly worse outcomes than PDT and surgery (2 RCTs)

- PDT (E) had significantly better outcomes than surgery, heat/cold, and radiation and nonsignificantly better outcomes than drugs (4 RCTs)

- Drugs (F) had better outcomes than surgery, heat/cold, and radiation, and nonsignificantly worse outcomes than PDT, but not statistically significantly so (1 $\mathrm{RCT})$

Moderate Possibly

consistent

(No robust

indications of

inconsistency)

Varies by

Mix of direct comparison from and indirect precise to data (most

imprecise. comparisons

Imprecise for based on

most

indirect data)

comparisons

1) Moderate

(2) [Insufficient]

(3) [Insufficient]

- $(A, B)$ Surgery had nonsignificantly better outcomes than radiation, significantly worse outcomes than PDT, and nonsignificantly worse outcomes than drugs, heat/cold, and placebo (4 RCTs, 1 NRCS)

- (C) Heat/cold interventions had significantly better outcomes than radiation, nonsignificantly better outcomes than surgery, and nonsignificantly worse outcomes than PDT, drugs, and placebo (1 RCT)

- Radiation (D) had significantly worse outcomes than heat/cold, PDT, drugs, and placebo, and nonsignificantly worse outcomes than surgery (1 RCT, 2 NRCS)

- PDT (E) had significantly better outcomes than surgery and radiation, nonsignificantly better outcomes than drugs and heat/cold, and nonsignificantly worse outcomes than placebo (7 RCTs, 1 NRCS)

- Drugs (F) had significantly better outcomes than radiation, nonsignificantly better outcomes than surgery and heat/cold, and nonsignificantly worse outcomes than PDT and placebo (1 RCT)
High

(selective reporting bias)
Unclear

(Consistency

cannot be

assessed)
Imprecise

elative effects. data (most

Forecasted comparisons

percentages of based on

patients with

adverse events

have wide $95 \%$

indirect data)
(1) Moderate

(2) Low
- For active interventions, the percentage of discontinuation of treatment, serious adverse events, and infection of the treatment site ranged from 0/not defined to $5.5 \%$. Forecast $\mathrm{Cls}$ are wide (as high as $29 \%$ )

- For active interventions, the percentage of pain after treatment ranged between 9.9 and $21.6 \%$. Forecast Cls are wide (as high as $88 \%$ ) 


\begin{tabular}{|c|c|c|c|c|c|c|}
\hline Conclusion statement & $\begin{array}{l}\text { RoB } \\
\text { (evidence } \\
\text {-base) }\end{array}$ & Consistency & Precision & Directness & $\begin{array}{l}\text { Overall } \\
\text { Rating }\end{array}$ & Comment \\
\hline & & & $\mathrm{Cls}$ & & & \\
\hline \multicolumn{7}{|l|}{ Other outcomes, all BCC } \\
\hline $\begin{array}{l}\text { [Evidence on quality of life, mental health, } \\
\text { patient satisfaction, mortality, cost and } \\
\text { resource use is reported in a minority of } \\
\text { studies and its strength not rated] } \\
\text { Other analyses }\end{array}$ & [Not rated] & [Not rated] & [Not rated] & [Not rated] & [Not rated] & [Not rated] \\
\hline $\begin{array}{l}\text { [Subgroup analyses and analyses focusing } \\
\text { on individual interventions are generally } \\
\text { sparse and are not rated] }\end{array}$ & [Not rated] & [Not rated] & [Not rated] & [Not rated] & [Not rated] & [Not rated] \\
\hline
\end{tabular}

Note: When a summary conclusion cannot be made, the description is given in square brackets.

RoB = risk of bias; $\mathrm{BCC}=$ basal cell carcinoma; SCC = squamous cell carcinoma; $\mathrm{MMS}=$ Mohs micrographic surgery; $\mathrm{PDT}$ = photodynamic therapy; RCT = randomized controlled trial; NRCS = nonrandomized comparative study; $\mathrm{CI}$ = confidence interval 
Table E. Summary conclusions for SCCIS lesions and strength of the relevant evidence

\begin{tabular}{|c|c|c|c|c|c|c|}
\hline Conclusion statement & $\begin{array}{l}\text { RoB } \\
\text { (evidence } \\
\text {-base) } \\
\end{array}$ & Consistency & Precision & Directness & Overall Rating & Comments \\
\hline \multicolumn{7}{|l|}{ Recurrence, SCCIS } \\
\hline \multicolumn{7}{|l|}{ Histologic clearance, SCCIS } \\
\hline $\begin{array}{l}\text { (1) [Laser (C5) + PDT with ALA (E2) results in } \\
\text { better histologic clearance over laser alone] } \\
\text { (2) 5-FU (F) results in better histologic } \\
\text { clearance than placebo }(I, J)\end{array}$ & $\begin{array}{l}\text { (1) Low } \\
\text { (2) High }\end{array}$ & [Not rated] & $\begin{array}{l}\text { (1) Imprecise } \\
\text { (2) Precise }\end{array}$ & $\begin{array}{l}\text { (1) Direct } \\
\text { (2) Direct }\end{array}$ & $\begin{array}{l}\text { (1) [Insufficient] } \\
\text { (2) Low }\end{array}$ & [2 RCTs, 50 patients.] \\
\hline $\begin{array}{l}\text { Clinical clearance, SCCIS } \\
\text { (1) Examined types of active interventions } \\
\text { (heat/cold [C], PDT (E), and drugs [5-FU, } \\
\text { imiquimod; F]) were associated with better } \\
\text { clinical outcomes than placebo } \\
\text { (2) [Imprecise data on relative comparisons } \\
\text { between types of active interventions] }\end{array}$ & Moderate & $\begin{array}{l}\text { Possibly } \\
\text { consistent } \\
\text { (No robust } \\
\text { indications of } \\
\text { inconsistency) }\end{array}$ & $\begin{array}{l}\text { Varies by } \\
\text { comparison from } \\
\text { precise to } \\
\text { imprecise. }\end{array}$ & $\begin{array}{l}\text { Mix of direct } \\
\text { and indirect } \\
\text { data }\end{array}$ & $\begin{array}{l}\text { (1) High } \\
\text { (2) [Insufficient] }\end{array}$ & $\begin{array}{l}\text { - Thermal interventions (C)performed significantly better than placebo, and } \\
\text { nonsignificantly better than drugs and PDT (4 RCTs) } \\
\text { - PDT (E) performed significantly better than placebo, nonsignificantly better than } \\
\text { drugs, and nonsignificantly worse than heat/cold ( } 5 \text { RCT) } \\
\text { - Drugs (F) (5-FU, imiquimod) performed significantly better than placebo, and } \\
\text { nonsignificantly worse than PDT and heat/cold (2 RCT) }\end{array}$ \\
\hline \multicolumn{7}{|l|}{$\begin{array}{l}\text { Observer-reported cosmetic outcomes, } \\
\text { SCCIS }\end{array}$} \\
\hline $\begin{array}{l}\text { (1) Cryotherapy plus } 5-\mathrm{FU}(\mathrm{C} 1+\mathrm{F} 1) \text { is } \\
\text { associated with better outcomes than } \\
\text { PDT (MAL) (E1) } \\
\text { (2) [No difference between laser pretreatment } \\
\text { of the lesion before PDT versus PDT } \\
\text { alone] }\end{array}$ & Low & $\begin{array}{l}\text { Unclear } \\
\text { (Consistency } \\
\text { cannot be } \\
\text { rated) }\end{array}$ & $\begin{array}{l}\text { (1) Precise } \\
\text { (2) Imprecise }\end{array}$ & $\begin{array}{l}\text { Mix of direct } \\
\text { and indirect } \\
\text { data }\end{array}$ & $\begin{array}{l}\text { (1) Moderate } \\
\text { (2) [Insufficient] }\end{array}$ & [2 RCTs, 204 patients.] \\
\hline \multicolumn{7}{|l|}{ Adverse effects, SSCIS } \\
\hline $\begin{array}{l}\text { (1) [Serious adverse events, adverse events } \\
\text { leading to discontinuation and infections } \\
\text { of the treated site are uncommon with } \\
\text { heat or cold (C), PDT (E) and drugs (F)] } \\
\text { (2) [On average, } 1 \text { in } 4 \text { and } 1 \text { in } 3 \text { patients } \\
\text { report experiencing pain after treatment } \\
\text { with PDT (E) and heat or cold (C), }\end{array}$ & $\begin{array}{l}\text { High } \\
\text { (selective } \\
\text { reporting } \\
\text { bias) }\end{array}$ & $\begin{array}{l}\text { Unclear } \\
\text { (Consistency } \\
\text { cannot be } \\
\text { assessed) }\end{array}$ & $\begin{array}{l}\text { Imprecise } \\
\text { We do not report } \\
\text { relative effects. } \\
\text { Forecasted } \\
\text { percentages of } \\
\text { patients with } \\
\text { adverse events }\end{array}$ & $\begin{array}{l}\text { Mix of direct } \\
\text { and indirect } \\
\text { data (most } \\
\text { comparisons } \\
\text { based on } \\
\text { indirect data) }\end{array}$ & $\begin{array}{l}\text { (1) [Insufficient] } \\
\text { (2) [Insufficient] }\end{array}$ & [3 RCTs 292 patients.] \\
\hline
\end{tabular}




\begin{tabular}{|c|c|c|c|c|c|c|}
\hline Conclusion statement & $\begin{array}{l}\text { RoB } \\
\text { (evidence } \\
\text {-base) }\end{array}$ & Consistency & Precision & Directness & Overall Rating & Comments \\
\hline respectively] & & & $\begin{array}{l}\text { have wide } 95 \% \\
\text { Cls }\end{array}$ & & & \\
\hline \multicolumn{7}{|l|}{ Other outcomes, SCCIS } \\
\hline $\begin{array}{l}\text { [Evidence on patient-reported cosmetic } \\
\text { outcomes, quality of life, mental health, } \\
\text { patient satisfaction, mortality, cost and } \\
\text { resource use id reported in a minority of } \\
\text { studies and its strength not rated] }\end{array}$ & $\begin{array}{l}\text { [Not } \\
\text { rated] }\end{array}$ & [Not rated] & [Not rated] & [Not rated] & [Not rated] & [Not rated] \\
\hline \multicolumn{7}{|l|}{ Other analyses } \\
\hline $\begin{array}{l}\text { [Subgroup analyses and analyses focusing on } \\
\text { individual interventions are generally } \\
\text { sparse and are not rated] }\end{array}$ & $\begin{array}{l}\text { [Not } \\
\text { rated] }\end{array}$ & [Not rated] & [Not rated] & [Not rated] & [Not rated] & [Not rated] \\
\hline
\end{tabular}

Note: When a summary conclusion cannot be made, the description is given in square brackets.

RoB = risk of bias; $\mathrm{BCC}$ = basal cell carcinoma; SCCIS = squamous cell carcinoma in situ; MMS = Mohs micrographic surgery; PDT = photodynamic therapy; RCT = randomized controlled trial; NRCS = nonrandomized comparative study; CI = confidence interval 


\section{References}

1. Centers for Disease Control and Prevention Skin Cancer Statistics. 2015.

http://www.cdc.gov/cancer/skin/statistics/index.ht $\mathrm{m}$.

2. Rogers HW, Weinstock MA, Feldman SR, et al. Incidence Estimate of Nonmelanoma Skin Cancer (Keratinocyte Carcinomas) in the US Population, 2012. JAMA Dermatol. 2015 Oct

1;151(10):1081-6. doi:

10.1001/jamadermatol.2015.1187. PMID: 25928283.

3. Bickers DR, Lim HW, Margolis D, et al. The burden of skin diseases: 2004 a joint project of the American Academy of Dermatology Association and the Society for Investigative Dermatology. J Am Acad Dermatol. 2006 Sep;55(3):490-500. doi: 10.1016/j.jaad.2006.05.048. PMID: 16908356.

4. Karimkhani C, Dellavalle RP, Coffeng LE, et al. Global Skin Disease Morbidity and Mortality: An Update From the Global Burden of Disease Study 2013. JAMA Dermatol. 2017 Mar 01doi: 10.1001/jamadermatol.2016.5538. PMID: 28249066.

5. Wu W, Weinstock MA. Trends of keratinocyte carcinoma mortality rates in the United States as reported on death certificates, 1999 through 2010. Dermatol Surg. 2014 Dec;40(12):1395-401. doi: 10.1097/DSS.0000000000000194. PMID: 25393350.

6. Karia PS, Han J, Schmults CD. Cutaneous squamous cell carcinoma: estimated incidence of disease, nodal metastasis, and deaths from disease in the United States, 2012. J Am Acad Dermatol. 2013 Jun;68(6):957-66. doi: 10.1016/j.jaad.2012.11.037. PMID: 23375456.

7. U. S. Department of Health and Human Services. Reports of the Surgeon General. The Surgeon General's Call to Action to Prevent Skin Cancer. Washington (DC): Office of the Surgeon General (US); 2014.

8. Cakir BO, Adamson P, Cingi C. Epidemiology and economic burden of nonmelanoma skin cancer. Facial Plast Surg Clin North Am. 2012 Nov;20(4):419-22. doi: 10.1016/j.fsc.2012.07.004. PMID: 23084294.
9. U.S. Department of Health Human Services. The surgeon general's call to action to prevent skin cancer. 2014.

https://www.ncbi.nlm.nih.gov/pubmed/25320835. PMID: 25320835.

10. Mudigonda T, Pearce DJ, Yentzer BA, et al. The economic impact of non-melanoma skin cancer: a review. J Natl Compr Canc Netw. 2010 Aug;8(8):888-96. PMID: 20870635.

11. AHRQ Methods for Effective Health Care. Methods Guide for Effectiveness and Comparative Effectiveness Reviews. Rockville (MD): Agency for Healthcare Research and Quality (US); 2011.

12. Higgins JP, Altman DG, Gotzsche PC, et al. The Cochrane Collaboration's tool for assessing risk of bias in randomised trials. Bmj. 2011;343:d5928. doi: 10.1136/bmj.d5928. PMID: 22008217.

13. Wells GAS, B.;O'Connell, D.;Peterson, J.; Welch, V.; Losos, M.; Tugwell, P. The Newcastle-Ottawa Scale (NOS) for assessing the quality of nonrandomised studies in metaanalyses. http://www.ohri.ca/programs/clinical_epidemiolo gy/oxford.asp.

14. Garcia-Martin E, Gil-Arribas LM, Idoipe M, et al. Comparison of imiquimod 5\% cream versus radiotherapy as treatment for eyelid basal cell carcinoma. Br J Ophthalmol. 2011 Oct;95(10):1393-6. doi: 10.1136/bjo.2010.193078. PMID: 21242584.

15. Choi SH, Kim KH, Song KH. Effect of Methyl Aminolevulinate Photodynamic Therapy With and Without Ablative Fractional Laser Treatment in Patients With Microinvasive Squamous Cell Carcinoma: A Randomized Clinical Trial. JAMA Dermatol. 2017 Feb 15doi: 10.1001/jamadermatol.2016.4463. PMID: 28199463.

16. Connolly SM, Baker DR, Coldiron BM, et al. AAD/ACMS/ASDSA/ASMS 2012 appropriate use criteria for Mohs micrographic surgery: a report of the American Academy of Dermatology, American College of Mohs Surgery, American Society for Dermatologic Surgery Association, and the American Society for Mohs Surgery. J Am Acad Dermatol. 2012 Oct;67(4):531-50. doi: 10.1016/j.jaad.2012.06.009. PMID: 22959232. 
17. Wilson LS, Pregenzer M, Basu R, et al. Fee comparisons of treatments for nonmelanoma skin cancer in a private practice academic setting. Dermatol Surg. 2012 Apr;38(4):570-84. doi: 10.1111/j.1524-4725.2011.02231.x. PMID: 22145798.

18. Mosterd K, Thissen MR, Nelemans P, et al. Fractionated 5-aminolaevulinic acidphotodynamic therapy vs. surgical excision in the treatment of nodular basal cell carcinoma: results of a randomized controlled trial. Br J Dermatol. 2008 Sep;159(4):864-70. doi: 10.1111/j.13652133.2008.08787.x. PMID: 18717680.

19. Merola JF, Armstrong AW, Saraiya A, et al. International Dermatology Outcome Measures Initiative as Applied to Psoriatic Disease Outcomes: An Update. J Rheumatol. 2016 May;43(5):959-60. doi: 10.3899/jrheum.160114. PMID: 27134269.

20. Schmitt J, Apfelbacher C, Spuls PI, et al. The Harmonizing Outcome Measures for Eczema (HOME) roadmap: a methodological framework to develop core sets of outcome measurements in dermatology. J Invest Dermatol. 2015 Jan;135(1):24-30. doi: 10.1038/jid.2014.320. PMID: 25186228.

21. Chan AW, Fung K, Tran JM, et al. Application of Recursive Partitioning to Derive and Validate a Claims-Based Algorithm for Identifying Keratinocyte Carcinoma (Nonmelanoma Skin Cancer). JAMA Dermatol. 2016 Oct 01;152(10):1122-7. doi: 10.1001/jamadermatol.2016.2609. PMID: 27533718. 


\section{Introduction}

\section{Background}

Skin cancers, including basal cell carcinoma (BCC) and squamous cell carcinoma (SCC), are the most common malignancies in the United States. ${ }^{1}$ BCC and SCC, the 2 most common skin cancers, are collectively referred to as keratinocyte carcinomas. Over 5.4 million of these cancers are diagnosed in 3.3 million people in the United States annually, ${ }^{2,3}$ and the global burden of disease from keratinocyte carcinomas is estimated at 12.9 disability-adjusted life years per 100,000 persons. ${ }^{4}$ Generally keratinocyte carcinomas are not aggressive and do not metastasize or kill as often as melanoma, which is the third most common skin cancer. ${ }^{5}$ However, SCC can metastasize and is estimated to kill between 3900 and 8800 people in the United States each year. ${ }^{6}$ Aggressive behavior is of particular concern in people who are immunosuppressed, including organ transplant recipients whose mortality is increased after being diagnosed with SCC. ${ }^{7}$ A more common problem is that basal and squamous cell carcinomas and their treatment may result in disfigurement or disability, which can adversely impact quality of life. ${ }^{3}$ The recent Surgeon General's call to action to prevent skin cancer at the population level emphasizes the public health importance of dealing with keratinocyte carcinomas. ${ }^{8}$ Because of their frequency, BCC and SCC are the fifth most expensive cancer at the population level, and, being more common in older adults, their management is of great importance to Medicare. ${ }^{2,3,9}$ It is estimated that in 2012 over 2 million Medicare beneficiaries underwent intervention for BCC or SCC. ${ }^{2}$

There are many potential management strategies for keratinocyte carcinoma, and they can be broadly grouped into eight main categories: (1) surgical excision without intraoperative evaluation of the margins, (2) surgical excision with intraoperative evaluation of the margins, (3) destruction via temperature gradients, (4) ionizing radiation, (5) photodynamic interventions, (6) medical therapies, along with (7) combinations of these therapies, and (8) watchful waiting. Surgical management is used most commonly, followed by radiation. ${ }^{10-12}$ In individuals over 65 , surgery is used to treat 61 percent of keratinocyte carcinomas (excision 42\% and Mohs micrographic surgery $19 \%$ ) followed by electrodessication and curettage (39\%). ${ }^{13}$ Specific surgical techniques include simple surgical excision with prespecified margins, surgery with intra-operative margin control (e.g. Mohs micrographic surgery or excision with examination of frozen sections), and curettage, which is usually combined with secondary destruction using electrodessication. ${ }^{14}$ Cryotherapy with liquid nitrogen is another destructive method. Ionizing radiation modalities include traditional external beam radiation as well as brachytherapy, in which radioactive implants are placed directly in the tumor. Topical medical treatments include topical chemotherapy (such as 5-fluorouracil) and topical immunomodulatory medications (such as imiquimod). Photodynamic therapy involves application of a topical photosensitizer (such as 5-aminoleveulinic acid (ALA) and methyl-ALA) followed by exposure to specific wavelengths of light to destroy tumor cells. New targeted systemic agents, such as vismodegib, for BCC ${ }^{15}$ are also available, but are reserved for advanced or metastatic cases and are used much less commonly than the modalities listed above. Additionally, active nonintervention (watchful waiting) has recently been advanced as a therapeutic strategy, particularly for patients with decreased life expectancy. ${ }^{16,17}$

The choice of management strategy for an individual patient with a specific keratinocyte carcinoma is complex. Factors that are important include patient factors (e.g. age, frailty, 
immunosuppression, and personal preference) and tumor factors (e.g. histologic subtype, size, and location). A lack of clarity regarding the comparative efficacy and safety of the available options overall and in specific circumstances further complicates the choice of treatment for both physicians and patients.

There is general agreement that surgical removal is the gold standard. However, despite several dozen randomized controlled trials (RCTs) and nonrandomized comparative studies (NRCS), it is not clear how various surgical techniques and other therapeutic options perform relative to each other (e.g., see references ${ }^{18-23}$ ). None of the over 30 systematic reviews and meta-analyses (e.g., see references ${ }^{24-31}$ ) on this topic to date includes all treatment modalities for both BCC and SCC. The Australian and Finnish clinical practice guidelines for keratinocyte carcinoma management allude to the difficulty in interpreting the existing evidence-base, which comprises comparisons among pairs of several available treatments. ${ }^{32,33}$ Furthermore, existing guidance is not based on systematic assessments of the evidence. It is hoped that the information in this review will be useful in the development of future guidelines, such as the guidelines on keratinocyte carcinomas from the American Academy of Dermatology, anticipated later in 2016.

Interventions for treating skin cancers differ substantially in cost and have a huge economic impact. ${ }^{3,}, 34,35$ Payers are faced with increased utilization of costly therapies, such as brachytherapy, without clear evidence for relative benefits to justify increased costs. ${ }^{36}$

Estimates of keratinocyte carcinoma treatments' comparative effectiveness and safety with respect to patient-relevant outcomes are needed to inform clinical decisionmaking and payer coverage decisions. The objective of this systematic review is to comprehensively collect and synthesize information on the comparative effectiveness and safety of each of the abovementioned therapeutic strategies for both BCC and SCC.

\section{Key Questions}

The review addresses two Key Questions for adult patients with basal cell or squamous cell carcinoma of the skin. Each Key Question will be answered separately for SCC and BCC:

Key Question 1: What is the comparative effectiveness of various interventions, overall and in subgroups of interest?

\section{Key Question 2: How do the adverse events associated with the various interventions compare overall and in subgroups of interest?}

\section{Analytic Framework}

The analytic framework in Figure 1 depicts the chain of logic that evidence must support to link the studied interventions. 
Figure 1. Analytic framework for treatments for basal cell and squamous cell carcinoma of the skin

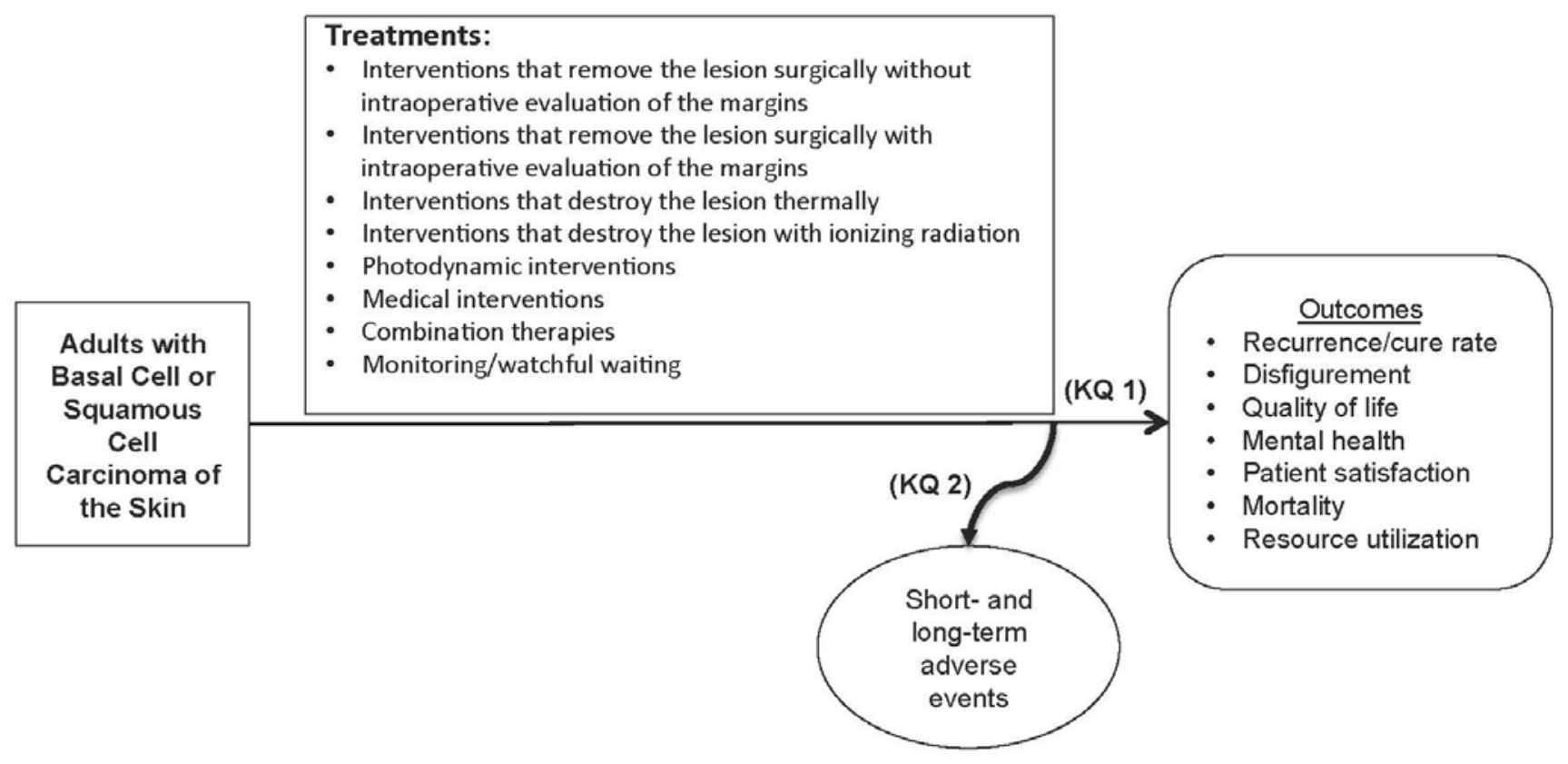




\section{Methods}

The Brown Evidence-based Practice Center (EPC) conducted this review based on a systematic review of the published scientific literature, using established methodologies as outlined in the Agency for Healthcare Research and Quality (AHRQ) Methods Guide for Effectiveness and Comparative Effectiveness Reviews. ${ }^{37}$ The Prospero registration number is CRD42016043353.

\section{Eligibility Criteria}

We use the population, intervention, comparator, outcomes, and designs (PICOTS) formalism to define the characteristics of the eligible studies for this review.

\section{Population}

The population of interest is people with primary squamous cell carcinoma (SCC) and basal cell carcinoma (BCC). This specifically excludes recurrent or metastatic disease. If populations were mixed, we included studies with at least 80 percent primary, nonmetastatic BCC or SCC. We excluded studies of recurrent or metastatic cancers in which it was not clear whether the advanced lesions were less than 20 percent of the total lesions studied.

We were also interested in the following specific subpopulations: (1) people who are immunocompromised, including those who have had a solid organ or bone marrow transplant, human immunodeficiency virus (HIV), chemotherapy, Chronic Lymphocytic Leukemia (CLL) or other leukemias and lymphomas, or other iatrogenic; (2) people with a limited life expectancy (e.g., the very elderly, those with terminal cancer, those with end stage renal disease). We have excluded subpopulations based on rare genetic factors (e.g., basal-cell nevus syndrome and xeroderma pigmentosa).

In addition, we were interested in the effects of treatments in subgroups as defined by location (e.g. face, hands, trunk, or extremities) and grade of lesion (e.g. superficial or nodular BCC or SCC in situ [Bowen's Disease] in SCC).

\section{Interventions}

The interventions of interest are organized into intervention categories (A through $\mathrm{J}$ ):

A. Surgical excision without intraoperative evaluation of the margins

B. Surgical excision with intraoperative evaluation of the margins

Mohs micrographically controlled surgery

Surgery with examination of frozen sections

C. Interventions that destroy the lesion via temperature gradients

(C1) Cryotherapy

(C2) Diathermy/electrodesiccation

(C3) Curettage of the lesion plus diathermy (cauterization) of margins

(C4) Curettage of the lesion plus cryotherapy

(C5) $\mathrm{CO}_{2}$ laser therapy

D. Interventions that destroy the lesion with ionizing radiation 
(D1) External beam radiation with photons ( $\mathrm{X}$ or gamma rays), electrons (beta rays), or positively charged particles (e.g., protons, helium nuclei/alpha rays), at orthovoltage or megavoltage energies, or using in-office radiation machines

(D2) Brachytherapy with superficial application or interstitial application (pleisiotherapy) of radiation sources (usually emitting beta or alpha rays)

E. Photodynamic interventions

(E1) 5-aminolevulinic acid (ALA) + blue light

(E2) Methyl aminolevulinate (MAL) + red light

(E3) Other forms of PDT

F. Medical interventions

(F1) 5-fluorouracil (5-FU)

(F2) Imiquimod

(F3) Interferon (IFN alpha-2a/2b or INF beta)

(F4) Ingenol mebutate

(F5) Other medical interventions, including BEC-5 cream, Bleomycin, Methotrexate, Diclofenac, and Hedgehog inhibitors (Vismodegib, Sonidegib)

G. Shave excision

H. Curettage without diathermy

I. Placebo

J. No treatment

\section{Outcomes}

We evaluated the outcomes in the following list. We did not use strict $a$ priori definitions of the outcomes, but included all reported outcomes as defined by study researchers. We evaluated outcomes at any and all time points given in a specific study. We used our best judgment to categorize outcomes when studies failed to clearly define their reported outcomes.

- Recurrence/cure rate (as defined in studies)

- Disfigurement/cosmetic outcome

- Quality of Life (only if they use validated instruments to measure - e.g. Short Form Health Survey-36, Skindex, Skin Cancer Index, Skin Cancer Quality of Life Impact Tool)

- Mental health, anxiety, depression, intrusive thoughts (only if they use validated instruments to measure - e.g. State-Trait Anxiety Inventory, Hospital Anxiety and Depression Scale, Impact of Event Scale)

- Patient satisfaction with treatment (only if they use validated instruments to measure e.g. Patient Satisfaction Questionnaire-18, Skin Cancer Index patient satisfaction subscale)

- Mortality

- Adverse events, including those that are reported by patients and clinically, as well as actively and passively. Both short-term (e.g. pain, skin irritation) and long-term (e.g. radiation exposure, scarring) adverse events were recorded. We systematically reviewed the following endpoints: "any serious adverse event" (leading to treatment discontinuation, or as defined by each study), "pain" and "infection". We enumerated the set of other reported events. 


\section{Design}

We evaluated all randomized controlled studies and all comparative nonrandomized controlled studies. We excluded studies enrolling fewer than 10 people total because they were unlikely to yield precise or broadly applicable conclusions. We excluded non-English studies, as there were very few of them and there is empirical evidence that excluding them typically has minimal impact on conclusions. ${ }^{38}$ Studies in any setting were acceptable.

As described by Linos et al., ${ }^{17}$ patient treatment is often determined by factors, such as disease stage, medical history, age and education, that could confound assessment of the outcomes of interest. Thus for the nonrandomized comparative studies (NRCSs), we required that studies included an analysis that accounted for confounders, such as inclusion in a multivariate model, balancing or quasi-randomization, or clearly matched groups. NRCSs that report only crude results were identified and tabulated but were excluded from the analysis in the full report.

\section{Evidence Identification}

We conducted literature searches of studies in PubMed, the Cochrane Central Trials Registry, the Cochrane Database of Systematic Reviews, and Embase to identify primary research studies meeting our criteria through March 8, 2017. These databases should adequately cover the published literature on this topic. The full search strategy for all databases is in Appendix A. We screened all references in published clinical practice guidelines, relevant narrative and systematic reviews, and Scientific Information Packages from manufacturers or other stakeholders. We searched ClinicalTrials.gov and the World Health Organization International Clinical Trials Registry Platform (ICTRP) for ongoing studies and studies that are not published in the medical literature. In addition, we searched the Food and Drug Administration drugs and devices portals for unpublished data. We did not find any studies with results that were not included in the published literature. Our requests to manufacturers for scientific information packets also did not yield any new data. We have extracted and incorporated all studies de novo and have not summarized or incorporated existing systematic reviews, per se. All articles identified through these sources have been screened for eligibility, using the same criteria as was used for articles identified through literature searches. The search will be updated upon submission of the draft report for peer and public review.

All citations found by literature searches and other sources were independently screened by two researchers. At the start of abstract screening, we implemented a training session, in which all researchers screened the same articles and conflicts were discussed. During title and abstract double-screening, we resolved conflicts as a group. All title and abstract screening was done in the open-source, online software Abstrackr (http://abstrackr.cebm.brown.edu/). ${ }^{39}$ All potentially relevant studies were rescreened in full text with double-screening to ensure eligibility.

\section{Data Extraction and Data Management}

Each study has been extracted by one member of the review team, which includes clinicians and methodologists. The extraction was reviewed and confirmed by at least one other experienced methodologist. Any disagreements were resolved by discussion among the team. Data was extracted into a customized form in Systematic Review Data Repository (SRDR) online system (http://srdr.ahrq.gov) designed to capture all elements relevant to the Key 
Questions. Upon completion of the review, the SRDR database will be made accessible to the general public (with capacity to read, download, and comment on data). The basic elements and design of the extraction form are the similar to those used for other AHRQ comparative effectiveness reviews and include elements that address population characteristics, including method of diagnosis; descriptions of the interventions, exposures, and comparators analyzed; outcome definitions; effect modifiers; enrolled and analyzed sample sizes; study design features; funding source; results; and risk of bias questions. If information was stratified by carcinoma subtype for BCC (e.g. superficial or nodular) and SCC (e.g. SCC in situ, well-differentiated, or poorly differentiated), we recorded that information as well.

\section{Assessment of Methodological Risk of Bias of Individual Studies}

We assessed the methodological quality of each study based on predefined criteria. For RCTs, we used the Cochrane risk of bias tool, ${ }^{40}$ which asks about risk of selection bias, performance bias, detection bias, attrition bias, reporting bias, and other potential biases. For observational studies, we used relevant questions from the Newcastle Ottawa Scale. ${ }^{41}$ For RCTs, the review team discussed each article, based on methodological (design and analysis) items that are related to the aforementioned biases for each outcome of each trial. To obtain information on (a lower bound of) the number of yet unpublished trials, we searched clinicaltrials.gov for completed trials, and examined the publication status of thus identified studies.

\section{Data Synthesis}

All included studies were summarized in narrative form and in summary tables that include the important features of the study populations, design, intervention, outcomes, and results. Lesions were divided by subtype (superficial, nodular, or high-risk BCC, SCC, or mixed populations) for analysis to ensure that the treatments would be most comparable. Where possible, lesions were also evaluated by size and location. Trial arms with fewer than 5 lesions were not included in the analysis, because they contribute minimal information, and in some instances, necessitated adding model parameters that were difficult to estimate.

We conducted pairwise and network meta-analyses with mixed effects (random intercepts and fixed intervention slopes) or full-random effects (random intercepts and random slopes) multilevel models within the generalized linear and latent mixed models. We used the normal approximation to discrete likelihoods with a canonical (logit) link function. Treatment effect estimates from such models are odds ratios. We fit models by maximizing the restricted likelihood. We explored clinical and methodological heterogeneity in subgroup analyses. We did not conduct dose-response meta-analyses because there was substantial heterogeneity in the definitions of intervention intensity (dose) across studies; instead, we summarized dose-response results qualitatively. To aid the interpretation of these analyses we also present model-based estimates for the mean frequency of an outcome in the examined interventions, as well as forecasts of the frequency of the outcome in a new setting (e.g., a new study, or in a population) that is similar to the studies in the meta-analysis. The forecasts' point estimate about the frequency of the outcome is very close to the point estimate of the mean frequency of the outcome over the meta-analyzed studies. However, the 95\% confidence interval (CI) for a forecast of the frequency of an outcome in a new setting accounts for between-study 
heterogeneity, and will, thus, be broader than the corresponding 95\% CI for the mean frequency of the outcome across the analyzed studies. See the next paragraph about the presentation of results. Inconsistency was assessed by comparing the fit of models that do not assume consistent intervention effects versus typical network meta-analysis models, that assume consistent treatment effects. Analyses did not identify statistical evidence of inconsistency. Because such analyses are known to be underpowered, we also compared qualitatively the agreement of estimates based only on direct data versus of estimates based on both direct and indirect data. Such estimates were deemed to be congruent.

\section{Presentation of Results}

We present results with plots and tables. We briefly describe three expository formats that are not commonly used in EPC reports, namely, evidence graphs, league tables, and relative effects tables.

\section{Evidence Graphs}

We use evidence graphs such as the one in Figure 2 to describe which interventions have been compared with others. An evidence graph comprises nodes, which represent interventions, and edges (depicted by a line linking nodes). Edges connect a pair of nodes only if the corresponding interventions have been compared in at least one head-to-head study. In Figure 2, nodes for interventions from the same intervention category are in a shaded area. For example, nodes E1 (corresponding to PDT with MAL) and E2 (corresponding to PDT with ALA) are within the same shaded area which represents PDT as the type of intervention), and analogously for other nodes and interventions in the figure. The organization of interventions in intervention categories has been described in the Interventions paragraph. We use the term connected subgraph to describe a set of nodes that are connected through one or more edges. For example, Figure 2 has 2 connected subgraphs, which include the following nodes:

1. $\mathrm{A}|\mathrm{B}, \mathrm{D} 1| \mathrm{D} 2$, and

2. all remaining nodes in the evidence graph, namely $\mathrm{A}, \mathrm{B}, \mathrm{A} \mid \mathrm{B}, \mathrm{B}+\mathrm{F} 3, \mathrm{D} 1, \mathrm{~F} 2, \mathrm{H}, \mathrm{C} 3$, C4, C1, C5+E1, E1, and E2.

If all the nodes in the graph were connected, then there would be a single connected subgraphwhich would be the whole graph. Identifying connected subgraphs is important, because we do not statistically compare interventions that belong to different connected subgraphs.

Figure 3 is an analogous representation of the comparisons between intervention categories for the same network of interventions depicted in Figure 2. When one considers intervention categories, comparisons between interventions that belong to the same type are not pertinent. Such comparisons are represented by edges enclosed in the shaded areas in the evidence graph in Figure 2. Observe also that comparing between intervention categories happened to result in a single connected subgraph in Figure 3. 
Figure 2. Example evidence graph depicting comparisons between individual interventions

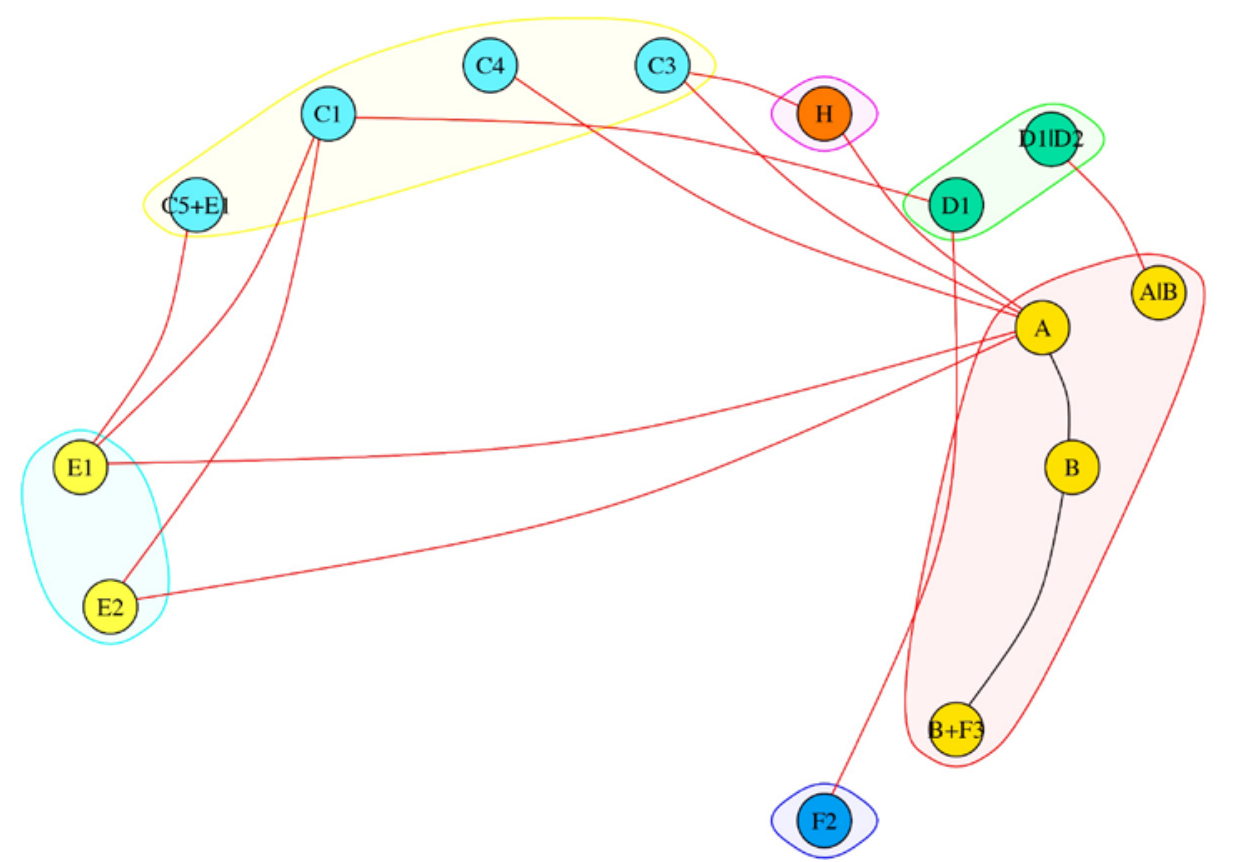

A: surgical excision

B: MMS

C1: cryotherapy

C3: curettage + diathermy

C4: curettage + cryotherapy

C5: laser

D1: external radiation

D2: brachytherapy

E1: PDT (MAL)

E2: PDT (ALA)

F1: 5-FU

F2: imiquimod

F3: INF

$\mathrm{F} 4$ : ingenol

F5: other medical

$\mathrm{H}$ : curettage

I: no treatment

$\mathrm{J}$ : placebo/sham

MMS = Mohs micrographic surgery; PDT = photodynamic therapy; ALA = 5-aminolevulinic acid; MAL = methyl aminolevulinate; FU = fluorouracil; INF = interferon

Figure 3. Evidence graph depicting comparisons between intervention categories

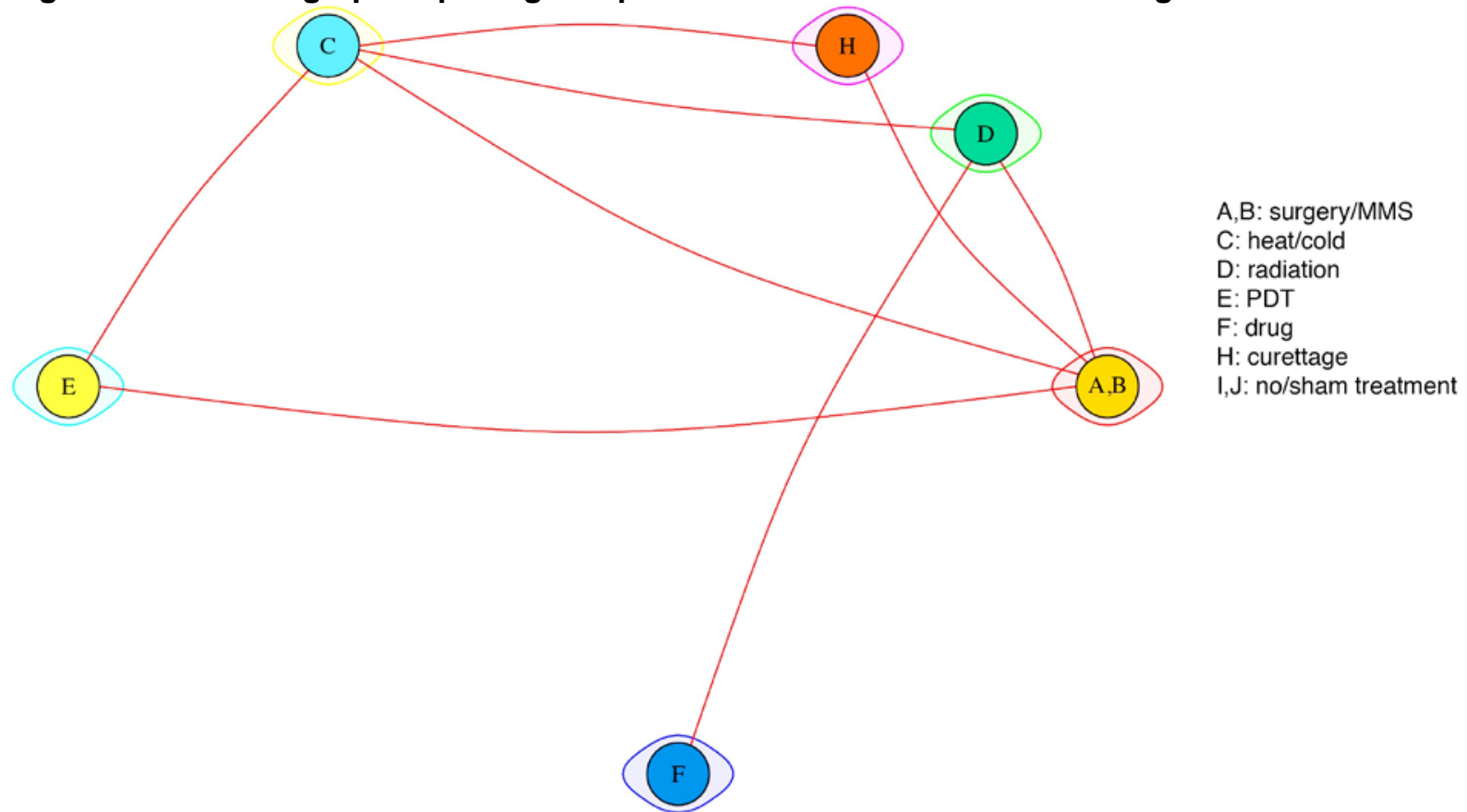

MMS = Mohs micrographic surgery; PDT = photodynamic therapy; ALA = 5-aminolevulinic acid; MAL= methyl aminolevulinate; FU = fluorouracil; INF = interferon 


\section{Relative Effects Tables}

Relative effects tables describe odds ratio estimates and 95\% CIs for all pairwise comparisons in a connected subgraph. Table 1 is an example; it is the analysis that corresponds to the evidence graph in Figure 3. Each cell has a (row, column) address, and reports the estimated odds ratio between the intervention in the row versus the intervention in the column. Consider the cell in the second row, fourth column: The odds ratio comparing interventions that destroy lesions with heat or cold (with code letter C; the intervention in the row) versus PDT (E; the intervention in the column) was 0.91 (95\% CI, 0.43 to 1.95). The cell in the fourth row, second column is the odds ratio for a comparison between the same interventions but in the other direction: 1.10 (95\% CI, 0.51, 2.34) is the odds ratio of PDT (E) versus interventions that destroy the lesion with heat or cold (C). The unshaded cells correspond to comparisons for which there is head-to-head information, i.e., there is an edge between these corresponding nodes in the evidence graph. The estimated treatment effects in these cells are informed by direct and indirect evidence. The shaded cells correspond to comparisons that have not been empirically observed (there is no edge between these corresponding nodes in the evidence graph), and are based only on indirect comparisons.

Table 1. Relative odds ratios for an outcome between intervention categories (Figure 3)

\begin{tabular}{|c|c|c|c|c|c|}
\hline $\begin{array}{c}\text { Surgery/MMS } \\
(A, B)\end{array}$ & $0.13(0.05,0.35)$ & $0.77(0.22,2.73)$ & $0.12(0.04,0.32)$ & $1.09(0.05,24.23)$ & $0.14(0.03,0.77)$ \\
\hline $7.71(2.83,20.98)$ & $\begin{array}{l}\text { Heat/cold } \\
\text { (C) }\end{array}$ & $5.95(2.03,17.4)$ & $0.91(0.43,1.95)$ & $\begin{array}{c}8.44(0.41 \\
173.75)\end{array}$ & $1.09(0.23,5.16)$ \\
\hline $1.3(0.37,4.59)$ & $0.17(0.06,0.49)$ & $\begin{array}{l}\text { Radiation } \\
\text { (D) }\end{array}$ & $0.15(0.05,0.45)$ & $1.42(0.06,32.2)$ & $0.18(0.03,1.04)$ \\
\hline $8.45(3.08,23.16)$ & $1.10(0.51,2.34)$ & $6.52(2.21,19.21)$ & $\begin{array}{l}\text { PDT } \\
\text { (E) }\end{array}$ & $\begin{array}{c}9.25(0.45, \\
190.91) \\
\end{array}$ & $1.19(0.25,5.68)$ \\
\hline $0.91(0.04,20.24)$ & $0.12(0.01,2.44)$ & $0.7(0.03,15.99)$ & $0.11(0.01,2.23)$ & $\begin{array}{l}\text { Drugs } \\
\text { (F) }\end{array}$ & $\begin{array}{c}0.13(<0.005 \\
3.56)\end{array}$ \\
\hline $7.08(1.3,38.49)$ & $0.92(0.19,4.35)$ & $5.46(0.96,31.02)$ & $0.84(0.18,3.99)$ & $\begin{array}{c}7.75(0.28, \\
214.11)\end{array}$ & $\begin{array}{l}\text { Curettage } \\
\text { (H) }\end{array}$ \\
\hline
\end{tabular}

Note: This example is for analyses of recurrence among patients with BCC lesions. Cells shaded gray indicate that the estimate is based only on indirect comparisons; bold-italic numbers indicate statistical significance. Results are given as odds ratios (95\% confidence intervals).

MMS = Mohs micrographic surgery; PDT = photodynamic therapy

\section{League Tables}

League tables such as Table 2, describe the mean fraction of lesions with the outcome of interest for each intervention (or intervention category) over the populations included in the meta-analysis, and the corresponding forecasted fraction in a new setting that is analogous to the settings of the analyzed studies. The results in the league table and the results in the relative effects table are from the same analysis. The league table explains what the relative effects imply about the probability of the outcome under each treatment. In the example, over the metaanalyzed studies the probability of the event with PDT (E) was 23.0 percent (95\% CI 14.8 to 33.9) and with interventions that destroy the lesion with heat or cold (C) it was 21.4 percent (95\% CI 13.8 to 31.6). The expected frequency of the event in a setting that is analogous to the settings in which the meta-analyzed studies where conducted is shown in the forecast column. 
Note that the confidence intervals for the forecast are always larger than the confidence intervals for the mean.

Imagine that you are hiking along a trail from east to west, through six camp sites. The camp sites serve as the analogue for the interventions. A table showing the signed distances ${ }^{\mathrm{a}}$ between pairs of campsites would be the analogue of the relative effects table. A table showing how far each campsite is from the easternmost end of the trail would be the analogue of the league table.

Table 2. Mean and forecasted event fractions by intervention category

\begin{tabular}{lcc}
\hline Intervention Type & $\begin{array}{c}\text { Mean } \\
\text { Percent }(95 \% \mathrm{Cl})\end{array}$ & $\begin{array}{c}\text { Forecast } \\
\text { Percent (95\% Cl) }\end{array}$ \\
\hline Surgery/MMS $(\mathrm{A}, \mathrm{B})$ & $3.4(1.5,7.6)$ & $3.4(1.0,11.4)$ \\
\hline Heat/cold $(\mathrm{C})$ & $21.4(13.8,31.6)$ & $21.4(8.3,45.1)$ \\
\hline Radiation (D) & $4.4(1.8,10.4)$ & $4.4(1.2,15.0)$ \\
\hline PDT (E) & $23.0(14.8,33.9)$ & $23.0(8.9,47.5)$ \\
\hline Drugs (F) & $3.1(0.2,38.8)$ & $3.1(0.1,42.5)$ \\
\hline Curettage $(\mathrm{H})$ & $20.0(5.5,51.9)$ & $20.0(4.1,59.1)$ \\
\hline MMS = Mohs micrographic surgery; PDT = photodynamic therapy; CI = confidence interval
\end{tabular}

\section{Grading the Strength of Evidence (SOE) for Major Comparisons and Outcomes}

We graded the strength of the body of evidence as per the AHRQ Methods Guide on assessing the strength of evidence. ${ }^{37}$ We assed the strength of evidence for each outcome. Following the standard AHRQ approach, for each intervention and comparison of intervention, and for each outcome, we assessed the number of studies, their study designs, the study limitations (i.e., risk of bias and overall methodological quality), the directness of the evidence to the KQs, the consistency of study results, the precision of any estimates of effect, the likelihood of reporting bias, and the overall findings across studies. Based on these assessments, we have assigned a strength of evidence rating as being either high, moderate, or low, or there being insufficient evidence to estimate an effect. The data sources, basic study characteristics, and each strength-of-evidence dimensional rating are summarized in a "Summary of Evidence Reviewed" table detailing our reasoning for arriving at the overall strength of evidence rating.

We assessed the applicability within and across studies with reference to demographics of enrolled participants (e.g. age and sex distributions), the location and severity of the lesions, and the availability of treatments (e.g. with respect to radiation treatments).

\section{Peer Review}

A draft version of this report was reviewed by invited and public reviewers. Revisions of the draft were made, where appropriate, based on their comments. The draft and final reports have also been reviewed by the Task Order Officer and an Associate Editor from another EPC. However, the findings and conclusions are those of the authors, who are responsible for the contents of the report.

\footnotetext{
${ }^{\mathrm{a}} \mathrm{A}$ signed distance encodes the direction of movement and the distance traveled.
} 


\section{Results}

\section{Summary of Studies}

The literature searches yielded 15813 citations (Figure 4), of which 15278 were excluded in abstract screening. A search of the reference lists of relevant systematic reviews yielded another 85 studies, which brought the total number screened in full text to 534. Appendix A presents the literature search strategies (for each database searched). Appendix B lists the articles that were reviewed in full text that were excluded, with their rejection reasons.

The 109 included studies (described in 125 papers) report 58 randomized controlled trials (RCTs) and 51 nonrandomized comparative studies (NRCSs). Two papers reported the results of two separate trials and were analyzed separately; another seven studies were reported in multiple papers. Among the 58 RCTs in 69 papers, ${ }^{19,20,22,42-105} 56$ were reported in full papers, and two were reported only as conference abstracts. ${ }^{42,43,45,64}$ Eighteen reported industry funding ${ }^{54,56,66,68 \text {, }}$ 69, 74, 75, 79, 83, 85, 92, 94, 96-98, 100, 104, 5 used materials supplied by industry, ${ }^{52,55,61,62,105} 165$ explicitly reported no industry support, ${ }^{19,42,49,51,53,57-59,70,72,73,76,81,91,99,102}$ and 19 did not provide funding information 20, 43, 45-48, 50, 63, 64, 67, 71, 80, 82, 89, 90, 93, 101 (Appendix C).

Eleven of the NRCS contained either matched cohorts or adjustments for known confounders, and they were included in the analysis; the remaining 34 have been tabulated in Appendix $\mathrm{G}^{106-140}$ Of the 11 NRCSs in 15 papers. ${ }^{141-155} 2$ reported industry funding, ${ }^{143,153} 6$ explicitly reported no industry support, ${ }^{142,144-148,150-152,155}$ and 3 did not provide funding information $^{141,149,154}$ (See Appendix C). Results from NRCSs are presented the end of each outcome section.

The studies primarily reported on basal cell carcinoma (BCC), with a minority reporting results for squamous cell carcinoma (SCC). Nearly all reported results for recurrence or cure rate outcomes and adverse events, and many reported results for cosmetic outcomes. Few studies reported results using validated instruments for quality of life, mental health, or patient satisfaction with treatment. Details about study design, baselines, and treatments are in Appendix C, D, and E, respectively. Risk of bias assessments are shown in Appendix F.

Because of the wide variety of adverse events reported (see Appendix $\mathrm{H}$ for a list of adverse events and how many studies reported each), we have limited the analysis to (1) adverse events that lead to treatment discontinuation, (2) any serious or severe adverse event (as defined by each study), (3) infections of the treatment site, and (4) pain after treatment. 
Figure 4. Literature flow diagram

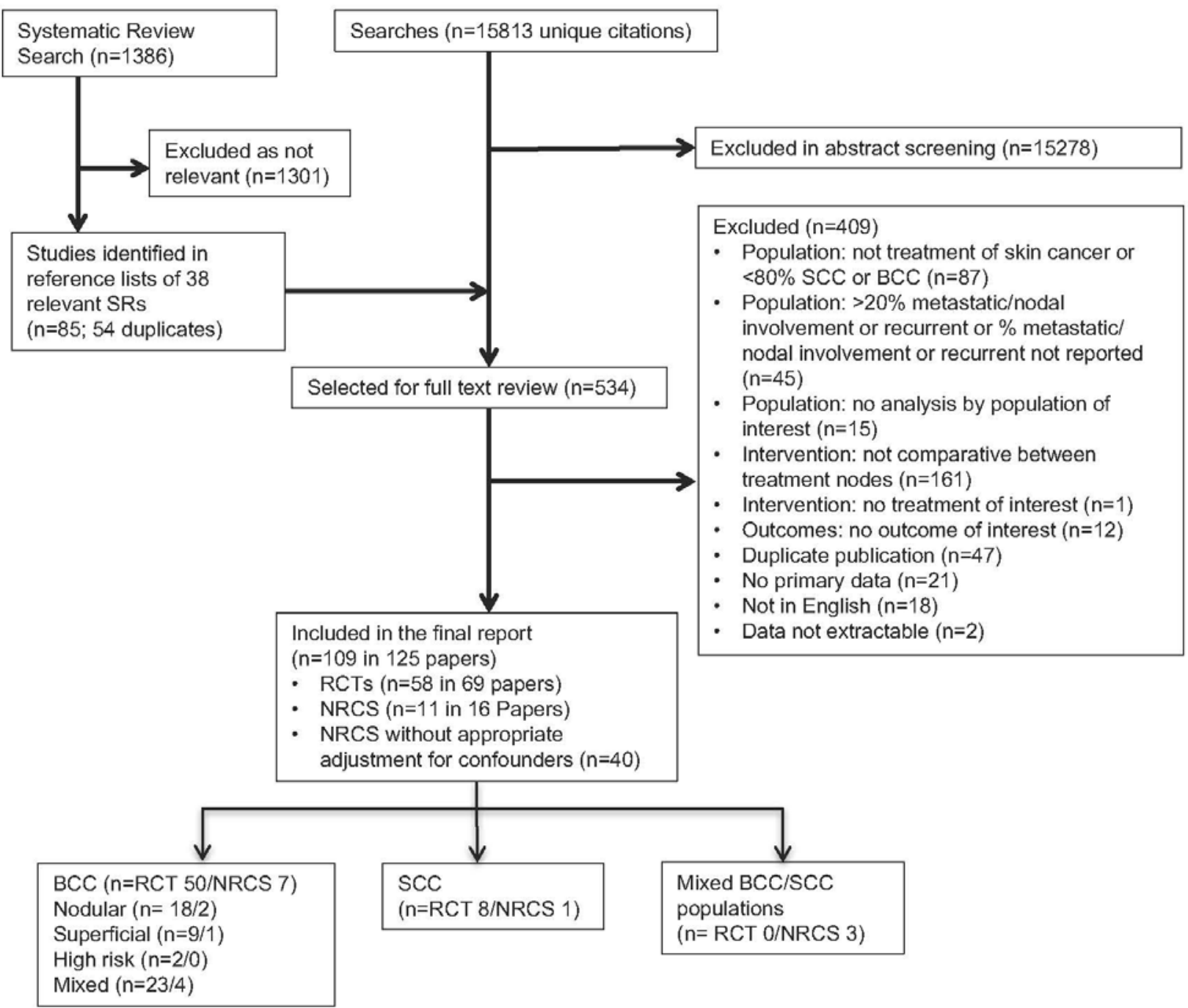

Note: Studies that enrolled both BCC and SCC populations are discussed in the BCC sections, because most enrolled lesions were BCCs.

$\mathrm{SR}$ = systematic review; SCC = squamous cell carcinoma; BCC = basal cell carcinoma; RCT = randomized controlled trial; NRCS = nonrandomized comparative study.

\section{Basal Cell Carcinoma}

The evidence graph in Figure 5 shows that there are 36 comparisons that have been observed between 29 interventions organized in 7 intervention categories.

This evidence graph suggests that limited conclusions can be drawn about which individual intervention is best (with respect to each outcome) for two reasons: 1) some interventions have never been compared with other interventions, directly or indirectly, and 2) the observed comparisons between individual interventions are relatively sparse.

Groups of interventions that have never been compared with other groups are readily identified in the Figure, because they are represented as connected subgraphs. For example, one 
connected subgraph comprises radiation therapy (external or brachytherapy, node D1|D2) versus surgery (surgical excision or Mohs micrographic surgery, node A|B). Another connected subgraph comprises laser ablation (C5) versus diclofenac and/or calcitriol (other medication F5) and versus no treatment (I). Four such subgraphs exist, and no conclusions can be drawn between interventions that belong to different subgraphs.

For individual interventions, the observed comparisons are relatively sparse: there are only 35 observed comparisons in the figure, out of the 378 that are possible among the 28 treatments. Further, information on each comparison is provided by at most three RCTs, and for most comparisons by only a single RCT. The evidence is even more sparse when one considers the information that is actually available for specific outcomes. Figure 6 shows the evidence graphs for the outcomes for which we have the most data, namely recurrence, lack of histologic clearance, and lack of clinical clearance. For these outcomes, no RCT data exist for 14, 8, and 14 of the 29 interventions, respectively. Evidence on other outcomes (quality of life, cosmetic outcomes, and costs or resource use) is even more sparse, as discussed in the following sections.

The evidence remains sparse at the level of individual interventions even after considering results from the seven eligible NRCSs, which are described separately from the RCTs.

Figure 5. Evidence graph depicting compared treatments in RCTs of BCC lesions

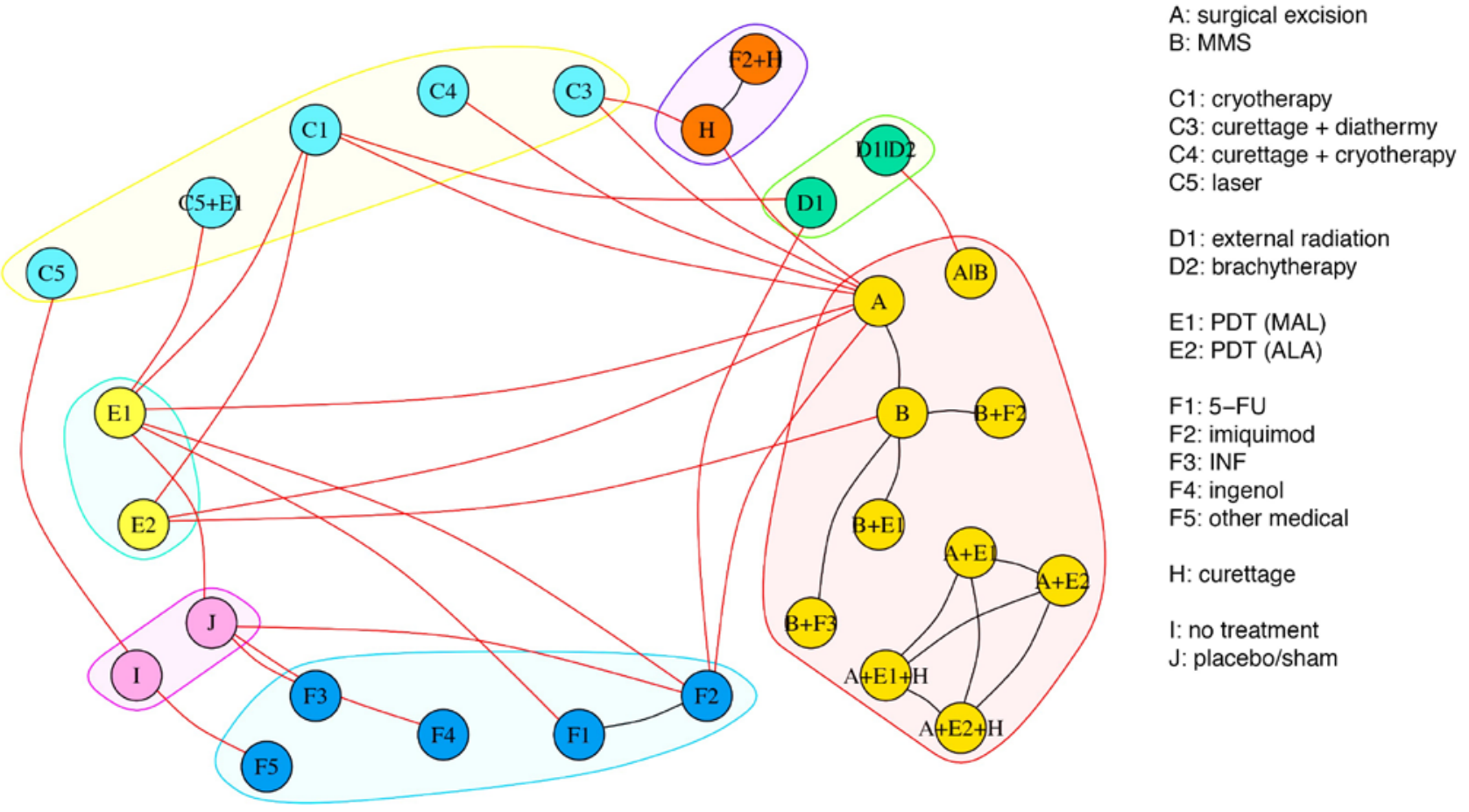

MMS = Mohs micrographic surgery; PDT = photodynamic therapy; ALA = 5-aminolevulinic acid; MAL = methyl aminolevulinate; FU = fluorouracil; INF = interferon 
Figure 6. Evidence graphs for recurrence, histologic clearance, and clinical clearance from RCTs of BCC lesions

\section{(A) Recurrence}

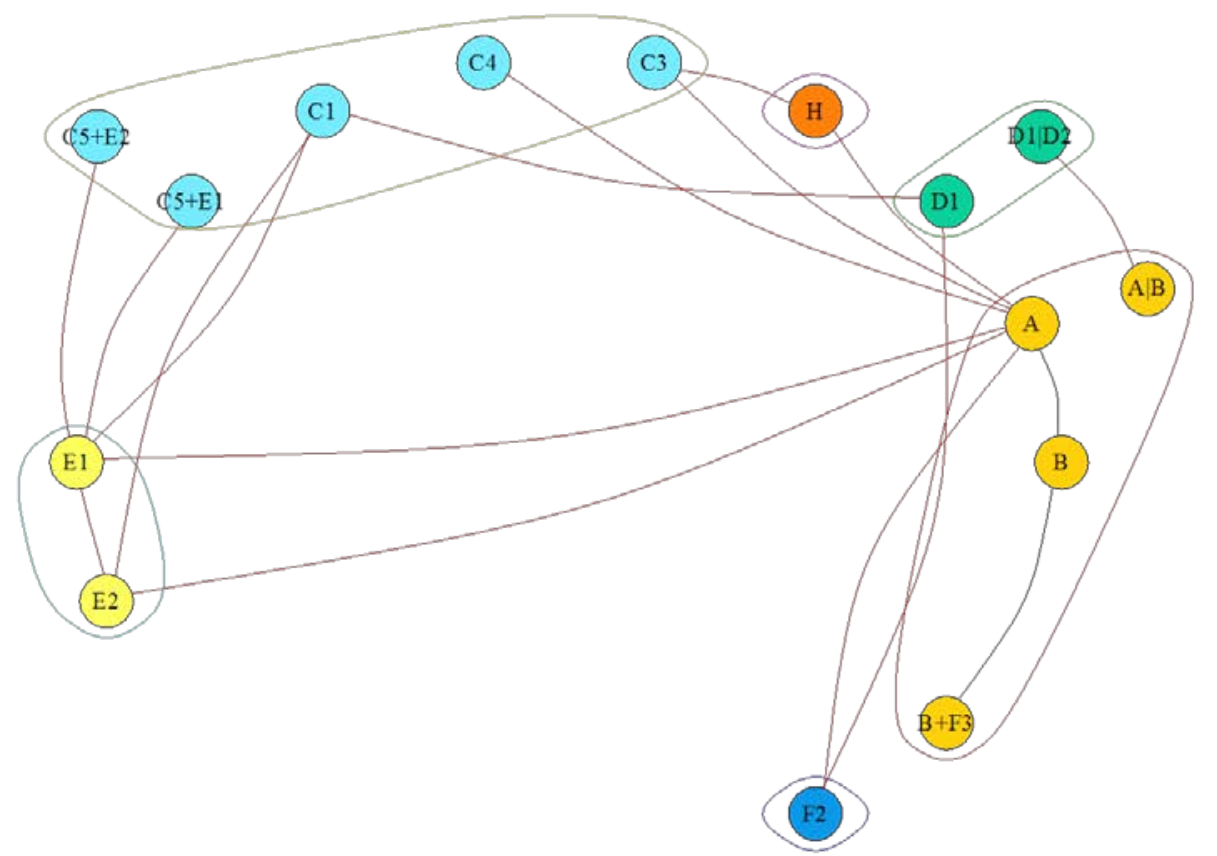

A: surgical excision

B: MMS

C1: cryotherapy

C3: curettage + diather my

C4: curettage + cr yotherapy

C5: laser

D1: external radiation

D2: brachytherapy

E1: PDT (MAL)

E2: PDT (ALA)

F1: 5-FU

F2: imiquimod

F3: INF

$\mathrm{F} 4$ : ingenol

F5: other medical

H: curettage

I: no treatment

J: placebo/sham

MMS = Mohs micrographic surgery; PDT = photodynamic therapy; ALA = 5-aminolevulinic acid; MAL= methyl aminolevulinate; FU = fluorouracil; INF = interferon

\section{(B) Lack of Histologic Clearance}

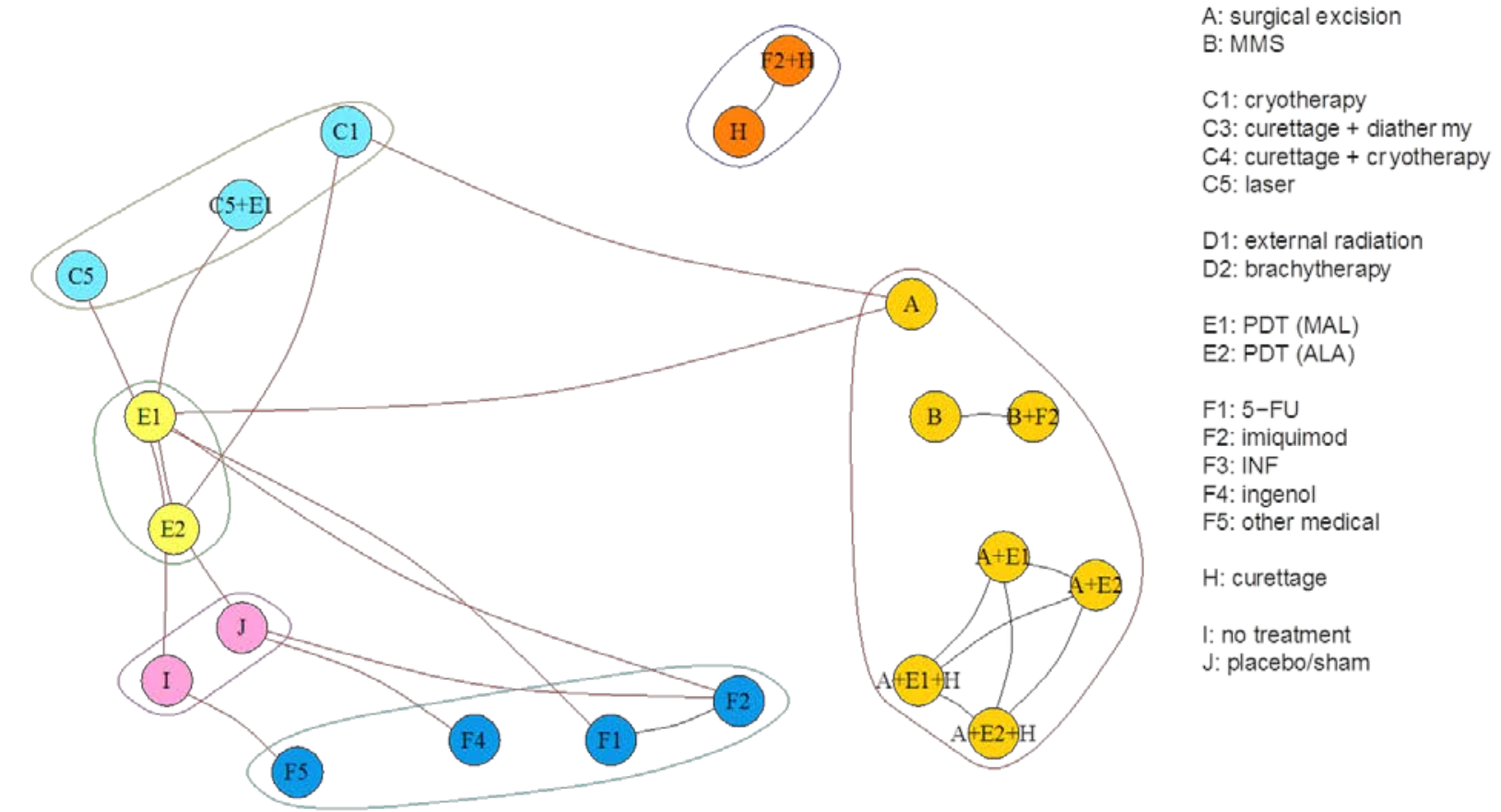

MMS = Mohs micrographic surgery; PDT = photodynamic therapy; ALA = 5-aminolevulinic acid; MAL = methyl aminolevulinate; FU = fluorouracil; INF = interferon 
(C) Lack of Clinical Clearance

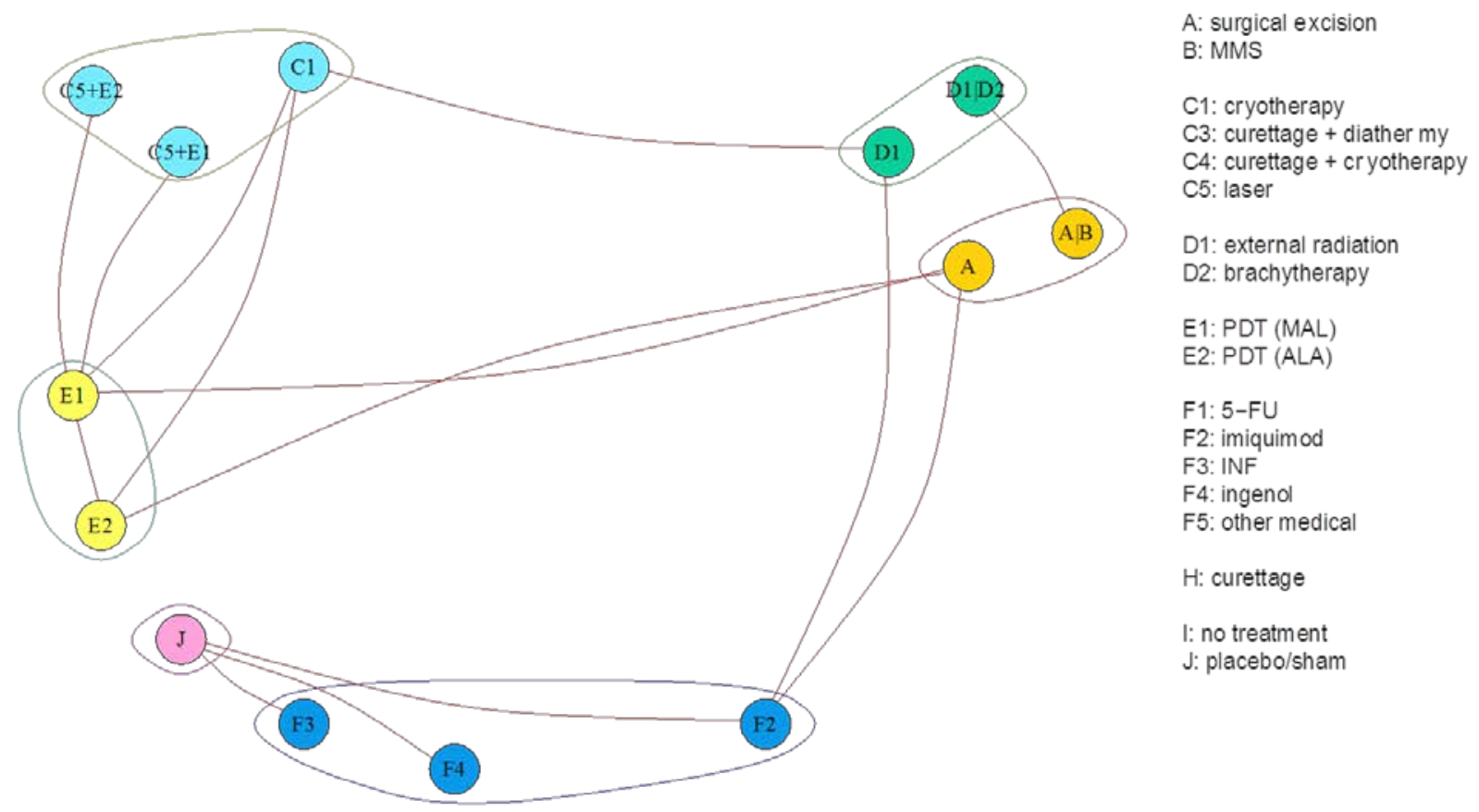

MMS = Mohs micrographic surgery; PDT = photodynamic therapy; ALA = 5-aminolevulinic acid; MAL = methyl aminolevulinate; FU = fluorouracil; INF = interferon

The characteristics of the included RCTs are summarized in Tables 3 through 6 , for RCTs on superficial $(n=9)$, nodular $(n=18)$, high-risk $(n=2)$, and mixed types $(n=21)$ of BCC lesions. RCTs that report stratified results for different types of lesions are listed in the mixed table.

Across all trials, the mean or median age of enrollees ranged between 55 and 75.3 (median: $64,25^{\text {th }}-75^{\text {th }}$ percentile: 61 to 67 ). The proportion of female patients ranged between 0 and 75 percent (median: $37,25^{\text {th }}-75^{\text {th }}$ percentile: 30 to 43 ). When reported, the mean or median lesion area was between 30.1 and $205 \mathrm{~mm}^{2}$, and the median maximum diameter was between 5.3 and $12 \mathrm{~mm}$. The majority of RCTs included lesions in various body locations, and only a few reported results stratified by lesion location (discussed separately). Based on this information, the RCTs included patients and lesions are typically encountered in clinical practice, but the lack of information on treatment effect heterogeneity with respect to patient-level factors hinders extrapolation to specific patient subgroups. No RCT focused on patients who were immunocompromised or had substantially limited life expectancy.

In terms of design characteristics, 29 RCTs had two arms, 5 had three arms, and 15 had four or more; the latter were primarily phase II studies, examining the tolerability of various doses or schedules of topically applied medications or alternative photodynamic treatment protocols. Such phase II studies are included in the comparisons between interventions only when they include a no intervention or placebo/sham intervention arm. Their findings with respect to different doses or protocols for the same intervention are summarized separately. Analyzed sample sizes ranged between 18 and 694 (median: 70, $25^{\text {th }}-75^{\text {th }}$ percentile: 31 to 126.5); sample sizes per RCT arm ranged between 3 and 408. 
Based on what was reported in the RCTs, we deemed that the allocation sequence was randomized using formal methods in 26 and successfully concealed in 25 RCTs, and that patients, providers, and outcome assessors were successfully blinded to the received treatments in 19, 13, and 19 RCTs, respectively. Our consensus assessment of the reported baseline characteristics across the compared arms in each RCT was that most RCTs $(n=28)$ had arms that were likely balanced at baseline. In 41 RCTs fewer than 20 percent of patients had missing outcomes for any eligible outcome in any arm. 
Table 3. Characteristics of RCTs of superficial BCCs

\begin{tabular}{|c|c|c|c|c|c|c|c|c|c|c|c|c|}
\hline Study & Arm & $\begin{array}{l}\text { Age, } \\
\text { Mean }\end{array}$ & $\begin{array}{l}\text { Female } \\
\%\end{array}$ & $\begin{array}{l}\text { Lesion } \\
\text { Size, } \\
\text { Mean }\end{array}$ & $\begin{array}{l}\text { Lesion location } \\
\text { (\%) }\end{array}$ & $\begin{array}{l}\mathbf{1}^{*} \\
\text { Adequate } \\
\text { Randomiz- } \\
\text { ation }\end{array}$ & $\begin{array}{l}2^{*} \\
\text { Allocation } \\
\text { Conceal- } \\
\text { ment }\end{array}$ & $\begin{array}{l}3^{*} \\
\text { Arms } \\
\text { Similar at } \\
\text { Baseline }\end{array}$ & $\begin{array}{l}4^{*} \\
\text { Patients } \\
\text { Blinded }\end{array}$ & $\begin{array}{l}5^{\star} \\
\text { Providers } \\
\text { Blinded }\end{array}$ & $\begin{array}{l}6^{*} \\
\text { Outcome } \\
\text { Assessors } \\
\text { Blinded }\end{array}$ & $\begin{array}{l}7^{*} \\
<20 \% \\
\text { Loss to } \\
\text { Followup }\end{array}$ \\
\hline \multirow[t]{3}{*}{$\begin{array}{l}\text { Arits } 2013 \\
23683751\end{array}$} & $\begin{array}{l}\text { MAL- } \\
\text { PDT }\end{array}$ & $\begin{array}{l}\text { median } \\
63\end{array}$ & 52 & NR & $\begin{array}{l}\text { head/neck } \\
\text { excluding H-zone } \\
\text { (12), extremities } \\
\text { (29), trunk (59), } \\
\text { upper extremities } \\
\text { (16), lower } \\
\text { extremities (13) }\end{array}$ & Yes & Yes & Yes & No & No & Yes & Yes \\
\hline & $\begin{array}{l}\text { Imiquim } \\
\text { od }\end{array}$ & $\begin{array}{l}\text { median } \\
62\end{array}$ & 49 & NR & $\begin{array}{l}\text { head/neck } \\
\text { excluding H-zone } \\
\text { (12), extremities } \\
\text { (27), trunk (61), } \\
\text { upper extremities } \\
\text { (13), lower } \\
\text { extremities (14) }\end{array}$ & & & & & & & \\
\hline & $\begin{array}{l}\text { Fluorour } \\
\text { acil }\end{array}$ & $\begin{array}{l}\text { median } \\
64\end{array}$ & 47 & NR & $\begin{array}{l}\text { head/neck } \\
\text { excluding H-zone } \\
\text { (15), extremities } \\
\text { (24), trunk (60), } \\
\text { upper extremities } \\
\text { (13), lower } \\
\text { extremities (11) }\end{array}$ & & & & & & & \\
\hline \multirow{2}{*}{$\begin{array}{l}\text { Basset- } \\
\text { Seguin } \\
2008 \\
18693158\end{array}$} & $\begin{array}{l}\text { MAL- } \\
\text { PDT }\end{array}$ & 62 & 33 & NR & $\begin{array}{l}\text { face/scalp (6), } \\
\text { extremities (22), } \\
\text { trunk/neck (72) }\end{array}$ & No & Yes & Yes & No & No & Unsure & Yes \\
\hline & $\begin{array}{l}\text { Cryothe } \\
\text { rapy }\end{array}$ & 64 & 47 & NR & $\begin{array}{l}\text { face/scalp (4), } \\
\text { extremities (20), } \\
\text { trunk/neck (76) }\end{array}$ & & & & & & & \\
\hline $\begin{array}{l}\text { Beutner } \\
1999 \\
10570388\end{array}$ & $\begin{array}{l}\text { imiquim } \\
\text { od } \\
3 x / \text { week }\end{array}$ & NR & NR & NR & $\begin{array}{l}\text { upper extremity } \\
(25), \text { anterior } \\
\text { upper trunk (25), } \\
\text { posterior upper } \\
\text { trunk (25), neck } \\
(25)\end{array}$ & No & No & No & Unsure & Yes & Unsure & Yes \\
\hline $\begin{array}{l}\text { Geisse } \\
2002 \\
12196749\end{array}$ & $\begin{array}{l}\text { Imiquim } \\
\text { od } \\
3 x / w k\end{array}$ & 62 & NR & $\begin{array}{l}\text { median } \\
1.0 \mathrm{~cm}^{2}\end{array}$ & $\begin{array}{l}\text { neck/face/forehea } \\
\text { d (4), upper } \\
\text { extremity (not }\end{array}$ & Yes & Yes & Yes & Yes & Yes & Yes & Yes \\
\hline
\end{tabular}




\begin{tabular}{|c|c|c|c|c|c|c|c|c|c|c|c|c|}
\hline Study & Arm & $\begin{array}{l}\text { Age, } \\
\text { Mean }\end{array}$ & $\begin{array}{l}\text { Female } \\
\%\end{array}$ & $\begin{array}{l}\text { Lesion } \\
\text { Size, } \\
\text { Mean }\end{array}$ & $\begin{array}{l}\text { Lesion location } \\
\text { (\%) }\end{array}$ & $\begin{array}{l}1^{*} \\
\text { Adequate } \\
\text { Randomiz- } \\
\text { ation } \\
\end{array}$ & $\begin{array}{l}2^{*} \\
\text { Allocation } \\
\text { Conceal- } \\
\text { ment } \\
\end{array}$ & $\begin{array}{l}3^{*} \\
\text { Arms } \\
\text { Similar at } \\
\text { Baseline } \\
\end{array}$ & $\begin{array}{l}4^{*} \\
\text { Patients } \\
\text { Blinded }\end{array}$ & $\begin{array}{l}5^{\star} \\
\text { Providers } \\
\text { Blinded }\end{array}$ & $\begin{array}{l}6^{*} \\
\text { Outcome } \\
\text { Assessors } \\
\text { Blinded } \\
\end{array}$ & $\begin{array}{l}7^{*} \\
<20 \% \\
\text { Loss to } \\
\text { Followup }\end{array}$ \\
\hline & & & & & $\begin{array}{l}\text { hand) (15), trunk } \\
\text { (73), lower } \\
\text { extremity/thigh } \\
\text { (not foot) (8) }\end{array}$ & & & & & & & \\
\hline & $\begin{array}{l}\text { Imiquim } \\
\text { od } \\
5 x / \text { wk }\end{array}$ & 55 & NR & $\begin{array}{l}\text { median } \\
0.6 \mathrm{~cm}^{2}\end{array}$ & $\begin{array}{l}\text { neck/face/forehea } \\
d(3), \text { upper } \\
\text { extremity (not } \\
\text { hand) (31), trunk } \\
\text { (55), lower } \\
\text { extremity/thigh } \\
\text { (not foot) (10) }\end{array}$ & & & & & & & \\
\hline & $\begin{array}{l}\text { Imiquim } \\
\text { od } \\
\text { 1x/day }\end{array}$ & 56 & NR & $\begin{array}{l}\text { median } \\
0.7 \mathrm{~cm}^{2}\end{array}$ & $\begin{array}{l}\text { neck/face/forehea } \\
d(7), \text { upper } \\
\text { extremity (not } \\
\text { hand) (21), trunk } \\
\text { (64), lower } \\
\text { extremity/thigh } \\
\text { (not foot) (7) }\end{array}$ & & & & & & & \\
\hline & $\begin{array}{l}\text { Imiquim } \\
\text { od } \\
2 x / \text { day }\end{array}$ & 69 & NR & $\begin{array}{l}\text { median } \\
1.0 \mathrm{~cm}^{2}\end{array}$ & $\begin{array}{l}\text { neck/face/forehea } \\
d \text { (8), upper } \\
\text { extremity (not } \\
\text { hand) (54), trunk } \\
\text { (31), lower } \\
\text { extremity/thigh } \\
\text { (not foot) (8) }\end{array}$ & & & & & & & \\
\hline & $\begin{array}{l}\text { vehicle } \\
\text { (control) }\end{array}$ & 58 & NR & $\begin{array}{l}\text { median } \\
0.8 \mathrm{~cm}^{2}\end{array}$ & $\begin{array}{l}\text { neck/face/forehea } \\
d(9), \text { upper } \\
\text { extremity (not } \\
\text { hand) (34), trunk } \\
\text { (47), lower } \\
\text { extremity/thigh } \\
\text { (not foot) (9) }\end{array}$ & & & & & & & \\
\hline \multirow[t]{2}{*}{$\begin{array}{l}\text { Schleier } \\
2007 \\
25047438\end{array}$} & $\begin{array}{l}\text { ALA- } \\
\text { thermog } \\
\text { el PDT }\end{array}$ & 69.9 & 46.15 & NR & $\begin{array}{l}\text { face (54.17), scalp } \\
\text { (20.83), lip (2.78), } \\
\text { eyelid (1.39), } \\
\text { extremities (9.72), } \\
\text { trunk/neck (11.11) }\end{array}$ & \multirow[t]{2}{*}{ Yes } & \multirow[t]{2}{*}{ No } & \multirow[t]{2}{*}{ Yes } & \multirow[t]{2}{*}{ Yes } & \multirow[t]{2}{*}{ Yes } & \multirow[t]{2}{*}{ Yes } & \multirow[t]{2}{*}{ Yes } \\
\hline & $\begin{array}{l}\text { Methyl- } \\
\text { ALA- }\end{array}$ & 71.8 & 36.36 & NR & $\begin{array}{l}\text { face (52.5), scalp } \\
\text { (30), extremities }\end{array}$ & & & & & & & \\
\hline
\end{tabular}




\begin{tabular}{|c|c|c|c|c|c|c|c|c|c|c|c|c|}
\hline Study & Arm & $\begin{array}{l}\text { Age, } \\
\text { Mean }\end{array}$ & $\begin{array}{l}\text { Female } \\
\%\end{array}$ & $\begin{array}{l}\text { Lesion } \\
\text { Size, } \\
\text { Mean }\end{array}$ & $\begin{array}{l}\text { Lesion location } \\
\text { (\%) }\end{array}$ & $\begin{array}{l}1^{*} \\
\text { Adequate } \\
\text { Randomiz- } \\
\text { ation } \\
\end{array}$ & $\begin{array}{l}2^{*} \\
\text { Allocation } \\
\text { Conceal- } \\
\text { ment } \\
\end{array}$ & $\begin{array}{l}3^{*} \\
\text { Arms } \\
\text { Similar at } \\
\text { Baseline } \\
\end{array}$ & $\begin{array}{l}4^{*} \\
\text { Patients } \\
\text { Blinded }\end{array}$ & $\begin{array}{l}5^{*} \\
\text { Providers } \\
\text { Blinded }\end{array}$ & $\begin{array}{l}6^{*} \\
\text { Outcome } \\
\text { Assessors } \\
\text { Blinded } \\
\end{array}$ & $\begin{array}{l}7^{*} \\
<20 \% \\
\text { Loss to } \\
\text { Followup }\end{array}$ \\
\hline & $\begin{array}{l}\text { thermog } \\
\text { el PDT }\end{array}$ & & & & $\begin{array}{l}\text { (5), trunk/neck } \\
(12.5)\end{array}$ & & & & & & & \\
\hline \multirow[t]{2}{*}{$\begin{array}{l}\text { Schulze } \\
2005 \\
15888150\end{array}$} & $\begin{array}{l}\text { imiquim } \\
\text { od 5\% }\end{array}$ & 64.3 & 39 & NR & $\begin{array}{l}\text { cheek (1), } \\
\text { forehead (0), } \\
\text { extremities } \\
\text { (including hand) } \\
\text { (20), trunk/neck } \\
\text { (70) }\end{array}$ & Yes & Yes & Yes & Yes & No & Unsure & No \\
\hline & vehicle & 64.5 & 39 & NR & $\begin{array}{l}\text { cheek (1), } \\
\text { forehead (5), } \\
\text { scalp (1), } \\
\text { extremities } \\
\text { (including hand) } \\
\text { (30), trunk/neck } \\
(61)\end{array}$ & & & & & & & \\
\hline $\begin{array}{l}\text { Siller } 2010 \\
20546215\end{array}$ & $\begin{array}{l}\text { Total } \\
\text { (ingenol } \\
\text { mebutat } \\
\text { e vs } \\
\text { placebo } \\
\end{array}$ & 59 & 27 & $9 \mathrm{~mm}$ & NR & Yes & Yes & Yes & Yes & Yes & Yes & Unsure \\
\hline \multirow[t]{3}{*}{$\begin{array}{l}\text { Sterry } 2002 \\
12452875 \\
\text { (superficial) }\end{array}$} & $\begin{array}{l}\text { Imiquim } \\
\text { od (2 } \\
\text { days/ } \\
\text { week) } \\
\text { with } \\
\text { occlusio } \\
\mathrm{n}\end{array}$ & 63 & 33 & $\begin{array}{l}\text { median } \\
1.5 \mathrm{~cm}^{2}\end{array}$ & $\begin{array}{l}\text { extremities (29), } \\
\text { trunk/neck (71) }\end{array}$ & Yes & Yes & No & No & No & Unsure & Yes \\
\hline & $\begin{array}{l}\text { Imiquim } \\
\text { od (3 } \\
\text { days/ } \\
\text { week) } \\
\text { with } \\
\text { occlusio } \\
\mathrm{n}\end{array}$ & 58 & 35 & $\begin{array}{l}\text { median } \\
1.2 \mathrm{~cm}^{2}\end{array}$ & $\begin{array}{l}\text { extremities (31), } \\
\text { trunk/neck (69) }\end{array}$ & & & & & & & \\
\hline & $\begin{array}{l}\text { Imiquim } \\
\text { od (2 } \\
\text { days/ } \\
\text { week) }\end{array}$ & 69 & 33 & $\begin{array}{l}\text { median } \\
1.0 \mathrm{~cm}^{2}\end{array}$ & $\begin{array}{l}\text { face }(8) . \\
\text { extremities (30), } \\
\text { trunk/neck (62) }\end{array}$ & & & & & & & \\
\hline
\end{tabular}




\begin{tabular}{|c|c|c|c|c|c|c|c|c|c|c|c|c|}
\hline Study & Arm & $\begin{array}{l}\text { Age, } \\
\text { Mean }\end{array}$ & $\begin{array}{l}\text { Female } \\
\%\end{array}$ & $\begin{array}{l}\text { Lesion } \\
\text { Size, } \\
\text { Mean }\end{array}$ & $\begin{array}{l}\text { Lesion location } \\
\text { (\%) }\end{array}$ & $\begin{array}{l}1^{*} \\
\text { Adequate } \\
\text { Randomiz- } \\
\text { ation } \\
\end{array}$ & $\begin{array}{l}2^{*} \\
\text { Allocation } \\
\text { Conceal- } \\
\text { ment } \\
\end{array}$ & $\begin{array}{l}* \\
\text { Arms } \\
\text { Similar at } \\
\text { Baseline }\end{array}$ & $\begin{array}{l}4^{*} \\
\text { Patients } \\
\text { Blinded }\end{array}$ & $\begin{array}{l}5^{\star} \\
\text { Providers } \\
\text { Blinded }\end{array}$ & $\begin{array}{l}6^{*} \\
\text { Outcome } \\
\text { Assessors } \\
\text { Blinded } \\
\end{array}$ & $\begin{array}{l}7^{*} \\
<20 \% \\
\text { Loss to } \\
\text { Followup }\end{array}$ \\
\hline & $\begin{array}{l}\text { without } \\
\text { occlusio } \\
\mathrm{n}\end{array}$ & & & & & & & & & & & \\
\hline & $\begin{array}{l}\text { Imiquim } \\
\text { od (3 } \\
\text { days/ } \\
\text { week) } \\
\text { without } \\
\text { occlusio } \\
\mathrm{n}\end{array}$ & 61 & 44 & $\begin{array}{l}\text { median } \\
1.0 \mathrm{~cm}^{2}\end{array}$ & $\begin{array}{l}\text { extremities (32), } \\
\text { trunk/neck (64), } \\
\text { genitals (4) }\end{array}$ & & & & & & & \\
\hline \multirow[t]{2}{*}{$\begin{array}{l}\text { Szeimies } \\
2008 \\
18624836\end{array}$} & $\begin{array}{l}\text { MAL- } \\
\text { PDT }\end{array}$ & 64.5 & 36.0 & $\begin{array}{l}12.5 \\
\mathrm{~mm}\end{array}$ & $\begin{array}{l}\text { face/scalp (11.1), } \\
\text { extremities (28.9), } \\
\text { trunk/neck (60) }\end{array}$ & \multirow[t]{2}{*}{ Yes } & \multirow[t]{2}{*}{ Yes } & \multirow[t]{2}{*}{ Yes } & \multirow[t]{2}{*}{ No } & \multirow[t]{2}{*}{ No } & \multirow[t]{2}{*}{ No } & \multirow[t]{2}{*}{ Yes } \\
\hline & excision & 63.1 & 31.3 & $\begin{array}{l}12.6 \\
\mathrm{~mm}\end{array}$ & $\begin{array}{l}\text { face/scalp (4.5), } \\
\text { extremities }(25.0) \text {, } \\
\text { trunk/neck }(70.5)\end{array}$ & & & & & & & \\
\hline
\end{tabular}

fluorouracil; INF = interferon; RCT = randomized controlled trial; NR = not reported; $\mathrm{x} / \mathrm{wk}=$ times per week

*Design items: 1: Adequate generation of a randomized sequence reported; 2: Adequate allocation concealment reported; 3: Group similarity at baseline; 4: Adequate blinding of patients reported; 5: Adequate blinding of providers reported; 6: Adequate blinding of outcome assessors reported; 7: Less than 20\% missing for any eligible outcome in any arm. 
Table 4. Characteristics of RCTs of nodular BCC

\begin{tabular}{|c|c|c|c|c|c|c|c|c|c|c|c|c|}
\hline Study & Arm & $\begin{array}{l}\text { Age, } \\
\text { Mean }\end{array}$ & $\begin{array}{l}\text { Female } \\
\%\end{array}$ & $\begin{array}{l}\text { Lesion } \\
\text { Size, } \\
\text { Mean }\end{array}$ & $\begin{array}{l}\text { Lesion } \\
\text { location (\%) }\end{array}$ & $\begin{array}{l}\mathbf{1}^{*} \\
\text { Adequa } \\
\text { te } \\
\text { Rando } \\
\text { mizatio } \\
\mathrm{n}\end{array}$ & $\begin{array}{l}\text { 2* } \\
\text { Allocati } \\
\text { on } \\
\text { Concea } \\
\text { Iment }\end{array}$ & $\begin{array}{l}3^{*} \\
\text { Arms } \\
\text { Similar at } \\
\text { Baseline }\end{array}$ & $\begin{array}{l}4^{*} \\
\text { Patien } \\
\text { ts } \\
\text { Blinde } \\
\text { d }\end{array}$ & $\begin{array}{l}5^{\star} \\
\text { Provide } \\
\text { rs } \\
\text { Blinded }\end{array}$ & $\begin{array}{l}6^{*} \\
\text { Outcome } \\
\text { Assessor } \\
\text { s Blinded }\end{array}$ & $\begin{array}{l}7^{*} \\
<20 \% \\
\text { Loss to } \\
\text { Followu } \\
\text { p }\end{array}$ \\
\hline \multirow[t]{2}{*}{ Abbade 2015} & $\begin{array}{l}\text { Surgical } \\
\text { excision }\end{array}$ & NR & NR & NR & $\begin{array}{l}\text { head and neck } \\
(100)\end{array}$ & No & No & Yes & No & No & unsure & Yes \\
\hline & MAL-PDT & NR & NR & NR & $\begin{array}{l}\text { head and neck } \\
(100)\end{array}$ & & & & & & & \\
\hline \multirow{2}{*}{$\begin{array}{l}\text { Al-Niaimi } \\
2015 \\
26157307\end{array}$} & $\begin{array}{l}\text { PDT + } \\
\text { MMS }\end{array}$ & 61.4 & 66.7 & $\begin{array}{l}200 \\
\mathrm{~mm}^{2}\end{array}$ & face (100) & No & Yes & Yes & No & No & Yes & No \\
\hline & MMS & 62.7 & 40 & $\begin{array}{l}201 \\
\mathrm{~mm} 2\end{array}$ & face (100) & & & & & & & \\
\hline $\begin{array}{l}\text { Berroeta } 2007 \\
17573890\end{array}$ & $\begin{array}{l}\text { Total (PDT } \\
\text { vs. } \\
\text { excision) }\end{array}$ & $\begin{array}{l}\text { median } \\
72\end{array}$ & NR & NR & NR & Yes & Yes & unsure & No & No & Yes & Yes \\
\hline \multirow[t]{2}{*}{$\begin{array}{l}\text { Butler } 2009 \\
19018814\end{array}$} & $\begin{array}{l}\text { Vehicle } \\
\text { group } \\
+ \text { MMS }\end{array}$ & 75.3 & 43.8 & $\begin{array}{l}30.1 \\
\mathrm{~mm} 2\end{array}$ & face (100) & Yes & Yes & Yes & Yes & Yes & Yes & Yes \\
\hline & $\begin{array}{l}\text { imiquimod } \\
5 \% \text { Cream } \\
\text { group } \\
+ \text { MMS }\end{array}$ & 73.3 & 66.7 & $\begin{array}{l}33.5 \\
\mathrm{~mm} 2\end{array}$ & hands (100) & & & & & & & \\
\hline \multirow[t]{2}{*}{$\begin{array}{l}\text { Choi } 2016 \\
26551044\end{array}$} & $\begin{array}{l}\text { Er:YAG } \\
\text { ablative } \\
\text { fractional } \\
\text { laser- } \\
\text { primed } \\
\text { MAL- PDT }\end{array}$ & NR & 55 & NR & NR & No & Yes & Yes & Yes & Yes & Yes & Yes \\
\hline & MAL-PDT & NR & 36.8 & NR & NR & & & & & & & \\
\hline $\begin{array}{l}\text { Eigentler } \\
2007 \\
17610993\end{array}$ & $\begin{array}{l}\text { imiquimod } \\
5 \% 8 \\
\text { weeks }\end{array}$ & $\begin{array}{l}\text { median } \\
65\end{array}$ & 27 & $\begin{array}{l}8.2 \\
\mathrm{~mm}\end{array}$ & $\begin{array}{l}\text { face (24.4), } \\
\text { scalp (2.2), ear } \\
(8.9), \\
\text { trunk/neck } \\
(4.4), \text { perioral } \\
(4.4), \\
\text { periorbital } \\
(8.9), \text { nose } \\
(42), \\
\text { arm/shoulder }\end{array}$ & No & No & Unsure & No & unsure & unsure & Yes \\
\hline
\end{tabular}




\begin{tabular}{|c|c|c|c|c|c|c|c|c|c|c|c|c|}
\hline Study & Arm & $\begin{array}{l}\text { Age, } \\
\text { Mean }\end{array}$ & $\begin{array}{l}\text { Female } \\
\%\end{array}$ & $\begin{array}{l}\text { Lesion } \\
\text { Size, } \\
\text { Mean }\end{array}$ & $\begin{array}{l}\text { Lesion } \\
\text { location (\%) }\end{array}$ & $\begin{array}{l}\mathbf{1}^{*} \\
\text { Adequa } \\
\text { te } \\
\text { Rando } \\
\text { mizatio } \\
\mathrm{n}\end{array}$ & $\begin{array}{l}2 * \\
\text { Allocati } \\
\text { on } \\
\text { Concea } \\
\text { Iment }\end{array}$ & $\begin{array}{l}3^{*} \\
\text { Arms } \\
\text { Similar at } \\
\text { Baseline }\end{array}$ & $\begin{array}{l}4^{*} \\
\text { Patien } \\
\text { ts } \\
\text { Blinde } \\
\text { d }\end{array}$ & $\begin{array}{l}5^{\star} \\
\text { Provide } \\
\text { rs } \\
\text { Blinded }\end{array}$ & $\begin{array}{l}6^{*} \\
\text { Outcome } \\
\text { Assessor } \\
\text { s Blinded }\end{array}$ & $\begin{array}{l}7^{*} \\
<20 \% \\
\text { Loss to } \\
\text { Followu } \\
p\end{array}$ \\
\hline & & & & & $(4.4)$ & & & & & & & \\
\hline & $\begin{array}{l}\text { imiquimod } \\
5 \% 12 \\
\text { weeks }\end{array}$ & $\begin{array}{l}\text { median } \\
63\end{array}$ & 33 & $\begin{array}{l}9.6 \\
\mathrm{~mm}\end{array}$ & $\begin{array}{l}\text { face (19.6), } \\
\text { scalp (2.2), ear } \\
(10.9), \\
\text { trunk/neck } \\
(8.7), \text { perioral } \\
(2.2), \\
\text { periorbital } \\
(6.5), \text { nose } \\
(37), \\
\text { arm/shoulder } \\
(4.4), \text { leg/hip } \\
(4.3)\end{array}$ & & & & & & & \\
\hline \multirow[t]{2}{*}{$\begin{array}{l}\text { Foley } 2009 \\
20064185\end{array}$} & $\begin{array}{l}\text { methyl- } \\
\text { aminolevuli } \\
\text { natePDT }\end{array}$ & 66 & 28.78 & $\begin{array}{l}8.8 \\
\mathrm{~mm}\end{array}$ & $\begin{array}{l}\text { face/scalp } \\
(25), \\
\text { extremities } \\
(20), \text { Trunk } 32 \\
(43 \%) \\
\text { Neck } 9(12 \%)\end{array}$ & Yes & Yes & unsure & Yes & Yes & Yes & Yes \\
\hline & $\begin{array}{l}\text { placebo } \\
\text { PDT }\end{array}$ & 67 & 20 & $\begin{array}{l}9.0 \\
\mathrm{~mm}\end{array}$ & $\begin{array}{l}\text { face/scalp } \\
(31), \\
\text { extremities } \\
(23), \text { Trunk } 34 \\
(45 \%) \\
\text { Neck } 1(1 \%)\end{array}$ & & & & & & & \\
\hline \multirow[t]{2}{*}{$\begin{array}{l}\text { Haak } 2015 \\
24903544\end{array}$} & MAL PDT & NR & 37.5 & $\begin{array}{l}\text { median } \\
8.5 \\
\mathrm{~mm}\end{array}$ & $\begin{array}{l}\text { nose (37), } \\
\text { forehead (31), } \\
\text { cheek (6), oral } \\
\text { area (13), } \\
\text { periorbital area } \\
(13)\end{array}$ & Yes & Yes & Yes & No & No & Yes & Yes \\
\hline & $\begin{array}{l}\text { AFXL MAL } \\
\text { PDT }\end{array}$ & NR & 68.8 & $\begin{array}{l}\text { median } \\
7 \mathrm{~mm}\end{array}$ & $\begin{array}{l}\text { nose (56), } \\
\text { forehead (19), } \\
\text { cheek (13), } \\
\text { oral area (6), } \\
\text { periorbital area } \\
(6)\end{array}$ & & & & & & & \\
\hline
\end{tabular}




\begin{tabular}{|c|c|c|c|c|c|c|c|c|c|c|c|c|}
\hline Study & Arm & $\begin{array}{l}\text { Age, } \\
\text { Mean }\end{array}$ & $\begin{array}{l}\text { Female } \\
\%\end{array}$ & $\begin{array}{l}\text { Lesion } \\
\text { Size, } \\
\text { Mean }\end{array}$ & $\begin{array}{l}\text { Lesion } \\
\text { location (\%) }\end{array}$ & $\begin{array}{l}\mathbf{1}^{*} \\
\text { Adequa } \\
\text { te } \\
\text { Rando } \\
\text { mizatio } \\
\mathrm{n}\end{array}$ & $\begin{array}{l}2^{*} \\
\text { Allocati } \\
\text { on } \\
\text { Concea } \\
\text { Iment }\end{array}$ & $\begin{array}{l}3^{*} \\
\text { Arms } \\
\text { Similar at } \\
\text { Baseline }\end{array}$ & $\begin{array}{l}* \\
\text { Patien } \\
\text { ts } \\
\text { Blinde } \\
\text { d }\end{array}$ & $\begin{array}{l}5^{*} \\
\text { Provide } \\
\text { rs } \\
\text { Blinded }\end{array}$ & $\begin{array}{l}6^{*} \\
\text { Outcome } \\
\text { Assessor } \\
\text { s Blinded }\end{array}$ & $\begin{array}{l}7^{*} \\
<20 \% \\
\text { Loss to } \\
\text { Followu } \\
p\end{array}$ \\
\hline \multirow[t]{6}{*}{$\begin{array}{l}\text { Kuijpers } 2006 \\
16865869\end{array}$} & $\begin{array}{l}\text { ALA-PDT } \\
\text { (total) }\end{array}$ & 68.4 & 34.9 & $\begin{array}{l}8.1 \\
\mathrm{~mm}\end{array}$ & $\begin{array}{l}\text { forehead/templ } \\
\text { e+nose/parana } \\
\text { sal }(36.4), \\
\text { cheek/chin/lips } \\
(9.1), \text { ears } \\
(9.1), \\
\text { extremities } \\
(9.1), \\
\text { trunk/neck } \\
(36.4)\end{array}$ & Yes & Yes & unsure & No & No & Unsure & No \\
\hline & $\begin{array}{l}\text { MAL-PDT } \\
\text { (total) }\end{array}$ & 68.4 & 34.9 & $\begin{array}{l}8.4 \\
\mathrm{~mm}\end{array}$ & $\begin{array}{l}\text { forehead/templ } \\
\text { e+nose/parana } \\
\text { sal (38.1), } \\
\text { cheek/chin/lips } \\
\text { (4.8), ears } \\
(14.3), \\
\text { extremities } \\
(4.8), \\
\text { trunk/neck } \\
(38.1)\end{array}$ & & & & & & & \\
\hline & $\begin{array}{l}\text { ALA-PDT } \\
\text { (debulking } \\
\text { subgroup) }\end{array}$ & 68.4 & 34.9 & NR & NR & & & & & & & \\
\hline & $\begin{array}{l}\text { ALA-PDT } \\
\text { (no } \\
\text { debulking } \\
\text { subgroup) }\end{array}$ & 68.4 & 34.9 & NR & NR & & & & & & & \\
\hline & $\begin{array}{l}\text { MAL-PDT } \\
\text { (debulking } \\
\text { subgroup) }\end{array}$ & 68.4 & 34.9 & NR & NR & & & & & & & \\
\hline & $\begin{array}{l}\text { MAL-PDT } \\
\text { (no } \\
\text { debulking } \\
\text { subgroup) }\end{array}$ & 68.4 & 34.9 & NR & NR & & & & & & & \\
\hline $\begin{array}{l}\text { Kuijpers } 2007 \\
17451581\end{array}$ & $\begin{array}{l}\text { Curettage + } \\
\text { Cryosurger } \\
\text { y }\end{array}$ & 67 & 43 & $\begin{array}{l}5.4 \\
\mathrm{~mm}\end{array}$ & $\begin{array}{l}\text { Forehead/temp } \\
\text { le, Cheek/chin, } \\
\text { Periocular } \\
(80) \text {, }\end{array}$ & No & No & Yes & $\begin{array}{l}\text { Unsur } \\
\text { e }\end{array}$ & Unsure & Yes & Yes \\
\hline
\end{tabular}




\begin{tabular}{|c|c|c|c|c|c|c|c|c|c|c|c|c|}
\hline Study & Arm & $\begin{array}{l}\text { Age, } \\
\text { Mean }\end{array}$ & $\begin{array}{l}\text { Female } \\
\%\end{array}$ & $\begin{array}{l}\text { Lesion } \\
\text { Size, } \\
\text { Mean }\end{array}$ & $\begin{array}{l}\text { Lesion } \\
\text { location (\%) }\end{array}$ & $\begin{array}{l}1^{*} \\
\text { Adequa } \\
\text { te } \\
\text { Rando } \\
\text { mizatio } \\
\mathrm{n}\end{array}$ & $\begin{array}{l}2^{*} \\
\text { Allocati } \\
\text { on } \\
\text { Concea } \\
\text { Iment }\end{array}$ & $\begin{array}{l}3^{*} \\
\text { Arms } \\
\text { Similar at } \\
\text { Baseline }\end{array}$ & $\begin{array}{l}* \\
\text { Patien } \\
\text { ts } \\
\text { Blinde } \\
\text { d }\end{array}$ & $\begin{array}{l}5^{*} \\
\text { Provide } \\
\text { rs } \\
\text { Blinded }\end{array}$ & $\begin{array}{l}6^{*} \\
\text { Outcome } \\
\text { Assessor } \\
\text { s Blinded }\end{array}$ & $\begin{array}{l}7^{*} \\
<20 \% \\
\text { Loss to } \\
\text { Followu } \\
p\end{array}$ \\
\hline & & & & & $\begin{array}{l}\text { Lips/mouth (4), } \\
\text { Ears/periauricu } \\
\text { lar (8), Neck, } \\
\text { chest/back (8) }\end{array}$ & & & & & & & \\
\hline & $\begin{array}{l}\text { Surgical } \\
\text { excision }\end{array}$ & 67 & 43 & $\begin{array}{l}5.3 \\
\mathrm{~mm}\end{array}$ & $\begin{array}{l}\text { Forehead/temp } \\
\text { le, Cheek/chin, } \\
\text { Periocular } \\
\text { (76), } \\
\text { Lips/mouth (6), } \\
\text { Ears/periauricu } \\
\text { lar (6), Neck, } \\
\text { chest/back } \\
\text { (12) }\end{array}$ & & & & & & & \\
\hline \multirow[t]{2}{*}{$\begin{array}{l}\text { Mosterd } 2008 \\
18717680\end{array}$} & ALA-PDT & 64 & 48.2 & $\begin{array}{l}8.9 \\
\mathrm{~mm}\end{array}$ & $\begin{array}{l}\text { face (53); "rest } \\
\text { of the body" } \\
(47 \%)\end{array}$ & Yes & Yes & Yes & Yes & No & No & No \\
\hline & $\begin{array}{l}\text { Surgical } \\
\text { excision }\end{array}$ & 65.1 & 50 & $\begin{array}{l}9.3 \\
\mathrm{~mm}\end{array}$ & $\begin{array}{l}\text { face }(51) ; \text { "rest } \\
\text { of the body" } \\
(49 \%)\end{array}$ & & & & & & & \\
\hline \multirow[t]{2}{*}{$\begin{array}{l}\text { Orenberg } \\
1992 \\
1430394\end{array}$} & $\begin{array}{l}7.5 \mathrm{mg} \mathrm{5-} \\
\text { FU }\end{array}$ & 60 & 5 & $\begin{array}{l}123.9 \\
\mathrm{~mm}^{2}\end{array}$ & $\begin{array}{l}\text { face }(30), \\
\text { extremities } \\
(30), \\
\text { trunk/neck (40) }\end{array}$ & unsure & unsure & No & yes & yes & yes & Yes \\
\hline & $15 \mathrm{mg} \mathrm{5-FU}$ & 60 & 5 & $\begin{array}{l}76.4 \\
\mathrm{~mm}^{2}\end{array}$ & $\begin{array}{l}\text { face (10), } \\
\text { scalp (10), lip } \\
(10), \text { ear (30), } \\
\text { extremities } \\
(10), \\
\text { trunk/neck (30) }\end{array}$ & & & & & & & \\
\hline \multirow[t]{2}{*}{$\begin{array}{l}\text { Rhodes } 2004 \\
14732655\end{array}$} & MAL PDT & 69 & 38 & NR & $\begin{array}{l}\text { face/scalp } \\
(40), \\
\text { extremities } \\
(11), \\
\text { trunk/neck (49) }\end{array}$ & Yes & Yes & No & No & No & No & No \\
\hline & excision & 67 & 41 & NR & $\begin{array}{l}\text { face/scalp } \\
\text { (58), } \\
\text { extremities (9), }\end{array}$ & & & & & & & \\
\hline
\end{tabular}




\begin{tabular}{|c|c|c|c|c|c|c|c|c|c|c|c|c|}
\hline Study & Arm & $\begin{array}{l}\text { Age, } \\
\text { Mean }\end{array}$ & $\begin{array}{l}\text { Female } \\
\%\end{array}$ & $\begin{array}{l}\text { Lesion } \\
\text { Size, } \\
\text { Mean }\end{array}$ & $\begin{array}{l}\text { Lesion } \\
\text { location (\%) }\end{array}$ & $\begin{array}{l}1^{*} \\
\text { Adequa } \\
\text { te } \\
\text { Rando } \\
\text { mizatio } \\
\mathrm{n}\end{array}$ & $\begin{array}{l}2^{*} \\
\text { Allocati } \\
\text { on } \\
\text { Concea } \\
\text { Iment }\end{array}$ & $\begin{array}{l}3^{*} \\
\text { Arms } \\
\text { Similar at } \\
\text { Baseline }\end{array}$ & $\begin{array}{l}4^{*} \\
\text { Patien } \\
\text { ts } \\
\text { Blinde } \\
\text { d }\end{array}$ & $\begin{array}{l}5^{\star} \\
\text { Provide } \\
\text { rs } \\
\text { Blinded }\end{array}$ & $\begin{array}{l}6^{*} \\
\text { Outcome } \\
\text { Assessor } \\
\text { s Blinded }\end{array}$ & $\begin{array}{l}7^{*} \\
<20 \% \\
\text { Loss to } \\
\text { Followu } \\
\text { p }\end{array}$ \\
\hline & & & & & trunk/neck (29) & & & & & & & \\
\hline \multirow[t]{5}{*}{$\begin{array}{l}\text { Shumack } \\
2002 \\
12224978 \text { (12 } \\
\text { weeks) }\end{array}$} & $\begin{array}{l}\text { vehicle } \\
\text { cream }\end{array}$ & NR & 42 & $\begin{array}{l}\text { median } \\
0.8 \\
\mathrm{~cm}^{2}\end{array}$ & $\begin{array}{l}\text { face (17), } \\
\text { trunk/neck } \\
\text { (54.2), upper } \\
\text { extremity (not } \\
\text { hand) (25), } \\
\text { lower extremity } \\
\text { (not foot) (4) }\end{array}$ & No & No & No & Yes & unsure & unsure & Yes \\
\hline & $\begin{array}{l}\text { imiquimod } \\
5 \% \text { cream - } \\
\text { Twice daily } \\
\text { for } 7 \text { days } \\
\text { per week }\end{array}$ & NR & 75 & $\begin{array}{l}\text { median } \\
0.8 \\
\mathrm{~cm} 2\end{array}$ & $\begin{array}{l}\text { face }(25) \\
\text { trunk/neck (75) }\end{array}$ & & & & & & & \\
\hline & $\begin{array}{l}\text { imiquimod } \\
5 \% \text { cream - } \\
\text { Once daily } \\
\text { for } 7 \text { days } \\
\text { per week }\end{array}$ & NR & 10 & $\begin{array}{l}\text { median } \\
0.7 \\
\mathrm{~cm} 2\end{array}$ & $\begin{array}{l}\text { face (29), } \\
\text { trunk/neck } \\
\text { (33), upper } \\
\text { extremity (not } \\
\text { hand) (19), } \\
\text { lower extremity } \\
\text { (not foot) (10) }\end{array}$ & & & & & & & \\
\hline & $\begin{array}{l}\text { imiquimod } \\
5 \% \text { cream - } \\
\text { Once daily } \\
\text { for } 5 \text { days } \\
\text { per week }\end{array}$ & NR & 35 & $\begin{array}{l}\text { median } \\
0.7 \\
\mathrm{~cm} 2\end{array}$ & $\begin{array}{l}\text { face (48), } \\
\text { trunk/neck } \\
\text { (26), Upper } \\
\text { extremity (not } \\
\text { hand) (17), } \\
\text { lower extremity } \\
\text { (not foot) (9) }\end{array}$ & & & & & & & \\
\hline & $\begin{array}{l}\text { imiquimod } \\
5 \% \text { cream - } \\
\text { Once daily } \\
\text { for } 3 \text { days } \\
\text { per week }\end{array}$ & NR & 30 & $\begin{array}{l}\text { median } \\
0.7 \\
\mathrm{~cm} 2\end{array}$ & $\begin{array}{l}\text { face (40), } \\
\text { trunk/neck } \\
\text { (35), upper } \\
\text { extremity (not } \\
\text { hand) (20), } \\
\text { lower extremity } \\
\text { (not foot) (5) }\end{array}$ & & & & & & & \\
\hline $\begin{array}{l}\text { Shumack } \\
2002 \\
12224978(6\end{array}$ & $\begin{array}{l}\text { imiquimod } \\
5 \% \text { cream - } \\
\text { Twice daily }\end{array}$ & NR & 0 & $\begin{array}{l}\text { median } \\
0.6 \\
\mathrm{~cm} 2\end{array}$ & face (100) & Yes & unsure & No & Yes & unsure & unsure & Yes \\
\hline
\end{tabular}




\begin{tabular}{|c|c|c|c|c|c|c|c|c|c|c|c|c|}
\hline Study & Arm & $\begin{array}{l}\text { Age, } \\
\text { Mean }\end{array}$ & $\begin{array}{l}\text { Female } \\
\%\end{array}$ & $\begin{array}{l}\text { Lesion } \\
\text { Size, } \\
\text { Mean }\end{array}$ & $\begin{array}{l}\text { Lesion } \\
\text { location (\%) }\end{array}$ & $\begin{array}{l}1^{*} \\
\text { Adequa } \\
\text { te } \\
\text { Rando } \\
\text { mizatio } \\
\mathrm{n}\end{array}$ & $\begin{array}{l}2^{*} \\
\text { Allocati } \\
\text { on } \\
\text { Concea } \\
\text { Iment }\end{array}$ & $\begin{array}{l}3^{*} \\
\text { Arms } \\
\text { Similar at } \\
\text { Baseline }\end{array}$ & $\begin{array}{l}4^{*} \\
\text { Patien } \\
\text { ts } \\
\text { Blinde } \\
\text { d }\end{array}$ & $\begin{array}{l}5^{\star} \\
\text { Provide } \\
\text { rs } \\
\text { Blinded }\end{array}$ & $\begin{array}{l}6^{*} \\
\text { Outcome } \\
\text { Assessor } \\
\text { s Blinded }\end{array}$ & $\begin{array}{l}7^{*} \\
<20 \% \\
\text { Loss to } \\
\text { Followu } \\
\text { p }\end{array}$ \\
\hline \multirow[t]{6}{*}{ weeks) } & $\begin{array}{l}\text { for } 7 \text { days } \\
\text { per week }\end{array}$ & & & & & & & & & & & \\
\hline & $\begin{array}{l}\text { imiquimod } \\
5 \% \text { cream - } \\
\text { Once daily } \\
\text { for } 3 \text { days } \\
\text { per week }\end{array}$ & 63 & 13 & $\begin{array}{l}\text { median } \\
0.8 \\
\mathrm{~cm} 2\end{array}$ & $\begin{array}{l}\text { face }(28), \\
\text { trunk/neck } \\
(11.11), \text { Upper } \\
\text { extremity (not } \\
\text { hand) (25), } \\
\text { lower extremity } \\
\text { (not foot) (13) }\end{array}$ & & & & & & & \\
\hline & $\begin{array}{l}\text { imiquimod } \\
5 \% \text { cream - } \\
\text { Twice daily } \\
\text { for } 7 \text { days } \\
\text { per week }\end{array}$ & 69 & 13 & $\begin{array}{l}\text { median } \\
0.8 \\
\mathrm{~cm} 2\end{array}$ & $\begin{array}{l}\text { face (32), } \\
\text { trunk/neck } \\
\text { (39), Upper } \\
\text { extremity (not } \\
\text { hand) (26), } \\
\text { lower extremity } \\
\text { (not foot) (3) }\end{array}$ & & & & & & & \\
\hline & $\begin{array}{l}\text { imiquimod } \\
5 \% \text { cream - } \\
\text { Once daily } \\
\text { for } 7 \text { days } \\
\text { per week }\end{array}$ & 66 & 29 & $\begin{array}{l}\text { median } \\
0.8 \\
\mathrm{~cm} 2\end{array}$ & $\begin{array}{l}\text { face (11), } \\
\text { trunk/neck } \\
\text { (48), Upper } \\
\text { extremity (not } \\
\text { hand) (26), } \\
\text { lower extremity } \\
\text { (not foot) (3) }\end{array}$ & & & & & & & \\
\hline & $\begin{array}{l}\text { imiquimod } \\
5 \%\end{array}$ & NR & 40 & NR & $\begin{array}{l}\text { face (60), ear } \\
(10), \\
\text { unspecified } \\
\text { other (30) }\end{array}$ & No & No & Unsure & $\begin{array}{l}\text { unsur } \\
\mathrm{e}\end{array}$ & unsure & No & Yes \\
\hline & vehicle & NR & 10 & NR & $\begin{array}{l}\text { face (50), ear } \\
(20), \\
\text { unspecified } \\
\text { other (30) }\end{array}$ & & & & & & & \\
\hline \multirow[t]{2}{*}{$\begin{array}{l}\text { Sterry } 2002 \\
12452875 \\
\text { (nodular) }\end{array}$} & $\begin{array}{l}\text { Imiquimod } \\
\text { ( } 2 \text { days/wk) } \\
\text { with } \\
\text { occlusion }\end{array}$ & 66 & 50 & $\begin{array}{l}\text { median } \\
: 0.6 \\
\mathrm{~cm} 2\end{array}$ & $\begin{array}{l}\text { Face (10), } \\
\text { Scalp (1), } \\
\text { extremities (2), } \\
\text { trunk/neck (9) }\end{array}$ & Yes & Yes & Yes & No & No & Unsure & Yes \\
\hline & $\begin{array}{l}\text { Imiquimod } \\
\text { (3 days/wk) }\end{array}$ & 66 & 30 & $\begin{array}{l}\text { median } \\
: 0.7 \\
\end{array}$ & $\begin{array}{l}\text { Face (18), } \\
\text { extremities (2), }\end{array}$ & & & & & & & \\
\hline
\end{tabular}




\begin{tabular}{|c|c|c|c|c|c|c|c|c|c|c|c|c|}
\hline Study & Arm & $\begin{array}{l}\text { Age, } \\
\text { Mean }\end{array}$ & $\begin{array}{l}\text { Female } \\
\%\end{array}$ & $\begin{array}{l}\text { Lesion } \\
\text { Size, } \\
\text { Mean }\end{array}$ & $\begin{array}{l}\text { Lesion } \\
\text { location (\%) }\end{array}$ & $\begin{array}{l}1^{*} \\
\text { Adequa } \\
\text { te } \\
\text { Rando } \\
\text { mizatio } \\
\mathrm{n}\end{array}$ & $\begin{array}{l}2^{*} \\
\text { Allocati } \\
\text { on } \\
\text { Concea } \\
\text { Iment }\end{array}$ & $\begin{array}{l}3^{*} \\
\text { Arms } \\
\text { Similar at } \\
\text { Baseline }\end{array}$ & $\begin{array}{l}* \\
\text { Patien } \\
\text { ts } \\
\text { Blinde } \\
\text { d }\end{array}$ & $\begin{array}{l}5^{*} \\
\text { Provide } \\
\text { rs } \\
\text { Blinded }\end{array}$ & $\begin{array}{l}6^{*} \\
\text { Outcome } \\
\text { Assessor } \\
\text { s Blinded }\end{array}$ & $\begin{array}{l}7^{*} \\
<20 \% \\
\text { Loss to } \\
\text { Followu } \\
\text { p }\end{array}$ \\
\hline & $\begin{array}{l}\text { with } \\
\text { occlusion }\end{array}$ & & & $\mathrm{cm} 2$ & trunk/neck (3) & & & & & & & \\
\hline & $\begin{array}{l}\text { Imiquimod } \\
\text { ( } 2 \text { days/wk) } \\
\text { without } \\
\text { occlusion }\end{array}$ & 67 & 24 & $\begin{array}{l}\text { median } \\
: 1.0 \\
\mathrm{~cm} 2\end{array}$ & $\begin{array}{l}\text { Face (9), } \\
\text { extremities (1), } \\
\text { trunk/neck (10) }\end{array}$ & & & & & & & \\
\hline & $\begin{array}{l}\text { Imiquimod } \\
\text { ( } 3 \text { days/wk) } \\
\text { without } \\
\text { occlusion }\end{array}$ & 66 & 46 & $\begin{array}{l}\text { median } \\
: 0.6 \\
\mathrm{~cm}^{2}\end{array}$ & $\begin{array}{l}\text { Face (11), } \\
\text { extremities (5), } \\
\text { trunk/neck (8) }\end{array}$ & & & & & & & \\
\hline \multirow[t]{2}{*}{$\begin{array}{l}\text { van der Geer } \\
2012 \\
22385074\end{array}$} & $\begin{array}{l}\text { Imiquimod } \\
+ \text { MMS }\end{array}$ & 69 & 37 & NR & $\begin{array}{l}\text { H-zone (57), } \\
\text { nose (23), ear } \\
4(11), \text { scalp + } \\
\text { frontal (23), } \\
\text { other regions } \\
\text { (cheek, } \\
\text { temporal, chin) } \\
(43)\end{array}$ & Yes & Yes & Yes & No & No & No & Yes \\
\hline & $\begin{array}{l}\text { no } \\
\text { treatment + } \\
\text { MMS }\end{array}$ & 68 & 31 & $\begin{array}{l}\text { median } \\
110 \\
\mathrm{~mm}^{2}\end{array}$ & $\begin{array}{l}\text { H-zone (66), } \\
\text { nose (26), ear } \\
(17), \text { scalp + } \\
\text { frontal (14), } \\
\text { other regions } \\
\text { (cheek, } \\
\text { temporal, chin) } \\
(43)\end{array}$ & & & & & & & \\
\hline \multirow[t]{2}{*}{$\begin{array}{l}\text { Wettstein } \\
2013 \\
23566745\end{array}$} & $\begin{array}{l}\text { Ringer's } \\
\text { lactate } \\
\text { (control } \\
\text { group) } \\
\end{array}$ & 59 & 26.67 & $\begin{array}{l}2.5 \\
\mathrm{~cm} 2\end{array}$ & $\begin{array}{l}\text { nose (46.2), } \\
\text { cheek (23.1), } \\
\text { frontal (7.7), } \\
\text { ear (23.1) }\end{array}$ & Yes & Unsure & Yes & Yes & Yes & Unsure & Yes \\
\hline & $\begin{array}{l}\text { interferon } \\
\text { alpha-2b }\end{array}$ & 59 & 26.67 & $\begin{array}{l}3.1 \\
\mathrm{~cm} 2\end{array}$ & $\begin{array}{l}\text { nose (50), } \\
\text { cheek (10), } \\
\text { frontal (20), } \\
\text { ear (20) }\end{array}$ & & & & & & & \\
\hline
\end{tabular}

BCC = basal cell carcinoma; MMS = Mohs micrographic surgery, PDT = photodynamic therapy; ALA = 5-aminolevulinic acid; MAL= methyl aminolevulinate; FU = fluorouracil; $\mathrm{INF}=$ interferon; NR = not reported; RCT = randomized controlled trial; $\mathrm{x} / \mathrm{wk}=$ times per week; AFXL = ablative fractional laser resurfacing 
*Design items: 1: Adequate generation of a randomized sequence reported; 2: Adequate allocation concealment reported; 3: Group similarity at baseline; 4: Adequate blinding of patients reported; 5: Adequate blinding of providers reported; 6: Adequate blinding of outcome assessors reported; 7: Less than $20 \%$ missing for any eligible outcome in any arm. 
Table 5. Characteristics of RCTs of high-risk BCC lesions

\begin{tabular}{|c|c|c|c|c|c|c|c|c|c|c|c|c|}
\hline Study & Arm & $\begin{array}{l}\text { Age, } \\
\text { Mean }\end{array}$ & $\begin{array}{l}\text { Female } \\
\%\end{array}$ & $\begin{array}{l}\text { Lesion } \\
\text { Size, } \\
\text { Mean }\end{array}$ & Lesion location (\%) & $\begin{array}{l}\text { 1* } \\
\text { Adequate } \\
\text { Randomiz- } \\
\text { ation }\end{array}$ & $\begin{array}{l}2^{*} \\
\text { Allocation } \\
\text { Conceal- } \\
\text { ment }\end{array}$ & $\begin{array}{l}* \\
\text { Arms } \\
\text { Similar } \\
\text { at } \\
\text { Baseline }\end{array}$ & $\begin{array}{l}4^{*} \\
\text { Patients } \\
\text { Blinded }\end{array}$ & $\begin{array}{l}\text { 5* } \\
\text { Providers } \\
\text { Blinded }\end{array}$ & $\begin{array}{l}6^{*} \\
\text { Outcome } \\
\text { Assessors } \\
\text { Blinded }\end{array}$ & $\begin{array}{l}\text { 7* } \\
<20 \% \\
\text { Loss to } \\
\text { Followup }\end{array}$ \\
\hline \multirow[t]{3}{*}{$\begin{array}{l}\text { Alpsoy } \\
1996 \\
8708151\end{array}$} & IFN alfa-2a & 58.7 & 53 & $\begin{array}{l}\text { median } \\
2.05 \\
\mathrm{~cm} 2\end{array}$ & $\begin{array}{l}\text { eyelid (27), nose (13), } \\
\text { zygoma (27), } \\
\text { forehead (13), cheek } \\
\text { (13), trunk (7) }\end{array}$ & \multirow[t]{3}{*}{ Yes } & \multirow[t]{3}{*}{ Yes } & \multirow[t]{3}{*}{ Unsure } & \multirow[t]{3}{*}{ Yes } & \multirow[t]{3}{*}{ Unsure } & \multirow[t]{3}{*}{ Unsure } & \multirow[t]{3}{*}{ Unsure } \\
\hline & IFN alfa-2b & 63.6 & 53 & $\begin{array}{l}\text { median } \\
1.82 \\
\mathrm{~cm} 2\end{array}$ & $\begin{array}{l}\text { eyelid (20), nose (7), } \\
\text { zygoma (20), } \\
\text { forehead (20), cheek } \\
(27), \text { trunk ( } 7)\end{array}$ & & & & & & & \\
\hline & $\begin{array}{l}\text { IFN alfa-2a } \\
+ \text { IFN alfa- } \\
2 b\end{array}$ & 60.3 & 40 & $\begin{array}{l}\text { median } \\
1.9 \mathrm{~cm} 2\end{array}$ & $\begin{array}{l}\text { eyelid (20), nose (13), } \\
\text { zygoma (27), } \\
\text { forehead (13), cheek } \\
(20), \text { trunk ( } 7)\end{array}$ & & & & & & & \\
\hline \multirow{2}{*}{$\begin{array}{l}\text { Migden } \\
2015 \\
25981810\end{array}$} & $\begin{array}{l}\text { Sonidegib } \\
200\end{array}$ & $\begin{array}{l}\text { media } \\
\text { n } 67\end{array}$ & 39 & NR & head and neck (100) & \multirow[t]{2}{*}{ Yes } & \multirow[t]{2}{*}{ Yes } & \multirow[t]{2}{*}{ Yes } & \multirow[t]{2}{*}{ Yes } & \multirow[t]{2}{*}{ Yes } & \multirow[t]{2}{*}{ Yes } & \multirow[t]{2}{*}{ Yes } \\
\hline & $\begin{array}{l}\text { Sonidegib } \\
800\end{array}$ & $\begin{array}{l}\text { media } \\
\mathrm{n} 65\end{array}$ & 36 & NR & head and neck (100) & & & & & & & \\
\hline
\end{tabular}

NR = not reported; IFN = interferon

*Design items: 1: Adequate generation of a randomized sequence reported; 2: Adequate allocation concealment reported; 3: Group similarity at baseline; 4: Adequate blinding of patients reported; 5: Adequate blinding of providers reported; 6: Adequate blinding of outcome assessors reported; 7: Less than 20\% missing for any eligible outcome in any arm.

\begin{tabular}{|c|c|c|c|c|c|c|c|c|c|c|c|c|}
\hline Study & Arm & $\begin{array}{l}\text { Age, } \\
\text { Mean }\end{array}$ & $\begin{array}{l}\text { Female } \\
\%\end{array}$ & $\begin{array}{l}\text { Lesion } \\
\text { Size, } \\
\text { Mean }\end{array}$ & $\begin{array}{l}\text { Lesion } \\
\text { Location (\%) }\end{array}$ & $\begin{array}{l}1^{*} \\
\text { Adequate } \\
\text { Randomiz- } \\
\text { ation } \\
\end{array}$ & $\begin{array}{l}\text { 2* } \\
\text { Allocation } \\
\text { Conceal- } \\
\text { ment }\end{array}$ & $\begin{array}{l}3^{*} \\
\text { Arms } \\
\text { Similar at } \\
\text { Baseline }\end{array}$ & $\begin{array}{l}4^{*} \\
\text { Patients } \\
\text { Blinded }\end{array}$ & $\begin{array}{l}5^{*} \\
\text { Providers } \\
\text { Blinded }\end{array}$ & $\begin{array}{l}6^{*} \\
\text { Outcome } \\
\text { Assessors } \\
\text { Blinded }\end{array}$ & $\begin{array}{l}7^{*} \\
<20 \% \\
\text { Loss to } \\
\text { Followup }\end{array}$ \\
\hline \multirow{2}{*}{$\begin{array}{l}\text { Allen } 1979 \\
298425\end{array}$} & cryotherapy & NR & NR & NR & NR & Yes & Yes & Unsure & Yes & Unsure & Unsure & Unsure \\
\hline & radiotherapy & NR & NR & NR & NR & & & & & & & \\
\hline \multirow[t]{2}{*}{$\begin{array}{l}\text { Alpsoy } \\
1996 \\
8708151\end{array}$} & IFN alfa-2a & 58.7 & 53 & $\begin{array}{l}\text { median } \\
2.05 \\
\mathrm{~cm} 2\end{array}$ & $\begin{array}{l}\text { eyelid (27), } \\
\text { nose (13), } \\
\text { zygoma (27), } \\
\text { forehead (13), } \\
\text { cheek (13), } \\
\text { trunk (7) }\end{array}$ & Unsure & Unsure & Yes & Unsure & Unsure & Unsure & Yes \\
\hline & IFN alfa-2b & 63.6 & 53 & median & eyelid (20), & & & & & & & \\
\hline
\end{tabular}




\begin{tabular}{|c|c|c|c|c|c|c|c|c|c|c|c|c|}
\hline Study & Arm & $\begin{array}{l}\text { Age, } \\
\text { Mean }\end{array}$ & $\begin{array}{l}\text { Female } \\
\%\end{array}$ & $\begin{array}{l}\text { Lesion } \\
\text { Size, } \\
\text { Mean }\end{array}$ & $\begin{array}{l}\text { Lesion } \\
\text { Location (\%) }\end{array}$ & $\begin{array}{l}1^{*} \\
\text { Adequate } \\
\text { Randomiz- } \\
\text { ation } \\
\end{array}$ & $\begin{array}{l}\text { 2* } \\
\text { Allocation } \\
\text { Conceal- } \\
\text { ment } \\
\end{array}$ & $\begin{array}{l}3^{*} \\
\text { Arms } \\
\text { Similar at } \\
\text { Baseline }\end{array}$ & $\begin{array}{l}4^{\star} \\
\text { Patients } \\
\text { Blinded }\end{array}$ & $\begin{array}{l}5^{*} \\
\text { Providers } \\
\text { Blinded }\end{array}$ & $\begin{array}{l}6^{*} \\
\text { Outcome } \\
\text { Assessors } \\
\text { Blinded } \\
\end{array}$ & $\begin{array}{l}7^{*} \\
<20 \% \\
\text { Loss to } \\
\text { Followup }\end{array}$ \\
\hline & & & & $\begin{array}{l}1.82 \\
\mathrm{~cm} 2\end{array}$ & $\begin{array}{l}\text { nose (7), } \\
\text { zygoma (20), } \\
\text { forehead (20), } \\
\text { cheek (27), } \\
\text { trunk (7) }\end{array}$ & & & & & & & \\
\hline & $\begin{array}{l}\text { IFN alfa-2a }+ \\
\text { IFN alfa-2b }\end{array}$ & 60.3 & 40 & $\begin{array}{l}\text { median } \\
1.9 \mathrm{~cm} 2\end{array}$ & $\begin{array}{l}\text { eyelid (20), } \\
\text { nose (13), } \\
\text { zygoma (27), } \\
\text { forehead (13), } \\
\text { cheek (20), } \\
\text { trunk (7) }\end{array}$ & & & & & & & \\
\hline \multirow[t]{2}{*}{$\begin{array}{l}\text { Avril } 1997 \\
9218740\end{array}$} & surgery & 66.5 & 54 & $\begin{array}{l}11.1 \\
\mathrm{~mm}\end{array}$ & $\begin{array}{l}\text { nose (53), } \\
\text { cheek, pre- and } \\
\text { retroauricular } \\
\text { areas (21), } \\
\text { eyelids, internal } \\
\text { and external } \\
\text { eye angles (19), } \\
\text { forehead, } \\
\text { temple, } \\
\text { between } \\
\text { eyebrows } 36 \\
\text { (21), chin, } \\
\text { cutaneous } \\
\text { superior lip } 10 \\
\text { (6), ear (3) }\end{array}$ & \multirow[t]{2}{*}{ No } & \multirow[t]{2}{*}{ Yes } & \multirow[t]{2}{*}{ Yes } & \multirow[t]{2}{*}{ No } & \multirow[t]{2}{*}{ No } & \multirow[t]{2}{*}{ No } & \multirow[t]{2}{*}{ No } \\
\hline & radiotherapy & 65.4 & 46 & $\begin{array}{l}11.7 \\
\mathrm{~mm}\end{array}$ & $\begin{array}{l}\text { nose (28), } \\
\text { cheek, pre- and } \\
\text { retroauricular } \\
\text { areas (24), } \\
\text { eyelids, internal } \\
\text { and external } \\
\text { eye angles (20), } \\
\text { forehead, } \\
\text { temple, } \\
\text { between } \\
\text { eyebrows (17), } \\
\text { chin, cutaneous } \\
\text { superior lip (7), }\end{array}$ & & & & & & & \\
\hline
\end{tabular}




\begin{tabular}{|c|c|c|c|c|c|c|c|c|c|c|c|c|}
\hline Study & Arm & $\begin{array}{l}\text { Age, } \\
\text { Mean }\end{array}$ & $\begin{array}{l}\text { Female } \\
\%\end{array}$ & $\begin{array}{l}\text { Lesion } \\
\text { Size, } \\
\text { Mean }\end{array}$ & $\begin{array}{l}\text { Lesion } \\
\text { Location (\%) }\end{array}$ & $\begin{array}{l}1^{*} \\
\text { Adequate } \\
\text { Randomiz- } \\
\text { ation } \\
\end{array}$ & $\begin{array}{l}2^{*} \\
\text { Allocation } \\
\text { Conceal- } \\
\text { ment } \\
\end{array}$ & $\begin{array}{l}3^{*} \\
\text { Arms } \\
\text { Similar at } \\
\text { Baseline } \\
\end{array}$ & $\begin{array}{l}4^{*} \\
\text { Patients } \\
\text { Blinded }\end{array}$ & $\begin{array}{l}5^{*} \\
\text { Providers } \\
\text { Blinded }\end{array}$ & $\begin{array}{l}6^{*} \\
\text { Outcome } \\
\text { Assessors } \\
\text { Blinded } \\
\end{array}$ & $\begin{array}{l}7^{*} \\
<20 \% \\
\text { Loss to } \\
\text { Followup }\end{array}$ \\
\hline & & & & & ear (3) & & & & & & & \\
\hline \multirow[t]{2}{*}{$\begin{array}{l}\text { Bath- } \\
\text { Hextall } \\
2014 \\
24332516\end{array}$} & Imiquimod & NR & 41 & $\begin{array}{l}\text { median } \\
12 \mathrm{~mm}\end{array}$ & $\begin{array}{l}\text { face (37), trunk } \\
(38), \text { neck (6), } \\
\text { arm (6), leg } \\
(10), \text { other (3) }\end{array}$ & Yes & Yes & Yes & No & No & Yes & No \\
\hline & excision & NR & 40 & $\begin{array}{l}\text { median } \\
10 \mathrm{~mm}\end{array}$ & $\begin{array}{l}\text { face (33), trunk } \\
(39), \text { neck (9), } \\
\text { arm (7), leg (9), } \\
\text { other (3) }\end{array}$ & & & & & & & \\
\hline \multirow[t]{5}{*}{$\begin{array}{l}\text { Beutner } \\
1999 \\
10570388\end{array}$} & $\begin{array}{l}\text { imiquimod } \\
2 x / \text { day }\end{array}$ & NR & NR & NR & $\begin{array}{l}\text { upper exremity } \\
\text { (57), anterior } \\
\text { upper trunk } \\
(14), \text { neck (29) }\end{array}$ & No & No & No & Unsure & Yes & unsure & Yes \\
\hline & $\begin{array}{l}\text { imiquimod } \\
\text { 1x/day }\end{array}$ & NR & NR & NR & $\begin{array}{l}\text { upper extremity } \\
\text { (50), anterior } \\
\text { upper trunk } \\
\text { (25), posterior } \\
\text { upper trunk (25) }\end{array}$ & & & & & & & \\
\hline & $\begin{array}{l}\text { imiquimod } \\
2 x / \text { week }\end{array}$ & NR & NR & NR & $\begin{array}{l}\text { lower extremity } \\
\text { (20), anterior } \\
\text { upper trunk } \\
\text { (40), posterior } \\
\text { upper trunk } \\
(20), \text { neck (20) }\end{array}$ & & & & & & & \\
\hline & $\begin{array}{l}\text { imiquimod } \\
\text { 1x/week }\end{array}$ & NR & NR & NR & $\begin{array}{l}\text { lower extremity } \\
\text { (50), anterior } \\
\text { upper trunk } \\
\text { (25), posterior } \\
\text { upper trunk (25) }\end{array}$ & & & & & & & \\
\hline & $\begin{array}{l}\text { vehicle (3 } \\
\text { 2x/day, } 2 \\
\text { 1x/day, } 2 \\
\text { 3x/week, } 2 \\
\text { 2x/week, } 2 \\
\text { 1x/week) }\end{array}$ & NR & NR & NR & $\begin{array}{l}\text { face (9), upper } \\
\text { extremity (46), } \\
\text { anterior upper } \\
\text { trunk (9), neck } \\
\text { (9), posterior } \\
\text { lower trunk (27) }\end{array}$ & & & & & & & \\
\hline $\begin{array}{l}\text { Brinkhuize } \\
\text { n } 2016 \\
27067393\end{array}$ & $\begin{array}{l}\text { Diclofenac } \\
\text { (results } \\
\text { superficial/nod } \\
\text { ular) } \\
\end{array}$ & $\begin{array}{l}63.0 / 7 \\
8.5\end{array}$ & 25 & $\begin{array}{l}61.7 / 49 . \\
5 \mathrm{~mm} 2\end{array}$ & $\begin{array}{l}\text { extremities (47), } \\
\text { trunk/neck (53) }\end{array}$ & Yes & Yes & No & No & No & Yes & Yes \\
\hline
\end{tabular}




\begin{tabular}{|c|c|c|c|c|c|c|c|c|c|c|c|c|}
\hline Study & Arm & $\begin{array}{l}\text { Age, } \\
\text { Mean }\end{array}$ & $\begin{array}{l}\text { Female } \\
\%\end{array}$ & $\begin{array}{l}\text { Lesion } \\
\text { Size, } \\
\text { Mean }\end{array}$ & $\begin{array}{l}\text { Lesion } \\
\text { Location (\%) }\end{array}$ & $\begin{array}{l}1^{*} \\
\text { Adequate } \\
\text { Randomiz- } \\
\text { ation } \\
\end{array}$ & $\begin{array}{l}2^{*} \\
\text { Allocation } \\
\text { Conceal- } \\
\text { ment } \\
\end{array}$ & $\begin{array}{l}3^{*} \\
\text { Arms } \\
\text { Similar at } \\
\text { Baseline } \\
\end{array}$ & $\begin{array}{l}4^{*} \\
\text { Patients } \\
\text { Blinded }\end{array}$ & $\begin{array}{l}5^{\star} \\
\text { Providers } \\
\text { Blinded }\end{array}$ & $\begin{array}{l}6^{*} \\
\text { Outcome } \\
\text { Assessors } \\
\text { Blinded } \\
\end{array}$ & $\begin{array}{l}7^{*} \\
<20 \% \\
\text { Loss to } \\
\text { Followup }\end{array}$ \\
\hline & $\begin{array}{l}\text { Calcitriol } \\
\text { (results } \\
\text { superficial/nod } \\
\text { ular) }\end{array}$ & $\begin{array}{l}65.5 / 6 \\
8.5\end{array}$ & 22 & $\begin{array}{l}54.2 / 59 . \\
7 \mathrm{~mm} 2\end{array}$ & $\begin{array}{l}\text { trunk/neck (59), } \\
\text { genetalia (41) }\end{array}$ & & & & & & & \\
\hline & $\begin{array}{l}\text { Diclofenac + } \\
\text { Calcitriol } \\
\text { (results } \\
\text { superficial/nod } \\
\text { ular) }\end{array}$ & $\begin{array}{l}67.5 / 7 \\
1\end{array}$ & 37.5 & $\begin{array}{l}46.7 / 44 . \\
8 \mathrm{~mm} 2\end{array}$ & $\begin{array}{l}\text { trunk/neck (50), } \\
\text { genetalia (44) }\end{array}$ & & & & & & & \\
\hline & $\begin{array}{l}\text { No treatment } \\
\text { (results } \\
\text { superficial/nod } \\
\text { ular) }\end{array}$ & $\begin{array}{l}61.5 / 6 \\
6\end{array}$ & 37.5 & $\begin{array}{l}59.7 / 53 . \\
4 \mathrm{~mm} 2\end{array}$ & $\begin{array}{l}\text { extremities (53), } \\
\text { trunk/neck (47) }\end{array}$ & & & & & & & \\
\hline \multirow[t]{2}{*}{$\begin{array}{l}\text { Carija } \\
2016 \\
27516420\end{array}$} & ALA-PDT & $\begin{array}{l}\text { Media } \\
\mathrm{n} 71\end{array}$ & 13.3 & $\begin{array}{l}255.4 \\
\mathrm{~mm} 2\end{array}$ & $\begin{array}{l}\text { extremities } \\
(3.6), \text { trunk/neck } \\
(96.4)\end{array}$ & No & No & Yes & unsure & Yes & Yes & Yes \\
\hline & $\begin{array}{l}\text { ALA-PDT + } \\
\text { PDL }\end{array}$ & $\begin{array}{l}\text { Media } \\
\mathrm{n} 71\end{array}$ & 13.3 & $\begin{array}{l}216 \\
\mathrm{~mm} 2\end{array}$ & $\begin{array}{l}\text { extremities } \\
(23.5), \\
\text { trunk/neck } \\
(76.5)\end{array}$ & & & & & & & \\
\hline \multirow[t]{2}{*}{$\begin{array}{l}\text { Cornell } \\
1990 \\
2229497\end{array}$} & interferon & 56 & 19 & $83 \mathrm{~mm} 2$ & $\begin{array}{l}\text { head and face } \\
(25), \text { extermities } \\
(12), \text { trunk/neck } \\
(63)\end{array}$ & Yes & No & Yes & Yes & No & Yes & Yes \\
\hline & placebo & 57 & 14 & $75 \mathrm{~mm} 2$ & $\begin{array}{l}\text { head and face } \\
(17), \text { extermities } \\
(14), \text { trunk/neck } \\
\text { (59) }\end{array}$ & & & & & & & \\
\hline \multirow{2}{*}{$\begin{array}{l}\text { Edwards } \\
1990 \\
2107219\end{array}$} & $\begin{array}{l}\text { interferon } \\
\text { gamma, } 0.01\end{array}$ & NR & NR & NR & NR & No & No & unsure & unsure & unsure & unsure & Yes \\
\hline & $\begin{array}{l}\text { interferon } \\
\text { gamma, } 0.05\end{array}$ & NR & NR & NR & NR & & & & & & & \\
\hline \multirow[t]{2}{*}{$\begin{array}{l}\text { Edwards } \\
1990 \\
2383027\end{array}$} & $\begin{array}{l}\text { Interferon alfa- } \\
2 \mathrm{~b}, 30 \text { million } \\
\mathrm{IU}\end{array}$ & NR & NR & NR & NR & No & No & unsure & Yes & Yes & Yes & Yes \\
\hline & $\begin{array}{l}\text { Interferon alfa- } \\
2 \mathrm{~b}, 10 \text { million } \\
\mathrm{IU}\end{array}$ & NR & NR & NR & NR & & & & & & & \\
\hline
\end{tabular}




\begin{tabular}{|c|c|c|c|c|c|c|c|c|c|c|c|c|}
\hline Study & Arm & $\begin{array}{l}\text { Age, } \\
\text { Mean }\end{array}$ & $\begin{array}{l}\text { Female } \\
\%\end{array}$ & $\begin{array}{l}\text { Lesion } \\
\text { Size, } \\
\text { Mean }\end{array}$ & $\begin{array}{l}\text { Lesion } \\
\text { Location (\%) }\end{array}$ & $\begin{array}{l}1^{*} \\
\text { Adequate } \\
\text { Randomiz- } \\
\text { ation } \\
\end{array}$ & $\begin{array}{l}2^{*} \\
\text { Allocation } \\
\text { Conceal- } \\
\text { ment } \\
\end{array}$ & $\begin{array}{l}3^{*} \\
\text { Arms } \\
\text { Similar at } \\
\text { Baseline }\end{array}$ & $\begin{array}{l}4^{*} \\
\text { Patients } \\
\text { Blinded }\end{array}$ & $\begin{array}{l}5^{*} \\
\text { Providers } \\
\text { Blinded }\end{array}$ & $\begin{array}{l}6^{*} \\
\text { Outcome } \\
\text { Assessors } \\
\text { Blinded } \\
\end{array}$ & $\begin{array}{l}7^{*} \\
<20 \% \\
\text { Loss to } \\
\text { Followup }\end{array}$ \\
\hline $\begin{array}{l}\text { Eimpunth } \\
2014\end{array}$ & $\begin{array}{l}\text { Laser vs. no } \\
\text { treatment }\end{array}$ & NR & 33 & NR & NR & No Data & unsure & unsure & No & unsure & unsure & Yes \\
\hline \multirow{2}{*}{$\begin{array}{l}\text { Garcia- } \\
\text { Martin } \\
2011 \\
21242584\end{array}$} & imiquimod 5\% & 73.1 & 33.3 & $7.6 \mathrm{~mm}$ & eyelid (100) & No & No & Yes & No & Unsure & Unsure & Yes \\
\hline & radiotherapy & 74.2 & 41.7 & $\begin{array}{l}7.41 \\
\mathrm{~mm}\end{array}$ & eyelid (100) & & & & & & & \\
\hline \multirow[t]{2}{*}{$\begin{array}{l}\text { Geisse } \\
2004 \\
15097956\end{array}$} & $\begin{array}{l}\text { Imiquimod } \\
\text { 5x/wk }\end{array}$ & 58.4 & 37 & NR & $\begin{array}{l}\text { neck (4), trunk: } \\
\text { anterior lower } \\
\text { (1), trunk: } \\
\text { anterior upper } \\
\text { (17), trunk: } \\
\text { posterior lower } \\
\text { (7), trunk: } \\
\text { posterior upper } \\
\text { (24), lower } \\
\text { extremity } \\
\text { (excluding foot) } \\
\text { (15), upper } \\
\text { extremity } \\
\text { (excluding } \\
\text { hand) (31), chin } \\
\text { (1), forehead (1) }\end{array}$ & Yes & Yes & No & Yes & Yes & Yes & Yes \\
\hline & $\begin{array}{l}\text { Vehicle } 5 x / w k \\
\text { or } 7 x / w k\end{array}$ & 58.7 & 38 & NR & $\begin{array}{l}\text { neck (1), trunk: } \\
\text { anterior lower } \\
\text { (1), trunk: } \\
\text { anterior upper } \\
\text { (20), trunk: } \\
\text { posterior lower } \\
\text { (6), trunk: } \\
\text { posterior upper } \\
\text { (20), lower } \\
\text { extremity } \\
\text { (excluding foot) } \\
\text { (10.5), upper } \\
\text { extremity } \\
\text { (excluding } \\
\text { hand) (39), }\end{array}$ & & & & & & & \\
\hline
\end{tabular}




\begin{tabular}{|c|c|c|c|c|c|c|c|c|c|c|c|c|}
\hline Study & Arm & $\begin{array}{l}\text { Age, } \\
\text { Mean }\end{array}$ & $\begin{array}{l}\text { Female } \\
\%\end{array}$ & $\begin{array}{l}\text { Lesion } \\
\text { Size, } \\
\text { Mean }\end{array}$ & $\begin{array}{l}\text { Lesion } \\
\text { Location (\%) }\end{array}$ & $\begin{array}{l}1^{*} \\
\text { Adequate } \\
\text { Randomiz- } \\
\text { ation } \\
\end{array}$ & $\begin{array}{l}\text { 2* } \\
\text { Allocation } \\
\text { Conceal- } \\
\text { ment } \\
\end{array}$ & $\begin{array}{l}3^{*} \\
\text { Arms } \\
\text { Similar at } \\
\text { Baseline } \\
\end{array}$ & $\begin{array}{l}4^{*} \\
\text { Patients } \\
\text { Blinded }\end{array}$ & $\begin{array}{l}5^{*} \\
\text { Providers } \\
\text { Blinded }\end{array}$ & $\begin{array}{l}6^{*} \\
\text { Outcome } \\
\text { Assessors } \\
\text { Blinded } \\
\end{array}$ & $\begin{array}{l}7^{*} \\
<20 \% \\
\text { Loss to } \\
\text { Followup }\end{array}$ \\
\hline & & & & & $\begin{array}{l}\text { cheek (1), chin } \\
(1), \text { forehead (1) }\end{array}$ & & & & & & & \\
\hline & $\begin{array}{l}\text { Imiquimod } \\
7 x / \text { wk }\end{array}$ & 59.4 & 41 & NR & $\begin{array}{l}\text { neck (5), trunk: } \\
\text { anterior lower } 3 \text {, } \\
\text { trunk: anterior } \\
\text { upper (13), } \\
\text { trunk: posterior } \\
\text { lower (8), trunk: } \\
\text { posterior upper } \\
\text { (26), lower } \\
\text { extremity } \\
\text { (excluding foot) } \\
\text { (11), upper } \\
\text { extremity } \\
\text { (excluding } \\
\text { hand) (33), } \\
\text { cheek (1), chin } \\
\text { (1), forehead (1) } \\
\text { Face: nose } 1 \\
\text { (1\%) }\end{array}$ & & & & & & & \\
\hline \multirow[t]{2}{*}{$\begin{array}{l}\text { Hall } 1986 \\
3514075\end{array}$} & Radiotherapy & NR & NR & NR & $\begin{array}{l}\text { face and neck } \\
(82), \text { eyelid (6), } \\
\text { trunk (12) }\end{array}$ & No & No & No & No & No & No & Unsure \\
\hline & Cryotherapy & NR & NR & NR & $\begin{array}{l}\text { face and neck } \\
(65), \text { eyelid (17), } \\
\text { trunk (17) }\end{array}$ & & & & & & & \\
\hline $\begin{array}{l}\text { Marks } \\
2001 \\
11312429\end{array}$ & Imiquimod & 61 & 27 & NR & $\begin{array}{l}\text { Upper } \\
\text { extremities (32), } \\
\text { upper trunk } \\
(28), \\
\text { head/neck/lower } \\
\text { limbs (40) }\end{array}$ & No & No & unsure & No & unsure & unsure & Yes \\
\hline \multirow{2}{*}{$\begin{array}{l}\text { Migden } \\
2015 \\
25981810\end{array}$} & sonidegib 200 & $\begin{array}{l}\text { media } \\
\text { n } 67\end{array}$ & 39 & NR & $\begin{array}{l}\text { head and neck } \\
(100)\end{array}$ & Yes & Yes & Yes & Yes & Yes & Yes & Yes \\
\hline & sonidegib 800 & $\begin{array}{l}\text { media } \\
\mathrm{n} 65\end{array}$ & 36 & NR & $\begin{array}{l}\text { head and neck } \\
(100)\end{array}$ & & & & & & & \\
\hline $\begin{array}{l}\text { Miller } 1997 \\
8996264\end{array}$ & $5-\mathrm{FU}$ & 61 & 20 & $80 \mathrm{~mm} 2$ & $\begin{array}{l}\text { head (7), } \\
\text { extremities (40), }\end{array}$ & No & No & unsure & Yes & Yes & Yes & No \\
\hline
\end{tabular}




\begin{tabular}{|c|c|c|c|c|c|c|c|c|c|c|c|c|}
\hline Study & Arm & $\begin{array}{l}\text { Age, } \\
\text { Mean }\end{array}$ & $\begin{array}{l}\text { Female } \\
\%\end{array}$ & $\begin{array}{l}\text { Lesion } \\
\text { Size, } \\
\text { Mean }\end{array}$ & $\begin{array}{l}\text { Lesion } \\
\text { Location (\%) }\end{array}$ & $\begin{array}{l}1^{*} \\
\text { Adequate } \\
\text { Randomiz- } \\
\text { ation } \\
\end{array}$ & $\begin{array}{l}2^{*} \\
\text { Allocation } \\
\text { Conceal- } \\
\text { ment } \\
\end{array}$ & $\begin{array}{l}3^{*} \\
\text { Arms } \\
\text { Similar at } \\
\text { Baseline } \\
\end{array}$ & $\begin{array}{l}4^{*} \\
\text { Patients } \\
\text { Blinded }\end{array}$ & $\begin{array}{l}5^{*} \\
\text { Providers } \\
\text { Blinded }\end{array}$ & $\begin{array}{l}6^{*} \\
\text { Outcome } \\
\text { Assessors } \\
\text { Blinded } \\
\end{array}$ & $\begin{array}{l}7^{*} \\
<20 \% \\
\text { Loss to } \\
\text { Followup }\end{array}$ \\
\hline & & & & & trunk/neck (52) & & & & & & & \\
\hline \multirow[t]{2}{*}{$\begin{array}{l}\text { Mosterd } \\
2008 \\
19010733\end{array}$} & MMS & 67.4 & 39.7 & $\begin{array}{l}1.28 \\
\mathrm{~cm} 2\end{array}$ & $\begin{array}{l}\text { frontal/temporal } \\
(26), \text { cheek/chin } \\
(9), \text { (peri)nasal } \\
(34), \\
\text { lips/perioral (7), } \\
\text { periocular (8), } \\
\text { ears (4), } \\
\text { periauricular } \\
(12)\end{array}$ & Yes & Yes & Unsure & No & No & No & No \\
\hline & $\begin{array}{l}\text { Surgical } \\
\text { excision }\end{array}$ & 68.7 & 38.2 & $\begin{array}{l}1.77 \\
\mathrm{~cm} 2\end{array}$ & $\begin{array}{l}\text { frontal/temporal } \\
\text { (32), cheek/chin } \\
\text { (8), (peri)nasal } \\
(30), \\
\text { lips/perioral (4), } \\
\text { periocular (8), } \\
\text { ears (8), } \\
\text { periauricular } \\
(10)\end{array}$ & & & & & & & \\
\hline \multirow[t]{3}{*}{$\begin{array}{l}\text { Salmanpo } \\
\text { or } 2012\end{array}$} & $\begin{array}{l}\text { Surgical } \\
\text { excision }\end{array}$ & 57.3 & 37 & NR & $\begin{array}{l}\text { face and scalp } \\
(100)\end{array}$ & No & No & unsure & Unsure & Unsure & Unsure & Yes \\
\hline & Curettage & 57.3 & 37 & NR & $\begin{array}{l}\text { face and scalp } \\
(100)\end{array}$ & & & & & & & \\
\hline & $\begin{array}{l}\text { Electodessicati } \\
\text { on and } \\
\text { curettage }\end{array}$ & 57.3 & 37 & NR & $\begin{array}{l}\text { face and scalp } \\
(100)\end{array}$ & & & & & & & \\
\hline \multirow[t]{2}{*}{$\begin{array}{l}\text { Thissen } \\
2000 \\
10940063\end{array}$} & cryotherapy & NR & NR & NR & $\begin{array}{l}\text { face (46), eyelid } \\
\text { (4), ear (4), } \\
\text { trunk/neck (6), } \\
\text { forehead/temple } \\
(34), \\
\text { chin/perioral (6) }\end{array}$ & No & No & Yes & No & Unsure & Unsure & Yes \\
\hline & $\begin{array}{l}\text { surgical } \\
\text { excision }\end{array}$ & NR & NR & NR & $\begin{array}{l}\text { face }(43), \text { eyelid } \\
(8), \text { trunk/neck } \\
(14), \\
\text { forehead/temple } \\
(25), \\
\text { chin/perioral } \\
(10)\end{array}$ & & & & & & & \\
\hline
\end{tabular}




\begin{tabular}{|c|c|c|c|c|c|c|c|c|c|c|c|c|}
\hline Study & Arm & $\begin{array}{l}\text { Age, } \\
\text { Mean }\end{array}$ & $\begin{array}{l}\text { Female } \\
\%\end{array}$ & $\begin{array}{l}\text { Lesion } \\
\text { Size, } \\
\text { Mean }\end{array}$ & $\begin{array}{l}\text { Lesion } \\
\text { Location (\%) }\end{array}$ & $\begin{array}{l}1^{*} \\
\text { Adequate } \\
\text { Randomiz- } \\
\text { ation } \\
\end{array}$ & $\begin{array}{l}2^{\star} \\
\text { Allocation } \\
\text { Conceal- } \\
\text { ment }\end{array}$ & $\begin{array}{l}3^{*} \\
\text { Arms } \\
\text { Similar at } \\
\text { Baseline }\end{array}$ & $\begin{array}{l}4^{\star} \\
\text { Patients } \\
\text { Blinded }\end{array}$ & $\begin{array}{l}5^{\star} \\
\text { Providers } \\
\text { Blinded }\end{array}$ & $\begin{array}{l}6^{*} \\
\text { Outcome } \\
\text { Assessors } \\
\text { Blinded } \\
\end{array}$ & $\begin{array}{l}7^{*} \\
<20 \% \\
\text { Loss to } \\
\text { Followup }\end{array}$ \\
\hline \multirow{4}{*}{$\begin{array}{l}\text { Torres } \\
2004 \\
15606733\end{array}$} & $\begin{array}{l}\text { imiquimod, } 2 \\
\text { weeks }\end{array}$ & NR & 33.3 & $\begin{array}{l}\text { median } \\
0.9 \mathrm{~cm} 2\end{array}$ & NR & \multirow[t]{4}{*}{ Yes } & \multirow[t]{4}{*}{ No } & \multirow[t]{4}{*}{ Yes } & \multirow[t]{4}{*}{ Yes } & \multirow[t]{4}{*}{ Yes } & \multirow[t]{4}{*}{ Unsure } & \multirow[t]{4}{*}{ Yes } \\
\hline & $\begin{array}{l}\text { imiquimod, } 4 \\
\text { weeks }\end{array}$ & NR & 41.7 & $\begin{array}{l}\text { median } \\
0.8 \mathrm{~cm} 2\end{array}$ & NR & & & & & & & \\
\hline & $\begin{array}{l}\text { imiquimod, } 6 \\
\text { weeks }\end{array}$ & NR & 33.3 & $\begin{array}{l}\text { median } \\
1.2 \mathrm{~cm} 2\end{array}$ & NR & & & & & & & \\
\hline & $\begin{array}{l}\text { vehicle } \\
\text { controlled- } \\
\text { pooled }\end{array}$ & NR & 19.4 & $\begin{array}{l}\text { median } \\
1.2 \mathrm{~cm} 2\end{array}$ & NR & & & & & & & \\
\hline \multirow[t]{3}{*}{$\begin{array}{l}\text { Tran } 2012 \\
22511036\end{array}$} & PDL 15 j/cm2 & NR & 57 & $88 \mathrm{~mm} 2$ & $\begin{array}{l}\text { extremities (12), } \\
\text { trunk/neck (88) }\end{array}$ & \multirow[t]{3}{*}{ No } & \multirow[t]{3}{*}{ No } & \multirow[t]{3}{*}{ No } & \multirow[t]{3}{*}{ Yes } & \multirow[t]{3}{*}{ No } & \multirow[t]{3}{*}{ No } & \multirow[t]{3}{*}{ Yes } \\
\hline & PDL $7.5 \mathrm{j} / \mathrm{cm} 2$ & NR & 43 & $\begin{array}{l}105 \\
\mathrm{~mm} 2\end{array}$ & $\begin{array}{l}\text { extremities (50), } \\
\text { trunk/neck (50) }\end{array}$ & & & & & & & \\
\hline & No treatment & NR & 43 & $94 \mathrm{~mm} 2$ & $\begin{array}{l}\text { extremities (43), } \\
\text { trunk/neck (57) }\end{array}$ & & & & & & & \\
\hline $\begin{array}{l}\text { Wang } \\
2001 \\
11298545\end{array}$ & $\begin{array}{l}\text { Total (ALA- } \\
\text { PDT vs. } \\
\text { Cryotherapy) }\end{array}$ & NR & 50 & NR & $\begin{array}{l}\text { legs (11), arms } \\
(7), \text { trunk (54), } \\
\text { head/neck (28) }\end{array}$ & Unsure & Unsure & Unsure & No & No & Unsure & Yes \\
\hline
\end{tabular}

fluorouracil; INF = interferon; $\mathrm{NR}=$ not reported; $\mathrm{PDL}=$ pulse dye laser; $\mathrm{x} / \mathrm{wk}=$ times per week

*Design items: 1: Adequate generation of a randomized sequence reported; 2: Adequate allocation concealment reported; 3: Group similarity at baseline; 4: Adequate blinding of patients reported; 5: Adequate blinding of providers reported; 6: Adequate blinding of outcome assessors reported; 7 : Less than 20\% missing for any eligible outcome in any arm. 


\section{Recurrence, All BCC Lesions}

The evidence graph for recurrence with respect to individual treatments is sparse (Figure 6 (A) - reproduced in Figure 7 (A) for ease of reference), and comprises two connected subgraphs. Detailed results at the RCT-level are in the appendix.

Figure 7. Evidence graph of RCTs evaluating recurrence in BCCs across $(A)$ individual interventions and (B) types of interventions

(A)

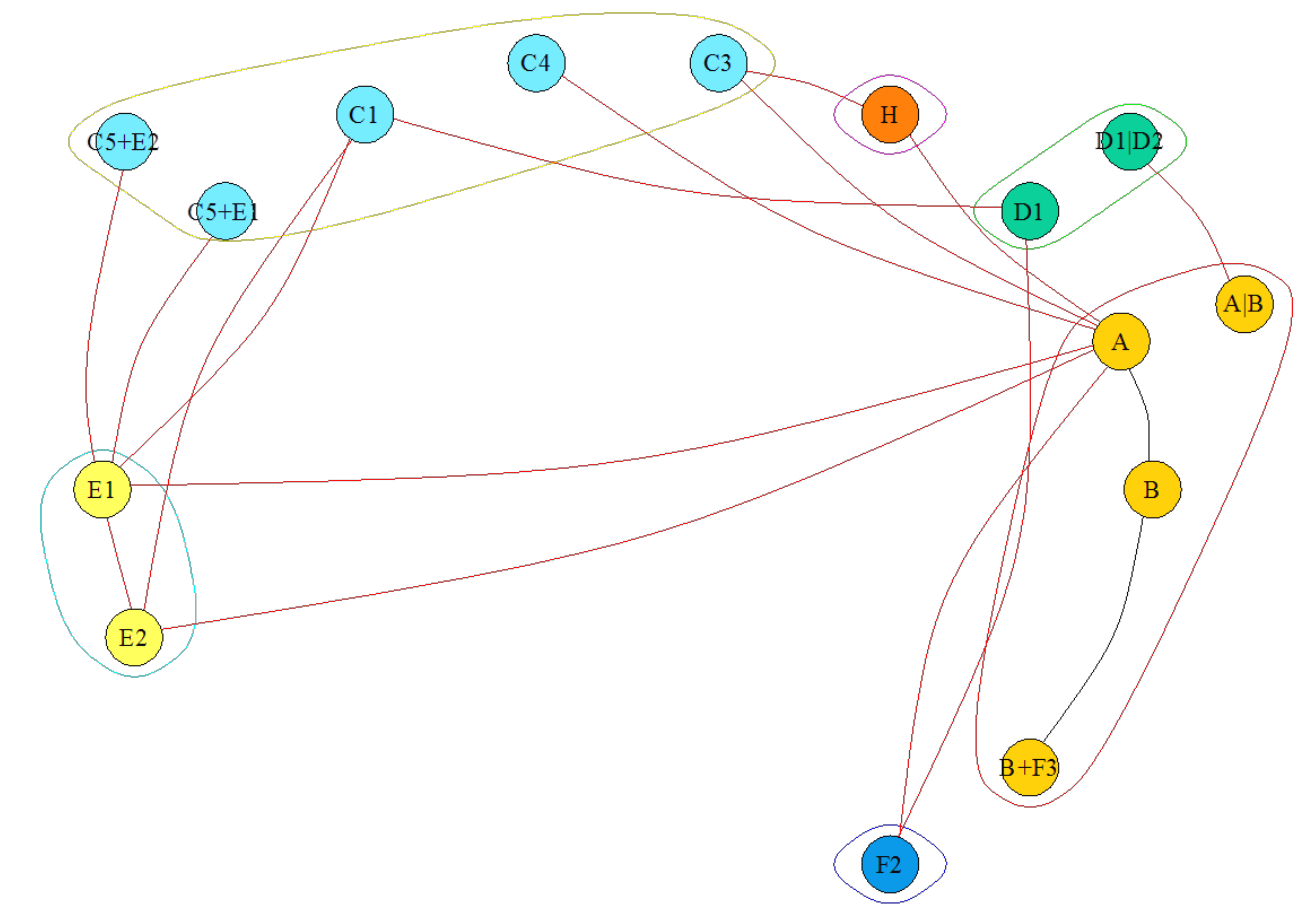

A: surgical excision
B: MMS
C1: cryotherapy
C3: curettage + diather my
C4: curettage + cr yotherapy
C5: laser
D1: external radiation
D2: brachytherapy
E1: PDT (MAL)
E2: PDT (ALA)
F1: 5-FU
F2: imiquimod
F3: INF
F4: ingenol
F5: other medical
H: curettage
I: no treatment
J: placebo/sham

MMS = Mohs micrographic surgery; PDT = photodynamic therapy; ALA = 5-aminolevulinic acid; MAL = methyl aminolevulinate; FU = fluorouracil; INF = interferon 
(B)

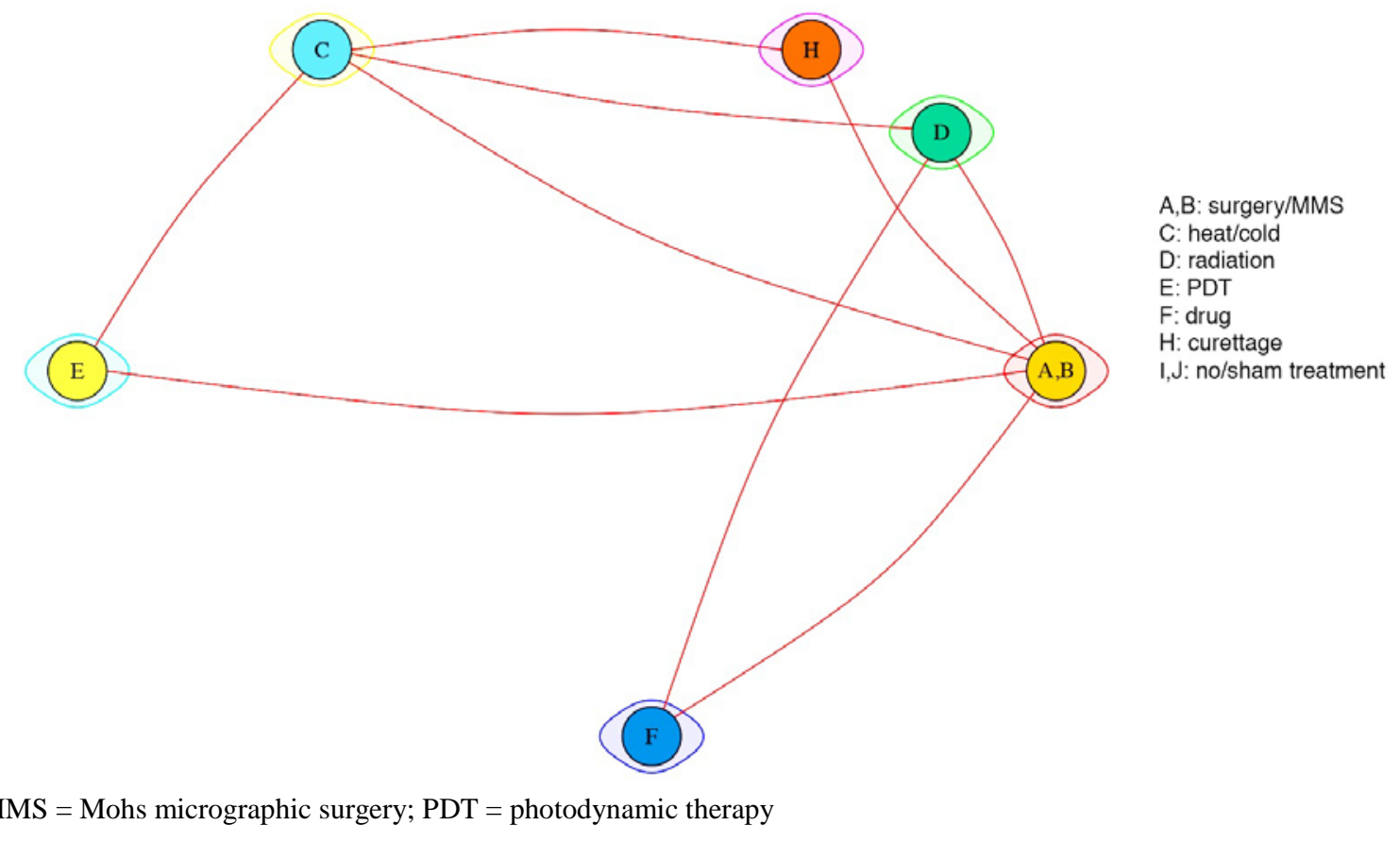

Note: The evidence graph for the individual treatments comprises 2 connected subgraphs defined by the following sets of nodes: $\mathrm{A}|\mathrm{B}, \mathrm{D} 1| \mathrm{D} 2$; and all remaining nodes.

\section{Comparisons Across Intervention Categories}

In total, 13 RCTs (1664 lesions) were included in this analysis. ${ }^{19,}$ 20, 42, 45, 50-52, 67, 70, 71, 85, 90, 104 Ten RCTs were deemed to be at low or moderate risk of bias. Cumulative sample sizes per comparison ranged from 27 to 347; for more details see Table 7.

Table 7. Sample information, recurrence (all BCC lesions, intervention categories)

\begin{tabular}{|c|c|}
\hline Studies (total sample) & $13(1664)$ \\
\hline Total sample by intervention & $(A, B): 580 ;(E): 329 ;(D): 234 ;(F): 221 ;(C): 280 ;(H): 20$ \\
\hline $\begin{array}{l}\text { Total sample by intervention, (min, } \\
\text { max) }\end{array}$ & 20,580 \\
\hline Data by comparison & $\begin{array}{l}\text { (A,B--E): } 3 \text { (305); (A,B--D): } 1 \text { (347); (A,B--C): } 2 \text { (134); (A,B--H): } 1 \text { (44); } \\
(\mathrm{A-F}): 1 \text { (203); (E--C): } 3 \text { (355); (D--F): } 1 \text { (27); (D--C): } 1(93) ;(C--H): 1 \\
(45)\end{array}$ \\
\hline Studies by comparison (min, max) & 1,3 \\
\hline $\begin{array}{l}\text { Total sample by comparison (min, } \\
\text { max) }\end{array}$ & 27,355 \\
\hline Followup median (min, max) & $28(3,96)$ months \\
\hline
\end{tabular}

A = surgical excision; B = Mohs micrographic surgery; BCC=basal cell carcinoma; C1 = cryotherapy; C3 = diathermy and curettage; C4 = cryotherapy and curettage; D1 = external radiation; E1 = MAL photodynamic therapy; E2 = ALA photodynamic therapy; F2 = Imiquimod; $\mathrm{H}$ = curettage

Table 8 shows the relative odds ratios for recurrence across intervention categories. Overall, surgical treatments (A,B), radiation (D), and drugs (F), appear to be better than interventions that destroy lesions with heat or cold (C), photodynamic therapies (E), or curettage $(\mathrm{H})$; and in many instances in the Table, statistically significantly so. There are no statistically significant 
differences among the intervention categories in the former set (namely, [A,B], D, F) or among those in the latter set (namely, C, E, H), but almost universally, the confidence intervals are broad and cannot exclude large differences in the odds of recurrence in either direction.

In Table 8, shaded cells correspond comparisons that have been inferred from the analysis model but have not been examined in the included RCTs. For example, comparisons of drugs (F) versus other intervention categories are mostly indirect, and drugs have been compared head-tohead only with radiation (D). Indirect comparisons are more uncertain than those for which headto-head data exist. The added uncertainty in indirect comparisons is partly reflected in the width of the respective 95 percent confidence intervals, which is (often much) broader for comparisons without direct data. For all comparisons that are empirically observed (all nonshaded cells in the Table), results using only head-to-head data agree well with the results from the network metaanalysis in Table 8 (see Appendix I).

Table 8. Relative odds ratios for recurrence between intervention categories (all BCC lesions, Figure 7B)

\begin{tabular}{cccccc}
\hline $\begin{array}{c}\text { Surgery/MMS } \\
(\mathrm{A}, \mathrm{B})\end{array}$ & $\mathbf{0 . 1 3 ( 0 . 0 5 , 0 . 3 5 )}$ & $0.77(0.21,2.74)$ & $0.13(0.05,0.36)$ & $1.09(0.05,24.26)$ & $0.14(0.03,0.77)$ \\
\hline $7.66(2.85,20.6)$ & $\begin{array}{c}\text { Heat/cold } \\
(\mathrm{C})\end{array}$ & $\mathbf{5 . 8 7 ( 2 . 0 2 , 1 7 . 0 8 )}$ & $1(0.48,2.08)$ & $8.33(0.4,171.71)$ & $1.07(0.23,5.1)$ \\
\hline $1.3(0.36,4.66)$ & $0.17(0.06,0.5)$ & $\begin{array}{c}\text { Radiation } \\
(\mathrm{D})\end{array}$ & $0.17(0.06,0.51)$ & $1.42(0.06,32.45)$ & $0.18(0.03,1.05)$ \\
\hline $7.63(2.79,20.9)$ & $1(0.48,2.07)$ & $\mathbf{5 . 8 5 ( 1 . 9 8 , 1 7 . 3 1 )}$ & $\begin{array}{c}\text { PDT } \\
(\mathrm{E})\end{array}$ & $8.3(0.4,172.17)$ & $1.07(0.22,5.14)$ \\
\hline $0.92(0.04,20.5)$ & $0.12(0.01,2.48)$ & $0.71(0.03,16.14)$ & $0.12(0.01,2.5)$ & $\begin{array}{c}\text { Drugs } \\
(\mathbf{F})\end{array}$ & $0.13(<0.005,3.6)$ \\
\hline $7.12(\mathbf{1 . 2 9}, 39.21)$ & $0.93(0.2,4.42)$ & $5.46(0.95,31.5)$ & $0.93(0.19,4.48)$ & $\begin{array}{c}7.75(0.28, \\
216.43)\end{array}$ & $\begin{array}{c}\text { Curettage } \\
(\mathrm{H})\end{array}$ \\
\hline
\end{tabular}

Note: Cells shaded gray indicate that the estimate is based only on indirect comparisons; bold-italic numbers indicate statistical significance. Results are given as odds ratios (95\% confidence intervals).

MMS = Mohs micrographic surgery; PDT = photodynamic therapy; BCC = basal cell carcinoma

Table 9 offers complementary information from the same analysis; for each intervention category, it shows the mean recurrence rate across the included RCTs. Surgical treatments, radiation, and drugs RCT arms had on average lower recurrence rates (3.1\% to 4.4\%) compared to photodynamic therapy, curettage, and interventions that destroy lesions with heat or cold, which had average recurrence in the 20 to 23 percent range.

Table 9. Mean and forecasted recurrence rates by intervention category (all BCC lesions)

\begin{tabular}{lcc}
\hline \multicolumn{1}{c}{ Intervention Type } & $\begin{array}{c}\text { Mean } \\
\text { Percent (95\% Cl) }\end{array}$ & $\begin{array}{c}\text { Forecast } \\
\text { Percent (95\% Cl) }\end{array}$ \\
\hline Surgery/MMS (A,B) & $3.4(1.5,7.6)$ & $3.4(0.9,11.5)$ \\
\hline Heat/cold $(\mathrm{C})$ & $21.2(14.0,30.7)$ & $21.2(8.2,44.8)$ \\
\hline Radiation (D) & $4.4(1.7,10.5)$ & $4.4(1.2,15.2)$ \\
\hline PDT (E) & $21.1(13.6,31.3)$ & $21.1(8.0,45.2)$ \\
\hline Drugs (F) & $3.1(0.2,39.0)$ & $3.1(0.1,42.8)$ \\
\hline Curettage $(\mathrm{H})$ & $20.0(5.4,52.2)$ & $20.0(4.1,59.6)$ \\
\hline
\end{tabular}

MMS = Mohs micrographic surgery; PDT = photodynamic therapy; BCC = basal cell carcinoma; $\mathrm{CI}=$ confidence interval 


\section{Comparisons Across Individual Interventions}

The results of the analyses of intervention categories are congruent with the corresponding results of the analyses of individual interventions. As evident from Figure 7, there are two connected subgraphs: a smaller one comprising the comparison between surgical treatments (surgical excision or MMS, [A,B]]) and external radiation of brachytherapy (D1|D2), and a larger one with all other interventions. In total, 14 RCTs (1772 lesions) were included in this analysis. ${ }^{19,}$ 20, 45, 50-52, 67, 70, 71, 81, 85, 90, 104, 105 They are described in Table 10.

Table 10. Sample information, recurrence (all BCC lesions, individual interventions)

\begin{tabular}{|c|c|c|}
\hline & $\begin{array}{c}\text { First subgraph }^{19,20,42,45,51,52,67,70,71,81,85,90}, \\
104,105\end{array}$ & Second Subgraph ${ }^{50}$ \\
\hline Studies (total sample) & $13(1425)$ & $1(347)$ \\
\hline $\begin{array}{l}\text { Total sample by } \\
\text { intervention }\end{array}$ & $\begin{array}{l}\text { (A): 475; (E2): 149; (D1): 61; (F2): 15; (C1): } \\
\text { 176; (C4): 38; (C3): 25; (H): 20; (B): 77; } \\
\text { (E1): } 206 \text { (F2); 180; (B+F3): 9; (C5+E1): } \\
\text { 16; (C5 + E2): } 25\end{array}$ & $(\mathrm{~A} \mid \mathrm{B}): 174 ;(\mathrm{D} 1 \mid \mathrm{D} 2): 173$ \\
\hline $\begin{array}{l}\text { Total sample by } \\
\text { intervention, (min, } \\
\text { max) }\end{array}$ & 9,298 & 173,174 \\
\hline Data by comparison & $\begin{array}{l}\text { (A--E2): } 1 \text { (171); (A--C4): } 1 \text { (85); (A--C3): } 1 \\
\text { (49); (A--H): } 1 \text { (44); (A--B): } 1 \text { (140); (A--E1): } \\
2 \text { (134); (A-F2): } 1 \text { (383); (E2--C1): } 1 \text { (83); } \\
(\mathrm{D} 1--F 2): 1(27) ;(D 1--C 1): 1 \text { (93); (C1--E1): } \\
1 \text { (193); (C3--H): } 1 \text { (45); (B--B+F3): } 1 \text { (15); } \\
\text { (E1--C5+E1): } 1 \text { (32); (E2-C5 + E2): } 47\end{array}$ & (A|B--D1|D2): 1 (347) \\
\hline $\begin{array}{l}\text { Studies by } \\
\text { comparison (min, } \\
\max \text { ) }\end{array}$ & 1,2 & 1,1 \\
\hline $\begin{array}{l}\text { Total sample by } \\
\text { comparison (min, } \\
\max \text { ) }\end{array}$ & 15,193 & 347,347 \\
\hline $\begin{array}{l}\text { Followup median } \\
(\min , \max )\end{array}$ & $28(3,>120)$ months & $41(41,41)$ months \\
\hline
\end{tabular}

Tables 11 and 12 show the relative effects for the larger and smaller subgraphs, respectively. Because the comparisons across individual interventions are sparse, however, the confidence intervals of the odds ratios for most indirect comparisons are very broad and cannot exclude very large differences between the compared interventions.

Table 13 shows, for each intervention, the mean recurrence rates across all RCTs; estimates for interventions in both subgraphs are listed in the table. One cannot compare statistically the estimated recurrence rates between an intervention in the first subgraph (e.g., cryotherapy [C1]) and the second subgraph (e.g., external radiation or brachytherapy [D1|D2]), because they come from disjoint analyses. The mean recurrence rates for individual interventions follow the same pattern as the corresponding recurrence rates for intervention categories. For example, the point estimates for the mean recurrence rate for surgical excision (A), MMS (B), and a combination of MMS and interferon (B+F3) ranged between 4.0 and 4.5 percent; and it was estimated at 3.4 percent for surgical interventions $(\mathrm{A}, \mathrm{B})$ in Table 8. 
Table 11. Relative odds ratios for recurrence between individual interventions (all BCC lesions, Figure 7A, first subgraph)

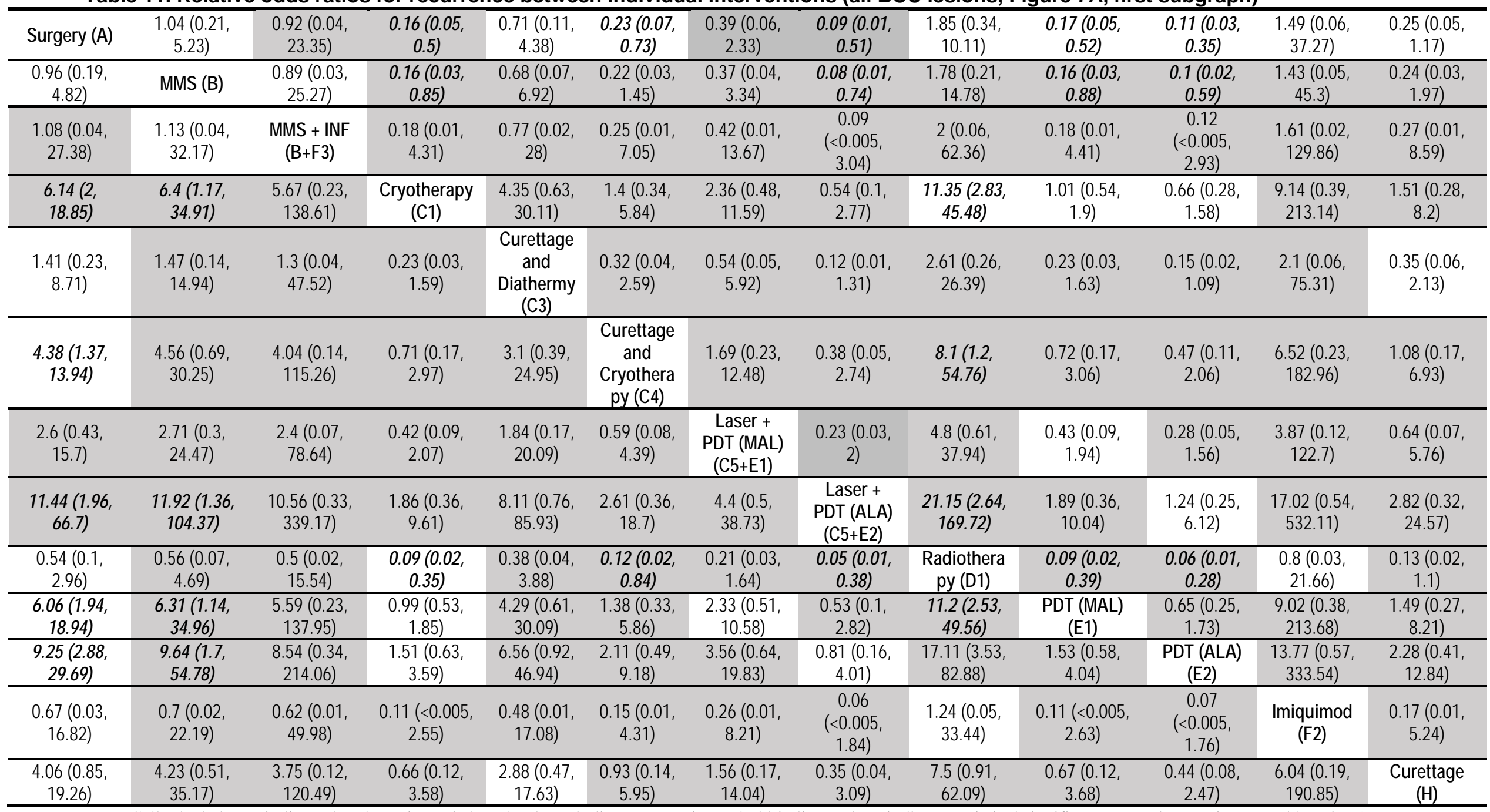

Note: Cells shaded gray indicate that the estimate is based only on indirect comparisons; bold-italic numbers indicate statistical significance. Results are given as odds ratios (95\% confidence intervals). 
ALA = 5-aminolevulinic acid; BCC = basal cell carcinoma; FU = fluorouracil; INF = interferon; MAL = methyl aminolevulinate; MMS = Mohs micrographic surgery; PDT = photodynamic therapy

Table 12. Relative odds ratios for recurrence between individual interventions (all BCC lesions, Figure 7A, second subgraph) Surgical excision 
Table 13. Mean recurrence rates by intervention (all BCC lesions)

\begin{tabular}{lcc}
\hline \multicolumn{1}{c}{ Intervention Type } & $\begin{array}{c}\text { Mean } \\
\text { Percent (95\% Cl) }\end{array}$ & $\begin{array}{c}\text { Forecast } \\
\text { Percent (95\% Cl) }\end{array}$ \\
\hline First subgraph (Figure 7) & & \\
\hline Surgical excision (A) & $4.2(1.7,9.7)$ & $4.2(0.8,18.5)$ \\
\hline MMS (B) & $4.0(0.9,16.4)$ & $4.0(0.5,24.9)$ \\
\hline MMS+INF (B+F3) & $4.5(0.2,51.4)$ & $4.5(0.2,58.7)$ \\
\hline Cryotherapy (C1) & $21.0(11.6,35.1)$ & $21.0(5.3,55.7)$ \\
\hline Diathermy+curettage (C3) & $5.8(1.0,27.2)$ & $5.8(0.6,37.3)$ \\
\hline Cryotherapy+Curettage (C4) & $15.9(5.1,40.0)$ & $15.9(2.8,55.1)$ \\
\hline Laser+PDT (MAL) (C5+E1) & $10.1(2.3,35.1)$ & $10.1(1.4,47.6)$ \\
\hline Laser+PDT (ALA) & $33.1(9.7,69.4)$ & $33.1(6.0,79.5)$ \\
\hline External radiation (D1) & $2.3(0.5,9.1)$ & $2.3(0.3,14.8)$ \\
\hline PDT (MAL) (E1) & $20.8(11.0,35.7)$ & $20.8(5.2,55.8)$ \\
\hline PDT (ALA) (E2) & $28.6(15.0,47.6)$ & $28.6(7.5,66.6)$ \\
\hline Imiquimod (F2) & $2.8(0.1,39.0)$ & $2.8(0.1,46.2)$ \\
\hline Curettage (H) & $14.9(3.6,45.2)$ & $14.9(2.2,58.2)$ \\
\hline Second subgraph (Figure 7) & & \\
\hline $\begin{array}{l}\text { Surgical excision or Mohs } \\
\text { (A|B) }\end{array}$ & $0.6(0.1,4.0)$ & NA \\
\hline $\begin{array}{l}\text { External radiation or } \\
\text { brachytherapy (D1|D2) }\end{array}$ & $4.6(2.3,9.0)$ & NA \\
\hline $\begin{array}{l}\text { ALA = 5-aminolevulinic acid; BCC = basal cell carcinoma; CI = confidence interval; FU = fluorouracil; INF = interferon; MAL } \\
\text { = methyl aminolevulinate; MMS = Mohs micrographic surgery; PDT = photodynamic therapy }\end{array}$
\end{tabular}




\section{Recurrence, Subgroup Analyses by Lesion Type}

We conducted subgroup analyses by the type of BCC lesion. We report analyses comparing intervention categories, but not analyses comparing individual treatments. The latter are very sparse, and their results are very similar to the pertinent comparisons in Tables 9 and 10.

Many subgroup analyses per lesion type are possible; we describe here analyses in RCTs of lower-risk lesions (strata of predominantly [>80\%] superficial BCCs, predominantly nodular BCCs, and superficial or nodular BCCs) overall, and broken down by lesion type; and of higherrisk lesions (morpheaform, micronodular, trabecular, infiltrative, or squamous differentiation).

Eleven RCTs ( $\mathrm{n}=1234$ lesions) included low risk BCCs (nodular and superficial subtypes). All results about comparisons among intervention categories are the same as in the previous section (Tables 7 and 8 ).

With respect to RCT strata of predominantly superficial lesions, a single RCT deemed to be at low risk of bias compared cryotherapy $(\mathrm{C} 1, \mathrm{n}=93)$ with PDT with MAL $(\mathrm{E} 1, \mathrm{n}=100) .{ }^{51} \mathrm{~A}$ second RCT, also deemed to be at low risk of bias, compared surgery to drug therapy, in this case Imiquimod. $^{52}$ Results are shown in Tables 14 and 15. Both studies had a followup of 60 months. Briefly, there was no statistically significant difference between the two interventions in ether study, but based on the width of the $95 \%$ confidence intervals, one cannot exclude differences in the odds of the outcome as large as $80 \%$ in either direction.

Table 14. Relative odds ratios for recurrence between interventions (predominantly superficial BCC lesions)

\begin{tabular}{|c|c|c|c|}
\hline $\begin{array}{c}\text { Heat/cold (C) } \\
{[\text { Cryotherapy (C1)] }}\end{array}$ & $0.91(0.46,1.82)$ & & \\
\hline \multirow[t]{3}{*}{$1.10(0.55,2.19)$} & $\begin{array}{c}\text { PDT (E) } \\
\text { [PDT (MAL) (E1)] }\end{array}$ & & \\
\hline & & Surgery|MMS (A|B) & $0.28(0.06,1.35)$ \\
\hline & & $3.57(0.74,17.26)$ & Drug (F) \\
\hline
\end{tabular}

MMS = Mohs micrographic surgery; PDT $($ MAL) = methyl aminolevulinate photodynamic therapy

Table 15. Mean recurrence rates by intervention category (predominantly superficial BCC lesions)

\begin{tabular}{lc}
\hline \multicolumn{1}{c}{ Intervention Type } & $\begin{array}{c}\text { Mean Recurrence } \\
\text { Rate }(\mathbf{9 5 \%} \mathbf{~ C l})\end{array}$ \\
\hline Subgraph 1 (Figure 7) & \\
\hline $\begin{array}{l}\text { Heat/cold (C) } \\
{[\text { Cryotherapy (C1)] }}\end{array}$ & $20.4(13.4,29.8)$ \\
\hline PDT (E) & $22.0(14.9,31.2)$ \\
\hline SPDT (MAL) (E1)] & \\
\hline Subgraph 2 (Figure 7) & $2.1(0.5,8.1)$ \\
\hline Surgery|MMS (A|B) & $7.2(3.6,18.8)$ \\
\hline
\end{tabular}

Note: Forecasted expected recurrence rates in groups of patients similar to the patients included in the analyzed RCTs are not given, because these results are from a fixed effects analysis

CI = confidence interval; MMS = Mohs micrographic surgery; PDT (MAL) = methyl aminolevulinate photodynamic therapy 
Information about the samples for predominantly nodular lesions is in Table 16 . The corresponding results are listed in Tables 17 and 18. These results are congruent with the corresponding results from the analyses in Tables 14 and 15.

Table 16. Sample information (predominantly nodular lesions)

\begin{tabular}{|c|c|}
\hline & Studies $^{20,45,52,67,70,85}$ \\
\hline Studies (total sample) & $6(747)$ \\
\hline $\begin{array}{l}\text { Total sample by } \\
\text { intervention }\end{array}$ & $(A, B): 335 ;(E): 163 ;(C): 16 ;(D): 12 ;(F): 221$ \\
\hline $\begin{array}{l}\text { Total sample by } \\
\text { intervention, (min, } \\
\operatorname{max)}\end{array}$ & 12,335 \\
\hline Data by comparison & $\begin{array}{l}\text { (A,B--E): } 3 \text { (305); (E--C): } 1 \text { (32); (A-F): 383; (D--F): } 1 \\
(27)\end{array}$ \\
\hline $\begin{array}{l}\text { Studies by } \\
\text { comparison (min, } \\
\text { max) }\end{array}$ & 1,3 \\
\hline $\begin{array}{l}\text { Total sample by } \\
\text { comparison (min, } \\
\text { max) }\end{array}$ & 27,383 \\
\hline $\begin{array}{l}\text { Followup median } \\
\text { (min, max) }\end{array}$ & $48(12,96)$ \\
\hline
\end{tabular}

Table 17. Relative odds ratios for recurrence between interventions (predominantly nodular BCC lesions)

\begin{tabular}{|c|c|c|c|c|}
\hline $\begin{array}{l}\text { Surgery|MMS } \\
\text { (A|B) }\end{array}$ & $\begin{array}{c}0.04(<0.005 \\
0.57)\end{array}$ & $0.23(0.01,9.06)$ & $\begin{array}{c}0.04(0.01 \\
0.28)\end{array}$ & $0.28(0.03,3.06)$ \\
\hline $\begin{array}{c}25.53(1.74 \\
374.42)\end{array}$ & $\begin{array}{l}\text { Heat or Cold } \\
\text { (C) }\end{array}$ & $\begin{array}{c}5.77(0.11 \\
295.02)\end{array}$ & $\begin{array}{l}1(0.09, \\
10.84)\end{array}$ & $7.1(0.45,111.9)$ \\
\hline $4.43(0.11,177.45)$ & $\begin{array}{c}0.17(<0.005 \\
8.86)\end{array}$ & $\begin{array}{l}\text { Radiotherapy } \\
\text { (D) }\end{array}$ & $\begin{array}{c}0.17(0.01 \\
5.61)\end{array}$ & $1.23(0.03,52.02)$ \\
\hline $\begin{array}{c}25.59(3.61 \\
181.57)\end{array}$ & $1(0.09,10.9)$ & $\begin{array}{c}5.78(0.18 \\
187.64)\end{array}$ & PDT (E) & $7.12(0.91,55.67)$ \\
\hline $3.6(0.33,39.57)$ & $\begin{array}{c}0.14(0.01, \\
2.22)\end{array}$ & $0.81(0.02,34.36)$ & $\begin{array}{c}0.14(0.02 \\
1.1)\end{array}$ & Drug (F) \\
\hline
\end{tabular}

Note: Results from comparisons in the first and second subgraphs are shown in the upper left and lower right blocks in this Table. Cells shaded gray indicate that the estimate is based only on indirect comparisons; bold-italic numbers indicate statistical significance. Results are given as odds ratios (95\% confidence intervals).

MMS = Mohs micrographic surgery; PDT = photodynamic therapy; $\mathrm{BCC}=$ basal cell carcinoma

Table 18. Mean and forecasted recurrence rates by intervention category (predominantly nodular BCC lesions)

\begin{tabular}{ccc}
\hline Intervention Type & $\begin{array}{c}\text { Mean } \\
\text { Percent (95\% Cl) }\end{array}$ & $\begin{array}{c}\text { Forecast } \\
\text { Percent (95\% Cl) }\end{array}$ \\
\hline First subgraph (Figure 7) & & \\
\hline Surgery/MMS (A,B) & $0.9(0.2,4.4)$ & $0.9(0.1,8.8)$ \\
\hline Heat/cold (C) & $18.7(2.7,66.0)$ & $18.7(1.5,78.1)$ \\
\hline
\end{tabular}




\begin{tabular}{ccc}
\hline Intervention Type & $\begin{array}{c}\text { Mean } \\
\text { Percent (95\% Cl) }\end{array}$ & $\begin{array}{c}\text { Forecast } \\
\text { Percent (95\% Cl) }\end{array}$ \\
\hline Radiation (D) & $3.8(0.1,52.2)$ & $3.8(0.1,62.5)$ \\
\hline PDT (E) & $18.8(7.3,40.4)$ & $18.8(3.0,63.8)$ \\
\hline Drugs (F) & $3.1(0.6,15.8)$ & $3.1(0.3,27.5)$ \\
\hline
\end{tabular}

MMS = Mohs micrographic surgery; PDT = photodynamic therapy; Drugs, in this case represents Imiquimod; CI = confidence interval

Finally, with respect to high risk lesions, a single RCT compared surgical excision (A) with MMS (B) in histologically aggressive facial lesions (morpheaform, micronodular, trabecular, infiltrative, or squamous differentiation). ${ }^{81}$ Although the average recurrence rate was smaller in the MMS arm (3.4\% [95\% CI 1.0\% to $11.0 \%$ ]) versus the surgical excision arm (4.8\% [95\% CI, $2.5 \%$ to $8.8 \%]$ ), it was not significantly so (odds ratio for surgical excision versus MMS 1.43 [95\% CI 0.35 to 5.95$])$.

\section{Recurrence, Other Subgroup Analyses (Lesion Location, Lesion Size)}

Table 19 summarizes results from two RCTs by lesion location and size. One RCT comparing surgical excision (A) versus MAL PDT (E) in predominantly nodular lesions ${ }^{22,85}$ examined subgroups defined by lesion diameter (<=10 mm versus 10 to $20 \mathrm{~mm}$ ) and found no evidence of effect modification by lesion size at one through 5 years of follow up. Another RCT comparing cryotherapy (C) to radiation therapy (D) in low-risk lesions (mixed superficial and nodular BCCs) found no evidence of effect modification by lesion size (smaller than $10 \mathrm{~mm}$, between 10 and $20 \mathrm{~mm}$, and larger than $20 \mathrm{~mm}$ ) or location (eyelids, face or neck, and trunk). ${ }^{71}$

Table 19. Subgroup results by lesion size and location for recurrence (BCC lesions)

\begin{tabular}{|c|c|c|c|c|c|c|}
\hline Study & Comparison & Timepoint & Subgroup & $\begin{array}{l}\text { n/N Arm } 1 \text { vs. } \\
\text { n/N Arm } 2\end{array}$ & $\begin{array}{l}\text { OR }(95 \% \mathrm{Cl}) ; \text { P- } \\
\text { Value Within }\end{array}$ & $\begin{array}{l}\text { P-Value } \\
\text { Between }\end{array}$ \\
\hline \multirow{10}{*}{$\begin{array}{l}\text { Rhodes } \\
2004 \\
14732655\end{array}$} & $\begin{array}{l}\text { Excision (A) vs } \\
\text { MAL-PDT (E) }\end{array}$ & \multirow[t]{2}{*}{12 months } & $\begin{array}{l}\text { lesion diameter: } \\
10-20 \mathrm{~mm}\end{array}$ & $0 / 14$ vs. $1 / 19$ & $\begin{array}{l}0.43(0.02,11.23) \\
p=1.00\end{array}$ & \multirow[t]{2}{*}{ NA } \\
\hline & & & $\begin{array}{l}\text { lesion diameter: } \\
<=10 \mathrm{~mm}\end{array}$ & $0 / 34$ vs $1 / 29$ & $\begin{array}{l}0.28(0.01,7.02) ; \\
p=0.46\end{array}$ & \\
\hline & & \multirow[t]{2}{*}{24 months } & $\begin{array}{l}\text { lesion diameter: } \\
10-20 \mathrm{~mm}\end{array}$ & $0 / 14$ vs. $0 / 19$ & NA & \multirow[t]{2}{*}{ NA } \\
\hline & & & $\begin{array}{l}\text { lesion diameter: } \\
<=10 \mathrm{~mm}\end{array}$ & $0 / 29$ vs. $3 / 29$ & $\begin{array}{l}0.13(0.01,2.60) ; \\
p=0.24\end{array}$ & \\
\hline & & \multirow[t]{2}{*}{36 months } & $\begin{array}{l}\text { lesion diameter: } \\
10-20 \mathrm{~mm}\end{array}$ & $1 / 14$ vs. $1 / 19$ & $\begin{array}{l}1.38(0.08,24.23) \\
p=1.00\end{array}$ & \multirow[t]{2}{*}{ NA } \\
\hline & & & $\begin{array}{l}\text { lesion diameter: } \\
<=10 \mathrm{~mm}\end{array}$ & $0 / 29$ vs. $1 / 29$ & $\begin{array}{l}0.32(0.01,8.24) \\
p=1.00\end{array}$ & \\
\hline & & \multirow[t]{2}{*}{48 months } & $\begin{array}{l}\text { lesion diameter: } \\
10-20 \mathrm{~mm}\end{array}$ & $1 / 14$ vs. $0 / 19$ & $\begin{array}{l}4.33(0.16,114.58) ; \\
p=0.42\end{array}$ & \multirow[t]{2}{*}{ NA } \\
\hline & & & $\begin{array}{l}\text { lesion diameter: } \\
<=10 \mathrm{~mm}\end{array}$ & $0 / 29$ vs. $0 / 29$ & NA & \\
\hline & & \multirow[t]{2}{*}{60 months } & $\begin{array}{l}\text { lesion diameter: } \\
10-20 \mathrm{~mm}\end{array}$ & 0/14 vs 0/19 & NA & \multirow[t]{2}{*}{ NA } \\
\hline & & & $\begin{array}{l}\text { lesion diameter: } \\
<=10 \mathrm{~mm}\end{array}$ & $0 / 29$ vs $0 / 29$ & NA & \\
\hline $\begin{array}{l}\text { Hall } 1986 \\
3514075\end{array}$ & $\begin{array}{l}\text { Cryotherapy } \\
\text { (E) vs } \\
\text { Radiation (D) }\end{array}$ & 12 months & $\begin{array}{l}\text { Lesion location: } \\
\text { eyelids }\end{array}$ & $3 / 6$ vs. $0 / 3$ & $\begin{array}{l}7.00(0.25,192.26) \\
p=0.464\end{array}$ & $p=0.97$ \\
\hline
\end{tabular}




\begin{tabular}{|c|c|c|c|c|c|c|}
\hline Study & Comparison & Timepoint & Subgroup & $\begin{array}{l}\text { n/N Arm } 1 \text { vs. } \\
\text { n/N Arm } 2\end{array}$ & $\begin{array}{l}\text { OR (95\% Cl); P- } \\
\text { Value Within }\end{array}$ & $\begin{array}{l}\text { P-Value } \\
\text { Between }\end{array}$ \\
\hline & therapy & & & & & \\
\hline & & & $\begin{array}{l}\text { Lesion location: } \\
\text { face/neck }\end{array}$ & $12 / 30$ vs. $2 / 40$ & $\begin{array}{l}12.67(2.56,62.65) \\
p<0.001\end{array}$ & \\
\hline & & & $\begin{array}{l}\text { Lesion location: } \\
\text { trunk }\end{array}$ & $2 / 8$ vs. $0 / 6$ & $\begin{array}{l}5.00(0.20,125.78) \\
p=0.473\end{array}$ & \\
\hline \multirow{4}{*}{$\begin{array}{l}\text { Hall } 1986 \\
3514075\end{array}$} & Cryotherapy & 12 months & Lesion diameter & $6 / 19$ vs. $0 / 19$ & $18.78(0.97$ & NA \\
\hline & & & $<10 \mathrm{~mm}$ & & $362.00) ; p=0.020$ & \\
\hline & & & $\begin{array}{l}\text { Lesion diameter } \\
10-20 \mathrm{~mm}\end{array}$ & $9 / 23$ vs. $2 / 25$ & $\begin{array}{l}7.39(1.39,39.27) ; \\
p=0.016\end{array}$ & \\
\hline & & & $\begin{array}{l}\text { Lesion diameter } \\
>20 \mathrm{~mm}\end{array}$ & $2 / 2$ vs. $0 / 5$ & $\begin{array}{l}55.00(0.83 \\
3650.69) ; p=0.048\end{array}$ & \\
\hline \multirow[t]{3}{*}{$\begin{array}{l}\text { Hall } 1986 \\
3514075\end{array}$} & $\begin{array}{l}\text { Cryotherapy } \\
\text { (E) vs }\end{array}$ & 24 months & $\begin{array}{l}\text { Lesion location: } \\
\text { eyelids }\end{array}$ & $3 / 6$ vs. $0 / 3$ & $\begin{array}{l}7.00(0.25,192.26) \\
p=0.464\end{array}$ & NA \\
\hline & & & $\begin{array}{l}\text { Lesion location: } \\
\text { face/neck }\end{array}$ & $12 / 30$ vs. $2 / 40$ & $\begin{array}{l}12.67(2.56,62.65) ; \\
p<0.001\end{array}$ & \\
\hline & & & $\begin{array}{l}\text { Lesion location: } \\
\text { trunk }\end{array}$ & $2 / 8$ vs. $0 / 6$ & $\begin{array}{l}5.00(0.20,125.78) ; \\
p=0.473\end{array}$ & \\
\hline \multirow{4}{*}{$\begin{array}{l}\text { Hall } 1986 \\
3514075\end{array}$} & Cryotherapy & 12 months & Lesion diameter & $6 / 19$ vs. $0 / 19$ & 18.78 (0.97, & NA \\
\hline & $\begin{array}{l}\text { (E) vs } \\
\text { Radiation (D) }\end{array}$ & & $<10 \mathrm{~mm}$ & & 362.00) $\mathrm{p}=0.020$ & \\
\hline & & & $\begin{array}{l}\text { Lesion diameter } \\
10-20 \mathrm{~mm}\end{array}$ & $9 / 23$ vs. $2 / 25$ & $\begin{array}{l}7.39(1.39,39.27) \\
p=0.016\end{array}$ & \\
\hline & & & $\begin{array}{l}\text { Lesion diameter } \\
>20 \mathrm{~mm}\end{array}$ & $2 / 2$ vs. $0 / 5$ & $\begin{array}{l}55.00(0.83 \\
3650.69) ; p=0.048\end{array}$ & \\
\hline
\end{tabular}

NA = not significant; PDT = photodynamic therapy; MAL = methyl aminolevulinate; $\mathrm{OR}$ = odds ratio; $\mathrm{CI}$ = confidence interval

\section{Recurrence, Results From Nonrandomized Studies (BCC Lesions)}

Two NRCSs reported on recurrence in populations with only BCC lesions. The first included 74 patients and reported on a matched population of 94 superficial (64\%) and nodular (36\%) BCCs 25 months after treatment. The study was rated as having a moderate risk of confounding bias because of lack of blinding, and unclear reporting. The mean age at baseline was 66 (range: 49 to 90), 47 percent of the population was female. Recurrence was similar across groups (4.2\% in the ALA-PDT group vs. 4.3\% in the surgical excision group; OR: 0.96 [95\% CI 0.13 to 7.09]). ${ }^{148}$ The second reported recurrence in 621 people (47\% female) with BCC lesions (38.5\% superficial, $17 \%$ nodular, and $44.5 \%$ infiltrative, micronodular, morpheaform, or sclerosing). This study was judged to have a high risk of confounding and selection bias because of lack of blinding, unclear distribution of dropouts, unclear results reporting, and uneven groups at baseline that were not accounted for in the analysis. Surgical excision had a higher rate of recurrence up to 5 years compared to Imiquimod (HR 2.13; 95\% CI 1.28 to 3.53). ${ }^{149}$

Two NRCS reported on recurrence in populations with both BCCs and SCC lesions. One reported on 1174 patients with 1488 lesions selected from a private, university-affiliated clinic and a nearby Veterans Affairs clinic. This study was deemed to have a low risk of bias, with 
balanced groups, consecutive recruitment, blinding of outcome assessors, and adequate accounting for people lost to followup. Most (75\%) of the lesions were BCCs; the other 25 percent were SCCs; 26 percent were female, 40 percent had a Fitzpatrick skin score of I or II, and 3 percent were immunocompromised due to prior solid-organ transplant. The lesions were treated by Mohs surgery (246; 65\% in the H-zone of the face), surgical excision (251; $26 \%$ in $\mathrm{H}$ zone of the face), and electrodessication and curettage (ED\&C) (136; 11\% in H-zone of the face). ED\&C had the highest rate of recurrence after 5 years (4.9\%), then excision (3.5\%), and finally Mohs (2.1\%). In a subsample of 240 pairs of tumors matched on propensity score, the difference in hazard of recurrence between Mohs and excision was not statistically significant $(0.61 ; 95 \%$ CI $0.3,1.24) .{ }^{142,144-147}$ A secondary analysis limited to 1483 lesions judged appropriate for Mohs surgery reported a similar nonsignificant 5-year adjusted hazard ratio for recurrence $\left(0.6\right.$; 95\% CI 0.3, 1.0), ${ }^{156}$ The second NRCS reported on two doses and schedules of orthovoltage radiotherapy. The population consisted of 436 lesions in 385 elderly people, with BCCs (71\%) and SCCs (29\%). The mean age was 78, and 42 percent were female. A lower dose of radiation (3675 cGy) had a nonsignificantly higher recurrence rate than the higher dose (4500 cGy) (HR: 0.483 ; $95 \%$ CI 0.065 to 3.58$){ }^{151}$

\section{Lack of Histologic Clearance (All BCC Lesions)}

The evidence graph for lack of histologic clearance with respect to individual treatments is sparse (Figure 6 (B) - reproduced in Figure 8 (A) for ease of reference) and comprises 5 connected subgraphs. Detailed results at the RCT-level are in Appendix I. 
Figure 8. Evidence graph of RCTs evaluating lack of histological clearance in BCCs across (A) individual interventions and (B) types of interventions

(A)

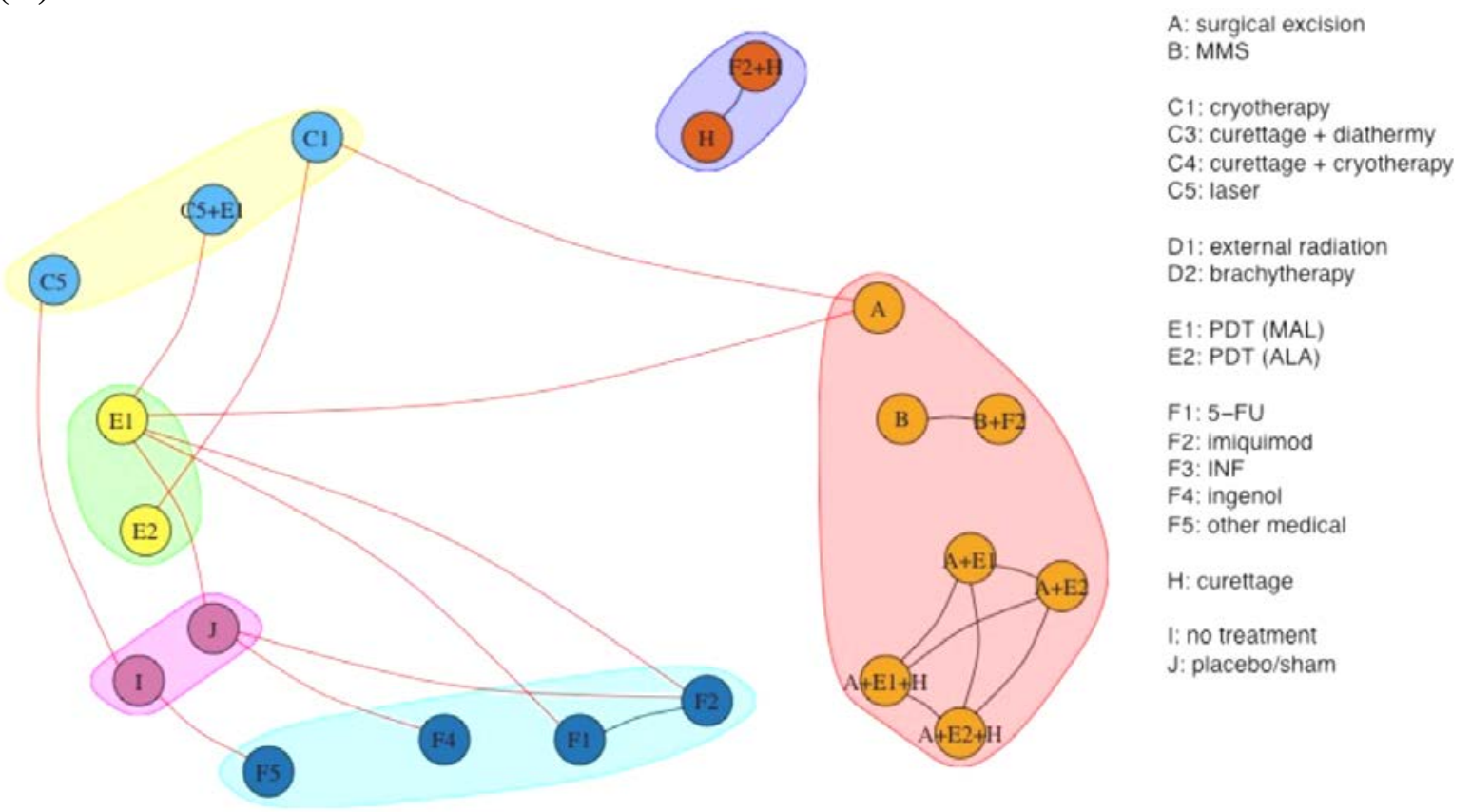

(B)

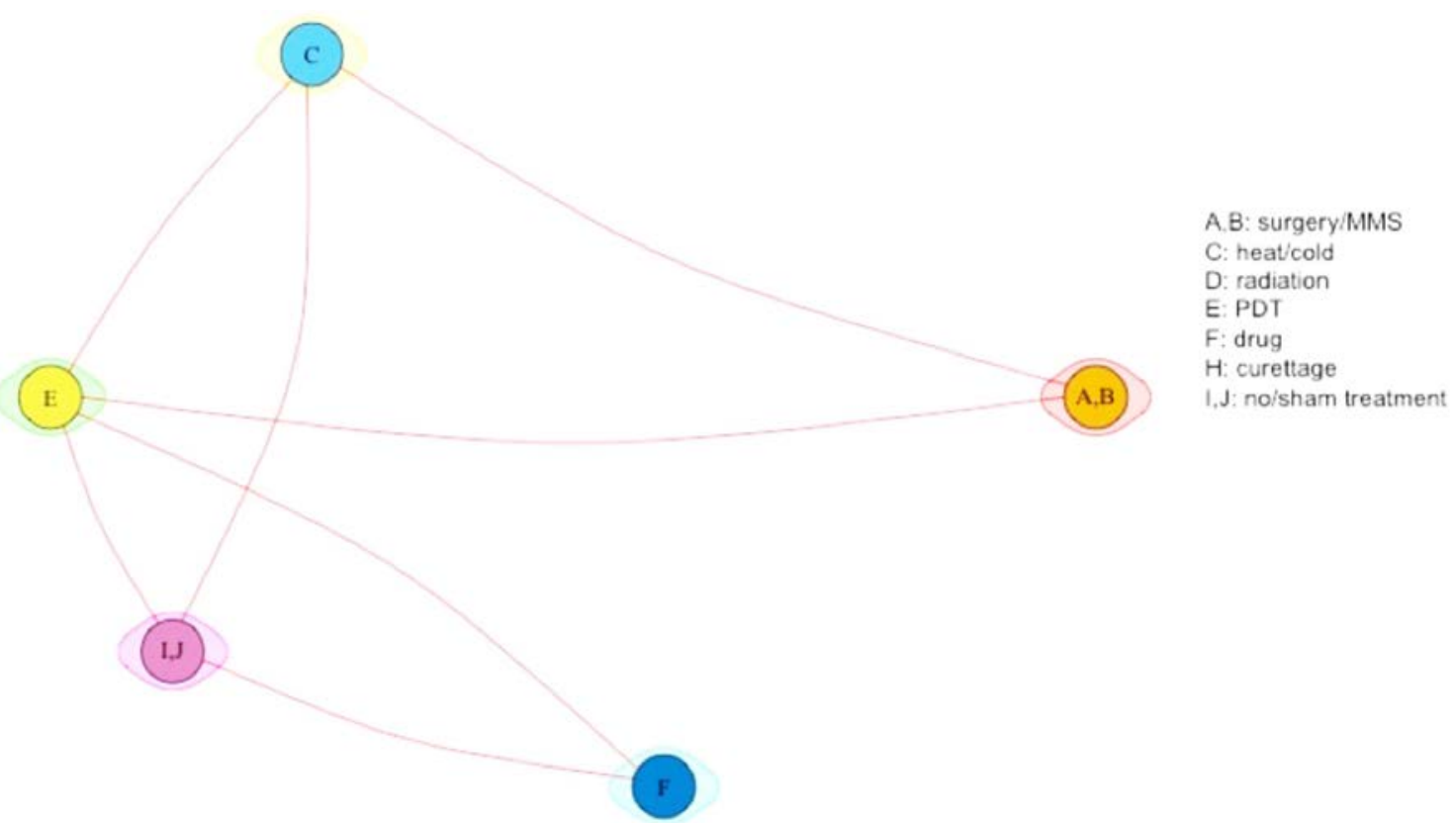

MMS = Mohs micrographic surgery; PDT = photodynamic therapy; ALA = 5-aminolevulinic acid; MAL = methyl aminolevulinate; FU = fluorouracil; INF = interferon

\section{Comparisons Across Intervention Categories}

In total, 15 RCTs (1940 lesions) were included in this analysis. 45, 49, 54, 55, 64, 66, 68-70, 92-94, 99, 101, ${ }^{104}$ Twelve RCTs were deemed to be at low or moderate risk of bias. Cumulative sample sizes per comparison ranged from 44 to 1196; for more details see Table 20. 
Table 20. Sample information, lack of histological clearance (all BCC lesions, intervention categories)

\begin{tabular}{|c|c|}
\hline Studies (total sample) & $15(1940)$ \\
\hline Total sample by intervention & (F): 825; (I,J): 607; (A,B): 83; (C): 131; (E): 294 \\
\hline $\begin{array}{l}\text { Total sample by intervention, (min, } \\
\text { max) }\end{array}$ & 83,825 \\
\hline Data by comparison & $\begin{array}{l}\text { (F--I,J): } 7 \text { (1196); (F--E): } 1 \text { (271); (I,J--C): } 2 \text { (44); (I,J--E): } 1 \text { (150); (A,B-- } \\
\text { C): } 1 \text { (96); (A,B--E): } 1 \text { (68); (C--E): } 2 \text { (115) }\end{array}$ \\
\hline Studies by comparison (min, max) & 1,7 \\
\hline $\begin{array}{l}\text { Total sample by comparison (min, } \\
\text { max) }\end{array}$ & 44,1196 \\
\hline Followup median (min, max) & $3(1.5,36)$ months \\
\hline
\end{tabular}

Table 21 shows the relative odds ratios for lack of histologic clearance across intervention categories. Overall, surgical treatments (A,B) were statistically significantly better than any other intervention category in terms of histological clearance. No or sham treatment (I,J) was statistically significantly worse that all other treatments. Among the other intervention categories, the odds ratios favor PDT (E) over interventions that destroy lesions with heat or cold (C), and the latter (C) over drugs (F), but these differences are not statistically significant. Further, the confidence intervals for the comparisons between the latter three treatments are broad and cannot exclude large effects in either direction.

In the table, shaded cells correspond to comparisons that have been inferred from the analysis model, but that have not been examined in the included RCTs. For example, comparisons of surgical treatments $(\mathrm{A}, \mathrm{B})$ or interventions that destroy lesions with heat or cold (C) versus drugs (F) or placebo (I,J) are indirect, through PDT (E) as the common comparator. Indirect comparisons are more uncertain that those for which head-to-head data exist. The added uncertainty in the indirect comparisons is partly reflected in the width of the respective 95 percent confidence intervals, which is (often much) broader for comparisons without versus with direct data. For all comparisons that have been empirically observed (all nonshaded cells in the table), results using only head-to-head data agree well with the results from the network metaanalysis in Table 17 (see Appendix I).

Table 21. Relative odds ratios for lack of histologic clearance between intervention categories (all BCC lesions, Figure 8B)

\begin{tabular}{|c|c|c|c|c|}
\hline $\begin{array}{c}\text { Surgery/MMS } \\
(A, B)\end{array}$ & $\begin{array}{c}0.04 \\
(<0.005,0.77)\end{array}$ & $\begin{array}{c}0.05 \\
(<0.005,1.03)\end{array}$ & $\begin{array}{c}0.02 \\
(<0.005,0.41)\end{array}$ & $\begin{array}{c}<0.005 \\
(<0.005,0.04)\end{array}$ \\
\hline $\begin{array}{c}27.5 \\
(1.3,579.51) \\
\end{array}$ & $\begin{array}{l}\text { Heat/cold } \\
\text { (C) }\end{array}$ & $\begin{array}{c}1.36 \\
(0.22,8.45) \\
\end{array}$ & $\begin{array}{c}0.6 \\
(0.11,3.16) \\
\end{array}$ & $\begin{array}{c}0.07 \\
(0.01,0.34) \\
\end{array}$ \\
\hline $\begin{array}{c}20.11 \\
(0.97,418.64) \\
\end{array}$ & $\begin{array}{c}0.73 \\
(0.12,4.54) \\
\end{array}$ & $\begin{array}{l}\text { PDT } \\
\text { (E) }\end{array}$ & $\begin{array}{c}0.44 \\
(0.09,2.25) \\
\end{array}$ & $\begin{array}{c}0.05 \\
(0.01,0.24) \\
\end{array}$ \\
\hline $\begin{array}{c}45.91 \\
(2.42,870.68)\end{array}$ & $\begin{array}{c}1.67 \\
(0.32,8.83)\end{array}$ & $\begin{array}{c}2.28 \\
(0.44,11.74)\end{array}$ & $\begin{array}{l}\text { Drugs } \\
\text { (F) }\end{array}$ & $\begin{array}{c}0.11 \\
(0.03,0.45)\end{array}$ \\
\hline $\begin{array}{c}418.6 \\
(22.48,7793.78)\end{array}$ & $\begin{array}{c}15.25 \\
(2.98,77.94)\end{array}$ & $\begin{array}{c}20.81 \\
(4.18,103.57)\end{array}$ & $\begin{array}{c}9.12 \\
(2.2,37.76)\end{array}$ & $\begin{array}{c}\text { No/sham treatment } \\
(\mathrm{I}, \mathrm{J})\end{array}$ \\
\hline
\end{tabular}

Note: Cells shaded gray indicate that the estimate is based only on indirect comparisons; bold-italic numbers indicate statistical significance. Results are given as odds ratios (95\% confidence intervals).

MMS = Mohs micrographic surgery; PDT = photodynamic therapy BCC = basal cell carcinoma 
Table 22 offers complementary information from the same analysis. For each intervention category, it shows the mean fraction of lesions without histologic clearance across the included RCTs. It also forecasts the expected fractions with lack of histologic clearance in each intervention category in groups of patients similar to the patients included in the analyzed RCTs. The average number of lesions with no histological clearance was 1.2 percent in the surgery arms, between 19.5 and 35.6 percent in other active intervention categories, and 83.5 percent for no or sham (placebo) treatment.

Table 22. Mean and forecasted lack of histologic clearance fractions by intervention category (all BCC lesions)

\begin{tabular}{lcc}
\hline \multicolumn{1}{c}{ Intervention Type } & $\begin{array}{c}\text { Mean Lack of Histological } \\
\text { Clearance Fraction } \\
\text { Percent } \mathbf{( 9 5 \% ~ C l )}\end{array}$ & $\begin{array}{c}\text { Forecasted Lack of } \\
\text { Histological Clearance } \\
\text { Fraction } \\
\text { Percent (95\% Cl) }\end{array}$ \\
\hline Surgery/MMS (A,B) & $1.2(0.1,15.9)$ & $1.2(<0.5,36.7)$ \\
\hline Heat/cold (C) & $24.9(8.2,55.0)$ & $24.9(1.6,87.1)$ \\
\hline PDT (E) & $19.5(6.4,46.4)$ & $19.5(1.2,83.0)$ \\
\hline Drugs (F) & $35.6(16.5,60.8)$ & $35.6(2.9,91.0)$ \\
\hline No/sham treatment $(\mathrm{I}, \mathrm{J})$ & $83.5(65.5,93.1)$ & $83.5(21.8,98.9)$ \\
\hline
\end{tabular}

MMS = Mohs micrographic surgery; PDT = photodynamic therapy; CI = confidence interval

\section{Comparisons Across Individual Interventions}

The results of the analyses of individual interventions are congruent with the analyses intervention categories are congruent with the corresponding results. As is evident from Figure 8, there are five connected subgraphs. Separate analyses are conducted for each connected subgraph. In total, 19 RCTs (2170 lesions) were included in these analyses, as summarized in the Table 23

Table 23. Sample information, lack of histological clearance (all BCC lesions, individual interventions)

\begin{tabular}{|c|c|c|c|c|c|}
\hline & $\begin{array}{l}\text { First subgraph } \\
68-70,49,54,66 \text {, } \\
\text { 68, 99, 104 }\end{array}$ & Second subgraph ${ }^{73}$ & $\begin{array}{l}\text { Third } \\
\text { subgraph } \\
55,64,101\end{array}$ & $\begin{array}{l}\text { Fourth } \\
\text { subgraph } \\
96\end{array}$ & $\begin{array}{l}\text { Fifth } \\
\text { subgraph } \\
6,100\end{array}$ \\
\hline $\begin{array}{l}\text { Studies (total } \\
\text { sample) }\end{array}$ & $12(2010)$ & $1(43)$ & $3(76)$ & $1(20)$ & $2(97)$ \\
\hline $\begin{array}{l}\text { Total sample by } \\
\text { intervention }\end{array}$ & $\begin{array}{l}\text { (F2): 761; (J): 575; (A): } \\
\text { 83; (C1): 87; (E2): 44; } \\
\text { (E1): 250; (F1): 146; } \\
\text { (F4): 48; (C5+E1): } 16\end{array}$ & $\begin{array}{l}\text { (A+E1): 11; }(A+E 1+H): \\
\text { 10; (A+E2): } 11 ; \\
(A+E 2+H): 11\end{array}$ & $\begin{array}{l}\text { (C5): } 28 ; \\
(\mathrm{I}): 32 ; \\
(\mathrm{F} 5): 16\end{array}$ & $\begin{array}{l}(\mathrm{F} 2+\mathrm{H}): \\
10 ;(\mathrm{H}): \\
10\end{array}$ & $\begin{array}{l}\text { (B): 50; } \\
\text { (B+F2): } 47\end{array}$ \\
\hline $\begin{array}{l}\text { Total sample by } \\
\text { intervention, } \\
\text { (min, max) }\end{array}$ & 16,761 & 10,11 & 16,32 & 10,10 & 47,50 \\
\hline $\begin{array}{l}\text { Data by } \\
\text { comparison }\end{array}$ & $\begin{array}{l}\text { (F2--J): } 5 \text { (1110); (F2-- } \\
\text { E1): } 1 \text { (271); (F2--F1): } 1 \\
\text { (291); (J--E1): } 1 \text { (150); } \\
\text { (J--F4): } 1 \text { (54); (A--C1): } 1 \\
\text { (96); (A--E1): } 1 \text { (68); (C1- } \\
-\mathrm{E} 2): 1 \text { (83); (E1--F1): } 1 \\
\text { (272); (E1--C5+E1): } 1 \\
\text { (32) }\end{array}$ & $\begin{array}{l}(A+E 1--A+E 1+H): 1(21) ; \\
(A+E 1--A+E 2): 1(22) ; \\
(A+E 1--A+E 2+H): 1(22) ; \\
(A+E 1+H--A+E 2): 1(21) ; \\
(A+E 1+H--A+E 2+H): 1 \\
(21) ;(A+E 2--A+E 2+H): 1 \\
(22)\end{array}$ & $\begin{array}{l}(C 5--I): 2 \\
(44) ;(1-- \\
\text { F5): } 1(32)\end{array}$ & $\begin{array}{l}\text { (F2+H-- } \\
\mathrm{H}): 1(20)\end{array}$ & $\begin{array}{l}(\mathrm{B}--\mathrm{B}+\mathrm{F} 2): \\
2(97)\end{array}$ \\
\hline $\begin{array}{l}\text { Studies by } \\
\text { comparison (min, }\end{array}$ & 1,5 & 1,1 & 1,2 & 1,1 & 2,2 \\
\hline
\end{tabular}




\begin{tabular}{|c|c|c|c|c|c|}
\hline & $\begin{array}{l}\text { First subgraph }{ }^{45,49,54,66,} \\
68-70,92-94,99,104\end{array}$ & Second subgraph ${ }^{73}$ & $\begin{array}{l}\text { Third } \\
\text { subgraph } \\
55,64,101\end{array}$ & $\begin{array}{l}\text { Fourth } \\
\text { subgraph } \\
96\end{array}$ & $\begin{array}{l}\text { Fifth } \\
\text { subgraph } \\
6,100\end{array}$ \\
\hline \multicolumn{6}{|l|}{$\max )$} \\
\hline $\begin{array}{l}\text { Total sample by } \\
\text { comparison (min, } \\
\max \text { ) }\end{array}$ & 32,1110 & 21,22 & 32,44 & 20,20 & 97,97 \\
\hline $\begin{array}{l}\text { Followup median } \\
\text { (min, max) }\end{array}$ & $3(3,36)$ months & $2.5(2.5,2.5)$ months & $\begin{array}{l}2(1.5,2) \\
\text { months }\end{array}$ & $\begin{array}{l}2(2,2) \\
\text { months }\end{array}$ & $\begin{array}{l}1.5(0.5 \\
2.5) \\
\text { months }\end{array}$ \\
\hline
\end{tabular}

A = surgical excision; B = Mohs micrographic surgery; BCC = basal cell carcinoma; C1 = cryotherapy; C3 = diathermy and curettage; C4 = cryotherapy and curettage; C5 = laser; D1 = external radiation; E1 = MAL photodynamic therapy; E2 = ALA photodynamic therapy; F1 = 5-FU; F2 = Imiquimod; F4 = Ingenol; H = curettage; $\mathrm{J}$ = placebo

Table 24 has results on the relative effects for the largest subgraph. Table 25 has the corresponding results for the other subgraphs: the one for the comparison of surgical excision with PDT with MAL or ALA, with or without curettage $(A+E 1$ versus $A+E 2$ versus, $A+E 1+H$ versus $\mathrm{A}+\mathrm{E} 2+\mathrm{H}$ ); and the one for the comparison between laser ablation (C5) versus diclofenac and/or calcitriol (other medication - F5) and versus no treatment (I). Table 26 shows the relative effects for the last two subgraphs, namely the one for the comparison between curettage alone $(\mathrm{H})$ versus curettage and imiquimod $(\mathrm{H}+\mathrm{F} 2)$; and the one for the comparison between MMS (B) and MMS with imiquimod $(\mathrm{B}+\mathrm{F} 2)$. In all three tables, comparisons across individual observations are sparse. The confidence intervals of the odds ratios for most indirect comparisons are very broad and cannot exclude very large differences between the compared interventions. The exception is for comparisons between surgical treatments and no intervention, which are statistically significant despite the wide confidence interval, because the relative effect is very large.

Table 27 shows, for each intervention, the mean fractions for lack of histologic clearance across all RCTs. Estimates for interventions in all five subgraphs are listed in the table. One should not compare statistically these fractions across the subgraphs, because they come from disjoint analyses. In general, the mean fractions for lack of histologic clearance for individual interventions are in congruence with the corresponding fractions estimated for intervention categories. For example, in the first subgraph, the average recurrence rates for PDT with MAL (E1) and ALA (E2) were 18.2 percent (95\% CI 5.1 to 48.0) and 25.0 percent (95\% CI 2.0 to 84.0), respectively, and the corresponding result from the analysis between intervention categories was 19.5 percent (95\% CI 6.4 to 46.4). The mean number of lesions with no histological clearance for the three medical interventions, namely 5-FU (F1), imiquimod (F2), and ingenol (F4), ranged between 5.5 and 77.1 percent, but the respective confidence intervals were very wide, and the corresponding odds ratios in Table 24 were not statistically significant. 
Table 24. Relative odds ratios for lack of histological clearance between individual interventions (all BCC lesions, Figure 8A, largest subgraph)

\begin{tabular}{|c|c|c|c|c|c|c|c|c|}
\hline $\begin{array}{c}\text { (A) } \\
\text { Surgery }\end{array}$ & $\begin{array}{c}0.11 \\
(<0.005,3.3) \\
\end{array}$ & $\begin{array}{c}0.02 \\
(<0.005,1.08) \\
\end{array}$ & $\begin{array}{c}0.05 \\
(<0.005,1.19)\end{array}$ & $\begin{array}{c}0.04 \\
(<0.005,1.81) \\
\end{array}$ & $\begin{array}{c}0.21 \\
(<0.005,10.47)\end{array}$ & $\begin{array}{c}0.04 \\
(<0.005,0.83) \\
\end{array}$ & $\begin{array}{c}<0.005 \\
(<0.005,0.18)\end{array}$ & $\begin{array}{c}<0.005 \\
(<0.005,0.05) \\
\end{array}$ \\
\hline $\begin{array}{c}9.31 \\
(0.3,285.72) \\
\end{array}$ & $\begin{array}{c}\text { (C1) } \\
\text { Cryotherapy }\end{array}$ & $\begin{array}{c}0.19 \\
(0.01,6.43) \\
\end{array}$ & $0.5(0.04,6.05)$ & $\begin{array}{c}0.34 \\
(0.01,10.68) \\
\end{array}$ & $\begin{array}{c}1.93 \\
(0.06,61.81) \\
\end{array}$ & $\begin{array}{c}0.39(0.04, \\
4.11)\end{array}$ & $\begin{array}{c}0.03 \\
(<0.005,1.06) \\
\end{array}$ & $\begin{array}{c}0.02 \\
(<0.005,0.23) \\
\end{array}$ \\
\hline $\begin{array}{c}49.8 \\
(0.93,2678.92)\end{array}$ & $\begin{array}{c}5.35 \\
(0.16,184.09)\end{array}$ & $\begin{array}{c}\text { (C5+E1) } \\
\text { Laser + PDT } \\
\text { (MAL) }\end{array}$ & $\begin{array}{c}2.69 \\
(0.11,67.1)\end{array}$ & $\begin{array}{c}1.8 \\
(0.03,99.7)\end{array}$ & $\begin{array}{c}10.35 \\
(0.19,576.29)\end{array}$ & $\begin{array}{c}2.1 \\
(0.09,47.08)\end{array}$ & $\begin{array}{c}0.18 \\
(<0.005,9.86)\end{array}$ & $\begin{array}{c}0.12 \\
(0.01,2.66)\end{array}$ \\
\hline $\begin{array}{c}18.52 \\
(0.84,407.72)\end{array}$ & $\begin{array}{c}1.99 \\
(0.17,23.98) \\
\end{array}$ & $\begin{array}{c}0.37 \\
(0.01,9.28) \\
\end{array}$ & $\begin{array}{c}\text { (E1) } \\
\text { PDT (MAL) } \\
\end{array}$ & $\begin{array}{c}0.67 \\
(0.03,15.3) \\
\end{array}$ & $\begin{array}{c}3.85 \\
(0.17,88.57) \\
\end{array}$ & $\begin{array}{c}0.78 \\
(0.13,4.86) \\
\end{array}$ & $\begin{array}{c}0.07 \\
(<0.005,1.51) \\
\end{array}$ & $\begin{array}{c}0.04 \\
(0.01,0.27) \\
\end{array}$ \\
\hline $\begin{array}{c}27.67 \\
(0.55,1386.65)\end{array}$ & $\begin{array}{c}2.97 \\
(0.09,94.42)\end{array}$ & $\begin{array}{c}0.56 \\
(0.01,30.77)\end{array}$ & $\begin{array}{c}1.49 \\
(0.07,34.14)\end{array}$ & $\begin{array}{c}\text { (E2) } \\
\text { PDT (ALA) }\end{array}$ & $\begin{array}{c}5.75 \\
(0.11,298.48)\end{array}$ & $\begin{array}{c}1.17 \\
(0.06,23.88)\end{array}$ & $\begin{array}{c}0.1 \\
(<0.005,5.11)\end{array}$ & $\begin{array}{c}0.07 \\
(<0.005,1.35)\end{array}$ \\
\hline $\begin{array}{c}4.81 \\
(0.1,242.47) \\
\end{array}$ & $\begin{array}{c}0.52 \\
(0.02,16.52) \\
\end{array}$ & $\begin{array}{c}0.1 \\
(<0.005,5.38) \\
\end{array}$ & $\begin{array}{c}0.26 \\
(0.01,5.98) \\
\end{array}$ & $\begin{array}{c}0.17 \\
(<0.005,9.03) \\
\end{array}$ & $\begin{array}{l}\text { (F1) } \\
5-F U\end{array}$ & $\begin{array}{c}0.2 \\
(0.01,4.18) \\
\end{array}$ & $\begin{array}{c}0.02 \\
(<0.005,0.89)\end{array}$ & $\begin{array}{c}0.01 \\
(<0.005,0.24)\end{array}$ \\
\hline $\begin{array}{c}23.66 \\
(1.2,464.54) \\
\end{array}$ & $\begin{array}{c}2.54 \\
(0.24,26.54) \\
\end{array}$ & $\begin{array}{c}0.48 \\
(0.02,10.63) \\
\end{array}$ & $\begin{array}{c}1.28 \\
(0.21,7.93) \\
\end{array}$ & $\begin{array}{c}0.86 \\
(0.04,17.46) \\
\end{array}$ & $\begin{array}{c}4.92 \\
(0.24,101.09) \\
\end{array}$ & $\begin{array}{c}\text { (F2) } \\
\text { Imiquimod }\end{array}$ & $\begin{array}{c}0.08 \\
(<0.005,1.73)\end{array}$ & $\begin{array}{c}0.06 \\
(0.01,0.28)\end{array}$ \\
\hline $\begin{array}{c}279.18 \\
(5.58, \\
13970.12) \\
\end{array}$ & $\begin{array}{c}30 \\
(0.95,951.08)\end{array}$ & $\begin{array}{c}5.61 \\
(0.1,310.02)\end{array}$ & $\begin{array}{c}15.07 \\
(0.66,343.77)\end{array}$ & $\begin{array}{c}10.09 \\
(0.2,520.18)\end{array}$ & $\begin{array}{c}58.02 \\
(1.12,3007.15)\end{array}$ & $\begin{array}{c}11.8 \\
(0.58,240.42)\end{array}$ & $\begin{array}{c}\text { (F4) } \\
\text { Ingenol }\end{array}$ & $\begin{array}{c}0.67 \\
(0.03,13.6)\end{array}$ \\
\hline $\begin{array}{c}414.45 \\
(21.31, \\
8061.19)\end{array}$ & $\begin{array}{c}44.53 \\
(4.32,459.3)\end{array}$ & $\begin{array}{c}8.32 \\
0.38,184.46)\end{array}$ & $\begin{array}{c}22.38 \\
(3.66,136.75)\end{array}$ & $\begin{array}{c}14.98 \\
(0.74,302.98)\end{array}$ & $\begin{array}{c}86.14 \\
(4.23,1754.46)\end{array}$ & $\begin{array}{c}17.52 \\
(3.51,87.41)\end{array}$ & $\begin{array}{c}1.48 \\
(0.07,29.96)\end{array}$ & $\begin{array}{c}\text { (J) } \\
\text { Placebo/sham }\end{array}$ \\
\hline
\end{tabular}

PDT=photodynamic therapy; ALA= 5-aminolevulinic acid, MAL=methyl aminolevulinate, FU= fluorouracil, BCC=basal cell carcinoma. Cells shaded gray indicate that the estimate is based only on indirect comparisons; bold-italic numbers indicate statistical significance. Results are given as odds ratios (95\% confidence intervals).

Table 25. Relative odds ratios for lack of histological clearance between individual interventions (all BCC lesions, Figure 8, other subgraphs)

\begin{tabular}{|c|c|c|c|c|c|c|}
\hline $\begin{array}{c}\text { (A_plus_E1) } \\
\text { Surgery + PDT (MAL) }\end{array}$ & $\begin{array}{c}2.29 \\
(0.32,16.51)\end{array}$ & $\begin{array}{c}1 \\
(0.18,5.68) \\
\end{array}$ & $\begin{array}{c}2.57 \\
(0.36,18.33) \\
\end{array}$ & & & \\
\hline $\begin{array}{c}0.44 \\
(0.06,3.16)\end{array}$ & $\begin{array}{c}\text { (A_plus_E1_plus_H) } \\
\text { Surgery + PDT (MAL) } \\
+ \text { curettage }\end{array}$ & $\begin{array}{c}0.44 \\
(0.06,3.16)\end{array}$ & $\begin{array}{c}1.13 \\
(0.13,9.94)\end{array}$ & & & \\
\hline $\begin{array}{c}1 \\
(0.18,5.68) \\
\end{array}$ & $\begin{array}{c}2.29 \\
(0.32,16.51) \\
\end{array}$ & $\begin{array}{c}\text { (A_plus_E2) } \\
\text { Surgery + PDT (MAL) }\end{array}$ & $\begin{array}{c}2.57 \\
(0.36,18.33) \\
\end{array}$ & & & \\
\hline \multirow[t]{3}{*}{$\begin{array}{c}0.39 \\
(0.05,2.77)\end{array}$} & $\begin{array}{c}0.89 \\
(0.1,7.86)\end{array}$ & $\begin{array}{c}0.39 \\
(0.05,2.77)\end{array}$ & $\begin{array}{c}\text { (A_plus_E2_plus_H) } \\
\text { Surgery + PDT (MAL) + } \\
\text { curettage }\end{array}$ & & & \\
\hline & & & & $\begin{array}{l}\text { (C5) } \\
\text { laser }\end{array}$ & $\begin{array}{c}0.02(<0.005 \\
0.56)\end{array}$ & $\begin{array}{c}0.17(0.03 \\
0.9)\end{array}$ \\
\hline & & & & $\begin{array}{c}43.95(1.77 \\
1090.16)\end{array}$ & $\begin{array}{c}\text { (F5) } \\
\text { Other } \\
\text { medical }\end{array}$ & $\begin{array}{l}7.49(0.29 \\
196.65)\end{array}$ \\
\hline
\end{tabular}


Note: Cells shaded gray indicate that the estimate is based only on indirect comparisons; bold-italic numbers indicate statistical significance. Results are given as odds ratios (95\% confidence intervals).

PDT = photodynamic therapy; ALA = 5-aminolevulinic acid; MAL = methyl aminolevulinate; FU = fluorouracil; BCC = basal cell carcinoma 
Table 26. Relative odds ratios for lack of histological clearance between individual interventions (all BCC lesions, Figure 8A, 2 more subgraphs)

(F2_plus_H) 0.17

Imiquimod + curettage $\quad(0.01,1.88)$

$6.00(0.53,67.65) \quad$ Curettage $\mathbf{~}(\mathbf{H})$

\begin{tabular}{cc} 
MMS (B) & $11.11(2.66,46.36)$ \\
\hline $0.09(0.02,0.38)$ & (B_plus_F2) \\
MMS + imiquimo
\end{tabular}

Note: Results are given as odds ratios (95\% confidence intervals). Bold-italic indicates statistical significance.

PDT = photodynamic therapy; ALA = 5-aminolevulinic acid; MAL = methyl aminolevulinate; FU = fluorouracil; $\mathrm{BCC}=$ basal cell carcinoma 
Table 27. Mean and forecasted lack of histological clearance fractions by intervention category (all BCC lesions)

\begin{tabular}{|c|c|c|}
\hline Intervention Type & $\begin{array}{c}\text { Mean Lack of } \\
\text { Histological Clearance } \\
\text { Fraction } \\
(95 \% \mathrm{Cl}) \\
\end{array}$ & $\begin{array}{c}\text { Forecasted Lack of } \\
\text { Histological Clearance } \\
\text { Fraction } \\
(95 \% \mathrm{Cl}) \\
\end{array}$ \\
\hline \multicolumn{3}{|l|}{ First subgraph } \\
\hline Surgical excision $(\mathrm{A})$ & $1.2(0.1,15.8)$ & $1.2(<0.5,36.3)$ \\
\hline Cryotherapy (C1) & $10.1(1.4,46.4)$ & $10.1(0.4,76.9)$ \\
\hline Laser (C5+E1) + PDT (MAL) & $37.5(3.2,91.5)$ & $37.5(1.1,96.9)$ \\
\hline PDT (MAL) (E1) & $18.2(5.1,48.0)$ & $18.2(1.0,82.5)$ \\
\hline PDT (ALA) (E2) & $25.0(2.0,84.4)$ & $25.0(0.7,94.2)$ \\
\hline 5-FU (F1) & $5.5(0.4,48.7)$ & $5.5(0.1,73.9)$ \\
\hline Imiquimod (F2) & $22.2(8.3,47.3)$ & $22.2(1.5,84.3)$ \\
\hline Ingenol (F4) & $77.1(17.2,98.2)$ & $77.1(6.5,99.4)$ \\
\hline Placebo (J) & $83.3(61.9,93.9)$ & $83.3(21.1,98.9)$ \\
\hline \multicolumn{3}{|l|}{ Second subgraph } \\
\hline Surgery + PDT (MAL) $(A+E 1)$ & $36.4(14.3,66.1)$ & NA \\
\hline $\begin{array}{c}\text { Surgery + PDT (MAL) + } \\
\text { curettage }(\mathrm{A}+\mathrm{E} 1+\mathrm{H})\end{array}$ & $20.0(5.0,54.1)$ & NA \\
\hline Surgery + PDT (ALA) (A+E2) & $36.4(14.3,66.1)$ & NA \\
\hline $\begin{array}{c}\text { Surgery + PDT }(\mathrm{ALA})+ \\
\text { curettage }(\mathrm{A}+\mathrm{E} 2+\mathrm{H}) \\
\end{array}$ & $18.2(4.6,50.7)$ & NA \\
\hline \multicolumn{3}{|l|}{ Third subgraph } \\
\hline Laser (C5) & $43.5(26.1,62.8)$ & NA \\
\hline $\begin{array}{l}\text { Other medical (diclofenac } \\
\text { and/or calcitriol) (F5) }\end{array}$ & $97.1(66.499 .8)$ & NA \\
\hline No treatment $(\mathrm{J})$ & $80.5(58.8,92.2)$ & NA \\
\hline \multicolumn{3}{|l|}{ Fourth subgraph } \\
\hline Imiquimod + curettage $(\mathrm{F} 2+\mathrm{H})$ & $10.0(1.4,46.7)$ & NA \\
\hline Curettage $(\mathrm{H})$ & $40.0(15.8,70.3)$ & NA \\
\hline \multicolumn{3}{|l|}{ Fifth subgraph } \\
\hline MMS (B) & $92.0(75.8,97.7)$ & NA \\
\hline MMS + imiquimod $(B+F 2)$ & $51.0(37.0,64.9)$ & NA \\
\hline
\end{tabular}

PDT = photodynamic therapy; ALA = 5-aminolevulinic acid; MAL = methyl aminolevulinate; $\mathrm{FU}=$ fluorouracil; $\mathrm{BCC}=$ basal cell carcinoma; $\mathrm{CI}$ = confidence interval; $\mathrm{NA}$ =forecasts are not available for the 4 smaller subgraphs, because they were analyzed with a fixed effects model 


\section{Incomplete Excision, All BCC Lesions}

Two RCTs reported incomplete excision outcomes in mixed BCC populations. In the first study, the average age was 68 (SD 12), and 39.7 percent were female. The average lesion size was $1.28 \mathrm{~cm}^{2}$ (SD 1.36) in the group randomized to receive Mohs surgery $(\mathrm{n}=198)$ and $1.77 \mathrm{~cm}^{2}$ (SD 1.28) in the surgical excision without intraoperative evaluation group ( $\mathrm{n}=199)$. In this study, about half of the BCCs were classified as aggressive. After the first excision, 35 of 199 lesions (17.6\%) were found to have been incompletely excised in the surgical excision without intraoperative margin assessment group; whereas none were found in the Mohs surgery group (0/198). Thirty-one of the lesions in the excision group were reexcised and of these four were found to have been incompletely excised (12.9\%). In the aggressive lesions, the incomplete excision rate was 21 of 88 (23.9\%) in the surgical excision group; none in the Mohs group $(\mathrm{n}=105){ }^{81}$

The second RCT reported incomplete excision and number of repeat procedures in people who had either surgical excision without intraoperative assessment of the margins or curettage and cryosurgery for BCCs on their face (90\%) or trunk/neck (10\%). The mean age was 67 (range 34 to 92), and 43 percent were women. In the curettage and cryosurgery group there were 51 lesions, all nodular, with an average diameter of $5.4 \mathrm{~mm}$ (SD 2.9). In the surgical excision arm, there were 49 lesions, 92 percent nodular and 8 percent superficial, with an average diameter of $5.3 \mathrm{~mm}$ (SD 2.6). There were no incomplete excisions in the curettage and cryosurgery group; and there were three in the surgical excision group (6\%). There were no repeat procedures in the curettage and cryosurgery group and four in the surgical excision group. ${ }^{19}$

\section{Lack of Histological Clearance, Subgroup Analyses by Lesion Type}

We conducted subgroup analyses by the type of BCC lesion. We report analyses comparing groups of interventions, but not analyses comparing individual treatments. The latter are very sparse, and their results are very similar to the pertinent comparisons in Tables 24, 25, and 26.

Many subgroup analyses per lesion type are possible. In this section, we describe analyses in RCTs of lower-risk lesions (strata of predominantly [>80\%] superficial BCCs, predominantly nodular BCCs, and superficial or nodular BCCs) overall, and broken down by lesion type, as well as higher-risk lesions (morpheaform, micronodular, trabecular, infiltrative, or squamous differentiation).

Fifteen RCTs ( $\mathrm{n}=1972$ lesions) included low-risk BCCs (nodular and superficial subtypes). ${ }^{45}$, 49, 54, 55, 64, 66, 68-70, 92-94, 99, 101, 104 Their results are very similar to the findings in Tables 22 and 23 in the previous section.

With respect to RCT strata of predominantly superficial lesions, six RCTs ( $\mathrm{n}=1300$ lesions) compared PDT (E) versus drugs (F) versus no or sham treatment. ${ }^{49,55,68,69,92,94}$ Table 28 provides details about the comparisons between these six RCTs. The results are shown in Tables 29 and 30. Briefly, there was no statistically significant difference between the two active intervention categories, but both were statistically significantly better than no or sham treatment.

Table 28. Sample information, lack of histological clearance (superficial lesions)

\begin{tabular}{ll}
\hline Studies (total sample) & $6(1300)$ \\
\hline Total sample by intervention & $(\mathrm{E}): 126 ;(\mathrm{F}): 693 ;(\mathrm{I}, \mathrm{J}): 481$ \\
\hline $\begin{array}{l}\text { Total sample by intervention, (min, } \\
\text { max) }\end{array}$ & 126,693 \\
\hline
\end{tabular}




\begin{tabular}{ll}
\hline Data by comparison & $(\mathrm{E}--\mathrm{F}): 1(271) ;(\mathrm{F}--\mathrm{I}, \mathrm{J}): 5(1029)$ \\
\hline Studies by comparison (min, max) & 1,5 \\
\hline $\begin{array}{l}\text { Total sample by comparison (min, } \\
\text { max) }\end{array}$ & 271,1029 \\
\hline Followup median (min, max) & $3(2,36)$ months \\
\hline $\begin{array}{l}\text { A = surgical excision; B = Mohs micrographic surgery; C = heat/cold; E = photodynamic therapy; F = drugs; } \mathrm{H}=\text { curettage; I = } \\
\text { no treatment; J = placebo }\end{array}$
\end{tabular}

Table 29. Relative odds ratios for lack of histological clearance between intervention categories (predominantly superficial BCC lesions)

\begin{tabular}{ccc}
\hline PDT (E) & $0.19(<0.005,9.84)$ & $0.01(<0.005,0.36)$ \\
\hline $5.17(0.1,263.01)$ & Drugs (F) & $0.03(<0.005,0.36)$ \\
\hline $150.98(2.78,8187.95)$ & $29.21(2.81,303.6)$ & $\begin{array}{c}\text { No/sham treatment } \\
(\mathrm{I}, \mathrm{J})\end{array}$ \\
\hline
\end{tabular}

Note: Cells shaded gray indicate that the estimate is based only on indirect comparisons; bold-italic numbers indicate statistical significance. Results are given as odds ratios (95\% confidence intervals).

PDT = photodynamic therapy; BCC = basal cell carcinoma

Table 30. Mean fraction of lesions without histological clearance by intervention category (predominantly superficial BCC lesions)

\begin{tabular}{lcc}
\multicolumn{1}{c}{ Intervention Type } & $\begin{array}{c}\text { Mean } \\
\text { Percent (95\% Cl) }\end{array}$ & $\begin{array}{c}\text { Forecast } \\
\text { Percent (95\% CI) }\end{array}$ \\
\hline PDT (E) & $7.9(0.2,75.9)$ & $7.9(0.1,93.1)$ \\
\hline Drugs (F) & $30.8(8.4,68.3)$ & $30.8(0.9,95.6)$ \\
\hline No/sham treatment (I,J) & $92.9(69.8,98.7)$ & $92.9(20.2,99.9)$ \\
\hline
\end{tabular}

PDT = photodynamic therapy; BCC = basal cell carcinoma; CI = confidence interval

With respect to the five RCT strata of predominantly nodular lesions ( $n=374), 45,55,66,70,93$ details on the comparisons are in Table 31. The corresponding results are listed in Tables 32 and 33. These results are qualitatively similar to the corresponding results from the analyses in Tables 22 and 23.

Table 31. Sample information, lack of histological clearance (nodular lesions)

\begin{tabular}{ll}
\hline Studies (total sample) & $5(374)$ \\
\hline Total sample by intervention & $(\mathrm{F}): 84 ;(\mathrm{I}, \mathrm{J}): 115 ;(\mathrm{A}, \mathrm{B}): 35 ;(\mathrm{E}): 124 ;(\mathrm{C}): 16$ \\
\hline $\begin{array}{l}\text { Total sample by intervention, (min, } \\
\text { max) }\end{array}$ & 16,124 \\
\hline Data by comparison & $(\mathrm{F}--\mathrm{I}, \mathrm{J}): 2$ (124); (I,J--E): 1 (150); (A,B--E): $1(68) ;(\mathrm{E}--\mathrm{C}): 1(32)$ \\
\hline Studies by comparison (min, max) & 1,2 \\
\hline $\begin{array}{l}\text { Total sample by comparison (min, } \\
\text { max) }\end{array}$ & 32,150 \\
\hline Followup median (min, max) & $3(2,12)$ months \\
\hline $\begin{array}{l}\text { A = surgical excision; B = Mohs micrographic surgery; C = heat/cold; E = photodynamic therapy; F = drugs; H = curettage; I = } \\
\text { no treatment; J = placebo }\end{array}$
\end{tabular}


Table 32. Relative odds ratios for lack of histological clearance between intervention categories (nodular BCC lesions)

\begin{tabular}{|c|c|c|c|c|}
\hline $\begin{array}{c}\text { Surgery/MMS } \\
(\mathrm{A}, \mathrm{B})\end{array}$ & $0.02(<0.005,1.48)$ & $0.04(<0.005,1.79)$ & $\begin{array}{c}0.01(<0.005 \\
0.44) \\
\end{array}$ & $\begin{array}{c}<0.005 \\
(<0.005,0.14)\end{array}$ \\
\hline $42.6(0.67,2692.89)$ & $\begin{array}{l}\text { Heat/cold } \\
\text { (C) }\end{array}$ & $1.9(0.14,26.18)$ & $\begin{array}{c}0.39(0.02 \\
6.78) \\
\end{array}$ & $\begin{array}{c}0.14(0.01 \\
1.99) \\
\end{array}$ \\
\hline $22.45(0.56,903.83)$ & $0.53(0.04,7.27)$ & $\begin{array}{l}\text { PDT } \\
\text { (E) }\end{array}$ & $\begin{array}{c}0.21(0.02 \\
1.76)\end{array}$ & $\begin{array}{c}0.08(0.01 \\
0.48)\end{array}$ \\
\hline $108.35(2.29,5127.73)$ & $2.54(0.15,43.87)$ & $4.83(0.57,40.95)$ & $\begin{array}{l}\text { Drugs } \\
\text { (F) }\end{array}$ & $\begin{array}{c}0.36(0.04 \\
3.13) \\
\end{array}$ \\
\hline $298.54(7.35,12122.67)$ & $7.01(0.5,97.88)$ & $13.3(2.1,84.38)$ & $\begin{array}{c}2.76(0.32 \\
23.73)\end{array}$ & $\begin{array}{c}\text { Nolsham } \\
\text { treatment } \\
(1, J)\end{array}$ \\
\hline
\end{tabular}

Note: Cells shaded gray indicate that the estimate is based only on indirect comparisons; bold-italic numbers indicate statistical significance. Results are given as odds ratios (95\% confidence intervals).

MMS = Mohs micrographic surgery; PDT = photodynamic therapy; BCC = basal cell carcinoma .

Table 33. Mean and forecasted lack of histological clearance fractions by intervention category (nodular BCC lesions)

\begin{tabular}{lcc}
\hline \multicolumn{1}{c}{ Intervention Type } & $\begin{array}{c}\text { Mean } \\
\text { Percent (95\% Cl) }\end{array}$ & $\begin{array}{c}\text { Forecast } \\
\text { Percent (95\% Cl) }\end{array}$ \\
\hline Surgery/MMS (A,B) & $1.4(<0.5,31.0)$ & $1.4(<0.5,44.0)$ \\
\hline Heat/cold $(\mathrm{C})$ & $37.5(5.8,85.5)$ & $37.5(2.7,92.8)$ \\
\hline PDT $(\mathrm{E})$ & $24.0(8.0,53.6)$ & $24.0(2.7,78.1)$ \\
\hline Drugs $(\mathrm{F})$ & $60.4(21.8,89.3)$ & $60.4(9.6,95.6)$ \\
\hline No/sham treatment $(\mathrm{I}, \mathrm{J})$ & $80.8(52.9,94.0)$ & $80.8(26.9,98.0)$ \\
\hline
\end{tabular}

MMS= Mohs micrographic surgery; PDT=photodynamic therapy; BCC=basal cell carcinoma; CI=confidence interval

\section{Incomplete Excision (a Related Outcome) in High-Risk BCCs}

We identified one RCT that measured the distinct, yet related, outcome of incomplete excision in 172 lesions, about half of which were on the face, the rest were elsewhere on the body. This study compared surgical excision (A) with MMS (B) in histologically aggressive facial lesions (morpheaform, micronodular, trabecular, infiltrative, or squamous differentiation). The average age was 65 years (standard deviation 13), and 43.3 percent were female. The average lesion diameter was $9.1 \mathrm{~mm}$ (standard deviation 4.1). In the 88 lesions that had surgical excision without intraoperative margin assessment, two had an incomplete excision. This outcome was not applicable to the other arm of the study (ALA-PDT). ${ }^{20}$

\section{Lack of Histological Clearance, Other Subgroup Analyses (Lesion Location, Lesion Size, Sex, Age)}

Table 34 shows results on subgroup analyses for two RCTs that reported treatment effects in subgroups of interest. The first RCT enrolled patients with predominantly superficial BCCs and found significant differences in treatment effects across a number of subgroups that include age, gender, lesion location, and lesion size. ${ }^{49,87,88}$ The second RCT reported subgroup results for lack of histological clearance in predominantly nodular BCC. There was no significant difference between or within subgroups based on lesion location or size. ${ }^{66}$ 
Table 34. Subgroup results for lack of histological clearance in superficial BCCs

\begin{tabular}{|c|c|c|c|c|c|c|}
\hline Study & Comparison & Timepoint & Subgroup & $\begin{array}{l}\text { n/N Arm } 1 \text { vs. } \\
\text { n/N Arm } 2\end{array}$ & $\begin{array}{l}\text { OR }(95 \% \mathrm{Cl}) ; \text { P. } \\
\text { Value Within }\end{array}$ & $\begin{array}{l}\text { P- Value } \\
\text { Between }\end{array}$ \\
\hline \multirow{3}{*}{$\begin{array}{l}\text { Arits } 2013 \\
23683751\end{array}$} & PDT (MAL) (E1) & \multirow[t]{3}{*}{12 months } & age: $<=60$ years & $25 / 81$ vs. $8 / 77$ & 3.85 (1.61, 9.20); & \multirow[t]{3}{*}{$p=0.032$} \\
\hline & vs. Imiquimod & & & & $p=0.002$ & \\
\hline & & & $\begin{array}{l}\text { age: }>60 \text { years } \\
\text { old }\end{array}$ & $27 / 115$ vs. $23 / 112$ & $\begin{array}{l}1.19(0.63,2.23) \\
p=0.633\end{array}$ & \\
\hline \multirow{3}{*}{$\begin{array}{l}\text { Arits } 2013 \\
23683751\end{array}$} & PDT (MAL) (E1) & \multirow[t]{3}{*}{12 months } & females & $29 / 103$ vs. $9 / 92$ & $3.61(1.61,8.13)$ & \multirow[t]{3}{*}{$p=0.029$} \\
\hline & vs. Imiquimod & & & & $p=0.002$ & \\
\hline & & & males & $23 / 93$ vs. $22 / 97$ & $\begin{array}{l}1.12(0.57,2.19) \\
p=0.865\end{array}$ & \\
\hline \multirow{5}{*}{$\begin{array}{l}\text { Arits } 2013 \\
23683751\end{array}$} & PDT (MAL) (E1) & \multirow[t]{5}{*}{12 months } & lesion location: & $9 / 24$ vs. $4 / 20$ & $2.40(0.61,9.47)$ & \multirow[t]{5}{*}{$p=0.047$} \\
\hline & $\begin{array}{l}\text { vs. Imiquimod } \\
\text { (F2) }\end{array}$ & & head/neck & & $p=0.321$ & \\
\hline & & & $\begin{array}{l}\text { lesion location: } \\
\text { trunk }\end{array}$ & $36 / 115$ vs. $12 / 116$ & $\begin{array}{l}3.95(1.93,8.08) \\
p<0.001\end{array}$ & \\
\hline & & & $\begin{array}{l}\text { lesion location: } \\
\text { lower } \\
\text { extremities }\end{array}$ & $2 / 26$ vs. $6 / 28$ & $\begin{array}{l}0.31(0.06,1.68) \\
p=0.253\end{array}$ & \\
\hline & & & $\begin{array}{l}\text { lesion location: } \\
\text { upper } \\
\text { extremities }\end{array}$ & $5 / 31$ vs. $3 / 25$ & $\begin{array}{l}1.41(0.30,6.58) \\
p=0.720\end{array}$ & \\
\hline \multirow{3}{*}{$\begin{array}{l}\text { Arits } 2013 \\
23683751\end{array}$} & PDT (MAL) (E1) & \multirow[t]{3}{*}{12 months } & lesion area: <= & $23 / 106$ vs. $18 / 90$ & $1.11(0.55,2.22)$ & \multirow[t]{3}{*}{$p=0.043$} \\
\hline & $\begin{array}{l}\text { vs. Imiquimod } \\
\text { (F2) }\end{array}$ & & $60 \mathrm{~mm} 2$ & & $p=0.861$ & \\
\hline & & & $\begin{array}{l}\text { lesion area: }>60 \\
\mathrm{~mm} 2\end{array}$ & $27 / 86$ vs. $12 / 96$ & $\begin{array}{l}3.20(1.50,6.83) \\
p=0.002\end{array}$ & \\
\hline \multirow[t]{4}{*}{$\begin{array}{l}\text { Foley } 2009 \\
20064185\end{array}$} & $\begin{array}{l}\text { PDT (MAL) (E1) } \\
\text { vs. sham PDT (J) }\end{array}$ & \multirow[t]{4}{*}{3 months } & $\begin{array}{l}\text { lesion location: } \\
\text { extremities }\end{array}$ & $5 / 15$ vs. $12 / 17$ & $\begin{array}{l}0.21(0.05,0.93) \\
p=0.074\end{array}$ & \multirow[t]{4}{*}{$p=0.437$} \\
\hline & & & $\begin{array}{l}\text { lesion location: } \\
\text { face/scalp }\end{array}$ & $3 / 19$ vs. $18 / 23$ & $\begin{array}{l}0.05(0.01,0.25) \\
p<0.001\end{array}$ & \\
\hline & & & $\begin{array}{l}\text { lesion location: } \\
\text { neck }\end{array}$ & $4 / 9$ vs. $1 / 1$ & $\begin{array}{l}0.27(0.01,8.46) \\
p=1.000\end{array}$ & \\
\hline & & & $\begin{array}{l}\text { lesion location: } \\
\text { trunk }\end{array}$ & $8 / 32$ vs. $24 / 34$ & $\begin{array}{l}0.14(0.05,0.41) \\
p<0.001\end{array}$ & \\
\hline \multirow{3}{*}{$\begin{array}{l}\text { Foley } 2009 \\
20064185\end{array}$} & PDT (MAL) (E1) & \multirow[t]{3}{*}{3 months } & lesion diameter: & $15 / 64$ vs. $43 / 61$ & $0.13(0.06,0.28)$ & \multirow[t]{3}{*}{$p=0.939$} \\
\hline & vs. sham PDT (J) & & $<10 \mathrm{~mm}$ & & $\mathrm{p}<0.001$ & \\
\hline & & & $\begin{array}{l}\text { lesion diameter: } \\
10-20 \mathrm{~mm}\end{array}$ & $5 / 11$ vs. $12 / 14$ & $\begin{array}{l}0.14(0.02,0.94) \\
p=0.081\end{array}$ & \\
\hline
\end{tabular}

NA = not significant; PDT = photodynamic therapy; BCC = basal cell carcinoma; MAL = methyl aminolevulinate; OR = odds ratio; $\mathrm{CI}=$ confidence interval

\section{Lack of Histological Clearance, Results From Nonrandomized Studies (BCC Lesions)}

We identified six NRCSs reporting lack of histological clearance in BCC or mixed BCC and SCC lesions. ${ }^{143,150-154}$ These are summarized narratively below.

The first NRCS included 12 patients with one superficial BCC each. After an initial excision surgery, six patients received imiquimod and six received placebo. The study was deemed to be at a high risk of confounding bias, because the arms were not balanced (there were only six patients per arm); both the dermatologist and pathologist were blinded, and the study followed all participants to the end. The mean lesion area was $52 \mathrm{~mm}^{2}$, and the lesions were located on the trunk or neck (67\%) or forearm (33\%). The mean age was 61 (range 52 to 78), and 33 percent 
were female. All lesions in the vehicle group had residual tumor at excision, as did four of the six treated with imiquimod. ${ }^{154}$

The second NRCS reported lack of clinical and histological clearance in 74 patients with one nodular BCC each, receiving different doses of vismodegib. The risk of bias of this study was judged to be moderate because of lack of blinding and inadequate baselines reporting that lead to ambiguity about how well balanced the arms were. The lesion diameter ranged from 1 to $3 \mathrm{~cm}$, and all were located in the scalp, head, neck, trunk, or limbs. The mean age was 63.6 (SD 12; range 40 to 89), and 22 percent were female; 99 percent were white. Twenty-four lesions were treated with vismodegib for 12 weeks then were excised; twenty-five were treated with vismodegib for 12 weeks then had a 24-week observation period before excision; and 25 were treated with vismodegib for 16 weeks then were excised. The 12-week groups had a much higher and statistically significant rate of lack of clinical clearance than the 16-week group (OR 10.42; 95\% CI 1.22 to 89.13). However, the lack of histological clearance was much closer between the two doses, and not significant (OR 1.57 ; $95 \%$ CI 0.49 to 5.01 ). ${ }^{153}$

The third NRCS reported on lack of histological clearance in 56 people with 56 nodular BCCs, who received ALA-PDT with or without surface preparation with a CO2 laser. This study was judged to have moderate to low risk of bias, primarily because of lack of blinding. The mean age was 62, and 43 percent were females. Most of the lesions (87.5\%) were on the head (not $\mathrm{H}$ zone or adjacent to the eyes or ears) or neck. The group with the surface preparation had a lower rate of lack of histological clearance than the group without surface preparation (OR 0.23; 95\% CI 0.07 to 0.75$){ }^{150}$

The fourth NRCS reported on a matched population of 40 patients treated with different doses of brachytherapy (36.6 versus $42 \mathrm{~Gy}$ ). This study was deemed to be at a moderate risk of confounding bias, primarily for lack of blinding and unclear reporting of baselines. The mean age was 75, 45 percent were female, and all had a Fitzpatrick skin score of I (47.5\%) or II (52.5\%). Forty-five percent of the BCCs were superficial, while 55 percent were nodular; 75 percent were on the head and neck and 25 percent on the trunk or extremities. The lower dose (36.6 Gy) had a higher rate of lack of histological clearance at up to a year than the higher dose (42 Gy), but this difference was not significant (OR 2.11; 95\% CI 0.18 to 25.35). ${ }^{143}$

The fifth NRCS reports on 20 BCC lesions (43\% superficial/multicentric, 47.5\% nodular, 9.5\% infiltrative/micronodular/morpheaform/scelorosing; $90.5 \%$ on the trunk/neck and $9.5 \%$ on the extremities) treated with pulse dye laser and 20 matched lesions that received no treatment. This study was deemed to be at a moderate risk of confounding bias, primarily for lack of blinding and unclear reporting of baselines. At surgical excision, approximately 2 weeks after the last treatment, 7 of the 20 lesions treated with pulse dye laser showed lack of histological clearance, compared to 18 of the lesions not treated (OR 0.06; 95\% CI 0.01 to 0.34). ${ }^{152}$

Finally, the sixth NRCS included both BCCs (71\%) and SCCs (29\%), and compared two doses of external radiation therapy. In the lower-dose (37 Gy) group 14 of 236 lesions (5.9\%) were not histologically clear compared to none of 149 (0\%) in the higher-dose (45 Gy) group. There was no adjusted analysis available for this outcome. ${ }^{151}$

\section{Lack of Clinical Clearance, All BCC Lesions}

The evidence graph for lack of clinical clearance with respect to individual treatments is sparse (Figure 6 (C) - reproduced in Figure 9 (A) for ease of reference), and comprises 3 connected subgraphs. Detailed results at the RCT-level are in Appendix I. 
Figure 9. Evidence graph of RCTs evaluating lack of clinical clearance in BCCs across (A) individual interventions and (B) types of interventions

(A)

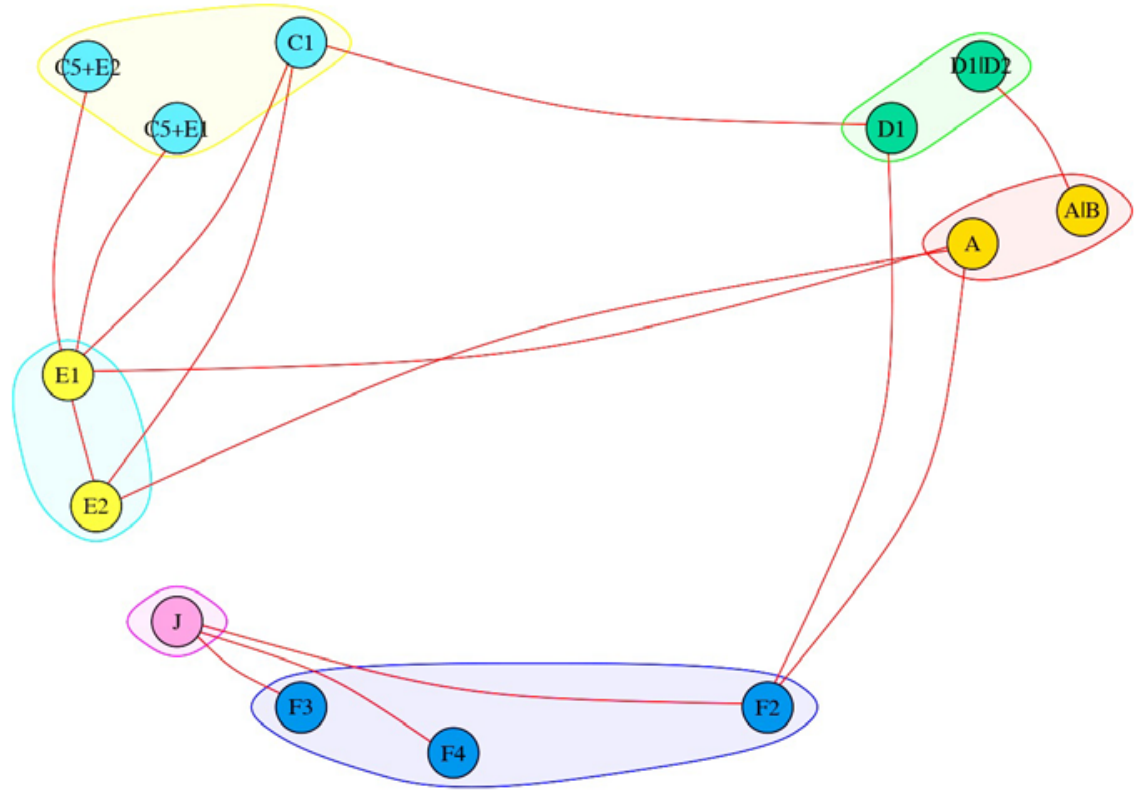

A: surgical excision B: MMS

C1: cryotherapy

C3: curettage + diathermy

C4: curettage + cryotherapy

C5: laser

D1: external radiation

D2: brachytherapy

E1: PDT (MAL)

E2: PDT (ALA)

F1: 5-FU

F2: imiquimod

F3: INF

$\mathrm{F} 4$ : ingenol

F5: other medical

$\mathrm{H}$ : curettage

I: no treatment

$\mathrm{J}$ : placebo/sham

(B)

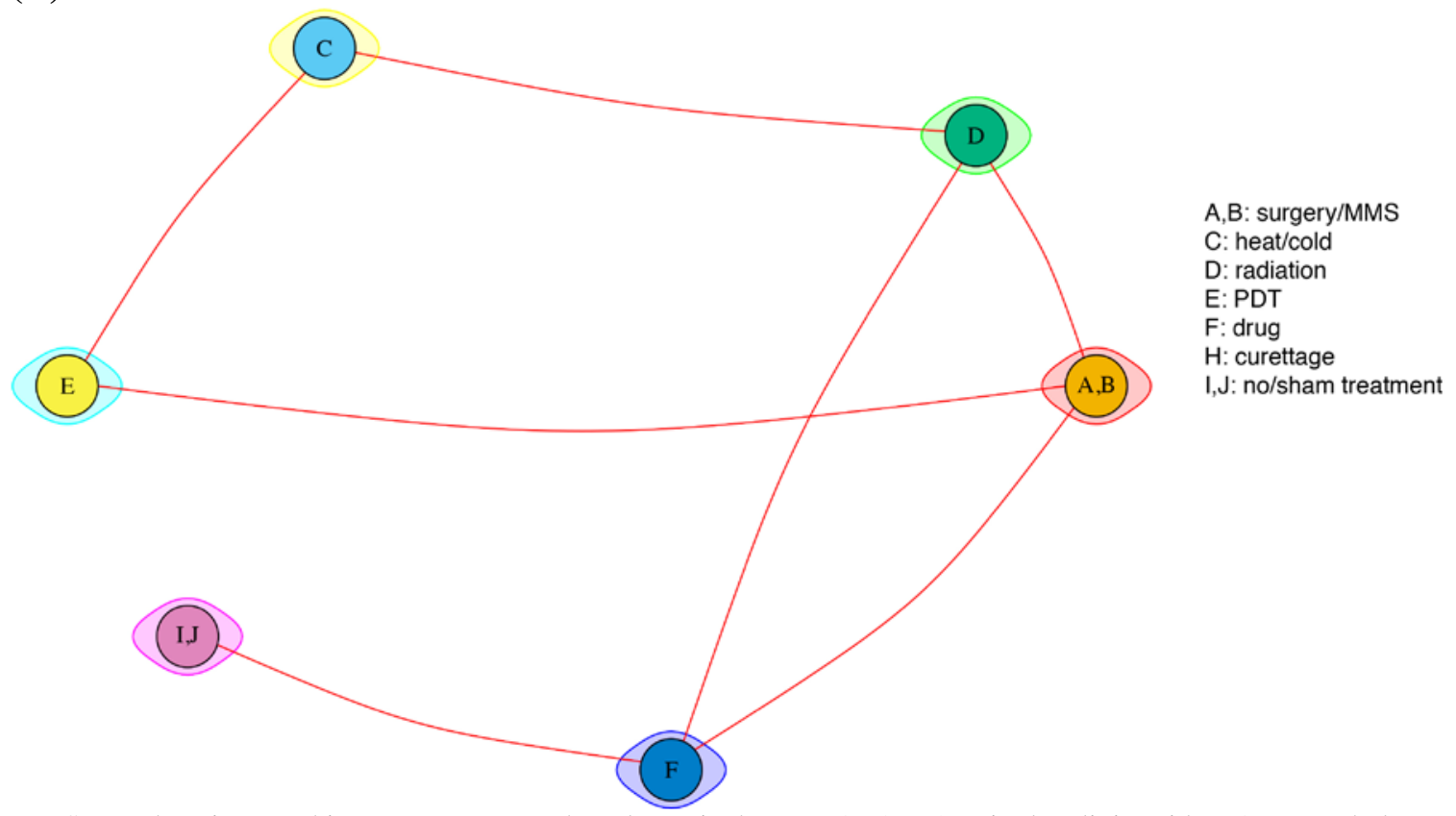

MMS = Mohs micrographic surgery; PDT = photodynamic therapy; ALA = 5-aminolevulinic acid; MAL = methyl aminolevulinate; FU = fluorouracil; INF = interferon 


\section{Comparisons Across Intervention Categories}

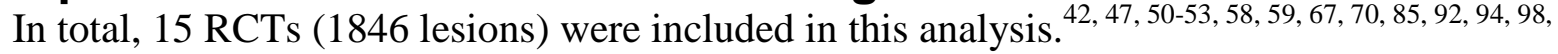

${ }^{104}$ Twelve RCTs were deemed to be at low or moderate risk of bias. Cumulative sample sizes per comparison ranged from 27 to 380; for more details see Table 35.

Table 35. Sample information, lack of clinical clearance (all BCC lesions, intervention categories)

\begin{tabular}{|c|c|}
\hline Studies (total sample) & $15(1846)$ \\
\hline Total sample by intervention & (D): 201; (F): 379; (A,B): 460; (C): 223; (I,J): 129; (E): 404 \\
\hline $\begin{array}{l}\text { Total sample by intervention, (min, } \\
\text { max) }\end{array}$ & 129,460 \\
\hline Data by comparison & $\begin{array}{l}\text { (D--F): } 1 \text { (27); (D--A,B): } 1 \text { (347); (D--C): } 1 \text { (31); (F--A,B): } 1 \text { (212); (F--I,J): } 3 \\
\text { (379); (A,B--E): } 3 \text { (380); (C--E): } 4 \text { (420) }\end{array}$ \\
\hline Studies by comparison (min, max) & 1,4 \\
\hline $\begin{array}{l}\text { Total sample by comparison (min, } \\
\text { max) }\end{array}$ & 27,420 \\
\hline Followup median (min, max) & $6(3,41)$ months \\
\hline
\end{tabular}

A = surgical excision, $\mathrm{B}=$ Mohs micrographic surgery; $\mathrm{BCC}=$ basal cell carcinoma; $\mathrm{C}=$ heat/cold; $\mathrm{D}=$ radiation; $\mathrm{E}=$ photodynamic therapy; $\mathrm{F}$ = drugs; $\mathrm{H}$ = curettage; $\mathrm{I}$ = no treatment; $\mathrm{J}$ = placebo

Table 36 shows the relative odds ratios for lack of clinical clearance across intervention categories. Overall, no or sham treatment $(\mathrm{I}, \mathrm{J})$ was statistically significantly worse than all active treatments. Surgical treatments (A,B) were statistically significantly better than PDT (E) and drugs (F) All other comparisons were statistically not significant; however, the confidence intervals were wide and could not exclude even large differences between the comparators.

In the Table, shaded cells correspond comparisons that have been inferred from the analysis model, but that have not been examined in the included RCTs. For example, comparisons of surgical treatments $(A, B)$ versus drugs $(F)$ are indirect. Indirect comparisons are more uncertain that those for which head-to-head data exist. The added uncertainty about indirect comparisons is partly reflected in the width of the respective 95\% confidence intervals, which is (often much) broader for comparisons without versus with direct data. For all comparisons that are empirically observed (all nonshaded cells in the Table), results using only head-to-head data agree well with the results from the network meta-analysis in Table 36 (see Appendix I).

Table 36. Relative odds ratios for lack of clinical clearance between intervention categories (all BCC lesions, Figure 9B)

\begin{tabular}{|c|c|c|c|c|c|}
\hline $\begin{array}{c}\text { Surgery|MMS } \\
(A \mid B)\end{array}$ & $0.23(0.05,1.11)$ & $\begin{array}{c}0.64(0.08, \\
5.11)\end{array}$ & $0.18(0.05,0.7)$ & $0.16(0.03,0.87)$ & $\begin{array}{c}0.01(<0.005, \\
0.04)\end{array}$ \\
\hline $4.29(0.9,20.48)$ & $\begin{array}{l}\text { Heat/cold } \\
\text { (C) }\end{array}$ & $\begin{array}{c}2.74(0.36, \\
20.9) \\
\end{array}$ & $0.78(0.28,2.2)$ & $0.68(0.13,3.56)$ & $\begin{array}{c}0.03(<0.005, \\
0.17)\end{array}$ \\
\hline $1.57(0.2,12.55)$ & $0.37(0.05,2.79)$ & $\begin{array}{l}\text { Radiation } \\
\text { (D) }\end{array}$ & $0.29(0.04,2.08)$ & $0.25(0.03,2.09)$ & $0.01(<0.005,0.1)$ \\
\hline $5.48(1.44,20.89)$ & $1.28(0.46,3.58)$ & $\begin{array}{c}3.5(0.48 \\
25.44)\end{array}$ & $\begin{array}{l}\begin{array}{l}\text { PDT } \\
(\mathrm{E})\end{array} \\
\end{array}$ & $0.86(0.18,4.1)$ & $0.03(0.01,0.2)$ \\
\hline $6.35(1.15,35.1)$ & $1.48(0.28,7.8)$ & $\begin{array}{c}4.05(0.48 \\
34.21)\end{array}$ & $1.16(0.24,5.51)$ & $\begin{array}{l}\text { Drugs } \\
\text { (F) }\end{array}$ & $0.04(0.01,0.14)$ \\
\hline $\begin{array}{c}171.32(23.53 \\
1247.4)\end{array}$ & $\begin{array}{l}39.94(5.86 \\
272.09)\end{array}$ & $\begin{array}{c}109.33 \\
(10.37, \\
1152.42)\end{array}$ & $31.28(5,195.56)$ & $\begin{array}{c}26.99(7.26 \\
100.4)\end{array}$ & $\begin{array}{l}\text { No/sham } \\
\text { treatment } \\
\text { (I|J) }\end{array}$ \\
\hline
\end{tabular}

Note: Cells shaded gray indicate that the estimate is based only on indirect comparisons; bold-italic numbers indicate statistical significance. Results are given as odds ratios (95\% confidence intervals). 
MMS = Mohs micrographic surgery; PDT = photodynamic therapy.

Table 37 offers complementary information from the same analysis. For each intervention category, it shows the mean fraction of lesions without clinical clearance across the included RCTs. It also forecasts expected fractions with each intervention category in groups of patients similar to the patients included in the analyzed RCTs. The average percentage of lesions with no clinical clearance was 2.9 percent in surgical treatment arms, between 4.5 and 16.5 percent in other active intervention categories, and 84.2 percent for no or sham treatment.

Table 37. Mean and forecasted lack of clinical clearance fractions by intervention category (all BCC lesions)

\begin{tabular}{lcc}
\hline \multicolumn{1}{c}{ Intervention Type } & $\begin{array}{c}\text { Mean Lack of Clinical Clearance } \\
\text { Fraction } \\
\text { Percent (95\% Cl) }\end{array}$ & $\begin{array}{c}\text { Forecasted Lack of Clinical } \\
\text { Clearance Fraction } \\
\text { Percent (95\% Cl) }\end{array}$ \\
\hline Surgery|MMS (A|B) & $3.0(0.8,10.7)$ & $3.0(0.2,38.7)$ \\
\hline Heat/cold (C) & $11.9(4.2,29.1)$ & $11.9(0.7,71.1)$ \\
\hline Radiation (D) & $4.7(0.8,23.4)$ & $4.7(0.2,55.8)$ \\
\hline PDT (E) & $14.7(6.1,31.3)$ & $14.7(1.0,74.9)$ \\
\hline Drugs (F) & $16.6(5.3,41.6)$ & $16.6(1.0,79.6)$ \\
\hline No/sham treatment $(I \mid J)$ & $84.3(52.5,96.3)$ & $84.3(19.2,99.2)$ \\
\hline MMS = Mohs micrographic surgery; PDT = photodynamic therapy; CI = confidence interval; BCC = basal cell carcinoma
\end{tabular}

\section{Comparisons Across Individual Interventions}

The results of the analyses of intervention categories are congruent with the corresponding results of analyses of individual interventions. As evident from Figure 9, there are 2 connected subgraphs for this outcome. Separate analyses are conducted for each connected subgraph. In total, 14 RCTs (1734 lesions) were included in these analyses, as summarized in Table 38.

Table 38. Sample information, lack of clinical clearance (all BCC lesions, individual interventions)

\begin{tabular}{|c|c|c|}
\hline & First subgraph ${ }^{42,47,51-53,58,59,67,70,85,92,94,98,104}$ & Second subgraph ${ }^{50}$ \\
\hline $\begin{array}{l}\text { Studies (total } \\
\text { sample) }\end{array}$ & $14(1449)$ & $1(347)$ \\
\hline $\begin{array}{l}\text { Total sample by } \\
\text { intervention }\end{array}$ & $\begin{array}{l}\text { (D1): 28; (F2): 213; (C1): 152; (F3): 118; (J): 129; (E2): 93; (A): } \\
\text { 286; (E1): 311; (F4): 48; (C5+E1): 37; (C5 + E2): } 34\end{array}$ & $(\mathrm{~A} \mid \mathrm{B}): 174 ;(\mathrm{D} 1 \mid \mathrm{D} 2): 173$ \\
\hline $\begin{array}{l}\text { Total sample by } \\
\text { intervention, } \\
\text { (min, max) }\end{array}$ & 28,311 & 173,174 \\
\hline $\begin{array}{l}\text { Data by } \\
\text { comparison }\end{array}$ & $\begin{array}{l}\text { (D1--F2): } 1 \text { (27); (D1--C1): } 1 \text { (31); (F2--J): } 1 \text { (166); (F2--A): } 1 \\
\text { (212); (C1--E2): } 1 \text { (83); (C1--E1): } 1 \text { (201); (F3--J): } 1 \text { (159); (J-- } \\
\text { F4): } 1 \text { (54); (E2--A): } 1 \text { (40); (A--E1): } 2 \text { (340); (E1--C5+E1): } 2 \\
\text { (74); (E2-C5 + E2): } 1 \text { (62) }\end{array}$ & (A|B--D1|D2): 1 (347) \\
\hline $\begin{array}{l}\text { Studies by } \\
\text { comparison (min, } \\
\max \text { ) }\end{array}$ & 1,2 & 1,1 \\
\hline $\begin{array}{l}\text { Total sample by } \\
\text { comparison (min, } \\
\text { max) }\end{array}$ & 27,340 & 347,347 \\
\hline $\begin{array}{l}\text { Followup median } \\
\text { (min, max) }\end{array}$ & $6(3,12)$ months & $41(41,41)$ months \\
\hline
\end{tabular}


A = surgical excision; $\mathrm{B}$ = Mohs micrographic surgery; BCC=basal cell carcinoma; $\mathrm{C} 1$ = cryotherapy; $\mathrm{C} 3$ = diathermy and curettage; C4 = cryotherapy and curettage; C5 = laser; D1 = external radiation; E1 = MAL photodynamic therapy; E2 = ALA photodynamic therapy; F1 = 5-FU; F2 = Imiquimod; F3 = Interferon; F4 = Ingenol; H = curettage; J = placebo

Table 39 has results on the relative effects for the largest subgraph. Table 40 has the corresponding results for the comparison of surgical excision or MMS (A|B) with external radiation therapy or brachytherapy (D1|D2). In Table 39 comparisons across individual observations are sparse; the majority of the pairwise comparisons are inferred from indirect data. The confidence intervals of the odds ratios for most indirect comparisons are very broad and cannot exclude very large differences between the comparators. The comparison in Table 40 was not statistically significant; the confidence interval was wide and could not exclude large differences between the comparators.

Table 41 shows, for each intervention, the mean fractions for lack of clinical clearance across all RCTs. Estimates for interventions in all three subgraphs are listed in the table. One should not statistically compare these fractions across the subgraphs, because they come from disjoint analyses. In general, the mean fractions of lack of clinical clearance for individual interventions are in congruence with the corresponding fractions estimated for intervention categories. 
Table 39. Relative odds ratios for lack of clinical clearance between individual interventions (all BCC lesions, Figure 9A, largest subgraph)

\begin{tabular}{|c|c|c|c|c|c|c|c|c|c|c|}
\hline Surgery (A) & $\begin{array}{c}0.17(0.04 \\
0.65)\end{array}$ & $\begin{array}{c}0.6(0.13 \\
2.72)\end{array}$ & $\begin{array}{c}0.28(0.07 \\
1.22)\end{array}$ & $\begin{array}{c}0.45(0.03 \\
6.02)\end{array}$ & $0.2(0.06,0.7)$ & $\begin{array}{c}0.41(0.14 \\
1.18)\end{array}$ & $\begin{array}{c}0.36(0.08 \\
1.74)\end{array}$ & $\begin{array}{c}0.27(0.05 \\
1.42) \\
\end{array}$ & $\begin{array}{c}0.07(0.01 \\
0.45)\end{array}$ & $0.06(0.01,0.29)$ \\
\hline $\begin{array}{c}5.95(1.53 \\
23.14)\end{array}$ & $\begin{array}{c}\text { Cryotherapy } \\
\text { (C1) }\end{array}$ & $\begin{array}{c}3.56(0.84, \\
15.01)\end{array}$ & $\begin{array}{c}1.68(0.34, \\
8.28) \\
\end{array}$ & $\begin{array}{c}2.67(0.23 \\
31.74) \\
\end{array}$ & $\begin{array}{c}1.21(0.39 \\
3.77) \\
\end{array}$ & $\begin{array}{c}2.44(0.7 \\
8.51) \\
\end{array}$ & $\begin{array}{c}2.16(0.41, \\
11.44)\end{array}$ & $\begin{array}{c}1.61(0.28 \\
9.21) \\
\end{array}$ & $\begin{array}{c}0.44(0.07 \\
2.84) \\
\end{array}$ & $0.35(0.07,1.88)$ \\
\hline $\begin{array}{c}1.67(0.37 \\
7.6)\end{array}$ & $\begin{array}{c}0.28(0.07 \\
1.18)\end{array}$ & $\begin{array}{c}\text { Laser + PDT } \\
\text { (MAL) } \\
\text { (C5+E1) }\end{array}$ & $\begin{array}{c}0.47(0.07 \\
2.99)\end{array}$ & $\begin{array}{c}0.75(0.05 \\
10.88)\end{array}$ & $\begin{array}{c}0.34(0.14 \\
0.84)\end{array}$ & $\begin{array}{c}0.69(0.14 \\
3.29)\end{array}$ & $\begin{array}{c}0.61(0.1 \\
3.69)\end{array}$ & $\begin{array}{c}0.45(0.07 \\
2.95)\end{array}$ & $\begin{array}{c}0.12(0.02 \\
0.9)\end{array}$ & $0.1(0.02,0.6)$ \\
\hline $\begin{array}{c}3.53(0.82 \\
15.24)\end{array}$ & $\begin{array}{c}0.59(0.12 \\
2.92)\end{array}$ & $\begin{array}{c}2.11(0.33 \\
13.37)\end{array}$ & $\begin{array}{c}\text { Laser + PDT } \\
\text { (ALA) } \\
(\mathrm{C} 5+\mathrm{E} 2)\end{array}$ & $\begin{array}{c}1.59(0.1 \\
25.01)\end{array}$ & $\begin{array}{c}0.72(0.14 \\
3.65)\end{array}$ & $\begin{array}{c}1.45(0.51 \\
4.16)\end{array}$ & $\begin{array}{c}1.28(0.19 \\
8.56)\end{array}$ & $\begin{array}{c}0.96(0.13 \\
6.85)\end{array}$ & $\begin{array}{c}0.26(0.03 \\
2.09)\end{array}$ & $0.21(0.03,1.41)$ \\
\hline $\begin{array}{c}2.22(0.17 \\
29.75)\end{array}$ & $\begin{array}{c}0.37(0.03 \\
4.44)\end{array}$ & $\begin{array}{c}1.33(0.09 \\
19.26)\end{array}$ & $\begin{array}{c}0.63(0.04 \\
9.9)\end{array}$ & $\begin{array}{l}\text { External } \\
\text { radiation } \\
\text { (D1) }\end{array}$ & $\begin{array}{c}0.45(0.04 \\
5.67)\end{array}$ & $\begin{array}{c}0.91(0.07 \\
12.11)\end{array}$ & $\begin{array}{c}0.81(0.06 \\
10.4)\end{array}$ & $\begin{array}{c}0.6(0.04 \\
8.25)\end{array}$ & $\begin{array}{c}0.16(0.01 \\
2.47)\end{array}$ & $0.13(0.01,1.72)$ \\
\hline $\begin{array}{c}4.93(1.43, \\
17.02)\end{array}$ & $\begin{array}{c}0.83(0.26 \\
2.6)\end{array}$ & $\begin{array}{c}2.95(1.2 \\
7.28)\end{array}$ & $\begin{array}{c}1.4(0.27 \\
7.12)\end{array}$ & $\begin{array}{c}2.22(0.18 \\
27.9)\end{array}$ & $\begin{array}{l}\text { PDT (MAL) } \\
\text { (E1) }\end{array}$ & $\begin{array}{c}2.02(0.55 \\
7.48)\end{array}$ & $\begin{array}{c}1.79(0.36 \\
8.8)\end{array}$ & $\begin{array}{c}1.33(0.25 \\
7.1)\end{array}$ & $\begin{array}{c}0.36(0.06, \\
2.2)\end{array}$ & $0.29(0.06,1.44)$ \\
\hline $\begin{array}{c}2.44(0.85 \\
7.01) \\
\end{array}$ & $\begin{array}{c}0.41(0.12 \\
1.43) \\
\end{array}$ & $1.46(0.3,7)$ & $\begin{array}{c}0.69(0.24 \\
1.98) \\
\end{array}$ & $\begin{array}{c}1.1(0.08, \\
14.53)\end{array}$ & $\begin{array}{c}0.49(0.13 \\
1.82) \\
\end{array}$ & $\begin{array}{l}\text { PDT (ALA) } \\
\text { (E2) }\end{array}$ & $\begin{array}{c}0.88(0.17 \\
4.57) \\
\end{array}$ & $\begin{array}{c}0.66(0.12 \\
3.7) \\
\end{array}$ & $\begin{array}{c}0.18(0.03, \\
1.15) \\
\end{array}$ & $0.14(0.03,0.75)$ \\
\hline $\begin{array}{c}2.75(0.58 \\
13.2)\end{array}$ & $\begin{array}{c}0.46(0.09 \\
2.46)\end{array}$ & $\begin{array}{c}1.65(0.27 \\
10.02)\end{array}$ & $\begin{array}{c}0.78(0.12 \\
5.21)\end{array}$ & $\begin{array}{c}1.24(0.1 \\
15.97)\end{array}$ & $\begin{array}{c}0.56(0.11 \\
2.75)\end{array}$ & $\begin{array}{c}1.13(0.22 \\
5.85)\end{array}$ & $\begin{array}{l}\text { Imiqumod } \\
\text { (F2) }\end{array}$ & $\begin{array}{c}0.74(0.35 \\
1.59)\end{array}$ & $\begin{array}{c}0.2(0.06, \\
0.63)\end{array}$ & $0.16(0.1,0.28)$ \\
\hline $\begin{array}{c}3.7(0.7 \\
19.48)\end{array}$ & $\begin{array}{c}0.62(0.11 \\
3.56)\end{array}$ & $\begin{array}{c}2.21(0.34, \\
14.44)\end{array}$ & $\begin{array}{c}1.05(0.15 \\
7.51) \\
\end{array}$ & $\begin{array}{c}1.66(0.12 \\
22.82) \\
\end{array}$ & $\begin{array}{c}0.75(0.14, \\
3.99) \\
\end{array}$ & $\begin{array}{c}1.52(0.27 \\
8.54) \\
\end{array}$ & $\begin{array}{c}1.34(0.63 \\
2.87) \\
\end{array}$ & INF (F3) & $\begin{array}{c}0.27(0.09, \\
0.87)\end{array}$ & $0.22(0.13,0.38)$ \\
\hline $\begin{array}{c}13.59(2.24 \\
82.53) \\
\end{array}$ & $\begin{array}{c}2.29(0.35, \\
14.85) \\
\end{array}$ & $\begin{array}{c}8.14(1.11 \\
59.66) \\
\end{array}$ & $\begin{array}{c}3.85(0.48, \\
30.95) \\
\end{array}$ & $\begin{array}{c}6.12(0.41 \\
92.23) \\
\end{array}$ & $\begin{array}{c}2.76(0.45, \\
16.72) \\
\end{array}$ & $\begin{array}{c}5.58(0.87 \\
35.77) \\
\end{array}$ & $\begin{array}{c}4.93(1.58, \\
15.4) \\
\end{array}$ & $\begin{array}{c}3.68(1.16, \\
11.69) \\
\end{array}$ & Ingenol (F4) & $0.81(0.29,2.24)$ \\
\hline $\begin{array}{c}16.86(3.47 \\
82.04)\end{array}$ & $\begin{array}{c}2.84(0.53, \\
15.08)\end{array}$ & $\begin{array}{c}10.09(1.66 \\
61.51)\end{array}$ & $\begin{array}{c}4.77(0.71 \\
32.06)\end{array}$ & $\begin{array}{c}7.59(0.58 \\
98.98)\end{array}$ & $\begin{array}{c}.42(0.69, \\
16.86)\end{array}$ & $\begin{array}{c}6.93(1.33 \\
36.11)\end{array}$ & $\begin{array}{c}6.12(3.61 \\
10.4)\end{array}$ & $\begin{array}{c}4.56(2.64, \\
7.88)\end{array}$ & $\begin{array}{c}1.24(0.45, \\
3.45)\end{array}$ & Placebo (J) \\
\hline
\end{tabular}

Note: Cells shaded gray indicate that the estimate is based only on indirect comparisons; bold-italic indicates a significant result. Results are given as odds ratios (95\% confidence intervals).

MMS = Mohs micrographic surgery; PDT = photodynamic therapy; INF = Interferon; $\mathrm{BCC}$ = basal cell carcinoma; ALA = 5-aminolevulinic acid; MAL = methyl aminolevulinate; $\mathrm{FU}=$ fluorouracil; $\mathrm{INF}=$ interferon. 
Table 40. Relative odds ratios for lack of clinical clearance between individual interventions (all BCC lesions, Figure 9A, remaining subgraphs)

Surgery or MMS

$$
(A \mid B)
$$

$6.16(0.31,123.87)$

BCC = basal cell carcinoma
$0.16(0.01,3.27)$

External radiation or

$$
\text { brachytherapy }
$$

(D1|D2) 
Table 41. Mean and forecasted lack of clinical clearance fractions by individual intervention (all BCC lesions)

\begin{tabular}{lll}
\hline \multicolumn{1}{c}{ Intervention Type } & $\begin{array}{c}\text { Mean } \\
\text { Percent }(95 \% \mathrm{Cl})\end{array}$ & $\begin{array}{c}\text { Forecast } \\
\text { Percent (95\% Cl) }\end{array}$ \\
\hline First subgraph (Figure 9) & & \\
\hline Surgical excision (A) & $0.5(0.2,1.8)$ & $0.5(0.0,9.8)$ \\
\hline Cryotherapy (C1) & $3.1(1.0,9.4)$ & $3.1(0.2,38.2)$ \\
\hline Laser + PDT (MAL) (C5+E1) & $0.9(0.2,3.4)$ & $0.9(0.0,16.2)$ \\
\hline Laser + PDT (ALA) & $1.8(0.4,7.5)$ & $1.8(0.1,29.1)$ \\
\hline External radiation (D1) & $1.2(0.1,11.5)$ & $1.2(0.0,31.1)$ \\
\hline PDT (MAL) (E1) & $2.6(0.9,7.1)$ & $2.6(0.1,32.9)$ \\
\hline PDT (ALA) (E2) & $1.3(0.4,4.2)$ & $1.3(0.1,20.6)$ \\
\hline Imiquimod (F2) & $1.6(0.4,5.2)$ & $1.6(0.1,24.0)$ \\
\hline IFN(F3) & $1.6(0.4,5.9)$ & $1.6(0.1,25.4)$ \\
\hline Ingenol (F4) & $5.5(1.5,18.4)$ & $5.5(0.3,54.9)$ \\
\hline No/sham treatment (J) & $6.6(2.0,19.7)$ & $6.6(0.4,58.6)$ \\
\hline Second subgraph (Figure 9) & & $\mathrm{NA}$ \\
\hline Surgical excision or MMS (A|B) & $0.3(0.0,4.4)$ & $\mathrm{NA}$ \\
\hline External radiation or \\
brachytherapy (D1|D2)
\end{tabular}

MMS = Mohs micrographic surgery; PDT = photodynamic therapy; ALA = 5-aminolevulinic acid; MAL = methyl aminolevulinate; INF = interferon; BCC = basal cell carcinoma; CI = confidence interval; NA = not applicable

\section{Lack of Clinical Clearance, Subgroup Analyses by Lesion Type}

We conducted subgroup analyses by the type of BCC lesion. We report analyses comparing groups of interventions, but not analyses comparing individual treatments. The latter are very sparse, and their results are similar to the pertinent comparisons in Tables 39 and 40.

Many subgroup analyses per lesion type are possible; we describe here analyses in RCTs of lower-risk lesions (strata of predominantly [>80\%] superficial BCCs, predominantly nodular BCCs, and superficial or nodular BCCs) overall, and broken down by lesion type, along with analyses of higher-risk lesions (morpheaform, micronodular, trabecular, infiltrative, or squamous differentiation).

All 14 RCTs reporting results on lack of clinical clearance enrolled patients with low-risk BCCs (nodular and superficial subtypes; $n=1922$ ). ${ }^{47,50-53,58,59,67,70,85,92,94,98,104}$ Thus, for the lower-risk BCCs subgroup the results are practically the same as in the previous section (Tables 36 and 37).

Table 42 summarizes characteristics of the five RCTs of patients with predominantly superficial BCC lesions (n=868). 51, 52, 92, 94, 98 Tables 43 and 44 show the results. Most comparisons in Table 43 are indirect, and the confidence intervals for these differences are too broad to allow drawing conclusions..

Table 42. Sample information, lack of clinical clearance (superficial BCC lesions)

\begin{tabular}{ll}
\hline Studies (total sample) & $5(868)$ \\
\hline Total sample by intervention & $(\mathrm{F}): 246 ;(\mathrm{I}, \mathrm{J}): 88 ;(\mathrm{A}, \mathrm{B}): 215 ;(\mathrm{E}): 221 ;(\mathrm{C}): 98$
\end{tabular}




\begin{tabular}{|c|c|}
\hline $\begin{array}{l}\text { Total sample by intervention, (min, } \\
\text { max) }\end{array}$ & 88,246 \\
\hline Data by comparison & (F--I,J): 2 (220); (F--A,B): 1 (212); (A,B--E): 1 (235); (E--C): 1 (201) \\
\hline Studies by comparison (min, max) & 1,2 \\
\hline $\begin{array}{l}\text { Total sample by comparison (min, } \\
\max \text { ) }\end{array}$ & 201,235 \\
\hline Followup median (min, max) & $3(3,36)$ months \\
\hline
\end{tabular}

Table 43. Relative odds ratios for lack of clinical clearance between intervention categories (superficial BCC lesions)

\begin{tabular}{ccccc}
\hline $\begin{array}{c}\text { Surgery/MMS } \\
(\mathrm{A}, \mathrm{B})\end{array}$ & $0.13(<0.005,10.81)$ & $0.12(<0.005,5.06)$ & $\begin{array}{c}0.02(<0.005, \\
0.79)\end{array}$ & $\begin{array}{c}<0.005(<0.005, \\
0.02)\end{array}$ \\
\hline $7.71(0.09,642.49)$ & $\begin{array}{c}\text { Heat/cold } \\
(\mathbf{C})\end{array}$ & $0.94(0.01,59.67)$ & $\begin{array}{c}0.19(<0.005, \\
9.58)\end{array}$ & $\begin{array}{c}<0.005(<0.005, \\
0.28)\end{array}$ \\
\hline $8.16(0.2,337.33)$ & $1.06(0.02,66.93)$ & $\begin{array}{c}\text { PDT } \\
(\mathrm{E})\end{array}$ & $0.2(0.01,4.49)$ & $\begin{array}{c}<0.005(<0.005, \\
0.14)\end{array}$ \\
\hline $40.1(1.27,1269.18)$ & $5.2(0.1,259.27)$ & $4.91(0.22,108.22)$ & $\begin{array}{c}\text { Drugs } \\
(\mathbf{F})\end{array}$ & $0.02(<0.005,0.53)$ \\
\hline $\begin{array}{c}2071.45(41.73, \\
102817.08)\end{array}$ & $268.7(3.6,20036.53)$ & $253.71(7.01,9177.18)$ & $\begin{array}{c}51.65(1.88, \\
1415.99)\end{array}$ & $\begin{array}{c}\text { No/sham treatment } \\
(\mathbf{I}, \mathbf{J})\end{array}$ \\
\hline
\end{tabular}

Note: Cells shaded gray indicate that the estimate is based only on indirect comparisons. Results are given as odds ratios (95\% confidence intervals).

BCC=basal cell carcinoma; MMS = Mohs micrographic surgery; PDT = photodynamic therapy

Table 44. Mean and forecasted lack of clinical clearance fractions by intervention category (superficial BCC lesions)

\begin{tabular}{lcc}
\hline Intervention Type & $\begin{array}{c}\text { Mean } \\
\text { Percent } \mathbf{( 9 5 \% ~ C l )}\end{array}$ & $\begin{array}{c}\text { Forecast } \\
\text { Percent } \mathbf{9 5 \%} \mathbf{~ C l )}\end{array}$ \\
\hline Surgery/MMS (A,B) & $0.7(<0.5,10.7)$ & $0.7(<0.5,34.6)$ \\
\hline Heat/cold (C) & $5.1(0.2,61.3)$ & $5.1(<0.5,85.5)$ \\
\hline PDT (E) & $5.4(0.5,38.5)$ & $5.4(0.1,76.5)$ \\
\hline Drugs (F) & $21.9(3.8,66.4)$ & $21.9(0.6,92.6)$ \\
\hline No/sham treatment $(\mathrm{I}, \mathrm{J})$ & $93.5(50.0,99.5)$ & $93.5(17.6,99.9)$ \\
\hline
\end{tabular}

MMS = Mohs micrographic surgery; PDT = photodynamic therapy; BCC = basal cell carcinoma; CI = confidence interval

Table 45 provides details on the comparisons of six RCTs of predominantly nodular lesions $(n=434)$. Results are given in Tables 46 and $47 .{ }^{52,53,58,67,70,85}$ These results very uncertain, and are based on at most two studies per comparison. The confidence intervals for differences between the intervention categories are generally very broad.

Table 45. Sample information, lack of clinical clearance (superficial BCC lesions)

\begin{tabular}{ll}
\hline Studies (total sample) & $6(434)$ \\
\hline Total sample by intervention & $(\mathrm{D}): 12 ;(\mathrm{F}): 113 ;(\mathrm{A}, \mathrm{B}): 161 ;(\mathrm{E}): 111 ;(\mathrm{C}): 37$ \\
\hline $\begin{array}{l}\text { Total sample by intervention, (min, } \\
\text { max) }\end{array}$ & 12,161 \\
\hline Data by comparison & $(\mathrm{D}--\mathrm{F}): 1(27) ;(\mathrm{F}-\mathrm{A}, \mathrm{B}): 1(188) ;(\mathrm{A}, \mathrm{B}-\mathrm{-E}): 2(145) ;(\mathrm{E}-\mathrm{-C}): 2(74)$ \\
\hline Studies by comparison (min, max) & 1,2 \\
\hline
\end{tabular}




\begin{tabular}{|c|c|c|c|c|}
\hline $\begin{array}{l}\text { Total sample by comparison (min, } \\
\text { max) }\end{array}$ & 27,188 & & & \\
\hline $\begin{array}{ll}\text { Followup median }(\min , \max ) & 8(3,36) \mathrm{m} \\
\mathrm{A}=\text { surgical excision } \cdot \mathrm{B}=\text { Mohs microoranhic suroerv } \mathrm{F} & \end{array}$ & \multicolumn{2}{|c|}{$8(3,36)$ months } & & \\
\hline \multicolumn{5}{|c|}{$\begin{array}{l}\text { A = surgical excision; } \mathrm{B}=\text { Mohs micrographic surgery; } \mathrm{BCC}=\text { basal cell carcinoma; } \mathrm{C}=\text { heat/cold; } \mathrm{E}=\text { photodynamic therapy; } \mathrm{F} \\
=\text { = drugs; } \mathrm{H} \text { = curettage; } \mathrm{I}=\text { no treatment; } \mathrm{J}=\text { placebo }\end{array}$} \\
\hline \multicolumn{5}{|c|}{$\begin{array}{l}\text { Table 46. Relative odds ratios between intervention categories for lack of clinical clearance } \\
\text { (nodular BCC lesions) }\end{array}$} \\
\hline $\begin{array}{l}\text { Surgery/MMS } \\
(A, B)\end{array}$ & $2.06(0.38,11.25)$ & $1.79(0.03,97.08)$ & $\begin{array}{c}0.28(0.09 \\
0.87)\end{array}$ & $\begin{array}{c}1.98(0.15 \\
26.6) \\
\end{array}$ \\
\hline $0.49(0.09,2.65)$ & $\begin{array}{l}\text { Heat/cold } \\
\text { (C) }\end{array}$ & $0.87(0.01,53.43)$ & $\begin{array}{c}0.13(0.04 \\
0.49)\end{array}$ & $\begin{array}{r}0.96(0.06 \\
16.76)\end{array}$ \\
\hline $0.56(0.01,30.25)$ & $1.15(0.02,70.67)$ & $\begin{array}{l}\text { Radiotherapy } \\
\text { (D) }\end{array}$ & $\begin{array}{c}0.15(<0.005 \\
8.16)\end{array}$ & $\begin{array}{r}1.11(0.03, \\
44.12)\end{array}$ \\
\hline $3.63(1.16,11.4)$ & $7.48(2.06,27.14)$ & $6.5(0.12,344.91)$ & $\begin{array}{c}\text { PDT } \\
\text { (E) }\end{array}$ & $\begin{array}{r}7.19(0.52 \\
99.37)\end{array}$ \\
\hline $0.5(0.04,6.77)$ & $1.04(0.06,18.11)$ & $0.9(0.02,36.04)$ & $\begin{array}{c}0.14(0.01 \\
1.92)\end{array}$ & $\begin{array}{l}\text { Drugs } \\
\text { (F) }\end{array}$ \\
\hline
\end{tabular}

Note: RCTs of predominantly nodular lesions. Cells shaded gray indicate that the estimate is based only on indirect comparisons. Results are given as odds ratios (95\% confidence intervals).

MMS = Mohs micrographic surgery; PDT = photodynamic therapy; $\mathrm{BCC}=$ basal cell carcinoma

Table 47. Mean fractions of lesions with no clinical clearance by intervention category (nodular BCC lesions)

\begin{tabular}{lcc}
\hline Intervention Type & $\begin{array}{c}\text { Mean } \\
\text { Percent (95\% Cl) }\end{array}$ & $\begin{array}{c}\text { Forecast } \\
\text { Percent (95\% Cl) }\end{array}$ \\
\hline Surgery/MMS (A,B) & $7.6(1.5,31.6)$ & $7.6(0.2,74.7)$ \\
\hline Heat/cold (C) & $3.9(0.6,20.6)$ & $3.9(0.1,60.6)$ \\
\hline Radiotherapy (D) & $4.4(0.1,66.7)$ & $4.4(0.0,86.1)$ \\
\hline PDT (E) & $23.0(6.0,58.5)$ & $23.0(0.9,90.8)$ \\
\hline Drugs (F) & $4.0(0.4,32.7)$ & $4.0(0.1,69.1)$ \\
\hline
\end{tabular}

Note: RCTs of predominantly nodular lesions.

MMS = Mohs micrographic surgery; PDT = photodynamic therapy; BCC = basal cell carcinoma; $\mathrm{CI}=$ confidence interval

\section{Lack of Clinical Clearance, Other Subgroup Analyses (Lesion Location, Lesion Size)}

Table 48 shows results on subgroup analyses for three RCTs that reported treatment effects in subgroups of interest, two in patients with predominantly superficial BCCs ${ }^{51,98}$ and one in patients with predominantly nodular BCCs. ${ }^{22,85}$ Neither lesion location nor size were associated with differences in the treatment effect beyond what is expected by chance. Only one outcome was statistically significant at a 0.05 level: surgical excision (A) performed better than PDT with MAL (E1) for lesions on the trunk and neck at 3 months; however by 12 months, this finding was no longer significant. ${ }^{98}$ 
Table 48. Subgroup results for lack of clinical clearance in BCC lesions

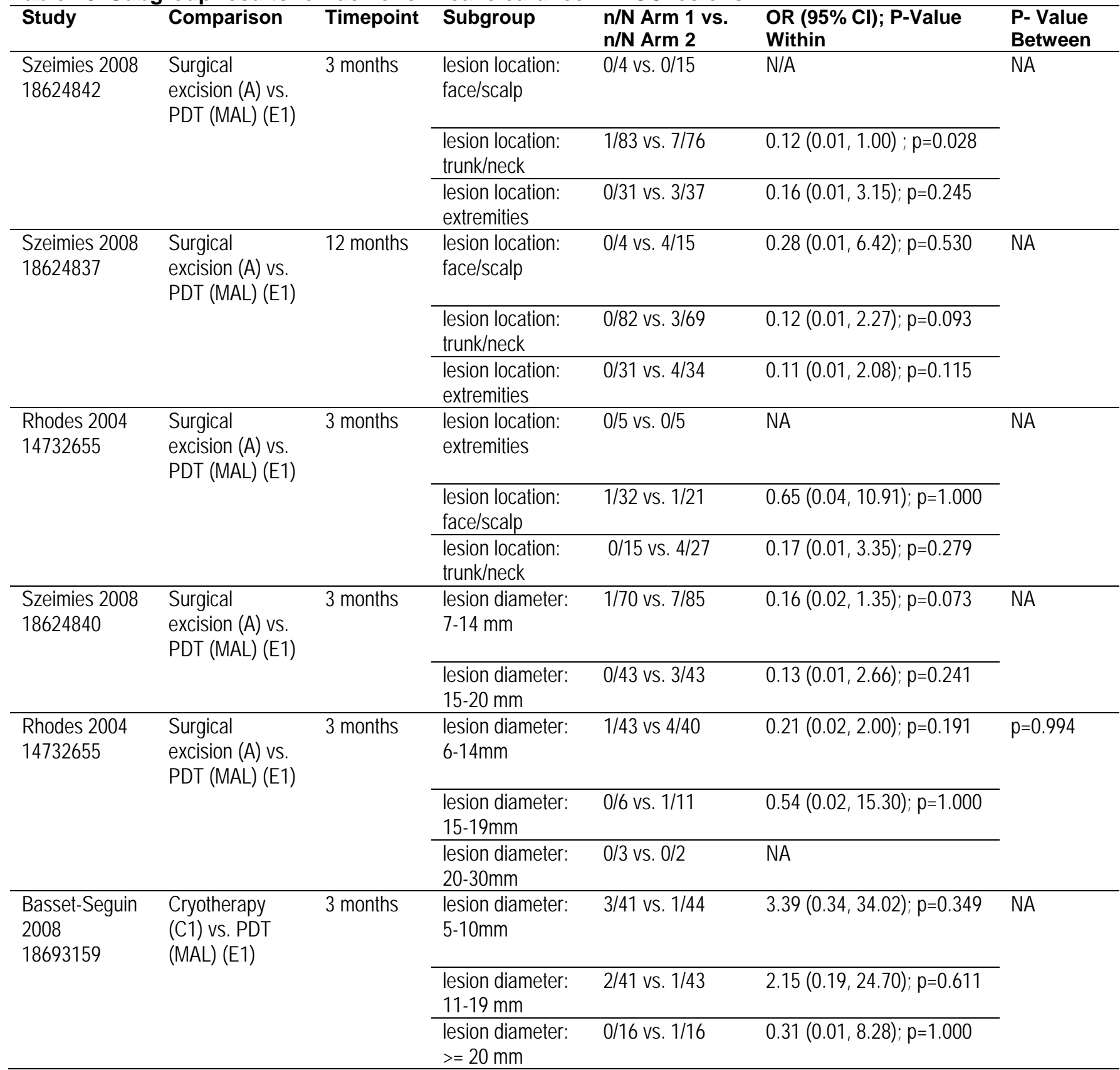

NA = not significant; PDT = photodynamic therapy; MAL = methyl aminolevulinate; $\mathrm{BCC}$ = basal cell carcinoma; $\mathrm{CI}=$ confidence interval

\section{Lack of Clinical Clearance, Results From Nonrandomized Studies (BCC Lesions)}

None of the eligible NRCSs reported data on lack of clinical clearance. 


\section{Various Outcomes in Patients With High-Risk BCC Lesions Treated With Hedgehog Inhibitors}

Hedgehog inhibitors, including vismodegib and sonidegib (F5, other drugs), are a group of systemic medications that are primarily used for advanced or metastatic BCC. Comparisons of outcomes in these high-risk populations with studies that include lower-risk BCCs are not clinically meaningful and so we report these separately.

One RCT ( $\mathrm{n}=230)$ compared 2 doses (200 vs. $800 \mathrm{mg}$ per os daily) of sonidegib for locally advanced BCC not amenable to surgery $(n=194)$ or radiation or metastatic BCC for which other options had been exhausted (n=36). Median age was 67 and 65, respectively, and over 90 percent were white. In the locally advanced group, 2 of 66 (3\%) participants in the $200 \mathrm{mg}$ arm achieved a complete response compared with none of $128(0 \%)$ in the $800 \mathrm{mg}$ arm. The number of participants experiencing any adverse event was high in both arms (75/79 [95\%] in the $200 \mathrm{mg}$ arm, 150/150 [100\%] in the $800 \mathrm{mg}$ arm. $^{75}$

\section{Patient-Reported Cosmetic Outcomes, All BCC Lesions}

For this outcome we describe only results between intervention categories, because data are sparse for the comparison of individual observations. In total, seven RCTs (752 lesions) were included in this analysis. ${ }^{50,51,67,70,85,98,99}$ Five RCTs were deemed to be at low or moderate risk of bias. The evidence graph in Figure 10 shows the observed comparisons based on RCTs that report patient assessments of "at least good" cosmetic outcome. The evidence graph is sparsely connected. Patients assessed cosmetic outcomes using different scales in each RCT, though often on scales of that included poor, fair, good, and excellent or similar. We provide analyses for an "at least good" cosmetic outcome. Details about the comparisons between these RCTs are in Table 49.

Table 49. Sample information, patient-reported cosmetic outcomes (all BCC lesions, intervention categories)

\begin{tabular}{ll}
\hline Studies (total sample) & $7(752)$ \\
\hline Total sample by intervention & $(\mathrm{D}): 125 ;(\mathrm{F}): 15 ;(\mathrm{A}, \mathrm{B}): 309 ;(\mathrm{C}): 113 ;(\mathrm{E}): 190$ \\
\hline Total sample by intervention, (min, & 15,309 \\
max) & $(\mathrm{D}--\mathrm{F}): 1$ (27); (D--A,B): 1 (244); (A,B--C): 1 (96); (A,B--E): 2 (254); (C--E): \\
\hline Data by comparison & $2(131)$ \\
\hline Studies by comparison (min, max) & 1,2 \\
\hline Total sample by comparison (min, & 27,254 \\
max) & $4(3,48)$ months \\
\hline Followup median (min, max) &
\end{tabular}

A = surgical excision; $\mathrm{B}=$ Mohs micrographic surgery; $\mathrm{BCC}=$ basal cell carcinoma; $\mathrm{C}=$ heat/cold; $\mathrm{D}=$ radiation; $\mathrm{E}=$ photodynamic therapy; $\mathrm{F}=$ drugs; $\mathrm{H}=$ curettage; $\mathrm{I}=$ no treatment; $\mathrm{J}=$ placebo 
Figure 10. Evidence graph of RCTs comparing patient-assessed cosmetic outcomes (all BCC lesions)

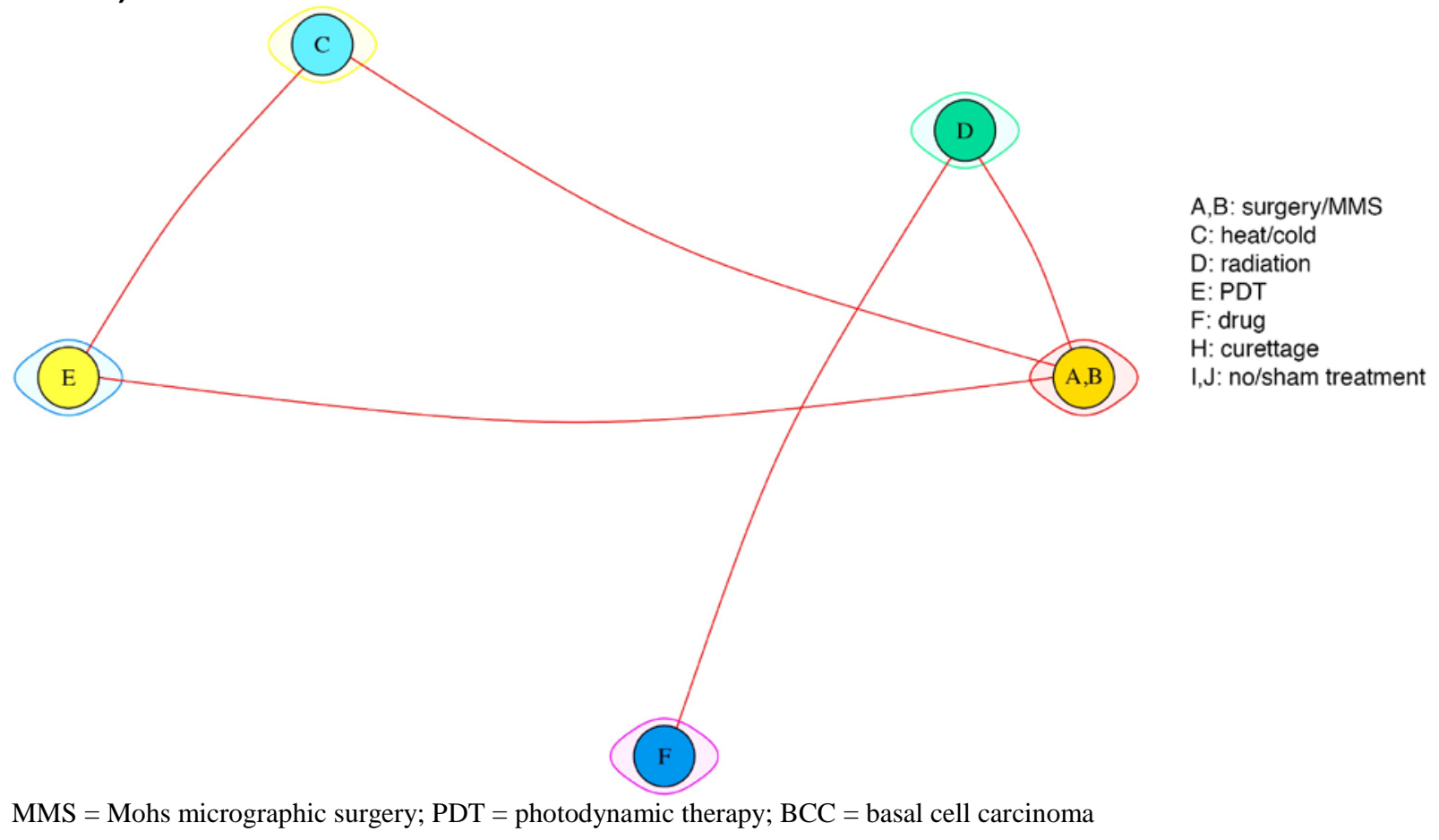

Table 50 shows the results of the comparisons between intervention categories based on a network meta-analysis. Most comparisons are indirect (denoted by shaded cells) and have wide confidence intervals. For comparisons with head-to-head data (denoted by unshaded cells) the numbers in the table are very similar whether information from indirect comparisons is included or excluded. Five of 10 comparisons are statistically significant.

Table 50. Relative odds ratios between intervention categories for at least good cosmetic outcome as assessed by patients (all BCC lesions, Figure 10)

\begin{tabular}{|c|c|c|c|c|}
\hline $\begin{array}{c}\text { Surgery/MMS } \\
(A, B)\end{array}$ & $5.2(1.37,19.79)$ & $2.1(1.18,3.72)$ & $0.17(0.06,0.46)$ & $0.49(0.02,14.01)$ \\
\hline $0.19(0.05,0.73)$ & Heat/cold (C) & $0.4(0.1,1.67)$ & $0.03(0.01,0.13)$ & $0.09(<0.005,3.01)$ \\
\hline $0.48(0.27,0.85)$ & $2.48(0.6,10.25)$ & Radiation (D) & $0.08(0.02,0.25)$ & $0.23(0.01,6.69)$ \\
\hline $6(2.16,16.69)$ & $\begin{array}{c}31.19(7.54 \\
128.97)\end{array}$ & $\begin{array}{c}12.58(3.95 \\
40.03)\end{array}$ & PDT (E) & $2.95(0.09,93.05)$ \\
\hline $2.03(0.07,58.01)$ & $\begin{array}{c}10.58(0.33 \\
336.76)\end{array}$ & $4.27(0.15,121.7)$ & $0.34(0.01,10.7)$ & ) \\
\hline
\end{tabular}

Note: Cells shaded gray indicate that the estimate is based only on indirect comparisons; bold-italic indicates that the result is statistically significant. Results are given as odds ratios (95\% confidence intervals).

MMS = Mohs micrographic surgery; PDT = photodynamic therapy; $\mathrm{BCC}=$ basal cell carcinoma

Table 51 shows the average percentage of patients with at least good cosmetic outcomes in the RCTs, based on the same network meta-analysis as Table 50. Drugs (F) and PDT (E) are associated with highest percentages, followed surgical treatments (A,B), radiation (D), interventions that use heat or cold to destroy the lesion (C). 
Table 51. Mean and forecasted fractions of lesions with at least good cosmetic outcome as assessed by patients (all BCC lesions)

\begin{tabular}{lcc}
\hline $\begin{array}{c}\text { Intervention } \\
\text { Type }\end{array}$ & $\begin{array}{c}\text { Mean } \\
\text { Percent (95\% Cl) }\end{array}$ & $\begin{array}{c}\text { Mean } \\
\text { Percent (95\% CI) }\end{array}$ \\
\hline $\begin{array}{l}\text { Surgery/MMS } \\
(\mathrm{A}, \mathrm{B})\end{array}$ & $88.8(73.7,95.7)$ & $88.8(44.3,98.8)$ \\
\hline Heat/cold (C) & $60.5(32.4,83.0)$ & $60.5(12.7,94.2)$ \\
\hline Radiation (D) & $79.1(55.2,92.1)$ & $79.1(26.8,97.5)$ \\
\hline PDT (E) & $97.9(93.1,99.4)$ & $97.9(81.1,99.8)$ \\
\hline Drugs (F) & $94.2(37.5,99.8)$ & $94.2(25.0,99.9)$ \\
\hline MMS = Mohs micrographic surgery; PDT = photodynamic therapy; BCC = basal cell carcinoma; CI = confidence interval
\end{tabular}

\section{Observer-Reported Cosmetic Outcomes, All BCC Lesions}

We describe only the results between intervention categories, because data are sparse for the comparison of individual observations. In total, 10 RCTs (1460 lesions) were included in this analysis. ${ }^{49-52,66,70,85,98,104}$ Nine RCTs were deemed to be at low or moderate risk of bias. The evidence graph in Figure 11 shows the observed comparisons based on RCTs that report observers' (investigators' or providers') assessments of “at least good” cosmetic outcome. The cosmetic outcome was assessed using different scales in each RCT, though often on scales of that included poor, fair, good, and excellent or similar. We provide analyses for an "at least good” cosmetic outcome. The evidence graph is sparsely connected. Details about the comparisons between these RCTs are in Table 52.

Table 52. Sample information, observer-reported cosmetic outcomes (all BCC lesions, intervention categories)

\begin{tabular}{|c|c|}
\hline Studies (total sample) & $10(1460)$ \\
\hline Total sample by intervention & $(\mathrm{A}, \mathrm{B}): 426 ;(\mathrm{D}): 113 ;(\mathrm{C}): 109 ;(\mathrm{E}): 443 ;(\mathrm{F}): 354 ;(\mathrm{I}, \mathrm{J}): 15$ \\
\hline $\begin{array}{l}\text { Total sample by intervention, (min, } \\
\text { max) }\end{array}$ & 15,443 \\
\hline Data by comparison & $\begin{array}{l}\text { (A,B--D): } 1 \text { (244); (A,B--E): } 2 \text { (235); (A,B--F): } 1 \text { (344); (C--E): } 4 \text { (209); (E-- } \\
\text { F): } 1 \text { (370); (E--I,J): } 1 \text { (58) }\end{array}$ \\
\hline Studies by comparison (min, $\max$ ) & 1,4 \\
\hline $\begin{array}{l}\text { Total sample by comparison (min, } \\
\text { max) }\end{array}$ & 58,370 \\
\hline Followup median (min, max) & $12(12,60)$ months \\
\hline
\end{tabular}


Figure 11. Evidence graph of RCTs comparing observer-assessed cosmetic outcomes (all BCC lesions)

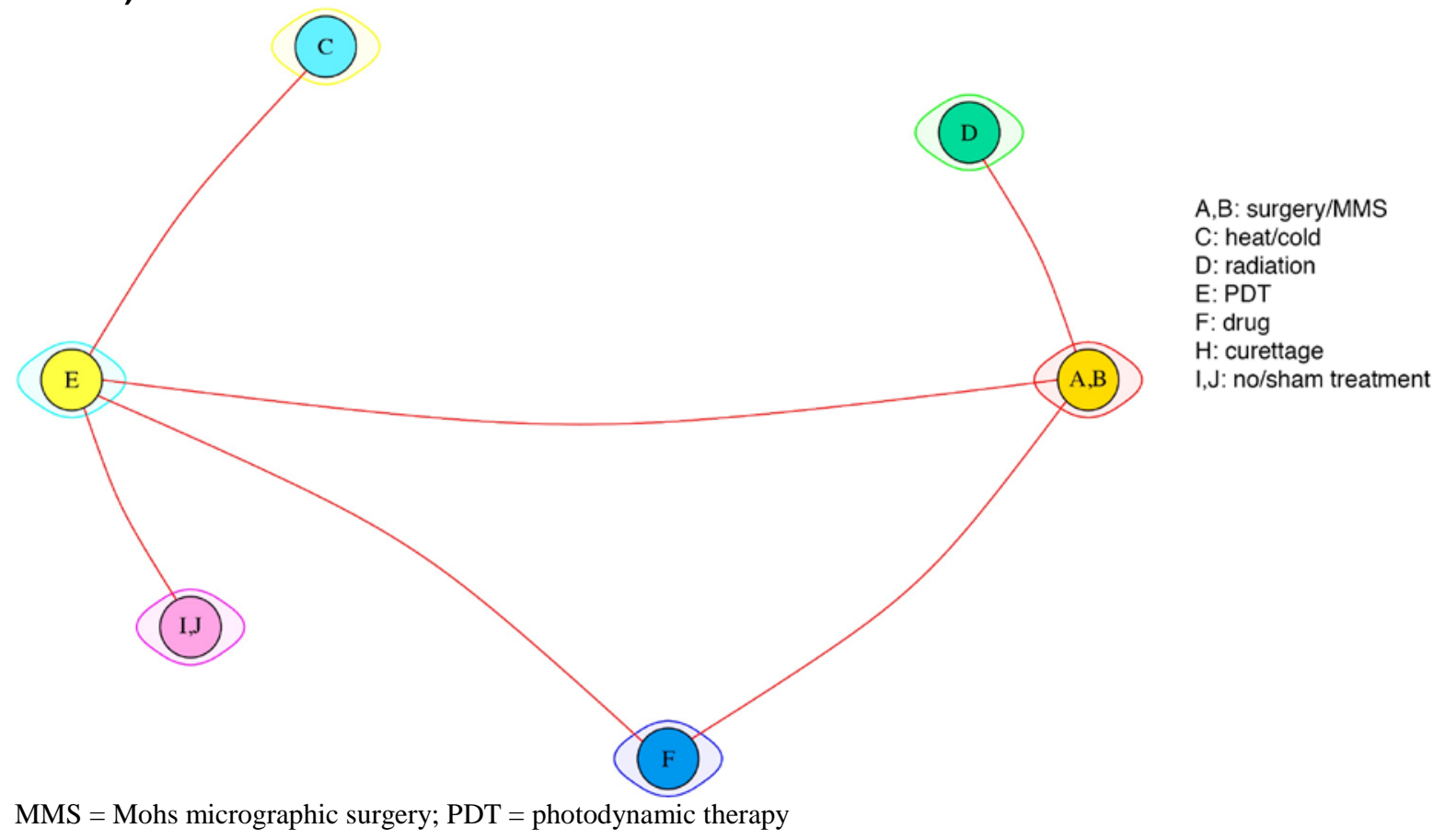

Table 53 has the results of the comparisons between intervention categories based on a network meta-analysis. Most comparisons are indirect (denoted by shaded cells) and have wide confidence intervals. For comparisons with head-to-head data (denoted by unshaded cells), the numbers in the table are very similar whether information from indirect comparisons is included or excluded. Overall, the results are compatible with the corresponding results for patient-rated cosmetic outcomes. Specifically, four out of 15 comparisons are statistically significant: For example, based only on indirect data, surgical interventions (A,B) are favored over radiation (D), and based on direct and indirect data, PDT $(\mathrm{E})$ is favored over surgical interventions $(\mathrm{A}, \mathrm{B})$.

Table 53. Relative odds ratios between intervention categories for at least good cosmetic outcome as assessed by an observer (all BCC lesions, Figure 11)

\begin{tabular}{|c|c|c|c|c|c|}
\hline $\begin{array}{c}\text { Surgery/MMS } \\
(\mathrm{A}, \mathrm{B})\end{array}$ & $0.42(0.12,1.47)$ & $\begin{array}{c}3.57(0.83 \\
15.36)\end{array}$ & $0.16(0.06,0.40)$ & $0.38(0.12,1.18)$ & $0.14(0.01,2.04)$ \\
\hline $\begin{array}{c}2.37(0.68 \\
8.25) \\
\end{array}$ & Heat/cold (C) & $8.45(1.42,50.4)$ & $0.37(0.13,1.06)$ & $0.90(0.22,3.64)$ & $0.33(0.02,5.11)$ \\
\hline $\begin{array}{c}0.28(0.07 \\
1.21) \\
\end{array}$ & $0.12(0.02,0.71)$ & Radiation (D) & $0.04(0.01,0.22)$ & $0.11(0.02,0.61)$ & $\begin{array}{c}0.04(<0.005 \\
0.76)\end{array}$ \\
\hline $\begin{array}{c}6.39(2.5, \\
16.35)\end{array}$ & $2.70(0.94,7.74)$ & $\begin{array}{c}22.81(4.56, \\
114.26) \\
\end{array}$ & PDT (E) & $2.43(0.81,7.33)$ & $0.89(0.06,12.23)$ \\
\hline $\begin{array}{c}2.63(0.85 \\
8.14)\end{array}$ & $1.11(0.27,4.48)$ & $\begin{array}{c}9.38(1.63 \\
54.02)\end{array}$ & $0.41(0.14,1.24)$ & Drugs (F) & $0.36(0.02,5.78)$ \\
\hline $\begin{array}{c}7.22(0.49 \\
106.52)\end{array}$ & $3.05(0.2,47.44)$ & $\begin{array}{c}25.76(1.31 \\
505.2)\end{array}$ & $\begin{array}{c}1.13(0.08, \\
15.59)\end{array}$ & $\begin{array}{c}2.75(0.17, \\
43.61)\end{array}$ & $\begin{array}{c}\text { No/sham } \\
\text { treatment } \\
(\mathrm{I}, \mathrm{J})\end{array}$ \\
\hline
\end{tabular}

Note: Cells shaded gray indicate that the estimate is based only on indirect comparisons; bold-italic indicates that the result is statistically significant. Results are given as odds ratios (95\% confidence intervals).

MMS = Mohs micrographic surgery PDT = photodynamic therapy; $\mathrm{BCC}=$ basal cell carcinoma 
Table 54 shows the average percentage of patients with at least good cosmetic outcomes in the RCTs, based on the same network meta-analysis as Table 53. The mean percentage of lesions with cosmetic outcome rated as good or excellent ranged between 74.3 and 89.8 percent for interventions that destroy the lesion with heat or cold (C), drugs (F), PDT (E) and no or sham treatment (I,J), and was 55.0 percent for surgical treatments (A,B). Radiation (D) had the smallest percentage of at least good cosmetic outcome. The confidence intervals for these proportions are wide. Refer to Table 53 for a pairwise comparison between these treatments.

Table 54. Mean fractions of lesions with at least good cosmetic outcome as assessed by an observer (all BCC lesions)

\begin{tabular}{lcc}
\hline Intervention Type & $\begin{array}{c}\text { Mean Fraction } \\
\text { Percent }(\mathbf{9 5 \%} \mathrm{Cl})\end{array}$ & $\begin{array}{c}\text { Forecasted Fraction } \\
\text { Percent }\end{array}$ \\
\hline Surgery/MMS $(\mathrm{A}, \mathrm{B})$ & $55.0(34.7,73.8)$ & $55.0(15.1,89.3)$ \\
\hline Heat/cold $(\mathrm{C})$ & $74.3(51.5,88.8)$ & $74.3(28.0,95.6)$ \\
\hline Radiation $(\mathrm{D})$ & $25.5(7.1,60.7)$ & $25.5(3.3,77.3)$ \\
\hline PDT $(\mathrm{E})$ & $88.7(78.9,94.2)$ & $88.7(54.2,98.1)$ \\
\hline Drugs $(\mathrm{F})$ & $76.3(52.8,90.2)$ & $76.3(29.6,96.1)$ \\
\hline No/sham treatment $(\mathrm{I}, \mathrm{J})$ & $89.8(40.1,99.1)$ & $89.8(28.3,99.5)$
\end{tabular}

MMS = Mohs micrographic surgery; PDT = photodynamic therapy; CI = confidence interval

\section{Evidence From NRCSs}

Three NRCS reported investigator-evaluated results for cosmetic outcomes. ${ }^{143,148,151}$

The first one compared surgical excision (A) and PDT with ALA (E2). It reported investigator-evaluated cosmetic outcomes in a matched population of 94 superficial (64\%) and nodular (36\%) BCCs in 74 patients at 12 months after treatment. The study was rated as having a moderate risk of bias due to lack of blinding and unclear reporting. The mean age was 66, with an age range of 49 to 90, 47 percent of the population was female. The group that received ALAPDT reported significantly better cosmetic results on a 4-level scale of poor to excellent (OR 10.2; $95 \%$ CI 4.0 to 26.1). ${ }^{148}$

A second NRCS reported whether an investigator saw pigmentation changes or alopecia in a small matched population of 40 patients treated with different doses of brachytherapy (36.6 versus $42 \mathrm{~Gy}$ ). The risk of bias of this study was determined to be moderate, primarily for lack of blinding and unclear reporting of baselines. The mean age was 75, 45 percent were female, and all had a Fitzpatrick skin score of I (47.5\%) or II (52.5\%). Forty-five percent of the BCCs were superficial, while 55 percent were nodular; 75 percent were on the head and neck and 25 percent on the trunk or extremities. The lower dose had one fewer patient with pigmentation changes or alopecia (OR 0.81, 95\% CI 0.23 to 2.86), but this difference was not significant. ${ }^{143}$

The third NRCS reported investigator-evaluated results for cosmetic outcomes to a median of 31.8 months after treatment, with two different doses and schedules of orthovoltage radiotherapy. The risk of bias was determined to be low with well-balanced arms, outcome assessors blinded, and full followup. The population consisted of 436 lesions in 385 elderly people, with BCCs (71\%) and SCCs (29\%). The mean age was 78, and 42 percent were female. A lower dose of radiation (37 Gy) had a slightly better cosmetic outcomes on a 4-level scale of poor to excellent than the higher dose (45 Gy), but this difference was not significant (RR: 1.048, $95 \%$ CI0.170 to 6.473$){ }^{151}$ 


\section{Quality of Life, All BCC Lesions}

One $\mathrm{RCT}^{65}$ and one NRCS ${ }^{142,144-147,156}$ reported eligible results. The former informs on the comparison between surgical excision (A) and MMS (B), and the latter on the comparison between excision (A), MMS (B), and electrodessication and curettage (C3).

\section{Evidence From RCTs}

The RCT reported on both quality of life and anxiety in a population of 408 primary BCCs (BCC) in 374 people, randomized to surgical excision (A; $n=204)$ or MMS (B; $n=204)$. The mean age of patients was 67.7. The majority of tumors were located in the H-zone (93\%) with the highest distribution in the frontal/temporal area (31\%). Approximately half of all lesions had an aggressive histological subtype (47\% BCC). Differences in tumor location or subtype were not significantly different between treatment groups. The Quality of life (emotional reactions, energy, pain, sleep, social isolation, and physical mobility) and level of anxiety of patients were measured at baseline and 6 months posttreatment, using the Nottingham Health Profile and the State-trait Anxiety Inventory, respectively. Both questionnaires were administered by a single researcher, and only patients with a single BCC were evaluated for these outcomes. At baseline and 6 months posttreatment, patients in both treatment groups showed good "health-related quality life" and a "minimum level of anxiety," with no observable statistically significant differences between the two groups for any measure. ${ }^{65}$

\section{Evidence From NRCSs}

The NRCS reported skin-specific quality of life in three domains: symptoms, emotion, and functioning in 1174 patients with 1488 lesions at two sites, a private, university-affiliated dermatology clinic (where majority of patients were recruited) and a nearby Veterans Affairs clinic. This study was deemed to have a low risk of bias, with balanced groups, consecutive recruitment, blinding of outcome assessors, and adequate accounting for people lost to followup. Most (75\%) of the lesions were BCCs; the other 25 percent were SCCs; 26 percent were female, 40 percent had a Fitzpatrick skin score of I or II, and 3 percent were immunocompromised due to prior solid-organ transplant. The lesions were treated by MMS (B; $n=246 ; 65 \%$ in H-zone of the face), surgical excision (A; $n=251 ; 26 \%$ in $H$-zone of the face), and electrodessication and curettage (ED\&C) (C3; $n=136 ; 11 \%$ in H-zone of the face). ${ }^{142,144-147}$

Table 55 shows the propensity-matched net differences between arms for improvement from baseline for each of the three reported Skindex domains (symptoms, emotions, and function) each measured on a scale from 0 (never bothered) to 100 (always bothered). The authors used a shortened, 16-item version of the Skindex (the current Skindex has 29-items), which they had previously validated in a similar population. ${ }^{157,158}$

The unadjusted results in a large population showed large and significant differences, primarily in favor of Mohs and surgical excision as compared to ED\&C, but no difference in improvement was observed comparing excision and Mohs surgery in any of the Skindex domains. ${ }^{145}$ However, these results are subject to residual confounding. The propensity-matched results include a smaller population, and thus, while they show potentially large differences, the differences cannot be distinguished from chance. ${ }^{142,144-147}$ 
Table 55. Quality of life measured with Skindex

\begin{tabular}{|c|c|c|c|c|c|c|}
\hline Outcome & Arm & N/arm & $\begin{array}{c}\text { Baseline } \\
\text { Score } \\
\text { Mean (SD) }\end{array}$ & Comparison & $\begin{array}{c}\text { Net Difference at } 2 \\
\text { Years }(95 \% \mathrm{Cl})\end{array}$ & $\begin{array}{l}\text { N Propensity- } \\
\text { Matched Pairs }\end{array}$ \\
\hline \multirow[t]{3}{*}{$\begin{array}{l}\text { QoL: Skindex } \\
\text { Symptoms }\end{array}$} & ED\&C & 136 & $19.6(23.6)$ & $\begin{array}{l}\text { excision vs } \\
\text { ED\&C }\end{array}$ & $-1.6(-9.8,6.7)$ & 51 \\
\hline & excision & 251 & $21.7(23.2)$ & $\begin{array}{l}\text { Mohs vs } \\
\text { ED\&C }\end{array}$ & $9.2(-2.1,20.5)$ & 24 \\
\hline & Mohs & 246 & $21.8(23.5)$ & $\begin{array}{l}\text { Mohs vs } \\
\text { excision }\end{array}$ & $4.0(-3.1,11.1)$ & 81 \\
\hline \multirow[t]{3}{*}{$\begin{array}{l}\text { QoL: Skindex } \\
\text { Emotions }\end{array}$} & ED\&C & 136 & $33.0(28.0)$ & $\begin{array}{l}\text { excision vs } \\
\text { ED\&C }\end{array}$ & $13.2(3.3,23.1)$ & 51 \\
\hline & excision & 251 & $38.9(30.4)$ & $\begin{array}{l}\text { Mohs vs } \\
\text { ED\&C }\end{array}$ & $23.6(10.1,37.2)$ & 24 \\
\hline & Mohs & 246 & $46.3(27.0)$ & $\begin{array}{l}\text { Mohs vs } \\
\text { excision }\end{array}$ & $3.4(-3.8,10.7)$ & 81 \\
\hline \multirow[t]{3}{*}{$\begin{array}{l}\text { QoL: Skindex } \\
\text { Functioning }\end{array}$} & ED\&C & 136 & $12.1(21.7)$ & $\begin{array}{l}\text { excision vs } \\
\text { ED\&C }\end{array}$ & $3.1(-3.5,9.8)$ & 51 \\
\hline & excision & 251 & $15.1(24.6)$ & $\begin{array}{l}\text { Mohs vs } \\
\text { ED\&C }\end{array}$ & $3.7(-4.6,12.0)$ & 24 \\
\hline & Mohs & 246 & $14.0(21.1)$ & $\begin{array}{l}\text { Mohs vs } \\
\text { excision }\end{array}$ & $4.2(-2.3,10.8)$ & 81 \\
\hline
\end{tabular}

ED\&C = electrodessication and curettage; QoL = Quality of Life; CI = confidence interval; SD = standard deviation

\section{Mental Health, All BCC Lesions}

A single RCT reported information on anxiety measured with the State-Trait Anxiety Inventory at 6 months, for a population of 408 primary BCCs (BCC) in 374 people randomized to surgical excision (A; $n=204$ ) or MMS (B; $n=204)$. No statistically significant differences were found between the comparators. This RCT is summarized in some more detail in the Quality of Life section, under Evidence from RCTs. ${ }^{65}$

\section{Patient Satisfaction, All BCC Lesions}

We did not identify eligible RCTs with results for this outcome.

\section{Mortality, All BCC Lesions}

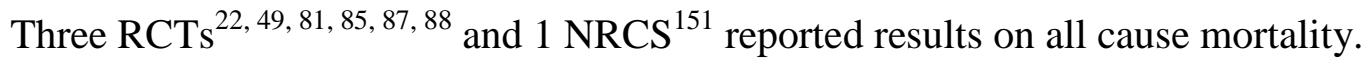

\section{Evidence From RCTs}

The first RCT reported mortality between 1 and 3 years in 501 people with 1 superficial BCC lesion each for the comparison of PDT with MAL (E1), 5-FU (F1), and imiquimod (F2). The risk of bias for this study was low, with randomization and allocation concealment adequately reported, blinding of outcome assessors, high similarity of groups at baseline, and low loss to followup. The median age was 63 (range 26 to 91), 49 percent were women, and most lesions were on the trunk (60\%), extremities (27\%), and face excluding the H-zone (13\%). All-cause mortality was recorded for 5 of 196 (2.6\%) patients in the PDT with MAL (E1) arm, 2 of 198 $(1.0 \%)$ in the 5-FU arm (F1), and 4 of 189 (2.1\%) in the imiquimod arm (F2). ${ }^{49,87,88}$

The second RCT compared surgical excision (A) $(n=49)$ to PDT with MAL (E1) (n=52). The average age was 68 (range 38 to 95), and 40 percent were female. Most (88\%) had Fitzpatrick skin types II (46.5\%) and III (41.5\%). The risk of bias for this study was judged to be relatively 
high because the groups were not similar at baseline, there was no blinding, and there was a high loss to followup after a year. Mortality at 1 and 2 years was not statistically significantly different in the excision (2/46, 4.3\%) and MAL-PDT groups $(2 / 50,4.0 \%){ }^{22,85}$

The third RCT compared surgical excision (A) without intraoperative evaluation of the excised margins $(n=199)$ versus MMS (B) $(n=198)$. It reported results for long-term mortality in people with unspecified BCCs on the face, about half of which were classified as an "aggressive histological subtype" between 18 months and 5 years. The average age was 68 (SD 12), and 39.7 percent were female. The average lesion size was $1.28 \mathrm{~cm}^{2}$ (SD 1.36) in the MMS arm and 1.77 $\mathrm{cm}^{2}$ (SD 1.28) in the surgical excision arm. The risk of bias was judged to be moderate to high because of lack of baseline details given, lack of blinding, and high loss to followup. Thirty-six (18\%) died in the MMS arm as compared to 34 (17\%) in the excision arm. None of the deaths were deemed to be related to the tumor or the treatment. ${ }^{81}$

\section{Evidence From NRCSs}

One NRCS reported results for long-term mortality, from 12 to a median of 31.8 months after treatment with two doses of external radiation (orthovoltage range) therapy. It was deemed that there was low risk of confounding or measurement bias based on the fact that arms were wellbalanced, outcome assessors were blinded, and no patients were lost to followup. The population consisted of 436 lesions in 385 elderly people, with BCCs (71\%) and SCCs (29\%). The mean age was 78, and 42 percent were female. The 45 Gy dose of radiation had a lower mortality (16.1\%) than the $37 \mathrm{~Gy}$ dose group (30.5\%), but the mean age in the lower dose group was significantly higher (81.3 vs. 73.3 years). Once adjusted for age, number of lesions per patient, histology, severity, and lesion site, the difference in mortality was not significant (Adjusted HR: 0.662 ; $95 \%$ CI 0.387 to 1.131$){ }^{151}$

\section{Costs and Resource Use, All BCC Lesions}

No RCTs informed on U.S. costs or on use of resources.

One NRCS reported cost and resource use outcomes in patients. ${ }^{155}$ It compared surgical excision (A), MMS (B), and electrodessication and curettage (C3). Among the 936 examined lesions, 80 percent $(n=748)$ were BCC and 20 percent $(n=188)$ were SCCs. The risk of confounding bias of this study was determined to be low with differences at baseline controlled for in multivariate analysis, and no loss to followup. Females accounted for 59.4 percent of the population. Overall, 60.1 percent $(n=563)$ of tumors in the study sample presented on the head and neck. Of these, the majority (56.3\%) was treated by MMS; the majority (69.3\%) of tumors presenting on the trunk and extremities were treated with electrodessication and curettage (ED\&C). Similarly, 31.5\% (295) of tumors presented in the H-zone, with the majority (80\%) of these treated with MMS, compared to a majority (36.8\% and 36.2\%) of tumors not in the H-zone treated with ED\&C and surgical excision, respectively. Differences in histology of the tumors and tumor diameter across treatment types were not observed to be statistically significant.

In both adjusted and unadjusted analyses of total surgical care, there was a statistically significant difference $(\mathrm{p}<0.001)$ in costs by treatment type. MMS treatments were observed to have the highest primary procedure and follow-up visit costs compared to excision (by, on average, \$857 in adjusted analyses). Excision had the second highest costs for both primary procedure and follow-up visit, and ED\&C had the lowest. Also, in both adjusted and unadjusted analyses, total fees for all surgical care were significantly higher for large tumors $(>10 \mathrm{~mm})$ and 
for H-zone locations. Independent predictors of higher total costs were determined using multivariate regression log models and included presentation of tumor at the head or neck, greater than $10 \mathrm{~mm}$ lesion diameter, and repair with flap or graft. However, the study did not take fees related to recurrence into account. ${ }^{155}$

\section{Adverse Events, All BCC Lesions}

In this section we describe only results between intervention categories, because data are sparse for the comparison of individual observations. Figure 12 shows the evidence graph for the comparison of the frequency of adverse events leading to discontinuation, serious adverse events, pain after treatment completion, and infection of the treated site. Reporting of adverse events was not consistent across RCTs. Appendix I enumerates other types of adverse events that were reported.

Figure 12. Evidence graph of RCTs comparing frequency of adverse events (all BCC lesions)

(A) Leading to treatment discontinuation

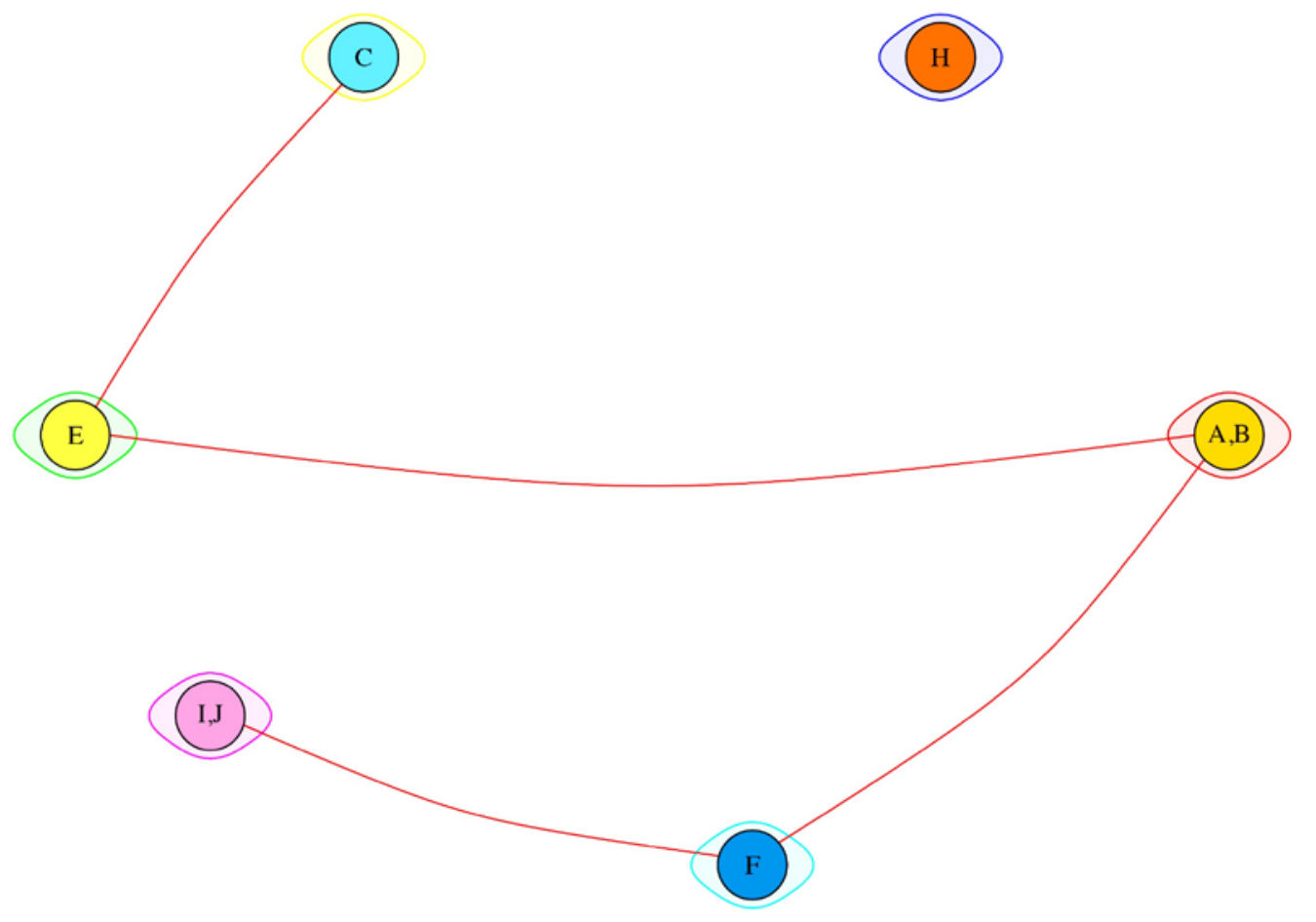


(B) Serious adverse events 
(C) Pain (after treatment completion)

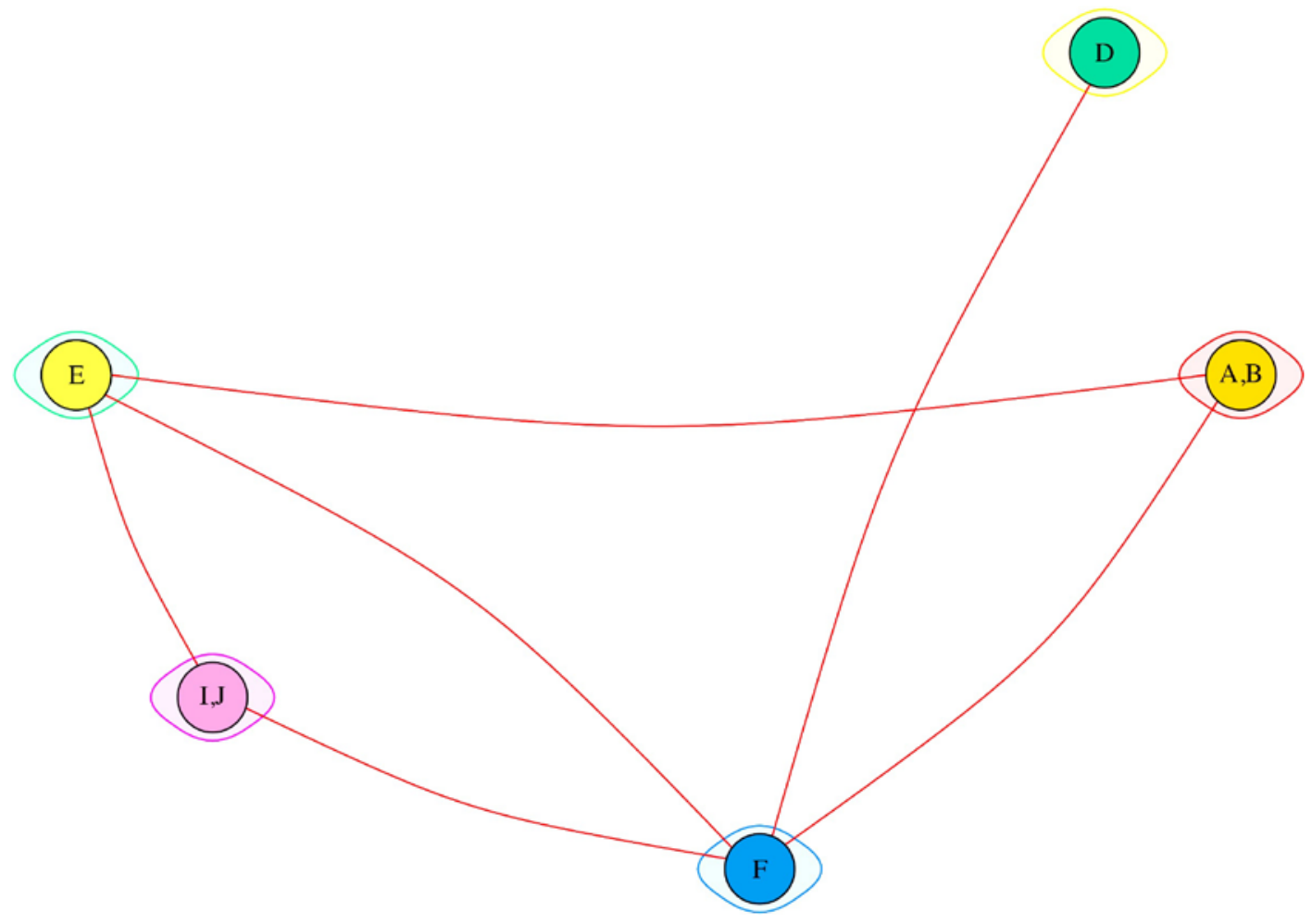

A,B: surgery/MMS

C: heat/cold

$\mathrm{D}$ : radiation

E: PDT

F: drug

$\mathrm{H}$ : curettage

$\mathrm{I}, \mathrm{J}:$ no/sham treatment

\section{(D) Infection of the treated site}

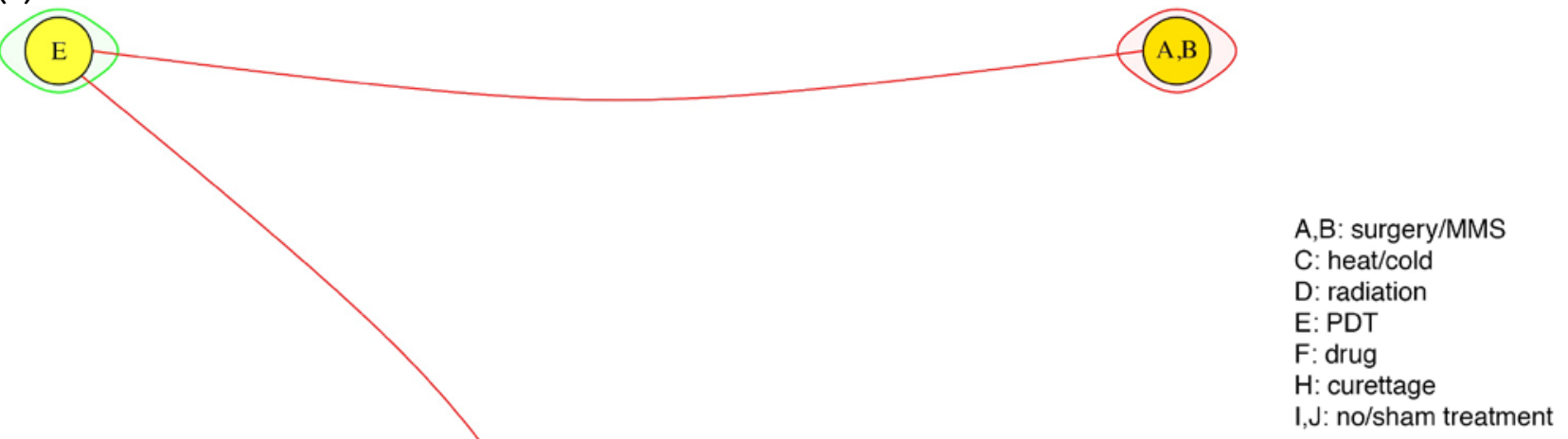

MMS = Mohs micrographic surgery; PDT = photodynamic therapy; $\mathrm{BCC}=$ basal cell carcinoma 
The evidence graphs in Figure 12 are sparsely connected. For parsimony, we do not report relative effects for comparisons of the frequency of each type of adverse event. Table 56 has details about the comparisons by type of adverse event.

Table 56. Sample information, adverse events (all BCC lesions, intervention categories)

\begin{tabular}{|c|c|c|c|c|}
\hline & $\begin{array}{l}\text { Adverse events leading to } \\
\text { treatment discontinuation }{ }^{51} \\
52,55,68,85,92,94\end{array}$ & $\begin{array}{l}\text { Serious adverse events }{ }^{20} \\
49,52,55,58,94,98\end{array}$ & $\begin{array}{l}\text { Pain after } \\
\text { treatment }^{49,} 52,54, \\
55,66,67,69,85,93,94, \\
98\end{array}$ & $\begin{array}{l}\text { Infection of treated } \\
\text { site }^{49,85,98}\end{array}$ \\
\hline $\begin{array}{l}\text { Studies (total } \\
\text { sample) }\end{array}$ & 7 (1733) & 7 (1395) & $12(1612)$ & $3(682)$ \\
\hline $\begin{array}{l}\text { Total sample by } \\
\text { intervention }\end{array}$ & $\begin{array}{l}(A, B): 287 ;(E): 120 ;(F): \\
782 ;(I, J): 486 ;(C): 58\end{array}$ & $\begin{array}{l}\text { (A,B): 413; (E): 397; (F): } \\
\text { 523; (I,J): 44; (C): } 18\end{array}$ & $\begin{array}{l}\text { (D): 12; (F): 705; } \\
(\mathrm{I}, \mathrm{J}): 176 ;(\mathrm{E}): 340 ; \\
(\mathrm{A}, \mathrm{B}): 351\end{array}$ & $\begin{array}{l}(E): 348 ;(F): 189 ; \\
(A, B): 145\end{array}$ \\
\hline $\begin{array}{l}\text { Total sample by } \\
\text { intervention, (min, } \\
\text { max) }\end{array}$ & 58,782 & 18,523 & 12,705 & 145,348 \\
\hline $\begin{array}{l}\text { Data by } \\
\text { comparison }\end{array}$ & $\begin{array}{l}\text { (A,B--E): } 1 \text { (118); (A,B--F): } \\
1 \text { (483); (E--C): } 1 \text { (118); (F-- } \\
\text { I,J): } 4 \text { (1014) }\end{array}$ & $\begin{array}{l}\text { (A,B--E): } 2(369) ;(A, B--F): \\
1(483) ;(E--F): 1 \text { (385); (E-- } \\
\text { C): } 1 \text { (34); (F--I,J): } 2 \text { (124) }\end{array}$ & $\begin{array}{l}\text { (D--F): } 1 \text { (27); (F-- } \\
\text { I,J): } 5 \text { (379); (F-- } \\
\text { E): } 1 \text { (339); (F-- } \\
\text { A,B): } 1 \text { (439); (I,J-- } \\
\text { E): } 1 \text { (131); (E--- } \\
\text { A,B): } 2 \text { (297) }\end{array}$ & $\begin{array}{l}\text { (E--F): } 1(385) ;(E-- \\
A, B): 2 \text { (297) }\end{array}$ \\
\hline $\begin{array}{l}\text { Studies by } \\
\text { comparison (min, } \\
\max \text { ) }\end{array}$ & 1,4 & 1,2 & 1,5 & 1,2 \\
\hline $\begin{array}{l}\text { Total sample by } \\
\text { comparison (min, } \\
\text { max) }\end{array}$ & 118,1014 & 34,483 & 27,439 & 297,385 \\
\hline $\begin{array}{l}\text { Followup median } \\
\text { (min, max) }\end{array}$ & [during treatment] & $12(1,60)$ months & $3(0.5,12)$ months & $3(1,12)$ months \\
\hline
\end{tabular}

We report mean fractions of adverse events per intervention category, based on a joint analysis of all RCTs reporting the same outcome. Most likely, adverse events were defined differently across studies, but these definitions were often not clearly described. Results for adverse events, as defined by each study, are in Table 57 and come from different analyses.

Drugs had the highest frequency of adverse events leading to treatment discontinuation was (4.9\%; 95\% CI, 2.0 to 20.1); for other interventions, it was less than 1.2 percent. Surgical interventions and PDT are one-time procedures and cannot be "discontinued"; for parsimony of exposition, however, in the descriptive analyses in Table 57 we assigned 0 discontinuation events to these interventions.

The frequency of adverse events characterized as "serious" by the investigators was smaller than 3.6 percent for all intervention categories.

Pain after treatment was most commonly encountered for surgical interventions $(21.5 \%)$ and for PDT (20.7\%), and was least common with sham treatments (2.9\%).

Infections at the treatment site were described in 5.5 percent of lesions with surgical treatments (95\% CI 28 to 10.7), and were reported in less than 1 percent for PDT (E) and drugs 
(F). No information on infections was available for treatments that destroy lesions with heat or cold (C) or for no (or sham) treatment.

Table 57. Mean fractions of adverse events, using each RCT's definitions (all BCC lesions)

\begin{tabular}{|c|c|c|c|c|c|c|}
\hline \multirow[t]{2}{*}{$\begin{array}{l}\text { Intervention } \\
\text { Type }\end{array}$} & \multicolumn{2}{|c|}{$\begin{array}{c}\text { Leading to } \\
\text { Discontinuation (Figure } \\
12 \mathrm{~A})\end{array}$} & \multirow{2}{*}{$\begin{array}{c}\begin{array}{c}\text { Serious* } \\
\text { (Figure 12B) }\end{array} \\
\text { Mean }\end{array}$} & \multicolumn{2}{|c|}{$\begin{array}{l}\text { Pain After Treatment } \\
\text { (Figure 12C) }\end{array}$} & \multirow{2}{*}{$\begin{array}{c}\text { Infection of } \\
\text { the Treated } \\
\text { Site* (Figure } \\
\text { 12D) } \\
\text { Mean } \\
\end{array}$} \\
\hline & Mean & Forecast & & Mean & Forecast & \\
\hline $\begin{array}{l}\text { Surgery/MMS } \\
(\mathrm{A}, \mathrm{B})\end{array}$ & $\begin{array}{c}\text { Not } \\
\text { defined }^{\star *}\end{array}$ & $\begin{array}{c}\text { Not } \\
\text { defined }^{\star *}\end{array}$ & $0.6(0.1,2.7)$ & $\begin{array}{c}21.5(8.1 \\
46.2) \\
\end{array}$ & $\begin{array}{c}21.5(1.7 \\
81.5) \\
\end{array}$ & $5.5(2.8,10.7)$ \\
\hline Heat/cold (C) & $\begin{array}{c}0.9(0.0 \\
20.1)\end{array}$ & $\begin{array}{c}0.9(0.0 \\
29.0)\end{array}$ & $2.6(0.1,36.7)$ & $\begin{array}{c}12.9(0.8 \\
73.1)\end{array}$ & $\begin{array}{c}12.9(0.3 \\
87.5)\end{array}$ & NA \\
\hline PDT (E) & $\begin{array}{c}\text { Not } \\
\text { defined }^{\star \star}\end{array}$ & $\begin{array}{c}\text { Not } \\
\text { defined }^{\star \star}\end{array}$ & $0.7(0.1,3.0)$ & $\begin{array}{c}20.7(8.2 \\
43.3)\end{array}$ & $\begin{array}{c}20.7(1.6 \\
80.3)\end{array}$ & $0.5(0.1,2.4)$ \\
\hline Drugs (F) & $\begin{array}{c}4.9(2.0 \\
11.6)\end{array}$ & $\begin{array}{c}4.9(0.6 \\
29.2)\end{array}$ & $2.2(0.8,6.4)$ & $\begin{array}{c}9.9(4.4 \\
20.9)\end{array}$ & $9.9(0.7,61.6)$ & $0.5(0.1,3.7)$ \\
\hline $\begin{array}{l}\text { No/sham } \\
\text { treatment }(\mathrm{I}, \mathrm{J})\end{array}$ & $\begin{array}{c}1.0(0.2 \\
4.4)\end{array}$ & $1.0(0.1,9.8)$ & $2.4(0.3,17.8)$ & $\begin{array}{c}2.9(0.9 \\
9.4)\end{array}$ & $2.9(0.2,33.5)$ & NA \\
\hline
\end{tabular}

Note: Results are given as percent and 95\% confidence interval.

MMM = Mohs micrographic surgery; PDT = photodynamic therapy; RCT = randomized controlled trial; $\mathrm{BCC}=$ basal cell carcinoma

* No forecasts for these outcomes (fixed effects analyses only); NA: not applicable.

** Surgical interventions and PDT are one-time procedures and cannot be "discontinued”; for parsimony of exposition, however, in the descriptive analyses in the Table we assigned 0 discontinuation events to these interventions.

\section{Evidence From NRCSs}

Results on the frequency of adverse events are reported in three NRCSs. ${ }^{151,153,154}$

The first NRCS reported on adverse events in 12 patients with 1 superficial BCC each. The mean lesion area was $52 \mathrm{~mm}^{2}$, and the lesions were located on the trunk or neck $(67 \%)$ or forearm (33\%). This study was deemed to have high risk of confounding bias, because of baseline imbalance. The mean age was 61 (range 52 to 78), and 33\% were female. Six lesions were treated with imiquimod (F2) and six with a vehicle (J). More people in the vehicle arm (3 of 6) reported application site adverse events than in the imiquimod arm (2 of 6) during treatment, both erythemas. ${ }^{154}$

The second NRCS reported on adverse events in 74 patients with 1 nodular BCC each, receiving different doses of vismodegib (F5, other drug). It was deemed that this study was at moderate risk of confounding bias; it was not blinded, and it was not possible to assess for baseline (im)balance, because pertinent information was not reported. The lesion diameter ranged from 10 to $30 \mathrm{~mm}$, and all were located in the scalp, head, neck, trunk or limbs. The mean age was 63.6 (SD 12; range 40 to 89), and 22 percent were female; 99 percent were white. Twenty-four lesions were treated with vismodegib for 12 weeks then were excised; twenty-five were treated with vismodegib for 12 weeks then had a 24-week observation period before excision; and 25 were treated with vismodegib for 16 weeks then were excised. Just about everyone (99\%) reported at least one adverse event, including muscle spasms (76\%), alopecia (58\%), and changes in tasting, namely dysgeusia (50\%) and ageusia (30\%). ${ }^{153}$

The third NRCS reported results for any adverse events, from 12 to a median of 31.8 months after treatment with two doses of (orthovoltage) radiation therapy (D1). The risk of bias was 
determined to be low; arms were well-balanced at baseline, outcome assessors were blinded, and no patients were lost to followup. The lower-dose group (36 Gy) had fewer adverse events (5.9\% as compared to $4.0 \%$ in the 45 Gy group), but no adjusted analysis was available for this outcome. ${ }^{151}$

\section{Dose Response Analyses for Drugs, All BCC Lesions}

Table 58 summarizes analyses from phase II or phase II/III trials on different doses or application schedules for drugs (F), stratified by whether the patients had superficial, nodular, or a mix of superficial and nodular BCC lesions in 16 studies. $^{48,54,61-63,68,69,74,76,82,93,94,97,100,101}$

Results cannot be combined across these studies in a straightforwardly interpretable way. Overall, the general pattern was that, with increasing intensity of treatment (higher doses or more applications) there was an apparent increase in the frequency of adverse events; but it is not always clearly reported whether this was statistically significant or not.

\section{Special Populations}

No studies reported comparative results in special populations of interest, specifically patients at the end of life or immunocompromised patients. 
Table 58. Summary of phase II or II/III trials comparing different doses or intensities of application schedules for drugs (all BCC lesions)

\begin{tabular}{|c|c|c|c|c|c|c|c|c|}
\hline $\begin{array}{l}\text { PMID } \\
\text { Author }\end{array}$ & $\operatorname{Arm1}(\mathrm{n})$ & Arm2 (n) & $\begin{array}{l}\text { Arm3 } \\
\text { (n) }\end{array}$ & Arm4 (n) & Arm5 (n) & Arm6 (n) & $\operatorname{Arm7}(\mathrm{n})$ & Authors' Conclusion \\
\hline \multicolumn{9}{|l|}{$\begin{array}{l}\text { Superficial } \\
\text { Lesions }\end{array}$} \\
\hline $\begin{array}{l}12196749 \\
\text { Geisse }\end{array}$ & $\begin{array}{l}\text { Vehicle } \\
(32)\end{array}$ & $\begin{array}{l}\text { Imiquimod } \\
5 \% 3 x / w k \\
(29)\end{array}$ & $\begin{array}{l}\text { Imiquim } \\
\text { od } 5 \% \\
5 x / w k \\
(26)\end{array}$ & $\begin{array}{l}\text { Imiquimo } \\
\text { d } 5 \% \\
1 x / \text { day } \\
(31)\end{array}$ & $\begin{array}{l}\text { Imiquimo } \\
\text { d 5\% } \\
2 x / \text { day } \\
(10)\end{array}$ & & & $\begin{array}{l}\text { "There was a positive association between dosing frequency and } \\
\text { complete response rate; higher response rates were associated } \\
\text { with more frequent dosing...An acceptable safety profile was seen } \\
\text { in } 3 \text { of the } 4 \text { imiquimod dosing regimens. Only the most frequent } \\
\text { dosing regimen, twice daily for } 12 \text { weeks, presented a safety profile } \\
\text { that was judged not acceptable because of severe local skin } \\
\text { reactions at the treatment site." }\end{array}$ \\
\hline $\begin{array}{l}15097956 \\
\text { Geisse }\end{array}$ & $\begin{array}{l}\text { Vehicle } \\
5 x / w k \\
(175)\end{array}$ & $\begin{array}{l}\text { Vehicle } \\
7 \times / \text { wk (171) }\end{array}$ & $\begin{array}{l}\text { Imiquim } \\
\text { od } 5 \% \\
5 \times / w k \\
(178)\end{array}$ & $\begin{array}{l}\text { Imiquimo } \\
\text { d } 5 \% \\
7 x / w k \\
(170)\end{array}$ & & & & $\begin{array}{l}\text { "The results from these Phase III studies confirm that imiquimod } \\
\text { has higher complete clearance rates than vehicle cream for each of } \\
\text { the active treatment groups. Additionally, there was not a } \\
\text { statistically significant or clinically meaningful difference in } \\
\text { complete clearance rate noted between the imiquimod 5/week and } \\
7 / \text { week (73\% composite and } 79 \% \text { histologic) treatment groups."69 }\end{array}$ \\
\hline $\begin{array}{l}11312429 \\
\text { Marks }\end{array}$ & $\begin{array}{l}\text { Imiquimod } \\
5 \% 1 x / \text { day } \\
\text { (33) }\end{array}$ & $\begin{array}{l}\text { Imiquimod } \\
5 \% 2 x / \text { day } \\
\text { (3) }\end{array}$ & $\begin{array}{l}\text { Imiquim } \\
\text { od } 5 \% \\
1 \times / \text { day } \\
3 x / \text { wk } \\
(33)\end{array}$ & $\begin{array}{l}\text { Imiquimo } \\
\text { d } 5 \% \\
2 x / \text { day } \\
3 x / \text { wk } \\
(30)\end{array}$ & & & & $\begin{array}{l}\text { "There was a dose-response } \\
\text { gradient varying from } 3 \text { of } 3(100 \%) \text { in the twice-every-day regimen } \\
\text { group to } 23 / 33(69.7 \%) \text { in the once-daily } 3 \text { times/week regimen } \\
\text { group...This study confirms previous work suggesting that } \\
\text { imiquimod } 5 \% \text { cream is likely to be of value in the treatment of } \\
\text { sBCC."." }\end{array}$ \\
\hline $\begin{array}{l}20546215 \\
\text { Siller }\end{array}$ & $\begin{array}{l}\text { Vehicle } \\
(12)\end{array}$ & $\begin{array}{l}\text { Ingenol } \\
\text { mebutate } \\
0.0025 \% \\
\text { Days } 1 \text { and } \\
2(8)\end{array}$ & $\begin{array}{l}\text { Ingenol } \\
\text { mebutat } \\
\text { e } 0.01 \% \\
\text { Days } 1 \\
\text { and } 2 \\
\text { (8) }\end{array}$ & $\begin{array}{l}\text { Ingenol } \\
\text { mebutate } \\
0.05 \% \\
\text { Days } 1 \\
\text { and } 2 \text { (8) }\end{array}$ & $\begin{array}{l}\text { Ingenol } \\
\text { mebutate } \\
0.0025 \% \\
\text { Days } 1 \\
\text { and } 8 \text { (8) }\end{array}$ & $\begin{array}{l}\text { Ingenol } \\
\text { mebutate } \\
0.01 \% \\
\text { Days } 1 \\
\text { and } 8 \text { (8) }\end{array}$ & $\begin{array}{l}\text { Ingenol } \\
\text { mebutate } \\
0.05 \% \text { Days } \\
1 \text { and } 8 \text { (8) }\end{array}$ & $\begin{array}{l}\text { The study was not powered to detect differences in treatment } \\
\text { concentration and schedule, but the clinical and histological } \\
\text { response was more common in } 0.05 \% 1 \& 2 \text { day application } \\
\text { compared to other doses or } 0.05 \% 1 \& 8 \text { day application. }{ }^{94}\end{array}$ \\
\hline $\begin{array}{l}12452875 \\
\text { Sterry }\end{array}$ & $\begin{array}{l}\text { Imiquimod } \\
5 \% 2 x / \text { wk } \\
\text { without } \\
\text { occlusion } \\
(24)\end{array}$ & $\begin{array}{l}\text { Imiquimod } \\
5 \% 2 x / \text { wk } \\
\text { with } \\
\text { occlusion } \\
(21)\end{array}$ & $\begin{array}{l}\text { Imiquim } \\
\text { od } 5 \% \\
3 \times / \text { wh } \\
\text { without } \\
\text { occlusio } \\
\mathrm{n}\end{array}$ & $\begin{array}{l}\text { Imiquimo } \\
\text { d } 5 \% \\
3 x / \text { wk } \\
\text { with } \\
\text { occlusion }\end{array}$ & & & & $\begin{array}{l}\text { "The complete response rate increased as dosing frequency } \\
\text { increased, both with and without occlusion. However, the only } \\
\text { statistically significant difference in response rate was seen when } \\
\text { comparing the } 2 \text { days per week with occlusion and } 3 \text { days per } \\
\text { week with occlusion groups }(P=0.004) . " \text { ". }\end{array}$ \\
\hline \multicolumn{9}{|l|}{$\begin{array}{l}\text { Nodular } \\
\text { lesions }\end{array}$} \\
\hline $\begin{array}{l}17610993 \\
\text { Eigentler }\end{array}$ & $\begin{array}{l}\text { Imiquimod } \\
5 \% 3 x / \text { wk } \\
\text { for } 8 \\
\text { weeks (45) }\end{array}$ & $\begin{array}{l}\text { Imiquimod } \\
5 \% 3 / \text { wk for } \\
12 \text { weeks } \\
\text { (45) }\end{array}$ & & & & & & $\begin{array}{l}\text { "There were no significant differences between the treatment arms } \\
\text { with respect to efficacy and tolerability." } 63\end{array}$ \\
\hline $\begin{array}{l}1430394 \\
\text { Orenberg }\end{array}$ & $\begin{array}{l}5-F U 7.5 \\
\mathrm{mg}\end{array}$ & $5-\mathrm{FU} 15 \mathrm{mg}$ & & & & & & $\begin{array}{l}\text { "Application of Fisher's exact test showed no differences in } \\
\text { response between the treatment groups." } 82\end{array}$ \\
\hline 12224977 & Vehicle & Imiquimod & Imiquim & Imiquimo & Imiquimo & & & "An increase in the complete response rate was seen with \\
\hline
\end{tabular}




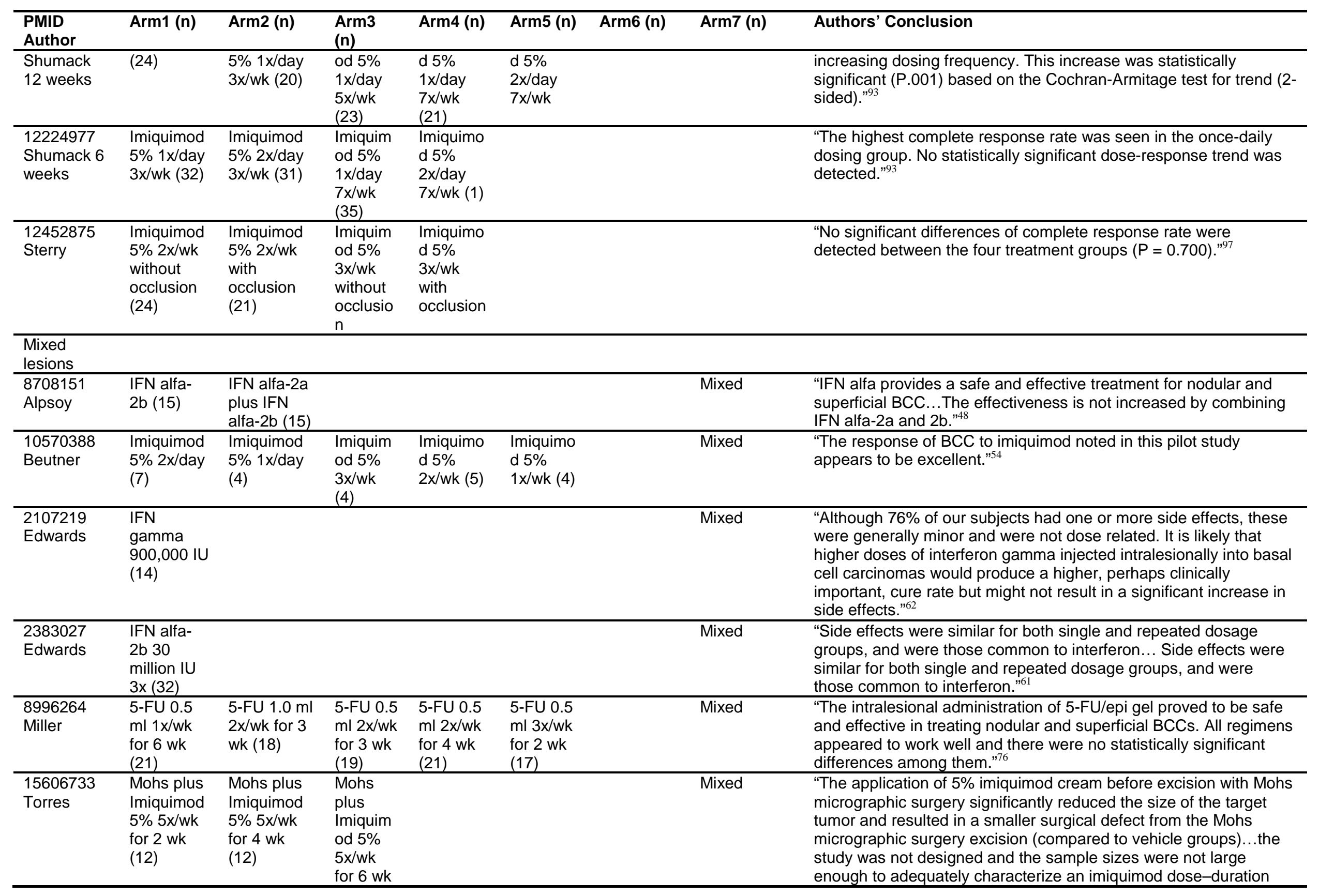




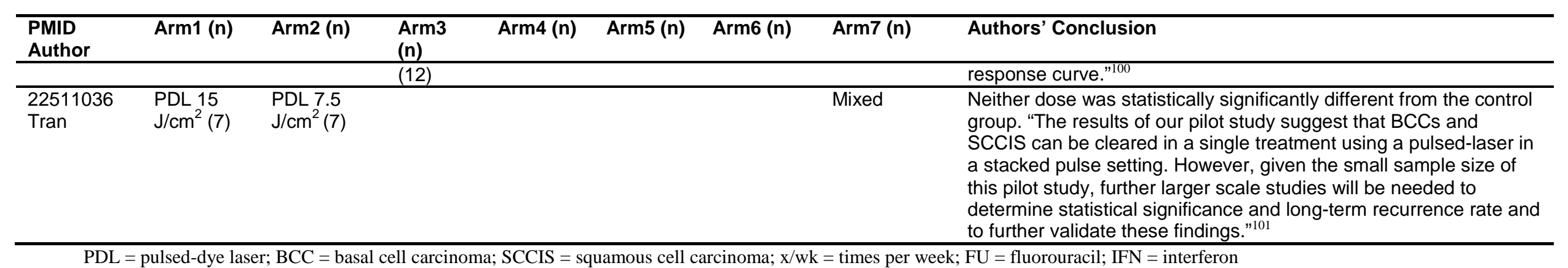

PDL = pulsed-dye laser; BCC = basal cell carcinoma; SCCIS = squamous cell carcinoma; x/wk = times per week; FU = fluorouracil; IFN = interferon 


\section{Squamous Cell Carcinoma}

The evidence graph in Figures 13 and 14 depict eight comparisons between 10 interventions organized in four intervention categories. Comparisons between individual interventions are sparse, suggesting that limited, if any, conclusions can be drawn about which individual treatment is best for each outcome. Figure 13 has two connected subgraphs. The smallest one compares a laser-based preparation of the lesion for PDT treatment (C5+E2) versus PDT alone (E2), and the other comprises all other treatments. Information on each comparison is provided by at most three RCTs, and for most comparisons, by a single RCT.

The evidence is sparser when one considers the information that is actually available for specific outcomes. Figure 15 shows the corresponding evidence graphs for the outcomes for which we have the most data, namely recurrence, lack of histologic clearance, and lack of clinical clearance. RCT data exists for only 7, 4, and 8 of the 28 interventions, respectively. Evidence on other outcomes (quality of life, cosmetic outcomes, costs or resource use) is even sparser.

We identified one NRCS comparing curettage $(\mathrm{H})$ versus cryotherapy $(\mathrm{C} 1)$ in patients with SCC lesions. This study is described separately. ${ }^{141}$

Figure 13. Evidence graph depicting compared treatments in RCTs of SCC lesions

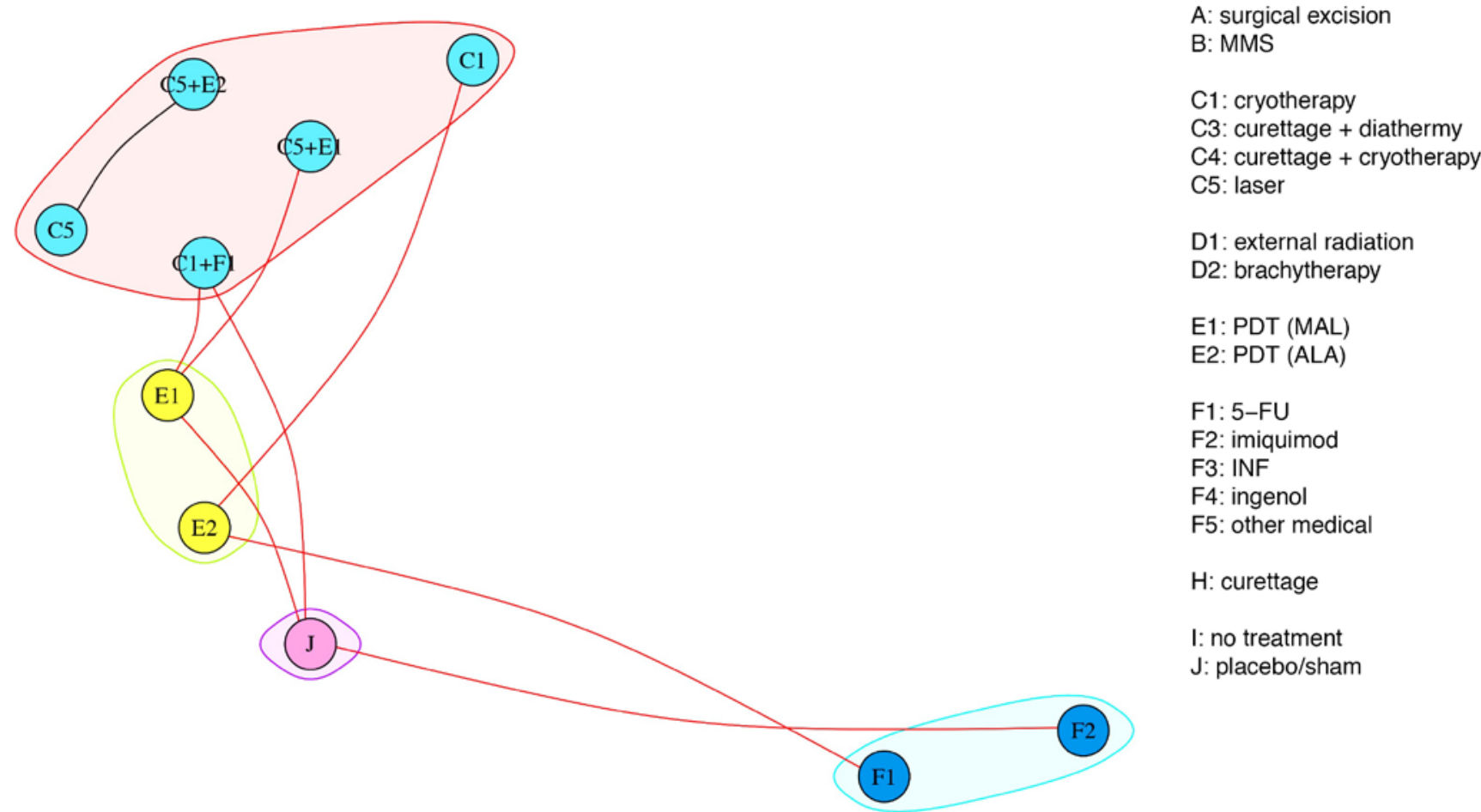

MMS = Mohs micrographic surgery; PDT = photodynamic therapy; ALA = 5-aminolevulinic acid; MAL = methyl aminolevulinate; $\mathrm{FU}=$ fluorouracil; INF = interferon 


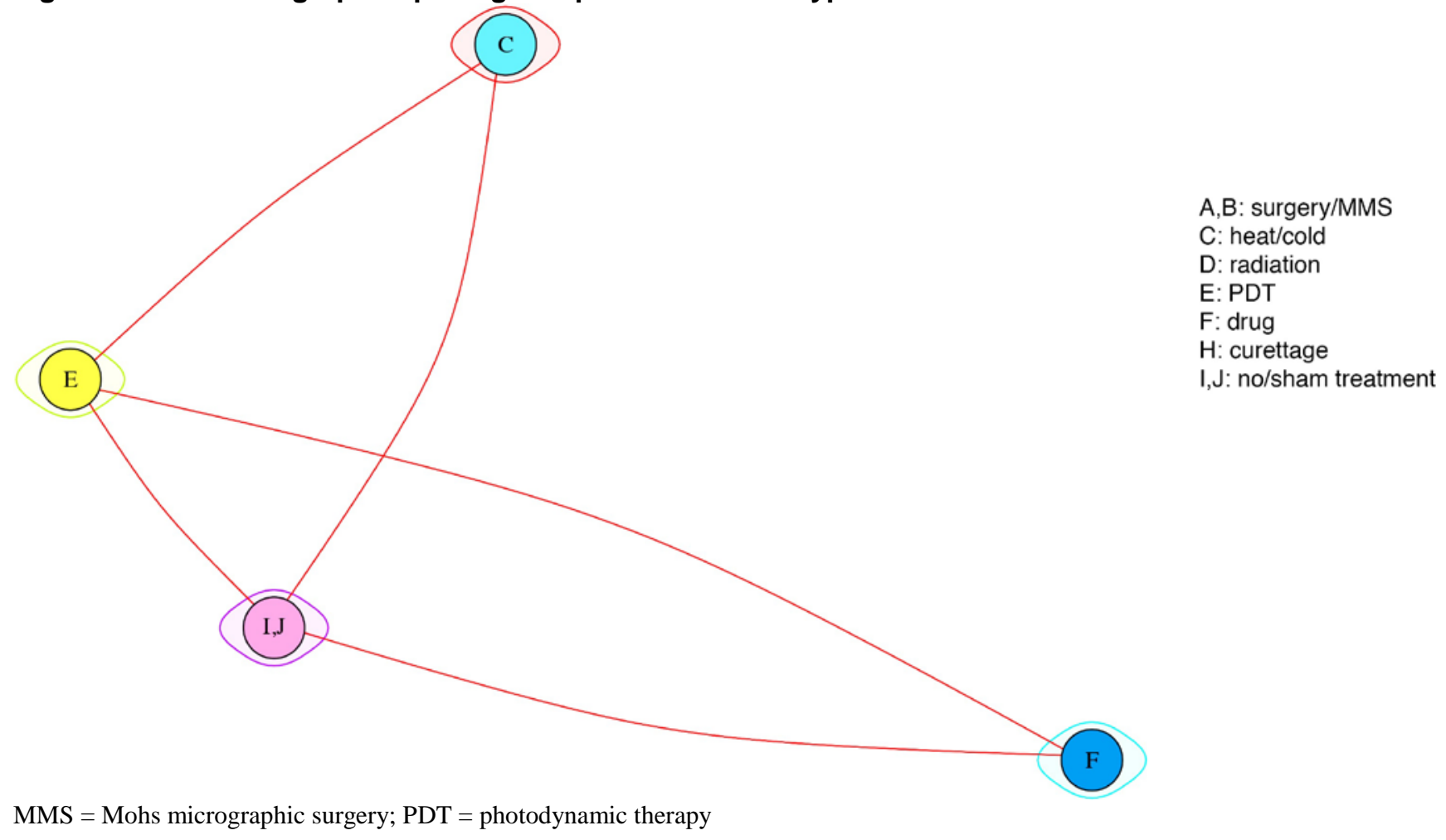

The characteristics of the six included RCTs are summarized in Table 59. All RCTS included only participants with SCC in situ (SCCIS).

Across all trials, the mean or median age of enrollees ranged between 68.9 and 76 (median 74, $25^{\text {th }}-75^{\text {th }}$ percentile: 72.4 to 76 ). The proportion of female patients ranged between 40 and 87.5 percent (median $62.8,25^{\text {th }}-75^{\text {th }}$ percentile: 54 to 80 ). When reported, the mean or median lesion area was between 82 and $429 \mathrm{~mm}^{2}$, and the maximum diameter was between 18.9 and $26.2 \mathrm{~mm}$. The majority of RCTs included lesions in various body locations, and only a few reported results stratified by lesion location (discussed separately). Based on this information, the RCTs included patients and lesions are typically encountered in clinical practice. No RCT focused on patients who were immunocompromised or had substantially limited life expectancy.

In terms of design characteristics, five RCTs had two arms and one had three arms. Analyzed sample sizes ranged between 18 and 209 (median $=23.5,25^{\text {th }}-75^{\text {th }}$ percentile: 18.25 to 37 ); sample sizes per RCT arm ranged between 11 and 91. Based on what was reported in the RCTs, we deemed that the allocation sequence was randomized using formal methods in one and successfully concealed in two RCTs, and that patients, providers, and outcome assessors were successfully blinded to the received treatments in one, two, and three RCTs, respectively. Our consensus assessment of the reported baseline characteristics across the compared arms in each RCT was that half of the RCTs $(n=3)$ had arms that were likely balanced at baseline. In four RCTs fewer than 20 percent of patients had missing outcomes for any eligible outcome in any arm. 
Figure 15. Evidence graphs for recurrence, histologic clearance, and clinical clearance for RCTs of SCC lesions

(A) Recurrence

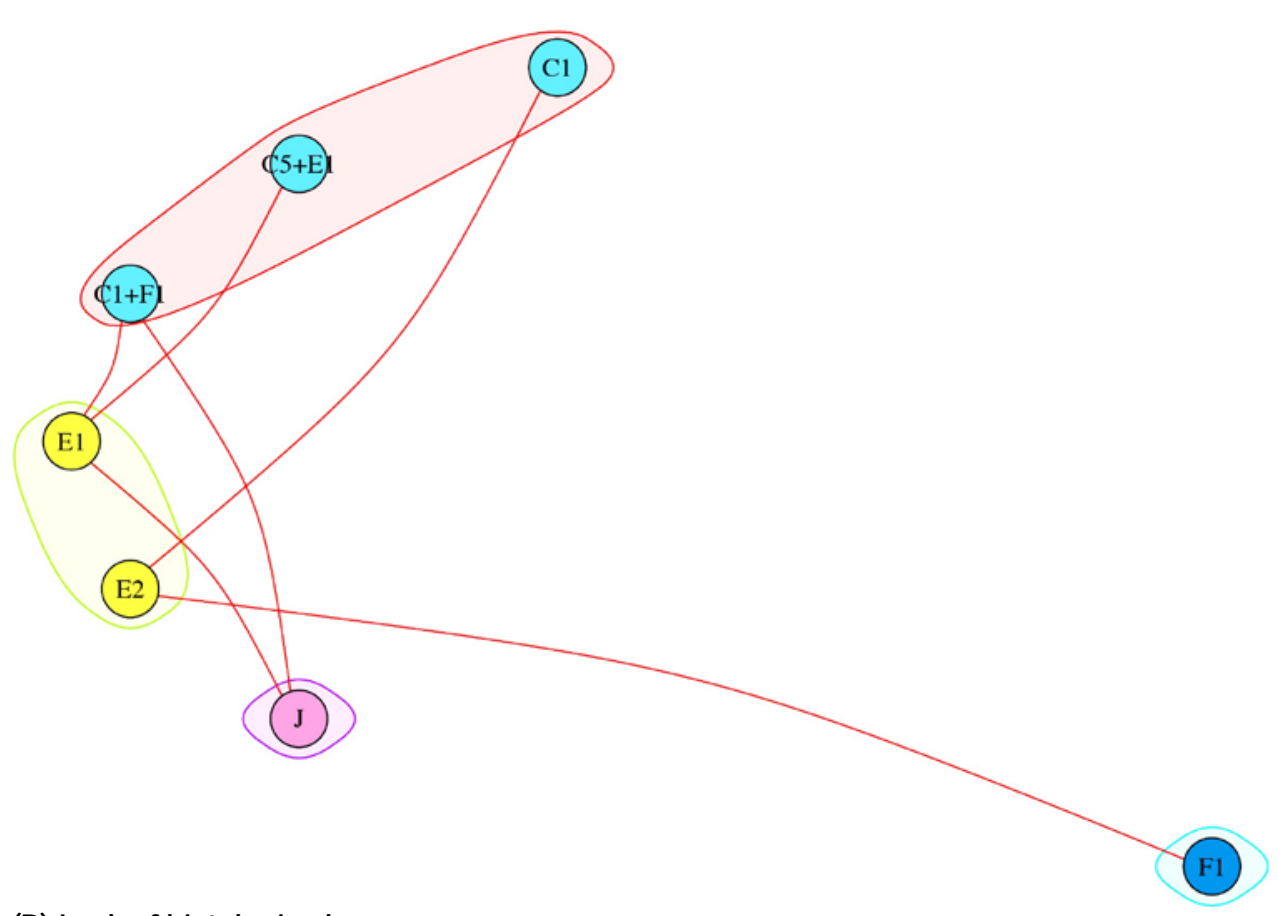

A: surgical excision

B: MMS

C1: cryotherapy

C3: curettage + diathermy

C4: curettage + cryotherapy

C5: laser

D1: external radiation

D2: brachytherapy

E1: PDT (MAL)

E2: PDT (ALA)

F1: 5-FU

F2: imiquimod

F3: INF

$\mathrm{F} 4$ : ingenol

F5: other medical

$\mathrm{H}$ : curettage

I: no treatment

J: placebo/sham

(B) Lack of histologic clearance

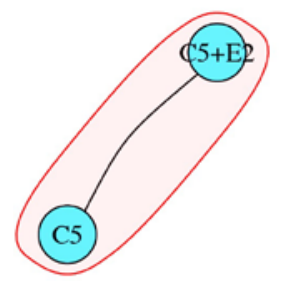

A: surgical excision

B: MMS

C1: cryotherapy

C3: curettage + diathermy

C4: curettage + cryotherapy

C5: laser

D1: external radiation

D2: brachytherapy

E1: PDT (MAL)

E2: PDT (ALA)

F1: 5-FU

F2: imiquimod

F3: INF

$\mathrm{F} 4$ : ingenol

F5: other medical

$\mathrm{H}$ : curettage

I: no treatment

$\mathrm{J}$ : placebo/sham

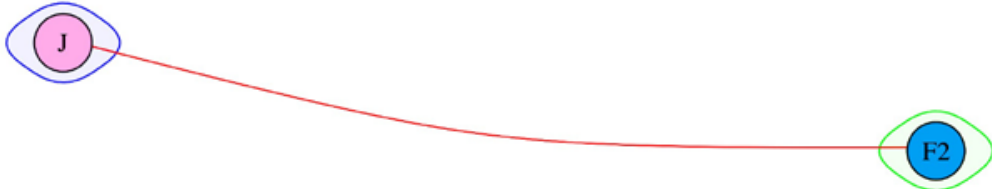


(C) Lack of clinical clearance

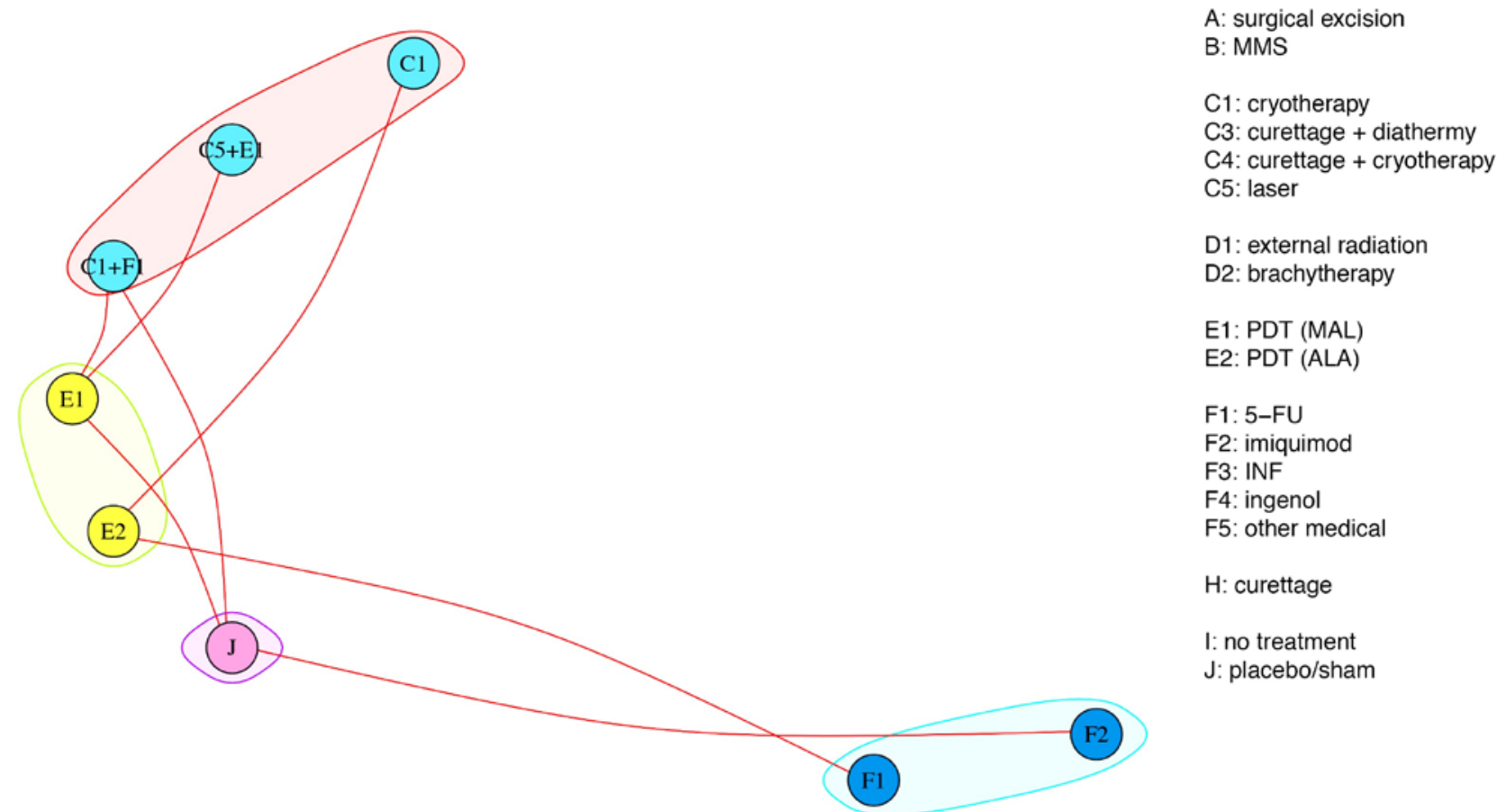

MMS = Mohs micrographic surgery; PDT = photodynamic therapy; ALA = 5-aminolevulinic acid; MAL = methyl aminolevulinate; $\mathrm{FU}$ = fluorouracil; INF = interferon 
Table 59. Characteristics of studies of SCC populations

\begin{tabular}{|c|c|c|c|c|c|c|c|c|c|c|c|c|}
\hline Study & Arm & $\begin{array}{l}\text { Age, } \\
\text { Mean }\end{array}$ & $\begin{array}{l}\text { Female } \\
\%\end{array}$ & $\begin{array}{l}\text { Lesion } \\
\text { Size, Mean }\end{array}$ & $\begin{array}{l}\text { Lesion Location } \\
\text { (\%) }\end{array}$ & $\begin{array}{l}\text { 1* } \\
\text { Adequate } \\
\text { Randomiz- } \\
\text { ation }\end{array}$ & $\begin{array}{l}2^{*} \\
\text { Allocation } \\
\text { Conceal- } \\
\text { ment }\end{array}$ & $\begin{array}{l}3^{*} \\
\text { Arms } \\
\text { Similar } \\
\text { at } \\
\text { Baseline }\end{array}$ & $\begin{array}{l}4^{*} \\
\text { Patients } \\
\text { Blinded }\end{array}$ & $\begin{array}{l}5^{\star} \\
\text { Providers } \\
\text { Blinded }\end{array}$ & $\begin{array}{l}6^{*} \\
\text { Outcome } \\
\text { Assessors } \\
\text { Blinded }\end{array}$ & $\begin{array}{l}7^{*} \\
<20 \% \\
\text { Loss to } \\
\text { Followup }\end{array}$ \\
\hline \multicolumn{13}{|l|}{ SCCIS } \\
\hline \multirow[t]{2}{*}{$\begin{array}{l}\text { Cai } 2015 \\
25899562\end{array}$} & $\begin{array}{l}\text { ALA-PDT } \\
+\mathrm{CO} 2 \\
\text { Laser }\end{array}$ & NR & 50 & $2.62 \mathrm{~cm}$ & NR & Unsure & Yes & Yes & Unsure & Yes & Yes & Yes \\
\hline & $\begin{array}{l}\text { CO2 } \\
\text { Laser }\end{array}$ & NR & 62.5 & $2.58 \mathrm{~cm}$ & NR & & & & & & & \\
\hline \multirow[t]{2}{*}{$\begin{array}{l}\text { Ko } 2014 \\
24102369\end{array}$} & $\begin{array}{l}\text { Er:YAG } \\
\text { AFL PDT }\end{array}$ & 68.9 & 52.4 & NR & extremities (100) & Unsure & No & Yes & No & Unsure & Yes & Yes \\
\hline & MAL-PDT & 68.9 & 52.4 & NR & extremities (100) & & & & & & & \\
\hline \multirow{2}{*}{$\begin{array}{l}\text { Morton } \\
1996 \\
8977678\end{array}$} & $\begin{array}{l}\text { cryotherap } \\
\text { y }\end{array}$ & 76 & 84 & $82 \mathrm{~mm}^{2}$ & $\begin{array}{l}\text { hands (5), face } \\
(15), \text { legs (80) }\end{array}$ & No & No & Yes & No & No & No & No \\
\hline & ALA-PDT & 76 & 84 & $150 \mathrm{~mm}^{2}$ & $\begin{array}{l}\text { hands (5), face } \\
(10), \text { legs (85) }\end{array}$ & & & & & & & \\
\hline \multirow[t]{4}{*}{$\begin{array}{l}\text { Morton } \\
2006 \\
16785375\end{array}$} & MAL PDT & 71.9 & 62 & $18.9 \mathrm{~mm}$ & $\begin{array}{l}\text { face/scalp (23), } \\
\text { extremities (65), } \\
\text { trunk/neck (12) }\end{array}$ & No & No & Unsure & No & No & No & Yes \\
\hline & $\begin{array}{l}\text { PDT } \\
\text { placebo }\end{array}$ & 73.4 & 65 & $19.3 \mathrm{~mm}$ & $\begin{array}{l}\text { face/scalp (25), } \\
\text { extremities (67), } \\
\text { trunk/neck (8) }\end{array}$ & & & & & & & \\
\hline & $\begin{array}{l}\text { Cryothera } \\
\text { py }\end{array}$ & 74 & 59 & $19.4 \mathrm{~mm}$ & $\begin{array}{l}\text { face/scalp (29), } \\
\text { extremities (57), } \\
\text { trunk/neck (14) }\end{array}$ & & & & & & & \\
\hline & $\begin{array}{l}\text { Fluorourac } \\
\text { il }\end{array}$ & 72.5 & 63 & $20.9 \mathrm{~mm}$ & $\begin{array}{l}\text { face/scalp (19), } \\
\text { extremities (69), } \\
\text { trunk/neck (11) }\end{array}$ & & & & & & & \\
\hline \multirow[t]{2}{*}{$\begin{array}{l}\text { Patel } 2006 \\
16713457\end{array}$} & $\begin{array}{l}\text { imiquimod } \\
5 \%\end{array}$ & 74 & 40 & $429 \mathrm{~mm}^{2}$ & NR & Yes & Yes & No & Yes & Yes & Yes & No \\
\hline & vehicle & 74 & 87.5 & $248 \mathrm{~mm}^{2}$ & NR & & & & & & & \\
\hline \multirow{2}{*}{$\begin{array}{l}\text { Salim } \\
2003 \\
12653747\end{array}$} & PDT & 76 & 80 & NR & extremities (100) & No & No & No & No & No & No & Yes \\
\hline & $5-F U$ & 76 & 80 & NR & $\begin{array}{l}\text { face }(12), \\
\text { extremities (88) }\end{array}$ & & & & & & & \\
\hline
\end{tabular}

SCC

microinva

sive 


\begin{tabular}{|c|c|c|c|c|c|c|c|c|c|c|c|c|}
\hline Study & Arm & $\begin{array}{l}\text { Age, } \\
\text { Mean }\end{array}$ & $\begin{array}{l}\text { Female } \\
\%\end{array}$ & $\begin{array}{l}\text { Lesion } \\
\text { Size, Mean }\end{array}$ & $\begin{array}{l}\text { Lesion Location } \\
\text { (\%) }\end{array}$ & $\begin{array}{l}\text { 1* } \\
\text { Adequate } \\
\text { Randomiz- } \\
\text { ation }\end{array}$ & $\begin{array}{l}2^{*} \\
\text { Allocation } \\
\text { Conceal- } \\
\text { ment }\end{array}$ & $\begin{array}{l}3^{*} \\
\text { Arms } \\
\text { Similar } \\
\text { at } \\
\text { Baseline }\end{array}$ & $\begin{array}{l}4^{*} \\
\text { Patients } \\
\text { Blinded }\end{array}$ & $\begin{array}{l}5^{*} \\
\text { Providers } \\
\text { Blinded }\end{array}$ & $\begin{array}{l}6^{*} \\
\text { Outcome } \\
\text { Assessors } \\
\text { Blinded }\end{array}$ & $\begin{array}{l}7^{*} \\
<20 \% \\
\text { Loss to } \\
\text { Followup }\end{array}$ \\
\hline \multirow[t]{2}{*}{$\begin{array}{l}\text { Choi } 2017 \\
28199463\end{array}$} & MAL-PDT & 75.1 & 54.6 & $11.8 \mathrm{~mm}$ & $\begin{array}{l}\text { Face or scalp } \\
(75.0), \text { extremities } \\
(16.7), \text { trunk/neck } \\
(8.3)\end{array}$ & \multirow[t]{2}{*}{ Yes } & \multirow[t]{2}{*}{ No } & \multirow[t]{2}{*}{ Yes } & \multirow[t]{2}{*}{ No } & \multirow[t]{2}{*}{ No } & \multirow[t]{2}{*}{ Yes } & \multirow[t]{2}{*}{ Yes } \\
\hline & $\begin{array}{l}\text { MAL-PDT } \\
+ \text { Er:YAG }\end{array}$ & 76.4 & 71.4 & $11.5 \mathrm{~mm}$ & $\begin{array}{l}\text { Face or scalp } \\
(76.2), \text { extremities } \\
(19.0) \text {, trunk/neck } \\
(4.8)\end{array}$ & & & & & & & \\
\hline
\end{tabular}

PDT = photodynamic therapy; ALA = 5-aminolevulinic acid; MAL = methyl aminolevulinate; FU = fluorouracil; INF = interferon; SCC = squamous cell carcinoma; Er:YAG = ablative fractional laser; NR = not reported

*Design items: 1: Adequate generation of a randomized sequence reported; 2: Adequate allocation concealment reported; 3: Group similarity at baseline; 4: Adequate blinding of patients reported; 5: Adequate blinding of providers reported; 6: Adequate blinding of outcome assessors reported; 7 : Less than $20 \%$ missing for any eligible outcome in any arm. PDT=photodynamic therapy. 


\section{Recurrence, SCCIS Lesions}

The evidence graph for recurrence with respect to individual treatments is sparse (Figure 15 (A) - reproduced in Figure 16 (A) for ease of reference). Detailed results at the RCT-level are in Appendix I.

\section{Figure 16. Evidence graph of RCTs evaluating recurrence in SCCIS across (A) individual} interventions and (B) types of interventions

(A)

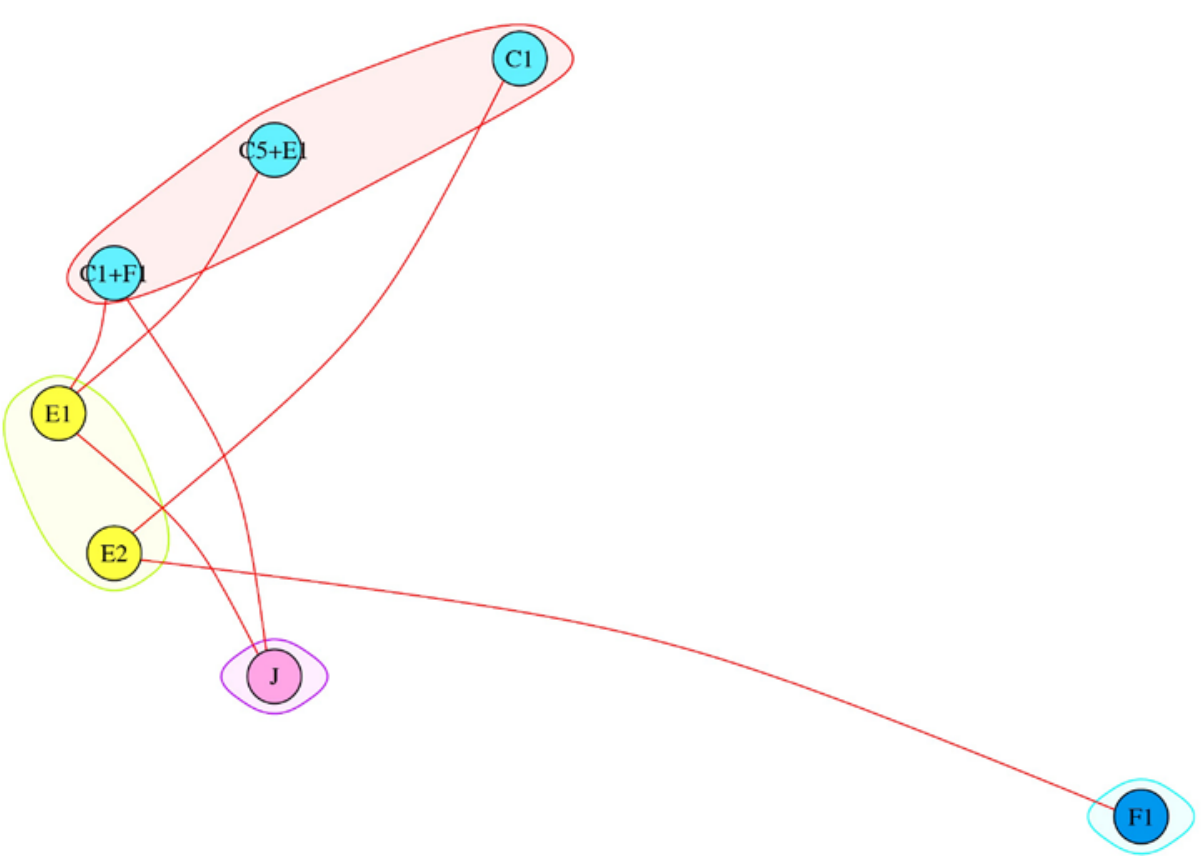

A: surgical excision
B: MMS
C1: cryotherapy
C3: curettage + diathermy
C4: curettage + cryotherapy
C5: laser
D1: external radiation
D2: brachytherapy
E1: PDT (MAL)
E2: PDT (ALA)
F1: 5-FU
F2: imiquimod
F3: INF
F4: ingenol
F5: other medical
H: curettage
I: no treatment
J: placebo/sham

(B)

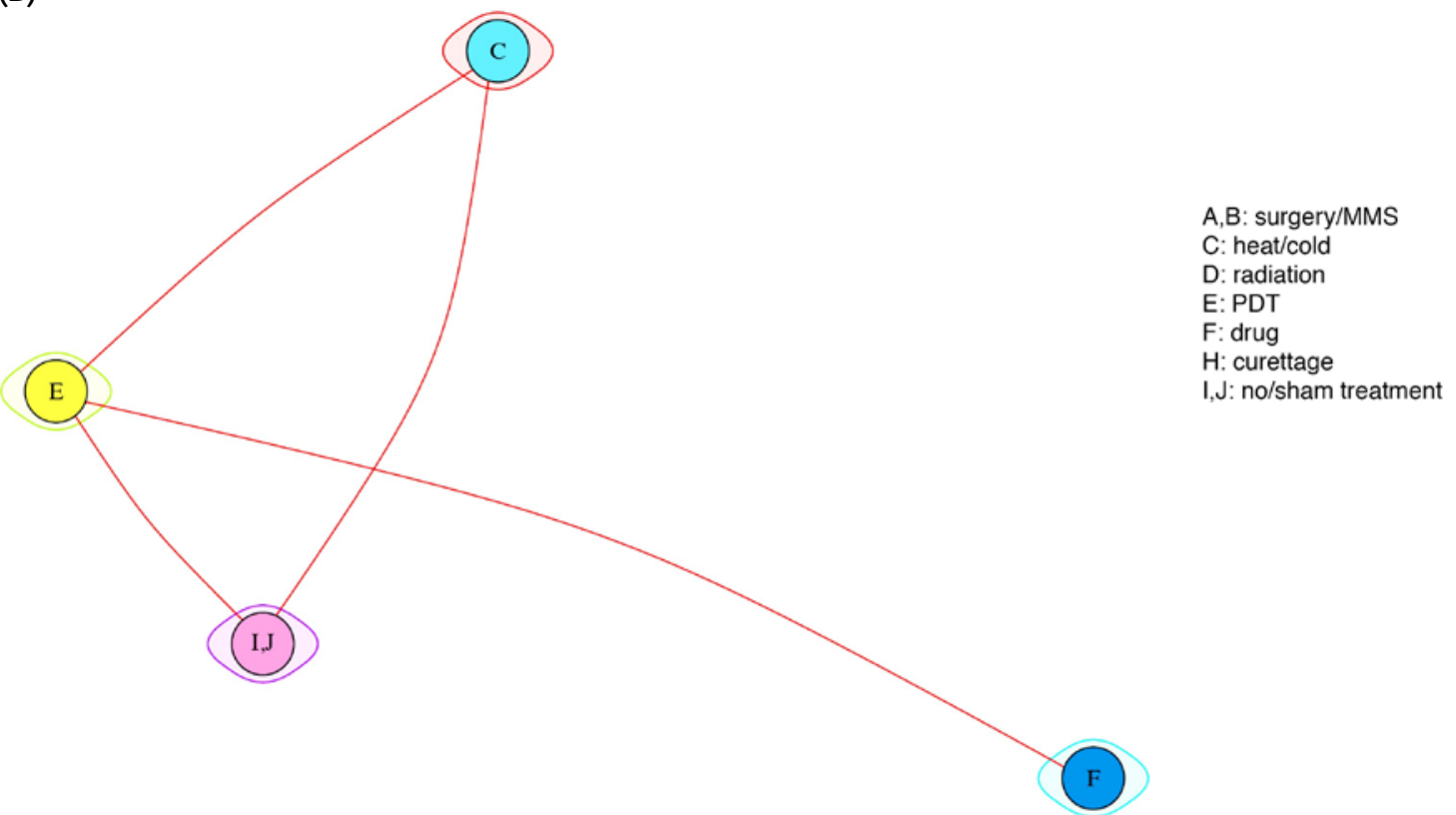

MMS = Mohs micrographic surgery; PDT = photodynamic therapy; ALA = 5-aminolevulinic acid; MAL = methyl aminolevulinate; FU = fluorouracil; INF = interferon; SCCIS = squamous cell carcinoma in situ 


\section{Comparisons Across Intervention Categories}

In total, 4 RCTs (348 lesions) were included in this analysis. ${ }^{72,79,80,89}$ Two RCTs were deemed to be at low or moderate risk of bias. The comparisons are described in Table 60.

Table 60. Sample information, recurrence (SCCIS, intervention categories)

\begin{tabular}{|c|c|}
\hline Studies (total sample) & $4(348)$ \\
\hline Total sample by intervention & (C): 136; (E): 175; (F): 33; (I,J): 4 \\
\hline $\begin{array}{l}\text { Total sample by intervention, (min, } \\
\text { max) }\end{array}$ & 4,175 \\
\hline Data by comparison & (C--E): 3 (278); (C--I,J): 1 (101); (E--F): 1 (66); (E--I,J): 1 (107) \\
\hline Studies by comparison (min, max) & 1,3 \\
\hline $\begin{array}{l}\text { Total sample by comparison (min, } \\
\text { max) }\end{array}$ & 66,278 \\
\hline Followup median (min, max) & $12(12,24)$ months \\
\hline
\end{tabular}

Table 61 shows the relative odds ratios for recurrence across intervention categories. Based on direct data, the odds ratio for recurrence is not statistically significantly different between interventions that destroy the lesions with heat or cold (C) and PDT (E); however, the confidence interval does not exclude differences in the odds as large as 50 percent in either direction. Based on direct data, the odds ratio between PDT (E) and drugs (F) is statistically significant, favoring PDT.

In the table, shaded cells correspond to comparisons that have been inferred from the analysis model, but that have not been examined in the included RCTs. For example, comparisons of drugs (F) and interventions that destroy the lesion with heat or cold (E) are indirect, and have very wide confidence intervals. For all comparisons that are empirically observed (all nonshaded cells in the table), results using only head-to-head data agree well with the results from the network meta-analysis in Table 61.

Table 61. Relative odds ratios for recurrence between intervention categories (SCCIS lesions, Figure 16B)

\begin{tabular}{cccc}
\hline $\begin{array}{c}\text { Heat/cold } \\
\text { (C) }\end{array}$ & $0.83(0.33,2.06)$ & $0.17(0.05,0.55)$ & $0.18(0.02,1.59)$ \\
\hline $1.21(0.49,3.01)$ & $\begin{array}{c}\text { PDT } \\
(\mathrm{E})\end{array}$ & $\mathbf{0 . 2 0}(\mathbf{0 . 0 7 , 0 . 6 2 )}$ & $0.22(0.03,1.86)$ \\
\hline $5.96(1.81,19.61)$ & $4.93(1.6,15.15)$ & $\begin{array}{c}\text { Drugs } \\
(\mathbf{F})\end{array}$ & $1.06(0.11,10.44)$ \\
\hline $5.61(0.63,50.1)$ & $4.64(0.54,39.96)$ & $0.94(0.1,9.25)$ & $\begin{array}{c}\text { No/sham treatment } \\
(\mathbf{I}, \mathrm{J})\end{array}$ \\
\hline
\end{tabular}

Note: Cells shaded gray indicate that the estimate is based only on indirect comparisons; bold-italic indicates that the result is statistically significant. Results are given as odds ratios (95\% confidence intervals).

PDT = photodynamic therapy; SCCIS = squamous cell carcinoma in situ.

Table 62 offers complementary information from the same analysis. For each intervention category, it shows the mean recurrence rate across the included RCTs. Interventions that destroy the lesion with heat or cold (C) and PDT (E) had on average lower recurrence rates $(15.1 \%$ and $17.7 \%$, respectively) compared to the other treatments. These estimates describe the outcome 
rates in the RCT arms, and are based on the relative effects in Table 61 and the observed baseline rates in the RCTs. Of note, the recurrence rate for drugs is 51.5 percent (95\% CI 28.9 to 73.5 ), reflecting the high recurrence rates observed in the single RCT comparing 5-FU with PDT (ALA) in this analysis.

Table 62. Mean recurrence rates by intervention category (SCCIS lesions)

\begin{tabular}{lcc}
\hline Intervention Type & $\begin{array}{c}\text { Mean } \\
\text { Percent (95\% Cl) }\end{array}$ & $\begin{array}{c}\text { Forecast } \\
\text { Percent (95\% Cl) }\end{array}$ \\
\hline Heat/cold (C) & $15.1(8.1,26.5)$ & $15.1(6.3,32.1)$ \\
\hline PDT (E) & $17.7(10.8,27.8)$ & $17.7(8.1,34.4)$ \\
\hline Drugs (F) & $51.5(28.9,73.5)$ & $51.5(24.7,77.5)$ \\
\hline No/sham treatment $(\mathrm{I}, \mathrm{J})$ & $50.0(11.2,88.8)$ & $50.0(10.1,89.9)$ \\
\hline
\end{tabular}

$\overline{\mathrm{PDT}}$ = photodynamic therapy; SCCIS = squamous cell carcinoma in situ; CI = confidence interval

\section{Comparisons Across Individual Interventions}

As is evident from Figure 16, there are two connected subgraphs for this outcome: a smaller one comprising the comparison among cryotherapy (C1), MAL with ALA (E2), and 5-FU (F1), and a larger one among PDT with MAL with and without laser preparation (E1 and C5+E1), cryotherapy with 5-FU (C1+F1), and placebo. In total, 4 RCTs (348 lesions) were included in these analyses, as summarized in Table 63.

Table 63. Sample information, recurrence (SCCIS, interventions)

\begin{tabular}{|c|c|c|}
\hline & First subgraph 72,79 & Second subgraph ${ }^{80,89}$ \\
\hline Studies (total sample) & $2(242)$ & $2(106)$ \\
\hline $\begin{array}{l}\text { Total sample by } \\
\text { intervention }\end{array}$ & (C5+E1): 19; (E1): 122; (C1+F1): 97; (J): 4 & (C1): 20; (E2): 53; (F1): 33 \\
\hline $\begin{array}{l}\text { Total sample by } \\
\text { intervention, (min, max) }\end{array}$ & 4,122 & 20,53 \\
\hline Data by comparison & $\begin{array}{l}\text { (C5+E1--E1): } 1 \text { (38); (E1--C1+F1): } 1 \text { (200); (E1-- } \\
\text { J): } 1 \text { (107); (C1+F1--J): } 1 \text { (101) }\end{array}$ & $\begin{array}{l}\text { (C1--E2): } 1 \text { (40); (E2--F1): } 1 \\
(66)\end{array}$ \\
\hline $\begin{array}{l}\text { Studies by comparison } \\
\text { (min, max) }\end{array}$ & 1,1 & 1,1 \\
\hline $\begin{array}{l}\text { Total sample by } \\
\text { comparison (min, max) }\end{array}$ & 38,200 & 40,66 \\
\hline Followup (min, max) & $(12,12)$ months & $(12,24)$ months \\
\hline
\end{tabular}

A = surgical excision; B = Mohs micrographic surgery; C1 = cryotherapy; C3 = diathermy and curettage; C4 = cryotherapy and curettage; C5 = laser; D1 = external radiation; E1 = MAL photodynamic therapy; E2 = ALA photodynamic therapy; F1 = 5-FU; F2 = Imiquimod; F3 = Interferon; F4 = Ingenol; H = curettage; J = placebo; SCCIS = squamous cell carcinoma in situ

Table 64 shows the relative effects for both subgraphs. Because the comparisons across individual observations are sparse, however, the confidence intervals of the odds ratios for most indirect comparisons are very broad and cannot exclude very large differences between the compared interventions.

Table 65 shows, for each intervention, the mean recurrence rates across all RCTs; estimates for interventions in both subgraphs are listed in the table. It was not possible to compare statistically the estimated recurrence rates between an intervention in the first subgraph (e.g., PDT with MAL [E1]) and the second subgraph (e.g., cryotherapy [C1]), because they come from disjoint analyses. 
Table 64. Relative odds ratios for recurrence between individual interventions (SCCIS lesions, Figure 16A)

\begin{tabular}{|c|c|c|c|c|c|c|}
\hline $\begin{array}{c}\text { Cryotherapy + 5-FU } \\
(\mathrm{C} 1+\mathrm{F} 1)\end{array}$ & $6.14(0.48,77.78)$ & $1.12(0.31,3.96)$ & $0.24(0.02,2.48)$ & & & \\
\hline $0.16(0.01,2.06)$ & $\begin{array}{c}\text { Laser + PDT (MAL) } \\
\text { (C5+E1) }\end{array}$ & $0.18(0.02,1.95)$ & $0.04(<0.005,0.94)$ & & & \\
\hline $0.9(0.25,3.18)$ & $5.5(0.51,58.9)$ & $\begin{array}{c}\text { PDT (MAL) } \\
\text { (E1) }\end{array}$ & $0.22(0.02,2.13)$ & & & \\
\hline \multirow[t]{4}{*}{$4.11(0.4,41.76)$} & $25.2(1.06,598.92)$ & $4.58(0.47,44.79)$ & $\begin{array}{c}\text { Placebo/sham } \\
(\mathrm{J})\end{array}$ & & & \\
\hline & & & & $\begin{array}{l}\text { Cryotherapy } \\
\text { (C1) }\end{array}$ & $\begin{array}{l}1.34(0.06 \\
28.22)\end{array}$ & $\begin{array}{c}0.19(0.01, \\
5.5)\end{array}$ \\
\hline & & & & $\begin{array}{c}0.75(0.04 \\
15.75) \\
\end{array}$ & $\begin{array}{l}\text { PDT (ALA) } \\
\text { (E2) }\end{array}$ & $\begin{array}{c}0.14(0.01, \\
1.55)\end{array}$ \\
\hline & & & & $\begin{array}{c}5.27(0.18 \\
153) \\
\end{array}$ & $\begin{array}{c}7.06(0.65 \\
77.1) \\
\end{array}$ & $\begin{array}{l}\text { 5-FU } \\
(\mathrm{F} 1)\end{array}$ \\
\hline
\end{tabular}

Note: Cells shaded gray indicate that the estimate is based only on indirect comparisons; bold-italic indicates that the result is statistically significant. Results are given as odds ratios (95\% confidence intervals).

PDT $($ MAL) = methyl aminolaevulinate photodynamic therapy; PDT (ALA ) = aminolevulinic acid photodynamic therapy; FU = fluorouracil; SCCIS = squamous cell carcinoma in situ

Table 65. Mean and forecasted recurrence rates by intervention category (SCCIS lesions)

\begin{tabular}{lcc}
\hline Intervention Type & $\begin{array}{c}\text { Mean } \\
\text { Percent (95\% Cl) }\end{array}$ & $\begin{array}{c}\text { Forecast } \\
\text { Percent (95\% Cl) }\end{array}$ \\
\hline First subgraph (Figure 16) & & \\
\hline Cryotherapy + 5-FU (C1+F1) & $22.4(8.0,48.8)$ & $22.4(5.3,60.0)$ \\
\hline Laser + PDT (MAL) (C5+E1) & $4.5(0.5,31.6)$ & $4.5(0.4,37.6)$ \\
\hline PDT (MAL) (E1) & $20.5(9.0,40.3)$ & $20.5(5.5,53.3)$ \\
\hline Placebo/sham (J) & $54.2(11.2,91.8)$ & $54.2(8.8,93.6)$ \\
\hline Second subgraph (Figure 16) & & \\
\hline Cryotherapy (C1) & $13.0(1.1,67.2)$ & $13.0(0.5,82.5)$ \\
\hline PDT (ALA) (E2) & $10.1(1.5,45.3)$ & $10.1(0.5,69.4)$ \\
\hline 5-FU (F1) & $44.1(7.5,88.5)$ & $44.1(3.1,95.1)$ \\
\hline
\end{tabular}

PDT $(\mathrm{MAL})$ = methyl aminolaevulinate photodynamic therapy; PDT (ALA) = aminolevulinic acid photodynamic therapy; FU = fluorouracil; SCCIS = squamous cell carcinoma in situ; $\mathrm{CI}=$ confidence interval

\section{Recurrence, Other Subgroup Analyses (Lesion Location, Lesion Size)}

\section{Evidence From RCTS}

Table 66 below shows results on subgroup analyses for a four-arm RCT. ${ }^{77,79}$ Neither lesion location nor size were associated with differences in the treatment effect beyond what is expected by chance.

Table 66. Subgroup analyses by lesion location and size: results for recurrence (SCCIS lesions)

\begin{tabular}{|c|c|c|c|c|c|c|}
\hline Study & Comparison & Timepoint & Subgroup & $\begin{array}{l}\text { n/N Arm } 1 \\
\text { vs. n/N Arm } \\
2 \text { vs. n/N arm } \\
3\end{array}$ & $\begin{array}{l}\text { OR }(95 \% \mathrm{Cl}) ; \mathrm{P}- \\
\text { Value Within }\end{array}$ & $\begin{array}{l}\text { P- Value } \\
\text { Between }\end{array}$ \\
\hline $\begin{array}{l}\text { Morton } 2006 \\
16785375\end{array}$ & $\begin{array}{l}\text { Cryotherapy (C1) } \\
\text { or 5-FU (F1) vs. }\end{array}$ & 12 months & $\begin{array}{l}\text { lesion location: } \\
\text { extremities }\end{array}$ & $\begin{array}{l}11 / 60 \text { vs. } \\
11 / 63 \text { vs. } 0 / 1\end{array}$ & $\begin{array}{l}1.06(0.42,2.67) ; \\
0.70(0.03,18.23)\end{array}$ & $p=0.483$ \\
\hline
\end{tabular}




\begin{tabular}{|c|c|c|c|c|c|c|}
\hline Study & Comparison & Timepoint & Subgroup & $\begin{array}{l}\text { n/N Arm } 1 \\
\text { vs. n/N Arm } \\
2 \text { vs. n/N arm } \\
3\end{array}$ & $\begin{array}{l}\text { OR }(95 \% \mathrm{Cl}) ; \text { P- } \\
\text { Value Within }\end{array}$ & $\begin{array}{l}\text { P- Value } \\
\text { Between }\end{array}$ \\
\hline & \multirow[t]{3}{*}{$\begin{array}{l}\text { MAL-PDT (E1) vs. } \\
\text { sham PDT (J) }\end{array}$} & & & & $\begin{array}{l}0.66(0.03,17.18) \\
p=1.000\end{array}$ & \\
\hline & & & $\begin{array}{l}\text { lesion location: } \\
\text { face/scalp }\end{array}$ & $\begin{array}{l}6 / 22 \text { vs. } 2 / 27 \\
\text { vs. } 1 / 2\end{array}$ & $\begin{array}{l}4.69(0.84,26.15) ; \\
0.38(0.02,7.00) ; \\
0.08(0.00,1.82) ; \\
p=0.084\end{array}$ & \\
\hline & & & $\begin{array}{l}\text { lesion location: } \\
\text { neck/trunk }\end{array}$ & $\begin{array}{l}2 / 15 \text { vs. } 2 / 13 \\
\text { vs. } 1 / 1\end{array}$ & $\begin{array}{l}0.85(0.10,7.04) \\
0.06(0.00,1.99) \\
0.07(0.00,2.35) \\
p=0.209\end{array}$ & \\
\hline \multirow[t]{4}{*}{$\begin{array}{l}\text { Morton } 2006 \\
16785375\end{array}$} & \multirow[t]{4}{*}{$\begin{array}{l}\text { Cryotherapy (C1) } \\
\text { or 5-FU (F1) vs. } \\
\text { MAL-PDT (E1) vs. } \\
\text { sham PDT (J) }\end{array}$} & \multirow[t]{4}{*}{12 months } & $\begin{array}{l}\text { lesion diameter: } \\
5-14 \mathrm{~mm}\end{array}$ & $\begin{array}{l}0 / 27 \text { vs. } 4 / 40 \\
\text { vs. } 1 / 1\end{array}$ & $\begin{array}{l}0.15(0.01,2.86) ; \\
0.01(0.00,0.43) \\
0.04(0.00,1.17) ; \\
p=0.018\end{array}$ & NA \\
\hline & & & lesion diameter: & $15 / 55$ vs. $5 / 43$ & $2.85(0.94,8.61) ;$ & \\
\hline & & & & & $\begin{array}{l}0.26(0.02,3.46) ; \\
p=0.093\end{array}$ & \\
\hline & & & $\begin{array}{l}\text { lesion diameter: } \\
>=30 \mathrm{~mm}\end{array}$ & $\begin{array}{l}3 / 12 \text { vs. } 6 / 20 \\
\text { vs. } 0 / 0\end{array}$ & $\begin{array}{l}0.78(0.15,3.93) ; N A ; \\
p=1.000\end{array}$ & \\
\hline
\end{tabular}

NA = not significant; PDT (MAL) = methyl aminolaevulinate photodynamic therapy; FU = fluorouracil; SCCIS = squamous cell carcinoma in situ

\section{Evidence From NRCSs}

One NRCS reported recurrence for 80 SCCIS lesions in 67 people, treated with either curettage (44 lesions) or cryotherapy (36 lesions). This study was deemed to be of high risk of bias, primarily for lack of reporting (baseline data and dropout numbers were not given by arm), but also for lack of blinding and for a high long-term dropout rate. The mean age was 74 (range: 46 to 89), and the mean lesion area was $336 \mathrm{~mm}^{2}$ (range 30 to $1890 \mathrm{~mm}^{2}$ ). Eighty-two percent were female, and the lesions were located on the extremities (84\%), trunk (7.5\%), and head/neck (8.5\%). The cryotherapy arm had a significantly higher rate of recurrence up to 22 months than the curettage arm (OR 5.65; 95\% CI 1.65 to 19.39). ${ }^{141}$

\section{Lack of Histological Clearance, SCCIS Lesions}

The evidence graph for recurrence with respect to individual treatments is sparse (Figure 15 (B) - reproduced in Figure 17 for ease of reference). For this outcome, one RCT compared between laser ablation (C5) versus a combination of laser ablation and PDT with ALA (C5+E2), and one RCT compared 5-FU (F2) versus placebo (J). An analysis of comparisons between intervention categories is superfluous, in that it would include the same evidence as in the latter comparison of 5-FU (F2) versus placebo (J). The comparisons in the two RCTs (50 lesions) are described in Table 67. 
Table 67. Sample information, lack of histological clearance (SCCIS)

\begin{tabular}{lll}
\hline & Figure 17, first subgraph & \\
\hline Studies (total sample) & $1(28)$ & $\begin{array}{l}\text { Figure 17, second } \\
\text { subgraph }^{57}\end{array}$ \\
\hline Total sample by intervention & $(\mathrm{F} 2): 12 ;(\mathrm{J}): 16$ & $1(22)$ \\
\hline $\begin{array}{l}\text { Total sample by intervention, (min, } \\
\text { max) }\end{array}$ & 12,16 & $(\mathrm{C} 5): 11 ;(\mathrm{C} 5+\mathrm{E} 2): 11$ \\
\hline Data by comparison & $(\mathrm{F} 2--\mathrm{J}): 1(28)$ & 11,11 \\
\hline Studies by comparison (min, max) & 1,1 & $(\mathrm{C} 5-\mathrm{C} 5+\mathrm{E} 2): 1(22)$ \\
\hline $\begin{array}{l}\text { Total sample by comparison (min, } \\
\text { max) }\end{array}$ & 28,28 & 1,1 \\
\hline Followup & 7 months & 22,22 \\
\hline
\end{tabular}

A = surgical excision; B = Mohs micrographic surgery; C1 = cryotherapy; C3 = diathermy and curettage; C4 = cryotherapy and curettage; C5 = laser; D1 = external radiation; E1 = MAL photodynamic therapy; E2 = ALA photodynamic therapy; F1 = 5-FU; F2 = Imiquimod; F3 = Interferon; F4 = Ingenol; H = curettage; J = placebo; SCCIS = squamous cell carcinoma in situ

Figure 17. Evidence graph of RCTs evaluating lack of histological clearance in SCCIS lesions across individual interventions
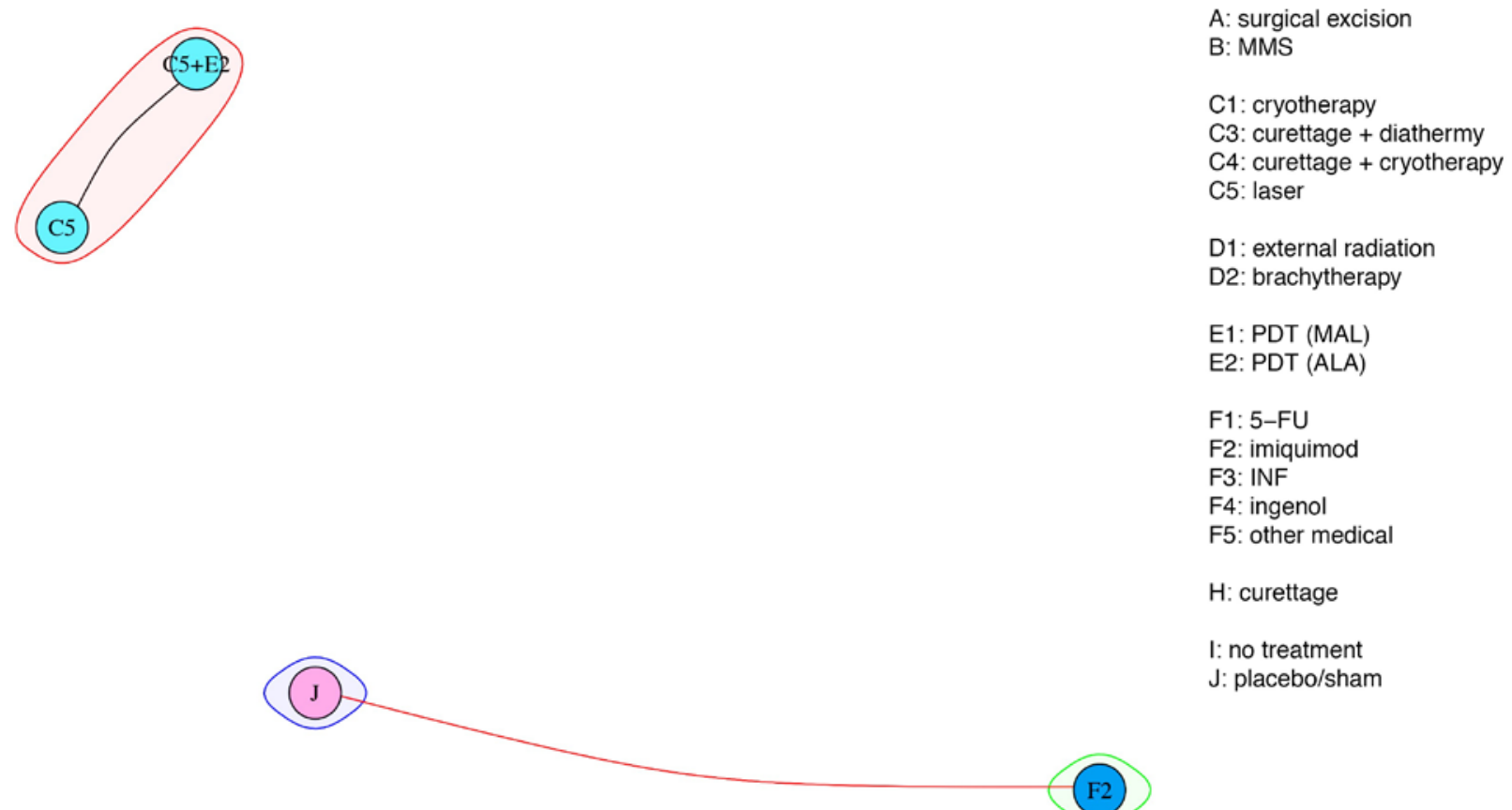

MMS = Mohs micrographic surgery; PDT = photodynamic therapy; ALA = 5-aminolevulinic acid; MAL = methyl aminolevulinate; FU = fluorouracil; INF = interferon; SCCIS = squamous cell carcinoma in situ

Table 68 shows the relative odds ratios for lack of histological clearance between individual interventions. Because of the very small sample sizes, the confidence intervals are very large. Table 69 has the respective fractions for lack of histological clearance in the two RCTs. 
Table 68. Relative odds ratios for lack of histological clearance between individual interventions (SCCIS lesions, Figure 17)

\begin{tabular}{cccc}
\hline (F1) & $\begin{array}{c}0.01 \\
\mathbf{5 - F U}\end{array}$ & & \\
\hline 99 & $(<0.005,0.22)$ & & \\
\cline { 1 - 3 }$(\mathbf{4 . 4 5}, \mathbf{2 2 0 2 . 2 3 )}$ & placebo & $(\mathrm{J} 5)$ & 8.33 \\
& & laser & $(0.78,89.47)$ \\
\cline { 2 - 3 } & & 0.12 & (C5+E2) \\
& & $(0.01,1.29)$ & laser + PDT (ALA) \\
\hline
\end{tabular}

Note: Bold-italic indicates that the result is statistically significant; Results are given in odds ratios and 95\% confidence intervals.

PDT = photodynamic therapy; ALA = 5-aminolevulinic acid; FU = fluorouracil; SCCIS = squamous cell carcinoma in situ

Table 69. Mean lack of histological clearance (all SCCIS lesions)

\begin{tabular}{lc}
\hline Intervention Type & $\begin{array}{c}\text { Mean } \\
\text { Percent }(\mathbf{9 5 \%} \mathbf{~ C l})\end{array}$ \\
\hline First comparison & $25.0(8.3,55.2)$ \\
\hline 5-FU (F1) & $97.1(66.4,99.8)$ \\
\hline Placebo (J) & \\
\hline Second comparison & $45.5(20.3,73.2)$ \\
\hline Laser (C5) & $9.1(1.3,43.9)$ \\
\hline $\begin{array}{l}\text { PDT = photodynamic therapy; ALA = 5-aminolevulinic acid; FU } \\
\text { confidence interval }\end{array}$
\end{tabular}

\section{Lack of Clinical Clearance, SCCIS Lesions}

The evidence graph for recurrence with respect to individual treatments is sparse (Figure 15 [C] - reproduced in Figure 18 [A] for ease of reference). Detailed results at the RCT-level are in Appendix I. 
Figure 18. Evidence graph of RCTs evaluating lack of clinical clearance in SCCIS lesions across $(A)$ individual interventions and $(B)$ types of interventions

(A)

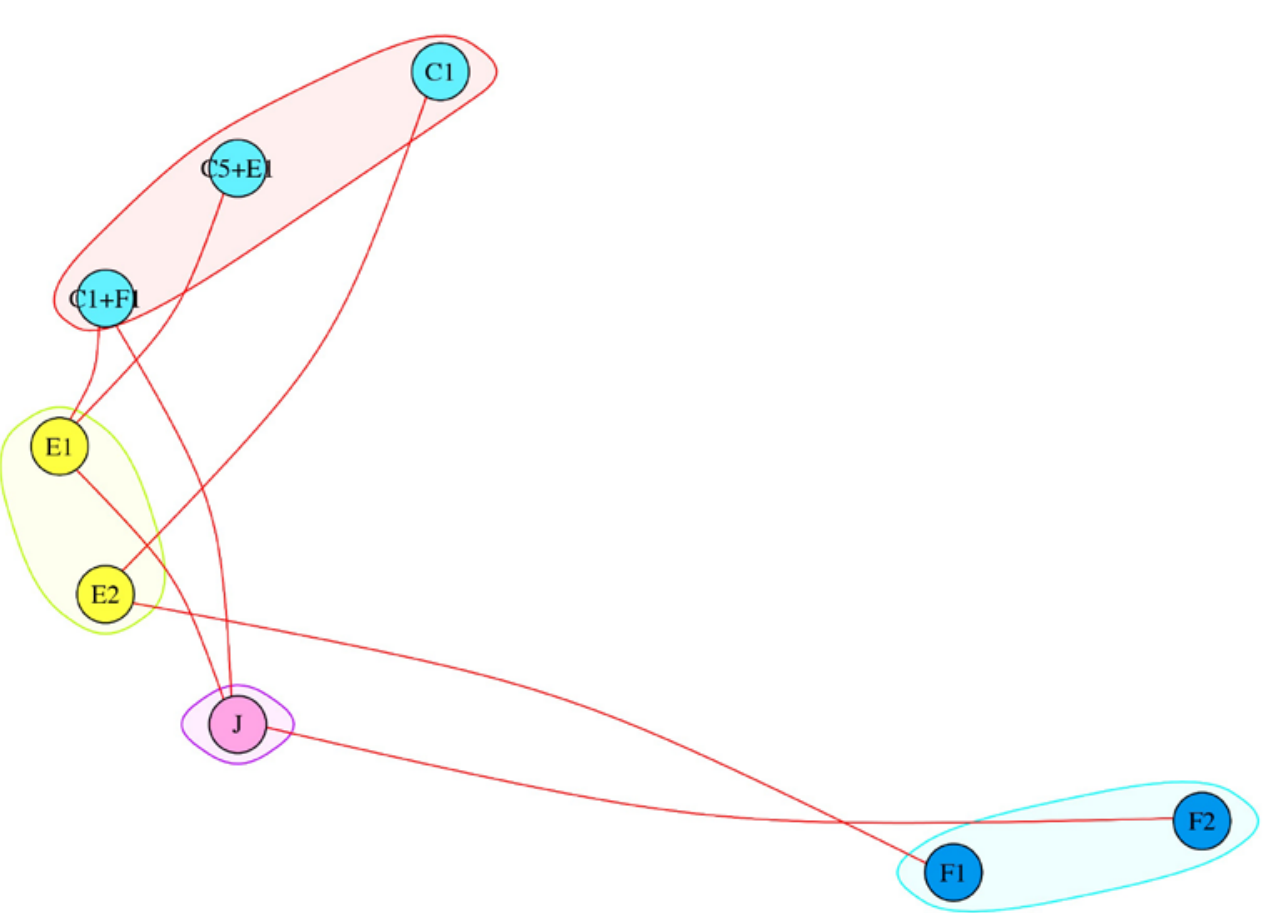

A: surgical excision

B: MMS

C1: cryotherapy

C3: curettage + diathermy

C4: curettage + cryotherapy

C5: laser

D1: external radiation

D2: brachytherapy

E1: PDT (MAL)

E2: PDT (ALA)

F1: 5-FU

F2: imiquimod

F3: INF

$\mathrm{F} 4$ : ingenol

F5: other medical

$\mathrm{H}$ : curettage

I: no treatment

J: placebo/sham 
(B)

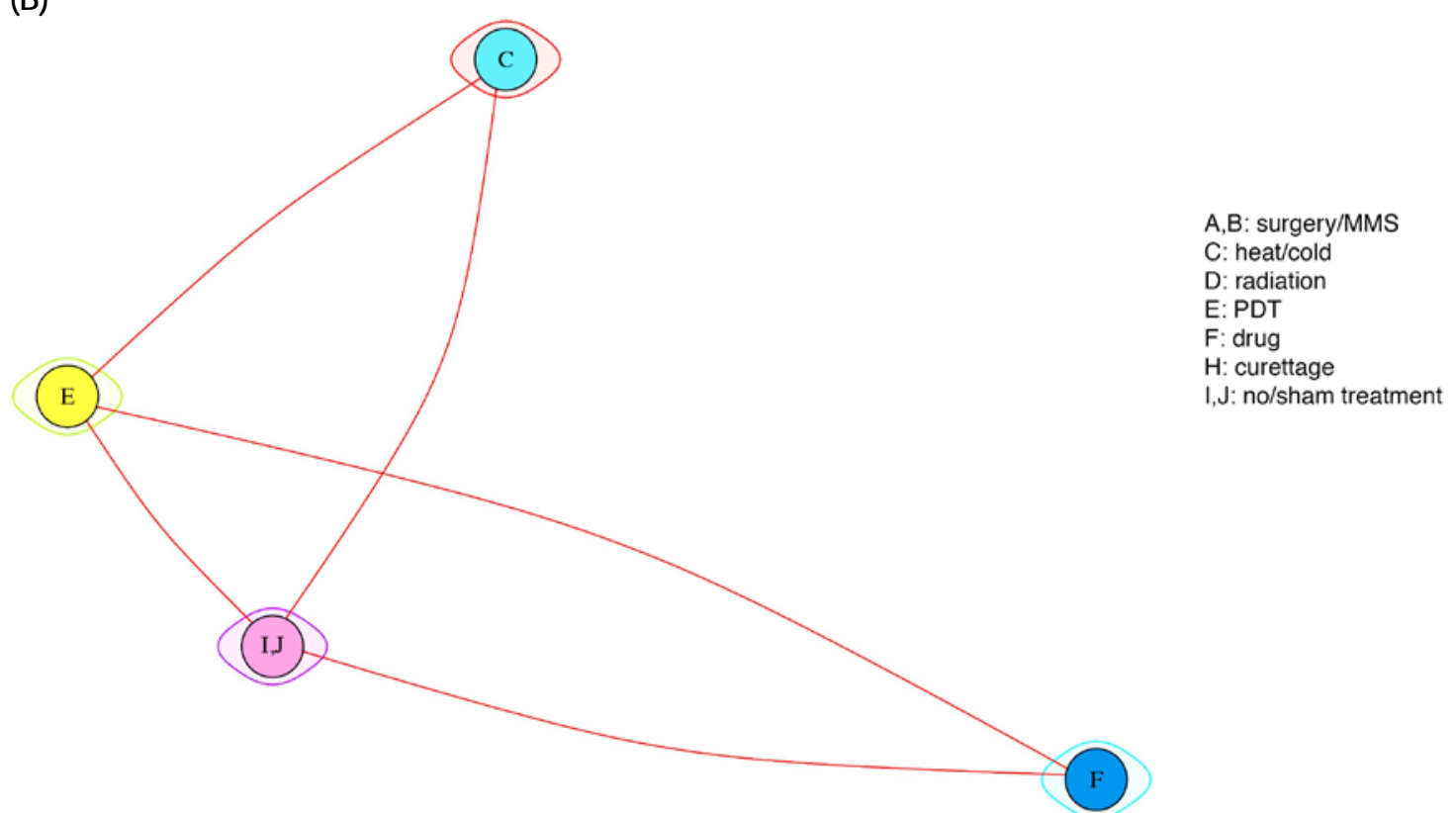

MMS = Mohs micrographic surgery; PDT = photodynamic therapy; ALA = 5-aminolevulinic acid; MAL=methyl aminolevulinate; FU = fluorouracil; INF = interferon; SCCIS = squamous cell carcinoma in situ

\section{Comparisons Across Intervention Categories}

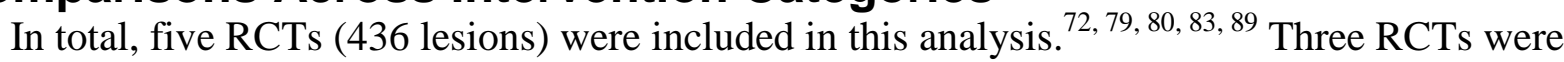
deemed to be at low or moderate risk of bias. The comparisons are described in Table 70.

Table 70. Sample information, lack of clinical clearance (SCCIS, intervention categories)

\begin{tabular}{ll}
\hline Studies (total sample) & $5(436)$ \\
\hline Total sample by intervention & $(\mathrm{C}): 166 ;(\mathrm{E}): 190 ;(\mathrm{F}): 45 ;(\mathrm{I}, \mathrm{J}): 35$ \\
\hline $\begin{array}{l}\text { Total sample by intervention, (min, } \\
\text { max) }\end{array}$ & 35,190 \\
\hline Data by comparison & $(\mathrm{C}--\mathrm{E}): 3(323) ;(\mathrm{C}--\mathrm{I}, \mathrm{J}): 1$ (133); (E--F): 1 (66); (E--I,J): $1(130) ;(\mathrm{F}--\mathrm{I}, \mathrm{J}): 1$ \\
& $(28)$ \\
\hline Studies by comparison (min, max) & 1,3 \\
\hline Total sample by comparison (min, & 28,323 \\
max) & $3(2,12)$ months \\
\hline Followup median (min, max) &
\end{tabular}

$\mathrm{A}$ = surgical excision; $\mathrm{B}=$ Mohs micrographic surgery; $\mathrm{C}=$ heat/cold; $\mathrm{D}=$ radiation; $\mathrm{E}=$ photodynamic therapy; $\mathrm{F}=$ drugs; $\mathrm{H}=$ curettage; $\mathrm{I}$ = no treatment; $\mathrm{J}$ = placebo; SCCIS = squamous cell carcinoma in situ

Table 71 shows the relative odds ratios for clinical clearance across intervention categories. There were no statistically significant differences between the active interventions, although the confidence intervals for the odds ratios were wide and could not exclude large differences in the odds of the outcome in either direction. Nevertheless, all active interventions were favored beyond chance versus placebo. 
Table 71. Relative odds ratios for lack of clinical clearance between intervention categories (SCCIS lesions, Figure 18B)

\begin{tabular}{|c|c|c|c|}
\hline $\begin{array}{l}\text { Heat/cold } \\
\text { (C) }\end{array}$ & $0.69(0.13,3.6)$ & $0.29(0.04,2.17)$ & $0.02(<0.005,0.15)$ \\
\hline $1.45(0.28,7.52)$ & $\begin{array}{l}\text { PDT } \\
\text { (E) }\end{array}$ & $0.42(0.07,2.65)$ & $0.02(<0.005,0.19)$ \\
\hline $3.42(0.46,25.34)$ & $2.37(0.38,14.83)$ & $\begin{array}{l}\text { Drugs } \\
\text { (F) }\end{array}$ & $0.06(0.01,0.58)$ \\
\hline $60.64(6.87,535.12)$ & $41.96(5.22,337.2)$ & $17.73(1.72,182.98)$ & $\begin{array}{c}\text { No/sham treatment } \\
(\mathrm{I}, \mathrm{J})\end{array}$ \\
\hline
\end{tabular}

Note: Cells shaded gray indicate that the estimate is based only on indirect comparisons; bold-italic indicates that the result is statistically significant. Results are given as odds ratios (95\% confidence intervals).

PDT = photodynamic therapy; SCCIS = squamous cell carcinoma in situ

Table 72 offers complementary information from the same analysis. The fraction of lesions without clinical clearance was between 10.8 and 29.2 percent in the active treatments and 88 percent with placebo. The confidence intervals for each estimate are wide.

Table 72. Mean and forecasted lack of clinical clearance fractions by intervention category (SCCIS lesions)

\begin{tabular}{ccc}
\hline Intervention Type & $\begin{array}{c}\text { Mean } \\
\text { Percent (95\% Cl) }\end{array}$ & $\begin{array}{c}\text { Forecast } \\
\text { Percent (95\% CI) }\end{array}$ \\
\hline Heat/cold (C) & $10.8(3.1,31.3)$ & $10.8(1.2,54.7)$ \\
\hline PDT (E) & $14.9(5.4,34.9)$ & $14.9(1.9,61.0)$ \\
\hline Drug (F) & $29.2(8.4,65.1)$ & $29.2(3.6,82.2)$ \\
\hline No/sham treatment $(\mathrm{I}, \mathrm{J})$ & $88.0(54.2,97.8)$ & $88.0(34.7,99.0)$ \\
\hline
\end{tabular}

PDT = photodynamic therapy; SCCIS = squamous cell carcinoma in situ

\section{Comparisons Across Individual Interventions}

As is evident from Figure 18, there are two connected subgraphs: a smaller one comprising the comparison between cryotherapy (C1), MAL with ALA (E2) and 5-FU (F1), and a larger one between PDT with MAL with and without laser preparation (E1 and C5+E1), cryotherapy with 5-FU (C1+F1), and placebo. In total, five RCTs (436 lesions) were included in these analyses, as summarized in Table 73.

Table 73. Sample information, lack of clinical clearance (SCCIS, interventions)

\begin{tabular}{lll}
\hline & First subgraph & \\
\hline Studies (total sample) & $3(330)$ & Second subgraph $^{80,89}$ \\
\hline Total sample by intervention & $\begin{array}{l}(\mathrm{C} 5+\mathrm{E} 1): 32 ;(\mathrm{E} 1): 137 ;(\mathrm{C} 1+\mathrm{F} 1): 114 ;(\mathrm{J}): 35 ; \\
(\mathrm{F} 2): 12\end{array}$ & $(\mathrm{C} 1): 20 ;(\mathrm{E} 2): 53 ;(\mathrm{F} 1): 33$ \\
\hline $\begin{array}{l}\text { Total sample by intervention, } \\
\text { (min, max) }\end{array}$ & 12,137 & 20,53 \\
\hline Data by comparison & $(\mathrm{C} 5+\mathrm{E} 1--\mathrm{E} 1): 1$ (58); (E1--C1+F1): 1 (225); (E1-- & $(\mathrm{C} 1--\mathrm{E} 2): 1(40) ;(\mathrm{E} 2--\mathrm{F} 1): 1$ \\
& $\mathrm{~J}): 1(130) ;(\mathrm{C} 1+\mathrm{F} 1--\mathrm{J}): 1(133) ;(\mathrm{J}-\mathrm{-F} 2): 1(28)$ & $(66)$ \\
\hline $\begin{array}{l}\text { Studies by comparison (min, } \\
\text { max) }\end{array}$ & 1,1 & 1,1 \\
\hline $\begin{array}{l}\text { Total sample by comparison } \\
\text { (min, max) }\end{array}$ & 28,225 & 40,66 \\
\hline Followup median (min, max) & $7(3,12)$ months & $2.5(2,3)$ months \\
\hline
\end{tabular}


A = surgical excision; $\mathrm{B}=$ Mohs micrographic surgery; $\mathrm{C} 1$ = cryotherapy; $\mathrm{C} 3$ = diathermy and curettage; C4 = cryotherapy and curettage; C5 = laser; D1 = external radiation; E1 = MAL photodynamic therapy; E2 = ALA photodynamic therapy; F1 = 5-FU; F2 = Imiquimod; F3 = Interferon; F4 = Ingenol; H = curettage; $\mathrm{J}=$ placebo; SCCIS = squamous cell carcinoma in situ

Table 74 shows the relative effects for both subgraphs, respectively. Because the comparisons across individual observations are sparse, however, the confidence intervals of the odds ratios for most indirect comparisons are broad and cannot exclude very large differences between the compared interventions.

Table 75 shows, for each intervention, the mean recurrence rates across all RCTs; estimates for interventions in both subgraphs are listed in Table 75. One cannot compare statistically the estimated recurrence rates between an intervention in the first subgraph (e.g., PDT with MAL [E1]) and the second subgraph (e.g., cryotherapy [C1]), because they come from disjoint analyses. 
Table 74. Relative odds ratios for lack of clinical clearance between individual interventions (SCCIS lesions, Figure 18A)

\begin{tabular}{|c|c|c|c|c|}
\hline (C) & $13.7(2.92$, & $2.11(0.88,5.06)$ & $3.04(0.21,44.58)$ & 0.04 \\
\hline .34) & ( & $0.15(0.0$ & 8) & 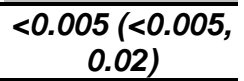 \\
\hline $0.47(0.2,1.14)$ & 3) & & 2) & 0.02 \\
\hline 0.3 & & $0.69(0$ & 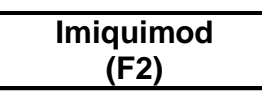 & 5, \\
\hline 22. & $310.05(51.68,1860.02)$ & 170.52) & $\begin{array}{c}68.87(5.18, \\
915.22) \\
\end{array}$ & $\begin{array}{c}\text { Placebo/s } \\
(\mathrm{J})\end{array}$ \\
\hline
\end{tabular}

\begin{tabular}{ccc}
$\begin{array}{c}\text { Cryotherapy } \\
\text { (C1) }\end{array}$ & $0.28(0.01$, & $0.06(<0.005$, \\
\hline 3.38$)$ & $1.59)$ \\
\hline 95.23$)$ & PDT (ALA) & $0.21(0.04$, \\
(E2) & $1.08)$ \\
\hline $16.9(0.63$, & $4.7(0.93$, & $\mathbf{5 - F U}$ \\
$453.4)$ & $23.88)$ & (F1) \\
\hline
\end{tabular}

Note: Cells shaded gray indicate that the estimate is based only on indirect comparisons; bold-italic indicates that the result is statistically significant. Results are given as odds ratios (95\% confidence intervals).

PDT = photodynamic therapy; ALA = 5-aminolevulinic acid; MAL = methyl aminolevulinate; FU = fluorouracil; SCCIS = squamous cell carcinoma in situ 
Table 75. Mean and forecasted lack of clinical clearance fractions by intervention (SCCIS lesions)

\begin{tabular}{lcc}
\hline Intervention Type & $\begin{array}{c}\text { Mean } \\
\text { Percent (95\% Cl) }\end{array}$ & $\begin{array}{c}\text { Forecast } \\
\text { Percent (95\% CI) }\end{array}$ \\
\hline First subgraph & & \\
\hline Cryotherapy + 5-FU (C1+F1) & $41.3(9.4,82.7)$ & $41.3(2.3,95.5)$ \\
\hline Laser + PDT (MAL) (C5+E1) & $4.9(0.6,31.0)$ & $4.9(0.1,64.4)$ \\
\hline PDT (MAL) (E1) & $25.0(4.9,68.5)$ & $25.0(1.1,90.8)$ \\
\hline Imiquimod (F2) & $18.8(1.7,75.5)$ & $18.8(0.5,91.4)$ \\
\hline Placebo (J) & $94.1(67.9,99.2)$ & $94.1(33.1,99.8)$ \\
\hline Second subgraph & & \\
\hline Cryotherapy (C1) & $2.6(0.1,35.5)$ & $2.6(0.1,40.3)$ \\
\hline PDT (ALA) (E2) & $8.8(2.4,27.6)$ & $8.8(1.6,36.4)$ \\
\hline 5-FU (F1) & $31.2(10.7,63.2)$ & $31.2(7.3,72.3)$ \\
\hline
\end{tabular}

PDT = photodynamic therapy; ALA = 5-aminolevulinic acid; MAL = methyl aminolevulinate; FU = fluorouracil; SCCIS = squamous cell carcinoma in situ; $\mathrm{CI}=$ confidence interval

\section{Lack of Clinical Clearance, Other Subgroup Analyses (Lesion Location, Lesion Size), SCCIS Lesions}

\section{Evidence From RCTS}

Table 76 shows results on subgroup analyses for a four-arm RCT. ${ }^{77,79}$ Neither lesion location nor size were associated with differences in the treatment effect beyond what is expected by chance.

Table 76. Subgroup analyses by lesion location and size: results for lack of clinical clearance (SCCIS lesions)

\begin{tabular}{|c|c|c|c|c|c|c|}
\hline Study & Comparison & Time Point & Subgroup & $\begin{array}{l}\text { n/N Arm } 1 \text { vs. } \\
\text { n/N Arm } 2 \text { vs. } \\
\text { n/N Arm } 3\end{array}$ & $\begin{array}{l}\text { OR }(95 \% \mathrm{Cl}) ; \mathrm{P}- \\
\text { Value Within }\end{array}$ & $\begin{array}{l}\text { P- Value } \\
\text { Between }\end{array}$ \\
\hline \multirow[t]{3}{*}{$\begin{array}{l}\text { Morton } 2006 \\
16785375\end{array}$} & \multirow[t]{3}{*}{$\begin{array}{l}\text { Cryotherapy (C1) or } \\
\text { 5-FU (F1) vs. MAL- } \\
\text { PDT (E1) vs. sham } \\
\text { PDT (J) }\end{array}$} & \multirow[t]{3}{*}{$\begin{array}{l}\text { after first } \\
\text { treatment }\end{array}$} & $\begin{array}{l}\text { lesion diameter: } \\
\text { 5-14 } \mathrm{mm}\end{array}$ & $\begin{array}{l}\text { 4/30 vs. } 5 / 42 \text { vs. } \\
6 / 7\end{array}$ & $\begin{array}{l}1.14(0.28,4.65) ; \\
0.03(0.00,0.27) ; \\
0.02(0.00,0.23) ; \\
p<0.001\end{array}$ & \multirow[t]{3}{*}{$p=0.457$} \\
\hline & & & $\begin{array}{l}\text { lesion diameter: } \\
15-29 \mathrm{~mm}\end{array}$ & $\begin{array}{l}21 / 65 \text { vs. } 11 / 48 \\
\text { vs. } 7 / 10\end{array}$ & $\begin{array}{l}1.61(0.69,3.76) ; \\
0.20(0.05,0.87) ; \\
0.13(0.03,0.58) ; \\
p=0.016\end{array}$ & \\
\hline & & & $\begin{array}{l}\text { lesion diameter: } \\
>=30 \mathrm{~mm}\end{array}$ & $\begin{array}{l}10 / 18 \text { vs. } 7 / 21 \\
\text { vs. } 2 / 2\end{array}$ & $\begin{array}{l}2.50(0.68,9.16) ; \\
0.25(0.01,5.87) ; \\
0.10(0.00,2.44) ; \\
p=0.102\end{array}$ & \\
\hline \multirow[t]{2}{*}{$\begin{array}{l}\text { Morton } 2006 \\
16785375\end{array}$} & \multirow[t]{2}{*}{$\begin{array}{l}\text { Cryotherapy (C1) or } \\
\text { 5-FU (F1) vs. MAL- } \\
\text { PDT (E1) vs. sham } \\
\text { PDT (J) }\end{array}$} & \multirow[t]{2}{*}{$\begin{array}{l}\text { after last } \\
\text { treatment }\end{array}$} & $\begin{array}{l}\text { lesion diameter: } \\
5-14 \mathrm{~mm}\end{array}$ & $\begin{array}{l}3 / 30 \text { vs. } 2 / 42 \text { vs. } \\
6 / 7\end{array}$ & $\begin{array}{l}2.22(0.35,14.20) \\
0.02(0.00,0.21) ; \\
0.01(0.00,0.11) ; \\
p<0.001\end{array}$ & \multirow[t]{2}{*}{$p=0.522$} \\
\hline & & & $\begin{array}{l}\text { lesion diameter: } \\
15-29 \mathrm{~mm}\end{array}$ & $\begin{array}{l}10 / 65 \text { vs. } 5 / 48 \\
\text { vs. } 7 / 10\end{array}$ & $\begin{array}{l}1.56(0.50,4.91) \\
0.08(0.02,0.35) \\
0.05(0.01,0.26) \\
p<0.001\end{array}$ & \\
\hline
\end{tabular}




\begin{tabular}{|c|c|c|c|c|c|c|}
\hline Study & Comparison & Time Point & Subgroup & $\begin{array}{l}\text { n/N Arm } 1 \text { vs. } \\
\text { n/N Arm } 2 \text { vs. } \\
\text { n/N Arm } 3\end{array}$ & $\begin{array}{l}\text { OR }(95 \% \mathrm{Cl}) ; \mathrm{P}- \\
\text { Value Within }\end{array}$ & $\begin{array}{l}\text { P- Value } \\
\text { Between }\end{array}$ \\
\hline & & & $\begin{array}{l}\text { lesion diameter: } \\
>=30 \mathrm{~mm}\end{array}$ & $\begin{array}{l}4 / 18 \text { vs. } 1 / 21 \text { vs. } \\
2 / 2\end{array}$ & $\begin{array}{l}5.71(0.58,56.73) \\
0.06(0.00,1.55) ; \\
0.01(0.00,0.47) ; \\
p=0.007\end{array}$ & \\
\hline \multirow[t]{3}{*}{$\begin{array}{l}\text { Morton } 2006 \\
16785375\end{array}$} & \multirow[t]{3}{*}{$\begin{array}{l}\text { Cryotherapy (C1) or } \\
\text { 5-FU (F1) vs. MAL- } \\
\text { PDT (E1) vs. sham } \\
\text { PDT (J) }\end{array}$} & \multirow[t]{3}{*}{$\begin{array}{l}\text { after last } \\
\text { treatment }\end{array}$} & $\begin{array}{l}\text { lesion location: } \\
\text { extremities }\end{array}$ & $\begin{array}{l}12 / 72 \text { vs. } 6 / 69 \\
\text { vs. } 11 / 12\end{array}$ & $\begin{array}{l}2.10(0.74,5.95) ; \\
0.02(0.00,0.15) ; \\
0.01(0.00,0.08) ; \\
p<0.001\end{array}$ & \multirow[t]{3}{*}{ NA } \\
\hline & & & $\begin{array}{l}\text { lesion location: } \\
\text { face/scalp }\end{array}$ & $\begin{array}{l}5 / 27 \text { vs. } 1 / 28 \text { vs. } \\
3 / 5\end{array}$ & $\begin{array}{l}1.56(0.50,56.48) \\
0.15(0.02,1.16) ; \\
0.02(0.00,0.36) ; \\
p=0.007\end{array}$ & \\
\hline & & & $\begin{array}{l}\text { lesion location: } \\
\text { neck/trunk }\end{array}$ & $\begin{array}{l}0 / 15 \text { vs. } 1 / 14 \text { vs. } \\
1 / 2\end{array}$ & $\begin{array}{l}0.29(0.01,7.74) ; \\
0.03(0.00,1.20) ; \\
0.08(0.00,2.39) ; \\
p=0.062\end{array}$ & \\
\hline
\end{tabular}

PDT = photodynamic therapy; ALA = 5-aminolevulinic acid; MAL = methyl aminolevulinate; FU = fluorouracil; NA = not significant; SCCIS = squamous cell carcinoma in situ

\section{Evidence From NRCSs}

One NRCS reported lack of clinical clearance for 80 SCCIS lesions in 67 people, treated with either curettage (44 lesions) or cryotherapy (36 lesions). This study was deemed to be of high risk of bias, primarily for lack of reporting (baseline data and dropout numbers were not given by arm), but also for lack of blinding. The mean age was 74 (range: 46 to 89), and the mean lesion area was $336 \mathrm{~mm}^{2}$ (range 30 to $1890 \mathrm{~mm}^{2}$ ). Eighty-two percent were female, and the lesions were located on the extremities (84\%), trunk (7.5\%), and head/neck (8.5\%). The cryotherapy arm had a higher rate of lack of clinical clearance at 2 weeks ( 2 of 36 vs. 0 of 44$){ }^{141}$

\section{Patient-Reported Cosmetic Outcomes, SCCIS Lesions}

We did not identify any studies with results for this outcome in this population.

\section{Observer-Reported Cosmetic Outcomes, SCCIS Lesions}

In this section, we describe only the results between intervention categories, because data are sparse for the comparison of individual observations. In total, two RCTs (204 lesions) were included in this analysis, both at low to moderate risk of bias for this outcome. ${ }^{72,79}$ The evidence graph in Figure 19 shows the observed comparisons based on RCTs that report observers' (investigators' or providers') assessments of “at least good” cosmetic outcome. The cosmetic outcome was assessed using different scales in each RCT. The evidence graph is sparsely connected. Details about the comparisons are in Table 77.

Table 77. Sample information, observer-reported cosmetic outcomes (SCCIS)

\begin{tabular}{ll}
\hline Studies (total sample) & $2(204)$ \\
\hline Total sample by intervention & $(\mathrm{C} 5+\mathrm{E} 1): 18 ;(\mathrm{E} 1): 100 ;(\mathrm{C} 1+\mathrm{F} 1): 86$ \\
\hline $\begin{array}{l}\text { Total sample by intervention, (min, } \\
\text { max) }\end{array}$ & 18,100 \\
\hline Data by comparison & (C5+E1--E1): 1 (36); (E1--C1+F1): $1(168)$ \\
\hline
\end{tabular}




\begin{tabular}{|c|c|}
\hline Studies by comparison (min, max) & 1,1 \\
\hline $\begin{array}{l}\text { Total sample by comparison (min, } \\
\text { max) }\end{array}$ & 36,168 \\
\hline Followup (min, max) & 12,12 months \\
\hline
\end{tabular}

Figure 19. Evidence graph of RCTs comparing observer-assessed cosmetic outcomes (all SCC lesions)

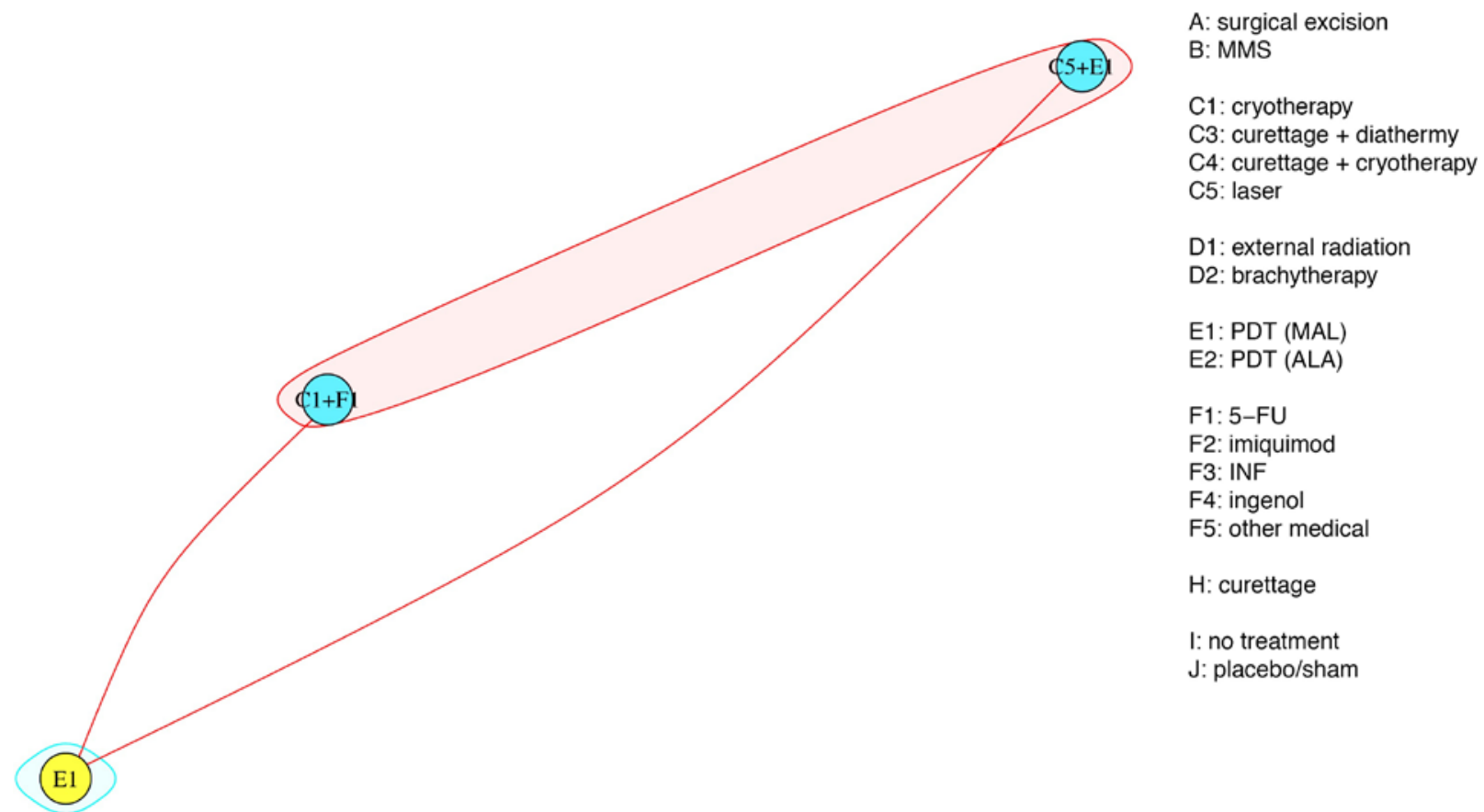

MMS = Mohs micrographic surgery; PDT = photodynamic therapy; ALA = 5-aminolevulinic acid; MAL = methyl aminolevulinate; FU = fluorouracil; INF = interferon

Table 78 has the results of the comparisons between intervention categories based on a network meta-analysis. Based on the odds ratios in Table 78, the combination of cryotherapy and 5-FU (C1+F1) had statistically significantly better observer-assessed cosmetic outcomes than PDT with MAL (E1). The other two comparisons were not statistically significant. However, based on their confidence intervals one could not exclude differences in the odds of the outcome as large as 50 percent in either direction.

Table 78. Relative odds ratios between interventions for at least good cosmetic outcome, as assessed by an observer (SCCIS lesions, Figure 19)

\begin{tabular}{|c|c|c|}
\hline $\begin{array}{c}\text { Cryotherapy+5-FU } \\
\text { (C1+F1) }\end{array}$ & $0.32(0.07,1.51)$ & $0.09(0.02,0.30)$ \\
\hline $3.1(0.66,14.5)$ & $\begin{array}{c}\text { Laser + PDT (MAL) } \\
\text { (C5+E1) }\end{array}$ & $0.26(0.04,1.71)$ \\
\hline $11.71(3.37,40.66)$ & $3.78(0.58,24.48)$ & $\begin{array}{l}\text { PDT (MAL) } \\
\text { (E1) }\end{array}$ \\
\hline
\end{tabular}


Table 79 shows the average percentage of patients with at least good cosmetic outcomes in the RCTs, based on the same network meta-analysis as the Table 78. The average number of lesions with cosmetic outcomes rated as good or excellent ranged between 72.1 and 96.8; however, the confidence intervals for these proportions were wide. Refer to Table 78 for a pairwise comparison between these treatments.

Table 79. Mean fractions of lesions with at least good cosmetic outcome, as assessed by an observer (SCCIS lesions)

\begin{tabular}{lc}
\hline Intervention & $\begin{array}{c}\text { Mean } \\
\text { percent }(95 \% \mathrm{Cl})\end{array}$ \\
\hline $\begin{array}{l}\text { Cryotherapy + 5-FU } \\
\text { (C1+F1) }\end{array}$ & $72.1(61.7,80.5)$ \\
\hline $\begin{array}{l}\text { Laser + PDT (MAL) } \\
\text { (C5+E1) }\end{array}$ & $88.9(64.8,97.2)$ \\
\hline PDT (MAL) (E1) & $96.8(90.5,99.0)$ \\
\hline
\end{tabular}

PDT = photodynamic therapy; MAL = methyl aminolevulinate; FU = fluorouracil; SCCIS = squamous cell carcinoma in situ; CI $=$ confidence interval

\section{Evidence From NRCSs}

No NRCS reported the outcome of interest in populations where the majority of lesions were SCCs. Refer to the section on this outcome in the BCC section for a description of an NRCS that included SCCs (29\%) and compared a lower dose of radiation (37 Gy) with a higher dose (45 Gy). For observer assessed cosmetic outcomes and among all lesions, the relative risk favored the lower dose, but not statistically significantly so. ${ }^{151}$

\section{Quality of Life, SCCIS Lesions}

We did not identify any studies with results for this outcome in this population.

\section{Mental Health, SCCIS Lesions}

We did not identify any studies with results for this outcome in this population.

\section{Patient Satisfaction, SCCIS Lesions}

We did not identify any studies with results for this outcome in this population.

\section{Mortality, SCCIS Lesions}

We did not identify any studies with results for this outcome in this population.

\section{Costs and Resource Use, SCCIS Lesions}

We did not identify any studies with results for this outcome in this population. 


\section{Adverse Events, All SCCIS Lesions}

We describe only results between intervention categories, because data are sparse for the comparison of individual observations. Figure 20 shows the evidence graph for the comparison of the frequency of adverse events leading to discontinuation, serious adverse events, pain after treatment completion, and infection of the treated site. Reporting of adverse events was not consistent across RCTs. Appendix I enumerates other types of adverse events that were reported.

Figure 20. Evidence graph of RCTs comparing frequency of adverse events (SCCIS lesions)

(A) Leading to treatment discontinuation

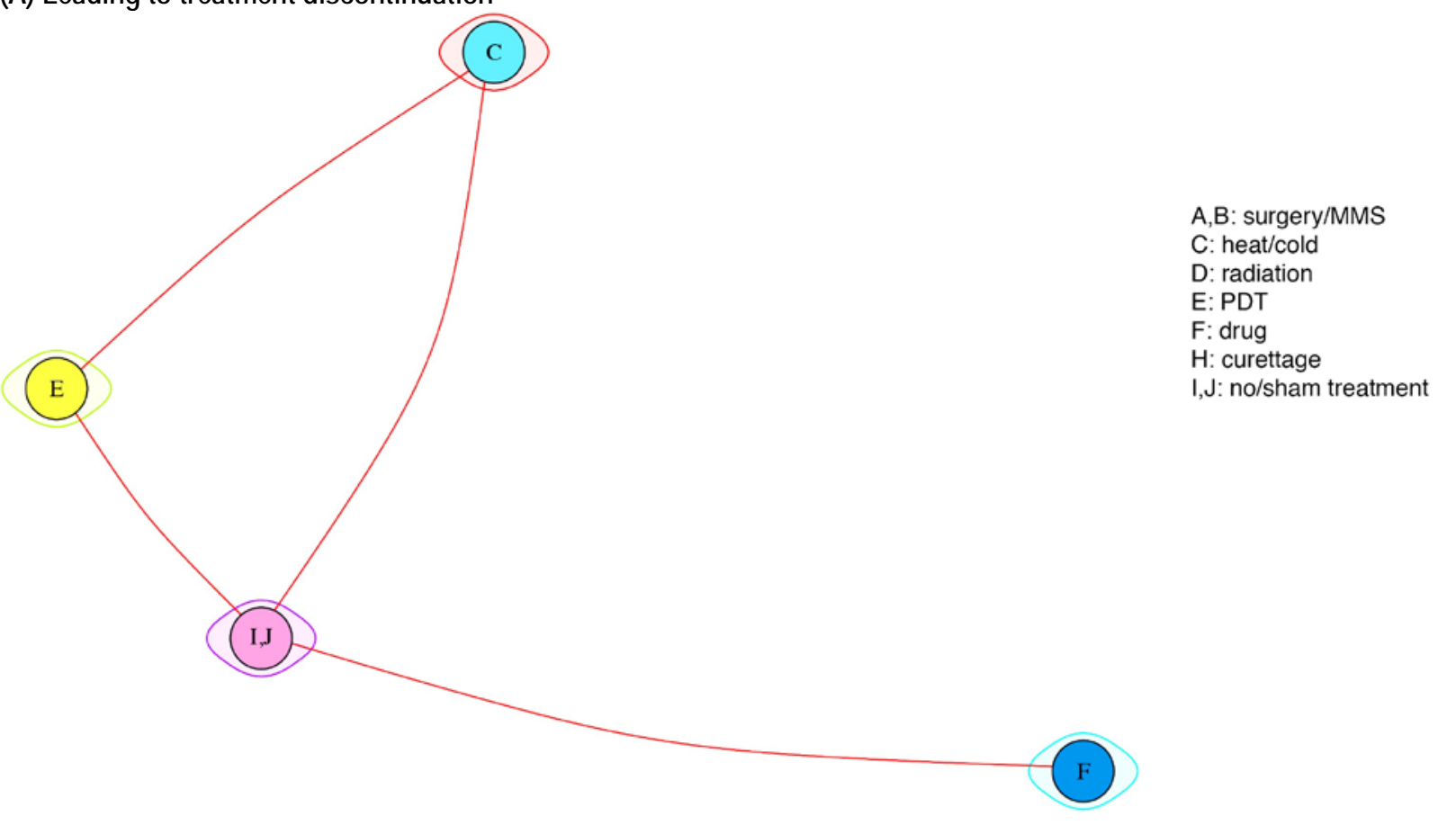


(B) Serious adverse events

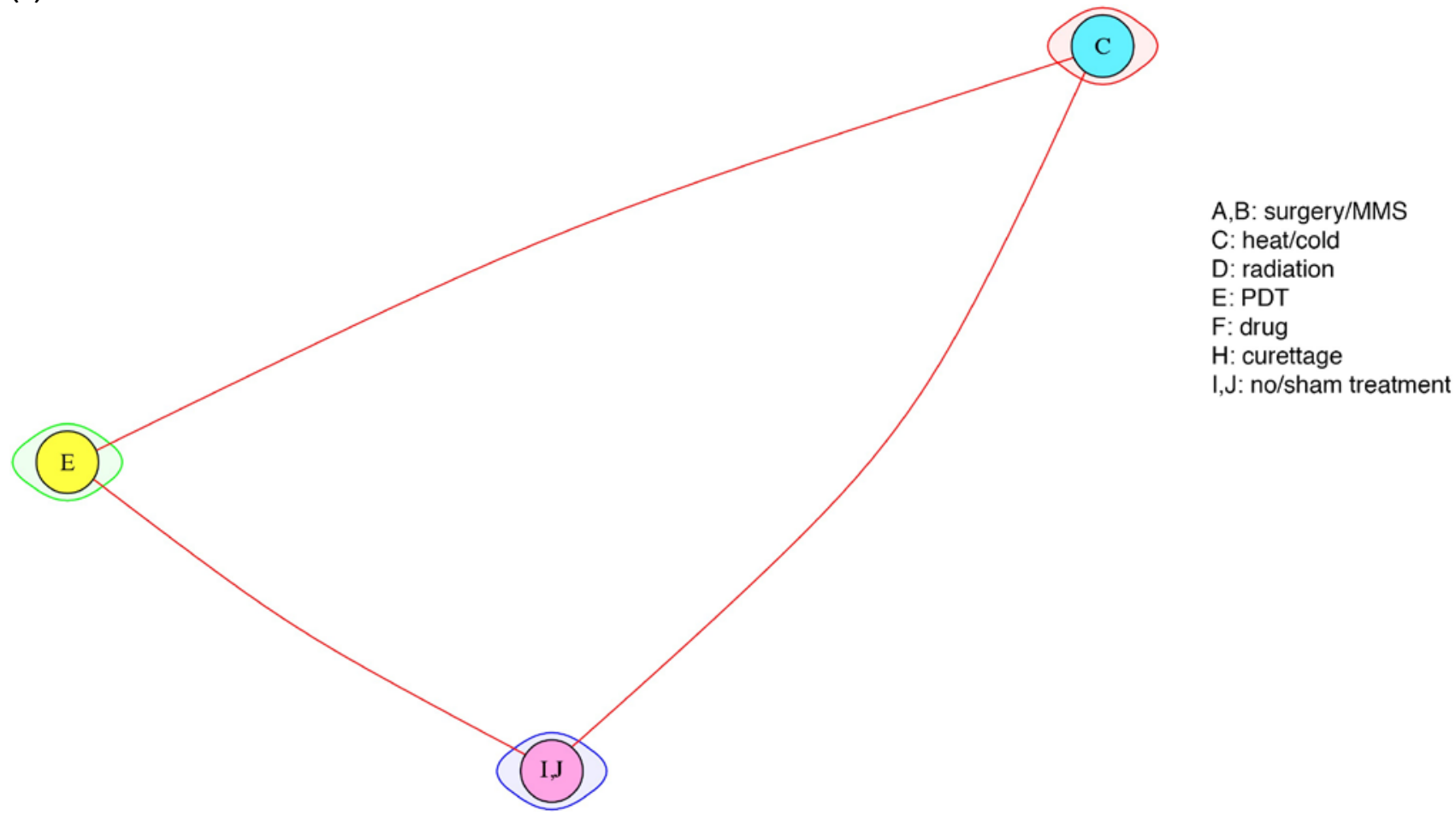

(C) Pain (after treatment completion)

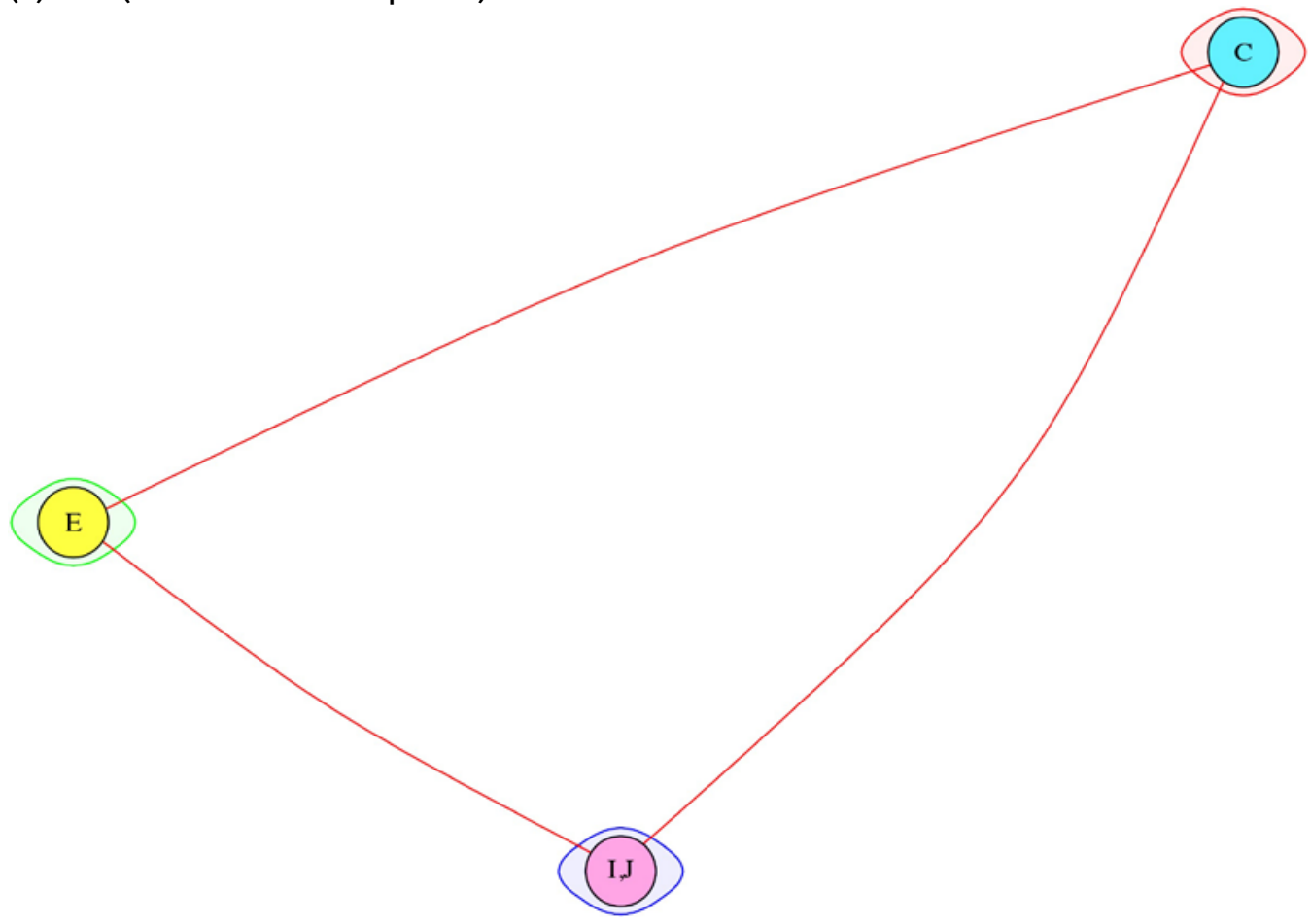

$\mathrm{A}, \mathrm{B}$ : surgery/MMS

C: heat/cold

D: radiation

E: PDT

$\mathrm{F}$ : drug

$\mathrm{H}$ : curettage

$\mathrm{I}, \mathrm{J}: \mathrm{no} /$ sham treatment 


\section{(D) Infection of the treated site}

MMS = Mohs micrographic surgery; PDT = photodynamic therapy; ALA = 5-aminolevulinic acid; $\mathrm{MAL}=$ methyl aminolevulinate; FU = fluorouracil; INF = interferon

The evidence graphs in Figure 20 are sparsely connected. For parsimony, we do not report relative effects for comparisons of the frequency of each type of adverse event. Table 80 has details about the comparisons by type of adverse event.

Table 80. Sample information, adverse events (SCCIS)

\begin{tabular}{|c|c|c|c|c|}
\hline & $\begin{array}{l}\text { Adverse Events } \\
\text { Leading to Treatment } \\
\text { Discontinuation }{ }^{72,79,83}\end{array}$ & $\begin{array}{l}\text { Serious Adverse } \\
\text { Events }^{79}\end{array}$ & $\begin{array}{l}\text { Pain After } \\
\text { Treatment }^{79,80}\end{array}$ & $\begin{array}{l}\text { Infection of } \\
\text { Treated Site }\end{array}$ \\
\hline $\begin{array}{l}\text { Studies (total } \\
\text { sample) }\end{array}$ & $3(292)$ & $1(225)$ & $2(265)$ & $1(36)$ \\
\hline $\begin{array}{l}\text { Total sample by } \\
\text { intervention }\end{array}$ & $\begin{array}{l}(\mathrm{C}): 130 ;(\mathrm{E}): 114 ;(\mathrm{I}, \mathrm{J}): \\
\text { 33; (F): } 15\end{array}$ & $(\mathrm{C}): 112 ;(\mathrm{E}): 96 ;(\mathrm{I}, \mathrm{J}): 17$ & $\begin{array}{l}\text { (C): 132; (E): } \\
116 ;(\mathrm{I}, \mathrm{J}): 17\end{array}$ & $(\mathrm{C}): 18 ;(\mathrm{E}): 18$ \\
\hline $\begin{array}{l}\text { Total sample by } \\
\text { intervention, } \\
\text { (min, max) }\end{array}$ & 15,130 & 17,112 & 17,132 & 18,18 \\
\hline $\begin{array}{l}\text { Data by } \\
\text { comparison }\end{array}$ & $\begin{array}{l}\text { (C--E): } 2(244) ;(C--I, J): 1 \\
(129) ;(E--I, J): 1 \text { (113); } \\
(I, J--F): 1(31)\end{array}$ & $\begin{array}{l}\text { (C--E): } 1(208) ;(C--I, J): 1 \\
(129) ;(E--I, J): 1 \text { (113) }\end{array}$ & $\begin{array}{l}\text { (C--E): } 2 \text { (248); } \\
(\mathrm{C}--\mathrm{I}, \mathrm{J}): 1(129) \\
(\mathrm{E}--\mathrm{I}, \mathrm{J}): 1 \text { (113) }\end{array}$ & (C--E): $1(36)$ \\
\hline $\begin{array}{l}\text { Studies by } \\
\text { comparison (min, } \\
\text { max) }\end{array}$ & 1,2 & 1,1 & 1,2 & 1,1 \\
\hline $\begin{array}{l}\text { Total sample by } \\
\text { comparison ( } \min , \\
\text { max) }\end{array}$ & 31,244 & 113,208 & 113,248 & 36,36 \\
\hline $\begin{array}{l}\text { Followup median } \\
\text { (min, max) }\end{array}$ & [during treatment] & $3(3,3)$ months & $\begin{array}{l}1.5(0.3,3) \\
\text { months }\end{array}$ & 1 week \\
\hline
\end{tabular}


We report rates of adverse events per intervention category, based on a joint analysis of all RCTs reporting the same outcome. Most likely, adverse events were defined differently across studies, but these definitions were often not clearly described. Results for different types of adverse events, as defined by each study, are in Table 81 and come from different analyses.

Drugs had the highest rate of adverse events leading to treatment discontinuation was (13.3\%; 95\% CI, 3.4 to 40.5); the rate for interventions destroying the lesion with heat or cold was 2.0 percent (C). This outcome was not applicable for PDT, because it is a one-time intervention.

The frequency of adverse events characterized as "serious” by the investigators was smaller than 1 percent for all intervention categories.

Rates of pain after treatment ranged between 23.4 and 34.1 percent (including sham treatments).

The outcome of infection at the treatment site was reported in a single RCT at 0 percent. $^{68}$

Table 81. Mean fractions of adverse events, using each RCT's definitions (SCCIS lesions)

\begin{tabular}{|c|c|c|c|c|c|c|}
\hline \multirow[t]{2}{*}{$\begin{array}{l}\text { Intervention } \\
\text { Type }\end{array}$} & \multicolumn{2}{|c|}{$\begin{array}{c}\text { Leading to } \\
\text { Discontinuation (Figure } \\
20 \mathrm{~A})\end{array}$} & \multirow{2}{*}{$\begin{array}{c}\text { Serious } \\
\text { (Figure } 20 \text { B)* } \\
\text { Mean }\end{array}$} & \multicolumn{2}{|c|}{$\begin{array}{l}\text { Pain After Treatment } \\
\text { (Figure } 20 \mathrm{C} \text { ) }\end{array}$} & \multirow{2}{*}{$\begin{array}{c}\text { Infection of } \\
\text { the Treated } \\
\text { Site } \\
\text { (Figure } 20 \\
\text { D)* } \\
\text { Mean }\end{array}$} \\
\hline & Mean & Forecast & & Mean & Forecast & \\
\hline Heat/cold (C) & $\begin{array}{c}1.9(0.6 \\
6.4)\end{array}$ & $1.9(0.6,6.4)$ & $0.9(0.1,6.1)$ & $\begin{array}{c}34.1(20.0 \\
51.6)\end{array}$ & $\begin{array}{c}34.1(14.7 \\
60.9)\end{array}$ & $0(0,31)$ \\
\hline PDT (E) & $\begin{array}{c}\text { Not } \\
\text { defined }^{* *}\end{array}$ & $\begin{array}{c}\text { Not } \\
\text { defined }^{\star *}\end{array}$ & $0.5(0.0,7.7)$ & $\begin{array}{c}23.4(12.4 \\
39.5)\end{array}$ & $\begin{array}{c}23.4(9.0 \\
48.5)\end{array}$ & $0(0,31)$ \\
\hline Drugs (F) & $\begin{array}{c}13.3(3.4 \\
40.5)\end{array}$ & $\begin{array}{c}13.3(3.4 \\
40.5)\end{array}$ & NA & NA & NA & NA \\
\hline $\begin{array}{l}\text { No/sham } \\
\text { treatment }(\mathrm{I}, \mathrm{J})\end{array}$ & $\begin{array}{c}4.7(0.9 \\
20.1)\end{array}$ & $\begin{array}{c}4.7(0.9, \\
20.1)\end{array}$ & $0(0,32.2)$ & $\begin{array}{c}28.4(9.7 \\
59.3)\end{array}$ & $\begin{array}{c}28.4(7.8 \\
65.0)\end{array}$ & NA \\
\hline
\end{tabular}

Note: Letters in outcomes refer to Figure 20. Results are given as percent and 95 percent confidence interval.

PDT = photodynamic therapy; NA = not applicable; SCCIS = squamous cell carcinoma in situ; RCT = randomized controlled trial

* No forecasts for these outcomes (fixed effects analyses only).

** PDT is a one-time treatment; discontinuation is not defined, but for parsimony, it was entered as 0 in the analysis.

\section{Evidence From NRCSs}

One NRCS reported pain for 80 SCCIS lesions in 67 people, treated with either curettage (44 lesions) or cryotherapy (36 lesions). The mean age was 74 (range: 46 to 89), and the mean lesion area was $336 \mathrm{~mm} 2$ (range 30 to $1890 \mathrm{~mm}$ ). Eighty-two percent were female, and the lesions were located on the extremities (84\%), trunk (7.5\%), and head/neck (8.5\%). The cryotherapy arm had a significantly higher patient-reported pain during the treatment to 1 day after the procedure (OR 10.4; P-value <0.001). ${ }^{141}$

\section{Microinvasive SCC Lesions}

We found one RCT that compared MAL-PDT with laser-primed PDT in 45 people. The mean age was 76 and the majority of lesions were on the face or scalp in both arms (75\% in the MAL-PDT arm and 76.2\% in the laser-primed PDT arm). The rest of the lesions were on the 
extremities (16.7\% and 19\%, respectively) and trunk/neck (8.3\% and $4.8 \%$, respectively). The majority of tumors were moderately differentiated (75\% in the MAL-PDT arm and $81 \%$ in the laser-primed PDT arm), while the rest were poorly differentiated. Risk of bias for this study was judged to be low (details in Table 59 ). ${ }^{43}$

\section{Recurrence, Microinvasive SCC Lesions}

At 12 months, recurrence rates were significantly lower in the laser-primed PDT arm (2 of 16 patients; $12.5 \%$; 95\% CI $0.1 \%$, 29.2\%) than in the MAL-PDT arm (7 of 11 patients; $63.6 \%$; $95 \%$ CI 33.8\%; 93.5\%; P between arms 0.006). At 24 months, the difference remained significantly lower in the laser-primed PDT arm (3 of 16 patients; 18.8\%; 95\% CI 0.1\%, 38.5\%) than in the MAL-PDT arm (8 of 11 patients; 95\% CI 45.1\%, 99.9\%; P between arms 0.005 ). ${ }^{43}$

\section{Lack of Histological Clearance, Microinvasive SCC Lesions}

Lack of histological clearance was measured at 3, 12, and 24 months. At each time point, lack of histological clearance was lower in the laser-primed arm. At three months, 3 of 19 participants in the laser-primed PDT arm showed a lack of histological clearance (15.8\%) compared to $47.6 \%$ in the MAL-PDT arm ( $\mathrm{P}=0.03)$. At 12 months, 5 of 19 participants in the laser-primed PDT arm showed a lack of histological clearance (26.3\%) compared to 17 of 21 (80.9\%) in the MAL-PDT arm ( $\mathrm{P}=0.001)$. Finally, at 24 months, 6 of 19 participants in the laserprimed PDT arm showed a lack of histological clearance (31.6\%) compared to 18 of 21 (85.7\%) in the MAL-PDT arm $(\mathrm{P}=0.001){ }^{43}$

\section{Observer-Reported Cosmetic Outcomes, Microinvasive SCC Lesions}

Cosmetic outcomes were only reported for patients who had a complete response at 24 months, so the sample sizes were too small to detect statistical significance. In the laser-primed PDT arm, observer-reported cosmetic outcome were at least good in 10 of 13 people (77\%). In the MAL-PDT arm, they were at least good in 2 of $3(67 \%) .{ }^{43}$

\section{Adverse Events, Microinvasive SCC Lesions}

Everyone in both arms reported at least one adverse event, which included erythema, crusting, hyperpigmentation, pruritus, edema, bullae, and burning sensations. A higher percentage of patients in the laser-primed PDT arm reported each adverse event than in the MAL-PDT arm, but the difference was not statistically significant.

Everyone in both arms also reported experiencing pain during the procedure, with similar mean VAS scores for laser-primed PDT (6.0; SD, 1.7) and MAL-PDT (5.7; SD, 1.7; P = 0.59). ${ }^{43}$ 


\section{Discussion}

\section{Evidence Summary}

Tables 82 and 83 summarize our conclusions on comparisons between types of intervention for treating basal cell carcinomas (BCCs) and squamous cell carcinoma in situ (SCCIS).

The conclusions in the Tables are general and do not cover all the analyses we explored. We estimated effects for 213 comparisons between intervention categories and 565 comparisons between individual interventions for the outcomes of interest, not counting information from dose-response analyses (e.g., Table 58) and from nonrandomized studies. Providing conclusions and rating the "strength of the evidence" for each of these hundreds of comparisons is not productive. Consumers of our report who have specific interests should consult the pertinent results.

Within the existing evidence, with respect to BCC recurrence, surgical treatments and radiation therapy appear to be (statistically significantly) better than interventions that destroy lesions with heat or cold, photodynamic therapy (PDT), or curettage. However, PDT was associated with improved cosmetic outcomes. With regards to drugs for the treatment of BCC, recurrence rates with imiquimod were not significantly different than with surgical excision in a single large randomized controlled trial (RCT) (Table 8).

Given that lack of recurrence is, essentially, cure from disease, these results support the use of surgical and radiation treatment for low-risk BCC. For SCCIS, the use of cryotherapy and PDT is supported over topical 5-fluorouracil with regards to recurrence. However, how these treatments perform for SCCIS compared with surgical treatments, which are commonly used in clinical practice, is not ascertainable based on the currently available evidence.

For patients and clinicians, though, cure is not the only important endpoint. Surgery, radiation and each of the other treatments under study are associated with benefits and drawbacks that patients and clinicians consider routinely. For example, while external beam radiation therapy is effective, its remote sequelae, such as skin atrophy and the development of secondary tumors, make it less advisable for younger patients. For patients for whom cosmesis is a primary concern, treatment with PDT may be preferable despite its higher recurrence rates. Despite sparse evidence on their ability to cure BCC and SCCIS, some patients may prefer the convenience provided by topical medical treatments such as 5-fluorouracil and imiquimod which can be applied by the patient at home; this contrasts with the multiple visits to hospitals or specialty clinics required for radiation therapy which are not be practical for some patients. Access to treatments will also impact clinical decisionmaking. Specialty care is not available in all communities; while primary care physicians can perform basic surgical procedures and prescribe topical medications, they do not have access to specialized treatments such as Mohs micrographic surgery (MMS), radiotherapy, and PDT.

We acknowledge that the clinical applicability of some of these results is limited. The analyses cannot adequately account for heterogeneity of the populations in included studies, particularly for low-risk BCCs, because, although the RCTs had comparable populations (see Tables 3-6), many did not stratify their results by histologic subtype (superficial or nodular) or location. Thus, we were unable to incorporate these important factors into the analyses. For example, radiation (because of its expense and poor cosmetic outcomes) is rarely used in routine clinical practice to treat low-risk BCC; its use is generally limited to patients with high risk or recurrent disease or for patients with contraindications to surgery. However, the four RCTs that 
included radiation arms did not differ significantly in population from the other studies included in the low-risk BCC network, with the exception that they included a larger percentage of lesions in high-risk (face, eyelids) areas. Conversely, use of topical drugs is generally limited to primary, superficial tumors. Therefore, comparisons of the efficacy of radiation and drugs for the low-risk BCCs included in our study may not be relevant in the clinical decision making for most patients and clinicians. That said, the analysis contains an RCT that looks at the direct comparison of radiation and imiquimod in a high-risk location (eyelids), so it might be that they are more relevant for low-risk lesions in high-risk locations. ${ }^{67}$

Perhaps the most striking observation is the dearth of information that is available comparing interventions for these very common cancers. For example, consider comparisons between interventions for BCC lesion recurrence (Figure 7), a most important outcome from a clinical, public health and cost perspective.

Within the existing evidence, with respect to BCC recurrence, surgical treatments and radiation therapy appear to be (statistically significantly) better than interventions that destroy lesions with heat or cold, PDT, or curettage. A comparison of surgery and imiquimod showed that surgery was not significantly better in terms of recurrence. Only 13 RCTs (n=1664 lesions) examining BCC recurrence were included, of which only 20 were treated with curettage. Further, the amount of evidence in the 10 comparisons with head to head data was limited: the number of RCTs per comparison ranged between 1 and 3 , and the cumulative number of lesions ranged between 27 and 355.

For SCCs, data on recurrence are even sparser. First, only one RCT examined invasive SCCs, the subgroup of lesions that are most likely to recur or metastasize, and thus most important to evaluate. In clinical practice, these lesions are routinely treated with surgical excision with or without intraoperative margin evaluation, and in most cases are considered appropriate for Mohs surgery in the American Academy of Dermatology appropriate use criteria. ${ }^{159}$ Radiation is also commonly used for invasive SCC. The lack of evidence comparing efficacy among these commonly used treatments is striking.

For SCCISs, only 4 RCTs (348 lesions) compared 4 types of interventions, namely a drug (imiquimod), interventions that destroy lesions with heat or cold, PDT, and sham treatments (Figure 16 (B) and Table 61). Note that surgical interventions, radiation therapy and curettage, therapies commonly used in clinical practice, were not examined. 
Table 82. Summary conclusions for BCC lesions and strength of the relevant evidence

\begin{tabular}{ll} 
Conclusion statement & $\begin{array}{l}\text { RoB } \\
\text { (evidence } \\
\text {-base) }\end{array}$ \\
\hline
\end{tabular}

nce, all BCC

(1) Surgical interventions $(A, B)$ and radiation (D) were associated with lower recurrence rates than interventions that destroy lesions with heat or cold (C), and PDT (E) (moderate to high strength of evidence)

(2) Curettage $(H)$ may have higher recurrence rates than surgical interventions $(A, B)$ or radiation (D)

(3) Imiquimod ( $F)$ was associated with recurrence rates that were not significantly different than that of surgical interventions $(A, B)$

(4) [Imprecise data on the comparison on curettage and interventions that destroy lesions with heat or cold (C) or PDT (E)]

Histologic clearance, all BCC

(1) Surgical interventions $(A, B)$ were associated with better histological clearance outcomes and were statistically significantly better than interventions that destroy lesions with heat or cold (C), PDT $(\mathrm{E})$, drugs $(\mathrm{F})$, and placebo $(\mathrm{l}, \mathrm{J})$.

(2) Interventions that destroy lesions with hea or cold (C), PDT (E), and drugs (F) have better histological outcomes than placebo $(\mathrm{I}, \mathrm{J})$

(3) [imprecise data on the relative comparisons of nonsurgical active interventions]

Clinical clearance, all BCC

(1) Surgical interventions $(A, B)$ were associated with better clinical clearance outcomes than PDT (E), drugs (F) and placebo $(I, J)$

(2) All active treatments were associated with better clinical clearance outcomes than placebo

(3) [Imprecise data on relative comparisons between nonsurgical active treatments]
Moderate

Possibly

consistent

(No robust

indications of

inconsistency)

Mix of direct

comparison from and indirect

precise to data

imprecise.

Moderate

Possibly

consistent

(No robust

indications of

inconsistency)

Varies by

mparison from and indirect

precise to

imprecise.

data
(1) Moderate to High

(2) LoW

(3) Low

(4) [Insufficient]
- Surgery/MMS $(A, B)$ had significantly fewer recurrences than heat/cold, PDT, and curettage; not significantly fewer than radiation; and not significantly more than drugs (7 RCTs; 2 NRCSs)

- Heat/cold (C) interventions had significantly more recurrences than surgery and radiation; not significantly more than drugs and curettage, and not significantly fewer than PDT (7 RCTs)

- Radiation (D) had significantly fewer recurrences than thermal interventions and PDT, not significantly fewer than curettage, and not significantly more than surgery and drugs (3 RCTs)

- PDT (E) had significantly more recurrences than radiation and surgery, and not significantly more than heat/cold, drugs, and curettage (6 RCTs, 1 NRCS)

- Imiquimod (F) had more recurrences than surgery, but not significantly so (1 RCT)

- Curettage $(\mathrm{H})$ had significantly more recurrences than surgery, not significantly more recurrences than drugs and radiation, and not significantly fewer recurrences than PDT and heat/cold (2 RCTs)

- Surgery $(A, B)$ performed significantly better than heat/cold, drugs, and placebo, and nonsignificantly better than PDT (2 RCTs)

- Thermal interventions (C) performed significantly better than placebo, nonsignificantly better than drugs, nonsignificantly worse than PDT, and significantly worse than surgery (2 RCTs)

- PDT (E) performed significantly better than placebo, nonsignificantly better than drugs and heat/cold, and nonsignificantly worse than surgery (7 RCTs, 1 NRCS)

- Drugs (F) performed significantly better than placebo, nonsignificantly worse than PDT and heat/cold, and significantly worse than surgery (8 RCTs, 2 (NRCSs)

$\begin{array}{llll}\text { Moderate } & \text { Possibly } & \text { Varies by } & \text { Mix of direct } \\ & \text { consistent } & \text { comparison from } & \text { and indirect } \\ \text { (No robust } & \text { precise to } & \text { data } \\ \text { indications of } & \text { imprecise. } & \\ \text { inconsistency) } & & \end{array}$

(1) High

(2) Moderate to

high

- Surgery $(A, B)$ performed statistically significantly better than drugs and placebo and nonsignificantly better than heat/cold and PDT (4 RCTs); this comparison is less relevant as surgery ought to achieve $100 \%$ clinical clearance

(3) [Insufficient] - Thermal interventions (C)performed statistically significantly better than plecebo nonsignificantly better than drugs and PDT, and nonsignificantly worse than surgery (3 RCTs)

- PDT (E) performed statistically significantly better than placebo, nonsignificantly better than drugs, and nonsignificantly worse than surgery and heat/cold (7 RCTs) 


\begin{tabular}{|c|c|c|c|c|c|c|}
\hline Conclusion statement & $\begin{array}{l}\text { RoB } \\
\text { (evidence } \\
\text {-base) } \\
\end{array}$ & Consistency & Precision & Directness & $\begin{array}{l}\text { Overall } \\
\text { Rating }\end{array}$ & Comments \\
\hline & & & & & & $\begin{array}{l}\text { Drugs (F) performed statistically significantly better than placebo, } \\
\text { nonsignificantly worse than PDT and heat/cold, and significantly worse than } \\
\text { surgery (5 RCTs) }\end{array}$ \\
\hline \multicolumn{7}{|l|}{ Patient-reported cosmetic outcomes, all BCC } \\
\hline $\begin{array}{l}\text { (1) PDT is associated with better cosmetic } \\
\text { outcomes than other intervention } \\
\text { categories } \\
\text { (2) [Imprecise data on relative comparisons } \\
\text { between nonsurgical active intervention } \\
\text { categories] }\end{array}$ & Moderate & $\begin{array}{l}\text { Possibly } \\
\text { consistent } \\
\text { (No robust } \\
\text { indications of } \\
\text { inconsistency) }\end{array}$ & $\begin{array}{l}\text { Varies by } \\
\text { comparison from } \\
\text { precise to } \\
\text { imprecise. } \\
\text { Imprecise for } \\
\text { most } \\
\text { comparisons }\end{array}$ & $\begin{array}{l}\text { Mix of direct } \\
\text { and indirect } \\
\text { data (most } \\
\text { comparisons } \\
\text { based on } \\
\text { indirect data) }\end{array}$ & $\begin{array}{l}\text { (1) Low } \\
\text { (2) Insufficient }\end{array}$ & $\begin{array}{l}\text { - Surgery(A,B) had significantly better outcomes than heat/cold and radiation, } \\
\text { significantly worse outcomes than PDT, and nonsignificantly worse outcomes } \\
\text { than drugs (4 RCTs) } \\
\text { - Thermal interventions (C)had significantly worse outcomes than surgery and } \\
\text { PDT and nonsignificantly worse than radiation and drugs ( } 2 \text { RCTs) } \\
\text { - Radiation (D) had nonsignificantly better outcomes than heat/cold, } \\
\text { nonsignificantly worse outcomes than drugs, and significantly worse outcomes } \\
\text { than PDT and surgery ( } 2 \text { RCTs) } \\
\text { - PDT (E) had significantly better outcomes than surgery, heat/cold, and radiation } \\
\text { and nonsignificantly better outcomes than drugs ( } 4 \text { RCTs) } \\
\text { - Drugs (F) had better outcomes than surgery, heat/cold, and radiation, and } \\
\text { nonsignificantly worse outcomes than PDT, but not statistically significantly so (1 } \\
\text { RCT) }\end{array}$ \\
\hline \multirow[b]{2}{*}{$\begin{array}{l}\text { Observer-reported cosmetic outcomes, all } \\
\text { BCC } \\
\text { (1) PDT is associated with significantly better } \\
\text { cosmetic outcomes than surgery }(A, B) \\
\text { (2) [PDT may be associated with better } \\
\text { cosmetic outcomes compared to } \\
\text { nonsurgical active intervention categories] } \\
\text { (3) [Imprecise data on relative comparisons } \\
\text { between heat/cold (C), radiation, and } \\
\text { drugs (D)] }\end{array}$} & & & & & & \\
\hline & Moderate & $\begin{array}{l}\text { Possibly } \\
\text { consistent } \\
\text { (No robust } \\
\text { indications of } \\
\text { inconsistency) }\end{array}$ & $\begin{array}{l}\text { Varies by } \\
\text { comparison from } \\
\text { precise to } \\
\text { imprecise. } \\
\text { Imprecise for } \\
\text { most } \\
\text { comparisons }\end{array}$ & $\begin{array}{l}\text { Mix of direct } \\
\text { and indirect } \\
\text { data (most } \\
\text { comparisons } \\
\text { based on } \\
\text { indirect data) }\end{array}$ & $\begin{array}{l}\text { (1) Moderate } \\
\text { (2) [Insufficient] } \\
\text { (3) [Insufficient] }\end{array}$ & $\begin{array}{l}\text { - Surgery(A,B) had nonsignificantly better outcomes than radiation, significantly } \\
\text { worse outcomes than PDT, and nonsignificantly worse outcomes than drugs, } \\
\text { heat/cold, and placebo (4 RCTs, } 1 \text { NRCS) } \\
\text { - Heat/cold interventions (C) had significantly better outcomes than radiation, } \\
\text { nonsignificantly better outcomes than surgery, and nonsignificantly worse } \\
\text { outcomes than PDT, drugs, and placebo (1 RCT) } \\
\text { - Radiation (D) had significantly worse outcomes than heat/cold, PDT, drugs, and } \\
\text { placebo, and nonsignificantly worse outcomes than surgery (1 RCT, 2 NRCS) } \\
\text { - PDT (E) had significantly better outcomes than surgery and radiation, } \\
\text { nonsignificantly better outcomes than drugs and heat/cold, and nonsignificantly } \\
\text { worse outcomes than placebo (7 RCTs, } 1 \text { NRCS) } \\
\text { - Drugs (F) had significantly better outcomes than radiation, nonsignificantly better } \\
\text { outcomes than surgery and heat/cold, and nonsignificantly worse outcomes than } \\
\text { PDT and placebo (1 RCT) }\end{array}$ \\
\hline Adverse effects, all BCC & & & & & & \\
\hline
\end{tabular}




\begin{tabular}{|c|c|c|c|c|c|c|}
\hline Conclusion statement & $\begin{array}{l}\text { RoB } \\
\text { (evidence } \\
\text {-base) }\end{array}$ & Consistency & Precision & Directness & $\begin{array}{l}\text { Overall } \\
\text { Rating }\end{array}$ & Comments \\
\hline $\begin{array}{l}\text { (1) Serious adverse events, adverse events } \\
\text { leading to discontinuation and infections } \\
\text { of the treated site are uncommon with } \\
\text { surgical interventions (A,B), heat or cold } \\
\text { (C), PDT (E) and drugs (F) } \\
\text { (2) For the interventions above, on average, } 1 \\
\text { in } 10 \text { to } 1 \text { in } 5 \text { patients report experiencing } \\
\text { pain after treatment }\end{array}$ & $\begin{array}{l}\text { High } \\
\text { (selective } \\
\text { reporting } \\
\text { bias) }\end{array}$ & $\begin{array}{l}\text { Unclear } \\
\text { (Consistency } \\
\text { cannot be } \\
\text { assessed) }\end{array}$ & $\begin{array}{l}\text { Imprecise } \\
\text { We do not report } \\
\text { relative effects. } \\
\text { Forecasted } \\
\text { percentages of } \\
\text { patients with } \\
\text { adverse events } \\
\text { have wide } 95 \% \\
\text { Cls }\end{array}$ & $\begin{array}{l}\text { Mix of direct } \\
\text { and indirect } \\
\text { data (most } \\
\text { comparisons } \\
\text { based on } \\
\text { indirect data) }\end{array}$ & $\begin{array}{l}\text { (1) Moderate } \\
\text { (2) Low }\end{array}$ & $\begin{array}{l}\text { - For active interventions, point estimates for percentage of discontinuation of } \\
\text { treatment, serious adverse events, and infection of the treatment site range from } \\
\text { O/not defined to 5.5\%. Forecast Cls are wide (as high as 29\%) } \\
\text { - For active interventions, point estimates for the percentage of pain after } \\
\text { treatment range between } 9.9 \text { and } 21.6 \% \text {. Forecast Cls are wide (as high as } \\
88 \% \text { ) }\end{array}$ \\
\hline \multicolumn{7}{|l|}{ Other outcomes, all BCC } \\
\hline $\begin{array}{l}\text { [Evidence on quality of life, mental health, } \\
\text { patient satisfaction, mortality, cost and } \\
\text { resource use is reported in a minority of } \\
\text { studies and its strength not rated] } \\
\text { Other analyses }\end{array}$ & [Not rated] & [Not rated] & [Not rated] & [Not rated] & [Not rated] & [Not rated] \\
\hline $\begin{array}{l}\text { [Subgroup analyses and analyses focusing on } \\
\text { individual interventions are generally } \\
\text { sparse and are not rated] }\end{array}$ & [Not rated] & [Not rated] & [Not rated] & [Not rated] & [Not rated] & [Not rated] \\
\hline
\end{tabular}

Note: When a summary conclusion cannot be made, the description is given in square brackets.

RoB = risk of bias; $\mathrm{BCC}=$ basal cell carcinoma; $\mathrm{SCC}=$ squamous cell carcinoma; MMS = Mohs micrographic surgery; PDT = photodynamic therapy; RCT = randomized controlled trial; NRCS = nonrandomized comparative study; $\mathrm{CI}$ = confidence interval 
Table 83. Summary conclusions for SCCIS lesions and strength of the relevant evidence

\begin{tabular}{|c|c|c|c|c|c|c|}
\hline Conclusion statement & $\begin{array}{l}\text { RoB } \\
\text { (evidence } \\
\text {-base) } \\
\end{array}$ & Consistency & Precision & Directness & $\begin{array}{l}\text { Overall } \\
\text { Rating }\end{array}$ & Comments \\
\hline \multicolumn{7}{|l|}{ Recurrence, SCCIS } \\
\hline $\begin{array}{l}\text { (1) Interventions that destroy the lesions with } \\
\text { heat or cold }(\mathrm{C}) \text { and PDT }(\mathrm{E}) \text { were } \\
\text { associated with lower recurrence rates } \\
\text { than } 5 \mathrm{FU}(\mathrm{F}) \\
\text { (2) [Imprecise data on the relative effect of } \\
\text { thermal interventions versus PDT] }\end{array}$ & Moderate & $\begin{array}{l}\text { Possibly } \\
\text { consistent } \\
\text { (No robust } \\
\text { indications of } \\
\text { inconsistency) }\end{array}$ & $\begin{array}{l}\text { Moderately } \\
\text { precise. } \\
\text { Varies by } \\
\text { comparison from } \\
\text { precise to } \\
\text { imprecise. }\end{array}$ & $\begin{array}{l}\text { Mix of direct } \\
\text { and indirect } \\
\text { data }\end{array}$ & $\begin{array}{l}\text { (1) Low } \\
\text { (2) [Insufficient] }\end{array}$ & $\begin{array}{l}\text { - Thermal interventions (C) had statistically significantly fewer recurrences than } \\
\text { drugs, and not significantly fewer than PDT or placebo (2 RCTs) } \\
\text { - PDT (E) had statistically significantly fewer recurrences than drugs, but not } \\
\text { statistically significantly fewer than placebo or more than heat/cold (4 RCTs) } \\
\text { - Drugs (F) had statistically significantly more recurrences than heat/cold and } \\
\text { PDT, and not significantly more than placebo (1 RCT) }\end{array}$ \\
\hline \multicolumn{7}{|l|}{ Histologic clearance, SCCIS } \\
\hline $\begin{array}{l}\text { (1) [Laser (C5) + PDT with ALA (E2) results in } \\
\text { better histologic clearance over laser alone] } \\
\text { (2) } 5 \text {-FU (F) results in better histologic } \\
\text { clearance than placebo }(I, J)\end{array}$ & $\begin{array}{l}\text { (1) Low } \\
\text { (2) High }\end{array}$ & [Not rated] & $\begin{array}{l}\text { (1) Imprecise } \\
\text { (2) Precise }\end{array}$ & $\begin{array}{l}\text { (1) Direct } \\
\text { (2) Direct }\end{array}$ & $\begin{array}{l}\text { (1) [Insufficient] } \\
\text { (2) Low }\end{array}$ & [2 RCTs, 50 patients.] \\
\hline $\begin{array}{l}\text { Clinical clearance, all SCCIS } \\
\text { (1) Examined types of active interventions } \\
\text { (heat/cold [C], PDT (E), and drugs [5-FU, } \\
\text { imiquimod; F]) were associated with better } \\
\text { clinical outcomes than placebo } \\
\text { (2) [Imprecise data on relative comparisons } \\
\text { between types of active interventions] }\end{array}$ & Moderate & $\begin{array}{l}\text { Possibly } \\
\text { consistent } \\
\text { (No robust } \\
\text { indications of } \\
\text { inconsistency) }\end{array}$ & $\begin{array}{l}\text { Varies by } \\
\text { comparison from } \\
\text { precise to } \\
\text { imprecise. }\end{array}$ & $\begin{array}{l}\text { Mix of direct } \\
\text { and indirect } \\
\text { data }\end{array}$ & $\begin{array}{l}\text { (1) High } \\
\text { (2) [Insufficient] }\end{array}$ & $\begin{array}{l}\text { - Thermal interventions (C) performed significantly better than placebo, and } \\
\text { nonsignificantly better than drugs and PDT (4 RCTs) } \\
\text { - PDT (E) performed significantly better than placebo, nonsignificantly better than } \\
\text { drugs, and nonsignificantly worse than heat/cold ( } 5 \text { RCT) } \\
\text { - Drugs (F) (5-FU, imiquimod) performed significantly better than placebo, and } \\
\text { nonsignificantly worse than PDT and heat/cold ( } 2 \text { RCT) }\end{array}$ \\
\hline $\begin{array}{l}\text { Observer-reported cosmetic outcomes, } \\
\text { SCCIS } \\
\text { (1) Cryotherapy plus 5-FU (C1+F1) is } \\
\text { associated with better outcomes than } \\
\text { PDT (MAL) (E1) } \\
\text { (2) [No difference between laser pretreatment } \\
\text { of the lesion before PDT versus PDT } \\
\text { alone] }\end{array}$ & Low & $\begin{array}{l}\text { Unclear } \\
\text { (Consistency } \\
\text { cannot be rated) }\end{array}$ & $\begin{array}{l}\text { (1) Precise } \\
\text { (2) Imprecise }\end{array}$ & $\begin{array}{l}\text { Mix of direct } \\
\text { and indirect } \\
\text { data }\end{array}$ & $\begin{array}{l}\text { (1) Moderate } \\
\text { (2) [Insufficient] }\end{array}$ & [2 RCTs, 204 patients.] \\
\hline \multicolumn{7}{|l|}{ Adverse effects, SSCIS } \\
\hline $\begin{array}{l}\text { (1) [Serious adverse events, adverse events } \\
\text { leading to discontinuation and infections } \\
\text { of the treated site are uncommon with } \\
\text { heat or cold }(C), \text { PDT }(E) \text { and drugs }(F)] \\
\text { (2) [On average, } 1 \text { in } 4 \text { and } 1 \text { in } 3 \text { patients } \\
\text { report experiencing pain after treatment } \\
\text { with PDT }(E) \text { and heat or cold }(C) \text {, } \\
\text { respectively] }\end{array}$ & $\begin{array}{l}\text { High } \\
\text { (selective } \\
\text { reporting } \\
\text { bias) }\end{array}$ & $\begin{array}{l}\text { Unclear } \\
\text { (Consistency } \\
\text { cannot be } \\
\text { assessed) }\end{array}$ & $\begin{array}{l}\text { Imprecise } \\
\text { We do not report } \\
\text { relative effects. } \\
\text { Forecasted } \\
\text { percentages of } \\
\text { patients with } \\
\text { adverse events } \\
\text { have wide } 95 \% \\
\text { Cls }\end{array}$ & $\begin{array}{l}\text { Mix of direct } \\
\text { and indirect } \\
\text { data (most } \\
\text { comparisons } \\
\text { based on } \\
\text { indirect data) }\end{array}$ & $\begin{array}{l}\text { (1) [Insufficient] } \\
\text { (2) [Insufficient] }\end{array}$ & [3 RCTs 292 patients.] \\
\hline
\end{tabular}




\begin{tabular}{|c|c|c|c|c|c|c|}
\hline Conclusion statement & $\begin{array}{l}\text { RoB } \\
\text { (evidence } \\
\text {-base) } \\
\end{array}$ & Consistency & Precision & Directness & $\begin{array}{l}\text { Overall } \\
\text { Rating }\end{array}$ & Comments \\
\hline \multicolumn{7}{|l|}{ Other outcomes, SCCIS } \\
\hline $\begin{array}{l}\text { [Evidence on patient-reported cosmetic } \\
\text { outcomes, quality of life, mental health, } \\
\text { patient satisfaction, mortality, cost and } \\
\text { resource use id reported in a minority of } \\
\text { studies and its strength not rated] } \\
\text { Other analyses }\end{array}$ & [Not rated] & [Not rated] & [Not rated] & [Not rated] & [Not rated] & [Not rated] \\
\hline $\begin{array}{l}\text { [Subgroup analyses and analyses focusing on } \\
\text { individual interventions are generally } \\
\text { sparse and are not rated] }\end{array}$ & [Not rated] & [Not rated] & [Not rated] & [Not rated] & [Not rated] & [Not rated] \\
\hline
\end{tabular}

Note: When a summary conclusion cannot be made, the description is given in square brackets.

RoB = risk of bias; $\mathrm{BCC}=$ basal cell carcinoma; $\mathrm{SCC}=$ squamous cell carcinoma; MMS = Mohs micrographic surgery; PDT = photodynamic therapy; RCT = randomized controlled trial; NRCS = nonrandomized comparative study; $\mathrm{CI}$ = confidence interval 


\section{Evidence Limitations}

With few exceptions and for most outcomes, individual studies were deemed to have at most moderate risk of confounding, selection, or measurement biases. The risk of bias of individual studies was not a major determinant for the conclusions in Tables 82 and 83 Assessing impact of the risk of bias of individual studies on the conclusions of a network meta-analysis is not straightforward. ${ }^{160}$ The comparison effects estimated from a network meta-analysis are a combination of the estimated effects from head-to-head studies and from studies contributing through indirect comparisons. For example, assume that there is a highly-biased study in a network meta-analysis: it would be a concern primarily for the comparison it directly informs on, it may be a smaller (or even negligible) concern for comparisons that it informs indirectly, and it will be no concern for comparisons to which it contributes zero information. ${ }^{161}$ In this analysis we deemed qualitatively that risk of bias concerns would not change our conclusions. While qualitative-only assessments are precarious, we opted for high-level conclusions that may be robust.

By far the major concern, however, is that the evidence is sparse when one considers the richness of the clinical questions that can be posed. Comparisons between intervention categories are not as informative as comparisons between individual interventions. We have provided analyses at the individual intervention level, but opt not to draw conclusions based on them, because most are based on indirect data and small numbers. In addition, sample sizes were small leading to concerns about generalization.

A second consequence of the paucity of the evidence base is that one cannot directly address questions that may have important health and cost implications for insurers and patients. For example, there are no studies on the effectiveness of external radiation therapy delivered with portable machines in the office setting versus radiation therapy delivered in specialized facilities or versus other interventions. Empirical data on this radiation therapy modality would be useful because there are only limited data on radiation therapy to extrapolate from. Adjuvant treatment in the case of positive margins post excision or in the case of high-risk features, such as adjuvant radiotherapy and new drugs (including epidermal growth factor receptor inhibitors, such as cetuximab and erlotinib) were not within the scope of this review but also have utility in treating BCC and SCC lesions.

Other large gaps remain in the knowledge base: There is no information on subgroups of patients who have limited life expectancy, are frail, or who are immunocompromised (e.g., have chronic lymphocytic leukemiaand other malignancies, immunodeficiency disorders, or who receive immunomodulating or immunosuppressive treatments). There is limited or no information on high risk BCC lesions, and on invasive SCCs. There is limited data on patientand lesion-specific modifiers of intervention effects.

The inconsistent reporting of adverse events was a challenge in this report. The specific adverse events reported and their definitions varied greatly among studies and treatment modalities. Because of the large number of individual adverse events reported, we grouped them for analysis. However, this can lead to misclassification, especially given that different treatments have different associated harms.

Finally, outcomes such as histological clearance and clinical clearance are surrogates for lesion recurrence. In particular, clinical clearance may be informative when comparing among PDT, medical, and radiation-based therapies, but is not an informative outcome for surgical 
interventions: any surgical treatment, regardless of margin control, removes all clinically visible tumor. Therefore, our conclusion in Table 82 that surgical interventions are better than all other interventions with respect to clinical clearance, while very likely to be true, is almost meaningless.

\section{Future Research Recommendations}

We have identified a number of important gaps in the medical literature on the topic of treating BCC and SCC. They are described briefly in the following paragraphs.

More trials are needed comparing commonly used treatment modalities such as simple excision, Mohs surgery, PDT and topical medical therapy. Further, in order to justify routine use of various forms of radiotherapy for these patients, more trials comparing radiotherapy with other modalities are needed. As it stands, the lack of evidence on radiotherapy has led the American Academy of Dermatology to discourage the use of superficial radiotherapy and electronic brachytherapy for keratinocyte carcinomas except in select patients. ${ }^{162,163}$ As these tumors are very common and generally have low morbidity and mortality, recruitment for such trials may not prove to be prohibitively difficult.

All trials for BCC and SCC should, where possible, use recurrent disease as a primary or secondary outcome as it is the most clinically important outcome. Trials should also attempt to incorporate measures of health care resource utilization, which were lacking in our review of the existing evidence save for one RCT and one NRCS. ${ }^{20,155}$ Future trials would also benefit from standardization and consistent definition of all outcomes, particularly adverse events and patientreported outcomes such as cosmesis. To this end, we encourage the development of a core outcome set as is being done for other skin diseases such as psoriasis (The International Dermatology Outcome Measures) ${ }^{164}$ and atopic dermatitis (Harmonising Outcome Measures for Eczema).

While more evidence is needed overall, future research should also focus on specific subgroups that have minimal evidence to date. Aggressive histologic subtypes of BCC, including infiltrative and sclerosing patterns, account for very little of the evidence found in our review. While their increased likelihood of recurrence has led to their inclusion as appropriate indications for Mohs surgery (except for lesions $\leq 0.5 \mathrm{~cm}$ on the trunk and extremities, whose appropriateness is rated as "uncertain"), there is scant evidence to support this. ${ }^{159}$ With regards to SCC, only one included RCT in this report concerns invasive SCC with the rest concerning in situ disease. Given that invasive SCC is responsible for mortality in 3900-8800 people in the United States each year, ${ }^{6}$ in addition to morbidity and health care costs, there is a clear need for comparative effectiveness research for invasive SCC treatments. No comparative evidence was found on keratinocyte carcinoma in high-risk groups such as organ transplant recipients and patients with other altered immune states such as HIV and Chronic Lymphocytic Leukemia (CLL). Patients with limited life-expectancy are another subgroup of interest who warrant study.

Given how common these tumors are and their burden on the health care system, research funding directed to determine the most effective and cost-effective measures for these tumors is needed. It is incumbent on funding agencies and health care payers to fund research examining important questions in this field. Patients, clinicians, payers, and research funders would benefit from a decision analysis of the management of BCC and SCC lesions.

Finally, better monitoring of population trends in BCCs and SCCs can help focus research on most consequential subtypes. Such monitoring can be performed by SEER (which currently 
ignores these cancers), the CDC, or large health organizations taking advantage of advances in health information technology. While the volume of these tumors makes surveillance logistically difficult and costly, advances in health information technology and big data analytic techniques should make it more feasible. ${ }^{165}$

\section{Conclusions}

Based on sparse evidence, surgical, radiation and some topical drug treatments have lower recurrence rates than other modalities for the treatment of low-risk BCC, and PDT appears to have superior cosmetic outcomes. Large gaps remain in the literature regarding the comparison of individual interventions, and very little or no information on immunocompromised patients, patients with limited life expectancy, and on patients with specific lesion categories, including high risk BCCs and invasive SCCs. In order for clinicians, patients and payers to make informed decisions regarding the treatment of these lesions, new RCT or high-quality NRCS evidence is needed. 


\section{References}

1. Centers for Disease Control and Prevention Skin Cancer Statistics. 2015. http://www.cdc.gov/cancer/skin/statistics/index.ht m.

2. Rogers HW, Weinstock MA, Feldman SR, et al. Incidence Estimate of Nonmelanoma Skin Cancer (Keratinocyte Carcinomas) in the US Population, 2012. JAMA Dermatol. 2015 Oct

1;151(10):1081-6. doi: 10.1001/jamadermatol.2015.1187. PMID: 25928283.

3. Bickers DR, Lim HW, Margolis D, et al. The burden of skin diseases: 2004 a joint project of the American Academy of Dermatology Association and the Society for Investigative Dermatology. J Am Acad Dermatol. 2006 Sep;55(3):490-500. doi: 10.1016/j.jaad.2006.05.048. PMID: 16908356.

4. Karimkhani C, Dellavalle RP, Coffeng LE, et al. Global Skin Disease Morbidity and Mortality: An Update From the Global Burden of Disease Study 2013. JAMA Dermatol. 2017 Mar 01doi: 10.1001/jamadermatol.2016.5538. PMID: 28249066.

5. Wu W, Weinstock MA. Trends of keratinocyte carcinoma mortality rates in the United States as reported on death certificates, 1999 through 2010. Dermatol Surg. 2014 Dec;40(12):1395-401. doi: 10.1097/DSS.0000000000000194. PMID: 25393350.

6. Karia PS, Han J, Schmults CD. Cutaneous squamous cell carcinoma: estimated incidence of disease, nodal metastasis, and deaths from disease in the United States, 2012. J Am Acad Dermatol. 2013 Jun;68(6):957-66. doi: 10.1016/j.jaad.2012.11.037. PMID: 23375456.

7. Oh CC, Hofbauer GF, Serra AL, et al. Painful skin lesions and squamous cell carcinoma predict overall mortality risk in organ transplant recipients: A cohort study. Br J Dermatol. 2016 Dec 23doi: 10.1111/bjd.15269. PMID: 28012178.

8. U. S. Department of Health and Human Services. Reports of the Surgeon General. The Surgeon General's Call to Action to Prevent Skin Cancer. Washington (DC): Office of the Surgeon General (US); 2014.
9. Cakir BO, Adamson P, Cingi C. Epidemiology and economic burden of nonmelanoma skin cancer. Facial Plast Surg Clin North Am. 2012 Nov;20(4):419-22. doi: 10.1016/j.fsc.2012.07.004. PMID: 23084294.

10. Casey S, Best L, Vujovic O, et al. Use of Protoporphyrin Fluorescence to Determine Clinical Target Volume for Non-melanotic Skin Cancers Treated with Primary Radiotherapy. Cureus. 2016 Sep 04;8(9):e767. doi: 10.7759/cureus.767. PMID: 27725923.

11. Lucena SR, Salazar N, Gracia-Cazana T, et al. Combined Treatments with Photodynamic Therapy for Non-Melanoma Skin Cancer. Int J Mol Sci. 2015 Oct 28;16(10):25912-33. doi: 10.3390/ijms161025912. PMID: 26516853.

12. Yoon J, Phibbs CS, Chow A, et al. Costs of Keratinocyte Carcinoma (Nonmelanoma Skin Cancer) and Actinic Keratosis Treatment in the Veterans Health Administration. Dermatol Surg. 2016 Sep;42(9):1041-7. doi: 10.1097/dss.0000000000000820. PMID: 27465252.

13. Linos E, Chren MM, Stijacic Cenzer I, et al. Skin Cancer in U.S. Elderly Adults: Does Life Expectancy Play a Role in Treatment Decisions? J Am Geriatr Soc. 2016 Aug;64(8):1610-5. doi: 10.1111/jgs.14202. PMID: 27303932.

14. Neville JA, Welch E, Leffell DJ. Management of nonmelanoma skin cancer in 2007. Nat Clin Pract Oncol. 2007 Aug;4(8):462-9. doi: 10.1038/ncponc0883. PMID: 17657251.

15. Jacobsen AA, Aldahan AS, Hughes OB, et al. Hedgehog Pathway Inhibitor Therapy for Locally Advanced and Metastatic Basal Cell Carcinoma: A Systematic Review and Pooled Analysis of Interventional Studies. JAMA Dermatol. 2016 Apr 20doi: 10.1001/jamadermatol.2016.0780. PMID: 27096888.

16. Jung JY, Linos E. Adding active surveillance as a treatment option for low risk skin cancers in patients with limited life expectancy. J Geriatr Oncol. 2016 May;7(3):221-2. doi: 10.1016/j.jgo.2016.03.008. PMID: 27133284. 
17. Linos E, Parvataneni R, Stuart SE, et al. Treatment of nonfatal conditions at the end of life: nonmelanoma skin cancer. JAMA Intern Med. 2013 Jun 10;173(11):1006-12. doi: 10.1001/jamainternmed.2013.639. PMID: 23699934.

18. Bath-Hextall F, Ozolins M, Armstrong SJ, et al. Surgical excision versus imiquimod 5\% cream for nodular and superficial basal-cell carcinoma (SINS): a multicentre, non-inferiority, randomised controlled trial. Lancet Oncol. 2014 Jan;15(1):96-105. doi: 10.1016/S14702045(13)70530-8. PMID: 24332516.

19. Kuijpers DI, Thissen MR, Berretty PJ, et al. Surgical excision versus curettage plus cryosurgery in the treatment of basal cell carcinoma. Dermatol Surg. 2007 May;33(5):57987. doi: 10.1111/j.1524-4725.2007.33117.x. PMID: 17451581.

20. Mosterd K, Thissen MR, Nelemans P, et al. Fractionated 5-aminolaevulinic acidphotodynamic therapy vs. surgical excision in the treatment of nodular basal cell carcinoma: results of a randomized controlled trial. Br J Dermatol. 2008 Sep;159(4):864-70. doi: 10.1111/j.13652133.2008.08787.x. PMID: 18717680.

21. Ozolins M, Williams HC, Armstrong SJ, et al. The SINS trial: a randomised controlled trial of excisional surgery versus imiquimod $5 \%$ cream for nodular and superficial basal cell carcinoma. Trials. 2010;11:42. doi: 10.1186/1745-6215-1142. PMID: 20409337.

22. Rhodes LE, de Rie MA, Leifsdottir R, et al. Fiveyear follow-up of a randomized, prospective trial of topical methyl aminolevulinate photodynamic therapy vs surgery for nodular basal cell carcinoma. Arch Dermatol. 2007

Sep;143(9):1131-6. doi: 10.1001/archderm.143.9.1131. PMID: 17875873.

23. Smucler R, Vlk M. Combination of Er:YAG laser and photodynamic therapy in the treatment of nodular basal cell carcinoma. Lasers Surg Med. 2008 Feb;40(2):153-8. doi: 10.1002/lsm.20606. PMID: 18306163.
24. Bath-Hextall FJ, Perkins W, Bong J, et al. Interventions for basal cell carcinoma of the skin. Cochrane Database Syst Rev.

2007(1):CD003412. doi: 10.1002/14651858.CD003412.pub2. PMID: 17253489.

25. Clark CM, Furniss M, Mackay-Wiggan JM. Basal cell carcinoma: an evidence-based treatment update. Am J Clin Dermatol. 2014 Jul;15(3):197-216. doi: 10.1007/s40257-0140070-z. PMID: 24733429.

26. Lansbury L, Bath-Hextall F, Perkins W, et al. Interventions for non-metastatic squamous cell carcinoma of the skin: systematic review and pooled analysis of observational studies. BMJ. 2013;347:f6153. doi: 10.1136/bmj.f6153. PMID: 24191270.

27. Lansbury L, Leonardi-Bee J, Perkins W, et al. Interventions for non-metastatic squamous cell carcinoma of the skin. Cochrane Database Syst Rev. 2010(4):CD007869. doi: 10.1002/14651858.CD007869.pub2. PMID: 20393962.

28. Love WE, Bernhard JD, Bordeaux JS. Topical imiquimod or fluorouracil therapy for basal and squamous cell carcinoma: a systematic review. Arch Dermatol. 2009 Dec;145(12):1431-8. doi: 10.1001/archdermatol.2009.291. PMID: 20026854.

29. Mosterd K, Arits AH, Thissen MR, et al. Histology-based treatment of basal cell carcinoma. Acta Derm Venereol. 2009;89(5):4548. doi: 10.2340/00015555-0710. PMID: 19734968.

30. Roozeboom MH, Arits AH, Nelemans PJ, et al. Overall treatment success after treatment of primary superficial basal cell carcinoma: a systematic review and meta-analysis of randomized and nonrandomized trials. $\mathrm{Br} \mathrm{J}$ Dermatol. 2012 Oct;167(4):733-56. doi: 10.1111/j.1365-2133.2012.11061.x. PMID: 22612571.

31. Thissen MR, Neumann MH, Schouten LJ. A systematic review of treatment modalities for primary basal cell carcinomas. Arch Dermatol. 1999 Oct;135(10):1177-83. PMID: 10522664. 
32. . Basal Cell Carcinoma, Squamous Cell Carcinoma, and Related Lesions: A Guide to Clinical Management in Australia. Cancer Council Australia and Australian Cancer Network. 2008.

33. Finnish Medical Society Duodecim. Naevi and skin cancer (basal cell and epidermoid carcinoma). In: EBM Guidelines. Evidence-Based Medicine [Internet]. Helsinki, Finland: Wiley Interscience. John Wiley \& Sons; 2010. .

34. US Department of Health Human Services. The surgeon general's call to action to prevent skin cancer. 2014.

35. Mudigonda T, Pearce DJ, Yentzer BA, et al. The economic impact of non-melanoma skin cancer: a review. J Natl Compr Canc Netw. 2010 Aug;8(8):888-96. PMID: 20870635.

36. Linos E, VanBeek M, Resneck JS, Jr. A Sudden and Concerning Increase in the Use of Electronic Brachytherapy for Skin Cancer. JAMA Dermatol. 2015 Jul;151(7):699-700. doi: 10.1001/jamadermatol.2015.0385. PMID: 25785818.

37. AHRQ Methods for Effective Health Care. Methods Guide for Effectiveness and Comparative Effectiveness Reviews. Rockville (MD): Agency for Healthcare Research and Quality (US); 2011.

38. Halladay CW, Trikalinos TA, Schmid IT, et al. Using data sources beyond PubMed has a modest impact on the results of systematic reviews of therapeutic interventions. J Clin Epidemiol. 2015 Sep;68(9):1076-84. doi: 10.1016/j.jclinepi.2014.12.017. PMID: 26279401.

39. . Deploying an interactive machine learning system in an evidence-based practice center: abstrackr. Proceedings of the 2nd ACM SIGHIT International Health Informatics Symposium; 2012. ACM.

40. Higgins JP, Altman DG, Gotzsche PC, et al. The Cochrane Collaboration's tool for assessing risk of bias in randomised trials. Bmj. 2011;343:d5928. doi: 10.1136/bmj.d5928. PMID: 22008217.
41. Wells GAS, B.;O'Connell, D.;Peterson, J.; Welch, V.; Losos, M.; Tugwell, P. The Newcastle-Ottawa Scale (NOS) for assessing the quality of nonrandomised studies in metaanalyses.

http://www.ohri.ca/programs/clinical_epidemiolo gy/oxford.asp.

42. Carija A, Puizina-Ivic N, Vukovic D, et al. Single treatment of low-risk basal cell carcinomas with pulsed dye laser-mediated photodynamic therapy (PDL-PDT) compared with photodynamic therapy (PDT): A controlled, investigatorblinded, intra-individual prospective study. Photodiagnosis Photodyn Ther. 2016 Dec;16:605. doi: 10.1016/j.pdpdt.2016.08.003. PMID: 27516420.

43. Choi SH, Kim KH, Song KH. Effect of Methyl Aminolevulinate Photodynamic Therapy With and Without Ablative Fractional Laser Treatment in Patients With Microinvasive Squamous Cell Carcinoma: A Randomized Clinical Trial. JAMA Dermatol. 2017 Feb 15doi: 10.1001/jamadermatol.2016.4463. PMID: 28199463.

44. Dummer R, Migden M, Guminski A, et al. Investigator-assessed efficacy and safety of sonidegib in patients with locally advanced basal cell carcinoma and metastatic basal cell carcinoma: Results of the BOLT 30-month analysis. Melanoma Research. 2016;26:e3. doi: 10.1097/CMR.0000000000000285.

45. Abbade L, Gige T, Amaral V, et al. Higher recurrence rates of head and neck nodular basal cell carcinoma treated with topical photodynamic therapy (MAL-PDT) compared to surgical excision: A randomized controlled study. Journal of the American Academy of Dermatology; 2015. p. Ab185.

46. Al-Niaimi F, Sheth N, Kurwa HA, et al. Photodynamic Therapy Followed by Mohs Micrographic Surgery Compared to Mohs Micrographic Surgery Alone for the Treatment of Basal Cell Carcinoma: Results of a Pilot SingleBlinded Randomised Controlled Trial. J Cutan Aesthet Surg. 2015 Apr-Jun;8(2):88-91. doi: 10.4103/0974-2077.158443. PMID: 26157307.

47. Allen ED, McGill JI, Hall VL, et al. Cryotherapy of basal cell lesions. Trans Ophthalmol Soc U K. 1979 Jul;99(2):264-8. PMID: 298425. 
48. Alpsoy E, Yilmaz E, Basaran E, et al. Comparison of the effects of intralesional interferon alfa-2a, $2 \mathrm{~b}$ and the combination of $2 \mathrm{a}$ and $2 \mathrm{~b}$ in the treatment of basal cell carcinoma. $\mathrm{J}$ Dermatol. 1996 Jun;23(6):394-6. PMID: 8708151.

49. Arits AH, Mosterd K, Essers BA, et al. Photodynamic therapy versus topical imiquimod versus topical fluorouracil for treatment of superficial basal-cell carcinoma: a single blind, non-inferiority, randomised controlled trial. Lancet Oncol. 2013 Jun;14(7):647-54. doi: 10.1016/s1470-2045(13)70143-8. PMID: 23683751.

50. Avril MF, Auperin A, Margulis A, et al. Basal cell carcinoma of the face: surgery or radiotherapy? Results of a randomized study. Br J Cancer. 1997;76(1):100-6. PMID: 9218740.

51. Basset-Seguin N, Ibbotson SH, Emtestam L, et al. Topical methyl aminolaevulinate photodynamic therapy versus cryotherapy for superficial basal cell carcinoma: a 5 year randomized trial. Eur J Dermatol. 2008 SepOct;18(5):547-53. doi: 10.1684/ejd.2008.0472. PMID: 18693158.

52. Bath-Hextall F, Ozolins M, Armstrong SJ, et al. Surgical excision versus imiquimod 5\% cream for nodular and superficial basal-cell carcinoma (SINS): a multicentre, non-inferiority, randomised controlled trial. Lancet Oncol. 2014 Jan;15(1):96-105. doi: 10.1016/s14702045(13)70530-8. PMID: 24332516.

53. Berroeta L, Clark C, Dawe RS, et al. A randomized study of minimal curettage followed by topical photodynamic therapy compared with surgical excision for low-risk nodular basal cell carcinoma. Br J Dermatol. 2007 Aug;157(2):4013. doi: 10.1111/j.1365-2133.2007.07996.x. PMID: 17573890.

54. Beutner KR, Geisse JK, Helman D, et al. Therapeutic response of basal cell carcinoma to the immune response modifier imiquimod 5\% cream. J Am Acad Dermatol. 1999 Dec;41(6):1002-7. PMID: 10570388.
55. Brinkhuizen T, Frencken KJ, Nelemans PJ, et al. The effect of topical diclofenac 3\% and calcitriol $3 \mathrm{mug} / \mathrm{g}$ on superficial basal cell carcinoma (sBCC) and nodular basal cell carcinoma (nBCC): A phase II, randomized controlled trial. J Am Acad Dermatol. 2016 Jul;75(1):126-34. doi: 10.1016/j.jaad.2016.01.050. PMID: 27067393.

56. Butler DF, Parekh PK, Lenis A. Imiquimod 5\% cream as adjunctive therapy for primary, solitary, nodular nasal basal cell carcinomas before Mohs micrographic surgery: a randomized, double blind, vehicle-controlled study. Dermatol Surg. 2009 Jan;35(1):24-9. doi: 10.1111/j.15244725.2008.34378.x. PMID: 19018814.

57. Cai H, Wang YX, Zheng JC, et al. Photodynamic therapy in combination with $\mathrm{CO} 2$ laser for the treatment of Bowen's disease. Lasers Med Sci. 2015 Jul;30(5):1505-10. doi: 10.1007/s10103015-1754-1. PMID: 25899562.

58. Choi SH, Kim KH, Song KH. Er:YAG ablative fractional laser-primed photodynamic therapy with methyl aminolevulinate as an alternative treatment option for patients with thin nodular basal cell carcinoma: 12-month follow-up results of a randomized, prospective, comparative trial. J Eur Acad Dermatol Venereol. 2016 May;30(5):783-8. doi: 10.1111/jdv.13453. PMID: 26551044.

59. Cornell RC, Greenway HT, Tucker SB, et al. Intralesional interferon therapy for basal cell carcinoma. J Am Acad Dermatol. 1990 Oct;23(4 Pt 1):694-700. PMID: 2229497.

60. Dummer R, Guminski A, Gutzmer R, et al. The 12-month analysis from Basal Cell Carcinoma Outcomes with LDE225 Treatment (BOLT): A phase II, randomized, double-blind study of sonidegib in patients with advanced basal cell carcinoma. J Am Acad Dermatol. 2016 Jul;75(1):113-25.e5. doi: 10.1016/j.jaad.2016.02.1226. PMID: 27067394.

61. Edwards L, Tucker SB, Perednia D, et al. The effect of an intralesional sustained-release formulation of interferon alfa-2b on basal cell carcinomas. Arch Dermatol. 1990 Aug;126(8):1029-32. PMID: 2383027.

62. Edwards L, Whiting D, Rogers D, et al. The effect of intralesional interferon gamma on basal cell carcinomas. J Am Acad Dermatol. 1990 Mar;22(3):496-500. PMID: 2107219. 
63. Eigentler TK, Kamin A, Weide BM, et al. A phase III, randomized, open label study to evaluate the safety and efficacy of imiquimod 5\% cream applied thrice weekly for 8 and 12 weeks in the treatment of low-risk nodular basal cell carcinoma. J Am Acad Dermatol. 2007 Oct;57(4):616-21. doi: 10.1016/j.jaad.2007.05.022. PMID: 17610993.

64. Eimpunth S, Oganesyan G, Hamman MS, et al. Effectiveness of a 595-nm pulsed dye laser for the treatment of basal cell carcinoma using 1 stackedpulse session. Journal of the American Academy of Dermatology; 2014. p. Ab134.

65. Essers BA, Dirksen CD, Nieman FH, et al. Costeffectiveness of Mohs Micrographic Surgery vs Surgical Excision for Basal Cell Carcinoma of the Face. Arch Dermatol. 2006 Feb;142(2):187-94. doi: 10.1001/archderm.142.2.187. PMID: 16490846.

66. Foley P, Freeman M, Menter A, et al. Photodynamic therapy with methyl aminolevulinate for primary nodular basal cell carcinoma: results of two randomized studies. Int J Dermatol. 2009 Nov;48(11):1236-45. doi: 10.1111/j.1365-4632.2008.04022.x. PMID: 20064185.

67. Garcia-Martin E, Gil-Arribas LM, Idoipe M, et al. Comparison of imiquimod 5\% cream versus radiotherapy as treatment for eyelid basal cell carcinoma. Br J Ophthalmol. 2011 Oct;95(10):1393-6. doi: 10.1136/bjo.2010.193078. PMID: 21242584.

68. Geisse J, Caro I, Lindholm J, et al. Imiquimod $5 \%$ cream for the treatment of superficial basal cell carcinoma: results from two phase III, randomized, vehicle-controlled studies. J Am Acad Dermatol. 2004 May;50(5):722-33. doi: 10.1016/j.jaad.2003.11.066. PMID: 15097956.

69. Geisse JK, Rich P, Pandya A, et al. Imiquimod $5 \%$ cream for the treatment of superficial basal cell carcinoma: a double-blind, randomized, vehicle-controlled study. J Am Acad Dermatol. 2002 Sep;47(3):390-8. PMID: 12196749.

70. Haak CS, Togsverd-Bo K, Thaysen-Petersen D, et al. Fractional laser-mediated photodynamic therapy of high-risk basal cell carcinomas--a randomized clinical trial. Br J Dermatol. 2015 Jan;172(1):215-22. doi: 10.1111/bjd.13166. PMID: 24903544.
71. Hall VL, Leppard BJ, McGill J, et al. Treatment of basal-cell carcinoma: comparison of radiotherapy and cryotherapy. Clin Radiol. 1986 Jan;37(1):33-4. PMID: 3514075.

72. Ko DY, Kim KH, Song KH. A randomized trial comparing methyl aminolaevulinate photodynamic therapy with and without Er:YAG ablative fractional laser treatment in Asian patients with lower extremity Bowen disease: results from a 12-month follow-up. $\mathrm{Br} \mathrm{J}$ Dermatol. 2014 Jan;170(1):165-72. doi: 10.1111/bjd.12627. PMID: 24102369.

73. Kuijpers DI, Thissen MR, Thissen CA, et al. Similar effectiveness of methyl aminolevulinate and 5-aminolevulinate in topical photodynamic therapy for nodular basal cell carcinoma. J Drugs Dermatol. 2006 Jul-Aug;5(7):642-5. PMID: 16865869.

74. Marks R, Gebauer K, Shumack S, et al. Imiquimod 5\% cream in the treatment of superficial basal cell carcinoma: results of a multicenter 6-week dose-response trial. J Am Acad Dermatol. 2001 May;44(5):807-13. doi: 10.1067/mjd.2001.113689. PMID: 11312429.

75. Migden MR, Guminski A, Gutzmer R, et al. Treatment with two different doses of sonidegib in patients with locally advanced or metastatic basal cell carcinoma (BOLT): a multicentre, randomised, double-blind phase 2 trial. Lancet Oncol. 2015 Jun;16(6):716-28. doi: 10.1016/s1470-2045(15)70100-2. PMID: 25981810.

76. Miller BH, Shavin JS, Cognetta A, et al. Nonsurgical treatment of basal cell carcinomas with intralesional 5-fluorouracil/epinephrine injectable gel. J Am Acad Dermatol. 1997 Jan;36(1):72-7. PMID: 8996264.

77. Morton C, Horn M, Lehman J, et al. A 24-month update of a placebo controlled European study comparing MAL-PDT with cryotherapy and 5fluoracil in patients with Bowen's disease (Abstract P08.73) The 14th Congress of the European Academy of Dermatology and Venereology, London,UK. 12-15th October 2005. Journal of the European Academy of Dermatology and Venereology : JEADV; 2005. p. 237-8. 
78. Morton C, Horn M, Leman J, et al. A placebo controlled European study controlling methylaminolaevulinate photodynamic therapy with cryotherapy and 5-fluorocaril in patients with Bowen's disease. Abstract P-33 The 85th BAD Annual Meeting 5-8th July 2005, Glasgow, UK. British journal of dermatology; 2005. p. 30.

79. Morton C, Horn M, Leman J, et al. Comparison of topical methyl aminolevulinate photodynamic therapy with cryotherapy or Fluorouracil for treatment of squamous cell carcinoma in situ: Results of a multicenter randomized trial. Arch Dermatol. 2006 Jun;142(6):729-35. doi: 10.1001/archderm.142.6.729. PMID: 16785375.

80. Morton CA, Whitehurst C, Moseley H, et al. Comparison of photodynamic therapy with cryotherapy in the treatment of Bowen's disease. Br J Dermatol. 1996 Nov;135(5):766-71. PMID: 8977678.

81. Mosterd K, Krekels GA, Nieman FH, et al. Surgical excision versus Mohs' micrographic surgery for primary and recurrent basal-cell carcinoma of the face: a prospective randomised controlled trial with 5-years' follow-up. Lancet Oncol. 2008 Dec;9(12):1149-56. doi: 10.1016/s1470-2045(08)70260-2. PMID: 19010733.

82. Orenberg EK, Miller BH, Greenway HT, et al. The effect of intralesional 5-fluorouracil therapeutic implant (MPI 5003) for treatment of basal cell carcinoma. J Am Acad Dermatol. 1992 Nov;27(5 Pt 1):723-8. PMID: 1430394.

83. Patel GK, Goodwin R, Chawla M, et al. Imiquimod 5\% cream monotherapy for cutaneous squamous cell carcinoma in situ (Bowen's disease): a randomized, double-blind, placebocontrolled trial. J Am Acad Dermatol. 2006 Jun;54(6):1025-32. doi: 10.1016/j.jaad.2006.01.055. PMID: 16713457.

84. Petit JY, Avril MF, Margulis A, et al. Evaluation of cosmetic results of a randomized trial comparing surgery and radiotherapy in the treatment of basal cell carcinoma of the face. Plast Reconstr Surg. 2000 Jun;105(7):2544-51. PMID: 10845311.
85. Rhodes LE, de Rie M, Enstrom Y, et al. Photodynamic therapy using topical methyl aminolevulinate vs surgery for nodular basal cell carcinoma: results of a multicenter randomized prospective trial. Arch Dermatol. 2004 Jan;140(1):17-23. doi: 10.1001/archderm.140.1.17. PMID: 14732655.

86. Roozeboom MH, Aardoom MA, Nelemans PJ, et al. Fractionated 5-aminolevulinic acid photodynamic therapy after partial debulking versus surgical excision for nodular basal cell carcinoma: a randomized controlled trial with at least 5-year follow-up. J Am Acad Dermatol. 2013 Aug;69(2):280-7. doi: 10.1016/j.jaad.2013.02.014. PMID: 23566914.

87. Roozeboom MH, Arits AH, Mosterd K, et al. Three-Year Follow-Up Results of Photodynamic Therapy vs. Imiquimod vs. Fluorouracil for Treatment of Superficial Basal Cell Carcinoma: A Single-Blind, Noninferiority, Randomized Controlled Trial. J Invest Dermatol. 2016 Aug;136(8):1568-74. doi: 10.1016/j.jid.2016.03.043. PMID: 27113429.

88. Roozeboom MH, Nelemans PJ, Mosterd K, et al. Photodynamic therapy vs. topical imiquimod for treatment of superficial basal cell carcinoma: a subgroup analysis within a noninferiority randomized controlled trial. Br J Dermatol. 2015 Mar;172(3):739-45. doi: 10.1111/bjd.13299. PMID: 25066012.

89. Salim A, Leman JA, McColl JH, et al. Randomized comparison of photodynamic therapy with topical 5-fluorouracil in Bowen's disease. Br J Dermatol. 2003 Mar;148(3):539-43. PMID: 12653747.

90. Salmanpoor R, Motevalli D, Saki N, et al. Efficacy of excisional surgery, curettage and combined curettage and electrodessication in treatment of basal cell carcinoma. Iranian Journal of Dermatology. 2012;15(60):66-7. PMID: 2013120389.

91. Schleier P, Berndt A, Kolossa S, et al. Comparison of aminolevulinic acid (ALA)thermogel-PDT with methyl-ALA-thermogelPDT in basal cell carcinoma. Photodiagnosis Photodyn Ther. 2007 Sep;4(3):197-201. doi: 10.1016/j.pdpdt.2007.04.004. PMID: 25047438. 
92. Schulze HJ, Cribier B, Requena L, et al. Imiquimod 5\% cream for the treatment of superficial basal cell carcinoma: results from a randomized vehicle-controlled phase III study in Europe. Br J Dermatol. 2005 May;152(5):939-47. doi: 10.1111/j.1365-2133.2005.06486.x. PMID: 15888150 .

93. Shumack S, Robinson J, Kossard S, et al. Efficacy of topical 5\% imiquimod cream for the treatment of nodular basal cell carcinoma: comparison of dosing regimens. Arch Dermatol. 2002 Sep;138(9):1165-71. PMID: 12224977.

94. Siller G, Rosen R, Freeman M, et al. PEP005 (ingenol mebutate) gel for the topical treatment of superficial basal cell carcinoma: results of a randomized phase IIa trial. Australas J Dermatol. 2010 May;51(2):99-105. doi: 10.1111/j.14400960.2010.00626.x. PMID: 20546215.

95. Smeets NW, Krekels GA, Ostertag JU, et al. Surgical excision vs Mohs' micrographic surgery for basal-cell carcinoma of the face: randomised controlled trial. Lancet. 2004 Nov 1319;364(9447):1766-72. doi: 10.1016/s01406736(04)17399-6. PMID: 15541449.

96. Spencer JM. Pilot study of imiquimod 5\% cream as adjunctive therapy to curettage and electrodesiccation for nodular basal cell carcinoma. Dermatol Surg. 2006 Jan;32(1):63-9. PMID: 16393600.

97. Sterry W, Ruzicka T, Herrera E, et al. Imiquimod $5 \%$ cream for the treatment of superficial and nodular basal cell carcinoma: randomized studies comparing low-frequency dosing with and without occlusion. Br J Dermatol. 2002 Dec;147(6):1227-36. PMID: 12452875.

98. Szeimies RM, Ibbotson S, Murrell DF, et al. A clinical study comparing methyl aminolevulinate photodynamic therapy and surgery in small superficial basal cell carcinoma (8-20 mm), with a 12-month follow-up. J Eur Acad Dermatol Venereol. 2008 Nov;22(11):1302-11. doi: 10.1111/j.1468-3083.2008.02803.x. PMID: 18624836.

99. Thissen MR, Nieman FH, Ideler AH, et al. Cosmetic results of cryosurgery versus surgical excision for primary uncomplicated basal cell carcinomas of the head and neck. Dermatol Surg. 2000 Aug;26(8):759-64. PMID: 10940063.
100. Torres A, Niemeyer A, Berkes B, et al. 5\% imiquimod cream and reflectance-mode confocal microscopy as adjunct modalities to Mohs micrographic surgery for treatment of basal cell carcinoma. Dermatol Surg. 2004 Dec;30(12 Pt 1):1462-9. doi: 10.1111/j.15244725.2004.30504.x. PMID: 15606733.

101. Tran HT, Lee RA, Oganesyan G, et al. Single treatment of non-melanoma skin cancers using a pulsed-dye laser with stacked pulses. Lasers Surg Med. 2012 Aug;44(6):459-67. doi: 10.1002/lsm.22032. PMID: 22511036.

102. van der Geer S, Martens J, van Roij J, et al. Imiquimod 5\% cream as pretreatment of Mohs micrographic surgery for nodular basal cell carcinoma in the face: a prospective randomized controlled study. Br J Dermatol. 2012 Jul;167(1):110-5. doi: 10.1111/j.13652133.2012.10924.x. PMID: 22385074.

103. van Loo E, Mosterd K, Krekels GA, et al. Surgical excision versus Mohs' micrographic surgery for basal cell carcinoma of the face: A randomised clinical trial with 10 year follow-up. Eur J Cancer. 2014 Nov;50(17):3011-20. doi: 10.1016/j.ejca.2014.08.018. PMID: 25262378.

104. Wang I, Bendsoe N, Klinteberg CA, et al. Photodynamic therapy vs. cryosurgery of basal cell carcinomas: results of a phase III clinical trial. Br J Dermatol. 2001 Apr;144(4):832-40. PMID: 11298545.

105. Wettstein R, Erba P, Itin P, et al. Treatment of basal cell carcinoma with surgical excision and perilesional interferon-alpha. J Plast Reconstr Aesthet Surg. 2013 Jul;66(7):912-6. doi: 10.1016/j.bjps.2013.03.008. PMID: 23566745.

106. . The use of laboratory animals. Notes for the guidance of research workers. Ann R Coll Surg Engl. 1973 Mar;52(3):193-7. PMID: 4700674.

107. Aguilar M, de Troya M, Martin L, et al. A cost analysis of photodynamic therapy with methyl aminolevulinate and imiquimod compared with conventional surgery for the treatment of superficial basal cell carcinoma and Bowen's disease of the lower extremities. J Eur Acad Dermatol Venereol. 2010 Dec;24(12):1431-6. doi: 10.1111/j.1468-3083.2010.03664.x. PMID: 20456549. 
108. Ashby MA, Smith J, Ainslie J, et al. Treatment of nonmelanoma skin cancer at a large Australian center. Cancer. 1989 May 01;63(9):1863-71. PMID: 2702595.

109. Avila J, Bosch A, Aristizabal S, et al. Carcinoma of the pinna. Cancer. 1977 Dec;40(6):2891-5. PMID: 589557.

110. Bean DJ, Rees RS, O'Leary JP, et al. Carcinoma of the hand: a 20-year experience. South Med J. 1984 Aug;77(8):998-1000. PMID: 6463702.

111. Cham BE, Daunter B, Evans RA. Topical treatment of malignant and premalignant skin lesions by very low concentrations of a standard mixture (BEC) of solasodine glycosides. Cancer Lett. 1991 Sep;59(3):183-92. PMID: 1913614.

112. Chernosky ME. Squamous cell and basal cell carcinomas: preliminary study of 3,817 primary skin cancers. South Med J. 1978 Jul;71(7):802-3, 6. PMID: 663726.

113. Cox NH, Dyson P. Wound healing on the lower leg after radiotherapy or cryotherapy of Bowen's disease and other malignant skin lesions. Br J Dermatol. 1995 Jul;133(1):60-5. PMID: 7669642.

114. Futoryan T, Grande D. Postoperative wound infection rates in dermatologic surgery. Dermatol Surg. 1995 Jun;21(6):509-14. PMID: 7773598.

115. Glass RL, Perez-Mesa CM. Management of inadequately excised epidermoid carcinoma. Arch Surg. 1974 Jan;108(1):50-1. PMID: 4808574.

116. Halnan KE, Britten MJ. Late functional and cosmetic results of treatment of eyelid tumours. Br J Ophthalmol. 1968 Jan;52(1):43-53. PMID: 5710508.

117. Hansen JP, Drake AL, Walling HW. Bowen's Disease: a four-year retrospective review of epidemiology and treatment at a university center. Dermatol Surg. 2008 Jul;34(7):878-83. doi: 10.1111/j.1524-4725.2008.34172.x. PMID: 18363722.

118. Harrison PV. Therapy of basal cell carcinoma-treatment in 1980-81 compared with 1985-86 and advantages of shave excision for smaller tumours. Br J Dermatol. 1987 Sep;117(3):349-57. PMID: 3676083.
119. Honeycutt WM, Jansen GT. Treatment of squamous cell carcinoma of the skin. Arch Dermatol. 1973 Nov;108(5):670-2. PMID: 4750203.

120. Ibbotson SH, Valentine R, Hearn R. Is the pain of topical photodynamic therapy with methyl aminolevulinate any different from that with 5aminolaevulinic acid? Photodermatol Photoimmunol Photomed. 2012 Oct;28(5):272-3. doi: 10.1111/j.1600-0781.2012.00684.x. PMID: 22971196.

121. Jebodhsingh KN, Calafati J, Farrokhyar F, et al. Recurrence rates of basal cell carcinoma of the periocular skin: what to do with patients who have positive margins after resection. Can J Ophthalmol. 2012 Apr;47(2):181-4. doi: 10.1016/j.jcjo.2012.01.024. PMID: 22560426.

122. Kadakia S, Ducic Y, Marra D, et al. Cutaneous squamous cell carcinoma of the scalp in the immunocompromised patient: review of 53 cases. Oral Maxillofac Surg. 2016 Jun;20(2):171-5. doi: 10.1007/s10006-016-0545-6. PMID: 26780196.

123. Knox JM, Freeman RG, Duncan WC, et al. Treatment of skin cancer. South Med J. 1967 Mar;60(3):241-6. PMID: 6020491.

124. Kowalzick L, Rogozinski T, Schober C, et al. Treatment of basal cell carcinoma with intralesional recombinant interferon beta: A dosefinding study. European Journal of Dermatology. 1994;4(6):430-3. PMID: 1994253804.

125. Marks R, Owens M, Walters SA. Efficacy and safely of 5\% imiquimod cream in treating patients with multiple superficial basal cell carcinomas. Archives of Dermatology. 2004 October;140(10):1284-5. PMID: 2004440056.

126. Mazeron JJ, Chassagne D, Crook J, et al. Radiation therapy of carcinomas of the skin of nose and nasal vestibule: a report of 1676 cases by the Groupe Europeen de Curietherapie. Radiother Oncol. 1988 Nov;13(3):165-73. PMID: 3146781.

127. McIntosh GS, Osborne DR, Li AK, et al. Basal cell carcinoma--a review of treatment results with special reference to cryotherapy. Postgrad Med J. 1983 Nov;59(697):698-701. PMID: 6647186.

128. Mebed AH, Soliman HO, Gad ZS, et al. Multimodality treatment for non melanoma skin cancer: a prospective study done on 120 egyptian patients. J Egypt Natl Canc Inst. 2010 Mar;22(1):49-55. PMID: 21503006. 
129. Nevrkla E, Newton KA. A survey of the treatment of 200 cases of basal cell carcinoma (1959-1966 inclusive). Br J Dermatol. 1974 Oct;91(4):429-33. PMID: 4425623.

130. Pereira CT, Kruger EA, Sayer G, et al. Mohs versus surgical excision in nonmelanoma skin cancers: does location matter? Ann Plast Surg. 2013 Apr;70(4):432-4. doi: 10.1097/SAP.0b013e3182834b47. PMID: 23486132.

131. Rank B. Surgery and skin cancer. Ann R Coll Surg Engl. 1973 Mar;52(3):148-64. PMID: 4700671.

132. Reschly MJ, Shenefelt PD. Controversies in skin surgery: electrodessication and curettage versus excision for low-risk, small, well-differentiated squamous cell carcinomas. J Drugs Dermatol. 2010 Jul;9(7):773-6. PMID: 20677531.

133. Shiffman NJ. Squamous cell carcinomas of the skin of the pinna. Can J Surg. 1975 May;18(3):279-83. PMID: 1125865.

134. Tarstedt M, Gillstedt M, Wennberg Larko AM, et al. Aminolevulinic acid and methyl aminolevulinate equally effective in topical photodynamic therapy for non-melanoma skin cancers. J Eur Acad Dermatol Venereol. 2016 Mar;30(3):420-3. doi: 10.1111/jdv.13558. PMID: 26841041.

135. Tourli I, Langner D, Haroske G, et al. BASAL CELL CARCINOMA OF THE HEAD-ANDNECK REGION: A SINGLE CENTER ANALYSIS OF 1,750 TUMORS. Georgian Med News. 2016 Jan(250):33-9. PMID: 26870972.

136. Valentine RM, Ibbotson SH, Brown CT, et al. A quantitative comparison of 5-aminolaevulinic acid- and methyl aminolevulinate-induced fluorescence, photobleaching and pain during photodynamic therapy. Photochem Photobiol. 2011 Jan-Feb;87(1):242-9. doi: 10.1111/j.17511097.2010.00829.x. PMID: 21077899.

137. Van Hezewijk M, Creutzberg CL, Putter H, et al. Efficacy of a hypofractionated schedule in electron beam radiotherapy for epithelial skin cancer: Analysis of 434 cases. Radiotherapy and Oncology. 2010 May;95(2):245-9. doi: http://dx.doi.org/10.1016/j.radonc.2010.02.024. PMID: 2012354969.
138. Wang Y, Lin Y, Zhang HG, et al. A photodynamic therapy combined with topical 5aminolevulinic acid and systemic hematoporphyrin derivative is more efficient but less phototoxic for cancer. Journal of Cancer Research and Clinical Oncology. 201601 Apr;142(4):813-21. doi: http://dx.doi.org/10.1007/s00432-015-2066-3. PMID: 2015524013.

139. Werlinger KD, Upton G, Moore AY. Recurrence rates of primary nonmelanoma skin cancers treated by surgical excision compared to electrodesiccation-curettage in a private dermatological practice. Dermatol Surg. 2002 Dec;28(12):1138-42; discussion 42. PMID: 12472494.

140. Yoon M, Chougule P, Dufresne R, et al. Localized carcinoma of the external ear is an unrecognized aggressive disease with a high propensity for local regional recurrence. Am J Surg. 1992 Dec;164(6):574-7. PMID: 1463102.

141. Ahmed I, Berth-Jones J, Charles-Holmes S, et al. Comparison of cryotherapy with curettage in the treatment of Bowen's disease: a prospective study. Br J Dermatol. 2000 Oct;143(4):759-66. PMID: 11069453.

142. Asgari MM, Bertenthal D, Sen S, et al. Patient satisfaction after treatment of nonmelanoma skin cancer. Dermatol Surg. 2009 Jul;35(7):1041-9. doi: 10.1111/j.1524-4725.2009.01181.x. PMID: 19438672.

143. Ballester-Sanchez R, Pons-Llanas O, CandelaJuan C, et al. Electronic brachytherapy for superficial and nodular basal cell carcinoma: a report of two prospective pilot trials using different doses. J Contemp Brachytherapy. 2016 Feb;8(1):48-55. doi: 10.5114/jcb.2016.57531. PMID: 26985197.

144. Chren MM, Linos E, Torres JS, et al. Tumor recurrence 5 years after treatment of cutaneous basal cell carcinoma and squamous cell carcinoma. J Invest Dermatol. 2013 May;133(5):1188-96. doi: 10.1038/jid.2012.403. PMID: 23190903.

145. Chren MM, Sahay AP, Bertenthal DS, et al. Quality-of-life outcomes of treatments for cutaneous basal cell carcinoma and squamous cell carcinoma. J Invest Dermatol. 2007 Jun;127(6):1351-7. doi: 10.1038/sj.jid.5700740. PMID: 17301830. 
146. Chren MM, Sahay AP, Sands LP, et al. Variation in care for nonmelanoma skin cancer in a private practice and a veterans affairs clinic. Med Care. 2004 Oct;42(10):1019-26. PMID: 15377935.

147. Chren MM, Torres JS, Stuart SE, et al. Recurrence after treatment of nonmelanoma skin cancer: a prospective cohort study. Arch Dermatol. 2011 May;147(5):540-6. doi: 10.1001/archdermatol.2011.109. PMID: 21576572.

148. Cosgarea R, Susan M, Crisan M, et al. Photodynamic therapy using topical 5aminolaevulinic acid vs. surgery for basal cell carcinoma. J Eur Acad Dermatol Venereol. 2013 Aug;27(8):980-4. doi: 10.1111/j.14683083.2012.04619.x. PMID: 22738399.

149. Graells J, Ojeda RM, Garcia-Cruz A. Effect of imiquimod as compared with surgery on the cancerization field in basal cell carcinoma. Actas Dermosifiliogr. 2014 Jan-Feb;105(1):53-9. doi: 10.1016/j.ad.2013.09.001. PMID: 24139468.

150. Lippert J, Smucler R, Vlk M. Fractional carbon dioxide laser improves nodular basal cell carcinoma treatment with photodynamic therapy with methyl 5-aminolevulinate. Dermatol Surg. 2013 Aug;39(8):1202-8. doi: 10.1111/dsu.12242. PMID: 23725586.

151. Pampena R, Palmieri T, Kyrgidis A, et al. Orthovoltage radiotherapy for nonmelanoma skin cancer (NMSC): Comparison between 2 different schedules. J Am Acad Dermatol. 2016 Feb;74(2):341-7. doi: 10.1016/j.jaad.2015.09.031. PMID: 26589877.

152. Shah SM, Konnikov N, Duncan LM, et al. The effect of $595 \mathrm{~nm}$ pulsed dye laser on superficial and nodular basal cell carcinomas. Lasers Surg Med. 2009 Aug;41(6):417-22. doi: 10.1002/lsm.20787. PMID: 19588534.

153. Sofen H, Gross KG, Goldberg LH, et al. A phase II, multicenter, open-label, 3-cohort trial evaluating the efficacy and safety of vismodegib in operable basal cell carcinoma. J Am Acad Dermatol. 2015 Jul;73(1):99-105.e1. doi: 10.1016/j.jaad.2015.03.013. PMID: 25913533.
154. Sullivan TP, Dearaujo T, Vincek V, et al. Evaluation of superficial basal cell carcinomas after treatment with imiquimod $5 \%$ cream or vehicle for apoptosis and lymphocyte phenotyping. Dermatol Surg. 2003 Dec;29(12):1181-6. PMID: 14725659.

155. Wilson LS, Pregenzer M, Basu R, et al. Fee comparisons of treatments for nonmelanoma skin cancer in a private practice academic setting. Dermatol Surg. 2012 Apr;38(4):570-84. doi: 10.1111/j.1524-4725.2011.02231.x. PMID: 22145798 .

156. Stuart SE, Schoen P, Jin C, et al. Tumor recurrence of keratinocyte carcinomas judged appropriate for Mohs micrographic surgery using Appropriate Use Criteria. J Am Acad Dermatol. 2017 Mar 29doi: 10.1016/j.jaad.2016.12.045. PMID: 28365039.

157. Chren MM, Lasek RJ, Sahay AP, et al. Measurement properties of Skindex-16: a brief quality-of-life measure for patients with skin diseases. J Cutan Med Surg. 2001 MarApr;5(2):105-10. doi: 10.1007/s102270000010. PMID: 11443481.

158. Chren MM, Lasek RJ, Quinn LM, et al. Skindex, a quality-of-life measure for patients with skin disease: reliability, validity, and responsiveness. J Invest Dermatol. 1996 Nov;107(5):707-13. PMID: 8875954.

159. Connolly SM, Baker DR, Coldiron BM, et al. AAD/ACMS/ASDSA/ASMS 2012 appropriate use criteria for Mohs micrographic surgery: a report of the American Academy of Dermatology, American College of Mohs Surgery, American Society for Dermatologic Surgery Association, and the American Society for Mohs Surgery. J Am Acad Dermatol. 2012 Oct;67(4):531-50. doi: 10.1016/j.jaad.2012.06.009. PMID: 22959232.

160. Chaimani A, Vasiliadis HS, Pandis N, et al. Effects of study precision and risk of bias in networks of interventions: a network metaepidemiological study. Int J Epidemiol. 2013 Aug;42(4):1120-31. doi: 10.1093/ije/dyt074. PMID: 23811232.

161. Salanti G, Del Giovane C, Chaimani A, et al. Evaluating the quality of evidence from a network meta-analysis. PLoS One. 2014;9(7):e99682. doi: 10.1371/journal.pone.0099682. PMID: 24992266. 
162. American Academy of Dermatology and AAD Association. Position Statement on Superficial Radiation Therapy for Basal Cell Carcinoma (BCC) and Squamous Cell Carcinomas (SCC). 2014.

163. American Academy of Dermatology and AAD Association. Position Statement on Electronic Surface Brachytherapy for Basal Cell Carcinoma (BCC) and Squamous Cell Carcinomas (SCC). 2016.

164. Merola JF, Armstrong AW, Saraiya A, et al. International Dermatology Outcome Measures Initiative as Applied to Psoriatic Disease Outcomes: An Update. J Rheumatol. 2016 May;43(5):959-60. doi: 10.3899/jrheum.160114. PMID: 27134269.

165. Chan AW, Fung K, Tran JM, et al. Application of Recursive Partitioning to Derive and Validate a Claims-Based Algorithm for Identifying Keratinocyte Carcinoma (Nonmelanoma Skin Cancer). JAMA Dermatol. 2016 Oct 01;152(10):1122-7. doi: 10.1001/jamadermatol.2016.2609. PMID: 27533718. 


\section{Treatments for Basal Cell and Squamous Cell Carcinoma of the Skin: BCC Addendum}

An updated search, using the same search strategy from the original report but limited to basal cell carcinoma (BCC), was conducted in May 2018. The methods used for this addendum were the same as those used in the original report. The updates are for individual interventions only.

\section{Summary of Studies}

The updated search yielded three new studies ${ }^{1-3}$ and a paper with 5-year results from a fourth. ${ }^{4}$ Study characteristics for the three new studies are summarized in Table 1 . Two compared photodynamic therapy (PDT) with 5-aminolevulinic acid (ALA) compared to PDT with methyl aminolevulinate (MAL). ${ }^{2} 3$ The third compared surgery, cryotherapy, and laser diathermy. ${ }^{1}$

Table 1. Characteristics of eligible randomized trials

\begin{tabular}{|c|c|c|c|c|c|c|c|c|c|c|c|c|c|c|c|}
\hline \multirow[t]{2}{*}{ Study } & \multirow[t]{2}{*}{ Amm } & \multirow{2}{*}{$\begin{array}{l}\mathrm{N} \\
\text { people }\end{array}$} & \multirow{2}{*}{$\begin{array}{l}\text { Age, } \\
\text { Mean }\end{array}$} & \multirow{2}{*}{$\begin{array}{l}\text { Female } \\
\%\end{array}$} & \multirow[b]{2}{*}{$\begin{array}{l}\text { Lesion A[rea] } \\
\left(\mathrm{mm}^{2}\right) \text {, } \\
\text { D[iameter] } \\
\text { (mm), or } \\
\text { T[hickness] } \\
\text { (mm) }\end{array}$} & \multirow[b]{2}{*}{$\begin{array}{l}\text { Head } \\
\text { and } \\
\text { neck } \\
\text { location } \\
(\%)\end{array}$} & \multirow{2}{*}{$\begin{array}{l}\text { Max } \\
\text { FU } \\
(\mathrm{mo})\end{array}$} & \multirow{2}{*}{$\begin{array}{l}1^{*} \\
\text { RNG }\end{array}$} & \multirow{2}{*}{$\frac{2^{*}}{A C}$} & \multirow{2}{*}{$\begin{array}{l}3^{*} \\
\text { Bal }\end{array}$} & \multirow{2}{*}{$\begin{array}{l}4^{*} \\
\mathrm{BI}-\mathrm{Pt}\end{array}$} & \multirow{2}{*}{$\begin{array}{l}5^{*} \\
\mathrm{Bl}-\mathrm{Dr}\end{array}$} & \multirow{2}{*}{$\begin{array}{l}6^{*} \\
\mathrm{Bl}- \\
\mathrm{As}\end{array}$} & \multirow{2}{*}{$\begin{array}{l}7^{\star} \\
<20 \% \\
\text { attrition }\end{array}$} & \multirow{2}{*}{$\begin{array}{l}\text { ROB } \\
\text { summary } \\
\text { (across all } \\
\text { outcomes) }\end{array}$} \\
\hline & & & & & & & & & & & & & & & \\
\hline \multirow{2}{*}{$\begin{array}{l}\text { Kessels } \\
2018 \\
28886209^{2}\end{array}$} & $\begin{array}{l}\text { PDT } \\
\text { (MAL), E1 }\end{array}$ & 80 & 64 & 56 & $D=11$ & 1 & \multirow[t]{2}{*}{12} & \multirow[t]{2}{*}{ Yes } & \multirow[t]{2}{*}{ No } & \multirow[t]{2}{*}{ Yes } & \multirow[t]{2}{*}{ No } & \multirow[t]{2}{*}{ Yes } & \multirow[t]{2}{*}{ No } & \multirow[t]{2}{*}{ Yes } & \multirow[t]{2}{*}{ Low } \\
\hline & $\begin{array}{l}\text { PDT (ALA), } \\
\text { E2 }\end{array}$ & 82 & 66 & 51 & $D=11$ & 8 & & & & & & & & & \\
\hline \multirow{2}{*}{$\begin{array}{l}\text { Morton } \\
2018 \\
29432644^{3}\end{array}$} & $\begin{array}{l}\text { PDT } \\
\text { (MAL), E1 }\end{array}$ & 133 & 67 & 50 & $T=0.46$ & $>=17^{\star \star}$ & \multirow[t]{2}{*}{12} & \multirow[t]{2}{*}{ Yes } & \multirow[t]{2}{*}{ Unsure } & \multirow[t]{2}{*}{ No } & \multirow[t]{2}{*}{ Yes } & \multirow[t]{2}{*}{ Yes } & \multirow[t]{2}{*}{ Yes } & \multirow[t]{2}{*}{ No } & \multirow[t]{2}{*}{ Low } \\
\hline & $\begin{array}{l}\text { PDT (ALA), } \\
\text { E2 }\end{array}$ & 129 & 67 & 37 & $\mathrm{~T}=0.41$ & $>=17^{\star \star}$ & & & & & & & & & \\
\hline \multirow[t]{3}{*}{$\begin{array}{l}\text { Zane } 2017 \\
28291062^{1}\end{array}$} & $\begin{array}{l}\text { Surgical } \\
\text { excision, A }\end{array}$ & 80 & 68 & 45 & NR & 0 & 3 & Yes & Yes & Yes & No & No & No & Yes & Low \\
\hline & $\begin{array}{l}\text { Cryotherap } \\
y, \mathrm{C} 1\end{array}$ & 80 & 69 & 41 & NR & 0 & & & & & & & & & \\
\hline & $\begin{array}{l}\text { Laser } \\
\text { diathermy, } \\
\text { C5 }\end{array}$ & 80 & 68 & 40 & NR & 0 & & & & & & & & & \\
\hline
\end{tabular}

*Design items: 1: RNG = Adequate generation of a randomized sequence reported; 2 : AC=Adequate allocation concealment reported; 3: Bal=Group similarity at baseline; 4: $\mathrm{Bl} \mathrm{Pt}=$ Adequate blinding of patients reported; 5 : $\mathrm{Bl} \mathrm{Dr}=$ Adequate blinding of providers reported; 6: $\mathrm{Bl} \mathrm{As} \mathrm{=} \mathrm{Adequate} \mathrm{blinding} \mathrm{of} \mathrm{outcome} \mathrm{assessors} \mathrm{reported;} \mathrm{7:} \mathrm{Less} \mathrm{than} \mathrm{20 \%} \mathrm{of} \mathrm{sample} \mathrm{size} \mathrm{missing} \mathrm{for} \mathrm{any}$ eligible outcome in any arm.

** Neck lesions are counted with torso/extremities.

Abbreviations: PDT=photodynamic therapy; ALA= 5-aminolevulinic acid, MAL=methyl aminolevulinate, FU= fluorouracil, INF=interferon; NR=not reported.

\section{Recurrence}

The updated evidence graph for recurrence with respect to individual treatments is shown in Figure 1. It replaces the graph shown in Figure 7 of the report. 
Figure 1. Evidence graph of RCTs evaluating recurrence in BCCs across individual interventions

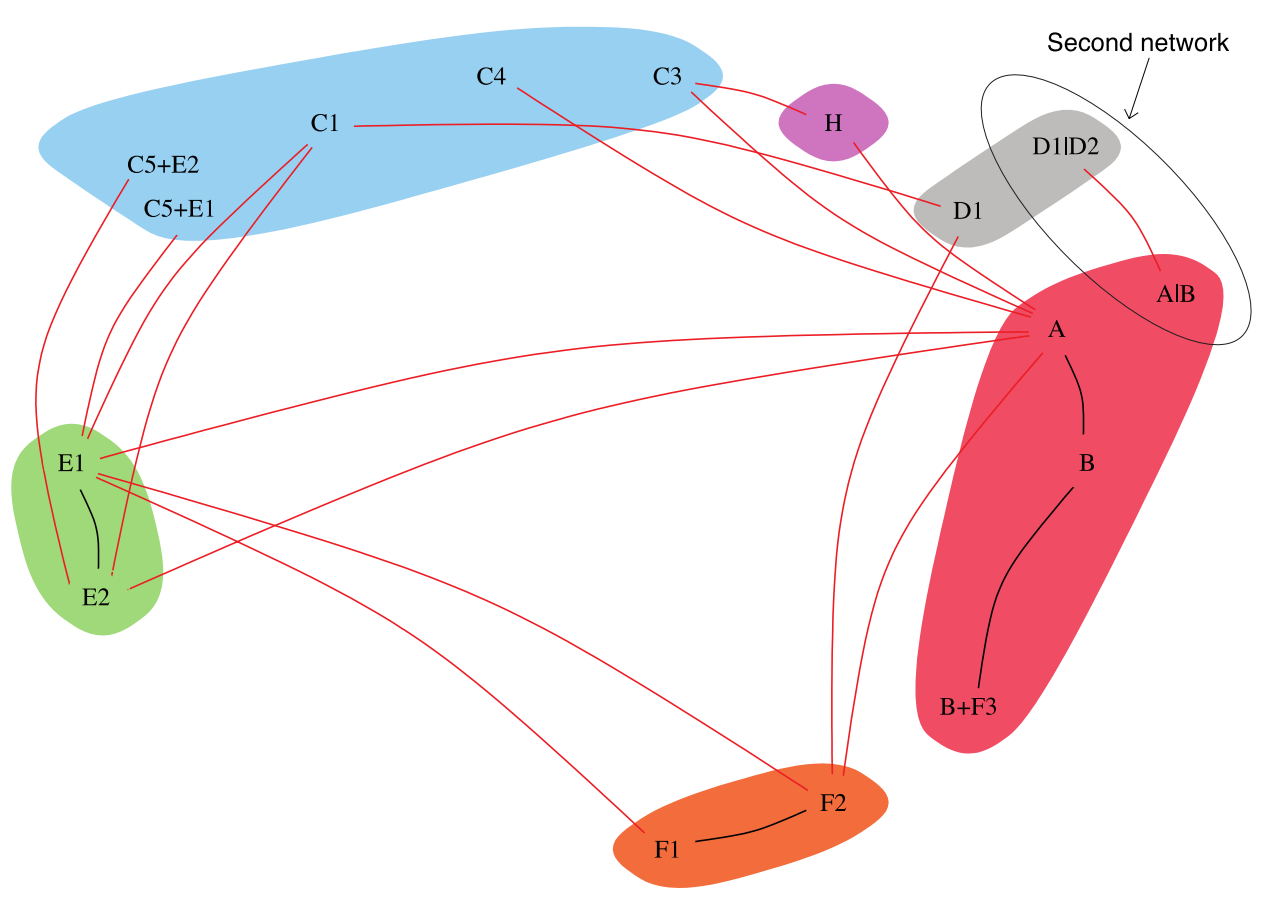

\author{
A: surgical excision \\ B: MMS \\ C1: cryotherapy \\ C3: curettage + diathermy \\ C4: curettage + cryotherapy \\ C5: laser \\ D1: external radiation \\ D2: brachytherapy \\ E1: PDT (MAL) \\ E2: PDT (ALA) \\ F1: 5-FU \\ F2: imiquimod \\ F3: INF \\ F4: ingenol \\ F5: other medical \\ $\mathrm{H}$ : curettage \\ I: no treatment \\ $\mathrm{J}$ : placebo/sham
}

Abbreviations: MMS=Mohs micrographic surgery, PDT=photodynamic therapy; ALA= 5-aminolevulinic acid, MAL=methyl aminolevulinate, FU= fluorouracil, INF=interferon. Layout as in Figure 1. This evidence graph comprises three connected networks. The first is the largest group of nodes and is not labeled explicitly. The second and third connected networks are labeled explicitly.

Table 2 replaces Table 11 from the original report, giving the relative effects for the larger subgraph. The results from the smaller network, given in Table 12 of the original report, were unaffected by this update. The new data changed the direction of the comparison for MALversus ALA-PDT from an odds ratio of 0.65 (95\% CI $0.25,1.73)$ to an odds ratio of 1.07 (95\% CI 0.34, 3.35). Nevertheless, the comparison remains nonsignificant, with wide confidence intervals. Similarly, surgery went from being better than Mohs surgery (MMS) (OR 1.04, 95\% CI $0.21,5.23$ ) to worse (OR 0.86; 95\% CI 0.13, 5.76), again with wide confidence intervals. MMS is no longer statistically significantly better than cryotherapy or PDT, with or without laser therapy. Other comparisons changed in magnitude, but not in direction or statistical significance. 
Table 2. Relative odds ratios for recurrence in the larger network in Figure 1

\begin{tabular}{|c|c|c|c|c|c|c|c|c|c|c|c|c|c|}
\hline \multirow[t]{14}{*}{$\begin{array}{l}\text { Sur- } \\
\text { gery }\end{array}$} & $\begin{array}{c}0.86(0.13 \\
5.76) \\
\end{array}$ & $\begin{array}{c}0.70(0.02 \\
21.26) \\
\end{array}$ & $\begin{array}{c}0.13(0.03, \\
0.48)\end{array}$ & $\begin{array}{c}0.53(0.06 \\
4.83) \\
\end{array}$ & $\begin{array}{c}0.16(0.03 \\
0.96)\end{array}$ & $\begin{array}{c}0.25(0.03 \\
2.18) \\
\end{array}$ & $\begin{array}{c}0.10(0.01 \\
0.78) \\
\end{array}$ & $\begin{array}{c}1.02(0.14 \\
7.39) \\
\end{array}$ & $\begin{array}{c}0.16(0.05 \\
0.5)\end{array}$ & $\begin{array}{c}0.17(0.05 \\
0.6)\end{array}$ & $\begin{array}{c}0.10(0.02 \\
0.56)\end{array}$ & $\begin{array}{c}0.20(0.05 \\
0.76)\end{array}$ & $\begin{array}{c}0.19(0.03, \\
1.36)\end{array}$ \\
\hline & MMS & $\begin{array}{c}0.82(0.02 \\
30.59)\end{array}$ & $\begin{array}{c}0.15(0.02 \\
1.13)\end{array}$ & $\begin{array}{c}0.62(0.04 \\
9.79)\end{array}$ & $\begin{array}{c}0.19(0.02 \\
2.2)\end{array}$ & $\begin{array}{c}0.29(0.02 \\
4.13)\end{array}$ & $\begin{array}{c}0.11(0.01 \\
1.5)\end{array}$ & $\begin{array}{c}1.19(0.1 \\
14.54)\end{array}$ & $\begin{array}{c}0.18(0.03 \\
1.26)\end{array}$ & $\begin{array}{c}0.19(0.03 \\
1.45)\end{array}$ & $\begin{array}{c}0.12(0.01 \\
1.19)\end{array}$ & $\begin{array}{c}0.24(0.03 \\
1.88)\end{array}$ & $\begin{array}{c}0.22(0.02 \\
2.89)\end{array}$ \\
\hline & & MMS + INF & $\begin{array}{c}0.18(0.01 \\
5.58)\end{array}$ & $\begin{array}{c}0.76(0.01 \\
38.1)\end{array}$ & $\begin{array}{c}0.23(0.01 \\
9.48)\end{array}$ & $\begin{array}{c}0.35(0.01 \\
16.35)\end{array}$ & $\begin{array}{c}0.14 \\
(<0.005 \\
6.06) \\
\end{array}$ & $\begin{array}{c}1.45(0.03 \\
60.47)\end{array}$ & $\begin{array}{c}0.22(0.01 \\
6.5)\end{array}$ & $\begin{array}{c}0.24(0.01 \\
7.24)\end{array}$ & $\begin{array}{c}0.15 \\
(<0.005 \\
5.33) \\
\end{array}$ & $\begin{array}{c}0.29(0.01 \\
9.21)\end{array}$ & $\begin{array}{c}0.26(0.01, \\
11.82)\end{array}$ \\
\hline & & & Cryotherapy & $\begin{array}{c}4.19(0.4 \\
43.92)\end{array}$ & $\begin{array}{c}1.29(0.18 \\
9.3)\end{array}$ & $\begin{array}{c}1.95(0.22 \\
17.33)\end{array}$ & $\begin{array}{c}0.76(0.09 \\
6.24)\end{array}$ & $\begin{array}{c}8.04(1.28 \\
50.51)\end{array}$ & $\begin{array}{c}1.23(0.39 \\
3.83)\end{array}$ & $\begin{array}{c}1.31(0.38 \\
4.56)\end{array}$ & $\begin{array}{c}0.81(0.14 \\
4.53)\end{array}$ & $\begin{array}{c}1.61(0.39 \\
6.66)\end{array}$ & $\begin{array}{c}1.46(0.17 \\
12.54)\end{array}$ \\
\hline & & & & $\begin{array}{c}\text { Curettage } \\
\text { and } \\
\text { Diathermy }\end{array}$ & $\begin{array}{c}0.31(0.02 \\
4.58)\end{array}$ & $\begin{array}{c}0.47(0.03 \\
8.55) \\
\end{array}$ & $\begin{array}{c}0.18(0.01 \\
3.12)\end{array}$ & $\begin{array}{c}1.92(0.12 \\
30.51)\end{array}$ & $\begin{array}{c}0.29(0.03 \\
2.82)\end{array}$ & $\begin{array}{c}0.31(0.03 \\
3.21)\end{array}$ & $\begin{array}{c}0.19(0.01 \\
2.55)\end{array}$ & $\begin{array}{c}0.38(0.04 \\
4.12)\end{array}$ & $\begin{array}{c}0.35(0.03 \\
3.94)\end{array}$ \\
\hline & & & & & $\begin{array}{l}\text { Curettage } \\
\text { and Cryo- } \\
\text { therapy }\end{array}$ & $\begin{array}{c}1.51(0.11 \\
20.74)\end{array}$ & $\begin{array}{c}0.59(0.05 \\
7.51)\end{array}$ & $\begin{array}{c}6.24(0.53 \\
72.8)\end{array}$ & $\begin{array}{c}0.95(0.15 \\
6.21)\end{array}$ & $\begin{array}{c}1.01(0.14 \\
7.16)\end{array}$ & $\begin{array}{c}0.63(0.07 \\
5.91)\end{array}$ & $\begin{array}{c}1.25(0.17 \\
9.21)\end{array}$ & $\begin{array}{c}1.13(0.09, \\
14.22)\end{array}$ \\
\hline & & & & & & $\begin{array}{c}\text { Laser + PDT } \\
\text { (MAL) }\end{array}$ & $\begin{array}{c}0.39(0.03 \\
5.96)\end{array}$ & $\begin{array}{c}4.12(0.29 \\
57.8)\end{array}$ & $\begin{array}{c}0.63(0.08 \\
4.68)\end{array}$ & $\begin{array}{c}0.67(0.08 \\
5.93)\end{array}$ & $\begin{array}{c}0.42(0.04 \\
4.64)\end{array}$ & $\begin{array}{c}0.83(0.09 \\
7.59)\end{array}$ & $\begin{array}{c}0.75(0.05 \\
11.74)\end{array}$ \\
\hline & & & & & & & $\begin{array}{l}\text { Laser + PDT } \\
\text { (ALA) }\end{array}$ & $\begin{array}{c}10.59(0.81 \\
138.59)\end{array}$ & $\begin{array}{c}1.62(0.21, \\
12.24)\end{array}$ & $\begin{array}{c}1.72(0.23 \\
12.82)\end{array}$ & $\begin{array}{c}1.07(0.1 \\
11.47)\end{array}$ & $\begin{array}{c}2.12(0.24 \\
18.39)\end{array}$ & $\begin{array}{c}1.92(0.13, \\
28.17)\end{array}$ \\
\hline & & & & & & & & $\begin{array}{c}\text { External } \\
\text { Radiotherap } \\
y\end{array}$ & $\begin{array}{c}0.15(0.02 \\
1.01) \\
\end{array}$ & $\begin{array}{c}0.16(0.02 \\
1.17) \\
\end{array}$ & $\begin{array}{c}0.10(0.01, \\
0.97)\end{array}$ & $\begin{array}{c}0.20(0.03 \\
1.52) \\
\end{array}$ & $\begin{array}{c}0.18(0.01 \\
2.44)\end{array}$ \\
\hline & & & & & & & & & PDT (MAL) & $\begin{array}{c}1.07(0.34 \\
3.35)\end{array}$ & $\begin{array}{c}0.66(0.15 \\
2.89)\end{array}$ & $\begin{array}{c}1.31(0.4 \\
4.28)\end{array}$ & $\begin{array}{c}1.19(0.15 \\
9.32)\end{array}$ \\
\hline & & & & & & & & & & PDT (ALA) & $\begin{array}{c}0.62(0.11 \\
3.44)\end{array}$ & $\begin{array}{c}1.23(0.3 \\
5.02)\end{array}$ & $\begin{array}{c}1.11(0.13, \\
9.41)\end{array}$ \\
\hline & & & & & & & & & & & $5-\mathrm{FU}$ & $\begin{array}{c}1.99(0.43, \\
9.21) \\
\end{array}$ & $\begin{array}{c}1.8(0.16 \\
19.87) \\
\end{array}$ \\
\hline & & & & & & & & & & & & Imiquimod & $\begin{array}{c}0.91(0.1 \\
7.99)\end{array}$ \\
\hline & & & & & & & & & & & & & Curettage \\
\hline
\end{tabular}

MMS=Mohs micrographic surgery, PDT=photodynamic therapy; ALA= 5-aminolevulinic acid, MAL=methyl aminolevulinate, 5-FU= 5-fluorouracil, INF=interferon, BCC=basal cell carcinoma. Cells shaded gray indicate that the estimate is based only on indirect comparisons; bold-italic numbers indicate statistical significance. Results are given as odds ratios (95\% confidence intervals). 
Table 3 replaces tables 13 and 27 from the main report. There is no change in the overall findings for recurrence, though the mean percentages changed some in magnitude.

Table 3. Mean outcome rates for specific interventions for basal cell carcinoma

\begin{tabular}{|c|c|c|c|}
\hline Intervention & $\begin{array}{c}\text { Recurrence } \\
\text { Mean Percent }(95 \% \mathrm{Cl})\end{array}$ & Intervention & $\begin{array}{l}\text { Lack of Histological Clearance } \\
\text { Mean Percent }(95 \% \text { Cl) }\end{array}$ \\
\hline First network in Figure 1 & & First network in Figure 2 & \\
\hline Surgical excision $(\mathrm{A})$ & $3.3(1.3,7.8)$ & Surgical excision (A) & $1.7(0.3,9.9)$ \\
\hline MMS (B) & $3.8(0.7,18.9)$ & Cryotherapy (C1) & $11.7(3.1,35.3)$ \\
\hline$M M S+I N F(B+F 3)$ & $4.6(0.2,56.2)$ & Laser (C5) & $33.7(10.9,67.9)$ \\
\hline Cryotherapy (C1) & $21.0(9.0,41.4)$ & Laser + PDT (MAL) (C5+E1) & $37.5(4.7,87.9)$ \\
\hline Diathermy+curettage (C3) & $5.9(0.7,34.9)$ & PDT (MAL) (E1) & $14.5(5.4,33.6)$ \\
\hline Cryotherapy+curettage $(\mathrm{C} 4)$ & $17.1(3.6,53.4)$ & PDT (ALA) (E2) & $11.0(2.1,41.4)$ \\
\hline Laser+PDT (MAL) (C5+E1) & $12.0(1.8,49.6)$ & 5-FU (F1) & $5.5(0.5,38.8)$ \\
\hline Laser+PDT (ALA) & $25.9(5.1,69.6)$ & Imiquimod (F2) & $28.6(14.6,48.6)$ \\
\hline External radiation (D1) & $3.2(0.6,16.1)$ & Ingenol (F4) & $77.1(23.7,97.3)$ \\
\hline PDT (MAL) (E1) & $17.8(9.1,31.8)$ & Other medical (F5) & $78.1(23.9,97.6)$ \\
\hline PDT (ALA) (E2) & $16.9(7.3,34.4)$ & No treatment (I) & $81.8(48.3,95.6)$ \\
\hline 5-fluorouracil (F1) & $24.7(7.1,58.4)$ & Placebo (J) & $86.3(72.1,93.9)$ \\
\hline Imiquimod (F2) & $14.1(5.4,32.4)$ & Second network in Figure 2 & \\
\hline Curettage $(H)$ & $15.4(2.6,55.3)$ & \multirow{3}{*}{$\begin{array}{l}\text { Surgery + PDT (MAL) (A+E1) } \\
\text { Surgery + PDT (MAL) + } \\
\text { curettage (A+E1+H) } \\
\text { Surgery + PDT (ALA) (A+E2) }\end{array}$} & $36.4(14.3,66.1)$ \\
\hline Second network in Figure 1 & & & $20.0(5.0,54.1)$ \\
\hline \multirow[t]{4}{*}{$\begin{array}{l}\text { Surgical excision or Mohs } \\
\text { (A|B) } \\
\text { External radiation or } \\
\text { brachytherapy (D1|D2) }\end{array}$} & \multirow[t]{4}{*}{$4.6(2.3,9.0)$} & & $\begin{array}{l}36.4(14.3,66.1) \\
18.2(4.6,50.7)\end{array}$ \\
\hline & & Third network in Figure 2 & \\
\hline & & Imiquimod + curettage $(\mathrm{F} 2+\mathrm{H})$ & $10.0(1.4,46.7)$ \\
\hline & & Curettage $(H)$ & \\
\hline
\end{tabular}

\section{Lack of Histologic Clearance}

The evidence graph for lack of histologic clearance with respect to individual treatments in Figure 2 replaces the one in Figure 8 of the main report 
Figure 2. Evidence graph for lack of BCC histological clearance

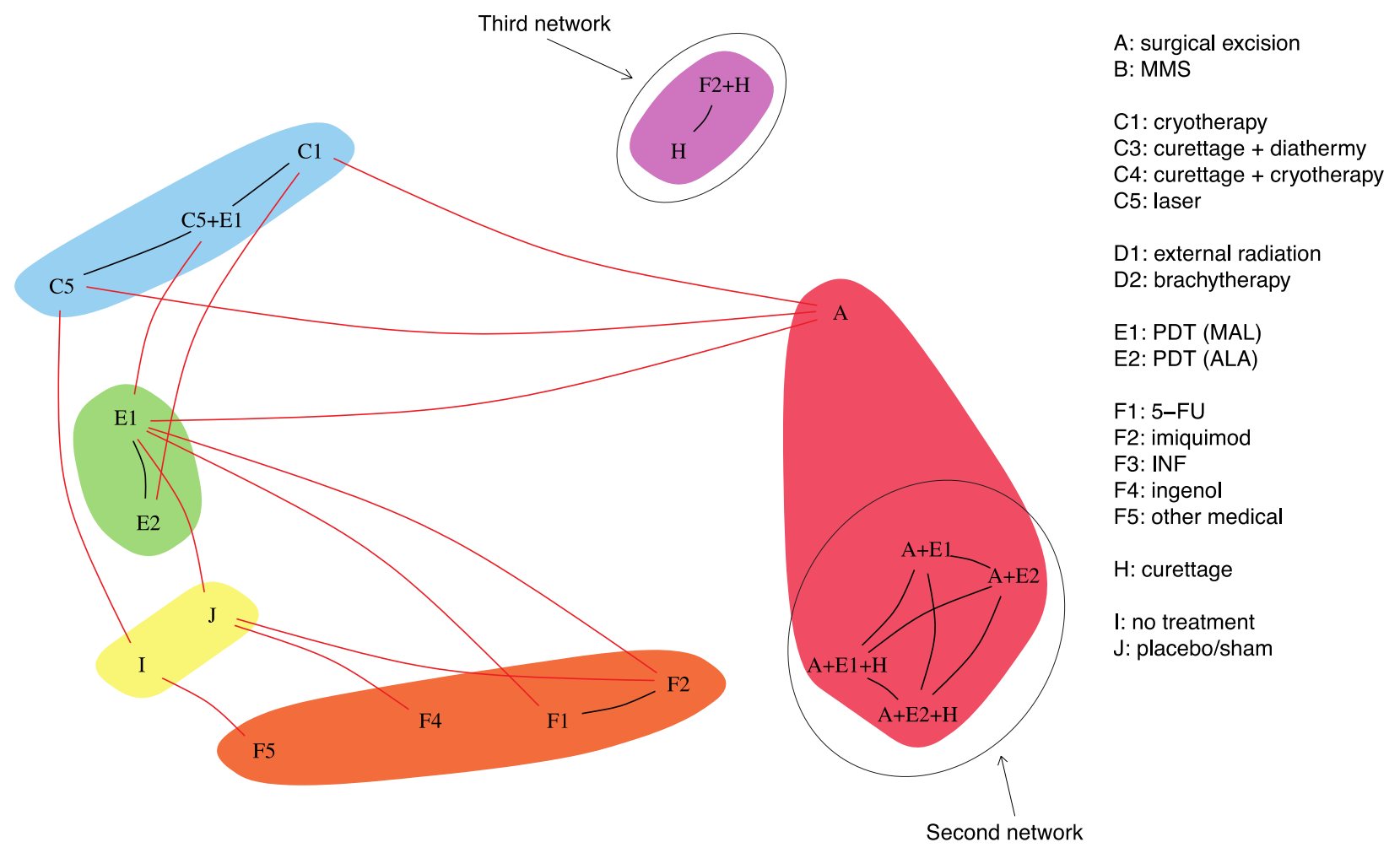

Abbreviations: MMS=Mohs micrographic surgery, PDT=photodynamic therapy; ALA=5-aminolevulinic acid, MAL=methyl aminolevulinate, $\mathrm{FU}=$ fluorouracil, $\mathrm{INF}=$ interferon. Layout as in Figure 1. This evidence graph comprises three connected networks. The first is the largest group of nodes and is not labeled explicitly. The second and third connected networks are labeled explicitly.

Table 4 replaces Table 24 of the main report. Tables 25 and 26 remain unchanged. Similar to what was seen in recurrence, MAL-PDT changed from performing non-significantly worse than ALA-PDT (OR 0.67; 95\% CI 0.30, 15.3) to non-significantly better (OR 1.37; 95\% CI 0.17, 10.74), with wide confidence intervals. Surgery's superiority over MAL-PDT with or without Laser, and Ingenol went from nonsignificant to significant. Ingenol performed statistically significantly worse than PDT and 5-FU. Other comparisons changed in magnitude, but not in direction or statistical significance. Table 3 replaces Table 27 from the report, but as was the case with recurrence, there are no changes, except in magnitude and precision. 
Table 4. Relative odds ratios for lack of histological clearance between individual interventions (large network in Figure 2)

\begin{tabular}{|c|c|c|c|c|c|c|c|c|c|c|c|}
\hline $\begin{array}{l}\text { Sur- } \\
\text { gery }\end{array}$ & $\begin{array}{c}0.13(0.01, \\
1.33) \\
\end{array}$ & $\begin{array}{c}0.03(<0.005, \\
0.35)\end{array}$ & $\begin{array}{c}0.03(<0.005 \\
0.64)\end{array}$ & $\begin{array}{c}0.1(0.01 \\
0.87) \\
\end{array}$ & $0.14(0.01,1.77)$ & $0.3(0.01,6.11)$ & $\begin{array}{c}0.04(0.01 \\
0.33) \\
\end{array}$ & $\begin{array}{c}0.01(<0.005 \\
0.1) \\
\end{array}$ & $\begin{array}{c}<0.005(<0.005, \\
0.1)\end{array}$ & $\begin{array}{c}<0.005 \\
(<0.005,0.04)\end{array}$ & $\begin{array}{c}<0.005 \\
(<0.005,0.02) \\
\end{array}$ \\
\hline & \multirow[t]{11}{*}{$\begin{array}{c}\text { Cryothera } \\
\text { py }\end{array}$} & $\begin{array}{c}0.26(0.03 \\
1.94) \\
\end{array}$ & $0.22(0.01,3.9)$ & $\begin{array}{c}0.78(0.13 \\
4.68) \\
\end{array}$ & $\begin{array}{c}1.07(0.11, \\
10.12) \\
\end{array}$ & $2.29(0.14,36.81)$ & $\begin{array}{c}0.33(0.06, \\
1.73) \\
\end{array}$ & $\begin{array}{c}0.04(<0.005, \\
0.63)\end{array}$ & $0.04(<0.005,0.62)$ & $\begin{array}{c}0.03(<0.005 \\
0.24) \\
\end{array}$ & $\begin{array}{c}0.02(<0.005 \\
0.11)\end{array}$ \\
\hline & & Laser & $\begin{array}{c}0.85(0.05, \\
15.04) \\
\end{array}$ & $\begin{array}{l}3(0.5 \\
18.11) \\
\end{array}$ & $4.11(0.43,39.1)$ & $\begin{array}{c}8.78(0.54, \\
142.06) \\
\end{array}$ & $\begin{array}{c}1.27(0.24 \\
6.69) \\
\end{array}$ & $0.15(0.01,2.42)$ & $0.14(0.01,2.39)$ & $\begin{array}{c}0.11(0.01 \\
0.94)\end{array}$ & $\begin{array}{c}0.08(0.02 \\
0.43)\end{array}$ \\
\hline & & & $\begin{array}{l}\text { Laser + PDT } \\
\text { (MAL) }\end{array}$ & $\begin{array}{c}3.54(0.23 \\
54.09) \\
\end{array}$ & $\begin{array}{c}4.85(0.23, \\
101.95) \\
\end{array}$ & $\begin{array}{c}10.35(0.33, \\
328.52)\end{array}$ & $\begin{array}{c}1.5(0.11 \\
20.96) \\
\end{array}$ & $0.18(0.01,5.62)$ & $0.17(0.01,5.48)$ & $\begin{array}{c}0.13(0.01 \\
2.55)\end{array}$ & $\begin{array}{c}0.1(0.01 \\
1.35) \\
\end{array}$ \\
\hline & & & & PDT (MAL) & $\begin{array}{c}1.37(0.17, \\
10.74) \\
\end{array}$ & $2.92(0.21,40.58)$ & $0.42(0.11,1.7)$ & $\begin{array}{c}0.05(<0.005, \\
0.69)\end{array}$ & $0.05(<0.005,0.68)$ & $\begin{array}{c}0.04(0.01 \\
0.26)\end{array}$ & $\begin{array}{c}0.03(0.01 \\
0.11) \\
\end{array}$ \\
\hline & & & & & PDT (ALA) & $2.13(0.11,41.16)$ & $\begin{array}{c}0.31(0.04, \\
2.15) \\
\end{array}$ & $\begin{array}{c}0.04(<0.005, \\
0.7)\end{array}$ & $0.03(<0.005,0.69)$ & $\begin{array}{c}0.03(<0.005 \\
0.29) \\
\end{array}$ & $\begin{array}{c}0.02(<0.005, \\
0.14)\end{array}$ \\
\hline & & & & & & 5-FU & $\begin{array}{c}0.14(0.01 \\
1.83) \\
\end{array}$ & $\begin{array}{c}0.02(<0.005 \\
0.5)\end{array}$ & $0.02(<0.005,0.49)$ & $\begin{array}{c}0.01(<0.005 \\
0.23) \\
\end{array}$ & $\begin{array}{c}0.01(<0.005 \\
0.12)\end{array}$ \\
\hline & & & & & & & Imiquimod & $0.12(0.01,1.5)$ & $0.11(0.01,1.48)$ & $\begin{array}{c}0.09(0.01 \\
0.53)\end{array}$ & $\begin{array}{c}0.06(0.02 \\
0.22) \\
\end{array}$ \\
\hline & & & & & & & & Ingenol & $0.94(0.03,28.3)$ & $\begin{array}{c}0.75(0.04, \\
12.94) \\
\end{array}$ & $\begin{array}{c}0.53(0.04 \\
6.78) \\
\end{array}$ \\
\hline & & & & & & & & & Other medical & $\begin{array}{c}0.79(0.04, \\
14.35) \\
\end{array}$ & $\begin{array}{c}0.57(0.04 \\
7.56) \\
\end{array}$ \\
\hline & & & & & & & & & & No treatment & $\begin{array}{c}0.71(0.12 \\
4.34)\end{array}$ \\
\hline & & & & & & & & & & & $\begin{array}{c}\text { Placebol } \\
\text { sham }\end{array}$ \\
\hline
\end{tabular}

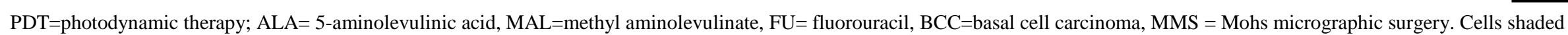
gray indicate that the estimate is based only on indirect comparisons; bold-italic numbers indicate statistical significance. Results are given as odds ratios (95\% confidence intervals). 


\section{Patient- and Observer-Reported Cosmetic Outcomes}

The report only gives results for intervention categories for these outcomes, so this data is additional. Figure 3 gives the evidence graph for patient-reported cosmetic outcomes, while Figure 4 gives the evidence graph for observer-reported cosmetic outcomes.

Figure 3. Evidence graph for patient-reported cosmetic outcomes of BCC treatment

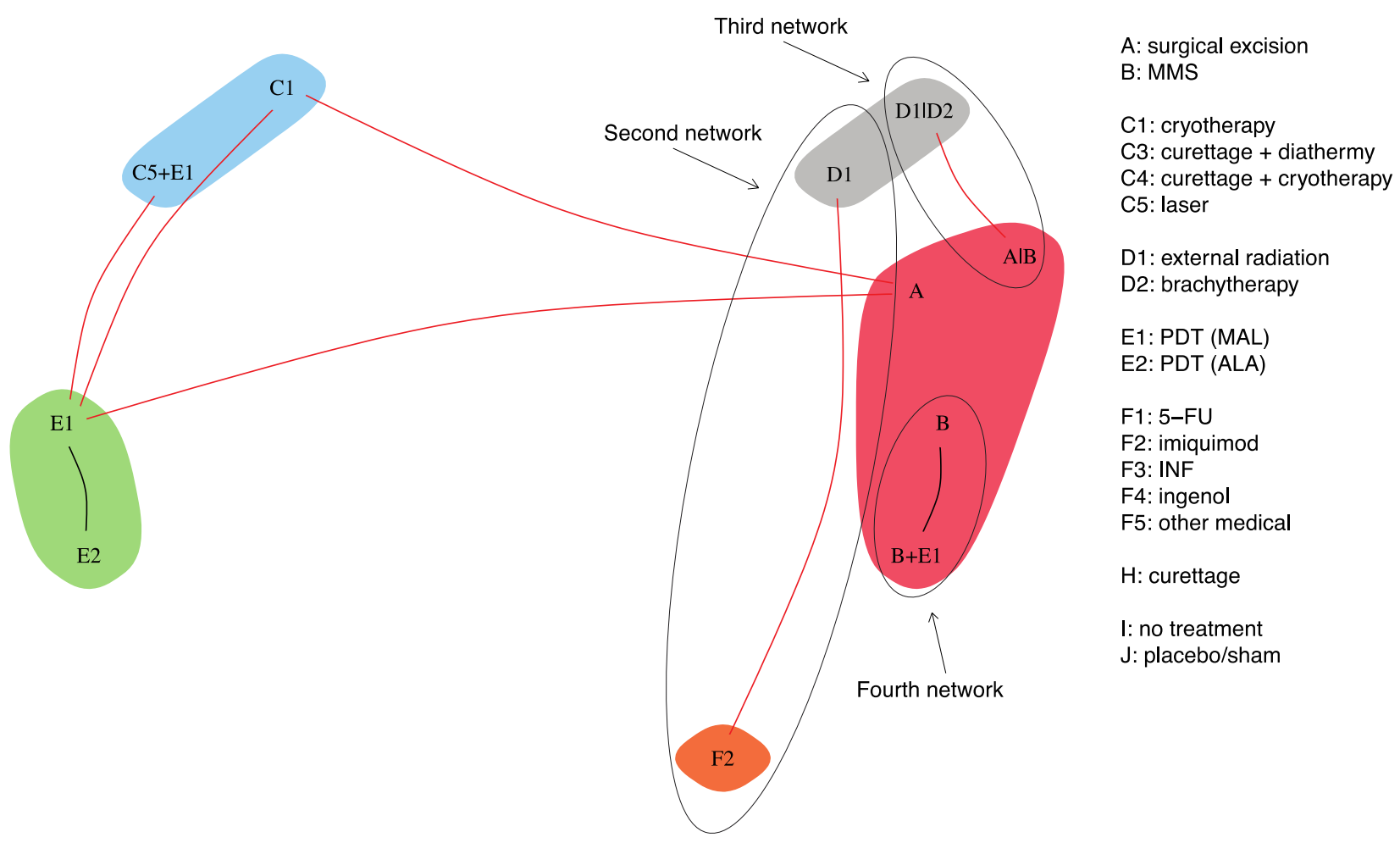

Abbreviations: MMS=Mohs micrographic surgery, PDT=photodynamic therapy; ALA= 5-aminolevulinic acid, MAL=methyl aminolevulinate, FU= fluorouracil, INF=interferon. Layout and naming of connected networks as in Figure 1. 
Figure 4. Evidence graph for observer-reported cosmetic outcomes of BCC treatments

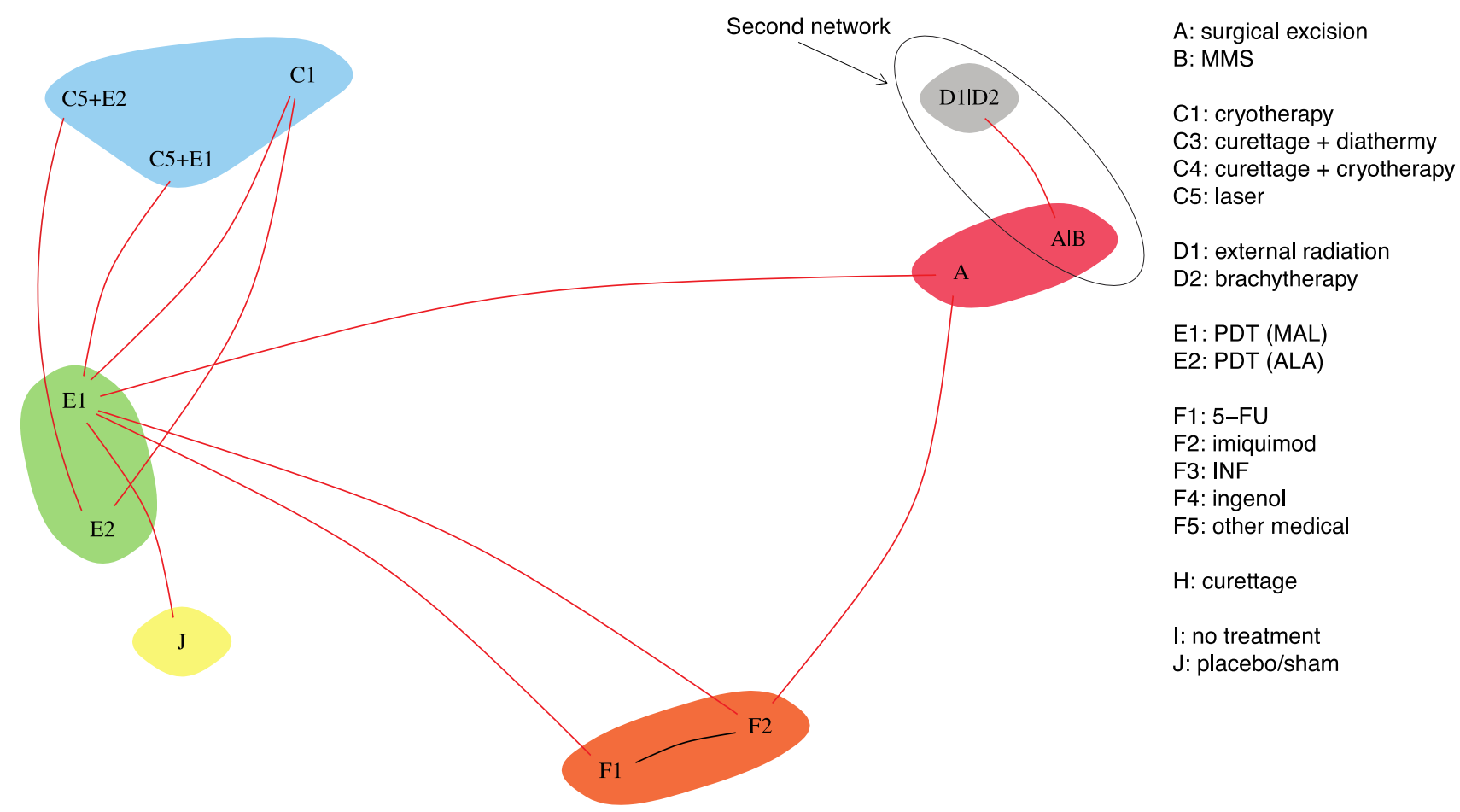

Abbreviations: MMS=Mohs micrographic surgery, PDT=photodynamic therapy; ALA= 5-aminolevulinic acid, MAL=methyl aminolevulinate, FU= fluorouracil, INF=interferon. Layout and naming of connected networks as in Figure 1.

As shown in Figure 3, patient-reported cosmetic outcomes comparisons in RCTs were sparse and involved four treatment networks. Table 5 gives the analysis of the largest network of 7 trials comparing 5 treatments (739 total lesions, range 23 to 169). Each of the other three networks comprised a single RCT. In one, an RCT ( $\mathrm{n}=27)$ did not find a difference between external radiation and imiquimod (OR 0.81, 95\% CI 0.01, 43.6). Another RCT favored surgical excision or Mohs micrographic surgery over external radiation/brachytherapy (n=244; OR 2.15, 95\% CI 1.2, 3.86). In the last RCT, 7/7 patients rated their outcome as good or better after methylaminolevulinic acid PDT followed by Mohs micrographic surgery and 10/10 after Mohs surgery alone.

Table 6 gives outcome rates for each intervention subgraph. In general, patients rated their cosmetic outcomes as good or better significantly more often with PDT using methylaminolevulinic acid (93.8\%, 95\% CI 79.2, 98.3) or aminolevulinic acid (95.8\%, 95\% CI 84.2, 99.0) compared with standard excision $(77.8 \%, 95 \%$ CI 44.8, 93.8), cryotherapy (51.1\%, 95\% CI $15.8,85.4)$ or PDT combined with laser preparation of the lesion (20\%, 95\% CI 1.9, 76.6). All other comparisons were statistically not significant and had wide confidence intervals. 
Table 5. Relative odds ratios for patient-reported cosmetic outcome

\begin{tabular}{ccccc}
\hline Surgery & $3.35(0.58,19.5)$ & $14.01(0.97,201.93)$ & $\mathbf{0 . 2 3}(0.08,0.65)$ & $0.16(0.05,0.49)$ \\
\hline & Cryotherapy & $4.18(0.23,77.64)$ & $\mathbf{0 . 0 7}(0.01,0.39)$ & $0.05(0.01,0.28)$ \\
\cline { 2 - 4 } & & Laser + PDT (MAL) & $\mathbf{0 . 0 2 ( < 0 . 0 0 5 , 0 . 2 1 )}$ & $0.01(<0.005,0.15)$ \\
\cline { 2 - 4 } & & PDT (MAL) & $0.67(0.38,1.15)$ \\
\cline { 3 - 5 } & & & PDT (ALA) \\
\cline { 3 - 4 }
\end{tabular}

PDT=photodynamic therapy; ALA= 5-aminolevulinic acid, MAL=methyl aminolevulinate. Cells shaded gray indicate that the estimate is based only on indirect comparisons; bold-italic numbers indicate statistical significance. Results are given as odds ratios (95\% confidence intervals).

Table 6. Mean outcome rates by specific intervention (all BCC lesions)

\begin{tabular}{|c|c|c|c|}
\hline Intervention & $\begin{array}{l}\text { Patient-reported good or } \\
\text { better cosmetic outcome } \\
\text { Mean Percent }(95 \% \mathrm{Cl})\end{array}$ & Intervention & $\begin{array}{l}\text { Observer-reported good or better cosmetic } \\
\text { outcome Mean Percent }(95 \% \mathrm{Cl})\end{array}$ \\
\hline First network in Figure 3 & & \multicolumn{2}{|l|}{ First network in Figure 4} \\
\hline Surgical excision $(\mathrm{A})$ & $77.8(44.8,93.8)$ & Surgical excision $(\mathrm{A})$ & $46.7(19.4,76.1)$ \\
\hline Cryotherapy (C1) & $51.1(15.8,85.4)$ & Cryotherapy (C1) & $60.1(23.1,88.3)$ \\
\hline Laser+PDT (MAL) (C5+E1) & $20.0(1.9,76.6)$ & Laser+PDT (MAL) (C5+E1) & $93.5(63.5,99.2)$ \\
\hline PDT (MAL) (E1) & $93.8(79.2,98.3)$ & Laser+PDT (ALA) (C5+E2) & $5.9(0.5,45.9)$ \\
\hline PDT (ALA) (E2) & $95.8(84.2,99.0)$ & PDT (MAL) (E1) & $87.9(73.3,95.1)$ \\
\hline \multicolumn{2}{|l|}{ Second network in Figure 3} & PDT (ALA) (E2) & $53.4(15.9,87.4)$ \\
\hline External radiation (D1) & $96.2(59.7,99.8)$ & 5-fluorouracil & $57.5(13.0,92.4)$ \\
\hline Imiquimod (F2) & $96.9(65.0,99.8)$ & \multirow{2}{*}{$\begin{array}{l}\text { Imiquimod } \\
\text { Placebo/sham }\end{array}$} & $61.0(24.8,88.1)$ \\
\hline Third network in Figure 3 & & & $93.3(41.5,99.6)$ \\
\hline Surgical excision or Mohs $(A \mid B)$ & $80.9(73.3,86.8)$ & \multicolumn{2}{|l|}{ Second network in Figure 4} \\
\hline $\begin{array}{l}\text { External radiation or } \\
\text { brachytherapy (D1|D2) }\end{array}$ & $66.4(57.2,74.5)$ & \multirow{4}{*}{$\begin{array}{l}\text { Surgical excision or Mohs }(A \mid B) \\
\text { External radiation or } \\
\text { brachytherapy (D1|D2) }\end{array}$} & $78.6(70.8,84.8)$ \\
\hline Fourth network in Figure 3 & & & \multirow[t]{3}{*}{$39.8(31.2,49.1)$} \\
\hline MMS (B) & $95.5(55.2,99.7)$ & & \\
\hline MMS +PDT (MAL) (B+E1) & $93.8(46.1,99.6)$ & & \\
\hline
\end{tabular}

MMS=Mohs micrographic surgery, PDT=photodynamic therapy; ALA=5-aminolevulinic acid, MAL=methyl aminolevulinate, FU= fluorouracil, INF=interferon; $\mathrm{BCC}=$ basal cell carcinoma; $\mathrm{CI}=$ confidence interval

Observer-reported cosmetic outcomes were evaluated in 11 RCTs and 1 NRCS. The larger network in Figure 4 consists of 10 RCTs that compare 9 treatments (3,505 total lesions, samples ranging from 23 to 563). As can be seen on Tables 6 and 7, good or better cosmetic outcomes were estimated to be more common for MAL-PDT than for ALA-PDT, surgical excision, cryotherapy, topical 5-fluorouracil, and imiquimod. The smaller network in Figure 4 was a single RCT that favored surgical excision or Mohs micrographic surgery over external radiation/brachytherapy $(\mathrm{n}=244$; odds ratio $5.56(3.17,9.76])$. 
Table 7. Relative odds ratios for observer-reported good or better cosmetic outcome

\begin{tabular}{|c|c|c|c|c|c|c|c|c|}
\hline $\begin{array}{l}\text { Sur- } \\
\text { gery }\end{array}$ & $\begin{array}{c}0.58(0.07 \\
4.58)\end{array}$ & $\begin{array}{c}0.06(0.01 \\
0.73)\end{array}$ & $\begin{array}{c}14.02(0.76, \\
257.68) \\
\end{array}$ & $\begin{array}{c}0.12(0.02 \\
0.61) \\
\end{array}$ & $0.76(0.08,7)$ & $\begin{array}{c}0.65(0.05 \\
8.31) \\
\end{array}$ & $\begin{array}{c}0.56(0.07 \\
4.24)\end{array}$ & $\begin{array}{c}0.06(<0.005 \\
1.61) \\
\end{array}$ \\
\hline & Cryotherapy & $\begin{array}{c}0.11(0.01 \\
1.49) \\
\end{array}$ & $\begin{array}{c}24.1(1.12, \\
517.07) \\
\end{array}$ & $\begin{array}{c}0.21(0.03 \\
1.36) \\
\end{array}$ & $\begin{array}{c}1.31(0.12 \\
14.7) \\
\end{array}$ & $\begin{array}{c}1.11(0.07 \\
17.02) \\
\end{array}$ & $\begin{array}{c}0.96(0.1 \\
9.05)\end{array}$ & $\begin{array}{c}0.11(<0.005 \\
3.18)\end{array}$ \\
\hline & & $\begin{array}{c}\text { Laser + PDT } \\
\text { (MAL) }\end{array}$ & $\begin{array}{c}228.74(7.99, \\
6544.69) \\
\end{array}$ & $\begin{array}{c}1.96(0.19 \\
20.04)\end{array}$ & $\begin{array}{c}12.48(0.78 \\
199.23)\end{array}$ & $\begin{array}{c}10.56(0.5 \\
222.5)\end{array}$ & $\begin{array}{c}9.14(0.66 \\
125.66)\end{array}$ & $\begin{array}{c}1.02(0.03 \\
39.29) \\
\end{array}$ \\
\hline & & & $\begin{array}{c}\text { Laser + PDT } \\
\text { (ALA) }\end{array}$ & $\begin{array}{c}0.01(<0.005, \\
0.14)\end{array}$ & $\begin{array}{c}0.05(<0.005, \\
1.3)\end{array}$ & $\begin{array}{c}0.05(<0.005, \\
1.4)\end{array}$ & $\begin{array}{c}0.04(<0.005, \\
0.83)\end{array}$ & $\begin{array}{c}<0.005(<0.005, \\
0.23)\end{array}$ \\
\hline & & & & PDT (MAL) & $\begin{array}{c}6.35(0.82 \\
49.22)\end{array}$ & $\begin{array}{c}5.38(0.48 \\
59.82)\end{array}$ & $\begin{array}{c}4.65(0.74 \\
29.3)\end{array}$ & $\begin{array}{c}0.52(0.02, \\
11.97)\end{array}$ \\
\hline & & & & & PDT (ALA) & $\begin{array}{c}0.85(0.05 \\
14.54)\end{array}$ & $\begin{array}{c}0.73(0.07 \\
7.92)\end{array}$ & $\begin{array}{c}0.08(<0.005 \\
2.66)\end{array}$ \\
\hline & & & & & & $5-\mathrm{FU}$ & $\begin{array}{c}0.87(0.06, \\
12.85)\end{array}$ & $\begin{array}{c}0.1(<0.005 \\
3.93)\end{array}$ \\
\hline & & & & & & & Imiquimod & $\begin{array}{c}0.11(<0.005 \\
3.23) \\
\end{array}$ \\
\hline & & & & & & & & $\begin{array}{c}\text { Placebol } \\
\text { sham }\end{array}$ \\
\hline
\end{tabular}

PDT=photodynamic therapy; ALA= 5-aminolevulinic acid, MAL=methyl aminolevulinate, FU= fluorouracil, BCC=basal cell carcinoma, MMS = Mohs micrographic surgery. Cells shaded gray indicate that the estimate is based only on indirect comparisons; bold-italic numbers indicate statistical significance. Results are given as odds ratios (95\% confidence intervals). 


\section{References}

1. Zane C, Facchinetti E, Arisi M, et al. Pulsed CO2 Laser Ablation of Superficial Basal Cell of Limbs and Trunk: A Comparative Randomized Clinical Trial With Cryotherapy and Surgical Ablation. Dermatol Surg. 2017 Jul;43(7):920-7. doi: 10.1097/dss.0000000000001106. PMID: 28291062.

2. Kessels J, Kreukels H, Nelemans PJ, et al. Treatment of superficial basal cell carcinoma by topical photodynamic therapy with fractionated 5-aminolaevulinic acid $20 \%$ vs. two-stage topical methyl aminolaevulinate: results of a randomized controlled trial. Br J Dermatol. 2017 Sep 8. doi: 10.1111/bjd.15967. PMID: 28886209.
3. Morton CA, Dominicus R, Radny P, et al. A randomized, multi-national, non-inferiority, phase III trial to evaluate the safety and efficacy of BF-200 ALA gel versus MAL cream in the treatment of non-aggressive basal cell carcinoma with photodynamic therapy (PDT). Br J Dermatol. 2018 Feb 12. doi: 10.1111/bjd.16441. PMID: 29432644.

4. Jansen MHE, Mosterd K, Arits A, et al. Five-Year Results of a Randomized Controlled Trial Comparing Effectiveness of Photodynamic Therapy, Topical Imiquimod, and Topical 5-Fluorouracil in Patients with Superficial Basal Cell Carcinoma. J Invest Dermatol. 2018 Mar;138(3):527-33. doi: 10.1016/j.jid.2017.09.033. PMID: 29045820. 


\section{PubMed (3/8/17)}

\section{Appendix A. Search Strategy}

(("Bowen's Disease"[Mesh] OR bowen’s Or “basal cell carcinoma” or "basal cell carcinomas” or "Carcinoma, Basal Cell"[Mesh] or BCC Or "squamous cell carcinoma” or "squamous cell carcinomas” OR "Carcinoma, Squamous Cell"[Mesh] or SCC OR ((keratinocyte* or "Keratinocytes"[Mesh]) and (carcinoma* or "Carcinoma"[Mesh])) OR “non-melanoma” OR “non melanoma” OR “nonmelanoma”) NOT (Oropharynx OR Oropharyngeal neoplasms or "Oropharyngeal Neoplasms"[Mesh] OR Pharynx OR Pharyngeal neoplasms OR "Pharyngeal Neoplasms"[Mesh] or "Lung Neoplasms"[Mesh] or "Urinary Bladder Neoplasms"[Mesh] or "Uterine Cervical Neoplasms"[Mesh] or "Esophageal Neoplasms"[Mesh] or "Laryngeal Neoplasms"[Mesh]))

AND

(((Surger* or surgic*) and (excision or removal)) Or "shave removal” Or "external beam radiation” Or "external-beam radiation” Or brachytherap* or "Brachytherapy"[Mesh] Or chemotherap* OR Sensus OR X-ray OR "X-Ray Therapy"[Mesh] OR radiotherapy OR "Radiotherapy"[Mesh] Or (topical and (medications or chemotherap*)) Or observation Or "watchful waiting” Or ((Mohs or micrographic*) and surgery) Or "Mohs Surgery"[Mesh] Or Curett* or "Curettage"[Mesh] Or diathermy or "Diathermy"[Mesh] Or cauterization or "Cautery"[Mesh] Or Cryotherapy or "Cryotherapy"[Mesh] Or electrodesiccation Or ((CO2 or "carbon dioxide”) and laser and therapy) Or "Laser Therapy"[Mesh] Or plesiotherapy Or "Methyl 5-aminolevulinate” or "methyl 5-aminolevulinate" [Supplementary Concept] OR MALA Or "5-aminolevulinic acid” or "Aminolevulinic Acid"[Mesh] Or ALA Or Photodynamic or "Photochemotherapy"[Mesh] or Photochemotherap* Or 5-fluorouracil Or 5-FU Or Methotrexate Or "Methotrexate"[Mesh] Or Bleomycin or "Bleomycin"[Mesh] Or imiquimod or "imiquimod" [Supplementary Concept] Or BEC-5 Or diclofenac or "Diclofenac"[Mesh] Or interferon or IFN Or "Ingenol mebutate" or "3-ingenyl angelate" [Supplementary Concept] or PEP005 or PEP-005 or "PEP 005” Or Vismodegib Or Erivedge or "HhAntag691"

[Supplementary Concept] or NSC747691 or NSC-747691 or "NSC 747691" or R-3616 or R3616 or "R 3616" or RG-3616 or RG3616 or "RG 3616" or GDC-0449 or GDC0449 or "GDC 0449" Or Sonidegib or Odomzo or "LDE225" [Supplementary Concept] or NVP-LDE225 Or Itraconazole or "Itraconazole"[Mesh] or Sporanox or Orungal or R51211 or R-51211 or "R 51211”)

AND

("Cohort Studies"[Mesh] OR cohort OR "Clinical Trial" [Publication Type] OR "Clinical Trials as Topic"[Mesh] OR (follow-up or followup) OR longitudinal OR "Placebos"[Mesh] OR placebo* OR "Research Design"[Mesh] OR "Evaluation Studies" [Publication Type] OR "Evaluation Studies as Topic"[Mesh] OR "Comparative Study" [Publication Type] OR ((comparative OR Intervention) AND study) OR pretest* OR pre test* OR posttest* OR post test* OR prepost* OR pre post* OR "before and after” OR interrupted time* OR time serie* OR intervention* OR ((quasi-experiment* OR quasiexperiment* OR quasi experiment*) and (method or study or trial or design*)) OR "Case-Control Studies"[Mesh] OR (case and control) OR Clinical Studies OR "Clinical Studies as Topic"[Mesh] OR random allocation [mh] OR double-blind method[mh] OR single-blind method[mh] OR random* OR "Clinical Trial" [Publication Type] OR "Clinical Trials as Topic"[Mesh] OR "Placebos"[Mesh] OR placebo OR ((clinical OR controlled) and trial*) OR ((singl* or doubl* or trebl* or tripl*) and (blind* or 
mask*)) OR rct OR "Randomized Controlled Trial" [Publication Type] OR "Controlled Clinical Trial" [Publication Type] OR randomized)

NOT

(“addresses”[pt] or “autobiography”[pt] or “bibliography”[pt] or "biography”[pt] or “case reports”[pt] or “comment”[pt] or “congresses”[pt] or “dictionary”[pt] or “directory”[pt] or “editorial”"[pt] or "festschrift”[pt] or "government publications”[pt] or "historical article”[pt] or “interview”[pt] or “lectures”[pt] or “legal cases”[pt] or “legislation”[pt] or “letter”[pt] or “news”[pt] or "newspaper article”[pt] or "patient education handout”[pt] or "periodical index”[pt] or "comment on" or "review”[pt] or “systematic”[sb] OR ("Animals"[Mesh] NOT "Humans"[Mesh]) OR rats[tw] or cow[tw] or cows[tw] or chicken*[tw] or horse[tw] or horses[tw] or mice[tw] or mouse[tw] or bovine[tw] or sheep or ovine or murinae)

\section{Cochrane (3/8/17)}

((bowen's Or bowens OR basal cell carcinoma or BCC Or squamous cell carcinoma or SCC OR keratinocyte* and carcinoma* OR “non-melanoma” OR “non melanoma” OR “nonmelanoma”) NOT (Oropharynx OR Oropharyngeal neoplasms OR Pharynx OR Pharyngeal neoplasms)) AND

(((Surger* or surgic*) and (excision or removal)) Or "shave removal” Or "external beam radiation” Or “external-beam radiation” Or brachytherap* Or chemotherap* OR Sensus OR X-ray OR radiotherapy Or (topical and (medications or chemotherap*)) Or observation Or "watchful waiting” Or ((Mohs or micrographic*) and surgery) Or Curett* Or diathermy or cauterization Or Cryotherapy Or electrodesiccation Or ((CO2 or "carbon dioxide”) and laser and therapy) Or plesiotherapy Or "Methyl 5-aminolevulinate” or "methyl 5-aminolevulinate" or MALA Or "5-aminolevulinic acid" or ALA Or Photodynamic or Photochemotherap* Or 5fluorouracil Or 5-FU Or Methotrexate Or Bleomycin Or imiquimod Or BEC-5 Or diclofenac Or interferon or IFN Or "Ingenol mebutate" or "3-ingenyl angelate" or PEP005 or PEP-005 or “PEP 005” Or Vismodegib Or Erivedge or NSC747691 or NSC-747691 or "NSC 747691" or R3616 or R3616 or "R 3616” or RG-3616 or RG3616 or “RG 3616” or GDC-0449 or GDC0449 or “GDC 0449” Or Sonidegib or Odomzo or NVP-LDE225 Or Itraconazole or Sporanox or Orungal or R51211 or R-51211 or “R 51211”)

\section{EMBASE (3/8/17)}

(bowen* OR basal cell carcinoma or BCC Or squamous cell carcinoma or SCC OR keratinocyte* and carcinoma* OR non-melanoma OR non melanoma OR nonmelanoma) NOT (Oropharynx OR Oropharyngeal neoplasms OR Pharynx OR Pharyngeal neoplasms) AND (((Surger* or surgic*) and (excision or removal)) Or "shave removal” Or "external beam radiation” Or "external-beam radiation” Or brachytherap* Or chemotherap* OR Sensus OR X-ray OR radiotherapy Or (topical and (medications or chemotherap*)) Or observation Or "watchful waiting” Or ((Mohs or micrographic*) and surgery) Or Curett* Or diathermy or cauterization Or Cryotherapy Or electrodesiccation Or ((CO2 or "carbon dioxide”) and laser and therapy) Or plesiotherapy Or "Methyl 5-aminolevulinate" or "methyl 5-aminolevulinate" or MALA Or "5-aminolevulinic acid" or ALA Or Photodynamic or Photochemotherap* Or 5fluorouracil Or 5-FU Or Methotrexate Or Bleomycin Or imiquimod Or BEC-5 Or diclofenac Or interferon or IFN Or "Ingenol mebutate" or "3-ingenyl angelate" or PEP005 or PEP-005 or “PEP 005” Or Vismodegib Or Erivedge or NSC747691 or NSC-747691 or “NSC 747691” or R- 
3616 or R3616 or "R 3616" or RG-3616 or RG3616 or "RG 3616” or GDC-0449 or GDC0449 or "GDC 0449" Or Sonidegib or Odomzo or NVP-LDE225 Or Itraconazole or Sporanox or Orungal or R51211 or R-51211 or "R 51211")

AND

(Clinical trial/ OR Randomized controlled trial/ OR Randomization/ OR Single blind procedure/ OR Double blind procedure/ OR Crossover procedure/ OR Placebo/ OR Randomi?ed controlled trial\$.tw. OR Rct.tw. OR Random allocation.tw. OR Randomly allocated.tw. OR Allocated randomly.tw. OR (allocated adj2 random).tw. OR Single blind\$.tw. OR Double blind\$.tw. OR ((treble or triple) adj blind\$).tw. OR Placebo\$.tw. OR Prospective study/ OR Clinical study/ OR Case control study OR Family study/ OR Longitudinal study/ OR Retrospective study/ OR Prospective study/ OR Randomized controlled trials/ OR Cohort analysis/ OR (Cohort adj (study or studies)).mp. OR (Case control adj (study or studies)).tw. OR (follow up adj (study or studies)).tw. OR (observational adj (study or studies)).tw. OR (epidemiologic\$ adj (study or studies)).tw. OR (cross sectional adj (study or studies)).tw.)

Limits: (human and english language and (adult $<18$ to 64 years $>$ or aged $<65+$ years $>$ ))

\section{ClinicalTrials.gov (8/25/16) 376 records}

(bowen's disease OR basal cell carcinoma OR BCC OR squamous cell carcinoma OR SCC OR keratinocyte carcinoma OR "non-melanoma")

AND (skin OR dermatology OR dermatological OR derma)

\section{ICTRP (8/25/16) 601 records}

bowen's disease OR basal cell carcinoma OR BCC OR squamous cell carcinoma AND skin OR SCC AND skin OR keratinocyte carcinoma OR non-melanoma AND skin 


\section{Appendix B. Excluded Studies}

Table B-1. Excluded studies

\begin{tabular}{|c|c|c|c|c|}
\hline UID & First Author & Title & Journal & $\begin{array}{l}\text { Reason for } \\
\text { Exclusion }\end{array}$ \\
\hline 4455059 & $\begin{array}{l}\text { Abad Iglesias, } \\
\text { R. }\end{array}$ & $\begin{array}{l}\text { [Topical treatment of basocellular epitheliomas } \\
\text { with } 5 \text {-fluorouracil and vinblastine. Radiobiologic } \\
\text { evaluation and comparison with its status with } \\
\text { radiotherapy] }\end{array}$ & $\begin{array}{l}\text { Actas } \\
\text { Dermosifili } \\
\text { ogr }\end{array}$ & Not English (Spanish) \\
\hline 24669636 & Afridi, R. A. & $\begin{array}{l}\text { Demographics of basal cell carcinoma and its } \\
\text { surgical management }\end{array}$ & $\begin{array}{l}\text { J Ayub } \\
\text { Med Coll } \\
\text { Abbottaba } \\
\text { d }\end{array}$ & $\begin{array}{l}\text { not comparative between } \\
\text { treatment nodes }\end{array}$ \\
\hline $\begin{array}{l}\mathrm{CN}- \\
00450646\end{array}$ & Ahmed, I. & $\begin{array}{l}\text { Comparison of cryotherapy versus curettage in } \\
\text { the treatment of Bowen's disease. Abstract }\end{array}$ & $\begin{array}{l}\text { British } \\
\text { journal of } \\
\text { dermatolo } \\
\text { gy }\end{array}$ & $\begin{array}{l}\text { duplicate/conference } \\
\text { abstract and we have full } \\
\text { publication }\end{array}$ \\
\hline $\begin{array}{l}\mathrm{CN}- \\
00400052\end{array}$ & $\begin{array}{l}\text { Almenar, D. F. } \\
\text { E. }\end{array}$ & $\begin{array}{l}\text { Comparative study of CDDP + 5-FU vs CDDP + } \\
\text { Ftorafur in advanced head and neck squamous- } \\
\text { cell carcinoma }\end{array}$ & $\begin{array}{l}\text { Libro de } \\
\text { ResÃmen } \\
\text { es. I } \\
\text { Congreso } \\
\text { Iberoameri } \\
\text { cano de } \\
\text { OncologÃ- } \\
\text { a }\end{array}$ & $\begin{array}{l}\text { not treatment of skin } \\
\text { cancer or }<80 \% \text { SCC or } \\
\text { BCC }\end{array}$ \\
\hline 8708151 & Alpsoy, E. & $\begin{array}{l}\text { Comparison of the effects of intralesional } \\
\text { interferon alfa- } 2 a, 2 b \text { and the combination of } 2 a \\
\text { and } 2 b \text { in the treatment of basal cell carcinoma }\end{array}$ & $\begin{array}{l}\mathrm{J} \\
\text { Dermatol }\end{array}$ & $\begin{array}{l}\text { duplicate/conference } \\
\text { abstract and we have full } \\
\text { publication }\end{array}$ \\
\hline 16374471 & $\begin{array}{l}\text { Angell- } \\
\text { Petersen, E. }\end{array}$ & $\begin{array}{l}\text { Porphyrin formation in actinic keratosis and } \\
\text { basal cell carcinoma after topical application of } \\
\text { methyl 5-aminolevulinate }\end{array}$ & $\begin{array}{l}\mathrm{J} \text { Invest } \\
\text { Dermatol }\end{array}$ & $\begin{array}{l}\text { not comparative between } \\
\text { treatment nodes }\end{array}$ \\
\hline 19863513 & Apalla, Z. & $\begin{array}{l}\text { Skin cancer: preventive photodynamic therapy } \\
\text { in patients with face and scalp cancerization. A } \\
\text { randomized placebo-controlled study }\end{array}$ & $\begin{array}{l}\mathrm{Br} \mathrm{J} \\
\text { Dermatol }\end{array}$ & $\begin{array}{l}\text { not treatment of skin } \\
\text { cancer or }<80 \% \text { SCC or } \\
\text { BCC }\end{array}$ \\
\hline 26489922 & Arenas, M. & $\begin{array}{l}\text { Hypofractionated high-dose-rate plesiotherapy } \\
\text { in nonmelanoma skin cancer treatment }\end{array}$ & $\begin{array}{l}\text { Brachythe } \\
\text { rapy }\end{array}$ & $\begin{array}{l}\text { not comparative between } \\
\text { treatment nodes }\end{array}$ \\
\hline 24749843 & Arits, A. H. & $\begin{array}{l}\text { Cost-effectiveness of topical imiquimod and } \\
\text { fluorouracil vs. photodynamic therapy for } \\
\text { treatment of superficial basal-cell carcinoma }\end{array}$ & $\begin{array}{l}\mathrm{Br} \mathrm{J} \\
\text { Dermatol }\end{array}$ & no outcomes of interest \\
\hline $\begin{array}{l}\text { CN- } \\
00789999\end{array}$ & Arits, Ahmm & $\begin{array}{l}\text { Three non-invasive treatment options for } \\
\text { superficial basal cell carcinoma: photodynamic } \\
\text { therapy versus imiquimod versus 5-fluorouracil. } \\
\text { TTOP-sBCC trial }\end{array}$ & $\begin{array}{l}\text { Melanoma } \\
\text { research }\end{array}$ & $\begin{array}{l}\text { duplicate/conference } \\
\text { abstract and we have full } \\
\text { publication }\end{array}$ \\
\hline 23930247 & Asilian, A. & $\begin{array}{l}\text { Comparison between examination with naked } \\
\text { eye, curretage and dermoscopy in determining } \\
\text { tumor extension before Mohs micrographic } \\
\text { surgery }\end{array}$ & $\begin{array}{l}\text { Adv } \\
\text { Biomed } \\
\text { Res }\end{array}$ & $\begin{array}{l}\text { not comparative between } \\
\text { treatment nodes }\end{array}$ \\
\hline 5450847 & Aurora, A. L. & $\begin{array}{l}\text { Reappraisal of basal cell carcinoma of the } \\
\text { eyelids }\end{array}$ & $\begin{array}{l}\text { Am J } \\
\text { Ophthalm } \\
\text { ol }\end{array}$ & $\begin{array}{l}>20 \% \text { recurrent or } \% \\
\text { recurrent not given }\end{array}$ \\
\hline 7917206 & Austin, J. R. & $\begin{array}{l}\text { Squamous cell carcinoma of the external } \\
\text { auditory canal. Therapeutic prognosis based on } \\
\text { a proposed staging system }\end{array}$ & $\begin{array}{l}\text { Arch } \\
\text { Otolaryng } \\
\text { ol Head } \\
\text { Neck Surg }\end{array}$ & $\begin{array}{l}\text { not treatment of skin } \\
\text { cancer or }<80 \% \text { SCC or } \\
\text { BCC }\end{array}$ \\
\hline $\begin{array}{l}\mathrm{CN}- \\
00465907\end{array}$ & Avril, M. & $\begin{array}{l}\text { Basal cell carcinoma of the face: surgery or } \\
\text { radiotherapy? Results of a randomised study } \\
\text { Abstract W12-6 The 7th Congress of the } \\
\text { European Academy of Dermatology and } \\
\text { Venereology, Nice, 7-11 Ocober } 1998\end{array}$ & $\begin{array}{l}\text { Journal of } \\
\text { the } \\
\text { European } \\
\text { Academy } \\
\text { of } \\
\text { Dermatolo }\end{array}$ & $\begin{array}{l}\text { duplicate/conference } \\
\text { abstract and we have full } \\
\text { publication }\end{array}$ \\
\hline
\end{tabular}




\begin{tabular}{|c|c|c|c|c|}
\hline UID & First Author & Title & Journal & $\begin{array}{l}\text { Reason for } \\
\text { Exclusion }\end{array}$ \\
\hline & & & $\begin{array}{l}\text { gy and } \\
\text { Venereolo } \\
\text { gy : } \\
\text { JEADV }\end{array}$ & \\
\hline 11453910 & Baas, $\mathrm{P}$. & $\begin{array}{l}\text { Photodynamic therapy with meta- } \\
\text { tetrahydroxyphenylchlorin for basal cell } \\
\text { carcinoma: a phase I/II study }\end{array}$ & $\begin{array}{l}\mathrm{Br} \mathrm{J} \\
\text { Dermatol }\end{array}$ & $\begin{array}{l}\text { not comparative between } \\
\text { treatment nodes }\end{array}$ \\
\hline 4782176 & $\begin{array}{l}\text { Babaiants, R. } \\
\text { S. }\end{array}$ & $\begin{array}{l}\text { [Clinical characteristics of skin cancer and } \\
\text { comparative characteristics of different methods } \\
\text { of its treatment at remote periods] }\end{array}$ & $\begin{array}{l}\text { Vestn } \\
\text { Dermatol } \\
\text { Venerol }\end{array}$ & Not English (Russian) \\
\hline 8985019 & $\begin{array}{l}\text { Bachaud, J. } \\
\text { M. }\end{array}$ & $\begin{array}{l}\text { Combined postoperative radiotherapy and } \\
\text { weekly cisplatin infusion for locally advanced } \\
\text { head and neck carcinoma: final report of a } \\
\text { randomized trial }\end{array}$ & $\begin{array}{l}\text { Int J } \\
\text { Radiat } \\
\text { Oncol Biol } \\
\text { Phys }\end{array}$ & $\begin{array}{l}\text { not treatment of skin } \\
\text { cancer or }<80 \% \text { SCC or } \\
\text { BCC }\end{array}$ \\
\hline 15933497 & $\begin{array}{l}\text { Backous, D. } \\
\text { D. }\end{array}$ & $\begin{array}{l}\text { Craniofacial resection for nonmelanoma skin } \\
\text { cancer of the head and neck }\end{array}$ & $\begin{array}{l}\text { Laryngosc } \\
\text { ope }\end{array}$ & $\begin{array}{l}\text { not comparative between } \\
\text { treatment nodes }\end{array}$ \\
\hline 23648439 & $\begin{array}{l}\text { Balamucki, C. } \\
\text { J. }\end{array}$ & $\begin{array}{l}\text { Impact of radiographic findings on for prognosis } \\
\text { skin cancer with perineural invasion }\end{array}$ & $\begin{array}{l}\text { Am J Clin } \\
\text { Oncol }\end{array}$ & $\begin{array}{l}\text { No analysis by } \\
\text { population of interest }\end{array}$ \\
\hline 26985197 & $\begin{array}{l}\text { Ballester- } \\
\text { Sanchez, R. }\end{array}$ & $\begin{array}{l}\text { Electronic brachytherapy for superficial and } \\
\text { nodular basal cell carcinoma: a report of two } \\
\text { prospective pilot trials using different doses }\end{array}$ & $\begin{array}{l}\text { J } \\
\text { Contemp } \\
\text { Brachythe } \\
\text { rapy }\end{array}$ & $\begin{array}{l}\text { duplicate/conference } \\
\text { abstract and we have full } \\
\text { publication }\end{array}$ \\
\hline 16836497 & Baptista, J. & $\begin{array}{l}\text { Our PDT experience in the treatment of non- } \\
\text { melanoma skin cancer over the last } 7 \text { years }\end{array}$ & $\begin{array}{l}\text { J Eur } \\
\text { Acad } \\
\text { Dermatol } \\
\text { Venereol }\end{array}$ & $\begin{array}{l}\text { not comparative between } \\
\text { treatment nodes }\end{array}$ \\
\hline $\begin{array}{l}\text { CN- } \\
00094532\end{array}$ & Bar-Am, A. & $\begin{array}{l}\text { High- and low-power CO2 lasers. Comparison } \\
\text { of results for three clinical indications }\end{array}$ & $\begin{array}{l}\text { The } \\
\text { Journal of } \\
\text { reproducti } \\
\text { ve } \\
\text { medicine } \\
\end{array}$ & $\begin{array}{l}\text { not treatment of skin } \\
\text { cancer or }<80 \% \text { SCC or } \\
\text { BCC }\end{array}$ \\
\hline $\begin{array}{l}\text { CN- } \\
00478464\end{array}$ & $\begin{array}{l}\text { Basset- } \\
\text { Seguin Net }\end{array}$ & $\begin{array}{l}\text { Photodynamic therapy using methyl } \\
\text { aminolaevulinate is as efficacious as } \\
\text { cryotherapy in basal cell carcinoma, with better } \\
\text { cosmetic results. British Association of } \\
\text { Dermatologists 83rd Annual Meeting. Abstract } \\
\text { P-66 }\end{array}$ & $\begin{array}{l}\text { British } \\
\text { journal of } \\
\text { dermatolo } \\
\text { gy }\end{array}$ & $\begin{array}{l}\text { duplicate/conference } \\
\text { abstract and we have full } \\
\text { publication }\end{array}$ \\
\hline $\begin{array}{l}\text { CN- } \\
00616027\end{array}$ & $\begin{array}{l}\text { Basset- } \\
\text { Seguin Net, al }\end{array}$ & $\begin{array}{l}\text { MAL-PDT Versus Cryotherapy for Treatment of } \\
\text { Primary Superficial Basal Cell Carcinoma: } \\
\text { Results of a Five Years Prospective } \\
\text { Randomized Trial Abstract PO7. 3rd Meeting of } \\
\text { the European Association of Dermato- } \\
\text { Oncology, Rome 23-25 June } 2006\end{array}$ & $\begin{array}{l}\text { Journal of } \\
\text { investigati } \\
\text { ve } \\
\text { dermatolo } \\
\text { gy }\end{array}$ & $\begin{array}{l}\text { duplicate/conference } \\
\text { abstract and we have full } \\
\text { publication }\end{array}$ \\
\hline NA & $\begin{array}{l}\text { Basset- } \\
\text { Seguin, N., et } \\
\text { al. }\end{array}$ & $\begin{array}{l}\text { Methyl aminolaevulinate photodynamic therapy } \\
\text { vs. cryotherapy in primary superficial basal cell } \\
\text { carcinoma: results of a } 36 \text {-month follow-up. }\end{array}$ & $\begin{array}{l}\text { British } \\
\text { Journal of } \\
\text { Dermatolo } \\
\text { gy } 153.1 \\
(2005): \\
29-29 .\end{array}$ & $\begin{array}{l}\text { duplicate/conference } \\
\text { abstract and we have full } \\
\text { publication }\end{array}$ \\
\hline $\begin{array}{l}\text { CN- } \\
00550836\end{array}$ & $\begin{array}{l}\text { Basset- } \\
\text { Sequin, N. }\end{array}$ & $\begin{array}{l}\text { Methyl aminolaevulinate photodynamic therapy } \\
\text { vs.cryotherapy in primary superficial basal cell } \\
\text { carcinoma: results of a 36-month follow-up } \\
\text { (Abstract P-30). The 85th BAD Annual Meeting } \\
\text { 5-8th July 2005, Glasgow, UK }\end{array}$ & $\begin{array}{l}\text { British } \\
\text { journal of } \\
\text { dermatolo } \\
\text { gy }\end{array}$ & $\begin{array}{l}\text { duplicate/conference } \\
\text { abstract and we have full } \\
\text { publication }\end{array}$ \\
\hline 21664850 & Ben Salah, $\mathrm{H}$. & $\begin{array}{l}\text { [Radiotherapy for cutaneous cancers with } \\
\text { xeroderma pigmentosum] }\end{array}$ & $\begin{array}{l}\text { Cancer } \\
\text { Radiother }\end{array}$ & $\begin{array}{l}\text { not treatment of skin } \\
\text { cancer or }<80 \% \text { SCC or } \\
\text { BCC }\end{array}$ \\
\hline 17657178 & Bernard, P. & $\begin{array}{l}\text { [Therapeutic modalities and economic } \\
\text { assessment in the treatment of superficial basal }\end{array}$ & $\begin{array}{l}\text { Ann } \\
\text { Dermatol }\end{array}$ & $\begin{array}{l}\text { not treatment of skin } \\
\text { cancer or }<80 \% \text { SCC or }\end{array}$ \\
\hline
\end{tabular}




\begin{tabular}{|c|c|c|c|c|}
\hline UID & First Author & Title & Journal & $\begin{array}{l}\text { Reason for } \\
\text { Exclusion }\end{array}$ \\
\hline & & $\begin{array}{l}\text { cell carcinomas and multiple actinic keratoses } \\
\text { by French dermatologists] }\end{array}$ & Venereol & $\mathrm{BCC}$ \\
\hline 9448970 & Berridge, J. K. & $\begin{array}{l}\text { A comparison of late cosmetic results following } \\
\text { two different radiotherapy techniques for } \\
\text { treating basal cell carcinoma }\end{array}$ & $\begin{array}{l}\text { Clin Oncol } \\
\text { (R Coll } \\
\text { Radiol) }\end{array}$ & $\begin{array}{l}>20 \% \text { recurrent or } \% \\
\text { recurrent not given }\end{array}$ \\
\hline $\begin{array}{l}\text { CN- } \\
00610206 \\
(17573890) \\
\end{array}$ & Berroeta, L. & $\begin{array}{l}\text { A randomized study of minimal curettage } \\
\text { followed by topical photodynamic therapy } \\
\text { compared with surgical excision for low-risk } \\
\text { nodular basal cell carcinoma }\end{array}$ & $\begin{array}{l}\text { The British } \\
\text { journal of } \\
\text { dermatolo } \\
\text { gy }\end{array}$ & $\begin{array}{l}\text { duplicate/conference } \\
\text { abstract and we have full } \\
\text { publication }\end{array}$ \\
\hline $\begin{array}{l}\text { CN- } \\
00550829\end{array}$ & Berroeta, L. & $\begin{array}{l}\text { Surgery versus debulking curettage plus topical } \\
\text { photodynamic therapy for low-risk nodular basal } \\
\text { cell carcinomas. Abstract DS-16 The 85th BAD } \\
\text { Annual Meeting 5-8th July 2005, Glasgow, UK }\end{array}$ & $\begin{array}{l}\text { British } \\
\text { journal of } \\
\text { dermatolo } \\
\text { gy }\end{array}$ & $\begin{array}{l}\text { duplicate/conference } \\
\text { abstract and we have full } \\
\text { publication }\end{array}$ \\
\hline 18563776 & Betz, C. S. & $\begin{array}{l}\text { Optimization of treatment parameters for } \\
\text { Foscan-PDT of basal cell carcinomas }\end{array}$ & $\begin{array}{l}\text { Lasers } \\
\text { Surg Med }\end{array}$ & $\begin{array}{l}\text { not comparative between } \\
\text { treatment nodes }\end{array}$ \\
\hline 15210467 & Bialy, T. L. & $\begin{array}{l}\text { Mohs micrographic surgery vs traditional } \\
\text { surgical excision: a cost comparison analysis }\end{array}$ & $\begin{array}{l}\text { Arch } \\
\text { Dermatol }\end{array}$ & $\begin{array}{l}\text { not comparative between } \\
\text { treatment nodes }\end{array}$ \\
\hline 5676901 & Binder, S. C. & Epidermoid carcinoma of the skin of the nose & Am J Surg & $\begin{array}{l}\text { not comparative between } \\
\text { treatment nodes }\end{array}$ \\
\hline 17598036 & $\begin{array}{l}\text { Bogelund, F. } \\
\text { S. }\end{array}$ & $\begin{array}{l}\text { Factors affecting the recurrence rate of basal } \\
\text { cell carcinoma }\end{array}$ & $\begin{array}{l}\text { Acta Derm } \\
\text { Venereol }\end{array}$ & data not extractable \\
\hline 25410443 & Borghi, A. & $\begin{array}{l}\text { Basal cell carcinoma incompletely excised: a } \\
\text { case-control study on recurrence }\end{array}$ & $\begin{array}{l}\text { G Ital } \\
\text { Dermatol } \\
\text { Venereol }\end{array}$ & $\begin{array}{l}\text { not comparative between } \\
\text { treatment nodes }\end{array}$ \\
\hline 3802321 & Brasseur, G. & $\begin{array}{l}\text { [Treatment of epithelioma of the eyelid by } \\
\text { interstitial radiotherapy. Long-term results. } \\
\text { Limitation of the method] }\end{array}$ & $\begin{array}{l}\text { Bull Soc } \\
\text { Ophtalmol } \\
\text { Fr }\end{array}$ & $\begin{array}{l}\text { not comparative between } \\
\text { treatment nodes }\end{array}$ \\
\hline 9243982 & Breuninger, $\mathrm{H}$. & $\begin{array}{l}\text { Micrographic surgery of malignant skin tumors: } \\
\text { a comparison of the frozen technique with } \\
\text { paraffin sectioning }\end{array}$ & $\begin{array}{l}\text { Facial } \\
\text { Plast Surg }\end{array}$ & $\begin{array}{l}\text { not comparative between } \\
\text { treatment nodes }\end{array}$ \\
\hline 17513803 & $\begin{array}{l}\text { Brewster, A. } \\
\text { M. }\end{array}$ & $\begin{array}{l}\text { Randomized trial of adjuvant 13-cis-retinoic acid } \\
\text { and interferon alfa for patients with aggressive } \\
\text { skin squamous cell carcinoma }\end{array}$ & $\begin{array}{l}\text { J Clin } \\
\text { Oncol }\end{array}$ & $\begin{array}{l}>20 \% \text { recurrent or } \% \\
\text { recurrent not given }\end{array}$ \\
\hline $\begin{array}{l}\text { CN- } \\
01056929\end{array}$ & $\begin{array}{l}\text { Brinkhuizen, } \\
\text { T. }\end{array}$ & $\begin{array}{l}\text { Topical Diclofenac and Vitamin D as treatment } \\
\text { for (micro)nodular and superficial basal cell } \\
\text { carcinoma }\end{array}$ & $\begin{array}{l}\text { Nederland } \\
\text { S } \\
\text { Tijdschrift } \\
\text { voor } \\
\text { Dermatolo } \\
\text { gie en } \\
\text { Venereolo } \\
\text { gie }\end{array}$ & $\begin{array}{l}\text { duplicate/conference } \\
\text { abstract and we have full } \\
\text { publication }\end{array}$ \\
\hline 16103328 & Brown, V. L. & $\begin{array}{l}\text { Safety and efficacy of } 5 \% \text { imiquimod cream for } \\
\text { the treatment of skin dysplasia in high-risk renal } \\
\text { transplant recipients: randomized, double-blind, } \\
\text { placebo-controlled trial }\end{array}$ & $\begin{array}{l}\text { Arch } \\
\text { Dermatol }\end{array}$ & $\begin{array}{l}\text { not treatment of skin } \\
\text { cancer or }<80 \% \text { SCC or } \\
\text { BCC }\end{array}$ \\
\hline 2217841 & $\begin{array}{l}\text { Brzezinska- } \\
\text { Wcislo, L. }\end{array}$ & $\begin{array}{l}\text { [Evaluation of the methods of treatment of } \\
\text { epithelioma basocellulare at the I Dermatology } \\
\text { Clinic, Silesian Medical Academy, in Katowice] }\end{array}$ & $\begin{array}{l}\text { Przegl } \\
\text { Dermatol }\end{array}$ & Not English (Polish) \\
\hline 1875617 & Budiak, V. A. & $\begin{array}{l}\text { [Effectiveness of some methods in the } \\
\text { treatment of primary squamous cell cancer of } \\
\text { the skin] }\end{array}$ & Klin Khir & Not English (Russian) \\
\hline 2013106063 & Caddick, J. & $\begin{array}{l}\text { Psychological outcomes following surgical } \\
\text { excision of facial skin cancers }\end{array}$ & $\begin{array}{l}\text { European } \\
\text { Journal of } \\
\text { Plastic } \\
\text { Surgery }\end{array}$ & $\begin{array}{l}\text { not comparative between } \\
\text { treatment nodes }\end{array}$ \\
\hline 1791498 & Calzavara, F. & $\begin{array}{l}\text { Photodynamic therapy: clinical experience at } \\
\text { the Department of Radiotherapy at Padova } \\
\text { General Hospital }\end{array}$ & $\begin{array}{l}\text { J } \\
\text { Photoche } \\
\text { m } \\
\text { Photobiol }\end{array}$ & $\begin{array}{l}\text { not comparative between } \\
\text { treatment nodes }\end{array}$ \\
\hline
\end{tabular}




\begin{tabular}{|c|c|c|c|c|}
\hline UID & First Author & Title & Journal & $\begin{array}{l}\text { Reason for } \\
\text { Exclusion }\end{array}$ \\
\hline & & & B & \\
\hline 18197827 & $\begin{array}{l}\text { Campbell, S. } \\
\text { M. }\end{array}$ & $\begin{array}{l}\text { A clinical investigation to determine the effect of } \\
\text { pressure injection on the penetration of topical } \\
\text { methyl aminolevulinate into nodular basal cell } \\
\text { carcinoma of the skin }\end{array}$ & $\begin{array}{l}\text { J Environ } \\
\text { Pathol } \\
\text { Toxicol } \\
\text { Oncol }\end{array}$ & $\begin{array}{l}\text { not comparative between } \\
\text { treatment nodes }\end{array}$ \\
\hline 18544077 & $\begin{array}{l}\text { Campbell, S. } \\
\text { M. }\end{array}$ & $\begin{array}{l}\text { Clinical investigation of the novel iron-chelating } \\
\text { agent, CP94, to enhance topical photodynamic } \\
\text { therapy of nodular basal cell carcinoma }\end{array}$ & $\begin{array}{l}\text { Br J } \\
\text { Dermatol }\end{array}$ & $\begin{array}{l}\text { not comparative between } \\
\text { treatment nodes }\end{array}$ \\
\hline 12410674 & Campolmi, P. & $\begin{array}{l}\text { Superpulsed CO2 laser treatment of basal cell } \\
\text { carcinoma with intraoperatory histopathologic } \\
\text { and cytologic examination }\end{array}$ & $\begin{array}{l}\text { Dermatol } \\
\text { Surg }\end{array}$ & $\begin{array}{l}\text { not comparative between } \\
\text { treatment nodes }\end{array}$ \\
\hline 21324035 & Carducci, M. & $\begin{array}{l}\text { Margin detection using digital dermatoscopy } \\
\text { improves the performance of traditional surgical } \\
\text { excision of basal cell carcinomas of the head } \\
\text { and neck }\end{array}$ & $\begin{array}{l}\text { Dermatol } \\
\text { Surg }\end{array}$ & $\begin{array}{l}\text { not comparative between } \\
\text { treatment nodes }\end{array}$ \\
\hline $\begin{array}{l}\text { CN- } \\
00478488\end{array}$ & Caro, I. & $\begin{array}{l}\text { Efficacy and safety of imiquimod } 5 \% \text { cream in } \\
\text { the treatment of superficial basal cell } \\
\text { carcinoma. Abstract P5-20 The 12th Congress } \\
\text { of the European Academy of Dermatology and } \\
\text { Venereology. Barcelona, Spain 15-18th October } \\
2003\end{array}$ & $\begin{array}{l}\text { Journal of } \\
\text { the } \\
\text { European } \\
\text { Academy } \\
\text { of } \\
\text { Dermatolo } \\
\text { gy and } \\
\text { Venereolo } \\
\text { gy : } \\
\text { JEADV }\end{array}$ & $\begin{array}{l}\text { duplicate/conference } \\
\text { abstract and we have full } \\
\text { publication }\end{array}$ \\
\hline 19418331 & Castineiras, I. & $\begin{array}{l}\text { Actinic cheilitis: evolution to squamous cell } \\
\text { carcinoma after carbon dioxide laser } \\
\text { vaporization. A study of } 43 \text { cases }\end{array}$ & $\begin{array}{l}\text { J } \\
\text { Dermatolo } \\
\text { g Treat }\end{array}$ & $\begin{array}{l}\text { not comparative between } \\
\text { treatment nodes }\end{array}$ \\
\hline 15752124 & Chan, A. L. & $\begin{array}{l}\text { Pharmacokinetics and clinical effects of mono- } \\
\text { L-aspartyl chlorin e6 (NPe6) photodynamic } \\
\text { therapy in adult patients with primary or } \\
\text { secondary cancer of the skin and mucosal } \\
\text { surfaces }\end{array}$ & $\begin{array}{l}\text { Photoder } \\
\text { matol } \\
\text { Photoimm } \\
\text { unol } \\
\text { Photomed }\end{array}$ & $\begin{array}{l}\text { not comparative between } \\
\text { treatment nodes }\end{array}$ \\
\hline 2012242601 & Chan, D. V. & $\begin{array}{l}\text { Radiation therapy in the management of } \\
\text { unilesional primary cutaneous T-cell lymphomas }\end{array}$ & $\begin{array}{l}\text { British } \\
\text { Journal of } \\
\text { Dermatolo } \\
\text { gy }\end{array}$ & $\begin{array}{l}\text { not treatment of skin } \\
\text { cancer or }<80 \% \text { SCC or } \\
\text { BCC }\end{array}$ \\
\hline 19027512 & Chang, C. H. & $\begin{array}{l}\text { Treatments and outcomes of malignant tumors } \\
\text { of external auditory canal }\end{array}$ & $\begin{array}{l}\text { Am J } \\
\text { Otolaryng } \\
\text { ol }\end{array}$ & $\begin{array}{l}\text { No analysis by } \\
\text { population of interest }\end{array}$ \\
\hline 7712447 & Chao, C. K. & $\begin{array}{l}\text { Reirradiation of recurrent skin cancer of the } \\
\text { face. A successful salvage modality }\end{array}$ & Cancer & $\begin{array}{l}>20 \% \text { recurrent or } \% \\
\text { recurrent not given }\end{array}$ \\
\hline 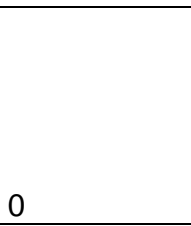 & Cheraghi, N. & $\begin{array}{l}\text { Retrospective study of punch scoring versus } \\
\text { freehand approach for first stage mohs } \\
\text { micrographic surgery }\end{array}$ & $\begin{array}{l}\text { Journal of } \\
\text { Clinical } \\
\text { and } \\
\text { Aesthetic } \\
\text { Dermatolo } \\
\text { gy }\end{array}$ & $\begin{array}{l}\text { not comparative between } \\
\text { treatment nodes }\end{array}$ \\
\hline 8171136 & Childers, B. J. & $\begin{array}{l}\text { Long-term results of irradiation for basal cell } \\
\text { carcinoma of the skin of the nose }\end{array}$ & $\begin{array}{l}\text { Plast } \\
\text { Reconstr } \\
\text { Surg }\end{array}$ & $\begin{array}{l}\text { not comparative between } \\
\text { treatment nodes }\end{array}$ \\
\hline 11074693 & Chiller, $\mathrm{K}$. & $\begin{array}{l}\text { Efficacy of curettage before excision in clearing } \\
\text { surgical margins of nonmelanoma skin cancer }\end{array}$ & $\begin{array}{l}\text { Arch } \\
\text { Dermatol }\end{array}$ & no outcomes of interest \\
\hline 10487003 & Cho, S. & $\begin{array}{l}\text { Clinical and histopathological characteristics of } \\
\text { basal cell carcinoma in Korean patients }\end{array}$ & $\begin{array}{l}\mathrm{J} \\
\text { Dermatol }\end{array}$ & $\begin{array}{l}\text { not treatment of skin } \\
\text { cancer or }<80 \% \text { SCC or } \\
\text { BCC }\end{array}$ \\
\hline $\begin{array}{l}\text { CN- } \\
01055091\end{array}$ & Choi, S. H. & $\begin{array}{l}\text { Efficacy of ablative fractional laser-assisted } \\
\text { photodynamic therapy for nodular basal cell } \\
\text { carcinoma: A prospective, randomized study }\end{array}$ & $\begin{array}{l}\text { Journal of } \\
\text { dermatolo } \\
\text { gy }\end{array}$ & $\begin{array}{l}\text { not comparative between } \\
\text { treatment nodes }\end{array}$ \\
\hline
\end{tabular}




\begin{tabular}{|c|c|c|c|c|}
\hline UID & First Author & Title & Journal & $\begin{array}{l}\text { Reason for } \\
\text { Exclusion }\end{array}$ \\
\hline & & with 12-month follow-up & & \\
\hline 18728281 & $\begin{array}{l}\text { Christian, J. } \\
\text { B. }\end{array}$ & $\begin{array}{l}\text { Association of ACE inhibitors and angiotensin } \\
\text { receptor blockers with keratinocyte cancer } \\
\text { prevention in the randomized VATTC trial }\end{array}$ & $\begin{array}{l}\text { J Natl } \\
\text { Cancer } \\
\text { Inst }\end{array}$ & $\begin{array}{l}\text { not treatment of skin } \\
\text { cancer or }<80 \% \text { SCC or } \\
\text { BCC }\end{array}$ \\
\hline 26207539 & $\begin{array}{l}\text { Christopoulos, } \\
\text { G. }\end{array}$ & $\begin{array}{l}\text { Surgical Treatment and Recurrence of } \\
\text { Cutaneous Nasal Malignancies: A 26-Year } \\
\text { Retrospective Review of } 1795 \text { Patients }\end{array}$ & $\begin{array}{l}\text { Ann Plast } \\
\text { Surg }\end{array}$ & $\begin{array}{l}\text { not comparative between } \\
\text { treatment nodes }\end{array}$ \\
\hline 12914598 & Clark, C. & $\begin{array}{l}\text { Topical 5-aminolaevulinic acid photodynamic } \\
\text { therapy for cutaneous lesions: outcome and } \\
\text { comparison of light sources }\end{array}$ & $\begin{array}{l}\text { Photoder } \\
\text { matol } \\
\text { Photoimm } \\
\text { unol } \\
\text { Photomed } \\
\end{array}$ & $\begin{array}{l}\text { not comparative between } \\
\text { treatment nodes }\end{array}$ \\
\hline $\begin{array}{l}\text { CN- } \\
00452483\end{array}$ & Clavel, M. & $\begin{array}{l}\text { Randomized trial of cisplatin }(C) \text {, methotrexate } \\
(A) \text {, bleomycin }(B) \text { and vincristine }(O) \text { vs } A B O \text { in } \\
\text { advanced squamous cell carcinoma of the head } \\
\text { and neck }\end{array}$ & $\begin{array}{l}\text { American } \\
\text { Society of } \\
\text { Clinical } \\
\text { Oncology } \\
\text { 19th } \\
\text { Annual } \\
\text { Meeting } \\
\text { (ASCO) . } \\
\text { San } \\
\text { Diego, } \\
\text { CA, 22-24 } \\
\text { May, 1983 }\end{array}$ & $\begin{array}{l}\text { not treatment of skin } \\
\text { cancer or }<80 \% \text { SCC or } \\
\text { BCC }\end{array}$ \\
\hline 16436340 & Clayton, T. H. & $\begin{array}{l}\text { Photodynamic therapy for superficial basal cell } \\
\text { carcinoma and Bowen's disease }\end{array}$ & $\begin{array}{l}\text { Eur J } \\
\text { Dermatol }\end{array}$ & $\begin{array}{l}\text { not comparative between } \\
\text { treatment nodes }\end{array}$ \\
\hline 2012167915 & Codazzi, D. & $\begin{array}{l}\text { A single-center retrospective study on } 3,957 \\
\text { consecutive excisions of basal cell carcinomas. } \\
\text { BCC behavior patterns: Retrospective statistical } \\
\text { analysis }\end{array}$ & $\begin{array}{l}\text { European } \\
\text { Journal of } \\
\text { Plastic } \\
\text { Surgery }\end{array}$ & $\begin{array}{l}\text { not comparative between } \\
\text { treatment nodes }\end{array}$ \\
\hline $\begin{array}{l}\mathrm{CN}- \\
00500580\end{array}$ & Cognetti, F. & $\begin{array}{l}\text { Randomized trial of sequential versus } \\
\text { simultaneous chemo and radiotherapy (CT- } \\
\text { XRT) in patients (PTS) with locally advanced } \\
\text { unresectable squamous cell carcinoma of the } \\
\text { head and neck (LAU-SCCHN). [abstract no: } 826 \\
\text { ] }\end{array}$ & $\begin{array}{l}\text { European } \\
\text { journal of } \\
\text { cancer }\end{array}$ & $\begin{array}{l}\text { not treatment of skin } \\
\text { cancer or }<80 \% \text { SCC or } \\
\text { BCC }\end{array}$ \\
\hline $\begin{array}{l}\mathrm{CN}- \\
00715090\end{array}$ & Cognetti, F. & $\begin{array}{l}\text { Preliminary results of a randomized trial of } \\
\text { sequential versus simultaneous chemo and } \\
\text { radiotherapy in patients with locally advanced } \\
\text { unresectable squamous cell carcinoma of the } \\
\text { head and neck [abstract] }\end{array}$ & $\begin{array}{l}\text { Proceedin } \\
\text { gs of the } \\
\text { American } \\
\text { Society of } \\
\text { Clinical } \\
\text { Oncology }\end{array}$ & $\begin{array}{l}\text { not treatment of skin } \\
\text { cancer or }<80 \% \text { SCC or } \\
\text { BCC }\end{array}$ \\
\hline 2013060222 & Comez, A. T. & Primary malignant tumors of the eyelids & $\begin{array}{l}\text { Turk } \\
\text { Oftalmoloij } \\
\text { i Dergisi }\end{array}$ & $\begin{array}{l}\text { not comparative between } \\
\text { treatment nodes }\end{array}$ \\
\hline 19182572 & Connelly, T. & $\begin{array}{l}\text { Delineating curettage as an adjunct to excision } \\
\text { of Basal cell carcinoma: results in } 334 \text { cases }\end{array}$ & $\begin{array}{l}\text { Plast } \\
\text { Reconstr } \\
\text { Surg }\end{array}$ & $\begin{array}{l}\text { not comparative between } \\
\text { treatment nodes }\end{array}$ \\
\hline 10201597 & $\begin{array}{l}\text { Cook, B. E., } \\
\text { Jr. }\end{array}$ & $\begin{array}{l}\text { Epidemiologic characteristics and clinical } \\
\text { course of patients with malignant eyelid tumors } \\
\text { in an incidence cohort in Olmsted County, } \\
\text { Minnesota }\end{array}$ & $\begin{array}{l}\text { Ophthalm } \\
\text { ology }\end{array}$ & $\begin{array}{l}>20 \% \text { metastatic/nodal } \\
\text { involvement }\end{array}$ \\
\hline 1994271944 & Dailey, J. R. & Squamous cell carcinoma of the eyelid & $\begin{array}{l}\text { Ophthalmi } \\
\text { c Plastic } \\
\text { and } \\
\text { Reconstru } \\
\text { ctive } \\
\text { Surgery }\end{array}$ & $\begin{array}{l}\text { No analysis by } \\
\text { population of interest }\end{array}$ \\
\hline
\end{tabular}




\begin{tabular}{|c|c|c|c|c|}
\hline UID & First Author & Title & Journal & $\begin{array}{l}\text { Reason for } \\
\text { Exclusion }\end{array}$ \\
\hline 10678347 & $\begin{array}{l}\text { Daum- } \\
\text { Sontrop, A. }\end{array}$ & $\begin{array}{l}\text { Treatment modalities for primary basal cell } \\
\text { carcinomas }\end{array}$ & $\begin{array}{l}\text { J Fam } \\
\text { Pract }\end{array}$ & no primary data \\
\hline 20729963 & David, P. & $\begin{array}{l}\text { Using a Hydroquinone/Tretinoin-based Skin } \\
\text { Care System Before and After } \\
\text { Electrodesiccation and Curettage of Superficial } \\
\text { Truncal Basal Cell Carcinoma: A Multicenter, } \\
\text { Randomized, Investigator-blind, Controlled } \\
\text { Study of Short-term Healing }\end{array}$ & $\begin{array}{l}\text { J Clin } \\
\text { Aesthet } \\
\text { Dermatol }\end{array}$ & $\begin{array}{l}\text { not comparative between } \\
\text { treatment nodes }\end{array}$ \\
\hline 16841035 & $\begin{array}{l}\text { de Haas, E. } \\
\text { R. }\end{array}$ & $\begin{array}{l}\text { Fractionated illumination significantly improves } \\
\text { the response of superficial basal cell carcinoma } \\
\text { to aminolevulinic acid photodynamic therapy }\end{array}$ & $\begin{array}{l}\text { J Invest } \\
\text { Dermatol }\end{array}$ & $\begin{array}{l}\text { not comparative between } \\
\text { treatment nodes }\end{array}$ \\
\hline 17310011 & $\begin{array}{l}\text { de Haas, } \\
\text { Ellen RM, et } \\
\text { al. }\end{array}$ & $\begin{array}{l}\text { "Response of Bowen disease to ALA-PDT using } \\
\text { a single and a 2-fold illumination scheme." }\end{array}$ & $\begin{array}{l}\text { Archives } \\
\text { of } \\
\text { dermatolo } \\
\text { gy } 143.2 \\
(2007) \text { : } \\
264-276 .\end{array}$ & $\begin{array}{l}\text { not comparative between } \\
\text { treatment nodes }\end{array}$ \\
\hline 22964973 & $\begin{array}{l}\text { de Vijlder, H. } \\
\text { C. }\end{array}$ & $\begin{array}{l}\text { Light fractionation significantly improves the } \\
\text { response of superficial basal cell carcinoma to } \\
\text { aminolaevulinic acid photodynamic therapy: } \\
\text { five-year follow-up of a randomized, prospective } \\
\text { trial }\end{array}$ & $\begin{array}{l}\text { Acta Derm } \\
\text { Venereol }\end{array}$ & $\begin{array}{l}\text { not comparative between } \\
\text { treatment nodes }\end{array}$ \\
\hline 2014322909 & $\begin{array}{l}\text { Demirseren, } \\
\text { D. D. }\end{array}$ & $\begin{array}{l}\text { Basal cell carcinoma of the head and neck } \\
\text { region: A retrospective analysis of completely } \\
\text { excised } 331 \text { cases }\end{array}$ & $\begin{array}{l}\text { Journal of } \\
\text { Skin } \\
\text { Cancer }\end{array}$ & $\begin{array}{l}\text { not comparative between } \\
\text { treatment nodes }\end{array}$ \\
\hline 8007618 & Denisov, L. E. & [Treatment of epitheliomas] & $\begin{array}{l}\text { Khirurgiia } \\
\text { (Mosk) }\end{array}$ & Not English (Russian) \\
\hline 2013198782 & Dirschka, T. & $\begin{array}{l}\text { Long-term ( } 6 \text { and } 12 \text { months) follow-up of two } \\
\text { prospective, randomized, controlled phase III } \\
\text { trials of photodynamic therapy with BF-200 ALA } \\
\text { and methyl aminolaevulinate for the treatment } \\
\text { of actinic keratosis }\end{array}$ & $\begin{array}{l}\text { British } \\
\text { Journal of } \\
\text { Dermatolo } \\
\text { gy }\end{array}$ & $\begin{array}{l}\text { not comparative between } \\
\text { treatment nodes }\end{array}$ \\
\hline 327370 & Dizon, R. V. & $\begin{array}{l}\text { Basal cell carcinoma recurrence: early } \\
\text { diagnosis and surgical treatment }\end{array}$ & $\begin{array}{l}\text { Ophthalmi } \\
\text { c Surg }\end{array}$ & $\begin{array}{l}>20 \% \text { recurrent or } \% \\
\text { recurrent not given }\end{array}$ \\
\hline 18818091 & Dognitz, N. & $\begin{array}{l}\text { Comparison of ALA- and ALA hexyl-ester- } \\
\text { induced PpIX depth distribution in human skin } \\
\text { carcinoma }\end{array}$ & $\begin{array}{l}\text { J } \\
\text { Photoche } \\
\text { m } \\
\text { Photobiol } \\
\text { B }\end{array}$ & no outcomes of interest \\
\hline $\begin{array}{l}\text { CN- } \\
00693262\end{array}$ & Domenge, $\mathrm{C}$. & $\begin{array}{l}\text { Randomized phase II study of all-trans retinoic } \\
\text { acid (ATRA) } \hat{A} \pm \text { a-interferon (IFN) in squamous } \\
\text { cell carcinoma (SCC) [abstract] }\end{array}$ & $\begin{array}{l}\text { Proceedin } \\
\text { gs of the } \\
\text { American } \\
\text { Society of } \\
\text { Clinical } \\
\text { Oncology }\end{array}$ & $\begin{array}{l}>20 \% \text { metastatic/nodal } \\
\text { involvement }\end{array}$ \\
\hline $\begin{array}{l}\text { CN- } \\
00305485\end{array}$ & Domenge, $\mathrm{C}$. & $\begin{array}{l}\text { All-trans retinoic acid (ATRA) +/- alfa interferon } \\
\text { (IFN) in squamous cell carci noma (SCC): A } \\
\text { randomized phase II study }\end{array}$ & Ann-Oncol & $\begin{array}{l}>20 \% \text { metastatic/nodal } \\
\text { involvement }\end{array}$ \\
\hline $\begin{array}{l}\text { CN- } \\
00691279\end{array}$ & Domenge, $\mathrm{C}$. & $\begin{array}{l}\text { Randomized phase II study of ALL-trans retinoic } \\
\text { acid (ATRA) +/- alpha-interferon (IFN) in } \\
\text { squamous cell carcinoma [abstract] }\end{array}$ & $\begin{array}{l}\text { Proceedin } \\
\text { gs of the } \\
\text { American } \\
\text { Society of } \\
\text { Clinical } \\
\text { Oncology }\end{array}$ & $\begin{array}{l}\text { duplicate/conference } \\
\text { abstract and we have full } \\
\text { publication }\end{array}$ \\
\hline 16334861 & $\begin{array}{l}\text { Donohue, K. } \\
\text { G. }\end{array}$ & $\begin{array}{l}\text { Safety and efficacy of a bilayered skin construct } \\
\text { in full-thickness surgical wounds }\end{array}$ & $\begin{array}{l}\text { J } \\
\text { Dermatol } \\
\end{array}$ & $\begin{array}{l}\text { not treatment of skin } \\
\text { cancer or }<80 \% \text { SCC or } \\
\text { BCC }\end{array}$ \\
\hline 28188086 & Dreno, B. & $\begin{array}{l}\text { Two intermittent vismodegib dosing regimens in } \\
\text { patients with multiple basal-cell carcinomas }\end{array}$ & $\begin{array}{l}\text { Lancet } \\
\text { Oncol }\end{array}$ & $\begin{array}{l}\text { not comparative between } \\
\text { treatment nodes }\end{array}$ \\
\hline
\end{tabular}




\begin{tabular}{|c|c|c|c|c|}
\hline UID & First Author & Title & Journal & $\begin{array}{l}\text { Reason for } \\
\text { Exclusion }\end{array}$ \\
\hline & & $\begin{array}{l}\text { (MIKIE): a randomised, regimen-controlled, } \\
\text { double-blind, phase } 2 \text { trial }\end{array}$ & & \\
\hline 0 & Dreno, B. & $\begin{array}{l}\text { Health-Related Quality of Life (HRQoL) analysis } \\
\text { by skindex-16 in MIKIE, a randomized phase } 2 \\
\text { study to assess the efficacy and safety of two } \\
\text { intermittent Vismodegib (VISMO) regimens in } \\
\text { patients (pts) with multiple basal cell } \\
\text { Carcinomas (BCCs) }\end{array}$ & $\begin{array}{l}\text { Melanoma } \\
\text { Research }\end{array}$ & $\begin{array}{l}\text { not comparative between } \\
\text { treatment nodes }\end{array}$ \\
\hline 21472887 & Ebrahimi, A. & $\begin{array}{l}\text { Metastatic head and neck cutaneous squamous } \\
\text { cell carcinoma: defining a low-risk patient }\end{array}$ & $\begin{array}{l}\text { Head } \\
\text { Neck }\end{array}$ & $\begin{array}{l}>20 \% \text { metastatic/nodal } \\
\text { involvement }\end{array}$ \\
\hline 6630599 & $\begin{array}{l}\text { Edens, B. L., } \\
\text { et al. }\end{array}$ & $\begin{array}{l}\text { "Effectiveness of curettage and } \\
\text { electrodesiccation in the removal of basal cell } \\
\text { carcinoma." }\end{array}$ & $\begin{array}{l}\text { Journal of } \\
\text { the } \\
\text { American } \\
\text { Academy } \\
\text { of } \\
\text { Dermatolo } \\
\text { gy } 9.3 \\
(1983) \text { : } \\
383-388 \text {. }\end{array}$ & $\begin{array}{l}\text { not comparative between } \\
\text { treatment nodes }\end{array}$ \\
\hline $\begin{array}{l}\text { CN- } \\
00726909\end{array}$ & $\begin{array}{l}\text { Eigentler, T. } \\
\mathrm{K} \text {. }\end{array}$ & $\begin{array}{l}\text { [A randomised, open therapy study to evaluate } \\
\text { the efficacy and safety of Imiquimod } 5 \% \text {-cream, } \\
\text { topically applied } 3 \text { times per week over an } 8 \text { or } \\
12 \text { week period to treat solid basal cell } \\
\text { carcinoma - an analysis of } 28 \text { patients] }\end{array}$ & $\begin{array}{l}\text { Aktuelle } \\
\text { Dermatolo } \\
\text { gie }\end{array}$ & $\begin{array}{l}\text { duplicate/conference } \\
\text { abstract and we have full } \\
\text { publication }\end{array}$ \\
\hline 27127144 & Espeli, V. & $\begin{array}{l}\text { Weekly Multi-agent Chemotherapy (CMF-b) for } \\
\text { Advanced Non-melanoma Skin Cancer }\end{array}$ & $\begin{array}{l}\text { Anticancer } \\
\text { Res }\end{array}$ & $\begin{array}{l}>20 \% \text { recurrent or } \% \\
\text { recurrent not given }\end{array}$ \\
\hline 17894707 & Essers, B. & $\begin{array}{l}\text { Perceptions of facial aesthetics in surgical } \\
\text { patients with basal cell carcinoma }\end{array}$ & $\begin{array}{l}\text { J Eur } \\
\text { Acad } \\
\text { Dermatol } \\
\text { Venereol }\end{array}$ & $\begin{array}{l}>20 \% \text { recurrent or } \% \\
\text { recurrent not given }\end{array}$ \\
\hline $\begin{array}{l}\text { CN- } \\
00493501\end{array}$ & Essers, B. & $\begin{array}{l}\text { Cost-effectiveness of Mohs' micrographic } \\
\text { surgery versus surgical excision for facial basal } \\
\text { cell carcinoma: results of a randomised clinical } \\
\text { trial [abstract] }\end{array}$ & $\begin{array}{l}\text { Proceedin } \\
\text { gs of the } \\
\text { First } \\
\text { Annual } \\
\text { Meeting of } \\
\text { the Health } \\
\text { Technolog } \\
\text { y } \\
\text { Assessme } \\
\text { nt } \\
\text { Internation } \\
\text { al (HTAi); } \\
2004 \text { May } \\
30 \text { - June } \\
2\end{array}$ & $\begin{array}{l}\text { duplicate/conference } \\
\text { abstract and we have full } \\
\text { publication }\end{array}$ \\
\hline 20387912 & Essers, B. A. & $\begin{array}{l}\text { Does the inclusion of a cost attribute result in } \\
\text { different preferences for the surgical treatment } \\
\text { of primary basal cell carcinoma?: a comparison } \\
\text { of two discrete-choice experiments }\end{array}$ & $\begin{array}{l}\text { Pharmaco } \\
\text { economics }\end{array}$ & $\begin{array}{l}\text { not treatment of skin } \\
\text { cancer or }<80 \% \text { SCC or } \\
\text { BCC }\end{array}$ \\
\hline 27283245 & Estall, V. & $\begin{array}{l}\text { Outcomes following management of squamous } \\
\text { cell carcinoma of the scalp: A retrospective } \\
\text { series of } 235 \text { patients treated at the Peter } \\
\text { MacCallum Cancer Centre }\end{array}$ & $\begin{array}{l}\text { Australas } \\
\mathrm{J} \\
\text { Dermatol }\end{array}$ & results not extractable \\
\hline $\begin{array}{l}\text { CN- } \\
01013219\end{array}$ & Euctr, G. B. & $\begin{array}{l}\text { An Open-label, International, Multi-Center, } \\
\text { Phase I/II, Dose-escalation Trial Investigating } \\
\text { the Safety of Zalutumumab, a Human } \\
\text { Monoclonal Epidermal Growth Factor Receptor } \\
\text { Antibody in Combination with Radiotherapy, in } \\
\text { Patients with Stage III, IVa or IVb Locally }\end{array}$ & $\begin{array}{l}\text { EUCTR } \\
\text { [www.clini } \\
\text { caltrialsre } \\
\text { gister.eu] }\end{array}$ & $\begin{array}{l}\text { not comparative between } \\
\text { treatment nodes }\end{array}$ \\
\hline
\end{tabular}




\begin{tabular}{|c|c|c|c|c|}
\hline UID & First Author & Title & Journal & $\begin{array}{l}\text { Reason for } \\
\text { Exclusion }\end{array}$ \\
\hline & & $\begin{array}{l}\text { Advanced Squamous Cell Carcinoma of the } \\
\text { Head and Neck Ineligible for Platinum based } \\
\text { Chemotherapy - Zalutumumab in combination } \\
\text { with radiotherapy in SCCHN patients ineligible } \\
\text { for platinum based chemoth }\end{array}$ & & \\
\hline 17917935 & Ezughah, F. I. & $\begin{array}{l}\text { A randomized parallel study to assess the } \\
\text { safety and efficacy of two different dosing } \\
\text { regimens of } 5 \% \text { imiquimod in the treatment of } \\
\text { superficial basal cell carcinoma }\end{array}$ & $\begin{array}{l}\text { J } \\
\text { Dermatolo } \\
\text { g Treat }\end{array}$ & $\begin{array}{l}\text { not comparative between } \\
\text { treatment nodes }\end{array}$ \\
\hline $\begin{array}{l}\mathrm{CN}- \\
00602233\end{array}$ & $\begin{array}{l}\text { Ezughah, Flet } \\
\text { al }\end{array}$ & $\begin{array}{l}\text { A randomized observer blinded study to assess } \\
\text { the safety and ef .cacy of two different dosing } \\
\text { regimens of } 5 \% \text { imiquimod cream in the } \\
\text { treatment of superficial basal cell carcinoma. } \\
\text { Abstract DS-13. British Association of } \\
\text { Dermatologists 86th Annual Meeting }\end{array}$ & $\begin{array}{l}\text { British } \\
\text { journal of } \\
\text { dermatolo } \\
\text { gy }\end{array}$ & $\begin{array}{l}\text { duplicate/conference } \\
\text { abstract and we have full } \\
\text { publication }\end{array}$ \\
\hline 25809617 & $\begin{array}{l}\text { Fargnoli, M. } \\
\text { C., et al. }\end{array}$ & $\begin{array}{l}\text { "Conventional vs. daylight methyl } \\
\text { aminolevulinate photodynamic therapy for } \\
\text { actinic keratosis of the face and scalp: an intra- } \\
\text { patient, prospective, comparison study in Italy." }\end{array}$ & $\begin{array}{l}\text { Journal of } \\
\text { the } \\
\text { European } \\
\text { Academy } \\
\text { of } \\
\text { Dermatolo } \\
\text { gy and } \\
\text { Venereolo } \\
\text { gy 29.10 } \\
(2015): \\
1926- \\
1932 \text {. }\end{array}$ & $\begin{array}{l}\text { not comparative between } \\
\text { treatment nodes }\end{array}$ \\
\hline 11382109 & $\begin{array}{l}\text { Federspil, P. } \\
\text { A. }\end{array}$ & $\begin{array}{l}\text { [Squamous epithelial carcinomas of the external } \\
\text { ear] }\end{array}$ & Hno & $\begin{array}{l}\text { not treatment of skin } \\
\text { cancer or }<80 \% \text { SCC or } \\
\text { BCC }\end{array}$ \\
\hline 23209908 & $\begin{array}{l}\text { Fernandez- } \\
\text { Guarino, M. }\end{array}$ & $\begin{array}{l}\text { Pulsed dye laser does not seem as effective as } \\
\text { red light in Basal cell carcinoma mal-pdt: a } \\
\text { small pilot study }\end{array}$ & $\begin{array}{l}\text { J Skin } \\
\text { Cancer }\end{array}$ & $\begin{array}{l}\text { not comparative between } \\
\text { treatment nodes }\end{array}$ \\
\hline 17062045 & $\begin{array}{l}\text { Fernandez- } \\
\text { Jorge, B. }\end{array}$ & $\begin{array}{l}\text { Outpatient dermatology major surgery: a 1-year } \\
\text { experience in a Spanish tertiary hospital }\end{array}$ & $\begin{array}{l}\text { J Eur } \\
\text { Acad } \\
\text { Dermatol } \\
\text { Venereol }\end{array}$ & $\begin{array}{l}\text { not comparative between } \\
\text { treatment nodes }\end{array}$ \\
\hline 22881585 & Ferrandiz, L. & $\begin{array}{l}\text { Assessing physicians' preferences on skin } \\
\text { cancer treatment in Europe }\end{array}$ & $\begin{array}{l}\mathrm{Br} \mathrm{J} \\
\text { Dermatol }\end{array}$ & no outcomes of interest \\
\hline 12004850 & Finizio, L. & $\begin{array}{l}\text { What is the current role of radiation therapy in } \\
\text { the treatment of skin carcinomas? }\end{array}$ & Tumori & $\begin{array}{l}\text { not comparative between } \\
\text { treatment nodes }\end{array}$ \\
\hline 23120649 & Fleiner, F. & $\begin{array}{l}\text { Cancer of the external auditory canal-diagnostic } \\
\text { and treatment }\end{array}$ & $\begin{array}{l}\text { Indian J } \\
\text { Otolaryng } \\
\text { ol Head } \\
\text { Neck Surg }\end{array}$ & $\begin{array}{l}\text { not treatment of skin } \\
\text { cancer or }<80 \% \text { SCC or } \\
\text { BCC }\end{array}$ \\
\hline $\begin{array}{l}\mathrm{CN}- \\
00478536\end{array}$ & Foley, P. & $\begin{array}{l}\text { A phase III randomized study comparing } \\
\text { photodynamic therapy (PDT) using methyl } \\
\text { aminolevulinate or placebo cream in nodular } \\
\text { basal cell carcinoma (NBCC). Abstract P9-14 } \\
\text { The 12th Congress of the European Academy } \\
\text { of Dermatology and Venereology. Barcelona, } \\
\text { Spain 15-18th October } 2003\end{array}$ & $\begin{array}{l}\text { Journal of } \\
\text { the } \\
\text { European } \\
\text { Academy } \\
\text { of } \\
\text { Dermatolo } \\
\text { gy and } \\
\text { Venereolo } \\
\text { gy: } \\
\text { JEADV }\end{array}$ & $\begin{array}{l}\text { duplicate/conference } \\
\text { abstract and we have full } \\
\text { publication }\end{array}$ \\
\hline 1583171 & Frankel, D. H. & $\begin{array}{l}\text { New primary nonmelanoma skin cancer in } \\
\text { patients with a history of squamous cell } \\
\text { carcinoma of the skin. Implications and } \\
\text { recommendations for follow-up }\end{array}$ & $\begin{array}{l}\text { J Am } \\
\text { Acad } \\
\text { Dermatol }\end{array}$ & $\begin{array}{l}\text { not treatment of skin } \\
\text { cancer or }<80 \% \text { SCC or } \\
\text { BCC }\end{array}$ \\
\hline
\end{tabular}




\begin{tabular}{|c|c|c|c|c|}
\hline UID & First Author & Title & Journal & $\begin{array}{l}\text { Reason for } \\
\text { Exclusion }\end{array}$ \\
\hline 8191597 & Gabriele, P. & $\begin{array}{l}\text { Carcinoma of the external auditory meatus and } \\
\text { middle ear. Results of the treatment of } 28 \text { cases }\end{array}$ & Tumori & $\begin{array}{l}\text { not treatment of skin } \\
\text { cancer or }<80 \% \text { SCC or } \\
\text { BCC }\end{array}$ \\
\hline 21926038 & Gaitanis, G. & $\begin{array}{l}\text { Cryosurgery is more effective in the treatment of } \\
\text { primary, non-superficial basal cell carcinomas } \\
\text { when applied during and not prior to a five week } \\
\text { imiquimod course: a randomized, prospective, } \\
\text { open-label study }\end{array}$ & $\begin{array}{l}\text { Eur J } \\
\text { Dermatol }\end{array}$ & $\begin{array}{l}\text { not comparative between } \\
\text { treatment nodes }\end{array}$ \\
\hline 424625 & Gajewska, B. & $\begin{array}{l}\text { [Comparative study of the results of surgical } \\
\text { and radiotherapy treatment of basal cell } \\
\text { epitheliomas and prickle cell carcinomas] }\end{array}$ & $\begin{array}{l}\text { Przegl } \\
\text { Dermatol }\end{array}$ & Not English (Polish) \\
\hline 15693020 & $\begin{array}{l}\text { Galloway, T. } \\
\text { J. }\end{array}$ & $\begin{array}{l}\text { Impact of radiographic findings on prognosis for } \\
\text { skin carcinoma with clinical perineural invasion }\end{array}$ & Cancer & $\begin{array}{l}\text { not comparative between } \\
\text { treatment nodes }\end{array}$ \\
\hline 26165629 & Gandhi, A. K. & $\begin{array}{l}\text { Treatment of squamous cell carcinoma of } \\
\text { external auditory canal: A tertiary cancer centre } \\
\text { experience }\end{array}$ & $\begin{array}{l}\text { Auris } \\
\text { Nasus } \\
\text { Larynx }\end{array}$ & $\begin{array}{l}\text { not treatment of skin } \\
\text { cancer or }<80 \% \text { SCC or } \\
\text { BCC }\end{array}$ \\
\hline 14648861 & $\begin{array}{l}\text { Garcia-Serra, } \\
\text { A. }\end{array}$ & Carcinoma of the skin with perineural invasion & $\begin{array}{l}\text { Head } \\
\text { Neck }\end{array}$ & $\begin{array}{l}\text { not comparative between } \\
\text { treatment nodes }\end{array}$ \\
\hline $\begin{array}{l}\mathrm{CN}- \\
00452584\end{array}$ & Garden, A. S. & $\begin{array}{l}\text { Preliminary results of RTOG } 9703 \text { - a phase II } \\
\text { randomized trial of concurrent radiation (RT) } \\
\text { and chemotherapy for advanced squamous cell } \\
\text { carcinomas (SCC) of the head and neck }\end{array}$ & $\begin{array}{l}\text { Proceedin } \\
\text { gs of the } \\
\text { American } \\
\text { Society of } \\
\text { Clinical } \\
\text { Oncology } \\
\text { (ASCO) }\end{array}$ & $\begin{array}{l}\text { not treatment of skin } \\
\text { cancer or }<80 \% \text { SCC or } \\
\text { BCC }\end{array}$ \\
\hline 11404627 & $\begin{array}{l}\text { Gayl } \\
\text { Schweitzer, V. }\end{array}$ & $\begin{array}{l}\text { Photofrin-mediated photodynamic therapy for } \\
\text { treatment of aggressive head and neck } \\
\text { nonmelanomatous skin tumors in elderly } \\
\text { patients }\end{array}$ & $\begin{array}{l}\text { Laryngosc } \\
\text { ope }\end{array}$ & $\begin{array}{l}>20 \% \text { recurrent or } \% \\
\text { recurrent not given }\end{array}$ \\
\hline $\begin{array}{l}\text { CN- } \\
00469536 \\
(15097956)\end{array}$ & Geisse, J. & $\begin{array}{l}\text { Imiquimod } 5 \% \text { cream for the treatment of } \\
\text { superficial basal cell carcinoma: results from } \\
\text { two phase III, randomized, vehicle-controlled } \\
\text { studies }\end{array}$ & $\begin{array}{l}\text { Journal of } \\
\text { the } \\
\text { American } \\
\text { Academy } \\
\text { of } \\
\text { Dermatolo } \\
\text { gy }\end{array}$ & $\begin{array}{l}\text { duplicate/conference } \\
\text { abstract and we have full } \\
\text { publication }\end{array}$ \\
\hline $\begin{array}{l}\text { CN- } \\
00520431\end{array}$ & Geisse, J. K. & $\begin{array}{l}\text { Imiquimod } 5 \% \text { cream for } 12 \text { weeks treating } \\
\text { superficial BCC [Abstract] }\end{array}$ & $\begin{array}{l}\text { 8th World } \\
\text { Congress } \\
\text { on Cancer } \\
\text { of the } \\
\text { Skin. } \\
\text { Zurich, } \\
\text { Switzerlan } \\
\text { d. July 18- } \\
21,2001 \\
\end{array}$ & $\begin{array}{l}\text { duplicate/conference } \\
\text { abstract and we have full } \\
\text { publication }\end{array}$ \\
\hline $\begin{array}{l}\text { CN- } \\
01013542\end{array}$ & $\begin{array}{l}\text { Ghosh- } \\
\text { Laskar, S. }\end{array}$ & $\begin{array}{l}\text { Phase II Study of 3-Dimensional Conformal } \\
\text { Radiotherapy (3D-CRT) vs Intensity Modulated } \\
\text { Radiotherapy (IMRT) for Squamous Cell } \\
\text { Carcinoma of the Head and Neck (HNSCC) }\end{array}$ & $\begin{array}{l}\text { Clinicaltria } \\
\text { Is.gov } \\
\text { [www.clini } \\
\text { caltrials.go } \\
\text { v] }\end{array}$ & $\begin{array}{l}\text { not treatment of skin } \\
\text { cancer or }<80 \% \text { SCC or } \\
\text { BCC }\end{array}$ \\
\hline $\begin{array}{l}\text { CN- } \\
00857249\end{array}$ & Giglio, R. & $\begin{array}{l}\text { No recurrences beyond the second year of } \\
\text { follow up in inoperable stage III and IV } \\
\text { squamous cell carcinoma of the head and neck } \\
\text { patients (IOHN). Final report of a randomized } \\
\text { trial of alternating chemotherapy (CT) + } \\
\text { hyperfractionated radiotherapy (RT) vS RT } \\
\text { alone }\end{array}$ & $\begin{array}{l}\text { Proceedin } \\
\text { gs of the } \\
\text { 35th } \\
\text { Annual } \\
\text { Meeting of } \\
\text { the } \\
\text { American } \\
\text { Society of } \\
\text { Clinical }\end{array}$ & $\begin{array}{l}\text { latment of skin } \\
\text { not treatm } \\
\text { cancer or }<80 \% \text { SCC or } \\
\text { BCC }\end{array}$ \\
\hline
\end{tabular}




\begin{tabular}{|c|c|c|c|c|}
\hline UID & First Author & Title & Journal & $\begin{array}{l}\text { Reason for } \\
\text { Exclusion }\end{array}$ \\
\hline & & & $\begin{array}{l}\text { Oncology; } \\
\text { 1999, May } \\
\text { 15-18; } \\
\text { Atlanta, } \\
\text { Georgia, } \\
\text { USA }\end{array}$ & \\
\hline 7961010 & $\begin{array}{l}\text { Glicksman, A. } \\
\text { S. }\end{array}$ & $\begin{array}{l}\text { Concurrent cis-platinum and radiation with or } \\
\text { without surgery for advanced head and neck } \\
\text { cancer }\end{array}$ & $\begin{array}{l}\text { Int J } \\
\text { Radiat } \\
\text { Oncol Biol } \\
\text { Phys }\end{array}$ & $\begin{array}{l}\text { not treatment of skin } \\
\text { cancer or }<80 \% \text { SCC or } \\
\text { BCC }\end{array}$ \\
\hline 18938044 & Gluck, I. & $\begin{array}{l}\text { Skin cancer of the head and neck with } \\
\text { perineural invasion: defining the clinical target } \\
\text { volumes based on the pattern of failure }\end{array}$ & $\begin{array}{l}\text { Int J } \\
\text { Radiat } \\
\text { Oncol Biol } \\
\text { Phys }\end{array}$ & $\begin{array}{l}\text { not comparative between } \\
\text { treatment nodes }\end{array}$ \\
\hline 15747068 & Graham, B. D. & $\begin{array}{l}\text { Topical 5-fluorouracil in the management of } \\
\text { extensive anal Bowen's disease: a preferred } \\
\text { approach }\end{array}$ & $\begin{array}{l}\text { Dis Colon } \\
\text { Rectum }\end{array}$ & $\begin{array}{l}\text { not treatment of skin } \\
\text { cancer or }<80 \% \text { SCC or } \\
\text { BCC }\end{array}$ \\
\hline 7635774 & Griep, C. & $\begin{array}{l}\text { Electron beam therapy is not inferior to } \\
\text { superficial x-ray therapy in the treatment of skin } \\
\text { carcinoma }\end{array}$ & $\begin{array}{l}\text { Int J } \\
\text { Radiat } \\
\text { Oncol Biol } \\
\text { Phys }\end{array}$ & $\begin{array}{l}>20 \% \text { recurrent or } \% \\
\text { recurrent not given }\end{array}$ \\
\hline 4701240 & Griffith, B. H. & $\begin{array}{l}\text { An appraisal of the treatment of basal cell } \\
\text { carcinoma of the skin }\end{array}$ & $\begin{array}{l}\text { Plast } \\
\text { Reconstr } \\
\text { Surg }\end{array}$ & $\begin{array}{l}\text { not comparative between } \\
\text { treatment nodes }\end{array}$ \\
\hline 20666811 & $\begin{array}{l}\text { Guardiano, R. } \\
\text { A. }\end{array}$ & $\begin{array}{l}\text { A direct comparison of visual inspection, } \\
\text { curettage, and epiluminescence microscopy in } \\
\text { determining tumor extent before the initial } \\
\text { margins are determined for Mohs micrographic } \\
\text { surgery }\end{array}$ & $\begin{array}{l}\text { Dermatol } \\
\text { Surg }\end{array}$ & $\begin{array}{l}\text { not comparative between } \\
\text { treatment nodes }\end{array}$ \\
\hline 7691784 & Haffty, B. G. & $\begin{array}{l}\text { Mitomycin C as an adjunct to postoperative } \\
\text { radiation therapy in squamous cell carcinoma of } \\
\text { the head and neck: results from two randomized } \\
\text { clinical trials }\end{array}$ & $\begin{array}{l}\text { Int J } \\
\text { Radiat } \\
\text { Oncol Biol } \\
\text { Phys }\end{array}$ & $\begin{array}{l}\text { not treatment of skin } \\
\text { cancer or }<80 \% \text { SCC or } \\
\text { BCC }\end{array}$ \\
\hline 8996152 & Haffty, B. G. & $\begin{array}{l}\text { Chemotherapy as an adjunct to radiation in the } \\
\text { treatment of squamous cell carcinoma of the } \\
\text { head and neck: results of the Yale Mitomycin } \\
\text { Randomized Trials }\end{array}$ & $\begin{array}{l}\text { J Clin } \\
\text { Oncol }\end{array}$ & $\begin{array}{l}\text { not treatment of skin } \\
\text { cancer or }<80 \% \text { SCC or } \\
\text { BCC }\end{array}$ \\
\hline 15629602 & Haffty, B. G. & $\begin{array}{l}\text { Concurrent chemo-radiotherapy with mitomycin } \\
\mathrm{C} \text { compared with porfiromycin in squamous cell } \\
\text { cancer of the head and neck: final results of a } \\
\text { randomized clinical trial }\end{array}$ & $\begin{array}{l}\text { Int J } \\
\text { Radiat } \\
\text { Oncol Biol } \\
\text { Phys }\end{array}$ & $\begin{array}{l}\text { not treatment of skin } \\
\text { cancer or }<80 \% \text { SCC or } \\
\text { BCC }\end{array}$ \\
\hline 3676083 & $\begin{array}{l}\text { Harrison, P. } \\
\text { V. }\end{array}$ & $\begin{array}{l}\text { Therapy of basal cell carcinoma--treatment in } \\
1980-81 \text { compared with } 1985-86 \text { and } \\
\text { advantages of shave excision for smaller } \\
\text { tumours }\end{array}$ & $\begin{array}{l}\mathrm{Br} \mathrm{J} \\
\text { Dermatol }\end{array}$ & $\begin{array}{l}\text { duplicate/conference } \\
\text { abstract and we have full } \\
\text { publication }\end{array}$ \\
\hline 10927141 & Hashi, N. & $\begin{array}{l}\text { The role of radiotherapy in treating squamous } \\
\text { cell carcinoma of the external auditory canal, } \\
\text { especially in early stages of disease }\end{array}$ & $\begin{array}{l}\text { Radiother } \\
\text { Oncol }\end{array}$ & $\begin{array}{l}\text { not treatment of skin } \\
\text { cancer or }<80 \% \text { SCC or } \\
\text { BCC }\end{array}$ \\
\hline 15534663 & Helsing, $\mathrm{P}$. & [Surgical treatment of basal cell carcinoma] & $\begin{array}{l}\text { Tidsskr } \\
\text { Nor } \\
\text { Laegefore } \\
\mathrm{n}\end{array}$ & Not English (Norwegian) \\
\hline $\begin{array}{l}\text { CN- } \\
00451395\end{array}$ & $\begin{array}{l}\text { Heyden, } \mathrm{H} \text {. } \\
\text { W. }\end{array}$ & $\begin{array}{l}\text { Chemotherapy (CT) of advanced squamous cell } \\
\text { carcinoma of the head and neck. A randomized } \\
\text { cross-over trial between cis-dichlorodiammine- } \\
\text { platinum (II) (CIS-DDP) and bleomycin (BLSM } \\
\text { vs. methotrexate (MTX) and vindesine (VDS) }\end{array}$ & $\begin{array}{l}\text { Journal of } \\
\text { cancer } \\
\text { research } \\
\text { and } \\
\text { clinical } \\
\text { oncology }\end{array}$ & $\begin{array}{l}\text { not treatment of skin } \\
\text { cancer or }<80 \% \text { SCC or } \\
\text { BCC }\end{array}$ \\
\hline 113627 & Hintz, B. & Randomized study of control of the primary & J Surg & not treatment of skin \\
\hline
\end{tabular}




\begin{tabular}{|c|c|c|c|c|}
\hline UID & First Author & Title & Journal & $\begin{array}{l}\text { Reason for } \\
\text { Exclusion }\end{array}$ \\
\hline & & $\begin{array}{l}\text { tumor and survival using preoperative radiation, } \\
\text { radiation alone, or surgery alone in head and } \\
\text { neck carcinomas }\end{array}$ & Oncol & $\begin{array}{l}\text { cancer or }<80 \% \text { SCC or } \\
\text { BCC }\end{array}$ \\
\hline 23241791 & $\begin{array}{l}\text { Hoefkens, M. } \\
\text { F. }\end{array}$ & $\begin{array}{l}\text { Does loupe magnification reduce the gap } \\
\text { between the macroscopic and microscopic } \\
\text { border of a Basal cell carcinoma?: a prospective } \\
\text { clinical study }\end{array}$ & $\begin{array}{l}\text { Ann Plast } \\
\text { Surg }\end{array}$ & $\begin{array}{l}\text { not comparative between } \\
\text { treatment nodes }\end{array}$ \\
\hline 25654948 & Hosokawa, S. & $\begin{array}{l}\text { Carcinoma of the external auditory canal: } \\
\text { histological and treatment groups }\end{array}$ & B-Ent & $\begin{array}{l}\text { not treatment of skin } \\
\text { cancer or }<80 \% \text { SCC or } \\
\text { BCC }\end{array}$ \\
\hline 2015376689 & Hsu, M. C. & $\begin{array}{l}\text { Secondary neoplasms arising from nevus } \\
\text { sebaceus: A retrospective study of } 450 \text { cases in } \\
\text { Taiwan }\end{array}$ & $\begin{array}{l}\text { Journal of } \\
\text { Dermatolo } \\
\text { gy }\end{array}$ & $\begin{array}{l}\text { not treatment of skin } \\
\text { cancer or }<80 \% \text { SCC or } \\
\text { BCC }\end{array}$ \\
\hline 15389195 & Huang, C. C. & $\begin{array}{l}\text { Randomized, controlled surgical trial of } \\
\text { preoperative tumor curettage of basal cell } \\
\text { carcinoma in Mohs micrographic surgery }\end{array}$ & $\begin{array}{l}\text { J Am } \\
\text { Acad } \\
\text { Dermatol }\end{array}$ & $\begin{array}{l}\text { not comparative between } \\
\text { treatment nodes }\end{array}$ \\
\hline 27109055 & Hussain, A. A. & $\begin{array}{l}\text { Adjunct use of optical coherence tomography } \\
\text { increases the detection of recurrent basal cell } \\
\text { carcinoma over clinical and dermoscopic } \\
\text { examination alone }\end{array}$ & $\begin{array}{l}\text { Photodiag } \\
\text { nosis } \\
\text { Photodyn } \\
\text { Ther }\end{array}$ & $\begin{array}{l}\text { not comparative between } \\
\text { treatment nodes }\end{array}$ \\
\hline 1955231 & Ikic, D. & Basal cell carcinoma treated with interferon & $\begin{array}{l}\text { Int J } \\
\text { Dermatol }\end{array}$ & $\begin{array}{l}\text { not comparative between } \\
\text { treatment nodes }\end{array}$ \\
\hline 1937994 & Ikic, D. & $\begin{array}{l}\text { Interferon therapy for basal cell carcinoma and } \\
\text { squamous cell carcinoma }\end{array}$ & $\begin{array}{l}\text { Int J Clin } \\
\text { Pharmacol } \\
\text { Ther } \\
\text { Toxicol }\end{array}$ & $\begin{array}{l}\text { not comparative between } \\
\text { treatment nodes }\end{array}$ \\
\hline 5618951 & Jackson, R. & $\begin{array}{l}\text { The team approach to the management of skin } \\
\text { cancer }\end{array}$ & $\begin{array}{l}\text { Med Serv } \\
\text { J Can }\end{array}$ & $\begin{array}{l}\text { No analysis by } \\
\text { population of interest }\end{array}$ \\
\hline 4750192 & $\begin{array}{l}\text { Jakobsson, P. } \\
\text { A. }\end{array}$ & $\begin{array}{l}\text { Fractionation scheme with low individual tumour } \\
\text { dose and high total dose }\end{array}$ & $\begin{array}{l}\text { Acta } \\
\text { Radiol } \\
\text { Ther Phys } \\
\text { Biol }\end{array}$ & $\begin{array}{l}\text { not comparative between } \\
\text { treatment nodes }\end{array}$ \\
\hline 24879468 & $\begin{array}{l}\text { Jarkowski, A., } \\
\text { 3rd }\end{array}$ & $\begin{array}{l}\text { Systemic Therapy in Advanced Cutaneous } \\
\text { Squamous Cell Carcinoma (CSCC): The } \\
\text { Roswell Park Experience and a Review of the } \\
\text { Literature }\end{array}$ & $\begin{array}{l}\text { Am J Clin } \\
\text { Oncol }\end{array}$ & $\begin{array}{l}\text { not comparative between } \\
\text { treatment nodes }\end{array}$ \\
\hline 24299572 & Jeon, S. Y. & $\begin{array}{l}\text { Efficacy of photodynamic diagnosis-guided } \\
\text { Mohs micrographic surgery in primary } \\
\text { squamous cell carcinoma }\end{array}$ & $\begin{array}{l}\text { Dermatol } \\
\text { Surg }\end{array}$ & $\begin{array}{l}\text { not comparative between } \\
\text { treatment nodes }\end{array}$ \\
\hline 10735893 & Jeremic, B. & $\begin{array}{l}\text { Hyperfractionated radiation therapy with or } \\
\text { without concurrent low-dose daily cisplatin in } \\
\text { locally advanced squamous cell carcinoma of } \\
\text { the head and neck: a prospective randomized } \\
\text { trial }\end{array}$ & $\begin{array}{l}\text { J Clin } \\
\text { Oncol }\end{array}$ & $\begin{array}{l}\text { not comparative between } \\
\text { treatment nodes }\end{array}$ \\
\hline $\begin{array}{l}\mathrm{CN}- \\
00742337\end{array}$ & Julian, C. & $\begin{array}{l}\text { A comparative study of the effects of disposable } \\
\text { and Volkmann spoon curettes in the treatment } \\
\text { of basal cell carcinoma }\end{array}$ & $\begin{array}{l}\text { The British } \\
\text { journal of } \\
\text { dermatolo } \\
\text { gy }\end{array}$ & $\begin{array}{l}>20 \% \text { recurrent or } \% \\
\text { recurrent not given }\end{array}$ \\
\hline 26362616 & $\begin{array}{l}\text { Kadouch, D. } \\
\text { J. }\end{array}$ & $\begin{array}{l}\text { Treatment of Basal Cell Carcinoma Using a } \\
\text { One-Stop-Shop With Reflectance Confocal } \\
\text { Microscopy: Study Design and Protocol of a } \\
\text { Randomized Controlled Multicenter Trial }\end{array}$ & $\begin{array}{l}\text { JMIR Res } \\
\text { Protoc }\end{array}$ & no outcomes of interest \\
\hline 23352886 & Khan, A. A. & $\begin{array}{l}\text { Guidelines for the excision of cutaneous } \\
\text { squamous cell cancers in the United Kingdom: } \\
\text { the best cut is the deepest }\end{array}$ & $\begin{array}{l}\text { J Plast } \\
\text { Reconstr } \\
\text { Aesthet } \\
\text { Surg }\end{array}$ & $\begin{array}{l}\text { not comparative between } \\
\text { treatment nodes }\end{array}$ \\
\hline 1999353368 & Khan, N. A. & $\begin{array}{l}\text { Role of elective irradiation to drainage sites in } \\
\text { squamous cell carcinoma of the skin trunk and } \\
\text { extremities }\end{array}$ & $\begin{array}{l}\text { JK } \\
\text { Practitione } \\
\mathrm{r}\end{array}$ & $\begin{array}{l}\text { not comparative between } \\
\text { treatment nodes }\end{array}$ \\
\hline
\end{tabular}




\begin{tabular}{|c|c|c|c|c|}
\hline UID & First Author & Title & Journal & $\begin{array}{l}\text { Reason for } \\
\text { Exclusion }\end{array}$ \\
\hline 25675868 & Khtibari, Z. & $\begin{array}{l}\text { [Squamous cell carcinoma of the eyelids. } \\
\text { Review of } 7 \text { years of experience of the adult } \\
\text { ophthalmology service of the Casablanca } \\
\text { university medical center] }\end{array}$ & $\begin{array}{l}\text { J Fr } \\
\text { Ophtalmol }\end{array}$ & Not English (French) \\
\hline 28027517 & Kim, S. A & $\begin{array}{l}\text { 18F-FDG PET/CT surveillance for the detection } \\
\text { of recurrence in patients with head and neck } \\
\text { cancer }\end{array}$ & $\begin{array}{l}\text { Eur J } \\
\text { Cancer }\end{array}$ & $\begin{array}{l}\text { not treatment of skin } \\
\text { cancer or }<80 \% \text { SCC or } \\
\text { BCC }\end{array}$ \\
\hline 7037180 & Kish, J. & $\begin{array}{l}\text { Clinical trial of cisplatin and 5-FU infusion as } \\
\text { initial treatment for advanced squamous cell } \\
\text { carcinoma of the head and neck }\end{array}$ & $\begin{array}{l}\text { Cancer } \\
\text { Treat Rep }\end{array}$ & $\begin{array}{l}\text { not treatment of skin } \\
\text { cancer or }<80 \% \text { SCC or } \\
\text { BCC }\end{array}$ \\
\hline 4764924 & Klein, E. & $\begin{array}{l}\text { Proceedings: Chemotherapy and } \\
\text { immunotherapy for cancer involving the skin }\end{array}$ & $\begin{array}{l}\text { Proc Natl } \\
\text { Cancer } \\
\text { Conf }\end{array}$ & no primary data \\
\hline 14290308 & Klein, E. & $\begin{array}{l}\text { TUMORS OF THE SKIN. IV. DOUBLE-BLIND } \\
\text { STUDY ON EFFECTS OF LOCAL } \\
\text { ADMINISTRATION OF ANTI-TUMOR AGENTS } \\
\text { IN BASAL CELL CARCINOMA }\end{array}$ & $\begin{array}{l}\text { J Invest } \\
\text { Dermatol }\end{array}$ & $\begin{array}{l}\text { not comparative between } \\
\text { treatment nodes }\end{array}$ \\
\hline 5321314 & Klein, E. & $\begin{array}{l}\text { Tumors of the skin. V. Local administration of } \\
\text { anti-tumor agents to multiple superficial basal } \\
\text { cell carcinomas }\end{array}$ & $\begin{array}{l}\text { J Invest } \\
\text { Dermatol }\end{array}$ & $\begin{array}{l}\text { not comparative between } \\
\text { treatment nodes }\end{array}$ \\
\hline 5387158 & $\begin{array}{l}\text { Kleine-Natrop, } \\
\text { H. E. }\end{array}$ & $\begin{array}{l}\text { [Clinical aspects and therapy of basal cell } \\
\text { epitheliomas and squamous cell carcinomas. A } \\
10 \text {-year analysis] }\end{array}$ & $\begin{array}{l}\text { Dermatol } \\
\text { Monatssc } \\
\mathrm{hr}\end{array}$ & $\begin{array}{l}>20 \% \text { recurrent or } \% \\
\text { recurrent not given }\end{array}$ \\
\hline 4850042 & $\begin{array}{l}\text { Kleine-Natrop, } \\
\text { H. E. }\end{array}$ & $\begin{array}{l}\text { [Treatment of recurrent basalioma (author's } \\
\text { transl)] }\end{array}$ & $\begin{array}{l}\text { Arch } \\
\text { Geschwul } \\
\text { stforsch }\end{array}$ & $\begin{array}{l}>20 \% \text { recurrent or } \% \\
\text { recurrent not given }\end{array}$ \\
\hline 9002265 & Koderhold, G. & $\begin{array}{l}\text { Experiences of photodynamic therapy in } \\
\text { dermatology }\end{array}$ & $\begin{array}{l}\text { J } \\
\text { Photoche } \\
\text { m } \\
\text { Photobiol } \\
\text { B }\end{array}$ & $\begin{array}{l}\text { not comparative between } \\
\text { treatment nodes }\end{array}$ \\
\hline 15611900 & Kollert, M. & $\begin{array}{l}\text { [Carcinoma of the external auditory canal and } \\
\text { middle ear: therapeutic strategy and follow up] }\end{array}$ & $\begin{array}{l}\text { Laryngorhi } \\
\text { nootologie }\end{array}$ & $\begin{array}{l}\text { No analysis by } \\
\text { population of interest }\end{array}$ \\
\hline 23532618 & Krema, $\mathrm{H}$. & $\begin{array}{l}\text { Orthovoltage radiotherapy in the management } \\
\text { of medial canthal basal cell carcinoma }\end{array}$ & $\begin{array}{l}\mathrm{Br} \mathrm{J} \\
\text { Ophthalm } \\
\text { ol }\end{array}$ & $\begin{array}{l}\text { not comparative between } \\
\text { treatment nodes }\end{array}$ \\
\hline 1145348 & Krenar, J. & [Surgery or irradiation of skin neoplasms?] & Rozhl Chir & Not English (Czech) \\
\hline 23415573 & Kropp, L. & $\begin{array}{l}\text { Mohs resection and postoperative radiotherapy } \\
\text { for head and neck cancers with incidental } \\
\text { perineural invasion }\end{array}$ & $\begin{array}{l}\text { Am J } \\
\text { Otolaryng } \\
\text { ol }\end{array}$ & $\begin{array}{l}>20 \% \text { recurrent or } \% \\
\text { recurrent not given }\end{array}$ \\
\hline $\begin{array}{l}\text { CN- } \\
01060149\end{array}$ & Kunstfeld, R. & $\begin{array}{l}\text { MIKIE: A randomized, double-blind, regimen- } \\
\text { controlled, phase II, multicenter study to assess } \\
\text { the efficacy and safety of two different } \\
\text { vismodegib regimens in patients with multiple } \\
\text { basal cell carcinomas }\end{array}$ & $\begin{array}{l}\text { Journal of } \\
\text { clinical } \\
\text { oncology }\end{array}$ & no primary data \\
\hline 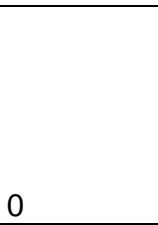 & Kunstfeld, R. & $\begin{array}{l}\text { Analysis of patients (pts) with and without basal } \\
\text { cell carcinoma nevus syndrome (BCCNS) in } \\
\text { MIKIE, a randomized phase } 2 \text { study to assess } \\
\text { the efficacy and safety of two intermittent } \\
\text { Vismodegib (VISMO) regimens in pts with } \\
\text { multiple Basal Cell Carcinomas (BCCs) }\end{array}$ & $\begin{array}{l}\text { Melanoma } \\
\text { Research }\end{array}$ & $\begin{array}{l}\text { not comparative between } \\
\text { treatment nodes }\end{array}$ \\
\hline 20338745 & Kyrgidis, A. & $\begin{array}{l}\text { Cutaneous squamous cell carcinoma (SCC) of } \\
\text { the head and neck: risk factors of overall and } \\
\text { recurrence-free survival }\end{array}$ & $\begin{array}{l}\text { Eur J } \\
\text { Cancer }\end{array}$ & $\begin{array}{l}>20 \% \text { metastatic/nodal } \\
\text { involvement }\end{array}$ \\
\hline 7857115 & Landthaler, M. & $\begin{array}{l}\text { Late irradiation damage to the skin caused by } \\
\text { soft X-ray radiation therapy of cutaneous tumors }\end{array}$ & $\begin{array}{l}\text { Arch } \\
\text { Dermatol }\end{array}$ & $\begin{array}{l}\text { not comparative between } \\
\text { treatment nodes }\end{array}$ \\
\hline $\begin{array}{l}\text { CN- } \\
00194309\end{array}$ & Landthaler, M. & $\begin{array}{l}\text { TDF factors in soft X-ray therapy. <ORIGINAL> } \\
\text { ANWENDUNG DES TDF-FAKTORS IN DER }\end{array}$ & $\begin{array}{l}\text { Der } \\
\text { Hautarzt; }\end{array}$ & $\begin{array}{l}\text { not comparative between } \\
\text { treatment nodes }\end{array}$ \\
\hline
\end{tabular}




\begin{tabular}{|c|c|c|c|c|}
\hline UID & First Author & Title & Journal & $\begin{array}{l}\text { Reason for } \\
\text { Exclusion }\end{array}$ \\
\hline & & RONTGENWEICHSTRAHLENTHERAPIE & $\begin{array}{l}\text { Zeitschrift } \\
\text { fur } \\
\text { Dermatolo } \\
\text { gie, } \\
\text { Venerologi } \\
\text { e, und } \\
\text { verwandte } \\
\text { Gebiete }\end{array}$ & \\
\hline 15275715 & $\begin{array}{l}\text { Langendijk, J. } \\
\text { A. }\end{array}$ & $\begin{array}{l}\text { Radiotherapy of squamous cell carcinoma of } \\
\text { the nasal vestibule }\end{array}$ & $\begin{array}{l}\text { Int J } \\
\text { Radiat } \\
\text { Oncol Biol } \\
\text { Phys }\end{array}$ & $\begin{array}{l}\text { not treatment of skin } \\
\text { cancer or }<80 \% \text { SCC or } \\
\text { BCC }\end{array}$ \\
\hline $\begin{array}{l}\text { CN- } \\
00888049\end{array}$ & Lansbury, L. & $\begin{array}{l}\text { Interventions for non-metastatic squamous cell } \\
\text { carcinoma of the skin: A summarised Cochrane } \\
\text { review }\end{array}$ & $\begin{array}{l}\text { Clinical } \\
\text { and } \\
\text { Experimen } \\
\text { tal } \\
\text { Dermatolo } \\
\text { gy }\end{array}$ & no primary data \\
\hline 19210500 & $\begin{array}{l}\text { Lawrence, } \mathrm{C} \text {. } \\
\text { M. }\end{array}$ & $\begin{array}{l}\text { Formalin-fixed tissue Mohs surgery (slow Mohs) } \\
\text { for basal cell carcinoma: 5-year follow-up data }\end{array}$ & $\begin{array}{l}\mathrm{Br} \mathrm{J} \\
\text { Dermatol }\end{array}$ & $\begin{array}{l}\text { not comparative between } \\
\text { treatment nodes }\end{array}$ \\
\hline 0 & Lear, J. & $\begin{array}{l}\text { Sonidegib safety in patients with locally } \\
\text { advanced Basal Cell Carcinoma and efficacy } \\
\text { based on tumor aggressiveness }\end{array}$ & $\begin{array}{l}\text { Melanoma } \\
\text { Research }\end{array}$ & $\begin{array}{l}\text { not comparative between } \\
\text { treatment nodes }\end{array}$ \\
\hline 24332515 & Lear, J. T. & $\begin{array}{l}\text { Evidence-based treatment for low-risk basal cell } \\
\text { carcinoma }\end{array}$ & $\begin{array}{l}\text { Lancet } \\
\text { Oncol }\end{array}$ & no primary data \\
\hline 25581584 & Lecluse LL & $\begin{array}{l}\text { Photodynamic therapy versus topical imiquimod } \\
\text { versus topical fluorouracil for treatment of } \\
\text { superficial basal-cell carcinoma: a single blind, } \\
\text { non-inferiority, randomised controlled trial: a } \\
\text { critical appraisal. }\end{array}$ & $\begin{array}{l}\mathrm{Br} \mathrm{J} \\
\text { Dermatol. }\end{array}$ & no primary data \\
\hline $\begin{array}{l}\text { CN- } \\
01039974\end{array}$ & $\begin{array}{l}\text { Lecluse, L. L. } \\
\text { A. }\end{array}$ & $\begin{array}{l}\text { Photodynamic therapy versus topical imiquimod } \\
\text { versus topical fluorouracil for treatment of } \\
\text { superficial basal-cell carcinoma: A single blind, } \\
\text { non-inferiority, randomised controlled trial: A } \\
\text { critical appraisal }\end{array}$ & $\begin{array}{l}\text { British } \\
\text { journal of } \\
\text { dermatolo } \\
\text { gy }\end{array}$ & no primary data \\
\hline 20840493 & Lee, C. Y. & $\begin{array}{l}\text { The efficacy of photodynamic diagnosis in } \\
\text { defining the lateral border between a tumor and } \\
\text { a tumor-free area during Mohs micrographic } \\
\text { surgery }\end{array}$ & $\begin{array}{l}\text { Dermatol } \\
\text { Surg }\end{array}$ & $\begin{array}{l}\text { not comparative between } \\
\text { treatment nodes }\end{array}$ \\
\hline 16710578 & Lindelof, B. & $\begin{array}{l}\text { Mortality and clinicopathological features of } \\
\text { cutaneous squamous cell carcinoma in organ } \\
\text { transplant recipients: a study of the Swedish } \\
\text { cohort }\end{array}$ & $\begin{array}{l}\text { Acta Derm } \\
\text { Venereol }\end{array}$ & $\begin{array}{l}\text { not comparative between } \\
\text { treatment nodes }\end{array}$ \\
\hline 23699934 & Linos, E. & $\begin{array}{l}\text { Treatment of nonfatal conditions at the end of } \\
\text { life: nonmelanoma skin cancer }\end{array}$ & $\begin{array}{l}\text { JAMA } \\
\text { Intern Med }\end{array}$ & $\begin{array}{l}\text { not comparative between } \\
\text { treatment nodes }\end{array}$ \\
\hline 4574777 & Littlewood, M. & $\begin{array}{l}\text { A clinical trial of the use of 5-fluouracil in the } \\
\text { treatment of some cutaneous malignancies }\end{array}$ & $\begin{array}{l}\text { Br J Plast } \\
\text { Surg }\end{array}$ & $\begin{array}{l}\text { not comparative between } \\
\text { treatment nodes }\end{array}$ \\
\hline 5091318 & Litwin, M. S. & $\begin{array}{l}\text { Treatment of basal and squamous cancers of } \\
\text { the nose and ear with 5-fluorouracil cream }\end{array}$ & $\begin{array}{l}\text { Laryngosc } \\
\text { ope }\end{array}$ & $\begin{array}{l}\text { not comparative between } \\
\text { treatment nodes }\end{array}$ \\
\hline 5110339 & Litwin, M. S. & $\begin{array}{l}\text { Topical chemotherapy of advanced cutaneous } \\
\text { malignancy with 5-Fluorouracil creme }\end{array}$ & $\begin{array}{l}\text { J Surg } \\
\text { Oncol }\end{array}$ & $\begin{array}{l}\text { not comparative between } \\
\text { treatment nodes }\end{array}$ \\
\hline 11360406 & Liu, C. H. & $\begin{array}{l}\text { The clinical features and surgical results of } \\
\text { malignant eyelid tumors }\end{array}$ & $\begin{array}{l}\text { Chang } \\
\text { Gung Med } \\
\text { J }\end{array}$ & $\begin{array}{l}\text { not comparative between } \\
\text { treatment nodes }\end{array}$ \\
\hline 1899855 & Liu, F. F. & $\begin{array}{l}\text { A management approach to incompletely } \\
\text { excised basal cell carcinomas of skin }\end{array}$ & $\begin{array}{l}\text { Int J } \\
\text { Radiat } \\
\text { Oncol Biol } \\
\text { Phys }\end{array}$ & $\begin{array}{l}\text { not comparative between } \\
\text { treatment nodes }\end{array}$ \\
\hline
\end{tabular}




\begin{tabular}{|c|c|c|c|c|}
\hline UID & First Author & Title & Journal & $\begin{array}{l}\text { Reason for } \\
\text { Exclusion }\end{array}$ \\
\hline 11697321 & Locke, J. & Radiotherapy for epithelial skin cancer & $\begin{array}{l}\text { Int J } \\
\text { Radiat } \\
\text { Oncol Biol } \\
\text { Phys }\end{array}$ & $\begin{array}{l}>20 \% \text { recurrent or } \% \\
\text { recurrent not given }\end{array}$ \\
\hline 1994344416 & Long, C. C. & $\begin{array}{l}\text { Curettage of small basal cell papillomas with the } \\
\text { disposable ring curette is superior to } \\
\text { conventional treatment [1] }\end{array}$ & $\begin{array}{l}\text { British } \\
\text { Journal of } \\
\text { Dermatolo } \\
\text { gy }\end{array}$ & $\begin{array}{l}\text { not comparative between } \\
\text { treatment nodes }\end{array}$ \\
\hline 4939510 & Lopes, C. F. & [Therapeutic trial with 5-fluorouracil ointment] & $\begin{array}{l}\text { Hospital } \\
\text { (Rio J) }\end{array}$ & $\begin{array}{l}\text { not treatment of skin } \\
\text { cancer or }<80 \% \text { SCC or } \\
\text { BCC }\end{array}$ \\
\hline 21300762 & $\begin{array}{l}\text { LoRusso, } \\
\text { Patricia M., et } \\
\text { al. }\end{array}$ & $\begin{array}{l}\text { "Phase I trial of hedgehog pathway inhibitor } \\
\text { vismodegib (GDC-0449) in patients with } \\
\text { refractory, locally advanced or metastatic solid } \\
\text { tumors." }\end{array}$ & $\begin{array}{l}\text { Clinical } \\
\text { Cancer } \\
\text { Research } \\
17.8 \\
(2011) \text { : } \\
2502- \\
2511 . \\
\end{array}$ & $\begin{array}{l}\text { not comparative between } \\
\text { treatment nodes }\end{array}$ \\
\hline 26353121 & Lu, S. M. & $\begin{array}{l}\text { Concurrent Radiotherapy With Cetuximab or } \\
\text { Platinum-based Chemotherapy for Locally } \\
\text { Advanced Cutaneous Squamous Cell } \\
\text { Carcinoma of the Head and Neck }\end{array}$ & $\begin{array}{l}\text { Am J Clin } \\
\text { Oncol }\end{array}$ & $\begin{array}{l}>20 \% \text { recurrent or } \% \\
\text { recurrent not given }\end{array}$ \\
\hline 14732656 & Lui, H. & $\begin{array}{l}\text { Photodynamic therapy of multiple } \\
\text { nonmelanoma skin cancers with verteporfin and } \\
\text { red light-emitting diodes: two-year results } \\
\text { evaluating tumor response and cosmetic } \\
\text { outcomes }\end{array}$ & $\begin{array}{l}\text { Arch } \\
\text { Dermatol }\end{array}$ & $\begin{array}{l}\text { not comparative between } \\
\text { treatment nodes }\end{array}$ \\
\hline 18704969 & Madsen, A. R. & $\begin{array}{l}\text { Cancer of the external auditory canal and } \\
\text { middle ear in Denmark from } 1992 \text { to } 2001\end{array}$ & $\begin{array}{l}\text { Head } \\
\text { Neck }\end{array}$ & $\begin{array}{l}\text { not comparative between } \\
\text { treatment nodes }\end{array}$ \\
\hline 17764086 & $\begin{array}{l}\text { Maghami, E. } \\
\text { G. }\end{array}$ & $\begin{array}{l}\text { Craniofacial surgery for nonmelanoma skin } \\
\text { malignancy: report of an international } \\
\text { collaborative study }\end{array}$ & $\begin{array}{l}\text { Head } \\
\text { Neck }\end{array}$ & $\begin{array}{l}\text { not comparative between } \\
\text { treatment nodes }\end{array}$ \\
\hline 7569812 & Mak, A. S. & $\begin{array}{l}\text { Audit of basal cell carcinoma in Princess } \\
\text { Margaret Hospital, Hong Kong: usefulness of } \\
\text { frozen section examination in surgical treatment }\end{array}$ & $\begin{array}{l}\text { Scand J } \\
\text { Plast } \\
\text { Reconstr } \\
\text { Surg Hand } \\
\text { Surg }\end{array}$ & no outcomes of interest \\
\hline $924616(8)$ & $\begin{array}{l}\text { Mallon E, } \\
\text { Dawbor E. }\end{array}$ & $\begin{array}{l}\text { Cryosurgery in the treatment of basal cell } \\
\text { carcinoma: assessment of one or two freeze- } \\
\text { thaw cycle schedules. }\end{array}$ & $\begin{array}{l}\text { Dermatol } \\
\text { Surg } \\
1996 ; 22: 8 \\
54-8 . \\
\end{array}$ & $\begin{array}{l}\text { not comparative between } \\
\text { treatment nodes }\end{array}$ \\
\hline 9246168 & Mallon, E. & $\begin{array}{l}\text { Cryosurgery in the treatment of basal cell } \\
\text { carcinoma. Assessment of one and two freeze- } \\
\text { thaw cycle schedules }\end{array}$ & $\begin{array}{l}\text { Dermatol } \\
\text { Surg }\end{array}$ & $\begin{array}{l}\text { not comparative between } \\
\text { treatment nodes }\end{array}$ \\
\hline 7096764 & Marchac, D. & $\begin{array}{l}\text { Curative and aesthetic results of surgical } \\
\text { treatment of } 138 \text { basal-cell carcinomas }\end{array}$ & $\begin{array}{l}\text { J } \\
\text { Dermatol } \\
\text { Surg } \\
\text { Oncol }\end{array}$ & $\begin{array}{l}\text { not comparative between } \\
\text { treatment nodes }\end{array}$ \\
\hline $\begin{array}{l}\mathrm{CN}- \\
00454540\end{array}$ & Marks, R. & $\begin{array}{l}\text { Optimal dosing duration and dosing regimen for } \\
\text { treatment of nodular BCC with imiquimod 5\% } \\
\text { cream }\end{array}$ & $\begin{array}{l}\text { Annales } \\
\text { de } \\
\text { Dermatolo } \\
\text { gie Et de } \\
\text { Venereolo } \\
\text { gie }\end{array}$ & Not English (French) \\
\hline 0 & Martin, I & $\begin{array}{l}\text { Patient preferences for treatment of basal cell } \\
\text { carcinoma: Importance of cure and cosmetic } \\
\text { outcome }\end{array}$ & $\begin{array}{l}\text { Acta } \\
\text { Dermato- } \\
\text { Venereolo } \\
\text { gica }\end{array}$ & $\begin{array}{l}\text { not treatment of skin } \\
\text { cancer or }<80 \% \text { SCC or } \\
\text { BCC }\end{array}$ \\
\hline 21742301 & Martorell- & [Intralesional infusion of methotrexate as & Actas & No analysis by \\
\hline
\end{tabular}




\begin{tabular}{|c|c|c|c|c|}
\hline UID & First Author & Title & Journal & $\begin{array}{l}\text { Reason for } \\
\text { Exclusion }\end{array}$ \\
\hline & Calatayud, A. & $\begin{array}{l}\text { neoadjuvant therapy improves the cosmetic and } \\
\text { functional results of surgery to treat } \\
\text { keratoacanthoma: results of a randomized trial] }\end{array}$ & $\begin{array}{l}\text { Dermosifili } \\
\text { ogr }\end{array}$ & population of interest \\
\hline 21843177 & Matthiesen, C. & $\begin{array}{l}\text { The role of radiotherapy for T4 non-melanoma } \\
\text { skin carcinoma }\end{array}$ & $\begin{array}{l}\text { J Med } \\
\text { Imaging } \\
\text { Radiat } \\
\text { Oncol }\end{array}$ & $\begin{array}{l}>20 \% \text { recurrent or } \% \\
\text { recurrent not given }\end{array}$ \\
\hline 24843224 & Mazzoni, A. & $\begin{array}{l}\text { Primary squamous cell carcinoma of the } \\
\text { external auditory canal: surgical treatment and } \\
\text { long-term outcomes }\end{array}$ & $\begin{array}{l}\text { Acta } \\
\text { Otorhinola } \\
\text { ryngol Ital }\end{array}$ & $\begin{array}{l}\text { No analysis by } \\
\text { population of interest }\end{array}$ \\
\hline 10078643 & $\begin{array}{l}\text { McCord, M. } \\
\text { W. }\end{array}$ & $\begin{array}{l}\text { Skin cancer of the head and neck with } \\
\text { incidental microscopic perineural invasion }\end{array}$ & $\begin{array}{l}\text { Int J } \\
\text { Radiat } \\
\text { Oncol Biol } \\
\text { Phys }\end{array}$ & $\begin{array}{l}>20 \% \text { recurrent or } \% \\
\text { recurrent not given }\end{array}$ \\
\hline 24927655 & $\begin{array}{l}\text { McKechnie, A. } \\
\text { J. }\end{array}$ & See-and-treat surgery for facial skin cancer & $\begin{array}{l}\text { Br J Oral } \\
\text { Maxillofac } \\
\text { Surg }\end{array}$ & $\begin{array}{l}\text { not comparative between } \\
\text { treatment nodes }\end{array}$ \\
\hline 2753698 & $\begin{array}{l}\text { Mendenhall, } \\
\text { W. M. }\end{array}$ & $\begin{array}{l}\text { Carcinoma of the skin of the head and neck with } \\
\text { perineural invasion }\end{array}$ & $\begin{array}{l}\text { Head } \\
\text { Neck }\end{array}$ & $\begin{array}{l}>20 \% \text { recurrent or } \% \\
\text { recurrent not given }\end{array}$ \\
\hline 1826208 & $\begin{array}{l}\text { Mendenhall, } \\
\text { W. M. }\end{array}$ & $\begin{array}{l}\text { Brachytherapy in head and neck cancer: } \\
\text { selection criteria and results at the University of } \\
\text { Florida }\end{array}$ & $\begin{array}{l}\text { Oncology } \\
\text { (Williston } \\
\text { Park) }\end{array}$ & no primary data \\
\hline 3597161 & $\begin{array}{l}\text { Mendenhall, } \\
\text { W. M. }\end{array}$ & $\begin{array}{l}\text { T2-T4 carcinoma of the skin of the head and } \\
\text { neck treated with radical irradiation }\end{array}$ & $\begin{array}{l}\text { Int J } \\
\text { Radiat } \\
\text { Oncol Biol } \\
\text { Phys }\end{array}$ & $\begin{array}{l}\text { not comparative between } \\
\text { treatment nodes }\end{array}$ \\
\hline 15825160 & $\begin{array}{l}\text { Mendenhall, } \\
\text { W. M. }\end{array}$ & $\begin{array}{l}\text { Retromolar trigone squamous cell carcinoma } \\
\text { treated with radiotherapy alone or combined } \\
\text { with surgery }\end{array}$ & Cancer & $\begin{array}{l}\text { not treatment of skin } \\
\text { cancer or }<80 \% \text { SCC or } \\
\text { BCC }\end{array}$ \\
\hline 5555851 & Menn, $\mathrm{H}$. & $\begin{array}{l}\text { The recurrent basal cell epithelioma. A study of } \\
100 \text { cases of recurrent, re-treated basal cell } \\
\text { epitheliomas }\end{array}$ & $\begin{array}{l}\text { Arch } \\
\text { Dermatol }\end{array}$ & $\begin{array}{l}>20 \% \text { recurrent or } \% \\
\text { recurrent not given }\end{array}$ \\
\hline $\begin{array}{l}\text { CN- } \\
00695148\end{array}$ & Merlano, M. & $\begin{array}{l}\text { Alternating chemotherapy and radiotherapy } \\
\text { (RT) vs RT in advanced inoperable SCC-HN: a } \\
\text { cooperative randomized trial [abstract] }\end{array}$ & $\begin{array}{l}\text { Proceedin } \\
\text { gs of the } \\
\text { American } \\
\text { Society of } \\
\text { Clinical } \\
\text { Oncology }\end{array}$ & $\begin{array}{l}\text { not treatment of skin } \\
\text { cancer or }<80 \% \text { SCC or } \\
\text { BCC }\end{array}$ \\
\hline $\begin{array}{l}\mathrm{CN}- \\
00353346\end{array}$ & Merlano, M. & $\begin{array}{l}\text { Alternating chemotherapy and radiotherapy in } \\
\text { advanced squamous cell carcinoma of the head } \\
\text { and neck. A randomized trial }\end{array}$ & $\begin{array}{l}\text { Proceedin } \\
\text { gs of the } \\
\text { American } \\
\text { Society of } \\
\text { Clinical } \\
\text { Oncology } \\
\text { (ASCO) }\end{array}$ & $\begin{array}{l}\text { not treatment of skin } \\
\text { cancer or }<80 \% \text { SCC or } \\
\text { BCC }\end{array}$ \\
\hline $\begin{array}{l}\text { CN- } \\
00715205\end{array}$ & Mickiewicz, R. & $\begin{array}{l}\text { No recurrences beyond the second year of } \\
\text { follow up in inoperable stage III and IV } \\
\text { squamous cell carcinoma of the head and neck } \\
\text { patients (IOHN). Final report of a randomized } \\
\text { trial of alternating chemotherapy (CT) + } \\
\text { hyperfractionated radiotherapy (RT) vs RT } \\
\text { alone [abstract] }\end{array}$ & $\begin{array}{l}\text { Proceedin } \\
\text { gs of the } \\
\text { American } \\
\text { Society of } \\
\text { Clinical } \\
\text { Oncology: } \\
\text { 35th } \\
\text { Annual } \\
\text { Meeting of } \\
\text { the } \\
\text { American } \\
\text { Society of } \\
\text { Clinical } \\
\text { Oncology; }\end{array}$ & $\begin{array}{l}\text { not treatment of skin } \\
\text { cancer or }<80 \% \text { SCC or } \\
\text { BCC }\end{array}$ \\
\hline
\end{tabular}




\begin{tabular}{|c|c|c|c|c|}
\hline UID & First Author & Title & Journal & $\begin{array}{l}\text { Reason for } \\
\text { Exclusion }\end{array}$ \\
\hline & & & $\begin{array}{l}\text { 15-18 May } \\
\text { 1999; } \\
\text { Atlanta, } \\
\text { Georgia, } \\
\text { USA }\end{array}$ & \\
\hline $\begin{array}{l}\text { CN- } \\
01088953\end{array}$ & Migden, M. & $\begin{array}{l}\text { Inhibition of the hedgehog pathway with } \\
\text { sonidegib (LDE225) in advanced basal cell } \\
\text { carcinoma }\end{array}$ & $\begin{array}{l}\text { Journal of } \\
\text { the } \\
\text { American } \\
\text { Academy } \\
\text { of } \\
\text { Dermatolo } \\
\text { gy }\end{array}$ & $\begin{array}{l}\text { duplicate/conference } \\
\text { abstract and we have full } \\
\text { publication }\end{array}$ \\
\hline $\begin{array}{l}\mathrm{CN}- \\
01088952\end{array}$ & Migden, M. & $\begin{array}{l}\text { Quality of life in patients with advanced basal } \\
\text { cell carcinoma treated with sonidegib (LDE225) }\end{array}$ & $\begin{array}{l}\text { Journal of } \\
\text { the } \\
\text { American } \\
\text { Academy } \\
\text { of } \\
\text { Dermatolo } \\
\text { gy }\end{array}$ & $\begin{array}{l}\text { duplicate/conference } \\
\text { abstract and we have full } \\
\text { publication }\end{array}$ \\
\hline $\begin{array}{l}\mathrm{CN}- \\
01088955\end{array}$ & Migden, M. & $\begin{array}{l}\text { A 12-month update of BOLT, a phase } 2 \text {, } \\
\text { randomized, double-blind study of sonidegib } \\
\text { (LDE225) in patients with locally advanced or } \\
\text { metastatic basal cell carcinoma }\end{array}$ & $\begin{array}{l}\text { Journal of } \\
\text { the } \\
\text { American } \\
\text { Academy } \\
\text { of } \\
\text { Dermatolo } \\
\text { gy }\end{array}$ & $\begin{array}{l}\text { duplicate/conference } \\
\text { abstract and we have full } \\
\text { publication }\end{array}$ \\
\hline 17509254 & Miller, S. J. & Basal cell and squamous cell skin cancers & $\begin{array}{l}\text { J Natl } \\
\text { Compr } \\
\text { Canc } \\
\text { Netw }\end{array}$ & no primary data \\
\hline 22548396 & Mizutani, K. & $\begin{array}{l}\text { Comparison of the efficacy of ALA-PDT using } \\
\text { an excimer-dye laser }(630 \mathrm{~nm}) \text { and a metal- } \\
\text { halide lamp ( } 600 \text { to } 740 \mathrm{~nm}) \text { for treatment of } \\
\text { Bowen's disease }\end{array}$ & $\begin{array}{l}\text { Photoder } \\
\text { matol } \\
\text { Photoimm } \\
\text { unol } \\
\text { Photomed }\end{array}$ & $\begin{array}{l}\text { not comparative between } \\
\text { treatment nodes }\end{array}$ \\
\hline $\begin{array}{l}\text { CN- } \\
00789893 \\
(20402949) \\
\end{array}$ & Moehrle, M. & $\begin{array}{l}\text { Imiquimod } 5 \% \text { cream as adjunctive therapy for } \\
\text { primary, solitary, nodular basal cell carcinomas } \\
\text { before mohs micrographic surgery: A } \\
\text { randomized, double-blind, vehicle-controlled } \\
\text { study }\end{array}$ & $\begin{array}{l}\text { Dermatolo } \\
\text { gic } \\
\text { surgery }\end{array}$ & no primary data \\
\hline 26442118 & Morley, G. L. & $\begin{array}{l}\text { A Comparative Study Examining the } \\
\text { Management of Bowen's Disease in the United } \\
\text { Kingdom and Australia }\end{array}$ & $\begin{array}{l}\text { Dermatol } \\
\text { Res Pract }\end{array}$ & no outcomes of interest \\
\hline $\begin{array}{l}\text { CN- } \\
00487882\end{array}$ & Morton, C. A. & $\begin{array}{l}\text { A placebo-controlled multicentre study } \\
\text { comparing photodynamic therapy using methyl } \\
\text { aminolaevulinate with cryotherapy and 5- } \\
\text { fluorouracil in BowenÂ's disease. Abstract O-4 } \\
\text { The 84th BAD Annual Meeting 6-9th July 2004, } \\
\text { Belfast,UK }\end{array}$ & $\begin{array}{l}\text { British } \\
\text { journal of } \\
\text { dermatolo } \\
\text { gy }\end{array}$ & $\begin{array}{l}\text { duplicate/conference } \\
\text { abstract and we have full } \\
\text { publication }\end{array}$ \\
\hline $\begin{array}{l}\text { CN- } \\
00318682\end{array}$ & Morton, C. A. & $\begin{array}{l}\text { Photodynamic therapy vs cryotherapy in the } \\
\text { treatment of Bowen's disease. (Abstract) }\end{array}$ & $\begin{array}{l}\text { Clinical } \\
\text { and } \\
\text { experimen } \\
\text { tal } \\
\text { dermatolo } \\
\text { gy }\end{array}$ & $\begin{array}{l}\text { duplicate/conference } \\
\text { abstract and we have full } \\
\text { publication }\end{array}$ \\
\hline $\begin{array}{l}\mathrm{CN}- \\
00416313\end{array}$ & Morton, C. A. & $\begin{array}{l}\text { Topical photodynamic therapy for Bowen's } \\
\text { disease and basal cell carcinoma- an effective } \\
\text { therapy? Abstract }\end{array}$ & $\begin{array}{l}\text { British } \\
\text { journal of } \\
\text { dermatolo } \\
\text { gy }\end{array}$ & $\begin{array}{l}\text { duplicate/conference } \\
\text { abstract and we have full } \\
\text { publication }\end{array}$ \\
\hline
\end{tabular}




\begin{tabular}{|c|c|c|c|c|}
\hline UID & First Author & Title & Journal & $\begin{array}{l}\text { Reason for } \\
\text { Exclusion }\end{array}$ \\
\hline 15859302 & Morton, C. A. & $\begin{array}{l}\text { Topical photodynamic therapy for Bowen's } \\
\text { disease }\end{array}$ & $\begin{array}{l}\text { Australas } \\
\mathrm{J} \\
\text { Dermatol }\end{array}$ & no primary data \\
\hline 11255332 & Morton, C. A. & $\begin{array}{l}\text { Photodynamic therapy for large or multiple } \\
\text { patches of Bowen disease and basal cell } \\
\text { carcinoma }\end{array}$ & $\begin{array}{l}\text { Arch } \\
\text { Dermatol }\end{array}$ & $\begin{array}{l}\text { not comparative between } \\
\text { treatment nodes }\end{array}$ \\
\hline 11069454 & Morton, C. A. & $\begin{array}{l}\text { Comparison of red and green light in the } \\
\text { treatment of Bowen's disease by photodynamic } \\
\text { therapy }\end{array}$ & $\begin{array}{l}\mathrm{Br} \mathrm{J} \\
\text { Dermatol }\end{array}$ & $\begin{array}{l}\text { not comparative between } \\
\text { treatment nodes }\end{array}$ \\
\hline $\begin{array}{l}\text { CN- } \\
00616044\end{array}$ & Morton, CA & $\begin{array}{l}\text { A Randomised, Placebo-Controlled, European } \\
\text { Study Comparing MALPDT with Cryotherapy } \\
\text { and 5-Fluorouracil in Subjects with BowenÂ's } \\
\text { Disease Abstract 13. 3rd Meeting of the } \\
\text { European Association of Dermato-Oncology, } \\
\text { Rome 23-25 June } 2006\end{array}$ & $\begin{array}{l}\text { Journal of } \\
\text { investigati } \\
\text { ve } \\
\text { dermatolo } \\
\text { gy }\end{array}$ & $\begin{array}{l}\text { duplicate/conference } \\
\text { abstract and we have full } \\
\text { publication }\end{array}$ \\
\hline 20497756 & Moscarelli, L. & $\begin{array}{l}\text { Keratinocyte cancer prevention with ACE } \\
\text { inhibitors, angiotensin receptor blockers or their } \\
\text { combination in renal transplant recipients }\end{array}$ & $\begin{array}{l}\text { Clin } \\
\text { Nephrol }\end{array}$ & $\begin{array}{l}\text { not treatment of skin } \\
\text { cancer or }<80 \% \text { SCC or } \\
\text { BCC }\end{array}$ \\
\hline 21056940 & Moskalik, K. & $\begin{array}{l}\text { Powerful neodymium laser radiation for the } \\
\text { treatment of facial carcinoma: } 5 \text { year follow-up } \\
\text { data }\end{array}$ & $\begin{array}{l}\text { Eur J } \\
\text { Dermatol }\end{array}$ & $\begin{array}{l}\text { not comparative between } \\
\text { treatment nodes }\end{array}$ \\
\hline 6419432 & $\begin{array}{l}\text { Moskalik, K. } \\
\text { G. }\end{array}$ & $\begin{array}{l}\text { [Late results and economic aspects of the } \\
\text { treatment of skin cancer with impulse laser } \\
\text { irradiation] }\end{array}$ & $\begin{array}{l}\text { Vestn Khir } \\
\text { Im I I Grek }\end{array}$ & $\begin{array}{l}>20 \% \text { recurrent or } \% \\
\text { recurrent not given }\end{array}$ \\
\hline 7189810 & $\begin{array}{l}\text { Moskalik, K. } \\
\text { G. }\end{array}$ & $\begin{array}{l}\text { [Comparative evaluation of treatment of skin } \\
\text { cancer by impulse laser irradiation, radiotherapy } \\
\text { or surgery] }\end{array}$ & $\begin{array}{l}\text { Med } \\
\text { Radiol } \\
\text { (Mosk) }\end{array}$ & Not English (Russian) \\
\hline $\begin{array}{l}\text { CN- } \\
00753875\end{array}$ & Mosterd, K. & $\begin{array}{l}\text { Mohs micrographic surgery for basal cell } \\
\text { carcinoma of the face: A randomized, controlled } \\
\text { trial. [Dutch] }\end{array}$ & $\begin{array}{l}\text { Nederland } \\
\text { s tijdschrift } \\
\text { voor } \\
\text { geneesku } \\
\text { nde }\end{array}$ & $\begin{array}{l}\text { duplicate/conference } \\
\text { abstract and we have full } \\
\text { publication }\end{array}$ \\
\hline $\begin{array}{l}\text { CN- } \\
00616039\end{array}$ & Muller, F. M. & $\begin{array}{l}\text { A randomized study comparing tissue } \\
\text { conservation in conventional vs.Mohs' surgery } \\
\text { of basal cell carcinoma. Abstract DS-3. The } \\
\text { 87th BAD Annual Meeting 10-13 July 2007, } \\
\text { Birmingham,UK }\end{array}$ & $\begin{array}{l}\text { British } \\
\text { journal of } \\
\text { dermatolo } \\
\text { gy }\end{array}$ & $\begin{array}{l}\text { duplicate/conference } \\
\text { abstract and we have full } \\
\text { publication }\end{array}$ \\
\hline 19500127 & Muller, F. M. & $\begin{array}{l}\text { Randomized comparison of Mohs micrographic } \\
\text { surgery and surgical excision for small nodular } \\
\text { basal cell carcinoma: tissue-sparing outcome }\end{array}$ & $\begin{array}{l}\text { Dermatol } \\
\text { Surg }\end{array}$ & no outcomes of interest \\
\hline 12705745 & Nagore, E. & $\begin{array}{l}\text { Positive margins in basal cell carcinoma: } \\
\text { relationship to clinical features and recurrence } \\
\text { risk. A retrospective study of } 248 \text { patients }\end{array}$ & $\begin{array}{l}\text { J Eur } \\
\text { Acad } \\
\text { Dermatol } \\
\text { Venereol }\end{array}$ & $\begin{array}{l}\text { not comparative between } \\
\text { treatment nodes }\end{array}$ \\
\hline 24411578 & Nanji, A. A. & $\begin{array}{l}\text { Surgical versus medical treatment of ocular } \\
\text { surface squamous neoplasia: a comparison of } \\
\text { recurrences and complications }\end{array}$ & $\begin{array}{l}\text { Ophthalm } \\
\text { ology }\end{array}$ & $\begin{array}{l}\text { No analysis by } \\
\text { population of interest }\end{array}$ \\
\hline $\begin{array}{l}\text { CN- } \\
00602168\end{array}$ & $\begin{array}{l}\text { Nasset- } \\
\text { Seguin }\end{array}$ & $\begin{array}{l}\text { Photodynamic therapy using topical methyl } \\
\text { aminolaevulinate versus cryotherapy for } \\
\text { treatment of primary superficial basal cell } \\
\text { carcinoma: results of a five-year prospective } \\
\text { randomized trial. Abstract P-80. British } \\
\text { Association of Dermatologists 86th Annual } \\
\text { Meeting }\end{array}$ & $\begin{array}{l}\text { British } \\
\text { journal of } \\
\text { dermatolo } \\
\text { gy }\end{array}$ & $\begin{array}{l}\text { duplicate/conference } \\
\text { abstract and we have full } \\
\text { publication }\end{array}$ \\
\hline $\begin{array}{l}\text { CN- } \\
01011816\end{array}$ & Naumann, P. & $\begin{array}{l}\text { Prophylaxis of acute radiation dermatitis with } \\
\text { topical R1 and R2: Interim results of a } \\
\text { multicenter, randomized, controlled trial } \\
\text { (CREAM-1) }\end{array}$ & $\begin{array}{l}\text { Supportive } \\
\text { care in } \\
\text { cancer }\end{array}$ & $\begin{array}{l}\text { not treatment of skin } \\
\text { cancer or }<80 \% \text { SCC or } \\
\text { BCC }\end{array}$ \\
\hline 25109244 & Neittaanmäki- & "Daylight photodynamic therapy for actinic & British & not treatment of skin \\
\hline
\end{tabular}




\begin{tabular}{|c|c|c|c|c|}
\hline UID & First Author & Title & Journal & $\begin{array}{l}\text { Reason for } \\
\text { Exclusion }\end{array}$ \\
\hline & $\begin{array}{l}\text { Perttu, N., et } \\
\text { al. }\end{array}$ & $\begin{array}{l}\text { keratoses: a randomized double-blinded } \\
\text { nonsponsored prospective study comparing 5- } \\
\text { aminolaevulinic acid nanoemulsion (BF-200) } \\
\text { with methyl-5-aminolaevulinate." }\end{array}$ & $\begin{array}{l}\text { Journal of } \\
\text { Dermatolo } \\
\text { gy } 171.5 \\
(2014): \\
1172- \\
1180 .\end{array}$ & $\begin{array}{l}\text { cancer or }<80 \% \text { SCC or } \\
\text { BCC }\end{array}$ \\
\hline 26011755 & $\begin{array}{l}\text { Neittaanmäki- } \\
\text { Perttu, N., et } \\
\text { al. }\end{array}$ & $\begin{array}{l}\text { "Hexyl-5-aminolaevulinate } 0 \cdot 2 \% \text { vs. methyl-5- } \\
\text { aminolaevulinate } 16 \% \text { daylight photodynamic } \\
\text { therapy for treatment of actinic keratoses: } \\
\text { results of a randomized double-blinded pilot } \\
\text { trial." }\end{array}$ & $\begin{array}{l}\text { British } \\
\text { Journal of } \\
\text { Dermatolo } \\
\text { gy (2015). }\end{array}$ & $\begin{array}{l}\text { not treatment of skin } \\
\text { cancer or }<80 \% \text { SCC or } \\
\text { BCC }\end{array}$ \\
\hline 16876511 & $\begin{array}{l}\text { Nemet AY, } \\
\text { Deckel Y, } \\
\text { Martin PA, } \\
\text { Kourt G, } \\
\text { Chilov M, } \\
\text { Sharma V, et } \\
\text { al. }\end{array}$ & $\begin{array}{l}\text { Management of periocular basal and squamous } \\
\text { cell carcinoma: a series of } 485 \text { cases. }\end{array}$ & $\begin{array}{l}\text { Am J } \\
\text { Ophthalm } \\
\text { ol2006;14 } \\
2: 293-7\end{array}$ & $\begin{array}{l}\text { not comparative between } \\
\text { treatment nodes }\end{array}$ \\
\hline 4439437 & Nemeth, G. & $\begin{array}{l}\text { [Experiences in the treatment of eyelid } \\
\text { carcinomas] }\end{array}$ & $\begin{array}{l}\text { Strahlenth } \\
\text { erapie }\end{array}$ & $\begin{array}{l}\text { not comparative between } \\
\text { treatment nodes }\end{array}$ \\
\hline 11774405 & $\begin{array}{l}\text { Newman, L. } \\
\text { A. }\end{array}$ & $\begin{array}{l}\text { Swallowing and speech ability after treatment } \\
\text { for head and neck cancer with targeted } \\
\text { intraarterial versus intravenous chemoradiation }\end{array}$ & $\begin{array}{l}\text { Head } \\
\text { Neck }\end{array}$ & $\begin{array}{l}\text { not treatment of skin } \\
\text { cancer or }<80 \% \text { SCC or } \\
\text { BCC }\end{array}$ \\
\hline 25687314 & Nguyen, B. T. & $\begin{array}{l}\text { Treatment of superficial basal cell carcinoma } \\
\text { and squamous cell carcinoma in situ on the } \\
\text { trunk and extremities with ablative fractional } \\
\text { laser-assisted delivery of topical fluorouracil }\end{array}$ & $\begin{array}{l}\text { J Am } \\
\text { Acad } \\
\text { Dermatol }\end{array}$ & $\begin{array}{l}\text { not comparative between } \\
\text { treatment nodes }\end{array}$ \\
\hline 25256352 & Nguyen, N. P. & $\begin{array}{l}\text { Effectiveness of radiotherapy for elderly patients } \\
\text { with non-melanoma skin cancer of the head }\end{array}$ & $\begin{array}{l}\text { Geriatr } \\
\text { Gerontol } \\
\text { Int }\end{array}$ & $\begin{array}{l}\text { not comparative between } \\
\text { treatment nodes }\end{array}$ \\
\hline 2013665813 & Nicoletti, G. & $\begin{array}{l}\text { Study to determine whether intraoperative } \\
\text { frozen section biopsy improves surgical } \\
\text { treatment of non-melanoma skin cancer }\end{array}$ & $\begin{array}{l}\text { Molecular } \\
\text { and } \\
\text { Clinical } \\
\text { Oncology }\end{array}$ & $\begin{array}{l}>20 \% \text { recurrent or } \% \\
\text { recurrent not given }\end{array}$ \\
\hline 288576 & $\begin{array}{l}\text { Niemczyk, H. } \\
\text { M. }\end{array}$ & $\begin{array}{l}\text { [Comparative study of surgical and radiological } \\
\text { treatment of basal cell carcinoma in head and } \\
\text { neck region] }\end{array}$ & $\begin{array}{l}\text { Dtsch } \\
\text { Zahnarztl } \\
\text { Z }\end{array}$ & Not English (German) \\
\hline 16398319 & Nikkels, A. F. & $\begin{array}{l}\text { Photodynamic therapy and imiquimod } \\
\text { immunotherapy for basal cell carcinomas }\end{array}$ & $\begin{array}{l}\text { Acta Clin } \\
\text { Belg }\end{array}$ & $\begin{array}{l}\text { not comparative between } \\
\text { treatment nodes }\end{array}$ \\
\hline 10233225 & Nordin, P. & $\begin{array}{l}\text { Curettage-cryosurgery for non-melanoma skin } \\
\text { cancer of the external ear: excellent 5-year } \\
\text { results }\end{array}$ & $\begin{array}{l}\mathrm{Br} \mathrm{J} \\
\text { Dermatol }\end{array}$ & $\begin{array}{l}\text { not comparative between } \\
\text { treatment nodes }\end{array}$ \\
\hline 23871719 & O'Bryan, K. & $\begin{array}{l}\text { An evolving paradigm for the workup and } \\
\text { management of high-risk cutaneous squamous } \\
\text { cell carcinoma }\end{array}$ & $\begin{array}{l}\text { J Am } \\
\text { Acad } \\
\text { Dermatol }\end{array}$ & $\begin{array}{l}>20 \% \text { recurrent or } \% \\
\text { recurrent not given }\end{array}$ \\
\hline 17446002 & Ogawa, K. & $\begin{array}{l}\text { Treatment and prognosis of squamous cell } \\
\text { carcinoma of the external auditory canal and } \\
\text { middle ear: a multi-institutional retrospective } \\
\text { review of } 87 \text { patients }\end{array}$ & $\begin{array}{l}\text { Int J } \\
\text { Radiat } \\
\text { Oncol Biol } \\
\text { Phys }\end{array}$ & $\begin{array}{l}\text { not treatment of skin } \\
\text { cancer or }<80 \% \text { SCC or } \\
\text { BCC }\end{array}$ \\
\hline $\begin{array}{l}\mathrm{CN}- \\
00622606 \\
\end{array}$ & Oosten, E. J. & $\begin{array}{l}\text { Different pain sensations in photodynamic } \\
\text { therapy of nodular basal cell carcinoma: Results } \\
\text { from a prospective trial and a review of the } \\
\text { literature }\end{array}$ & $\begin{array}{l}\text { Photodiag } \\
\text { nosis and } \\
\text { photodyna } \\
\text { mic } \\
\text { therapy }\end{array}$ & $\begin{array}{l}\text { duplicate/conference } \\
\text { abstract and we have full } \\
\text { publication }\end{array}$ \\
\hline 16788928 & Oseroff, A. R. & $\begin{array}{l}\text { A dose ranging study of photodynamic therapy } \\
\text { with porfimer sodium (Photofrin) for treatment of } \\
\text { basal cell carcinoma }\end{array}$ & $\begin{array}{l}\text { Lasers } \\
\text { Surg Med }\end{array}$ & $\begin{array}{l}>20 \% \text { recurrent or } \% \\
\text { recurrent not given }\end{array}$ \\
\hline 22293891 & Osiecka, B. & $\begin{array}{l}\text { The application of Levulan-based photodynamic } \\
\text { therapy with imiquimod in the treatment of }\end{array}$ & $\begin{array}{l}\text { Med Sci } \\
\text { Monit }\end{array}$ & $\begin{array}{l}>20 \% \text { recurrent or } \% \\
\text { recurrent not given }\end{array}$ \\
\hline
\end{tabular}




\begin{tabular}{|c|c|c|c|c|}
\hline UID & First Author & Title & Journal & $\begin{array}{l}\text { Reason for } \\
\text { Exclusion }\end{array}$ \\
\hline & & \multicolumn{3}{|l|}{ recurrent basal cell carcinoma } \\
\hline $\begin{array}{l}\mathrm{CN}- \\
00452810\end{array}$ & Overgaard, J. & $\begin{array}{l}\text { The Danish Head and Neck Cancer Study } \\
\text { Group DAHANCA } 6 \& 7 \text { randomized trial of } 5 \\
\text { versus } 6 \text { fractions per week of conventional } \\
\text { radiotherapy of squamous cell carcinoma of the } \\
\text { head and neck }\end{array}$ & $\begin{array}{l}\text { Proceedin } \\
\text { gs of the } \\
\text { American } \\
\text { Society of } \\
\text { Clinical } \\
\text { Oncology } \\
\text { (ASCO). } \\
\text { Chicago, } \\
\text { Illinois, } 31 \\
\text { May-3 } \\
\text { June, } \\
2003\end{array}$ & $\begin{array}{l}\text { not comparative between } \\
\text { treatment nodes }\end{array}$ \\
\hline 20409337 & Ozolins, M. & $\begin{array}{l}\text { The SINS trial: a randomised controlled trial of } \\
\text { excisional surgery versus imiquimod } 5 \% \text { cream } \\
\text { for nodular and superficial basal cell carcinoma }\end{array}$ & Trials & no primary data \\
\hline 8538187 & Palo, G. & $\begin{array}{l}\text { Controlled clinical trials with fenretinide in breast } \\
\text { cancer, basal cell carcinoma and oral } \\
\text { leukoplakia }\end{array}$ & $\begin{array}{l}\text { Journal of } \\
\text { cellular } \\
\text { biochemist } \\
\text { ry. } \\
\text { Suppleme } \\
\text { nt }\end{array}$ & $\begin{array}{l}\text { not treatment of skin } \\
\text { cancer or }<80 \% \text { SCC or } \\
\text { BCC }\end{array}$ \\
\hline 12828747 & Palsson, S. & $\begin{array}{l}\text { Kinetics of the superficial perfusion and } \\
\text { temperature in connection with photodynamic } \\
\text { therapy of basal cell carcinomas using esterified } \\
\text { and non-esterified 5-aminolaevulinic acid }\end{array}$ & $\begin{array}{l}\mathrm{Br} \mathrm{J} \\
\text { Dermatol }\end{array}$ & $\begin{array}{l}\text { not comparative between } \\
\text { treatment nodes }\end{array}$ \\
\hline 26589877 & Pampena, R. & $\begin{array}{l}\text { Orthovoltage radiotherapy for nonmelanoma } \\
\text { skin cancer (NMSC): Comparison between } 2 \\
\text { different schedules }\end{array}$ & $\begin{array}{l}\text { J Am } \\
\text { Acad } \\
\text { Dermatol }\end{array}$ & $\begin{array}{l}\text { duplicate/conference } \\
\text { abstract and we have full } \\
\text { publication }\end{array}$ \\
\hline 2010372046 & Pariser, D. & $\begin{array}{l}\text { Using a hydroquinone/tretinoin-based skin care } \\
\text { system before and after electrodesiccation and } \\
\text { curettage of superficial truncal basal cell } \\
\text { carcinoma }\end{array}$ & $\begin{array}{l}\text { Journal of } \\
\text { Clinical } \\
\text { and } \\
\text { Aesthetic } \\
\text { Dermatolo } \\
\text { gy }\end{array}$ & $\begin{array}{l}\text { not comparative between } \\
\text { treatment nodes }\end{array}$ \\
\hline $\begin{array}{l}\mathrm{CN}- \\
00130587\end{array}$ & Parsons, J. T. & $\begin{array}{l}\text { Re: Five-year update of a randomized trial of } \\
\text { alternating radiotherapy and chemotherapy } \\
\text { compared with radiotherapy alone in treatment } \\
\text { of unresectable squamous cell carcinoma of the } \\
\text { head and neck }\end{array}$ & $\begin{array}{l}\text { Journal of } \\
\text { the } \\
\text { National } \\
\text { Cancer } \\
\text { Institute }\end{array}$ & no primary data \\
\hline 2420153 & $\begin{array}{l}\text { Parvinen, L. } \\
\text { M. }\end{array}$ & $\begin{array}{l}\text { Combined bleomycin treatment and radiation } \\
\text { therapy in squamous cell carcinoma of the head } \\
\text { and neck region }\end{array}$ & $\begin{array}{l}\text { Acta } \\
\text { Radiol } \\
\text { Oncol }\end{array}$ & $\begin{array}{l}\text { not treatment of skin } \\
\text { cancer or }<80 \% \text { SCC or } \\
\text { BCC }\end{array}$ \\
\hline 10496562 & $\begin{array}{l}\text { Paterson, C. } \\
\text { A. }\end{array}$ & $\begin{array}{l}\text { Basal cell carcinoma of the perianal region: } 20- \\
\text { year experience }\end{array}$ & $\begin{array}{l}\text { Dis Colon } \\
\text { Rectum }\end{array}$ & $\begin{array}{l}\text { not treatment of skin } \\
\text { cancer or }<80 \% \text { SCC or } \\
\text { BCC }\end{array}$ \\
\hline 21668511 & Pauwels, C. & $\begin{array}{l}\text { Topical methyl aminolevulinate photodynamic } \\
\text { therapy for management of basal cell } \\
\text { carcinomas in patients with basal cell nevus } \\
\text { syndrome improves patient's satisfaction and } \\
\text { reduces the need for surgical procedures }\end{array}$ & $\begin{array}{l}\text { J Eur } \\
\text { Acad } \\
\text { Dermatol } \\
\text { Venereol }\end{array}$ & $\begin{array}{l}\text { not comparative between } \\
\text { treatment nodes }\end{array}$ \\
\hline 22748679 & Pazdrowski, J. & $\begin{array}{l}\text { [The recurrence of facial basal cell carcinoma in } \\
\text { patients treated at the Head and Neck Surgery } \\
\text { Ward and Laryngological Oncology Clinic of the } \\
\text { Greater Poland Cancer Centre in the years } \\
\text { 2007-2010] }\end{array}$ & $\begin{array}{l}\text { Otolaryng } \\
\text { ol Pol }\end{array}$ & Not English (Polish) \\
\hline 19625138 & $\begin{array}{l}\text { Penagaricano, } \\
\text { J. A. }\end{array}$ & $\begin{array}{l}\text { Evaluation of spatially fractionated radiotherapy } \\
\text { (GRID) and definitive chemoradiotherapy with } \\
\text { curative intent for locally advanced squamous }\end{array}$ & $\begin{array}{l}\text { Int J } \\
\text { Radiat } \\
\text { Oncol Biol }\end{array}$ & $\begin{array}{l}\text { not treatment of skin } \\
\text { cancer or }<80 \% \text { SCC or } \\
\text { BCC }\end{array}$ \\
\hline
\end{tabular}




\begin{tabular}{|c|c|c|c|c|}
\hline UID & First Author & Title & Journal & $\begin{array}{l}\text { Reason for } \\
\text { Exclusion }\end{array}$ \\
\hline & & $\begin{array}{l}\text { cell carcinoma of the head and neck: initial } \\
\text { response rates and toxicity }\end{array}$ & Phys & \\
\hline 11566277 & Peng, Q. & $\begin{array}{l}\text { Selective distribution of porphyrins in skin thick } \\
\text { basal cell carcinoma after topical application of } \\
\text { methyl } 5 \text {-aminolevulinate }\end{array}$ & $\begin{array}{l}\text { J } \\
\text { Photoche } \\
\text { m } \\
\text { Photobiol } \\
\text { B }\end{array}$ & $\begin{array}{l}\text { not comparative between } \\
\text { treatment nodes }\end{array}$ \\
\hline 6182982 & $\begin{array}{l}\text { Pennacchio, } \\
\text { J. L. }\end{array}$ & $\begin{array}{l}\text { Combination of cis-platinum and bleomycin prior } \\
\text { to surgery and/or radiotherapy compared with } \\
\text { radiotherapy alone for the treatment of } \\
\text { advanced squamous cell carcinoma of the head } \\
\text { and neck }\end{array}$ & Cancer & $\begin{array}{l}\text { not treatment of skin } \\
\text { cancer or }<80 \% \text { SCC or } \\
\text { BCC }\end{array}$ \\
\hline 1908427 & Perez, C. A. & $\begin{array}{l}\text { Electron beam and } x \text {-rays in the treatment of } \\
\text { epithelial skin cancer: dosimetric considerations } \\
\text { and clinical results }\end{array}$ & $\begin{array}{l}\text { Front } \\
\text { Radiat } \\
\text { Ther } \\
\text { Oncol } \\
\end{array}$ & $\begin{array}{l}>20 \% \text { recurrent or } \% \\
\text { recurrent not given }\end{array}$ \\
\hline 1903023 & Perez, C. A. & $\begin{array}{l}\text { Randomized phase III study comparing } \\
\text { irradiation and hyperthermia with irradiation } \\
\text { alone in superficial measurable tumors. Final } \\
\text { report by the Radiation Therapy Oncology } \\
\text { Group }\end{array}$ & $\begin{array}{l}\text { Am J Clin } \\
\text { Oncol }\end{array}$ & $\begin{array}{l}\text { No analysis by } \\
\text { population of interest }\end{array}$ \\
\hline 15923570 & Perki & $\begin{array}{l}\text { Nonmelanoma skin cancer in survivors of } \\
\text { childhood and adolescent cancer: a report from } \\
\text { the childhood cancer survivor study }\end{array}$ & $\begin{array}{l}\text { J Clin } \\
\text { Oncol }\end{array}$ & no primary data \\
\hline 17223873 & Perrett, C. M. & $\begin{array}{l}\text { Treatment of post-transplant premalignant skin } \\
\text { disease: a randomized intrapatient comparative } \\
\text { study of 5-fluorouracil cream and topical } \\
\text { photodynamic therapy }\end{array}$ & $\begin{array}{l}\mathrm{Br} \mathrm{J} \\
\text { Dermatol }\end{array}$ & $\begin{array}{l}\text { not treatment of skin } \\
\text { cancer or }<80 \% \text { SCC or } \\
\text { BCC }\end{array}$ \\
\hline $\begin{array}{l}\text { CN- } \\
00602171\end{array}$ & Perrett, C. M. & $\begin{array}{l}\text { A comparative study of topical 5-fluorouracil and } \\
\text { topical photodynamic therapy using } \\
\text { methylaminolevulinate for actinic keratosis and } \\
\text { BowenÂ's disease in organ transplant recipients } \\
\text { (Abstract P26) American Academy of } \\
\text { Dermatology 64th Annual Meeting March 3-7, } \\
2006\end{array}$ & $\begin{array}{l}\text { Journal of } \\
\text { the } \\
\text { American } \\
\text { Academy } \\
\text { of } \\
\text { Dermatolo } \\
\text { gy }\end{array}$ & $\begin{array}{l}\text { not treatment of skin } \\
\text { cancer or }<80 \% \text { SCC or } \\
\text { BCC }\end{array}$ \\
\hline 12271300 & $\begin{array}{l}\text { Perse } \\
\text { N. }\end{array}$ & $\begin{array}{l}\text { Clinical effect of imiquimod } 5 \% \text { cream in the } \\
\text { treatment of actinic keratosis }\end{array}$ & $\begin{array}{l}\text { J Am } \\
\text { Acad } \\
\text { Dermatol }\end{array}$ & $\begin{array}{l}\text { not treatment of skin } \\
\text { cancer or }<80 \% \text { SCC or } \\
\text { BCC }\end{array}$ \\
\hline 12395436 & Pesic, Z. & $\begin{array}{l}\text { [Ultrasonography and surgical treatment of } \\
\text { facial skin neoplasms] }\end{array}$ & $\begin{array}{l}\text { Srp Arh } \\
\text { Celok Lek }\end{array}$ & $\begin{array}{l}\text { not treatment of skin } \\
\text { cancer or }<80 \% \text { SCC or } \\
\text { BCC }\end{array}$ \\
\hline 15125510 & $\begin{array}{l}\text { Pichardo- } \\
\text { Velazquez, } \mathrm{P} .\end{array}$ & Surgical option for nonmelanoma skin cancer & $\begin{array}{l}\text { Int J } \\
\text { Dermatol }\end{array}$ & $\begin{array}{l}\text { not comparative between } \\
\text { treatment nodes }\end{array}$ \\
\hline 6665189 & Placek, W. & $\begin{array}{l}\text { [Comparative evaluation of } 2 \text { methods of } \\
\text { fractionated soft X-ray therapy of basal cell } \\
\text { carcinoma of the skin] }\end{array}$ & $\begin{array}{l}\text { Przegl } \\
\text { Dermatol }\end{array}$ & Not English (Polish) \\
\hline 4012422 & Pletnev, S. D. & $\begin{array}{l}\text { [Treatment of recurrent basal-cell skin cancer } \\
\text { with laser irradiation] }\end{array}$ & Sov Med & $\begin{array}{l}>20 \% \text { recurrent or } \% \\
\text { recurrent not given }\end{array}$ \\
\hline 24666361 & Pomerantz, $\mathrm{H}$. & $\begin{array}{l}\text { Predictors of local adverse effects caused by } \\
\text { topical tretinoin cream } 0.1 \% \text { in the Veterans } \\
\text { Affairs Topical Tretinoin Chemoprevention trial }\end{array}$ & $\begin{array}{l}\mathrm{Br} \mathrm{J} \\
\text { Dermatol }\end{array}$ & $\begin{array}{l}\text { not treatment of skin } \\
\text { cancer or }<80 \% \text { SCC or } \\
\text { BCC }\end{array}$ \\
\hline 1223143 & Popkin, G. L. & $\begin{array}{l}\text { Excision versus curettage and } \\
\text { electrodesiccation as dermatologic office } \\
\text { procedures for the treatment of basal-cell } \\
\text { carcinomas }\end{array}$ & $\begin{array}{l}\text { J } \\
\text { Dermatol } \\
\text { Surg }\end{array}$ & no primary data \\
\hline 10901965 & Poulsen, M. & $\begin{array}{l}\text { Acute toxicity and cost analysis of a phase III } \\
\text { randomized trial of accelerated and } \\
\text { conventional radiotherapy for squamous } \\
\text { carcinoma of the head and neck: a Trans- }\end{array}$ & $\begin{array}{l}\text { Australas } \\
\text { Radiol }\end{array}$ & $\begin{array}{l}\text { not treatment of skin } \\
\text { cancer or }<80 \% \text { SCC or } \\
\text { BCC }\end{array}$ \\
\hline
\end{tabular}




\begin{tabular}{|c|c|c|c|c|}
\hline UID & First Author & Title & Journal & $\begin{array}{l}\text { Reason for } \\
\text { Exclusion }\end{array}$ \\
\hline & & Tasman Radiation Oncology Group study & & \\
\hline 19398900 & Prabhu, R. & $\begin{array}{l}\text { Squamous cell carcinoma of the external } \\
\text { auditory canal: long-term clinical outcomes } \\
\text { using surgery and external-beam radiotherapy }\end{array}$ & $\begin{array}{l}\text { Am J Clin } \\
\text { Oncol }\end{array}$ & $\begin{array}{l}\text { not comparative between } \\
\text { treatment nodes }\end{array}$ \\
\hline 19138010 & $\begin{array}{l}\text { Puizina-Ivic, } \\
\text { N. }\end{array}$ & $\begin{array}{l}\text { Fractionated illumination improves the outcome } \\
\text { in the treatment of precancerous lesions with } \\
\text { photodynamic therapy }\end{array}$ & $\begin{array}{l}\text { Coll } \\
\text { Antropol } \\
\end{array}$ & $\begin{array}{l}\text { not treatment of skin } \\
\text { cancer or }<80 \% \text { SCC or } \\
\text { BCC }\end{array}$ \\
\hline 18173610 & Punjabi, S. & $\begin{array}{l}\text { Solasodine glycoalkaloids: a novel topical } \\
\text { therapy for basal cell carcinoma. A double-blind, } \\
\text { randomized, placebo-controlled, parallel group, } \\
\text { multicenter study }\end{array}$ & $\begin{array}{l}\text { Int J } \\
\text { Dermatol }\end{array}$ & $\begin{array}{l}\text { not treatment of interest } \\
\text { (Solasodine } \\
\text { glycoalkaloids) }\end{array}$ \\
\hline 17034468 & $\begin{array}{l}\text { Quirk, Chris, } \\
\text { et al. }\end{array}$ & $\begin{array}{l}\text { "Two-year interim results from a 5-year study } \\
\text { evaluating clinical recurrence of superficial } \\
\text { basal cell carcinoma after treatment with } \\
\text { imiquimod } 5 \% \text { cream daily for } 6 \text { weeks." }\end{array}$ & $\begin{array}{l}\text { Australasi } \\
\text { an journal } \\
\text { of } \\
\text { dermatolo } \\
\text { gy47.4 } \\
(2006) \text { : } \\
258-265 \text {. }\end{array}$ & $\begin{array}{l}\text { not comparative between } \\
\text { treatment nodes }\end{array}$ \\
\hline 12828745 & $\begin{array}{l}\text { Ramrakha- } \\
\text { Jones, V. S. }\end{array}$ & $\begin{array}{l}\text { Treating Bowen's disease: a cost-minimization } \\
\text { study }\end{array}$ & $\begin{array}{l}\mathrm{Br} \mathrm{J} \\
\text { Dermatol }\end{array}$ & no primary data \\
\hline 25704233 & Reigneau, M. & $\begin{array}{l}\text { Efficacy of neoadjuvant cetuximab alone or with } \\
\text { platinum salt for the treatment of unresectable } \\
\text { advanced nonmetastatic cutaneous squamous } \\
\text { cell carcinomas }\end{array}$ & $\begin{array}{l}\mathrm{Br} \mathrm{J} \\
\text { Dermatol }\end{array}$ & $\begin{array}{l}>20 \% \text { recurrent or } \% \\
\text { recurrent not given }\end{array}$ \\
\hline 4919323 & Reymann, F. & $\begin{array}{l}\text { Treatment of basal cell carcinoma with 5- } \\
\text { fluorouracil (5-FU) ointment }\end{array}$ & $\begin{array}{l}\text { Dermatolo } \\
\text { gica }\end{array}$ & $\begin{array}{l}\text { not comparative between } \\
\text { treatment nodes }\end{array}$ \\
\hline 5555850 & Reymann, F. & $\begin{array}{l}\text { Treatment of basal cell carcinoma of the skin } \\
\text { with curettage }\end{array}$ & $\begin{array}{l}\text { Arch } \\
\text { Dermatol }\end{array}$ & $\begin{array}{l}\text { not comparative between } \\
\text { treatment nodes }\end{array}$ \\
\hline 6515862 & Reymann, F. & [Treatment of basal cell carcinoma of the skin] & $\begin{array}{l}\text { Ugeskr } \\
\text { Laeger }\end{array}$ & $\begin{array}{l}\text { not comparative between } \\
\text { treatment nodes }\end{array}$ \\
\hline $\begin{array}{l}\mathrm{CN}- \\
00454623\end{array}$ & Rhodes Let, al & $\begin{array}{l}\text { A randomized comparison of excision surgery } \\
\text { and PDT using methyl aminolevulinate in } \\
\text { nodular BCC Abstract }\end{array}$ & $\begin{array}{l}\text { Annales } \\
\text { de } \\
\text { dermatolo } \\
\text { gie et de } \\
\text { venereolo } \\
\text { gie }\end{array}$ & $\begin{array}{l}\text { duplicate/conference } \\
\text { abstract and we have full } \\
\text { publication }\end{array}$ \\
\hline $\begin{array}{l}\mathrm{CN}- \\
00478736\end{array}$ & Rhodes Let, al & $\begin{array}{l}\text { A randomized comparison of excision surgery } \\
\text { and photodynamic therapy using methyl } \\
\text { aminolaevulinate in nodular basal cell } \\
\text { carcinoma. British Association of } \\
\text { Dermatologists 83rd Annual Meeting. Abstract } \\
\text { P-68 }\end{array}$ & $\begin{array}{l}\text { British } \\
\text { journal of } \\
\text { dermatolo } \\
\text { gy }\end{array}$ & $\begin{array}{l}\text { duplicate/conference } \\
\text { abstract and we have full } \\
\text { publication }\end{array}$ \\
\hline $\begin{array}{l}\mathrm{CN}- \\
00602507\end{array}$ & Rhodes, L. & $\begin{array}{l}\text { A randomized European comparison of excision } \\
\text { surgery and MAL-PDT in nodular basal cell } \\
\text { carcinoma: results from a 36-month follow-up. } \\
\text { Abstract P08.69. The 14th Congress of the } \\
\text { European Academy of Dermatology and } \\
\text { Venereology, London,UK. 12-15th October } \\
2005\end{array}$ & $\begin{array}{l}\text { Journal of } \\
\text { the } \\
\text { European } \\
\text { Academy } \\
\text { of } \\
\text { Dermatolo } \\
\text { gy and } \\
\text { Venereolo } \\
\text { gy : } \\
\text { JEADV }\end{array}$ & $\begin{array}{l}\text { duplicate/conference } \\
\text { abstract and we have full } \\
\text { publication }\end{array}$ \\
\hline $\begin{array}{l}\text { CN- } \\
00612111 \\
(17875873)\end{array}$ & Rhodes, L. E. & $\begin{array}{l}\text { Five-year follow-up of a randomized, } \\
\text { prospective trial of topical methyl } \\
\text { aminolevulinate photodynamic therapy vs } \\
\text { surgery for nodular basal cell carcinoma }\end{array}$ & $\begin{array}{l}\text { Archives } \\
\text { of } \\
\text { dermatolo } \\
\text { gy }\end{array}$ & $\begin{array}{l}\text { duplicate/conference } \\
\text { abstract and we have full } \\
\text { publication }\end{array}$ \\
\hline $\begin{array}{l}\text { CN- } \\
00527223\end{array}$ & Rhodes, L. E. & $\begin{array}{l}\text { A randomized European comparison of MAL- } \\
\text { PDT and excision surgery in nodular basal cell } \\
\text { carcinoma. Abstract P-29 The 85th BAD Annual }\end{array}$ & $\begin{array}{l}\text { British } \\
\text { journal of } \\
\text { dermatolo }\end{array}$ & $\begin{array}{l}\text { duplicate/conference } \\
\text { abstract and we have full } \\
\text { publication }\end{array}$ \\
\hline
\end{tabular}




\begin{tabular}{|c|c|c|c|c|}
\hline UID & First Author & Title & Journal & $\begin{array}{l}\text { Reason for } \\
\text { Exclusion }\end{array}$ \\
\hline & & Meeting 5-8th July 2005, Glasgow, UK & gy & \\
\hline $\begin{array}{l}\mathrm{CN}- \\
00602236\end{array}$ & Rhodes, L. E. & $\begin{array}{l}\text { A randomized european comparison of mal-pdt } \\
\text { and excision surgery in nodular basal cell } \\
\text { carcinoma }\end{array}$ & $\begin{array}{l}\text { 7th Asian } \\
\text { Congress } \\
\text { of } \\
\text { Dermatolo } \\
\text { gy } \\
\text { Incorporati } \\
\text { ng the 5th } \\
\text { Regional } \\
\text { Conferenc } \\
\text { e of } \\
\text { Paediatric } \\
\text { Dermatolo } \\
\text { gy Kuala } \\
\text { Lumpur, } \\
\text { Malaysia } \\
\text { 28th } \\
\text { Septembe } \\
\text { r -1st } \\
\text { October, } \\
\text { 2005 }\end{array}$ & $\begin{array}{l}\text { duplicate/conference } \\
\text { abstract and we have full } \\
\text { publication }\end{array}$ \\
\hline $\begin{array}{l}\text { CN- } \\
00616002\end{array}$ & Rhodes, LE & $\begin{array}{l}\text { A Randomized European Comparison of MAL- } \\
\text { PDT and Excision Surgery in Nodular Basal Cell } \\
\text { Carcinoma: Results From a } 60 \text { Month Follow- } \\
\text { Up Study. Abstract PO6. 3rd Meeting of the } \\
\text { European Association of Dermato-Oncology, } \\
\text { Rome } 23-25 \text { June } 2006\end{array}$ & $\begin{array}{l}\text { Journal of } \\
\text { investigati } \\
\text { ve } \\
\text { dermatolo } \\
\text { gy }\end{array}$ & $\begin{array}{l}\text { duplicate/conference } \\
\text { abstract and we have full } \\
\text { publication }\end{array}$ \\
\hline 15927410 & Rio, E. & $\begin{array}{l}\text { Interstitial brachytherapy of periorificial skin } \\
\text { carcinomas of the face: a retrospective study of } \\
97 \text { cases }\end{array}$ & $\begin{array}{l}\text { Int J } \\
\text { Radiat } \\
\text { Oncol Biol } \\
\text { Phys }\end{array}$ & $\begin{array}{l}\text { not comparative between } \\
\text { treatment nodes }\end{array}$ \\
\hline 16529964 & Rio, E. & $\begin{array}{l}\text { [Interstitial brachytherapy of peri-orificial skin } \\
\text { carcinomas on the face] }\end{array}$ & $\begin{array}{l}\text { Cancer } \\
\text { Radiother }\end{array}$ & $\begin{array}{l}\text { not comparative between } \\
\text { treatment nodes }\end{array}$ \\
\hline 15625362 & Rischin, D. & $\begin{array}{l}\text { Tirapazamine, Cisplatin, and Radiation versus } \\
\text { Fluorouracil, Cisplatin, and Radiation in patients } \\
\text { with locally advanced head and neck cancer: a } \\
\text { randomized phase II trial of the Trans-Tasman } \\
\text { Radiation Oncology Group (TROG 98.02) }\end{array}$ & $\begin{array}{l}\text { J Clin } \\
\text { Oncol }\end{array}$ & $\begin{array}{l}\text { not treatment of skin } \\
\text { cancer or }<80 \% \text { SCC or } \\
\text { BCC }\end{array}$ \\
\hline $\begin{array}{l}\text { CN- } \\
00775868\end{array}$ & Rischin, D. & $\begin{array}{l}\text { Preliminary results of TROG } 98.02 \text { - a } \\
\text { randomized phase II study of 5-fluorouracil, } \\
\text { cisplatin and radiation versus tirapazamine, } \\
\text { cisplatin and radiation for advanced squamous } \\
\text { cell carcinoma of the head and neck }\end{array}$ & $\begin{array}{l}\text { Proceedin } \\
\text { gs of the } \\
\text { American } \\
\text { Society of } \\
\text { Clinical } \\
\text { Oncology }\end{array}$ & $\begin{array}{l}\text { not treatment of skin } \\
\text { cancer or }<80 \% \text { SCC or } \\
\text { BCC }\end{array}$ \\
\hline $\begin{array}{l}\text { CN- } \\
00478739\end{array}$ & $\begin{array}{l}\text { Robinson, } \mathrm{J} . \\
\mathrm{K} .\end{array}$ & $\begin{array}{l}\text { Imiquimod } 5 \% \text { cream for } 12 \text { weeks treating } \\
\text { nodular BCC [Abstract] }\end{array}$ & $\begin{array}{l}\text { 8th World } \\
\text { Congress } \\
\text { on Cancer } \\
\text { of the } \\
\text { Skin. } \\
\text { Zurich, } \\
\text { Switzerlan } \\
\text { d. July 18- } \\
21,2001\end{array}$ & $\begin{array}{l}\text { duplicate/conference } \\
\text { abstract and we have full } \\
\text { publication }\end{array}$ \\
\hline 21576573 & $\begin{array}{l}\text { Robinson, J. } \\
\text { K. }\end{array}$ & Evidence-based choice of treatment of NMSC & $\begin{array}{l}\text { Arch } \\
\text { Dermatol }\end{array}$ & no primary data \\
\hline $\begin{array}{l}\text { CN- } \\
00641211\end{array}$ & Rocher, C. & $\begin{array}{l}\text { Imiquimod } 5 \% \text { in the treatment of basal cell } \\
\text { carcinoma: Assessment of efficacy and } \\
\text { tolerability. [Spanish] }\end{array}$ & $\begin{array}{l}\text { Dermatolo } \\
\text { gia } \\
\text { Revista } \\
\text { Mexicana }\end{array}$ & $\begin{array}{l}\text { not comparative between } \\
\text { treatment nodes }\end{array}$ \\
\hline
\end{tabular}




\begin{tabular}{|c|c|c|c|c|}
\hline UID & First Author & Title & Journal & $\begin{array}{l}\text { Reason for } \\
\text { Exclusion }\end{array}$ \\
\hline 15605806 & Rodrigo, J. P. & $\begin{array}{l}\text { [Efficacy of postoperative radiation therapy for } \\
\text { squamous cell carcinoma of the head and neck: } \\
\text { results of a prospective randomised clinical trial] }\end{array}$ & $\begin{array}{l}\text { Acta } \\
\text { Otorrinolar } \\
\text { ingol Esp }\end{array}$ & $\begin{array}{l}\text { not treatment of skin } \\
\text { cancer or }<80 \% \text { SCC or } \\
\text { BCC }\end{array}$ \\
\hline 2894839 & $\begin{array}{l}\text { Rodriguez- } \\
\text { Sains, R. S. }\end{array}$ & $\begin{array}{l}\text { Radiotherapy of periocular basal cell } \\
\text { carcinomas: recurrence rates and treatment } \\
\text { with special attention to the medical canthus }\end{array}$ & $\begin{array}{l}\text { Br J } \\
\text { Ophthalm } \\
\text { ol }\end{array}$ & $\begin{array}{l}>20 \% \text { recurrent or } \% \\
\text { recurrent not given }\end{array}$ \\
\hline 17190625 & $\begin{array}{l}\text { Rodriguez- } \\
\text { Vigil, T. }\end{array}$ & $\begin{array}{l}\text { Recurrence rates of primary basal cell } \\
\text { carcinoma in facial risk areas treated with } \\
\text { curettage and electrodesiccation }\end{array}$ & $\begin{array}{l}\text { J Am } \\
\text { Acad } \\
\text { Dermatol }\end{array}$ & $\begin{array}{l}\text { not comparative between } \\
\text { treatment nodes }\end{array}$ \\
\hline 1390484 & $\begin{array}{l}\text { Rodriguez, J. } \\
\text { M. }\end{array}$ & $\begin{array}{l}\text { The treatment of periocular basal cell } \\
\text { carcinomas by radiotherapy }\end{array}$ & $\begin{array}{l}\text { Br J } \\
\text { Ophthalm } \\
\text { ol }\end{array}$ & $\begin{array}{l}\text { not comparative between } \\
\text { treatment nodes }\end{array}$ \\
\hline $\begin{array}{l}\mathrm{CN}- \\
00193051\end{array}$ & $\begin{array}{l}\text { Rogozinski, T. } \\
\text { T. }\end{array}$ & $\begin{array}{l}\text { Intralesional treatment with recombinant } \\
\text { interferon beta is an effective alternative for the } \\
\text { treatment of basal cell carcinoma. Double-blind, } \\
\text { placebo-controlled study. <ORIGINAL> } \\
\text { DOOGNISKOWE PODAWANIE } \\
\text { REKOMBINANTOWEGO INTERFERONU } \\
\text { BETA. SKUTECZNA ALTERNATYWA W } \\
\text { LECZENIU BASALIOMA (WYNIKI } \\
\text { PODWOJNIE SLEPEJ PROBY) }\end{array}$ & $\begin{array}{l}\text { Przeglad } \\
\text { dermatolo } \\
\text { giczny }\end{array}$ & $\begin{array}{l}\text { not comparative between } \\
\text { treatment nodes }\end{array}$ \\
\hline 10759821 & $\begin{array}{l}\text { Romagosa, } \\
\text { Ricardo, et al. }\end{array}$ & $\begin{array}{l}\text { "A Pilot Study to Evaluate the Treatment of } \\
\text { Basal Cell Carcinoma with 5-Fluorouracil Using } \\
\text { Phosphatidyl Choline as a Transepidermal } \\
\text { Carrier." }\end{array}$ & $\begin{array}{l}\text { Dermatolo } \\
\text { gic } \\
\text { surgery } \\
26.4 \\
(2000) \text { : } \\
338-340 \text {. }\end{array}$ & $\begin{array}{l}\text { not comparative between } \\
\text { treatment nodes }\end{array}$ \\
\hline 0 & $\begin{array}{l}\text { Romanko, Yu } \\
\text { S. }\end{array}$ & $\begin{array}{l}\text { Efficacy of photodynamic therapy for basal cell } \\
\text { carcinoma using photosensitizers of different } \\
\text { classes }\end{array}$ & $\begin{array}{l}\text { Voprosy } \\
\text { Onkologii }\end{array}$ & $\begin{array}{l}\text { not comparative between } \\
\text { treatment nodes }\end{array}$ \\
\hline 25935596 & $\begin{array}{l}\text { Roozeboom, } \\
\text { M. H. }\end{array}$ & $\begin{array}{l}\text { Tumor thickness and adnexal extension of } \\
\text { superficial basal cell carcinoma (sBCC) as } \\
\text { determinants of treatment failure for } \\
\text { methylaminolevulinate (MAL)-photodynamic } \\
\text { therapy (PDT), imiquimod, and 5-fluorouracil } \\
\text { (FU) }\end{array}$ & $\begin{array}{l}\text { J Am } \\
\text { Acad } \\
\text { Dermatol }\end{array}$ & no outcomes of interest \\
\hline 26376042 & Rotunno, R. & $\begin{array}{l}\text { Electrochemotherapy in non-melanoma head } \\
\text { and neck skin cancers: a three centers } \\
\text { experience and literature review }\end{array}$ & $\begin{array}{l}\text { G Ital } \\
\text { Dermatol } \\
\text { Venereol }\end{array}$ & $\begin{array}{l}\text { not comparative between } \\
\text { treatment nodes }\end{array}$ \\
\hline 24861492 & $\begin{array}{l}\text { Rubel, D. M., } \\
\text { et al. }\end{array}$ & $\begin{array}{l}\text { "Daylight photodynamic therapy with methyl } \\
\text { aminolevulinate cream as a convenient, } \\
\text { similarly effective, nearly painless alternative to } \\
\text { conventional photodynamic therapy in actinic } \\
\text { keratosis treatment: a randomized controlled } \\
\text { trial." }\end{array}$ & $\begin{array}{l}\text { British } \\
\text { Journal of } \\
\text { Dermatolo } \\
\text { gy171.5 } \\
(2014): \\
1164- \\
1171 .\end{array}$ & $\begin{array}{l}\text { not treatment of skin } \\
\text { cancer or }<80 \% \text { SCC or } \\
\text { BCC }\end{array}$ \\
\hline 4848330 & $\begin{array}{l}\text { Rubisz- } \\
\text { Brzezinska, J. }\end{array}$ & $\begin{array}{l}\text { [Comparative appraisal of results of treatment of } \\
\text { basal cell epithelioma with various methods] }\end{array}$ & $\begin{array}{l}\text { Przegl } \\
\text { Dermatol }\end{array}$ & Not English (Polish) \\
\hline 424458 & Sakura, C. Y. & $\begin{array}{l}\text { Comparison of treatment modalities for } \\
\text { recurrent basal cell carcinoma }\end{array}$ & $\begin{array}{l}\text { Plast } \\
\text { Reconstr } \\
\text { Surg }\end{array}$ & $\begin{array}{l}>20 \% \text { recurrent or } \% \\
\text { recurrent not given }\end{array}$ \\
\hline $\begin{array}{l}\text { CN- } \\
00429205\end{array}$ & Salim, A. & $\begin{array}{l}\text { Comparison of photodynamic therapy with } \\
\text { topical 5-8uorouracil in Bowen's disease } \\
\text { Abstract }\end{array}$ & $\begin{array}{l}\text { British } \\
\text { journal of } \\
\text { dermatolo } \\
\text { gy }\end{array}$ & $\begin{array}{l}\text { duplicate/conference } \\
\text { abstract and we have full } \\
\text { publication }\end{array}$ \\
\hline 25354233 & Samain, A. & $\begin{array}{l}\text { Cryosurgery and curettage-cryosurgery for } \\
\text { basal cell carcinomas of the mid-face }\end{array}$ & $\begin{array}{l}\text { J Eur } \\
\text { Acad } \\
\text { Dermatol } \\
\text { Venereol }\end{array}$ & $\begin{array}{l}\text { not comparative between } \\
\text { treatment nodes }\end{array}$ \\
\hline
\end{tabular}




\begin{tabular}{|c|c|c|c|c|}
\hline UID & First Author & Title & Journal & $\begin{array}{l}\text { Reason for } \\
\text { Exclusion }\end{array}$ \\
\hline 25136458 & $\begin{array}{l}\text { Samstein, R. } \\
\text { M. }\end{array}$ & $\begin{array}{l}\text { Locally advanced and unresectable cutaneous } \\
\text { squamous cell carcinoma: outcomes of } \\
\text { concurrent cetuximab and radiotherapy }\end{array}$ & $\begin{array}{l}\text { J Skin } \\
\text { Cancer }\end{array}$ & $\begin{array}{l}\text { not treatment of skin } \\
\text { cancer or }<80 \% \text { SCC or } \\
\text { BCC }\end{array}$ \\
\hline 25030404 & Samy, N. A. & $\begin{array}{l}\text { Effect of methylene blue-mediated } \\
\text { photodynamic therapy for treatment of basal cell } \\
\text { carcinoma }\end{array}$ & $\begin{array}{l}\text { Lasers } \\
\text { Med Sci }\end{array}$ & $\begin{array}{l}\text { not comparative between } \\
\text { treatment nodes }\end{array}$ \\
\hline 8720817 & $\begin{array}{l}\text { Scholten, A. } \\
\text { N. }\end{array}$ & $\begin{array}{l}\text { [Electron beam irradiation is effective in the } \\
\text { treatment of skin carcinomas; a comparison } \\
\text { with superficial roentgen therapy] }\end{array}$ & $\begin{array}{l}\text { Ned } \\
\text { Tijdschr } \\
\text { Geneeskd }\end{array}$ & $\begin{array}{l}\text { No analysis by } \\
\text { population of interest }\end{array}$ \\
\hline 16310060 & Schulte, K. W. & $\begin{array}{l}\text { Soft x-ray therapy for cutaneous basal cell and } \\
\text { squamous cell carcinomas }\end{array}$ & $\begin{array}{l}\text { J Am } \\
\text { Acad } \\
\text { Dermatol }\end{array}$ & $\begin{array}{l}\text { not comparative between } \\
\text { treatment nodes }\end{array}$ \\
\hline 26449347 & Schulze, B. & $\begin{array}{l}\text { Hedgehog pathway inhibitor in combination with } \\
\text { radiation therapy for basal cell carcinomas of } \\
\text { the head and neck : First clinical experience } \\
\text { with vismodegib for locally advanced disease }\end{array}$ & $\begin{array}{l}\text { Strahlenth } \\
\text { er Onkol }\end{array}$ & $\begin{array}{l}\text { not comparative between } \\
\text { treatment nodes }\end{array}$ \\
\hline 11383121 & Schwager, K. & $\begin{array}{l}\text { [Carcinoma of the external ear canal and middle } \\
\text { ear as interdisciplinary challenge for ear surgery } \\
\text { and radiotherapy] }\end{array}$ & $\begin{array}{l}\text { Laryngorhi } \\
\text { nootologie }\end{array}$ & $\begin{array}{l}\text { No analysis by } \\
\text { population of interest }\end{array}$ \\
\hline 27110895 & $\begin{array}{l}\text { Sebaratnam, } \\
\text { D. F. }\end{array}$ & $\begin{array}{l}\text { Direct Cost-Analysis of Mohs Micrographic } \\
\text { Surgery and Traditional Excision for Basal Cell } \\
\text { Carcinoma at Initial Margin Clearance }\end{array}$ & $\begin{array}{l}\text { Dermatol } \\
\text { Surg }\end{array}$ & $\begin{array}{l}\text { No analysis by } \\
\text { population of interest }\end{array}$ \\
\hline 20946582 & Segura, S. & $\begin{array}{l}\text { Non-invasive management of non-melanoma } \\
\text { skin cancer in patients with cancer } \\
\text { predisposition genodermatosis: a role for } \\
\text { confocal microscopy and photodynamic therapy }\end{array}$ & $\begin{array}{l}\text { J Eur } \\
\text { Acad } \\
\text { Dermatol } \\
\text { Venereol }\end{array}$ & $\begin{array}{l}>20 \% \text { recurrent or } \% \\
\text { recurrent not given }\end{array}$ \\
\hline 19737291 & Seidler, A. M. & $\begin{array}{l}\text { Mohs versus traditional surgical excision for } \\
\text { facial and auricular nonmelanoma skin cancer: } \\
\text { an analysis of cost-effectiveness }\end{array}$ & $\begin{array}{l}\text { Dermatol } \\
\text { Surg }\end{array}$ & $\begin{array}{l}\text { not comparative between } \\
\text { treatment nodes }\end{array}$ \\
\hline 22670903 & $\begin{array}{l}\text { Sekulic, } \\
\text { Aleksandar, et } \\
\text { al. }\end{array}$ & $\begin{array}{l}\text { "Efficacy and safety of vismodegib in advanced } \\
\text { basal-cell carcinoma." }\end{array}$ & $\begin{array}{l}\text { New } \\
\text { England } \\
\text { Journal of } \\
\text { Medicine } \\
366.23 \\
(2012) \text { : } \\
2171- \\
2179 \text {. }\end{array}$ & $\begin{array}{l}\text { not comparative between } \\
\text { treatment nodes }\end{array}$ \\
\hline 11786562 & Shin, D. M. & $\begin{array}{l}\text { Phase II and biologic study of interferon alfa, } \\
\text { retinoic acid, and cisplatin in advanced } \\
\text { squamous skin cancer }\end{array}$ & $\begin{array}{l}\text { J Clin } \\
\text { Oncol }\end{array}$ & $\begin{array}{l}>20 \% \text { recurrent or } \% \\
\text { recurrent not given }\end{array}$ \\
\hline $\begin{array}{l}\text { CN- } \\
00261581\end{array}$ & $\begin{array}{l}\text { Shuttleworth, } \\
\text { D. }\end{array}$ & $\begin{array}{l}\text { A comparison of the effects of intralesional } \\
\text { interferon alpha-2b and topical 5\% 5-fluorouracil } \\
\text { cream in the treatment of solar keratoses and } \\
\text { Bowen's disease }\end{array}$ & $\begin{array}{l}\text { Journal of } \\
\text { dermatolo } \\
\text { gical } \\
\text { treatment }\end{array}$ & $\begin{array}{l}\text { not treatment of skin } \\
\text { cancer or }<80 \% \text { SCC or } \\
\text { BCC }\end{array}$ \\
\hline 10802373 & Silva, J. J. & $\begin{array}{l}\text { Results of radiotherapy for epithelial skin cancer } \\
\text { of the pinna: the Princess Margaret Hospital } \\
\text { experience, 1982-1993 }\end{array}$ & $\begin{array}{l}\text { Int J } \\
\text { Radiat } \\
\text { Oncol Biol } \\
\text { Phys } \\
\end{array}$ & $\begin{array}{l}\text { No analysis by } \\
\text { population of interest }\end{array}$ \\
\hline 1890243 & $\begin{array}{l}\text { Silverman, M. } \\
\mathrm{K} \text {. }\end{array}$ & $\begin{array}{l}\text { Recurrence rates of treated basal cell } \\
\text { carcinomas. Part 1: Overview }\end{array}$ & $\begin{array}{l}\text { J } \\
\text { Dermatol } \\
\text { Surg } \\
\text { Oncol }\end{array}$ & $\begin{array}{l}\text { not comparative between } \\
\text { treatment nodes }\end{array}$ \\
\hline 1624628 & $\begin{array}{l}\text { Silverman, M. } \\
\mathrm{K} \text {. }\end{array}$ & $\begin{array}{l}\text { Recurrence rates of treated basal cell } \\
\text { carcinomas. Part } 4: \text { X-ray therapy }\end{array}$ & $\begin{array}{l}\text { J } \\
\text { Dermatol } \\
\text { Surg } \\
\text { Oncol }\end{array}$ & $\begin{array}{l}\text { not comparative between } \\
\text { treatment nodes }\end{array}$ \\
\hline 18306163 & Smucler, R. & $\begin{array}{l}\text { Combination of Er:YAG laser and photodynamic } \\
\text { therapy in the treatment of nodular basal cell } \\
\text { carcinoma }\end{array}$ & $\begin{array}{l}\text { Lasers } \\
\text { Surg Med }\end{array}$ & $\begin{array}{l}>20 \% \text { recurrent or } \% \\
\text { recurrent not given }\end{array}$ \\
\hline
\end{tabular}




\begin{tabular}{|c|c|c|c|c|}
\hline UID & First Author & Title & Journal & $\begin{array}{l}\text { Reason for } \\
\text { Exclusion }\end{array}$ \\
\hline 1622958 & Smyth, A. G. & $\begin{array}{l}\text { A prospective study of } 134 \text { consecutive patients } \\
\text { requiring diagnosis, excision and repair of a } \\
\text { facial cutaneous lesion }\end{array}$ & $\begin{array}{l}\text { Br J Oral } \\
\text { Maxillofac } \\
\text { Surg }\end{array}$ & $\begin{array}{l}\text { not comparative between } \\
\text { treatment nodes }\end{array}$ \\
\hline 11093368 & Soler, A. M. & $\begin{array}{l}\text { Photodynamic therapy of residual or recurrent } \\
\text { basal cell carcinoma after radiotherapy using } \\
\text { topical 5-aminolevulinic acid or methylester } \\
\text { aminolevulinic acid }\end{array}$ & $\begin{array}{l}\text { Acta } \\
\text { Oncol }\end{array}$ & $\begin{array}{l}>20 \% \text { recurrent or } \% \\
\text { recurrent not given }\end{array}$ \\
\hline 10857368 & Soler, A. M. & $\begin{array}{l}\text { Photodynamic therapy of superficial basal cell } \\
\text { carcinoma with 5-aminolevulinic acid with } \\
\text { dimethylsulfoxide and ethylendiaminetetraacetic } \\
\text { acid: a comparison of two light sources }\end{array}$ & $\begin{array}{l}\text { Photoche } \\
\text { m } \\
\text { Photobiol }\end{array}$ & $\begin{array}{l}\text { not comparative between } \\
\text { treatment nodes }\end{array}$ \\
\hline 11531838 & Soler, A. M. & $\begin{array}{l}\text { A follow-up study of recurrence and cosmesis in } \\
\text { completely responding superficial and nodular } \\
\text { basal cell carcinomas treated with methyl 5- } \\
\text { aminolaevulinate-based photodynamic therapy } \\
\text { alone and with prior curettage }\end{array}$ & $\begin{array}{l}\mathrm{Br} \mathrm{J} \\
\text { Dermatol }\end{array}$ & $\begin{array}{l}\text { not comparative between } \\
\text { treatment nodes }\end{array}$ \\
\hline 16230937 & Soriano, E. & $\begin{array}{l}\text { [Course and prognosis of basaloid squamous } \\
\text { cell carcinoma: case-control study of } 49 \\
\text { patients] }\end{array}$ & $\begin{array}{l}\text { Ann } \\
\text { Otolaryng } \\
\text { ol Chir } \\
\text { Cervicofac }\end{array}$ & $\begin{array}{l}\text { not comparative between } \\
\text { treatment nodes }\end{array}$ \\
\hline 24754529 & Sotiriou, E. & $\begin{array}{l}\text { Photodynamic therapy vs. imiquimod } 5 \% \text { cream } \\
\text { as skin cancer preventive strategies in patients } \\
\text { with field changes: a randomized intraindividual } \\
\text { comparison study }\end{array}$ & $\begin{array}{l}\text { J Eur } \\
\text { Acad } \\
\text { Dermatol } \\
\text { Venereol }\end{array}$ & $\begin{array}{l}\text { No analysis by } \\
\text { population of interest }\end{array}$ \\
\hline 17020898 & Soysal, H. G. & $\begin{array}{l}\text { Invasive squamous cell carcinoma of the } \\
\text { eyelids and periorbital region }\end{array}$ & $\begin{array}{l}\text { Br J } \\
\text { Ophthalm } \\
\text { ol }\end{array}$ & $\begin{array}{l}>20 \% \text { recurrent or } \% \\
\text { recurrent not given }\end{array}$ \\
\hline 18520835 & Soysal, H. G. & $\begin{array}{l}\text { Basal cell carcinoma of the eyelids and } \\
\text { periorbital region in a Turkish population }\end{array}$ & $\begin{array}{l}\text { Ophthal } \\
\text { Plast } \\
\text { Reconstr } \\
\text { Surg }\end{array}$ & $\begin{array}{l}>20 \% \text { recurrent or } \% \\
\text { recurrent not given }\end{array}$ \\
\hline $\begin{array}{l}\text { CN- } \\
01059263\end{array}$ & Spelman, L. & $\begin{array}{l}\text { Ingenol mebutate } 0.05 \% \text { gel with full occlusion } \\
\text { effectively treats sBCC }\end{array}$ & $\begin{array}{l}\text { JDDG - } \\
\text { Journal of } \\
\text { the } \\
\text { German } \\
\text { Society of } \\
\text { Dermatolo } \\
\text { gy }\end{array}$ & $\begin{array}{l}\text { not comparative between } \\
\text { treatment nodes }\end{array}$ \\
\hline 19839887 & Stafanous, S. & $\begin{array}{l}\text { Five-year cycle of basal cell carcinoma } \\
\text { management re-audit }\end{array}$ & Orbit & $\begin{array}{l}>20 \% \text { recurrent or } \% \\
\text { recurrent not given }\end{array}$ \\
\hline 19639112 & $\begin{array}{l}\text { Steinbauer, J. } \\
\text { M. }\end{array}$ & $\begin{array}{l}\text { Topical photodynamic therapy with porphyrin } \\
\text { precursors--assessment of treatment- } \\
\text { associated pain in a retrospective study }\end{array}$ & $\begin{array}{l}\text { Photoche } \\
\text { m } \\
\text { Photobiol } \\
\text { Sci }\end{array}$ & $\begin{array}{l}\text { not treatment of skin } \\
\text { cancer or }<80 \% \text { SCC or } \\
\text { BCC }\end{array}$ \\
\hline 6709010 & Stern, R. S. & $\begin{array}{l}\text { Cutaneous squamous-cell carcinoma in patients } \\
\text { treated with PUVA }\end{array}$ & $\begin{array}{l}\text { N Engl J } \\
\text { Med }\end{array}$ & $\begin{array}{l}\text { not comparative between } \\
\text { treatment nodes }\end{array}$ \\
\hline 22494856 & Stockfleth, E. & $\begin{array}{l}\text { Recurrence rates and patient assessed } \\
\text { outcomes of } 0.5 \% 5 \text {-fluorouracil in combination } \\
\text { with salicylic acid treating actinic keratoses }\end{array}$ & $\begin{array}{l}\text { Eur J } \\
\text { Dermatol }\end{array}$ & $\begin{array}{l}\text { not treatment of skin } \\
\text { cancer or }<80 \% \text { SCC or } \\
\text { BCC }\end{array}$ \\
\hline 16650155 & Streeton, C. L. & $\begin{array}{l}\text { Treatment of basal cell carcinomas by general } \\
\text { practitioners in Australia }\end{array}$ & $\begin{array}{l}\text { Int J } \\
\text { Dermatol }\end{array}$ & $\begin{array}{l}\text { not comparative between } \\
\text { treatment nodes }\end{array}$ \\
\hline 6556694 & $\begin{array}{l}\text { Swanson, N. } \\
\text { A. }\end{array}$ & $\begin{array}{l}\text { Basal cell carcinoma. Treatment modalities and } \\
\text { recommendations }\end{array}$ & Prim Care & no primary data \\
\hline 4088894 & Szymczyk, W. & $\begin{array}{l}\text { [Effect of dose fractionation on } 3 \text { years results } \\
\text { of roentgenotherapy of skin cancer] }\end{array}$ & $\begin{array}{l}\text { Nowotwor } \\
\mathrm{y}\end{array}$ & Not English (Polish) \\
\hline 17322605 & Taherian, $\mathrm{K}$. & $\begin{array}{l}\text { Surgical excision of periocular basal cell } \\
\text { carcinomas }\end{array}$ & $\begin{array}{l}\text { Indian J } \\
\text { Ophthalm } \\
\text { ol }\end{array}$ & $\begin{array}{l}\text { not comparative between } \\
\text { treatment nodes }\end{array}$ \\
\hline
\end{tabular}




\begin{tabular}{|c|c|c|c|c|}
\hline UID & First Author & Title & Journal & $\begin{array}{l}\text { Reason for } \\
\text { Exclusion }\end{array}$ \\
\hline 15061853 & Tan, S. R. & $\begin{array}{l}\text { Effect of acitretin on wound healing in organ } \\
\text { transplant recipients }\end{array}$ & $\begin{array}{l}\text { Dermatol } \\
\text { Surg }\end{array}$ & $\begin{array}{l}\text { not comparative between } \\
\text { treatment nodes }\end{array}$ \\
\hline 22547009 & Tang, C. & $\begin{array}{l}\text { Stereotactic radiosurgery for retreatment of } \\
\text { gross perineural invasion in recurrent cutaneous } \\
\text { squamous cell carcinoma of the head and neck }\end{array}$ & $\begin{array}{l}\text { Am J Clin } \\
\text { Oncol }\end{array}$ & $\begin{array}{l}\text { not comparative between } \\
\text { treatment nodes }\end{array}$ \\
\hline 24441673 & Tang, J. Y. & $\begin{array}{l}\text { Tazarotene: randomized, double-blind, vehicle- } \\
\text { controlled, and open-label concurrent trials for } \\
\text { basal cell carcinoma prevention and therapy in } \\
\text { patients with basal cell nevus syndrome }\end{array}$ & $\begin{array}{l}\text { Cancer } \\
\text { prevention } \\
\text { research } \\
\text { (Philadelp } \\
\text { hia, } \mathrm{Pa} \text {.) } \\
\end{array}$ & $\begin{array}{l}\text { not comparative between } \\
\text { treatment nodes }\end{array}$ \\
\hline 22670904 & Tang, J. Y. & $\begin{array}{l}\text { Inhibiting the hedgehog pathway in patients with } \\
\text { the basal-cell nevus syndrome }\end{array}$ & $\begin{array}{l}\text { N Engl J } \\
\text { Med }\end{array}$ & $\begin{array}{l}\text { not treatment of skin } \\
\text { cancer or }<80 \% \text { SCC or } \\
\text { BCC }\end{array}$ \\
\hline 24623654 & $\begin{array}{l}\text { Tanvetyanon, } \\
\text { Tawee, et al. }\end{array}$ & $\begin{array}{l}\text { "Postoperative concurrent chemotherapy and } \\
\text { radiotherapy for high-risk cutaneous squamous } \\
\text { cell carcinoma of the head and neck." }\end{array}$ & $\begin{array}{l}\text { Head \& } \\
\text { neck 37.6 } \\
(2015): \\
840-845 . \\
\end{array}$ & $\begin{array}{l}>20 \% \text { metastatic/nodal } \\
\text { involvement }\end{array}$ \\
\hline 1080961 & Tarpley, J. L. & $\begin{array}{l}\text { High dose methotrexate as a preoperative } \\
\text { adjuvant in the treatment of epidermoid } \\
\text { carcinoma of the head and neck. A feasibility } \\
\text { study and clinical trial }\end{array}$ & Am J Surg & $\begin{array}{l}\text { not treatment of skin } \\
\text { cancer or }<80 \% \text { SCC or } \\
\text { BCC }\end{array}$ \\
\hline 20101335 & Teli, M. A. & $\begin{array}{l}\text { Recurrence pattern in squamous cell carcinoma } \\
\text { of skin of lower extremities and abdominal wall } \\
\text { (Kangri cancer) in Kashmir valley of Indian } \\
\text { subcontinent: impact of various treatment } \\
\text { modalities }\end{array}$ & $\begin{array}{l}\text { Indian } \mathrm{J} \\
\text { Dermatol }\end{array}$ & $\begin{array}{l}>20 \% \text { recurrent or } \% \\
\text { recurrent not given }\end{array}$ \\
\hline 12832877 & Thomas, D. J. & Excision margins for nonmelanotic skin cancer & $\begin{array}{l}\text { Plast } \\
\text { Reconstr } \\
\text { Surg }\end{array}$ & $\begin{array}{l}\text { not comparative between } \\
\text { treatment nodes }\end{array}$ \\
\hline 19852120 & Tierney, E. P. & $\begin{array}{l}\text { Cost effectiveness of Mohs micrographic } \\
\text { surgery: review of the literature }\end{array}$ & $\begin{array}{l}\text { J Drugs } \\
\text { Dermatol }\end{array}$ & no primary data \\
\hline 2090402 & Tijl, J.W. & $\begin{array}{l}\text { The optimal follow-up time for a basal cell } \\
\text { carcinoma of the eyelid }\end{array}$ & $\begin{array}{l}\text { Doc } \\
\text { Ophthalm } \\
\text { ol }\end{array}$ & $\begin{array}{l}>20 \% \text { recurrent or } \% \\
\text { recurrent not given }\end{array}$ \\
\hline 17700732 & $\begin{array}{l}\text { Tindholdt, } \mathrm{T} \text {. } \\
\mathrm{T} \text {. }\end{array}$ & $\begin{array}{l}\text { [Photodynamic therapy of facial basal cell } \\
\text { carcinoma] }\end{array}$ & $\begin{array}{l}\text { Tidsskr } \\
\text { Nor } \\
\text { Laegefore } \\
\mathrm{n}\end{array}$ & $\begin{array}{l}\text { not comparative between } \\
\text { treatment nodes }\end{array}$ \\
\hline 23035730 & Tinelli, M. & $\begin{array}{l}\text { What determines patient preferences for } \\
\text { treating low risk basal cell carcinoma when } \\
\text { comparing surgery vs imiquimod? A discrete } \\
\text { choice experiment survey from the SINS trial }\end{array}$ & $\begin{array}{l}\text { BMC } \\
\text { Dermatol }\end{array}$ & $\begin{array}{l}\text { not treatment of skin } \\
\text { cancer or }<80 \% \text { SCC or } \\
\text { BCC }\end{array}$ \\
\hline 24975199 & $\begin{array}{l}\text { Togsverd-Bo, } \\
\text { Katrine, et al. }\end{array}$ & $\begin{array}{l}\text { "Combination of ablative fractional laser and } \\
\text { daylight-mediated photodynamic therapy for } \\
\text { actinic keratosis in organ transplant recipients-a } \\
\text { randomized controlled trial." }\end{array}$ & $\begin{array}{l}\text { British } \\
\text { Journal of } \\
\text { Dermatolo } \\
\text { gy } 172.2 \\
\text { (2015): } \\
467-474 . \\
\end{array}$ & $\begin{array}{l}\text { not treatment of skin } \\
\text { cancer or }<80 \% \text { SCC or } \\
\text { BCC }\end{array}$ \\
\hline 9487802 & Tope, W. D. & $\begin{array}{l}\text { Protoporphyrin IX fluorescence induced in basal } \\
\text { cell carcinoma by oral delta-aminolevulinic acid }\end{array}$ & $\begin{array}{l}\text { Photoche } \\
\text { m } \\
\text { Photobiol }\end{array}$ & no outcomes of interest \\
\hline $\begin{array}{l}\mathrm{CN}- \\
00454732\end{array}$ & Torres, A. & $\begin{array}{l}\text { Imiquimod 5\% cream preceeding surgery for } \\
\text { BCC monitoring with confocal microscopy }\end{array}$ & $\begin{array}{l}\text { Annales } \\
\text { de } \\
\text { Dermatolo } \\
\text { gie Et de } \\
\text { Venereolo } \\
\text { gie }\end{array}$ & $\begin{array}{l}\text { duplicate/conference } \\
\text { abstract and we have full } \\
\text { publication }\end{array}$ \\
\hline $\begin{array}{l}\text { CN- } \\
00478784\end{array}$ & Torres, A. & $\begin{array}{l}\text { Treatment of basal cell carcinoma using } \\
\text { imiquimod } 5 \% \text { cream as an adjuvant therapy to }\end{array}$ & $\begin{array}{l}\text { Journal of } \\
\text { the }\end{array}$ & $\begin{array}{l}\text { duplicate/conference } \\
\text { abstract and we have full }\end{array}$ \\
\hline
\end{tabular}




\begin{tabular}{|c|c|c|c|c|}
\hline UID & First Author & Title & Journal & $\begin{array}{l}\text { Reason for } \\
\text { Exclusion }\end{array}$ \\
\hline & & $\begin{array}{l}\text { Mohs micrographic surgery. Abstract P5-19 The } \\
\text { 12th Congress of the European Academy of } \\
\text { Dermatology and Venereology. Barcelona, } \\
\text { Spain 15-18th October } 2003\end{array}$ & $\begin{array}{l}\text { European } \\
\text { Academy } \\
\text { of } \\
\text { Dermatolo } \\
\text { gy and } \\
\text { Venereolo } \\
\text { gy : } \\
\text { JEADV }\end{array}$ & publication \\
\hline 16984216 & $\begin{array}{l}\text { Triesscheijn, } \\
\text { M. }\end{array}$ & $\begin{array}{l}\text { Optimizing meso-tetra-hydroxyphenyl-chlorin- } \\
\text { mediated photodynamic therapy for basal cell } \\
\text { carcinoma }\end{array}$ & $\begin{array}{l}\text { Photoche } \\
\text { m } \\
\text { Photobiol }\end{array}$ & $\begin{array}{l}\text { not comparative between } \\
\text { treatment nodes }\end{array}$ \\
\hline 2015753178 & Trone, J. C. & $\begin{array}{l}\text { Skin Cancers in Nonagenarian Patients: Special } \\
\text { Focus on Radiotherapy }\end{array}$ & $\begin{array}{l}\text { Clinical } \\
\text { Oncology }\end{array}$ & $\begin{array}{l}\text { not comparative between } \\
\text { treatment nodes }\end{array}$ \\
\hline 11958891 & Tsao, M. N. & $\begin{array}{l}\text { Radiotherapy management for squamous cell } \\
\text { carcinoma of the nasal skin: the Princess } \\
\text { Margaret Hospital experience }\end{array}$ & $\begin{array}{l}\text { Int J } \\
\text { Radiat } \\
\text { Oncol Biol } \\
\text { Phys }\end{array}$ & $\begin{array}{l}\text { not comparative between } \\
\text { treatment nodes }\end{array}$ \\
\hline 20033810 & Tsukuda, M. & $\begin{array}{l}\text { Randomized controlled phase II comparison } \\
\text { study of concurrent chemoradiotherapy with } \\
\text { docetaxel, cisplatin, and 5-fluorouracil versus } \\
\text { CCRT with cisplatin, 5-fluorouracil, } \\
\text { methotrexate and leucovorin in patients with } \\
\text { locally advanced squamous cell carcinoma of } \\
\text { the head and neck }\end{array}$ & $\begin{array}{l}\text { Cancer } \\
\text { Chemothe } \\
\text { r } \\
\text { Pharmacol }\end{array}$ & $\begin{array}{l}\text { not treatment of skin } \\
\text { cancer or }<80 \% \text { SCC or } \\
\text { BCC }\end{array}$ \\
\hline 24397256 & Tuerdi, M. & $\begin{array}{l}\text { Standard surgical excision and reconstruction of } \\
\text { giant basal cell carcinoma of the face: may be } \\
\text { an alternative to the Mohs micrographic surgery }\end{array}$ & $\begin{array}{l}\text { Journal of } \\
\text { the } \\
\text { European } \\
\text { Academy } \\
\text { of } \\
\text { Dermatolo } \\
\text { gy and } \\
\text { Venereolo } \\
\text { gy: } \\
\text { JEADV }\end{array}$ & $\begin{array}{l}\text { not comparative between } \\
\text { treatment nodes }\end{array}$ \\
\hline 10219440 & Tufano, R. P. & $\begin{array}{l}\text { Malignant tumors of the nose and paranasal } \\
\text { sinuses: hospital of the University of } \\
\text { Pennsylvania experience 1990-1997 }\end{array}$ & $\begin{array}{l}\text { Am J } \\
\text { Rhinol }\end{array}$ & $\begin{array}{l}\text { not treatment of skin } \\
\text { cancer or }<80 \% \text { SCC or } \\
\text { BCC }\end{array}$ \\
\hline 21277787 & Tyrrell, J. & $\begin{array}{l}\text { The effect of air cooling pain relief on } \\
\text { protoporphyrin IX photobleaching and clinical } \\
\text { efficacy during dermatological photodynamic } \\
\text { therapy }\end{array}$ & $\begin{array}{l}\text { J } \\
\text { Photoche } \\
\text { m } \\
\text { Photobiol } \\
\text { B }\end{array}$ & $\begin{array}{l}\text { not comparative between } \\
\text { treatment nodes }\end{array}$ \\
\hline 19881375 & Unlu, R. E. & $\begin{array}{l}\text { Is it really necessary to make wide excisions for } \\
\text { basal cell carcinoma treatment? }\end{array}$ & $\begin{array}{l}\text { J } \\
\text { Craniofac } \\
\text { Surg }\end{array}$ & $\begin{array}{l}\text { not comparative between } \\
\text { treatment nodes }\end{array}$ \\
\hline 2218385 & Vaillant, L. & $\begin{array}{l}\text { [Skin carcinoma of the face: surgery or } \\
\text { radiotherapy?] }\end{array}$ & $\begin{array}{l}\text { Rev } \\
\text { Stomatol } \\
\text { Chir } \\
\text { Maxillofac }\end{array}$ & Not English (French) \\
\hline 22170313 & $\begin{array}{l}\text { van der Beek, } \\
\text { N. }\end{array}$ & $\begin{array}{l}\text { PpIX fluorescence combined with auto- } \\
\text { fluorescence is more accurate than PpIX } \\
\text { fluorescence alone in fluorescence detection of } \\
\text { non-melanoma skin cancer: an intra-patient } \\
\text { direct comparison study }\end{array}$ & $\begin{array}{l}\text { Lasers } \\
\text { Surg Med }\end{array}$ & $\begin{array}{l}\text { not treatment of skin } \\
\text { cancer or }<80 \% \text { SCC or } \\
\text { BCC }\end{array}$ \\
\hline 21046543 & $\begin{array}{l}\text { van der } \\
\text { Eerden, P. A. }\end{array}$ & $\begin{array}{l}\text { Eighteen years of experience in Mohs } \\
\text { micrographic surgery and conventional excision } \\
\text { for nonmelanoma skin cancer treated by a } \\
\text { single facial plastic surgeon and pathologist }\end{array}$ & $\begin{array}{l}\text { Laryngosc } \\
\text { ope }\end{array}$ & $\begin{array}{l}>20 \% \text { recurrent or } \% \\
\text { recurrent not given }\end{array}$ \\
\hline 11494691 & van der Meer, & [Low 5-year recurrence rate after surgical & Ned & Not English (Dutch) \\
\hline
\end{tabular}




\begin{tabular}{|c|c|c|c|c|}
\hline UID & First Author & Title & Journal & $\begin{array}{l}\text { Reason for } \\
\text { Exclusion }\end{array}$ \\
\hline & G. T. & $\begin{array}{l}\text { excision of } 126 \text { basal cell carcinomas with } \\
\text { frozen section analysis upon indication] }\end{array}$ & $\begin{array}{l}\text { Tijdschr } \\
\text { Geneeskd }\end{array}$ & \\
\hline 25049028 & $\begin{array}{l}\text { van Oosten, } \\
\text { E. J. }\end{array}$ & $\begin{array}{l}\text { Different pain sensations in photodynamic } \\
\text { therapy of nodular basal cell carcinoma Results } \\
\text { from a prospective trial and a review of the } \\
\text { literature }\end{array}$ & $\begin{array}{l}\text { Photodiag } \\
\text { nosis } \\
\text { Photodyn } \\
\text { Ther }\end{array}$ & $\begin{array}{l}\text { not comparative between } \\
\text { treatment nodes }\end{array}$ \\
\hline 2001338148 & $\begin{array}{l}\text { Van Zuuren, } \\
\text { E. J. }\end{array}$ & $\begin{array}{l}\text { Basal cell carcinoma on the dorsum of the } \\
\text { hand: Report of } 11 \text { cases }\end{array}$ & $\begin{array}{l}\text { Journal of } \\
\text { the } \\
\text { European } \\
\text { Academy } \\
\text { of } \\
\text { Dermatolo } \\
\text { gy and } \\
\text { Venereolo } \\
\text { gy }\end{array}$ & $\begin{array}{l}\text { not comparative between } \\
\text { treatment nodes }\end{array}$ \\
\hline 9012035 & Veien, K. & $\begin{array}{l}\text { [Results of treatment of non-melanoma skin } \\
\text { cancer in a dermatologic practice. A prospective } \\
\text { study] }\end{array}$ & $\begin{array}{l}\text { Ugeskr } \\
\text { Laeger }\end{array}$ & $\begin{array}{l}\text { not comparative between } \\
\text { treatment nodes }\end{array}$ \\
\hline 21707774 & Veronese, F. & $\begin{array}{l}\text { Basal cell carcinoma of the head region: } \\
\text { therapeutical results of } 350 \text { lesions treated with } \\
\text { Mohs micrographic surgery }\end{array}$ & $\begin{array}{l}\text { J Eur } \\
\text { Acad } \\
\text { Dermatol } \\
\text { Venereol }\end{array}$ & $\begin{array}{l}\text { not comparative between } \\
\text { treatment nodes }\end{array}$ \\
\hline 15377354 & Vidal, D. & $\begin{array}{l}\text { Efficacy of imiquimod for the expression of Bcl- } \\
2, \mathrm{Ki} 67, \text { p53 and basal cell carcinoma apoptosis }\end{array}$ & $\begin{array}{l}\mathrm{Br} \mathrm{J} \\
\text { Dermatol }\end{array}$ & no outcomes of interest \\
\hline 15115500 & Vidal, D. & $\begin{array}{l}\text { Efficacy of imiquimod } 5 \% \text { cream for basal cell } \\
\text { carcinoma in transplant patients }\end{array}$ & $\begin{array}{l}\text { Clin Exp } \\
\text { Dermatol }\end{array}$ & $\begin{array}{l}\text { not comparative between } \\
\text { treatment nodes }\end{array}$ \\
\hline 15347339 & Vidal, D. & $\begin{array}{l}\text { Open study of the efficacy and mechanism of } \\
\text { action of topical imiquimod in basal cell } \\
\text { carcinoma }\end{array}$ & $\begin{array}{l}\text { Clin Exp } \\
\text { Dermatol }\end{array}$ & $\begin{array}{l}\text { not comparative between } \\
\text { treatment nodes }\end{array}$ \\
\hline 17310012 & $\begin{array}{l}\text { Vidal, David, } \\
\text { Xavier Matías- } \\
\text { Guiu, and } \\
\text { Agustín } \\
\text { Alomar. }\end{array}$ & $\begin{array}{l}\text { "Fifty-five basal cell carcinomas treated with } \\
\text { topical imiquimod: outcome at 5-year follow-up." }\end{array}$ & $\begin{array}{l}\text { Archives } \\
\text { of } \\
\text { dermatolo } \\
\text { gy } 143.2 \\
(2007) \text { : } \\
264-276 . \\
\end{array}$ & $\begin{array}{l}\text { not comparative between } \\
\text { treatment nodes }\end{array}$ \\
\hline 22508870 & Viola, K. V. & $\begin{array}{l}\text { Mohs micrographic surgery and surgical } \\
\text { excision for nonmelanoma skin cancer } \\
\text { treatment in the Medicare population }\end{array}$ & $\begin{array}{l}\text { Arch } \\
\text { Dermatol }\end{array}$ & no outcomes of interest \\
\hline 19726763 & $\begin{array}{l}\text { Von Hoff, } \\
\text { Daniel D., et } \\
\text { al. }\end{array}$ & $\begin{array}{l}\text { "Inhibition of the hedgehog pathway in } \\
\text { advanced basal-cell carcinoma." }\end{array}$ & $\begin{array}{l}\text { New } \\
\text { England } \\
\text { Journal of } \\
\text { Medicine } \\
361.12 \\
(2009) \text { : } \\
1164- \\
1172 .\end{array}$ & $\begin{array}{l}\text { not comparative between } \\
\text { treatment nodes }\end{array}$ \\
\hline 25925162 & $\begin{array}{l}\text { Waalboer- } \\
\text { Spuij, R. }\end{array}$ & $\begin{array}{l}\text { Patient Perception of Imiquimod Treatment for } \\
\text { Actinic Keratosis and Superficial Basal Cell } \\
\text { Carcinoma in } 202 \text { Patients }\end{array}$ & $\begin{array}{l}\text { Dermatolo } \\
\text { gy }\end{array}$ & $\begin{array}{l}\text { not comparative between } \\
\text { treatment nodes }\end{array}$ \\
\hline 25865716 & Wang, L. & $\begin{array}{l}\text { Outcomes of Primary Squamous Cell } \\
\text { Carcinoma of Major Salivary Glands Treated by } \\
\text { Surgery With or Without Postoperative } \\
\text { Radiotherapy }\end{array}$ & $\begin{array}{l}\text { J Oral } \\
\text { Maxillofac } \\
\text { Surg }\end{array}$ & $\begin{array}{l}\text { not treatment of skin } \\
\text { cancer or }<80 \% \text { SCC or } \\
\text { BCC }\end{array}$ \\
\hline $\begin{array}{l}\text { CN- } \\
01027501\end{array}$ & $\begin{array}{l}\text { Weinstock, M. } \\
\text { A. }\end{array}$ & $\begin{array}{l}\text { The veterans affairs topical tretinoin } \\
\text { chemoprevention (VATTC) trial }\end{array}$ & $\begin{array}{l}\text { British } \\
\text { journal of } \\
\text { dermatolo } \\
\text { gy }\end{array}$ & $\begin{array}{l}\text { not treatment of skin } \\
\text { cancer or }<80 \% \text { SCC or } \\
\text { BCC }\end{array}$ \\
\hline $\begin{array}{l}\mathrm{CN}- \\
00178586\end{array}$ & $\begin{array}{l}\text { Weissberg, J. } \\
\text { B. }\end{array}$ & $\begin{array}{l}\text { Radiation therapy (RT) and mitomycin C (MC) } \\
\text { in the treatment of head and neck cancer: }\end{array}$ & $\begin{array}{l}\text { Proc-Am- } \\
\text { Assoc- }\end{array}$ & $\begin{array}{l}\text { not treatment of skin } \\
\text { cancer or }<80 \% \text { SCC or }\end{array}$ \\
\hline
\end{tabular}




\begin{tabular}{|c|c|c|c|c|}
\hline UID & First Author & Title & Journal & $\begin{array}{l}\text { Reason for } \\
\text { Exclusion }\end{array}$ \\
\hline & & Prospective randomized trial & $\begin{array}{l}\text { Cancer- } \\
\text { Res }\end{array}$ & $\mathrm{BCC}$ \\
\hline 18698246 & $\begin{array}{l}\text { Wennberg, A. } \\
\text { M. }\end{array}$ & $\begin{array}{l}\text { Photodynamic therapy with methyl } \\
\text { aminolevulinate for prevention of new skin } \\
\text { lesions in transplant recipients: a randomized } \\
\text { study }\end{array}$ & $\begin{array}{l}\text { Transplant } \\
\text { ation }\end{array}$ & $\begin{array}{l}\text { not treatment of skin } \\
\text { cancer or }<80 \% \text { SCC or } \\
\text { BCC }\end{array}$ \\
\hline $\begin{array}{l}\mathrm{CN}- \\
00602278\end{array}$ & $\begin{array}{l}\text { Wennberg, } \\
\text { AM }\end{array}$ & $\begin{array}{l}\text { Results from a 15-month update of a } \\
\text { multicentre study of methyl aminolaevulinate } \\
\text { photodynamic therapy in immunocompromised } \\
\text { organ transplant recipients with nonmelanoma } \\
\text { skin cancer. Abstract P-79. British Association } \\
\text { of Dermatologists 86th Annual Meeting }\end{array}$ & $\begin{array}{l}\text { British } \\
\text { journal of } \\
\text { dermatolo } \\
\text { gy }\end{array}$ & $\begin{array}{l}\text { duplicate/conference } \\
\text { abstract and we have full } \\
\text { publication }\end{array}$ \\
\hline 5587022 & $\begin{array}{l}\text { Wernsdorfer, } \\
\text { R. }\end{array}$ & $\begin{array}{l}\text { [Carcinomas of the external ear. Report on } 170 \\
\text { cases] }\end{array}$ & $\begin{array}{l}\text { Z Haut } \\
\text { Geschlech } \\
\text { tskr }\end{array}$ & no primary data \\
\hline 23760141 & White, G. M. & $\begin{array}{l}\text { Biopsy followed by immediate curettage and } \\
\text { electrodesiccation of suspected basal cell } \\
\text { carcinomas at the first visit }\end{array}$ & $\begin{array}{l}\text { JAMA } \\
\text { Dermatol }\end{array}$ & $\begin{array}{l}\text { not comparative between } \\
\text { treatment nodes }\end{array}$ \\
\hline 21219287 & $\begin{array}{l}\text { Wiegell, S. R., } \\
\text { et al. }\end{array}$ & $\begin{array}{l}\text { "A randomized, multicentre study of directed } \\
\text { daylight exposure times of } 1 \frac{11 / 2 \mathrm{Vs} .}{21 / 2 \mathrm{~h} \text { in }} \\
\text { daylight-mediated photodynamic therapy with } \\
\text { methyl aminolaevulinate in patients with multiple } \\
\text { thin actinic keratoses of the face and scalp." }\end{array}$ & $\begin{array}{l}\text { British } \\
\text { Journal of } \\
\text { Dermatolo } \\
\text { gy } 164.5 \\
(2011): \\
1083- \\
1090 .\end{array}$ & $\begin{array}{l}\text { not treatment of skin } \\
\text { cancer or }<80 \% \text { SCC or } \\
\text { BCC }\end{array}$ \\
\hline 18294318 & $\begin{array}{l}\text { Wiegell, S. R., } \\
\text { et al. }\end{array}$ & $\begin{array}{l}\text { "Continuous activation of PpIX by daylight is as } \\
\text { effective as and less painful than conventional } \\
\text { photodynamic therapy for actinic keratoses; a } \\
\text { randomized, controlled, single-blinded study." }\end{array}$ & $\begin{array}{l}\text { British } \\
\text { Journal of } \\
\text { Dermatolo } \\
\text { gy } 158.4 \\
\text { (2008): } \\
740-746 .\end{array}$ & $\begin{array}{l}\text { not treatment of skin } \\
\text { cancer or }<80 \% \text { SCC or } \\
\text { BCC }\end{array}$ \\
\hline 19416257 & $\begin{array}{l}\text { Wiegell, S. R., } \\
\text { et al. }\end{array}$ & $\begin{array}{l}\text { "Photodynamic therapy of actinic keratoses } \\
\text { with } 8 \% \text { and } 16 \% \text { methyl aminolaevulinate and } \\
\text { home-based daylight exposure: a double- } \\
\text { blinded randomized clinical trial." }\end{array}$ & $\begin{array}{l}\text { British } \\
\text { Journal of } \\
\text { Dermatolo } \\
\text { gy160.6 } \\
\text { (2009): } \\
1308- \\
1314 .\end{array}$ & $\begin{array}{l}\text { not treatment of skin } \\
\text { cancer or }<80 \% \text { SCC or } \\
\text { BCC }\end{array}$ \\
\hline 22250644 & $\begin{array}{l}\text { Wiegell, S. R., } \\
\text { et al. }\end{array}$ & $\begin{array}{l}\text { "Daylight-mediated photodynamic therapy of } \\
\text { moderate to thick actinic keratoses of the face } \\
\text { and scalp: a randomized multicentre study." }\end{array}$ & $\begin{array}{l}\text { British } \\
\text { Journal of } \\
\text { Dermatolo } \\
\text { gy } 166.6 \\
(2012): \\
1327- \\
1332 . \\
\end{array}$ & $\begin{array}{l}\text { not treatment of skin } \\
\text { cancer or }<80 \% \text { SCC or } \\
\text { BCC }\end{array}$ \\
\hline 1913451 & Wilder, R. B. & $\begin{array}{l}\text { Basal cell carcinoma treated with radiation } \\
\text { therapy }\end{array}$ & Cancer & $\begin{array}{l}\text { not comparative between } \\
\text { treatment nodes }\end{array}$ \\
\hline $\begin{array}{l}\text { CN- } \\
01007534 \\
\end{array}$ & $\begin{array}{l}\text { Williams, } \mathrm{H} \text {. } \\
\text { C. }\end{array}$ & $\begin{array}{l}\text { Surgical excision versus imiquimod } 5 \% \text { cream } \\
\text { for basal-cell carcinoma (SINS): A multi-centre } \\
\text { non-inferiority randomised controlled trial }\end{array}$ & $\begin{array}{l}\text { Journal of } \\
\text { investigati } \\
\text { ve } \\
\text { dermatolo } \\
\text { gy }\end{array}$ & $\begin{array}{l}\text { duplicate/conference } \\
\text { abstract and we have full } \\
\text { publication }\end{array}$ \\
\hline $\begin{array}{l}\text { CN- } \\
00873112\end{array}$ & $\begin{array}{l}\text { Williams, } \mathrm{H} \text {. } \\
\text { C. }\end{array}$ & $\begin{array}{l}\text { Surgical excision vs. imiquimod 5\% cream for } \\
\text { basal cell carcinoma: A multicentre } \\
\text { noninferiority randomized controlled trial } \\
\text { (Abstract DS03). 93rd Annual Meeting of the } \\
\text { British Association of Dermatologists Liverpool } \\
\text { United Kingdom. Conference Start: } 20130709 \\
\text { Conference End: } 20130711\end{array}$ & $\begin{array}{l}\text { British } \\
\text { journal of } \\
\text { dermatolo } \\
\text { gy }\end{array}$ & $\begin{array}{l}\text { duplicate/conference } \\
\text { abstract and we have full } \\
\text { publication }\end{array}$ \\
\hline
\end{tabular}




\begin{tabular}{|c|c|c|c|c|}
\hline UID & First Author & Title & Journal & $\begin{array}{l}\text { Reason for } \\
\text { Exclusion }\end{array}$ \\
\hline 15225948 & Wilson, A. W. & $\begin{array}{l}\text { Surgical management of incompletely excised } \\
\text { basal cell carcinomas of the head and neck }\end{array}$ & $\begin{array}{l}\text { Br J Oral } \\
\text { Maxillofac } \\
\text { Surg }\end{array}$ & $\begin{array}{l}\text { not comparative between } \\
\text { treatment nodes }\end{array}$ \\
\hline $\begin{array}{l}\mathrm{CN}- \\
00452999\end{array}$ & Woods, R. L. & $\begin{array}{l}\text { Chemotherapy (CT for advanced squamous cell } \\
\text { carcinomas) (SCCS) of head and neck: A } \\
\text { randomised comparison of high dose versus } \\
\text { low dose cis platinum (CIS DDP) in combination } \\
\text { with bleomycin and methotrexate }\end{array}$ & $\begin{array}{l}\text { Proceedin } \\
\text { gs of the } \\
\text { American } \\
\text { Associatio } \\
\text { n for } \\
\text { Cancer } \\
\text { Research, } \\
\text { 75th } \\
\text { Annual } \\
\text { Meeting . } \\
\text { Toronto, } \\
\text { Ontario, 9- } \\
\text { 12 May, } \\
1984\end{array}$ & $\begin{array}{l}\text { not treatment of skin } \\
\text { cancer or }<80 \% \text { SCC or } \\
\text { BCC }\end{array}$ \\
\hline 16431060 & Yin, $M$. & $\begin{array}{l}\text { Analysis of } 95 \text { cases of squamous cell } \\
\text { carcinoma of the external and middle ear }\end{array}$ & $\begin{array}{l}\text { Auris } \\
\text { Nasus } \\
\text { Larynx }\end{array}$ & $\begin{array}{l}\text { not treatment of skin } \\
\text { cancer or }<80 \% \text { SCC or } \\
\text { BCC }\end{array}$ \\
\hline 1587735 & Zablow, A. I. & $\begin{array}{l}\text { Electron beam therapy for skin cancer of the } \\
\text { head and neck }\end{array}$ & $\begin{array}{l}\text { Head } \\
\text { Neck }\end{array}$ & $\begin{array}{l}\text { not comparative between } \\
\text { treatment nodes }\end{array}$ \\
\hline 25393353 & Zeitouni, N. C. & $\begin{array}{l}\text { A prospective study of pain control by a 2-step } \\
\text { irradiance schedule during topical } \\
\text { photodynamic therapy of nonmelanoma skin } \\
\text { cancer }\end{array}$ & $\begin{array}{l}\text { Dermatol } \\
\text { Surg }\end{array}$ & $\begin{array}{l}\text { not comparative between } \\
\text { treatment nodes }\end{array}$ \\
\hline 21055053 & Zhang, Z. X. & $\begin{array}{l}\text { [Clinical analysis of } 60 \text { cases with maxillary } \\
\text { squamous cell carcinoma] }\end{array}$ & $\begin{array}{l}\text { Zhonghua } \\
\text { Er Bi Yan } \\
\text { Hou Tou } \\
\text { Jing Wai } \\
\text { Ke Za Zhi }\end{array}$ & $\begin{array}{l}\text { not treatment of skin } \\
\text { cancer or }<80 \% \text { SCC or } \\
\text { BCC }\end{array}$ \\
\hline 55288 & & $\begin{array}{l}\text { Bleomycin in advanced squamous cell } \\
\text { carcinoma: a random controlled trial. Report of } \\
\text { Medical Research Council Working Party on } \\
\text { Bleomycin }\end{array}$ & Br Med J & $\begin{array}{l}\text { No analysis by } \\
\text { population of interest }\end{array}$ \\
\hline 22777303 & & Vismodegib (Erivedge) for basal cell carcinoma & $\begin{array}{l}\text { Med Lett } \\
\text { Drugs } \\
\text { Ther }\end{array}$ & no primary data \\
\hline
\end{tabular}




\section{Appendix C. Design Details}

Table C-1. Design details

\begin{tabular}{|c|c|c|c|c|c|c|c|}
\hline $\begin{array}{l}\text { Author Year } \\
\text { PMID } \\
\text { (Country/Region) }\end{array}$ & $\begin{array}{l}\text { Number } \\
\text { of } \\
\text { centers }\end{array}$ & Funding & $\begin{array}{l}\text { Inclusion } \\
\text { criteria* }\end{array}$ & Exclusion criteria* & $\begin{array}{l}\text { Method of } \\
\text { diagnosis }\end{array}$ & $\begin{array}{l}\text { Preoperative } \\
\text { tumor size } \\
\text { assessment }\end{array}$ & $\begin{array}{l}\mathrm{N} \text { enrolled } / \\
\text { randomized } / \\
\text { analyzed }\end{array}$ \\
\hline \multicolumn{8}{|l|}{ RCT } \\
\hline $\begin{array}{l}\text { Abbade } 2015 \\
\text { (Conference } \\
\text { abstract) (Brazil) }\end{array}$ & Unclear & Not reported & $\begin{array}{l}\text { primary nodular } \\
\text { BCC in the head and } \\
\text { neck, }<=2 \text { in } \varnothing\end{array}$ & $\begin{array}{l}\text { no histologic confirmation of } \\
\text { nodular BCC, Gorlin } \\
\text { syndrome or contraindication } \\
\text { to surgical resection or PDT. }\end{array}$ & $\begin{array}{l}\text { Biopsy/pathologic } \\
\text { confirmed }\end{array}$ & $\begin{array}{l}\text { Method of } \\
\text { assessment not } \\
\text { reported }\end{array}$ & $\begin{array}{l}92 \text { lesions/68 } \\
\text { lesions/68 } \\
\text { lesions }\end{array}$ \\
\hline $\begin{array}{l}\text { Al-Niaimi } 2015 \\
26157307 \text { (UK) }\end{array}$ & $\begin{array}{l}\text { Single } \\
\text { center }\end{array}$ & Not reported & $\begin{array}{l}>18 \text { y/o, } \mathrm{BCC}>1 \times 1 \\
\mathrm{~cm} 2 \text { requiring } \\
\text { treatment with MMS }\end{array}$ & $\begin{array}{l}\text { morphoeic, infiltrative and } \\
\text { subtypes, a photosensitive } \\
\text { skin disorder, hypersensitivity } \\
\text { to MAL, participation in } \\
\text { another investigational drug } \\
\text { or research study within } 30 \\
\text { days, and females of child- } \\
\text { bearing potential }\end{array}$ & $\begin{array}{l}\text { Method of } \\
\text { diagnosis not } \\
\text { reported }\end{array}$ & $\begin{array}{l}\text { Visual } \\
\text { assessment }\end{array}$ & 19/19/19 \\
\hline $\begin{array}{l}\text { Allen } 1979298425 \\
\text { (UK) }\end{array}$ & $\begin{array}{l}\text { Single } \\
\text { center }\end{array}$ & Not reported & $\begin{array}{l}\text { BCC anywhere in } \\
\text { the body }\end{array}$ & $\begin{array}{l}<18 \text { y/o; previous deep x-ray } \\
\text { tx or cryotherapy; lesion near } \\
\text { the eye when the other eye } \\
\text { sees less than } 6 / 18 \text {. }\end{array}$ & $\begin{array}{l}\text { Method of } \\
\text { diagnosis not } \\
\text { reported }\end{array}$ & $\begin{array}{l}\text { Method of } \\
\text { assessment not } \\
\text { reported }\end{array}$ & $31 / 31 / 31$ \\
\hline $\begin{array}{l}\text { Alpsoy } 1996 \\
8708151 \text { (Turkey) }\end{array}$ & Unclear & Not reported & $\begin{array}{l}\text { adults with } \\
\text { histologically } \\
\text { confirmed BCC }\end{array}$ & $\begin{array}{l}\text { recurrent lesions, genetic or } \\
\text { nevoid conditions, or lesions } \\
\text { with deep tissue involvement }\end{array}$ & $\begin{array}{l}\text { Biopsy/pathologic } \\
\text { confirmed: } \\
\text { histologically } \\
\text { confirmed BCC }\end{array}$ & $\begin{array}{l}\text { Method of } \\
\text { assessment not } \\
\text { reported }\end{array}$ & $45 / 45 / 45$ \\
\hline $\begin{array}{l}\text { Arits } 201323683751 \\
\text { (Netherlands) }\end{array}$ & Multicenter & $\begin{array}{l}\text { No industry } \\
\text { support }\end{array}$ & $\begin{array}{l}1 \text { primary, } \\
\text { histologically proven } \\
\text { superficial BCC per } \\
\text { patient }\end{array}$ & $\begin{array}{l}\text { using immunosuppressive } \\
\text { drugs, had genetic skin } \\
\text { cancer disorders, tumour was } \\
\text { located in the H zone or } \\
\text { scalp, or were breastfeeding } \\
\text { or pregnant }\end{array}$ & $\begin{array}{l}\text { Biopsy/pathologic } \\
\text { confirmed: } 3 \mathrm{~mm} \\
\text { punch biopsy and } \\
\text { was assessed by } \\
\text { consensus }\end{array}$ & $\begin{array}{l}\text { Method of } \\
\text { assessment not } \\
\text { reported }\end{array}$ & 601/601/583 \\
\hline $\begin{array}{l}\text { Avril } 19979218740 \\
\text { (France) }\end{array}$ & Unclear & Not reported & $\begin{array}{l}\text { previously untreated } \\
\mathrm{BCC} \text { of the face, } \varnothing< \\
4 \mathrm{~cm} .\end{array}$ & $\begin{array}{l}\text { contraindication to surgery } \\
\text { and radiotherapy, BCC on } \\
\text { the scalp or the neck, pts } \\
\text { with total removal of BCC at } \\
\text { biopsy, pts w/ >=5 BCCs, LE } \\
<3 \text { yrs }\end{array}$ & $\begin{array}{l}\text { Biopsy/pathologic } \\
\text { confirmed }\end{array}$ & $\begin{array}{l}\text { Method of } \\
\text { assessment not } \\
\text { reported }\end{array}$ & $360 / 360 / 347$ \\
\hline $\begin{array}{l}\text { Basset-Seguin } 2008 \\
18693158(13 \\
\text { centers in } 7\end{array}$ & Multicenter & $\begin{array}{l}\text { No industry } \\
\text { support }\end{array}$ & $\begin{array}{l}>=18 \text { y/o w/ } \\
\text { previously untreated } \\
\text { primary superficial }\end{array}$ & $\begin{array}{l}\text { xeroderma pigmentosum, } \\
\text { porphyria, Gorlin's syndrome, } \\
\text { history of arsenic exposure, }\end{array}$ & $\begin{array}{l}\text { Biopsy/pathologic } \\
\text { confirmed }\end{array}$ & $\begin{array}{l}\text { Method of } \\
\text { assessment not } \\
\text { reported }\end{array}$ & $120 / 118 / 115$ \\
\hline
\end{tabular}




\begin{tabular}{|c|c|c|c|c|c|c|c|}
\hline $\begin{array}{l}\text { Author Year } \\
\text { PMID } \\
\text { (Country/Region) }\end{array}$ & $\begin{array}{l}\text { Number } \\
\text { of } \\
\text { centers }\end{array}$ & Funding & $\begin{array}{l}\text { Inclusion } \\
\text { criteria* }\end{array}$ & Exclusion criteria* & $\begin{array}{l}\text { Method of } \\
\text { diagnosis }\end{array}$ & $\begin{array}{l}\text { Preoperative } \\
\text { tumor size } \\
\text { assessment }\end{array}$ & $\begin{array}{l}\mathrm{N} \text { enrolled } I \\
\text { randomized } / \\
\text { analyzed }\end{array}$ \\
\hline european countries) & & & $\begin{array}{l}\text { BCC lesions suitable } \\
\text { for cryotherapy. } \\
\text { confirmed by } \\
\text { histology using } 4 \\
\text { mm punch biopsy. } \\
<=10 \text { eligible } \\
\text { lesions. } \varnothing 6-15 \mathrm{~mm} \\
\text { on the face or scalp, } \\
<20 \text { mm on the } \\
\text { extremities or neck } \\
\text { and <30 mm on the } \\
\text { trunk, which were } \\
\text { not pigmented, } \\
\text { morpheaform or } \\
\text { infiltrating. }\end{array}$ & $\begin{array}{l}\text { allergy to MAL or other } \\
\text { topical photosensitizers or } \\
\text { excipients of the cream, } \\
\text { participated in other } \\
\text { investigational studies in last } \\
30 \text { days and pregnant or } \\
\text { breast-feeding women. } \\
\text { Concomitant treatment with } \\
\text { immunosuppressive } \\
\text { medication }\end{array}$ & & & \\
\hline $\begin{array}{l}\text { Bath-Hextall } 2014 \\
24332516 \text { (UK) }\end{array}$ & Multicenter & $\begin{array}{l}\text { Industry } \\
\text { supplied } \\
\text { materials }\end{array}$ & $\begin{array}{l}\text { histologically } \\
\text { confirmed, primary, } \\
\text { previously untreated, } \\
\text { nodular or superficial } \\
\text { BCC not arising at } \\
\text { sites at high risk for } \\
\text { subclinical tumour } \\
\text { spread }\end{array}$ & $\begin{array}{l}\text { morphoeic or recurrent BCC } \\
\text { and those with Gorlin } \\
\text { syndrome }\end{array}$ & $\begin{array}{l}\text { Biopsy/pathologic } \\
\text { confirmed: } \\
\text { Histologically } \\
\text { proven BCC } \\
\text { (usually a punch or } \\
\text { shave biopsy } \\
\text { specimen of no } \\
\text { more than } 25 \% \text { of } \\
\text { the total lesion, } \\
\text { though sometimes } \\
\text { at surgery) }\end{array}$ & $\begin{array}{l}\text { Method of } \\
\text { assessment not } \\
\text { reported }\end{array}$ & $501 / 501 / 485$ \\
\hline $\begin{array}{l}\text { Berroeta } 2007 \\
17573890 \text { (United } \\
\text { Kingdom) }\end{array}$ & $\begin{array}{l}\text { Single } \\
\text { center }\end{array}$ & $\begin{array}{l}\text { No industry } \\
\text { support }\end{array}$ & $\begin{array}{l}<=2 \mathrm{~cm} \text {, well-define, } \\
\text { nodular BCC on } \\
\text { anatomically } \\
\text { noncritical sites }\end{array}$ & $\begin{array}{l}<18 \text { y/o; pregnancy; } \\
\text { photosensitivity; morphoeic } \\
\text { BCCs; high-risk site; } \\
\text { recurrent BCCs; } \\
\text { immunodeficiency; size > } 2 \\
\mathrm{~cm} \text {. }\end{array}$ & $\begin{array}{l}\text { Method of } \\
\text { diagnosis not } \\
\text { reported }\end{array}$ & $\begin{array}{l}\text { Visual } \\
\text { assessment: well } \\
\text { defined }<=2 \mathrm{~cm}\end{array}$ & $31 / 31 / 31$ \\
\hline $\begin{array}{l}\text { Beutner } 1999 \\
10570388 \text { (USA) }\end{array}$ & Unclear & $\begin{array}{l}\text { Industry } \\
\text { funded }\end{array}$ & $\begin{array}{l}\text { biopsy-confirmed } \\
\text { BCC with clearly } \\
\text { visible margins, } \\
\text { nodular w/ area } 0.5 \text { - } \\
1.5 \mathrm{~cm}^{\wedge} 2 \text {, or } \\
\text { superficial w/ area of } \\
0.5-2 \mathrm{~cm}^{\wedge} 2 \text {, and that } \\
\text { was suitable for tx } \\
\text { by surgical excision. }\end{array}$ & central facial/periorificial sites & $\begin{array}{l}\text { Biopsy/pathologic } \\
\text { confirmed }\end{array}$ & $\begin{array}{l}\text { Method of } \\
\text { assessment not } \\
\text { reported }\end{array}$ & $35 / 35 / 35$ \\
\hline
\end{tabular}




\begin{tabular}{|c|c|c|c|c|c|c|c|}
\hline $\begin{array}{l}\text { Author Year } \\
\text { PMID } \\
\text { (Country/Region) }\end{array}$ & $\begin{array}{l}\text { Number } \\
\text { of } \\
\text { centers }\end{array}$ & Funding & $\begin{array}{l}\text { Inclusion } \\
\text { criteria* }\end{array}$ & Exclusion criteria* & $\begin{array}{l}\text { Method of } \\
\text { diagnosis }\end{array}$ & $\begin{array}{l}\text { Preoperative } \\
\text { tumor size } \\
\text { assessment }\end{array}$ & $\begin{array}{l}\mathrm{N} \text { enrolled } l \\
\text { randomized } / \\
\text { analyzed }\end{array}$ \\
\hline $\begin{array}{l}\text { Brinkhuizen } 2016 \\
27067393 \\
\text { (Netherlands) }\end{array}$ & $\begin{array}{l}\text { Single } \\
\text { center }\end{array}$ & $\begin{array}{l}\text { Industry } \\
\text { supplied } \\
\text { materials }\end{array}$ & $\begin{array}{l}\text { Patients with } \\
\text { histologically proven } \\
\text { primary SBCC or } \\
\text { (micro) nBCC >=4 } \\
\mathrm{mm} \text {, not located on } \\
\text { the face or on the } \\
\text { hairy scalp }\end{array}$ & & $\begin{array}{l}\text { Biopsy/pathologic } \\
\text { confirmed }\end{array}$ & $\begin{array}{l}\text { Visual } \\
\text { assessment }\end{array}$ & $128 / 128 / 119$ \\
\hline $\begin{array}{l}\text { Butler } 2009 \\
\text { 19018814 (texas, } \\
\text { usa) }\end{array}$ & $\begin{array}{l}\text { Single } \\
\text { center }\end{array}$ & $\begin{array}{l}\text { Industry } \\
\text { funded }\end{array}$ & $\begin{array}{l}\text { Immunocompetent, } \\
\text { non-pregnant, }>=18 \\
\text { y/o, primary nodular } \\
\text { nasal BCCs, }<1 \mathrm{~cm}\end{array}$ & $\begin{array}{l}\text { superficial, morpheaform, or } \\
\text { micronodular histologic BCC }\end{array}$ & $\begin{array}{l}\text { Biopsy/pathologic } \\
\text { confirmed: } \\
\text { histological } \\
\text { confirmation of } \\
\text { BCC before study } \\
\text { enrollment with a } \\
2-m m \text { punch } \\
\text { biopsy by a pathol- } \\
\text { ogist. }\end{array}$ & $\begin{array}{l}\text { Method of } \\
\text { assessment not } \\
\text { reported }\end{array}$ & $\begin{array}{l}31 / 31 / 31(\mathrm{ITT}) \\
28 \text { actual }\end{array}$ \\
\hline $\begin{array}{l}\text { Cai } 201525899562 \\
\text { (china) }\end{array}$ & $\begin{array}{l}\text { Single } \\
\text { center }\end{array}$ & $\begin{array}{l}\text { No industry } \\
\text { support }\end{array}$ & $\begin{array}{l}\text { having skin BD upon } \\
\text { biopsy }\end{array}$ & porphyria or photosensitivity & $\begin{array}{l}\text { Biopsy/pathologic } \\
\text { confirmed }\end{array}$ & $\begin{array}{l}\text { Method of } \\
\text { assessment not } \\
\text { reported }\end{array}$ & $18 / 18 / 18$ \\
\hline $\begin{array}{l}\text { Carija } 2016 \\
27516420\end{array}$ & $\begin{array}{l}\text { Single } \\
\text { center }\end{array}$ & $\begin{array}{l}\text { No industry } \\
\text { support }\end{array}$ & $\begin{array}{l}>18 \text { years, > } \mathrm{BCCs}, \\
\text { biopsy proven }\end{array}$ & $\begin{array}{l}\text { lactating and pregnant } \\
\text { women, heavily pigmented } \\
\text { BCC, diagnosed porphyria }\end{array}$ & $\begin{array}{l}\text { Biopsy/pathologic } \\
\text { confirmed }\end{array}$ & $\begin{array}{l}\text { assessment: } \\
\text { traced onto } \\
\text { graph paper and } \\
\text { count squares }\end{array}$ & $15 / 15 / 15$ \\
\hline $\begin{array}{l}\text { Choi } 201626551044 \\
\text { (korea) }\end{array}$ & $\begin{array}{l}\text { Single } \\
\text { center }\end{array}$ & $\begin{array}{l}\text { No industry } \\
\text { support }\end{array}$ & $\begin{array}{l}\geq 18 \text { y/o, untreated } \\
\text { thin primary nBCC, } \\
\text { maximum tumour } \\
\text { depth of } 2 \mathrm{~mm} \text { in a } \\
\text { biopsy specimen } \\
\text { and clinical } \\
\text { evaluation, surgical } \\
\text { excision would be } \\
\text { difficult because of } \\
\text { bleeding } \\
\text { abnormalities or } \\
\text { cardiac problems. }\end{array}$ & $\begin{array}{l}\text { >5 eligible lesions; lesions } \\
\text { located in the midface region, } \\
\text { nose, orbital areas or ears; } \varnothing \\
>15 \text { mm; non-nodular; known } \\
\text { allergies to MAL or lidocaine; } \\
\text { pregnancy or lactation; active } \\
\text { systemic infectious disease; } \\
\text { immunosuppressive } \\
\text { treatment; personal history of } \\
\text { malignant melanoma; } \\
\text { tendency toward melasma or } \\
\text { keloid formation; any } \\
\text { indication of poor } \\
\text { compliance. }\end{array}$ & $\begin{array}{l}\text { Biopsy/pathologic } \\
\text { confirmed }\end{array}$ & $\begin{array}{l}\text { Method of } \\
\text { assessment not } \\
\text { reported }\end{array}$ & $\begin{array}{l}39(42 \\
\text { lesions)/39 (42 } \\
\text { lesions)/34 } \\
\text { patients (37 } \\
\text { lesions) }\end{array}$ \\
\hline Choi 201728199463 & $\begin{array}{l}\text { Single } \\
\text { center }\end{array}$ & Not reported & $\begin{array}{l}>18 \text { years with } \\
\text { previously untreated } \\
\text { microinvasive SCC, }\end{array}$ & $\begin{array}{l}\text { pregnancy or lactation; active } \\
\text { systemic infectious disease; } \\
\text { other inflammatory, }\end{array}$ & $\begin{array}{l}\text { Biopsy/pathologic } \\
\text { confirmed: } 4 \mathrm{~mm} \\
\text { punch biopsy }\end{array}$ & $\begin{array}{l}\text { Visual } \\
\text { assessment: } \\
\text { photographed for }\end{array}$ & $45 / 45 / 40$ \\
\hline
\end{tabular}




\begin{tabular}{|c|c|c|c|c|c|c|c|}
\hline $\begin{array}{l}\text { Author Year } \\
\text { PMID } \\
\text { (Country/Region) }\end{array}$ & $\begin{array}{l}\text { Number } \\
\text { of } \\
\text { centers }\end{array}$ & Funding & $\begin{array}{l}\text { Inclusion } \\
\text { criteria* }\end{array}$ & Exclusion criteria* & $\begin{array}{l}\text { Method of } \\
\text { diagnosis }\end{array}$ & $\begin{array}{l}\text { Preoperative } \\
\text { tumor size } \\
\text { assessment }\end{array}$ & $\begin{array}{l}\mathrm{N} \text { enrolled } / \\
\text { randomized } / \\
\text { analyzed }\end{array}$ \\
\hline & & & $\begin{array}{l}\text { tumor invasion into } \\
\text { the papillary dermis } \\
\text { (Clark level II) } \\
\text { according to a } \\
\text { biopsy specimen } \\
\text { and difficulty in } \\
\text { surgical excision } \\
\text { because of health } \\
\text { problems }\end{array}$ & $\begin{array}{l}\text { infectious, or neoplastic skin } \\
\text { diseases in the treated area; } \\
\text { allergy to MAL,other topical } \\
\text { photosensitizers, or } \\
\text { excipients of the cream; } \\
\text { history of photosensitivity; } \\
\text { use of immunosuppressive or } \\
\text { photosensitizing drugs; } \\
\text { participation in any other } \\
\text { investigational study in the } \\
\text { preceding } 30 \text { days; history or } \\
\text { indicators of poor } \\
\text { compliance. Histological } \\
\text { findings of acantholysis, } \\
\text { desmoplasia, perineural or } \\
\text { lymphovascular invasion, and } \\
\text { echographic features of } \\
\text { regional lymph node } \\
\text { metastasis }\end{array}$ & & $\begin{array}{l}\text { baseline } \\
\text { measurement }\end{array}$ & \\
\hline $\begin{array}{l}\text { Cornell } 1990 \\
2229497 \text { (US) }\end{array}$ & Multicenter & $\begin{array}{l}\text { No industry } \\
\text { support }\end{array}$ & $\begin{array}{l}\text { superficial or } \\
\text { noduloulcerative } \\
\text { BCC confirmed by } \\
\text { biopsy, } 32-70 \text { y/o, } \\
\text { not pregnant, and in } \\
\text { good general health. }\end{array}$ & $\begin{array}{l}\text { previous therapy to the test } \\
\text { lesion, immunosuppressive } \\
\text { or cytotoxic therapy (within } \\
\text { the prior } 4 \text { wks), or } \\
\text { exogenous } \\
\text { interferon/interferon-inducer } \\
\text { except interferonalfa-2b } \\
\text { (Intron A), BCC located in the } \\
\text { perioral or central area of the } \\
\text { face or penetrating to deep } \\
\text { tissue }\end{array}$ & $\begin{array}{l}\text { Biopsy/pathologic } \\
\text { confirmed: punch } \\
\text { or shave biopsy }\end{array}$ & $\begin{array}{l}\text { Visual } \\
\text { assessment: } \\
\text { photographed } \\
\text { and its size and } \\
\text { anatomic location } \\
\text { were precisely } \\
\text { defined. }\end{array}$ & $172 / 172 / 165$ \\
\hline $\begin{array}{l}\text { Edwards } 1990 \\
2107219 \text { (U.S.) }\end{array}$ & Unclear & $\begin{array}{l}\text { Industry } \\
\text { supplied } \\
\text { materials }\end{array}$ & $\begin{array}{l}\text { clinically typical, } \\
\text { sharply defined BCC } \\
\text { easily excisable at } \\
\text { the end of the study }\end{array}$ & $\begin{array}{l}\text { any serious or debilitating } \\
\text { illness, history of } \\
\text { thromboembolic phenomena } \\
\text { or CVD, received rt to the test } \\
\text { site area or who had a history } \\
\text { of arsenic ingestion, pregnant } \\
\text { or nursing women, } \\
\text { immunosuppressed, pts } \\
\text { taking nonsteroidal anti- } \\
\text { inflammatory medications }\end{array}$ & $\begin{array}{l}\text { Biopsy/pathologic } \\
\text { confirmed }\end{array}$ & $\begin{array}{l}\text { Visual } \\
\text { assessment: at } \\
\text { randomization, } \\
\text { immediately } \\
\text { before treatment } \\
\text { and at the } \\
\text { beginning of } \\
\text { each treatment } \\
\text { week }\end{array}$ & $29 / 29 / 29$ \\
\hline Edwards 1990 & Unclear & Industry & otherwise healthy & Morpheic BCC, recurrent & Biopsy/pathologic & Visual & $65 / 65 / 63$ \\
\hline
\end{tabular}




\begin{tabular}{|c|c|c|c|c|c|c|c|}
\hline $\begin{array}{l}\text { Author Year } \\
\text { PMID } \\
\text { (Country/Region) }\end{array}$ & $\begin{array}{l}\text { Number } \\
\text { of } \\
\text { centers }\end{array}$ & Funding & $\begin{array}{l}\text { Inclusion } \\
\text { criteria* }\end{array}$ & Exclusion criteria* & $\begin{array}{l}\text { Method of } \\
\text { diagnosis }\end{array}$ & $\begin{array}{l}\text { Preoperative } \\
\text { tumor size } \\
\text { assessment }\end{array}$ & $\begin{array}{l}\mathrm{N} \text { enrolled } / \\
\text { randomized } / \\
\text { analyzed }\end{array}$ \\
\hline 2383027 (U.S.) & & $\begin{array}{l}\text { supplied } \\
\text { materials }\end{array}$ & $\begin{array}{l}35-65 \text { y/o; } 1 \text { clinically } \\
\text { typical, sharply } \\
\text { defined basal cell } \\
\text { carcinoma with } \\
\text { clearly visible } \\
\text { margins, } \varnothing 0.5 \text { to } \\
1.5 \mathrm{~cm} \text { for nodular } \\
\text { tumors or } 2 \mathrm{~cm} \text { for } \\
\text { superficial lesions, } \\
\text { per pt. }\end{array}$ & $\begin{array}{l}\text { cancers, deeply invasive } \\
\text { lesions, periorificial tumors, } \\
\text { and central facial BCC; } \\
\text { serious or debilitating illness, } \\
\text { a history of thromboembolic } \\
\text { or CVD, rt to the test site } \\
\text { area, or a history of arsenic } \\
\text { ingestion. Pregnancy, breast- } \\
\text { feeding, and } \\
\text { immunosuppression as a } \\
\text { result of medication or illness, } \\
\text { nonsteroidal anti- } \\
\text { inflammatory medications }\end{array}$ & $\begin{array}{l}\text { confirmed: } \\
\text { confirmatory } \\
\text { diagnostic shave } \\
\text { or punch skin } \\
\text { biopsy that } \\
\text { removed less than } \\
25 \% \text { of the lesion }\end{array}$ & $\begin{array}{l}\text { assessment: The } \\
\text { size and a } \\
\text { clinical } \\
\text { description of } \\
\text { each basal cell } \\
\text { carcinoma were } \\
\text { recorded. The } \\
\text { lesion was then } \\
\text { photographed. }\end{array}$ & \\
\hline $\begin{array}{l}\text { Eigentler } 2007 \\
17610993 \\
\text { (Germany) }\end{array}$ & Unclear & Not reported & $\begin{array}{l}\text { adults } w />=1 \\
\text { clinically typical and } \\
\text { histologically } \\
\text { confirmed primary } \\
\text { nBCC } \varnothing<=1.5 \mathrm{~cm}\end{array}$ & $\begin{array}{l}\text { micronodular, infiltrative, } \\
\text { superficial, or morpheic BCC, } \\
\text { BCCs w/ multicentric growth } \\
\text { pattern, w/in } 0.5 \mathrm{~cm} \text { of the } \\
\text { eyes }\end{array}$ & $\begin{array}{l}\text { Biopsy/pathologic } \\
\text { confirmed }\end{array}$ & $\begin{array}{l}\text { Visual } \\
\text { assessment: the } \\
\text { lesion was } \\
\text { documented by } \\
\text { photography and } \\
\text { the silhouette } \\
\text { was traced on a } \\
\text { plastic film. }\end{array}$ & $102 / 102 / 90$ \\
\hline $\begin{array}{l}\text { Eimpunth } 2014 \\
\text { (Conference } \\
\text { abstract) (unclear) }\end{array}$ & Unclear & Not reported & $\begin{array}{l}\text { biopsy proven, } \\
\text { superficial or nodular } \\
\text { BCCs located on } \\
\text { trunk or extremities }\end{array}$ & & $\begin{array}{l}\text { Biopsy/pathologic } \\
\text { confirmed }\end{array}$ & $\begin{array}{l}\text { Method of } \\
\text { assessment not } \\
\text { reported }\end{array}$ & $24 / 24 / 24$ \\
\hline $\begin{array}{l}\text { Foley } 2009 \\
20064185 \text { (U.S. and } \\
\text { australia) }\end{array}$ & Multicenter & $\begin{array}{l}\text { Industry } \\
\text { funded }\end{array}$ & $\begin{array}{l}18 \text { y/o, primary } \\
\text { nodular BCC verified } \\
\text { by local histologic } \\
\text { exam of } 2-3 \mathrm{~mm} \\
\text { punch biopsy and } \\
\text { suitable for a simple } \\
\text { excision surgery. }\end{array}$ & $\begin{array}{l}\text { periorbital area, ears, } \\
\text { nasaolabial fold; } \varnothing<6 \mathrm{~mm} \\
\text { (any site) or }>15 \mathrm{~mm} \text { (face or } \\
\text { scalp), > } 20 \mathrm{~mm} \text { (extremities } \\
\text { or neck), or }>30 \mathrm{~mm} \text { (trunk); } \\
\text { pigmented, morpheaform or } \\
\text { infiltrating pattern. porphyria, } \\
\text { Gorlin's syndrome, } \\
\text { xeroderma pigmentosum, } \\
\text { history of arsenic exposure or } \\
\text { allergy to MAL, ALA, or } \\
\text { excipients, participated in any } \\
\text { other investigational study in } \\
\text { the previous } 30 \text { days or were } \\
\text { likely to be poorly compliant, } \\
\text { pregnant or breast-feeding. }\end{array}$ & $\begin{array}{l}\text { Biopsy/pathologic } \\
\text { confirmed }\end{array}$ & $\begin{array}{l}\text { Method of } \\
\text { assessment not } \\
\text { reported }\end{array}$ & $\begin{array}{l}131(160 \\
\text { lesions }) / 131 \\
(160 \\
\text { lesions)/128 }\end{array}$ \\
\hline
\end{tabular}




\begin{tabular}{|c|c|c|c|c|c|c|c|}
\hline $\begin{array}{l}\text { Author Year } \\
\text { PMID } \\
\text { (Country/Region) }\end{array}$ & $\begin{array}{l}\text { Number } \\
\text { of } \\
\text { centers }\end{array}$ & Funding & $\begin{array}{l}\text { Inclusion } \\
\text { criteria* }\end{array}$ & Exclusion criteria* & $\begin{array}{l}\text { Method of } \\
\text { diagnosis }\end{array}$ & $\begin{array}{l}\text { Preoperative } \\
\text { tumor size } \\
\text { assessment }\end{array}$ & $\begin{array}{l}\mathrm{N} \text { enrolled } / \\
\text { randomized } / \\
\text { analyzed }\end{array}$ \\
\hline & & & & $\begin{array}{l}\text { concomitant treatment with } \\
\text { any immunosuppressive } \\
\text { medication was prohibited. }\end{array}$ & & & \\
\hline $\begin{array}{l}\text { Garcia-Martin } 2011 \\
21242584 \text { (Spain) }\end{array}$ & Unclear & Not reported & $\begin{array}{l}\text { nodular BCC on the } \\
\text { eyelid }\end{array}$ & $\begin{array}{l}\text { previous tx, other } \\
\text { dermatological diseases such } \\
\text { as Gorlin syndrome or } \\
\text { psoriasis, } \\
\text { immunocompromised status, } \\
\text { aggressive varieties of BCC } \\
\text { such as morpheaform } \\
\text { (sclerosing or infiltrative) } \\
\text { BCC }\end{array}$ & $\begin{array}{l}\text { Biopsy/pathologic } \\
\text { confirmed: punch } \\
\text { of diameter } 4 \mathrm{~mm}\end{array}$ & $\begin{array}{l}\text { Visual } \\
\text { assessment }\end{array}$ & $27 / 27 / 27$ \\
\hline $\begin{array}{l}\text { Geisse } 2002 \\
12196749 \text { (U.S.) }\end{array}$ & Multicenter & $\begin{array}{l}\text { Industry } \\
\text { funded }\end{array}$ & $\begin{array}{l}\text { >=18 y/o, } \\
\text { histologically } \\
\text { confirmed superficial } \\
\text { BCC } 0.5-2.0 \mathrm{~cm}^{\wedge} 2\end{array}$ & $\begin{array}{l}\text { w/in } 1 \mathrm{~cm} \text { of the hairline, } \\
\text { eyes, nose, mouth, or ears; } \\
\text { the anogenital area; hands } \\
\text { and feet, previously treated, } \\
\text { recurrent, or w/in } 5 \mathrm{~cm} \text { of } \\
\text { another BCC tumor }\end{array}$ & $\begin{array}{l}\text { Biopsy/pathologic } \\
\text { confirmed: A } \\
\text { biopsy specimen } \\
\text { of no more than } \\
25 \% \text { of the tumor } \\
\text { area was taken for } \\
\text { histologic } \\
\text { confirmation of } \\
\text { sBCC. }\end{array}$ & $\begin{array}{l}\text { Visual } \\
\text { assessment }\end{array}$ & $128 / 128 / 125$ \\
\hline $\begin{array}{l}\text { Geisse } 2004 \\
15097956 \text { (U.S.) }\end{array}$ & Multicenter & $\begin{array}{l}\text { Industry } \\
\text { funded }\end{array}$ & $\begin{array}{l}>=18 \text { y/o, primary, } \\
\text { histologically- } \\
\text { confirmed superficial } \\
\text { BCC }>=0.5 \mathrm{~cm} 2, \varnothing \\
<=2.0 \mathrm{~cm} \text { on the } \\
\text { limbs, trunk } \\
\text { (excluding the } \\
\text { anogenital area), } \\
\text { neck, or head } \\
\text { (excluding the H- } \\
\text { zone) }\end{array}$ & $\begin{array}{l}\text { any dermatological disease in } \\
\text { the target SBCC site or } \\
\text { surrounding area that could } \\
\text { be exacerbated by imiquimod } \\
\text { or cause difficulty with } \\
\text { examination (such as } \\
\text { subjects with nevoid basal } \\
\text { cell carcinoma syndrome) }\end{array}$ & $\begin{array}{l}\text { Biopsy/pathologic } \\
\text { confirmed: } \\
\text { confirmatory punch } \\
\text { or shave biopsy < } \\
25 \% \text { of the tumor } \\
\text { area }\end{array}$ & $\begin{array}{l}\text { Visual } \\
\text { assessment: } \\
\text { clinically evident } \\
\text { tumor margins } \\
\text { and local } \\
\text { landmarks }\end{array}$ & $724 / 724 / 694$ \\
\hline $\begin{array}{l}\text { Haak } 2015 \\
24903544 \\
\text { (Denmark) }\end{array}$ & $\begin{array}{l}\text { Single } \\
\text { center }\end{array}$ & $\begin{array}{l}\text { No industry } \\
\text { support }\end{array}$ & $\begin{array}{l}>=18 \text { y/o, previously } \\
\text { untreated facial } \\
\text { tumours. } \\
\text { histologically verified } \\
\text { nBCC either: } \varnothing>15 \\
\text { mm, located in the } \\
\text { H-zone, located on } \\
\text { severely sun- } \\
\text { damaged skin with }\end{array}$ & $\begin{array}{l}\text { lactating or pregnant women, } \\
\text { porphyria, known allergy to } \\
\text { MAL, Gorlin syndrome, } \\
\text { immunosuppressive } \\
\text { treatment, Fitzpatrick skin } \\
\text { type IV-VI, history of keloid } \\
\text { formation and conditions } \\
\text { associated with risk of poor } \\
\text { compliance }\end{array}$ & $\begin{array}{l}\text { Biopsy/pathologic } \\
\text { confirmed: } \\
\text { histologically } \\
\text { verified }\end{array}$ & $\begin{array}{l}\text { Visual } \\
\text { assessment: } \\
\text { photographed } \\
\text { and mapped on a } \\
\text { template }\end{array}$ & $32 / 32 / 32$ \\
\hline
\end{tabular}




\begin{tabular}{|c|c|c|c|c|c|c|c|}
\hline $\begin{array}{l}\text { Author Year } \\
\text { PMID } \\
\text { (Country/Region) }\end{array}$ & $\begin{array}{l}\text { Number } \\
\text { of } \\
\text { centers }\end{array}$ & Funding & $\begin{array}{l}\text { Inclusion } \\
\text { criteria* }\end{array}$ & Exclusion criteria* & $\begin{array}{l}\text { Method of } \\
\text { diagnosis }\end{array}$ & $\begin{array}{l}\text { Preoperative } \\
\text { tumor size } \\
\text { assessment }\end{array}$ & $\begin{array}{l}\mathrm{N} \text { enrolled } / \\
\text { randomized } / \\
\text { analyzed }\end{array}$ \\
\hline & & & $\begin{array}{l}\text { one or more co- } \\
\text { existing actinic } \\
\text { lesions requiring } \\
\text { treatment }\end{array}$ & & & & \\
\hline $\begin{array}{l}\text { Hall } 19863514075 \\
\text { (UK) }\end{array}$ & $\begin{array}{l}\text { Single } \\
\text { center }\end{array}$ & Not reported & $\begin{array}{l}\text { BCC proven by } \\
\text { biopsy, considered } \\
\text { suitable for tx w/ rt }\end{array}$ & $\begin{array}{l}\text { Recurrent tumors, location on } \\
\text { nose or pinna, electrons } \\
\text { considered Tx of choice, } \\
\text { lesion near eye and vision in } \\
\text { contralateral eye }<6 / 18\end{array}$ & $\begin{array}{l}\text { Biopsy/pathologic } \\
\text { confirmed: "Proven } \\
\text { by biopsy" }\end{array}$ & $\begin{array}{l}\text { Method of } \\
\text { assessment not } \\
\text { reported }\end{array}$ & $105 / 105 / 93$ \\
\hline $\begin{array}{l}\text { Ko } 201424102369 \\
\text { (Korea) }\end{array}$ & $\begin{array}{l}\text { Single } \\
\text { center }\end{array}$ & $\begin{array}{l}\text { No industry } \\
\text { support }\end{array}$ & $\begin{array}{l}\text { Korean, } \geq 18 \text { y/o, } \\
\text { biopsy-confirmed } \\
\text { Bowen's Disease } \\
\text { lesions on lower } \\
\text { extremities, >=2 } \\
\text { comparable } \\
\text { symmetrical lesions } \\
\text { of similar severity } \\
\text { and <=twofold } \\
\text { difference in number } \\
\text { of lesions between } \\
\text { the right and left } \\
\text { sides. }\end{array}$ & $\begin{array}{l}\text { porphyria, known allergies to } \\
\text { the MAL cream or lidocaine, } \\
\text { pregnancy, lactation, any } \\
\text { active systemic infectious } \\
\text { disease, immunosuppressive } \\
\text { treatment, personal history of } \\
\text { malignant melanoma, } \\
\text { tendency towards melasma } \\
\text { or keloid formation, prior } \\
\text { treatment of the lesions w/in } \\
4 \text { wks, and any indication of } \\
\text { poor compliance. }\end{array}$ & $\begin{array}{l}\text { Biopsy/pathologic } \\
\text { confirmed }\end{array}$ & $\begin{array}{l}\text { Visual } \\
\text { assessment: } \\
\text { photographed, } \\
\text { mapped and } \\
\text { numbered }\end{array}$ & $21 / 19 / 18$ \\
\hline $\begin{array}{l}\text { Kuijpers } 2006 \\
16865869 \\
\text { (Netherlands) }\end{array}$ & $\begin{array}{l}\text { Single } \\
\text { center }\end{array}$ & $\begin{array}{l}\text { No industry } \\
\text { support }\end{array}$ & $\begin{array}{l}\text { nodular, primary } \\
\text { BCC located } \\
\text { anywhere but } \\
\text { periocular area and } \\
\text { hairy scalp; clinical } \\
\varnothing<20 \mathrm{~mm} \text {. }\end{array}$ & $\begin{array}{l}\text { pigmented BCC; contra- } \\
\text { indications to surgery; } \\
\text { hypersensitivity to daylight or } \\
\text { creams; porphyria; >5 BCCs. }\end{array}$ & $\begin{array}{l}\text { Method of } \\
\text { diagnosis not } \\
\text { reported }\end{array}$ & $\begin{array}{l}\text { Method of } \\
\text { assessment not } \\
\text { reported }\end{array}$ & $43 / 43 / 43$ \\
\hline $\begin{array}{l}\text { Kuijpers } 2007 \\
17451581 \\
\text { (Netherlands) }\end{array}$ & $\begin{array}{l}\text { Single } \\
\text { center }\end{array}$ & $\begin{array}{l}\text { No industry } \\
\text { support }\end{array}$ & $\begin{array}{l}>=18 \text { y/o, untreated, } \\
\text { primary histologically } \\
\text { proven BCC, } \\
\text { nodular or } \\
\text { superficial, on the } \\
\text { head and neck, } \\
<20 \mathrm{~mm} \varnothing\end{array}$ & $\begin{array}{l}\text { Recurrent, not superficial or } \\
\text { nodular, }>20 \mathrm{~mm} \varnothing \text {, } \\
\text { contraindications to either } \\
\text { procedure, presence of } 5+ \\
\text { BCCs }\end{array}$ & $\begin{array}{l}\text { Biopsy/pathologic } \\
\text { confirmed }\end{array}$ & $\begin{array}{l}\text { Method of } \\
\text { assessment not } \\
\text { reported }\end{array}$ & $88 / 88 / 88$ \\
\hline $\begin{array}{l}\text { Marks } 2001 \\
11312429 \text { (Australia } \\
\text { and New Zealand) }\end{array}$ & Multicenter & $\begin{array}{l}\text { Industry } \\
\text { funded }\end{array}$ & $\begin{array}{l}\text { >=18 y/o, biopsy- } \\
\text { proven superficial } \\
\text { BCC on head, neck, } \\
\text { trunk or limbs, SA } \\
0.5-2 \mathrm{~cm}^{\wedge} 2, \text { primary } \\
\text { tumor, biopsy }<25 \%\end{array}$ & $\begin{array}{l}\text { Infection, recurrent, w/in } 1 \mathrm{~cm} \\
\text { of the hairline, eyes, nose, } \\
\text { mouth, ears, anogenital } \\
\text { region, hands, and feet }\end{array}$ & $\begin{array}{l}\text { Biopsy/pathologic } \\
\text { confirmed }\end{array}$ & $\begin{array}{l}\text { Method of } \\
\text { assessment not } \\
\text { reported }\end{array}$ & 99/99/99 \\
\hline
\end{tabular}




\begin{tabular}{|c|c|c|c|c|c|c|c|}
\hline $\begin{array}{l}\text { Author Year } \\
\text { PMID } \\
\text { (Country/Region) }\end{array}$ & $\begin{array}{l}\text { Number } \\
\text { of } \\
\text { centers }\end{array}$ & Funding & $\begin{array}{l}\text { Inclusion } \\
\text { criteria* }\end{array}$ & Exclusion criteria* & $\begin{array}{l}\text { Method of } \\
\text { diagnosis }\end{array}$ & $\begin{array}{l}\text { Preoperative } \\
\text { tumor size } \\
\text { assessment }\end{array}$ & $\begin{array}{l}\mathrm{N} \text { enrolled } / \\
\text { randomized } / \\
\text { analyzed }\end{array}$ \\
\hline & & & of the lesion & & & & \\
\hline $\begin{array}{l}\text { Migden } 2015 \\
25981810 \\
\text { (worldwide) }\end{array}$ & Multicenter & $\begin{array}{l}\text { Industry } \\
\text { funded }\end{array}$ & $\begin{array}{l}\text { >= } 18 \text { y/o; } \\
\text { histologically } \\
\text { confirmed, locally } \\
\text { advanced BCC not } \\
\text { amenable to rt or } \\
\text { curative surgery; } \\
\text { adequate bone } \\
\text { marrow, liver } \\
\text { function, and renal } \\
\text { function }\end{array}$ & $\begin{array}{l}\text { previous tx with sonidegib or } \\
\text { another Hedgehog pathway } \\
\text { inhibitor, major surgery, other } \\
\text { antineoplastic therapy, taken } \\
\text { an investigational agent w/in } \\
4 \text { wks before the start of the } \\
\text { study, currently taking strong } \\
\text { inhibitors or inducers of } \\
\text { CYP3A4 or CYP3A5 } \\
\text { expression or drugs } \\
\text { metabolised by CYP2B6 or } \\
\text { CYP2C9; gastrointestinal } \\
\text { dysfunction or known } \\
\text { malabsorption syndromes, } \\
\text { neuromuscular disorders, or } \\
\text { other uncontrolled medical } \\
\text { disorders; treatment with } \\
\text { drugs known to cause } \\
\text { rhabdomyolysis (pravastatin } \\
\text { allowed w/ extra caution); } \\
\text { pregnancy or breastfeeding }\end{array}$ & $\begin{array}{l}\text { Biopsy/pathologic } \\
\text { confirmed }\end{array}$ & $\begin{array}{l}\text { Visual } \\
\text { assessment: } \\
\text { standard } \\
\text { annotated } \\
\text { photography }\end{array}$ & $269 / 230 / 230$ \\
\hline $\begin{array}{l}\text { Miller } 19978996264 \\
\text { (USA) }\end{array}$ & Multicenter & $\begin{array}{l}\text { No industry } \\
\text { support }\end{array}$ & $\begin{array}{l}6-15 \mathrm{~mm} \varnothing, \text { well- } \\
\text { defined margins, } \\
<=50 \mathrm{~mm} \text { from any } \\
\text { other malignancy } \\
\text { that would otherwise } \\
\text { be treated with } \\
\text { surgery or } \\
\text { curettage/electrodesi } \\
\text { ccation }\end{array}$ & $\begin{array}{l}\text { lesions already received tx, } \\
\text { high-risk sites, tumors } \\
\text { considered to be more } \\
\text { appropriately treated w/ } \\
\text { Mohs, deep tissue involved } \\
\text { lesions, morpheaform } \\
\text { lesions, lesions associated } \\
\text { with basal cell nevus } \\
\text { syndrome, known } \\
\text { hypersensitivities or allergies } \\
\text { to 5-FU, sulfites, epinephrine, } \\
\text { or bovine collagen; history of } \\
\text { autoimmune disease or } \\
\text { immunosuppression; women } \\
\text { who were pregnant or } \\
\text { lactating }\end{array}$ & $\begin{array}{l}\text { Biopsy/pathologic } \\
\text { confirmed: punch } \\
\text { or shave biopsy of } \\
\text { no more than } 25 \% \\
\text { of total legion }\end{array}$ & $\begin{array}{l}\text { Visual } \\
\text { assessment: 6- } \\
\text { 15mm in largest } \\
\text { diameter, well- } \\
\text { defined margins }\end{array}$ & $122 / 122 / 116$ \\
\hline $\begin{array}{l}\text { Morton } 1996 \\
8977678 \text { (Scotland) }\end{array}$ & Unclear & Not reported & $<=21 \mathrm{~mm} \varnothing$ & & $\begin{array}{l}\text { Biopsy/pathologic } \\
\text { confirmed: 4-mm } \\
\text { punch biopsy }\end{array}$ & $\begin{array}{l}\text { Method of } \\
\text { assessment not } \\
\text { reported }\end{array}$ & $19 / 19 / 19$ \\
\hline
\end{tabular}




\begin{tabular}{|c|c|c|c|c|c|c|c|}
\hline $\begin{array}{l}\text { Author Year } \\
\text { PMID } \\
\text { (Country/Region) }\end{array}$ & $\begin{array}{l}\text { Number } \\
\text { of } \\
\text { centers }\end{array}$ & Funding & $\begin{array}{l}\text { Inclusion } \\
\text { criteria* }\end{array}$ & Exclusion criteria* & $\begin{array}{l}\text { Method of } \\
\text { diagnosis }\end{array}$ & $\begin{array}{l}\text { Preoperative } \\
\text { tumor size } \\
\text { assessment }\end{array}$ & $\begin{array}{l}\mathrm{N} \text { enrolled } l \\
\text { randomized } / \\
\text { analyzed }\end{array}$ \\
\hline $\begin{array}{l}\text { Morton } 2006 \\
16785375 \text { (Europe) }\end{array}$ & Multicenter & $\begin{array}{l}\text { Industry } \\
\text { funded }\end{array}$ & $\begin{array}{l}>=18 \text { y/o, } \\
\text { histologically } \\
\text { confirmed SCC in } \\
\text { situ }\end{array}$ & $\begin{array}{l}\text { treated w/in the previous } 3 \\
\text { mo or strongly pigmented, } \\
<6 \mathrm{~mm} \text { or }>40 \mathrm{~mm} \varnothing \text {, located } \\
\text { on the genitalia }\end{array}$ & $\begin{array}{l}\text { Biopsy/pathologic } \\
\text { confirmed: biopsy } \\
\text { specimen taken } \\
\text { within } 5 \text { months, } \\
\text { and with no } \\
\text { evidence of any } \\
\text { change in } \\
\text { appearance } \\
\text { suggestive of } \\
\text { lesion progression }\end{array}$ & $\begin{array}{l}\text { Visual } \\
\text { assessment }\end{array}$ & $229 / 229 / 209$ \\
\hline $\begin{array}{l}\text { Mosterd } 2008 \\
18717680 \\
\text { (Netherlands) }\end{array}$ & $\begin{array}{l}\text { Single } \\
\text { center }\end{array}$ & Not reported & $\begin{array}{l}>18 \text { y/o, untreated } \\
\mathrm{nBCC} \text { w/ } \varnothing<=20 \\
\mathrm{~mm}\end{array}$ & $\begin{array}{l}\text { Pregnancy, LE }<5 \text { years, } \\
\text { known skin cancer } \\
\text { syndromes, use of phototoxic } \\
\text { photosensitive drugs, } \\
\text { hypersensitivity to light or } \\
\text { ALA cream, recurrent or } \\
\text { pigmented BCC, not nodular } \\
\text { BBC, and a localization on } \\
\text { concave areas or hairy skin }\end{array}$ & $\begin{array}{l}\text { Biopsy/pathologic } \\
\text { confirmed: } 3 \mathrm{~mm} \\
\text { punch biopsy }\end{array}$ & $\begin{array}{l}\text { Visual } \\
\text { assessment }\end{array}$ & $151 / 149 / 149$ \\
\hline $\begin{array}{l}\text { Mosterd } 2008 \\
19010733 \\
\text { (Netherlands) }\end{array}$ & Multicenter & $\begin{array}{l}\text { No industry } \\
\text { support }\end{array}$ & $\begin{array}{l}>=1 \text { untreated, } \\
\text { histologically } \\
\text { confirmed primary } \\
\text { BCC >=1cm } \varnothing \\
\text { located in the } \mathrm{H} \text { - } \\
\text { zone or a facial } \\
\text { primary BCC of an } \\
\text { aggressive } \\
\text { histological subtype } \\
\text { (ie, morpheaform, } \\
\text { micronodular, } \\
\text { trabecular, } \\
\text { infiltrative, or BCC } \\
\text { with squamous } \\
\text { differentiation) }\end{array}$ & LE $<3$ yrs & $\begin{array}{l}\text { Biopsy/pathologic } \\
\text { confirmed }\end{array}$ & $\begin{array}{l}\text { Visual } \\
\text { assessment: } \\
\text { overall and } \\
\text { close-up } \\
\text { photographs } \\
\text { were taken } \\
\text { before each } \\
\text { treatment }\end{array}$ & $443 / 374 / 251$ \\
\hline $\begin{array}{l}\text { Orenberg } 1992 \\
1430394 \text { (USA) }\end{array}$ & Unclear & Not reported & $\begin{array}{l}\text { Biopsy-proven } \\
\text { nodular BCC, 06-1.5 } \\
\mathrm{cm} \varnothing\end{array}$ & $\begin{array}{l}\text { Previous local tx or systemic } \\
\text { cancer therapy w/in } 6 \text { mo; } \\
\text { Gorlin's syndrome, } \\
\text { morpheaform, pigmented or } \\
\text { deeply invasive lesions; any } \\
\text { serious or debilitating illness, } \\
\text { chronic respiratory disease, }\end{array}$ & $\begin{array}{l}\text { Biopsy/pathologic } \\
\text { confirmed }\end{array}$ & $\begin{array}{l}\text { Method of } \\
\text { assessment not } \\
\text { reported }\end{array}$ & $20 / 20 / 20$ \\
\hline
\end{tabular}




\begin{tabular}{|c|c|c|c|c|c|c|c|}
\hline $\begin{array}{l}\text { Author Year } \\
\text { PMID } \\
\text { (Country/Region) }\end{array}$ & $\begin{array}{l}\text { Number } \\
\text { of } \\
\text { centers }\end{array}$ & Funding & $\begin{array}{l}\text { Inclusion } \\
\text { criteria* }\end{array}$ & Exclusion criteria* & $\begin{array}{l}\text { Method of } \\
\text { diagnosis }\end{array}$ & $\begin{array}{l}\text { Preoperative } \\
\text { tumor size } \\
\text { assessment }\end{array}$ & $\begin{array}{l}\mathrm{N} \text { enrolled } / \\
\text { randomized } / \\
\text { analyzed }\end{array}$ \\
\hline & & & & $\begin{array}{l}\text { depressed bone marrow, } \\
\text { autoimmunedisease, or w/ } \\
\text { hypersensitivity to 5-FU, } \\
\text { epinephrine, or bovine } \\
\text { couagen; Pregnant or } \\
\text { lactating women and subjects } \\
\text { requiring the use of } \\
\text { nonsteroidal antiinflammatory } \\
\text { drugs, nonselective beta- } \\
\text { blocking drugs, aspirin, and } \\
\text { topical or systemic steroids }\end{array}$ & & & \\
\hline $\begin{array}{l}\text { Patel } 2006 \\
16713457 \text { (United } \\
\text { Kingdom) }\end{array}$ & $\begin{array}{l}\text { Single } \\
\text { center }\end{array}$ & $\begin{array}{l}\text { Industry } \\
\text { funded }\end{array}$ & $\begin{array}{l}\text { biopsy-proven } \\
\text { cutaneous SCC in } \\
\text { situ; full-thickness } \\
\text { epidermal dysplasia; } \\
\text { no active treatment } \\
1 \text { mo; post-biopsy } \\
\text { legion } 1-20 \mathrm{~cm}^{\wedge} 2 ; \\
>=1 \mathrm{~cm} \text { away from } \\
\text { eye; had to be able } \\
\text { to attend clinical } \\
\text { trials room. }\end{array}$ & & $\begin{array}{l}\text { Biopsy/pathologic } \\
\text { confirmed: biopsy } \\
\text { specimen, which } \\
\text { by conventional } \\
\text { histologic } \\
\text { examination } \\
\text { showed full- } \\
\text { thickness } \\
\text { epidermal } \\
\text { dysplasia }\end{array}$ & $\begin{array}{l}\text { Method of } \\
\text { assessment not } \\
\text { reported }\end{array}$ & $31 / 31 / 28$ \\
\hline $\begin{array}{l}\text { Rhodes } 2004 \\
14732655 \text { (Europe) }\end{array}$ & Multicenter & $\begin{array}{l}\text { Industry } \\
\text { funded }\end{array}$ & $\begin{array}{l}\text { >=18 y/o w/ } \\
\text { perviously untreated } \\
\text { primary nodular } \\
\text { BCC suitable for } \\
\text { simple excision } \\
\text { surgery }\end{array}$ & $\begin{array}{l}>10 \text { eligible lesions; lesions } \\
\text { in midface region, orbital } \\
\text { areas, or ears; } 6 \mathrm{~mm}-15 \mathrm{~mm} \varnothing \\
\text { (face and scalp), > 20mm } \varnothing \\
\text { (extremities or neck), >30mm } \\
\varnothing \text { (trunk); pigmented or } \\
\text { morpheaform BCCs; } \\
\text { polyphyria; Gorlin syndrome; } \\
\text { history of arsenic exposure; } \\
\text { in another study in past } 30 \\
\text { days; likely to be poor } \\
\text { compliers; taking } \\
\text { immunosuppresive } \\
\text { medication; pregnant or } \\
\text { breasfeeding }\end{array}$ & $\begin{array}{l}\text { Biopsy/pathologic } \\
\text { confirmed }\end{array}$ & $\begin{array}{l}\text { Visual } \\
\text { assessment }\end{array}$ & $103 / 103 / 101$ \\
\hline $\begin{array}{l}\text { Salim } 2003 \\
12653747 \text { (UK) }\end{array}$ & Multicenter & Not reported & Bowen's disease & Not reported & $\begin{array}{l}\text { Biopsy/pathologic } \\
\text { confirmed }\end{array}$ & $\begin{array}{l}\text { Method of } \\
\text { assessment not } \\
\text { reported }\end{array}$ & $49 / 40 / 40$ \\
\hline Salmanpoor 2012 & Single & Not reported & Pathologically & Tumors with indications for & Biopsy/pathologic & Method of & $55 / 55 / 55$ \\
\hline
\end{tabular}




\begin{tabular}{|c|c|c|c|c|c|c|c|}
\hline $\begin{array}{l}\text { Author Year } \\
\text { PMID } \\
\text { (Country/Region) }\end{array}$ & $\begin{array}{l}\text { Number } \\
\text { of } \\
\text { centers }\end{array}$ & Funding & $\begin{array}{l}\text { Inclusion } \\
\text { criteria* }\end{array}$ & Exclusion criteria* & $\begin{array}{l}\text { Method of } \\
\text { diagnosis }\end{array}$ & $\begin{array}{l}\text { Preoperative } \\
\text { tumor size } \\
\text { assessment }\end{array}$ & $\begin{array}{l}\mathrm{N} \text { enrolled } / \\
\text { randomized } / \\
\text { analyzed }\end{array}$ \\
\hline (Iran) & center & & confirmed BCC & Mohs & confirmed & $\begin{array}{l}\text { assessment not } \\
\text { reported }\end{array}$ & \\
\hline $\begin{array}{l}\text { Schleier } 2007 \\
25047438 \text { (Germany } \\
\text { (Friedrich-Schiller } \\
\text { University Jena)) }\end{array}$ & $\begin{array}{l}\text { Single } \\
\text { center }\end{array}$ & $\begin{array}{l}\text { No industry } \\
\text { support }\end{array}$ & $\begin{array}{l}\text { histologically verified } \\
\text { superficial BCC w/ } \\
\text { no deep infiltration } \\
(<2 \mathrm{~mm}) \text {, no } \\
\text { morpheic and } \\
\text { pigmented BCC, and } \\
\text { good compliance. }\end{array}$ & $\begin{array}{l}\text { unclear histology, clinically } \\
\text { nodular BCC, expected poor } \\
\text { compliance, untreated } \\
\text { diabetes mellitus, and } \\
\text { pregnancy }\end{array}$ & $\begin{array}{l}\text { Biopsy/pathologic } \\
\text { confirmed }\end{array}$ & $\begin{array}{l}\text { Method of } \\
\text { assessment not } \\
\text { reported }\end{array}$ & $24 / 24 / 24$ \\
\hline $\begin{array}{l}\text { Schulze } 2005 \\
15888150 \text { (Europe) }\end{array}$ & Multicenter & $\begin{array}{l}\text { Industry } \\
\text { funded }\end{array}$ & $\begin{array}{l}\text { non-pregnant, >= } 18 \\
\text { y/o; histologically } \\
\text { confirmed primary } \\
\text { sBCC on limbs, } \\
\text { trunk, neck, or head; } \\
\text { area }>=0.5 \mathrm{~cm}^{\wedge} 2 \\
\text { and } \varnothing<=2.0 \mathrm{~cm} \\
\text { prior to biopsy. }\end{array}$ & $\begin{array}{l}\text { clinically significant, unstable } \\
\text { medical conditions; } \\
\text { metastatic tumor or tumor } \\
\text { with high probability of } \\
\text { metastatic spread; tumor on } \\
\text { anogenital area or w/in } 1 \mathrm{~cm} \\
\text { of the hairline, nose, mouth, } \\
\text { ears, and eyes; histological } \\
\text { evidence morphoeic, severe } \\
\text { squamous metaplasia, or any } \\
\text { infliltrative or desmoplastic } \\
\text { features; dermatological } \\
\text { disease w/in } 5 \text { cm of target } \\
\text { site margins that would be } \\
\text { exacerbated by treatment } \\
\text { and would affect assessment. }\end{array}$ & $\begin{array}{l}\text { Biopsy/pathologic } \\
\text { confirmed }\end{array}$ & $\begin{array}{l}\text { Visual } \\
\text { assessment: } \\
\text { multiplying the } \\
\text { two largest } \\
\text { diameters } \\
\text { perpendicular to } \\
\text { each other }\end{array}$ & $166 / 166 / 166$ \\
\hline $\begin{array}{l}\text { Shumack } 2002 \\
12224977 \text { (12 } \\
\text { weeks) (Australia } \\
\text { and New Zealand; } \\
\text { And United States) }\end{array}$ & Multicenter & Not reported & $\begin{array}{l}>=18 \text { y/o, primary } \\
\text { target tumor, } \\
\text { histologically } \\
\text { confirmed as } \\
\text { nodular BCC. } 0.5- \\
1.5 \mathrm{~cm}^{\wedge} 2 \text { area and } \\
>1 \mathrm{~cm} \text { from the } \\
\text { eyes, nose, mouth, } \\
\text { ear, and hairline. }\end{array}$ & $\begin{array}{l}\text { BCC with morpheic infiltrating } \\
\text { and micronodular patterns }\end{array}$ & $\begin{array}{l}\text { Biopsy/pathologic } \\
\text { confirmed: punch } \\
\text { or shave biopsy of } \\
\text { the target tumor. }\end{array}$ & $\begin{array}{l}\text { Visual } \\
\text { assessment: } \\
\text { Target tumors } \\
\text { were measured } \\
\text { and } \\
\text { photographed } \\
\text { prior to the } \\
\text { prestudy biopsy } \\
\text { and } \\
\text { rephotographed } \\
\text { prior to treatment } \\
\text { initiation and at } \\
\text { each interval } \\
\text { visit. }\end{array}$ & $92 / 92 / 77$ \\
\hline $\begin{array}{l}\text { Shumack } 2002 \\
12224977 \text { (6 weeks) }\end{array}$ & Multicenter & Not reported & $\begin{array}{l}>=18 \text { y/o, primary } \\
\text { target tumor, }\end{array}$ & $\begin{array}{l}\text { BCC with morpheic infiltrating } \\
\text { and micronodular patterns }\end{array}$ & $\begin{array}{l}\text { Biopsy/pathologic } \\
\text { confirmed: punch }\end{array}$ & $\begin{array}{l}\text { Visual } \\
\text { assessment: }\end{array}$ & $2 / 92 / 77$ \\
\hline
\end{tabular}




\begin{tabular}{|c|c|c|c|c|c|c|c|}
\hline $\begin{array}{l}\text { Author Year } \\
\text { PMID } \\
\text { (Country/Region) }\end{array}$ & $\begin{array}{l}\text { Number } \\
\text { of } \\
\text { centers }\end{array}$ & Funding & $\begin{array}{l}\text { Inclusion } \\
\text { criteria* }\end{array}$ & Exclusion criteria* & $\begin{array}{l}\text { Method of } \\
\text { diagnosis }\end{array}$ & $\begin{array}{l}\text { Preoperative } \\
\text { tumor size } \\
\text { assessment }\end{array}$ & $\begin{array}{l}\mathrm{N} \text { enrolled } / \\
\text { randomized } / \\
\text { analyzed }\end{array}$ \\
\hline $\begin{array}{l}\text { (Australia and New } \\
\text { Zealand; And United } \\
\text { States) }\end{array}$ & & & $\begin{array}{l}\text { histologically } \\
\text { confirmed as } \\
\text { nodular BCC. 0.5- } \\
1.5 \mathrm{~cm}^{\wedge} 2 \text { area and } \\
>1 \mathrm{~cm} \text { from the } \\
\text { eyes, nose, mouth, } \\
\text { ear, and hairline. }\end{array}$ & & $\begin{array}{l}\text { or shave biopsy of } \\
\text { the target tumor. }\end{array}$ & $\begin{array}{l}\text { Target tumors } \\
\text { were measured } \\
\text { and } \\
\text { photographed } \\
\text { prior to the } \\
\text { prestudy biopsy } \\
\text { and re- } \\
\text { photographed } \\
\text { prior to treatment } \\
\text { initiation and at } \\
\text { each interval } \\
\text { visit. }\end{array}$ & \\
\hline $\begin{array}{l}\text { Siller } 2010 \\
20546215 \text { (8 private } \\
\text { dermatology clinics } \\
\text { Australia) }\end{array}$ & Multicenter & $\begin{array}{l}\text { Industry } \\
\text { funded }\end{array}$ & $\begin{array}{l}\text { >=18 y/o, with one } \\
\text { sBCC lesion suitable } \\
\text { for surgical excision } \\
\text { on the arm, } \\
\text { shoulder, chest, } \\
\text { face, neck, } \\
\text { abdomen, back, leg } \\
\text { or scalp. Lesions } \\
\text { with pre- and post- } \\
\text { biopsy } \varnothing 4-15 \text { mm } \\
\text { and thickness <=4 } \\
\text { mm }\end{array}$ & $\begin{array}{l}\text { women of childbearing } \\
\text { potential; recurrent or atypical } \\
\text { lesions, immunosuppression, } \\
\text { and prior, concomitant or } \\
\text { anticipated therapy with the } \\
\text { potential to confound the } \\
\text { study results. }\end{array}$ & $\begin{array}{l}\text { Biopsy/pathologic } \\
\text { confirmed }\end{array}$ & $\begin{array}{l}\text { Visual } \\
\text { assessment }\end{array}$ & $60 / 60 / 60$ \\
\hline $\begin{array}{l}\text { Spencer } 2006 \\
16393600 \text { (United } \\
\text { States) }\end{array}$ & $\begin{array}{l}\text { Single } \\
\text { center }\end{array}$ & $\begin{array}{l}\text { Industry } \\
\text { funded }\end{array}$ & $\begin{array}{l}\text { >= } 18 \text { y/o; previously } \\
\text { untreated } \\
\text { histologically } \\
\text { confirmed nBCC. }\end{array}$ & $\begin{array}{l}\text { comorbidities that would } \\
\text { interfere with or be } \\
\text { exacerbated by treatment. }\end{array}$ & $\begin{array}{l}\text { Biopsy/pathologic } \\
\text { confirmed: } \\
\text { histologically } \\
\text { confirmed }\end{array}$ & $\begin{array}{l}\text { Visual } \\
\text { assessment }\end{array}$ & $20 / 20 / 20$ \\
\hline $\begin{array}{l}\text { Sterry } 2002 \\
12452875 \text { (nodular) } \\
\text { (Europe) }\end{array}$ & Multicenter & $\begin{array}{l}\text { Industry } \\
\text { funded }\end{array}$ & $\begin{array}{l}>=18 \text { y/o, primary } \\
\text { tumour, } \\
\text { histologically } \\
\text { confirmed superficial } \\
\text { or nodular BCC, } \\
\text { area } 0.5 \mathrm{~cm}^{\wedge} 2-2.0 \\
\mathrm{~cm}^{\wedge} 2 \text { for superficial } \\
\text { or } 0.25 \mathrm{~cm}^{\wedge} 2-1.5 \\
\mathrm{~cm}^{\wedge} 2 \text { for nodular }\end{array}$ & $\begin{array}{l}\text { previous therapy to the target } \\
\text { tumour or any dermatological } \\
\text { conditions that would } \\
\text { interfere with local } \\
\text { assessments. }\end{array}$ & $\begin{array}{l}\text { Biopsy/pathologic } \\
\text { confirmed: } \\
\text { prestudy } \\
\text { confirmatory } \\
\text { punch, deep } \\
\text { shave, or wedge } \\
\text { biopsy that } \\
\text { removed no more } \\
\text { than approximately } \\
25 \% \text { of the tumour }\end{array}$ & $\begin{array}{l}\text { Visual } \\
\text { assessment: } \\
\text { measuring and } \\
\text { multiplying the } \\
\text { two largest } \\
\text { perpendicular } \\
\text { dimensions of } \\
\text { the tumour. The } \\
\text { tumour site and } \\
\text { appropriate } \\
\text { anatomic } \\
\text { landmarks were } \\
\text { mapped using a }\end{array}$ & $183 / 177$ \\
\hline
\end{tabular}




\begin{tabular}{|c|c|c|c|c|c|c|c|}
\hline $\begin{array}{l}\text { Author Year } \\
\text { PMID } \\
\text { (Country/Region) }\end{array}$ & $\begin{array}{l}\text { Number } \\
\text { of } \\
\text { centers }\end{array}$ & Funding & $\begin{array}{l}\text { Inclusion } \\
\text { criteria* }\end{array}$ & Exclusion criteria* & $\begin{array}{l}\text { Method of } \\
\text { diagnosis }\end{array}$ & $\begin{array}{l}\text { Preoperative } \\
\text { tumor size } \\
\text { assessment }\end{array}$ & $\begin{array}{l}\mathrm{N} \text { enrolled } / \\
\text { randomized/ } \\
\text { analyzed }\end{array}$ \\
\hline & & & & & & $\begin{array}{l}\text { clear plastic } \\
\text { sheet as a } \\
\text { template to guide } \\
\text { the excision at } \\
\text { the end of the } \\
\text { study }\end{array}$ & \\
\hline $\begin{array}{l}\text { Sterry } 2002 \\
12452875 \\
\text { (superficial) } \\
\text { (Europe) }\end{array}$ & Multicenter & $\begin{array}{l}\text { Industry } \\
\text { funded }\end{array}$ & $\begin{array}{l}>=18 \text { y/o, primary } \\
\text { tumour, } \\
\text { histologically } \\
\text { confirmed superficial } \\
\text { or nodular BCC, } \\
\text { area } 0.5 \mathrm{~cm}^{\wedge} 2-2.0 \\
\mathrm{~cm}^{\wedge} 2 \text { for superficial } \\
\text { or } 0.25 \mathrm{~cm}^{\wedge} 2-1.5 \\
\mathrm{~cm}^{\wedge} 2 \text { for nodular }\end{array}$ & $\begin{array}{l}\text { previous therapy to the target } \\
\text { tumour or any dermatological } \\
\text { conditions that would } \\
\text { interfere with local } \\
\text { assessments. }\end{array}$ & $\begin{array}{l}\text { Biopsy/pathologic } \\
\text { confirmed: } \\
\text { prestudy } \\
\text { confirmatory } \\
\text { punch, deep } \\
\text { shave, or wedge } \\
\text { biopsy that } \\
\text { removed no more } \\
\text { than approximately } \\
25 \% \text { of the tumour }\end{array}$ & $\begin{array}{l}\text { Visual } \\
\text { assessment: } \\
\text { measuring and } \\
\text { multiplying the } \\
\text { two largest } \\
\text { perpendicular } \\
\text { dimensions of } \\
\text { the tumour. The } \\
\text { tumour site and } \\
\text { appropriate } \\
\text { anatomic } \\
\text { landmarks were } \\
\text { mapped using a } \\
\text { clear plastic } \\
\text { sheet as a } \\
\text { template to guide } \\
\text { the excision at } \\
\text { the end of the } \\
\text { study }\end{array}$ & $183 / 177$ \\
\hline $\begin{array}{l}\text { Szeimies } 2008 \\
18624836 \text { (United } \\
\text { Kingdom/Germany/S } \\
\text { witzerland/Australia) }\end{array}$ & Multicenter & $\begin{array}{l}\text { Industry } \\
\text { funded }\end{array}$ & $\begin{array}{l}\text { >= } 18 \text { y/o; primary } \\
\text { sBCC suitable for } \\
\text { simple excision } \\
\text { surgery; confirmed } \\
\text { by histology; no } \\
\text { histological evidence } \\
\text { of aggressive growth } \\
\text { patterns }\end{array}$ & $\begin{array}{l}>5 \text { eligible lesions; lesions } \\
\text { located in nose, nasolabial, } \\
\text { or orbial areas; lesions w/ } \varnothing \\
<8 \mathrm{~mm} \text { or }>20 \mathrm{~mm} \text {; recurrent } \\
\text { lesions; lesions located in } \\
\text { severely sun-damaged skin } \\
\text { where surgery was not } \\
\text { suitable due to frequent } \\
\text { recurrence/ occurrence of } \\
\text { other BCCs in the same area; } \\
\text { lesions located close to or } \\
\text { involving a scar of SCC; } \\
\text { pigmented, morpheaform or } \\
\text { infiltrating lesions on the } \\
\text { treated area; at risk in terms } \\
\text { of precautions, warnings, and }\end{array}$ & $\begin{array}{l}\text { Biopsy/pathologic } \\
\text { confirmed: biopsy } \\
\text { at screening }\end{array}$ & $\begin{array}{l}\text { Method of } \\
\text { assessment not } \\
\text { reported }\end{array}$ & $196 / 196 / 196$ \\
\hline
\end{tabular}




\begin{tabular}{|c|c|c|c|c|c|c|c|}
\hline $\begin{array}{l}\text { Author Year } \\
\text { PMID } \\
\text { (Country/Region) }\end{array}$ & $\begin{array}{l}\text { Number } \\
\text { of } \\
\text { centers }\end{array}$ & Funding & $\begin{array}{l}\text { Inclusion } \\
\text { criteria* }\end{array}$ & Exclusion criteria* & $\begin{array}{l}\text { Method of } \\
\text { diagnosis }\end{array}$ & $\begin{array}{l}\text { Preoperative } \\
\text { tumor size } \\
\text { assessment }\end{array}$ & $\begin{array}{l}\mathrm{N} \text { enrolled } / \\
\text { randomized } / \\
\text { analyzed }\end{array}$ \\
\hline & & & & $\begin{array}{l}\text { contraindications as indicated } \\
\text { in MAL-PDT package insert; } \\
\text { pregnant or breastfeeding } \\
\text { women. }\end{array}$ & & & \\
\hline $\begin{array}{l}\text { Thissen } 2000 \\
10940063 \\
\text { (Netherlands) }\end{array}$ & $\begin{array}{l}\text { Single } \\
\text { center }\end{array}$ & $\begin{array}{l}\text { No industry } \\
\text { support }\end{array}$ & $\begin{array}{l}\text { superficial or nodular } \\
\text { BCCs, clinically }<2 \\
\mathrm{~cm} \varnothing \text {, localized } \\
\text { anywhere in the } \\
\text { head and neck area }\end{array}$ & $\begin{array}{l}\text { recurrent BCCs, histologic } \\
\text { subtypes not nodular or } \\
\text { superficial, }>2 \mathrm{~cm} \varnothing,>=5 \\
\text { BCCs, and contraindications } \\
\text { to surgery or cryosurgery (eg, } \\
\text { cold intolerance). LE }<1 \text { yr. }\end{array}$ & $\begin{array}{l}\text { Method of } \\
\text { diagnosis not } \\
\text { reported }\end{array}$ & $\begin{array}{l}\text { Visual } \\
\text { assessment: } \\
\text { Before treatment, } \\
\text { the tumors were } \\
\text { documented with } \\
\text { photographs } \\
\end{array}$ & 96/96/96 \\
\hline $\begin{array}{l}\text { Torres } 2004 \\
15606733 \text { (loma } \\
\text { linda, CA; boston, } \\
\text { MA) }\end{array}$ & Multicenter & $\begin{array}{l}\text { Industry } \\
\text { funded }\end{array}$ & $\begin{array}{l}\text { biopsy proven BCC; } \\
<=25 \% \text { of the lesion } \\
\text { removed at time of } \\
\text { biopsy. } 18 \text { y/o, } \\
\text { histologically } \\
\text { confirmed, primary, } \\
\text { superficial, nodular, } \\
\text { or mixed superficial } \\
\text { and nodular BCC. } \\
\text { Target tumor } \\
\text { consistent w/ BCC } \\
\text { w/ no histologic } \\
\text { evidence of } \\
\text { aggressive growth } \\
\text { patterns, including } \\
\text { severe squamous } \\
\text { metaplasia, } \\
\text { morpheaform or } \\
\text { infiltrative/desmopla } \\
\text { stic features, or } \\
\text { basosquamous } \\
\text { features, and } \\
\text { suitable for } \\
\text { treatment with Mohs. } \\
\text { area >=0.5 cm2 and } \\
\varnothing<2.0 \mathrm{~cm} \text { and could } \\
\text { be located on an } \\
\text { acceptable area of } \\
\text { the body as } \\
\text { determined by the } \\
\text { investigator. }\end{array}$ & $\begin{array}{l}\text { previous therapy to the target } \\
\text { tumor or dermatologic } \\
\text { conditions that could interfere } \\
\text { with skin assessments. }\end{array}$ & $\begin{array}{l}\text { Biopsy/pathologic } \\
\text { confirmed }\end{array}$ & $\begin{array}{l}\text { Visual } \\
\text { assessment: use } \\
\text { of tattoo in center } \\
\text { of lesion }\end{array}$ & $72 / 72 / 69$ \\
\hline
\end{tabular}




\begin{tabular}{|c|c|c|c|c|c|c|c|}
\hline $\begin{array}{l}\text { Author Year } \\
\text { PMID } \\
\text { (Country/Region) }\end{array}$ & $\begin{array}{l}\text { Number } \\
\text { of } \\
\text { centers }\end{array}$ & Funding & $\begin{array}{l}\text { Inclusion } \\
\text { criteria* }\end{array}$ & Exclusion criteria* & $\begin{array}{l}\text { Method of } \\
\text { diagnosis }\end{array}$ & $\begin{array}{l}\text { Preoperative } \\
\text { tumor size } \\
\text { assessment }\end{array}$ & $\begin{array}{l}\mathrm{N} \text { enrolled } / \\
\text { randomized } / \\
\text { analyzed }\end{array}$ \\
\hline $\begin{array}{l}\text { Tran } 201222511036 \\
\text { (US) }\end{array}$ & $\begin{array}{l}\text { Single } \\
\text { center }\end{array}$ & Not reported & $\begin{array}{l}\text { Caucasian, } \\
\text { Fitzpatrick skin type } \\
\text { I or II, } 46-84 \text { y/o. } \\
\text { Superficial, nodular, } \\
\text { multicentric BCCs, } \\
\text { and SCCIS 0.4-3 } \\
\mathrm{cm}\end{array}$ & $\begin{array}{l}\text { Morpheaform, infiltrating, and } \\
\text { recurrent BCCs and invasive } \\
\text { SCCs or lesions on the head } \\
\text { and neck, hands, feet, and } \\
\text { genital areas. }\end{array}$ & $\begin{array}{l}\text { Biopsy/pathologic } \\
\text { confirmed }\end{array}$ & $\begin{array}{l}\text { Visual } \\
\text { assessment }\end{array}$ & $20 / 20 / 20$ \\
\hline $\begin{array}{l}\text { van der Geer } 2012 \\
22385074 \\
\text { (Netherlands) }\end{array}$ & $\begin{array}{l}\text { Single } \\
\text { center }\end{array}$ & $\begin{array}{l}\text { No industry } \\
\text { support }\end{array}$ & $\begin{array}{l}>18 \text { y/0ears w/ } \\
\text { nodular (or nodular } \\
\text { and partially } \\
\text { superficial) BCC } 1-5 \\
\mathrm{~cm} \varnothing \text { in the face }\end{array}$ & $\begin{array}{l}\text { pregnant women, women } \\
\text { who were breastfeeding, } \\
\text { recurrent BCC, aggressive } \\
\text { growth pattern, pts w/ BCC } \\
\text { w/in } 1 \mathrm{~cm} \text { from the eyes, lips } \\
\text { or mucosa of the nose, } \\
\text { another skin tumour w/in } 5 \\
\mathrm{~cm} \text { of the target tumour, and } \\
\text { allergy to imiquimod } 5 \% \\
\text { cream or components of the } \\
\text { cream }\end{array}$ & $\begin{array}{l}\text { Biopsy/pathologic } \\
\text { confirmed }\end{array}$ & $\begin{array}{l}\text { Phtotgraphy and } \\
\text { computer } \\
\text { assesment }\end{array}$ & $70 / 70 / 70$ \\
\hline $\begin{array}{l}\text { Wang } 2001 \\
11298545 \text { (England) }\end{array}$ & $\begin{array}{l}\text { Single } \\
\text { center }\end{array}$ & $\begin{array}{l}\text { Industry } \\
\text { funded }\end{array}$ & $\begin{array}{l}\text { histopathologically } \\
\text { verified BCC } \\
\text { suitable for PDT and } \\
\text { cryosurgery, 20-90 } \\
\text { y/o }\end{array}$ & $\begin{array}{l}\text { pregnancy/lactation; severe } \\
\text { malignancies; daily intake of } \\
\text { vitamins E or C, b-carotene, } \\
\text { iron preparations, non- } \\
\text { steroidal anti-inflammatory } \\
\text { agents or strong analgesics } \\
\text { in higher than specified } \\
\text { doses; BCC on the nose; } \\
\text { morphoeic growth; porphyria; } \\
\text { abdominal pain of unknown } \\
\text { aetiology; } \\
\text { photosensitivity;and } \\
\text { treatment of the BCC with } \\
\text { topical steroids type III or IV } \\
\text { within the last month. }\end{array}$ & $\begin{array}{l}\text { Biopsy/pathologic } \\
\text { confirmed }\end{array}$ & $\begin{array}{l}\text { Method of } \\
\text { assessment not } \\
\text { reported }\end{array}$ & $88 / 88 / 83$ \\
\hline $\begin{array}{l}\text { Wettstein } 2013 \\
23566745 \\
\text { (Switzerland) }\end{array}$ & $\begin{array}{l}\text { Single } \\
\text { center }\end{array}$ & $\begin{array}{l}\text { Industry } \\
\text { supplied } \\
\text { materials }\end{array}$ & $\begin{array}{l}\text { diagnosed clinically } \\
\text { or by biopsy wl } \\
\text { primary nodular } \\
\text { BCC of the face } \\
\text { presenting at the } \\
\text { University Hospital } \\
\text { Basel between June } \\
2007 \text { and February }\end{array}$ & $\begin{array}{l}\text { patients under steroid } \\
\text { medication or } \\
\text { immunosuppressive therapy; } \\
\text { patients with direct defect } \\
\text { closure; pathological analysis } \\
\text { revealed incomplete tumour } \\
\text { resection or another BCC } \\
\text { sub-type than solid/nodular }\end{array}$ & $\begin{array}{l}\text { Biopsy/pathologic } \\
\text { confirmed }\end{array}$ & $\begin{array}{l}\text { Confocal } \\
\text { assessment }\end{array}$ & $32 / 23 / 23$ \\
\hline
\end{tabular}




\begin{tabular}{|c|c|c|c|c|c|c|c|}
\hline $\begin{array}{l}\text { Author Year } \\
\text { PMID } \\
\text { (Country/Region) }\end{array}$ & $\begin{array}{l}\text { Number } \\
\text { of } \\
\text { centers }\end{array}$ & Funding & $\begin{array}{l}\text { Inclusion } \\
\text { criteria* }\end{array}$ & Exclusion criteria* & $\begin{array}{l}\text { Method of } \\
\text { diagnosis }\end{array}$ & $\begin{array}{l}\text { Preoperative } \\
\text { tumor size } \\
\text { assessment }\end{array}$ & $\begin{array}{l}\mathrm{N} \text { enrolled } / \\
\text { randomized } / \\
\text { analyzed }\end{array}$ \\
\hline & & & 2008 & & & & \\
\hline \multicolumn{8}{|l|}{ NRCS } \\
\hline $\begin{array}{l}\text { Ahmed } 2000 \\
11069453 \text { (UK) }\end{array}$ & Multicenter & Not reported & $\begin{array}{l}\text { clinical diagnosis of } \\
\text { Bowen's Disease }\end{array}$ & $\begin{array}{l}\text { Patients with recurrent } \\
\text { lesions and those on } \\
\text { immunosuppression }\end{array}$ & $\begin{array}{l}\text { Biopsy/pathologic } \\
\text { confirmed: biopsy- } \\
\text { proven }\end{array}$ & $\begin{array}{l}\text { Method of } \\
\text { assessment not } \\
\text { reported }\end{array}$ & $73 / 67$ \\
\hline $\begin{array}{l}\text { Ballester-Sanchez } \\
201626985197 \\
\text { (Spain) }\end{array}$ & $\begin{array}{l}\text { Single } \\
\text { center }\end{array}$ & $\begin{array}{l}\text { Industry } \\
\text { funded }\end{array}$ & $\begin{array}{l}\text { adults, primary } \\
\text { superficial or nodular } \\
\text { BCC w/ T1 and T2 } \\
\text { clinical stage }\end{array}$ & $\begin{array}{l}\varnothing>20 \mathrm{~mm}, \text { depth }>4 \mathrm{~mm}, \text { or } \\
\text { located on irregular surfaces }\end{array}$ & $\begin{array}{l}\text { Biopsy/pathologic } \\
\text { confirmed: } \\
\text { histopathologic } \\
\text { examination }\end{array}$ & $\begin{array}{l}\text { Visual } \\
\text { assessment: } \\
\text { clinically aided by } \\
\text { dermoscope } \\
\end{array}$ & $40 / 40$ \\
\hline $\begin{array}{l}\text { Chren } 2013 \\
23190903 \text { (U.S.) }\end{array}$ & Multicenter & $\begin{array}{l}\text { No industry } \\
\text { support }\end{array}$ & $\begin{array}{l}\text { consecutive patients } \\
\text { with nonrecurrent } \\
\text { NMSC diagnosed in } \\
1999 \text { and } 2000 \text { and } \\
\text { treated in } 2 \text { sites, a } \\
\text { university-affiliated } \\
\text { private dermatology } \\
\text { practice and the } \\
\text { dermatology clinic at } \\
\text { the nearby VA } \\
\text { medical center } \\
\text { affiliated with the } \\
\text { university }\end{array}$ & & $\begin{array}{l}\text { Biopsy/pathologic } \\
\text { confirmed: } \\
\text { Biopsies were } \\
\text { performed either } \\
\text { by dermatology } \\
\text { faculty members or } \\
\text { by dermatology } \\
\text { residents }\end{array}$ & $\begin{array}{l}\text { Method of } \\
\text { assessment not } \\
\text { reported }\end{array}$ & $1253 / 1174$ \\
\hline $\begin{array}{l}\text { Cosgarea } 2012 \\
22738399 \\
\text { (Romania) }\end{array}$ & $\begin{array}{l}\text { Single } \\
\text { center }\end{array}$ & $\begin{array}{l}\text { No industry } \\
\text { support }\end{array}$ & $\begin{array}{l}\text { Men or women }>18 \\
\text { y/o, clinically } \\
\text { diagnosised primary } \\
\text { BCC, superficial or } \\
\text { nodular BCC, with a } \\
\text { maximum } 3 \text { mm } \\
\text { above the skin level }\end{array}$ & $\begin{array}{l}\text { recurrent, pigmented or } \\
\text { morpheaform lesions; use of } \\
\text { phototoxic/photosensitive } \\
\text { drugs, hypersensitivity to light } \\
\text { or ALA cream, pregnant or } \\
\text { breastfeeding women }\end{array}$ & $\begin{array}{l}\text { Biopsy/pathologic } \\
\text { confirmed: } \\
\text { histologically } \\
\text { confirmed }\end{array}$ & $\begin{array}{l}\text { Method of } \\
\text { assessment not } \\
\text { reported }\end{array}$ & $72 / 72$ \\
\hline $\begin{array}{l}\text { Graells } 2014 \\
24139468 \text { (Spain) }\end{array}$ & $\begin{array}{l}\text { Single } \\
\text { center }\end{array}$ & Not reported & $\begin{array}{l}\text { patients treated for } \\
\text { their first BCC at the } \\
\text { hospital between } \\
\text { January } 2003 \text { and } \\
\text { December } 2011\end{array}$ & $\begin{array}{l}\text { patients followed for less than } \\
3 \text { months }\end{array}$ & $\begin{array}{l}\text { Biopsy/pathologic } \\
\text { confirmed: } \\
\text { histologically } \\
\text { confirmed BCCs }\end{array}$ & $\begin{array}{l}\text { Method of } \\
\text { assessment not } \\
\text { reported }\end{array}$ & $623 / 621$ \\
\hline $\begin{array}{l}\text { Lippert } 2013 \\
23725586 \text { (Czech } \\
\text { Republic) }\end{array}$ & $\begin{array}{l}\text { Single } \\
\text { center }\end{array}$ & $\begin{array}{l}\text { No industry } \\
\text { support }\end{array}$ & $\begin{array}{l}\text { one confirmed } \\
\text { nBCC, and there } \\
\text { was one tested } \\
\text { nBCC per person, } \varnothing \\
20-30 \mathrm{~mm}\end{array}$ & $\begin{array}{l}\text { tumors in the middle portion } \\
\text { of the face and areas } \\
\text { adjacent to the eyes and ears }\end{array}$ & $\begin{array}{l}\text { Biopsy/pathologic } \\
\text { confirmed: Verified } \\
\text { by biopsy sample } \\
\text { from the peripheral } \\
\text { portion of the } \\
\text { tumor, which was }\end{array}$ & $\begin{array}{l}\text { Other: thickness } \\
\text { measured using } \\
\text { high-resolution } \\
\text { ultrasound }\end{array}$ & $56 / 56$ \\
\hline
\end{tabular}




\begin{tabular}{|c|c|c|c|c|c|c|c|}
\hline $\begin{array}{l}\text { Author Year } \\
\text { PMID } \\
\text { (Country/Region) }\end{array}$ & $\begin{array}{l}\text { Number } \\
\text { of } \\
\text { centers }\end{array}$ & Funding & $\begin{array}{l}\text { Inclusion } \\
\text { criteria* }\end{array}$ & Exclusion criteria* & $\begin{array}{l}\text { Method of } \\
\text { diagnosis }\end{array}$ & $\begin{array}{l}\text { Preoperative } \\
\text { tumor size } \\
\text { assessment }\end{array}$ & $\begin{array}{l}\mathrm{N} \text { enrolled } / \\
\text { randomized } / \\
\text { analyzed }\end{array}$ \\
\hline & & & & & $\begin{array}{l}\text { as small as } \\
\text { possible so that } \\
\text { the area intended } \\
\text { for the experiment } \\
\text { was not reduced, }\end{array}$ & & \\
\hline $\begin{array}{l}\text { Pampena } 2016 \\
26589877 \text { (Italy) }\end{array}$ & $\begin{array}{l}\text { Single } \\
\text { center }\end{array}$ & $\begin{array}{l}\text { No industry } \\
\text { support }\end{array}$ & $\begin{array}{l}\text { Histologically } \\
\text { verified NMSC }\end{array}$ & $\begin{array}{l}\text { lymphatic or visceral } \\
\text { metastases }\end{array}$ & $\begin{array}{l}\text { Biopsy/pathologic } \\
\text { confirmed: } \\
\text { histologically } \\
\text { confirmed }\end{array}$ & $\begin{array}{l}\text { Method of } \\
\text { assessment not } \\
\text { reported }\end{array}$ & $385 / 385$ \\
\hline $\begin{array}{l}\text { Shah } 2009 \\
19588534 \text { (U.S.) }\end{array}$ & $\begin{array}{l}\text { Single } \\
\text { center }\end{array}$ & $\begin{array}{l}\text { No industry } \\
\text { support }\end{array}$ & $\begin{array}{l}\text { male patients w/ } \\
\text { biopsy-proven BCCs } \\
\text { on the trunk and } \\
\text { extremities }\end{array}$ & $\begin{array}{l}\text { Morpheaform, infiltrative, and } \\
\text { recurrent BCCs }\end{array}$ & $\begin{array}{l}\text { Biopsy/pathologic } \\
\text { confirmed: biopsy- } \\
\text { proven }\end{array}$ & $\begin{array}{l}\text { Method of } \\
\text { assessment not } \\
\text { reported }\end{array}$ & $32 / 32$ \\
\hline $\begin{array}{l}\text { Sofen } 2015 \\
25913533 \text { (U.S.) }\end{array}$ & Multicenter & $\begin{array}{l}\text { Industry } \\
\text { funded }\end{array}$ & $\begin{array}{l}\text { >=21 y/o, new, } \\
\text { operable, biopsy- } \\
\text { confirmed, nodular } \\
\text { BCC and willing to } \\
\text { delay excision }\end{array}$ & & $\begin{array}{l}\text { Biopsy/pathologic } \\
\text { confirmed: biopsy- } \\
\text { confirmed }\end{array}$ & $\begin{array}{l}\text { Method of } \\
\text { assessment not } \\
\text { reported }\end{array}$ & $74 / 49$ \\
\hline $\begin{array}{l}\text { Sullivan } 2003 \\
14725659 \text { (US) }\end{array}$ & $\begin{array}{l}\text { Single } \\
\text { center }\end{array}$ & Not reported & $\begin{array}{l}\text { biopsy confirmed } \\
\text { superficial BCC, } \varnothing \\
0.8-2.0 \mathrm{~cm} \text { on the } \\
\text { neck, trunk, or limbs. }\end{array}$ & $\begin{array}{l}\text { recurrent or previously } \\
\text { treated tumors or tumors } \\
\text { located on the head }\end{array}$ & $\begin{array}{l}\text { Biopsy/pathologic } \\
\text { confirmed }\end{array}$ & $\begin{array}{l}\text { Method of } \\
\text { assessment not } \\
\text { reported }\end{array}$ & $12 / 12$ \\
\hline $\begin{array}{l}\text { Wilson } 2012 \\
22145798 \text { (U.S.) }\end{array}$ & Multicenter & $\begin{array}{l}\text { No industry } \\
\text { support }\end{array}$ & $\begin{array}{l}\text { NMSCs identified by } \\
\text { daily review of } \\
\text { pathology records } \\
\text { and defined } \\
\text { according to final } \\
\text { histopathologic } \\
\text { diagnosis of BCC or } \\
\text { SCC. }\end{array}$ & $\begin{array}{l}\text { No "recurrent" or "possibly } \\
\text { recurrent" skin cancers }\end{array}$ & $\begin{array}{l}\text { Biopsy/pathologic } \\
\text { confirmed }\end{array}$ & $\begin{array}{l}\text { Method of } \\
\text { assessment not } \\
\text { reported }\end{array}$ & $1777 / 1777$ \\
\hline
\end{tabular}




\section{Appendix D. Baselines}

Table D-1. Baselines

\begin{tabular}{|c|c|c|c|c|c|c|c|}
\hline $\begin{array}{l}\text { Author Year } \\
\text { PMID }\end{array}$ & Arm & $\begin{array}{l}\text { Age, mean } \\
\text { (SD); range }\end{array}$ & $\begin{array}{l}\text { Lesion area, } \\
\text { mean (SD); } \\
\text { range }\end{array}$ & $\begin{array}{l}\text { Female, } \\
\%\end{array}$ & $\begin{array}{l}\text { Fitzpatrick } \\
\text { score \% }\end{array}$ & Lesion type (\%) & Lesion location (\%) \\
\hline \multicolumn{8}{|l|}{ RCT } \\
\hline Abbade 2015 & $\begin{array}{l}\text { Surgical } \\
\text { excision }\end{array}$ & NR & & NR & & BCC: nodular (100) & head and neck (100) \\
\hline Abbade 2015 & MAL-PDT & NR & & MR & & BCC: nodular (100) & head and neck (100) \\
\hline $\begin{array}{l}\text { Al-Niaimi } 2015 \\
26157307\end{array}$ & $\mathrm{PDT}+\mathrm{MMS}$ & $\begin{array}{l}61.4(\mathrm{NR}) ; \\
\text { range }(44,84)\end{array}$ & $\begin{array}{l}200 \mathrm{~mm} 2 ; \text { range } \\
(100-459)\end{array}$ & 66.7 & & BCC: nodular (100) & face (100) \\
\hline $\begin{array}{l}\text { Al-Niaimi } 2015 \\
26157307\end{array}$ & MMS & $\begin{array}{l}62.7(\mathrm{NR}) ; \\
\text { range }(41,89)\end{array}$ & $\begin{array}{l}201 \mathrm{~mm} 2 ; \text { range } \\
(120,408)\end{array}$ & 40 & & BCC: nodular (100) & face (100) \\
\hline Allen 1979298425 & cryotherapy & NR & & NR & & BCC: unspecified (100) & NR \\
\hline Allen 1979298425 & radiotherapy & NR & & NR & & BCC: unspecified (100) & NR \\
\hline $\begin{array}{l}\text { Alpsoy } 1996 \\
8708151\end{array}$ & IFN alfa-2a & $\begin{array}{l}58.7(\mathrm{NR}) ; \\
\text { range }(48,73)\end{array}$ & $\begin{array}{l}\text { median } 2.05 \mathrm{~cm} 2 \\
\text { range }(0.5,8.75)\end{array}$ & 53 & & $\begin{array}{l}\text { BCC: superficial(14), } \\
\text { nodular (79), } \\
\text { morphealike (7) }\end{array}$ & $\begin{array}{l}\text { eyelid (27), nose (13), } \\
\text { zygoma (27), forehead } \\
(13), \text { cheek (13), trunk (7) }\end{array}$ \\
\hline $\begin{array}{l}\text { Alpsoy } 1996 \\
8708151\end{array}$ & IFN alfa-2b & $\begin{array}{l}63.6(\mathrm{NR}) ; \\
\text { range }(38,70)\end{array}$ & $\begin{array}{l}\text { median } 1.82 \mathrm{~cm} 2 \\
\text { range }(0.6,8.2)\end{array}$ & 53 & & $\begin{array}{l}\text { BCC: superficial(7), } \\
\text { nodular (86), } \\
\text { morphealike (7) }\end{array}$ & $\begin{array}{l}\text { eyelid (20), nose (7), } \\
\text { zygoma (20), forehead } \\
(20), \text { cheek (27), trunk (7) }\end{array}$ \\
\hline $\begin{array}{l}\text { Alpsoy } 1996 \\
8708151 \\
\end{array}$ & $\begin{array}{l}\text { IFN alfa-2a } \\
+ \text { IFN alfa- } \\
2 \mathrm{~b}\end{array}$ & $\begin{array}{l}60.3(\mathrm{NR}) \\
\text { range }(39,74)\end{array}$ & $\begin{array}{l}\text { median } 1.9 \mathrm{~cm} 2 \\
\text { range }(0.5,8.9)\end{array}$ & 40 & & $\begin{array}{l}\text { BCC: superficial(7), } \\
\text { nodular (79), } \\
\text { morphealike (14) }\end{array}$ & $\begin{array}{l}\text { eyelid (20), nose (13), } \\
\text { zygoma (27), forehead } \\
(13), \text { cheek (20), trunk (7) }\end{array}$ \\
\hline Arits 201323683751 & MAL-PDT & $\begin{array}{l}\text { median } 63 ; \\
\text { range }(26,87)\end{array}$ & & 52 & & BCC: superficial (100) & $\begin{array}{l}\text { head/neck excluding H- } \\
\text { zone (12), extremities } \\
\text { (29), trunk (59), upper } \\
\text { extremities (16), lower } \\
\text { extremities (13) }\end{array}$ \\
\hline Arits 201323683751 & Imiquimod & $\begin{array}{l}\text { median } 62 ; \\
\text { range }(30,91)\end{array}$ & & 49 & & BCC: superficial (100) & $\begin{array}{l}\text { head/neck excluding } \mathrm{H}- \\
\text { zone (12), extremities } \\
\text { (27), trunk (61), upper } \\
\text { extremities (13), lower } \\
\text { extremities (14) }\end{array}$ \\
\hline Arits 201323683751 & Fluorouacil & $\begin{array}{l}\text { median } 64 ; \\
\text { range }(35,86)\end{array}$ & & 47 & & BCC: superficial (100) & $\begin{array}{l}\text { head/neck excluding } \mathrm{H}- \\
\text { zone (15), extremities } \\
\text { (24), trunk (60), upper } \\
\text { extremities (13), lower } \\
\text { extremities (11) }\end{array}$ \\
\hline Avril 19979218740 & surgery & $66.5(12.6)$ & diameter: 11.1 & 54 & & BCC: superficial (21), & nose (53), cheek, pre- and \\
\hline
\end{tabular}




\begin{tabular}{|c|c|c|c|c|c|c|c|}
\hline $\begin{array}{l}\text { Author Year } \\
\text { PMID }\end{array}$ & Arm & $\begin{array}{l}\text { Age, mean } \\
\text { (SD); range }\end{array}$ & $\begin{array}{l}\text { Lesion area, } \\
\text { mean (SD); } \\
\text { range }\end{array}$ & $\begin{array}{l}\text { Female, } \\
\%\end{array}$ & $\begin{array}{l}\text { Fitzpatrick } \\
\text { score } \%\end{array}$ & Lesion type (\%) & Lesion location (\%) \\
\hline & & & $\mathrm{mm}(5.7)$ & & & $\begin{array}{l}\text { ulcerated (30), nodular } \\
\text { (45), sclerosing (4) }\end{array}$ & $\begin{array}{l}\text { retroauricular areas (21), } \\
\text { eyelids, internal and } \\
\text { external eye angles (19), } \\
\text { forehead, temple, } \\
\text { between eyebrows } 36 \\
\text { (21), chin, cutaneous } \\
\text { superior lip } 10 \text { (6), ear (3) }\end{array}$ \\
\hline Avril 19979218740 & radiotherapy & $65,4(11.5)$ & $\begin{array}{l}\text { diameter: } 11.7 \\
(5.7)\end{array}$ & 46 & & $\begin{array}{l}\text { BCC: superficial (23), } \\
\text { ulcerated ( } 29), \text { nodular } \\
\text { (43), sclerosing (5) }\end{array}$ & $\begin{array}{l}\text { nose (28), cheek, pre- and } \\
\text { retroauricular areas (24), } \\
\text { eyelids, internal and } \\
\text { external eye angles (20), } \\
\text { forehead, temple, } \\
\text { between eyebrows (17), } \\
\text { chin, cutaneous superior } \\
\text { lip (7), ear (3) }\end{array}$ \\
\hline $\begin{array}{l}\text { Basset-Seguin } 2008 \\
18693158\end{array}$ & MAL-PDT & $\begin{array}{l}62(\mathrm{NR}) ; \text { range } \\
(25,86)\end{array}$ & & 33 & $\begin{array}{l}\text { I 5; II 57; III } \\
33 ; \text { IV } 5\end{array}$ & BCC: superficial (100) & $\begin{array}{l}\text { face/scalp (6), extremities } \\
(22), \text { trunk/neck (72) }\end{array}$ \\
\hline $\begin{array}{l}\text { Basset-Seguin } 2008 \\
18693158\end{array}$ & Cryotherapy & $\begin{array}{l}64 \text { (NR); range } \\
(38,90)\end{array}$ & & 47 & $\begin{array}{l}\text { I 5; II 63; III } \\
30 ; \text { IV } 2\end{array}$ & BCC: superficial (100) & $\begin{array}{l}\text { face/scalp (4), extremities } \\
(20), \text { trunk/neck (76) }\end{array}$ \\
\hline $\begin{array}{l}\text { Bath-Hextall } 2014 \\
24332516\end{array}$ & Imiquimod & NR & $\begin{array}{l}\text { diameter: median } \\
12 \mathrm{~mm} \text { (IQR 9, } \\
\text { 16) }\end{array}$ & 41 & $\begin{array}{l}\text { I 14; II 37; III } \\
42 ; \text { IV } 6\end{array}$ & $\begin{array}{l}\text { BCC: superficial (52), } \\
\text { nodular (48) }\end{array}$ & $\begin{array}{l}\text { face (37), trunk (38), neck } \\
\text { (6), arm (6), leg (10), other } \\
\text { (3) }\end{array}$ \\
\hline $\begin{array}{l}\text { Bath-Hextall } 2014 \\
24332516\end{array}$ & excision & NR & $\begin{array}{l}\text { diameter: median } \\
10 \text { mm (IQR 8, } \\
\text { 15) }\end{array}$ & 40 & $\begin{array}{l}\text { I 13; II 46; III } \\
35 ; \text { IV } 6\end{array}$ & $\begin{array}{l}\text { BCC: superficial (50), } \\
\text { nodular }(50)\end{array}$ & $\begin{array}{l}\text { face (33), trunk (39), neck } \\
\text { (9), arm (7), leg (9), other } \\
\text { (3) }\end{array}$ \\
\hline $\begin{array}{l}\text { Berroeta } 2007 \\
17573890\end{array}$ & Total & $\begin{array}{l}\text { median } 72 ; \\
\text { range }(50,89)\end{array}$ & & NR & & BCC: nodular (100) & NR \\
\hline $\begin{array}{l}\text { Beutner } 1999 \\
10570388\end{array}$ & $\begin{array}{l}\text { imiquimod } \\
\text { 2x/day }\end{array}$ & range $(37,81)$ & & NR & & $\begin{array}{l}\text { BCC: superficial (86), } \\
\text { nodular (14) }\end{array}$ & $\begin{array}{l}\text { upper exremity (57), } \\
\text { anterior upper trunk (14), } \\
\text { neck (29) }\end{array}$ \\
\hline $\begin{array}{l}\text { Beutner } 1999 \\
10570388\end{array}$ & $\begin{array}{l}\text { imiquimod } \\
\text { 1x/day }\end{array}$ & range $(37,81)$ & & NR & & $\begin{array}{l}\text { BCC: superficial (75), } \\
\text { nodular }(25)\end{array}$ & $\begin{array}{l}\text { upper extremity (50), } \\
\text { anterior upper trunk (25), } \\
\text { posterior upper trunk (25) }\end{array}$ \\
\hline $\begin{array}{l}\text { Beutner } 1999 \\
10570388\end{array}$ & $\begin{array}{l}\text { imiquimod } \\
\text { 3x/week }\end{array}$ & range $(37,81)$ & & NR & & BCC: superficial (100) & $\begin{array}{l}\text { upper extremity (25), } \\
\text { anterior upper trunk (25), } \\
\text { posterior upper trunk (25), } \\
\text { neck (25) }\end{array}$ \\
\hline $\begin{array}{l}\text { Beutner } 1999 \\
10570388\end{array}$ & $\begin{array}{l}\text { imiquimod } \\
\text { 2x/week }\end{array}$ & range $(37,81)$ & & NR & & $\begin{array}{l}\text { BCC: superficial (60), } \\
\text { nodular }(40)\end{array}$ & $\begin{array}{l}\text { lower extremity (20), } \\
\text { anterior upper trunk (40), } \\
\text { posterior upper trunk (20), } \\
\text { neck (20) }\end{array}$ \\
\hline
\end{tabular}




\begin{tabular}{|c|c|c|c|c|c|c|c|}
\hline $\begin{array}{l}\text { Author Year } \\
\text { PMID }\end{array}$ & Arm & $\begin{array}{l}\text { Age, mean } \\
\text { (SD); range }\end{array}$ & $\begin{array}{l}\text { Lesion area, } \\
\text { mean (SD); } \\
\text { range }\end{array}$ & $\begin{array}{l}\text { Female, } \\
\%\end{array}$ & $\begin{array}{l}\text { Fitzpatrick } \\
\text { score } \%\end{array}$ & Lesion type (\%) & Lesion location (\%) \\
\hline $\begin{array}{l}\text { Beutner } 1999 \\
10570388\end{array}$ & $\begin{array}{l}\text { imiquimod } \\
\text { 1x/week }\end{array}$ & range $(37,81)$ & & NR & & $\begin{array}{l}\text { BCC: superficial (50), } \\
\text { nodular }(50)\end{array}$ & $\begin{array}{l}\text { lower extremity (50), } \\
\text { anterior upper trunk (25), } \\
\text { posterior upper trunk (25) }\end{array}$ \\
\hline $\begin{array}{l}\text { Beutner } 1999 \\
10570388\end{array}$ & $\begin{array}{l}\text { vehicle (3 } \\
2 x / \text { day, } 2 \\
1 x / \text { day, } 2 \\
3 x / \text { week, } 2 \\
2 x / \text { week, } 2 \\
1 x / \text { week) }\end{array}$ & range $(37,81)$ & & NR & & $\begin{array}{l}\text { BCC: superficial (91), } \\
\text { nodular (9) }\end{array}$ & $\begin{array}{l}\text { face (9), upper extremity } \\
(46) \text {, anterior upper trunk } \\
\text { (9), neck (9), posterior } \\
\text { lower trunk (27) }\end{array}$ \\
\hline $\begin{array}{l}\text { Brinkhuizen } 2016 \\
27067393\end{array}$ & $\begin{array}{l}\text { Diclofenac } \\
\text { (results } \\
\text { superficial/n } \\
\text { odular) }\end{array}$ & $\begin{array}{l}63.0 / 78.5(\mathrm{NR}) \\
\text { range }(54,82)\end{array}$ & $\begin{array}{l}61.7 / 49.5 \mathrm{~mm} 2 \\
\text { range }(30.0,84.4)\end{array}$ & 25 & & $\begin{array}{l}\text { BCC: superficial (50), } \\
\text { nodular }(50)\end{array}$ & $\begin{array}{l}\text { extremities (47), } \\
\text { trunk/neck (53) }\end{array}$ \\
\hline $\begin{array}{l}\text { Brinkhuizen } 2016 \\
27067393\end{array}$ & $\begin{array}{l}\text { Calcitriol } \\
\text { (results } \\
\text { superficial/n } \\
\text { odular) } \\
\end{array}$ & $\begin{array}{l}65.5 / 68.5(\mathrm{NR}) \\
\text { range }(55,75)\end{array}$ & $\begin{array}{l}54.2 / 59.7 \mathrm{~mm} 2 \\
\text { range }(34.3,87.6)\end{array}$ & 22 & & $\begin{array}{l}\text { BCC: superficial }(50), \\
\text { nodular ( } 41) \text {; } \\
\text { micronodular or mixed } \\
(9)\end{array}$ & $\begin{array}{l}\text { trunk/neck (59), genetalia } \\
\text { (41) }\end{array}$ \\
\hline $\begin{array}{l}\text { Brinkhuizen } 2016 \\
27067393\end{array}$ & $\begin{array}{l}\text { Diclofenac + } \\
\text { Calcitriol } \\
\text { (results } \\
\text { superficial/n } \\
\text { odular) }\end{array}$ & $\begin{array}{l}67.5 / 71(\mathrm{NR}) \\
\text { range }(60,79)\end{array}$ & $\begin{array}{l}46.7 / 44.8 \mathrm{~mm} 2 \\
\text { range }(33.0 \\
101.3)\end{array}$ & 37.5 & & $\begin{array}{l}\text { BCC: superficial (50), } \\
\text { nodular (50) }\end{array}$ & $\begin{array}{l}\text { trunk/neck (50), genetalia } \\
(44)\end{array}$ \\
\hline $\begin{array}{l}\text { Brinkhuizen } 2016 \\
27067393\end{array}$ & $\begin{array}{l}\text { No treatment } \\
\text { (results } \\
\text { superficial/n } \\
\text { odular) }\end{array}$ & $\begin{array}{l}61.5 / 66(\mathrm{NR}) \\
\text { range }(49,73)\end{array}$ & $\begin{array}{l}59.7 / 53.4 \mathrm{~mm} 2 ; \\
\text { range }(39.1,98.4)\end{array}$ & 37.5 & & $\begin{array}{l}\text { BCC: superficial (50), } \\
\text { nodular (44), } \\
\text { micronodular or mixed } \\
(6)\end{array}$ & $\begin{array}{l}\text { extremities (53), } \\
\text { trunk/neck (47) }\end{array}$ \\
\hline $\begin{array}{l}\text { Butler } 2009 \\
19018814\end{array}$ & $\begin{array}{l}\text { Vehicle } \\
\text { group+MOH } \\
\mathrm{s}\end{array}$ & $\begin{array}{l}75.3(11.4) ; \\
\text { range }(48,93)\end{array}$ & $\begin{array}{l}30.1 \mathrm{~mm} 2(9.5) ; \\
\text { range }(19.2,50.4)\end{array}$ & 43.8 & & BCC: nodular (100) & face (100) \\
\hline $\begin{array}{l}\text { Butler } 2009 \\
19018814\end{array}$ & $\begin{array}{l}\text { imiquimod } \\
5 \% \text { Cream } \\
\text { group+MOH } \\
\mathrm{s}\end{array}$ & $\begin{array}{l}73.3(10.5) ; \\
\text { range }(42,85)\end{array}$ & $\begin{array}{l}33.5 \mathrm{~mm} 2(12.8) \\
\text { range }(14.1,57.6)\end{array}$ & 66.7 & & BCC: nodular (100) & hands (100) \\
\hline Cai 201525899562 & $\begin{array}{l}\text { ALA-PDT + } \\
\text { CO2 Laser }\end{array}$ & NR & $\begin{array}{l}\text { diameter: } 2.62 \\
\mathrm{~cm}(0.94)\end{array}$ & 50 & & SCC: Bowen's (100) & NR \\
\hline Cai 201525899562 & CO2 Laser & NR & $\begin{array}{l}\text { diameter: } 2.58 \\
\mathrm{~cm}(0.86)\end{array}$ & 62.5 & & SCC: Bowen's (100) & NR \\
\hline $\begin{array}{l}\text { Carija } 2016 \\
27516420\end{array}$ & ALA-PDT & $\begin{array}{l}\text { Median } 71 \\
\text { (range } 55,78 \text { ) }\end{array}$ & $\begin{array}{l}255.4 \mathrm{~mm} 2 \\
(209.2)\end{array}$ & 13.3 & II and III 100 & $\begin{array}{l}\text { BCC: superficial (79), } \\
\text { nodular }(21)\end{array}$ & $\begin{array}{l}\text { Trunk/neck (96), } \\
\text { extremities (4) }\end{array}$ \\
\hline Carija 2016 & ALA-PDT + & Median 71 & $216 \mathrm{~mm} 2(154.3$ & 13.3 & II and III 100 & BCC: superficial (82), & Trunk/neck (76.5), \\
\hline
\end{tabular}




\begin{tabular}{|c|c|c|c|c|c|c|c|}
\hline $\begin{array}{l}\text { Author Year } \\
\text { PMID }\end{array}$ & Arm & $\begin{array}{l}\text { Age, mean } \\
\text { (SD); range }\end{array}$ & $\begin{array}{l}\text { Lesion area, } \\
\text { mean (SD); } \\
\text { range }\end{array}$ & $\begin{array}{l}\text { Female, } \\
\%\end{array}$ & $\begin{array}{l}\text { Fitzpatrick } \\
\text { score } \%\end{array}$ & Lesion type (\%) & Lesion location (\%) \\
\hline 27516420 & PDL & (range 55,78 ) & & & & nodular (18) & extremities (23.5) \\
\hline Choi 201626551044 & $\begin{array}{l}\text { Er:YAG } \\
\text { ablative } \\
\text { fractional } \\
\text { laser-primed } \\
\text { MAL- PDT }\end{array}$ & NR & & 55 & $\begin{array}{l}\text { III 15; IV 65; } \\
\text { V } 20\end{array}$ & BCC: nodular (100) & NR \\
\hline Choi 201626551044 & MAL-PDT & NR & & 36.8 & $\begin{array}{l}\text { III 10.5; IV } \\
74.7 ; \text { V } 15.8\end{array}$ & BCC: nodular (100) & NR \\
\hline Choi 201728199463 & $\begin{array}{l}\text { Er:YAG } \\
\text { ablative } \\
\text { fractional } \\
\text { laser-primed } \\
\text { MAL- PDT }\end{array}$ & $76.4(6.2)$ & $\begin{array}{l}\text { Diameter } 11.5 \\
\mathrm{~mm}(3.8)\end{array}$ & 71.4 & $\begin{array}{l}\text { III: } 28.6 ; \text { IV } \\
57.1 ; \mathrm{V}: 14.3\end{array}$ & $\begin{array}{l}\text { SCC: moderately } \\
\text { differentiated (81), } \\
\text { poorly differentiated } \\
\text { (19) }\end{array}$ & $\begin{array}{l}\text { Face/scalp (76.2), } \\
\text { extremities (19.0), } \\
\text { trunk/neck (4.8) }\end{array}$ \\
\hline Choi 201728199463 & MAL-PDT & $75.1(6.2)$ & $\begin{array}{l}\text { Diameter } 11.8 \\
\mathrm{~mm}(4.1)\end{array}$ & 54.6 & $\begin{array}{l}\text { III: 29.1, IV: } \\
54.2, \text { V: } 16.7\end{array}$ & $\begin{array}{l}\text { SCC: moderately } \\
\text { differentiated (75), } \\
\text { poorly differentiated } \\
(25)\end{array}$ & $\begin{array}{l}\text { Face/scalp (75.0), } \\
\text { extremities (16.7), } \\
\text { trunk/neck (8.3) }\end{array}$ \\
\hline $\begin{array}{l}\text { Cornell } 1990 \\
2229497\end{array}$ & interferon & 56 & $83 \mathrm{~mm} 2$ & 19 & & $\begin{array}{l}\text { BCC: superficial (46), } \\
\text { noduloulcerative (54) }\end{array}$ & $\begin{array}{l}\text { head and face (25), } \\
\text { extermities (12), } \\
\text { trunk/neck (63) }\end{array}$ \\
\hline $\begin{array}{l}\text { Cornell } 1990 \\
2229497\end{array}$ & placebo & 57 & $75 \mathrm{~mm} 2$ & 14 & & $\begin{array}{l}\text { BCC: superficial (45), } \\
\text { noduloulcerative (55) }\end{array}$ & $\begin{array}{l}\text { head and face (17), } \\
\text { extermities (14), } \\
\text { trunk/neck (59) }\end{array}$ \\
\hline $\begin{array}{l}\text { Edwards } 1990 \\
2107219\end{array}$ & $\begin{array}{l}\text { interferon } \\
\text { gamma, } \\
0.01\end{array}$ & range $(37,69)$ & & NR & & $\begin{array}{l}\text { BCC: superficial (47), } \\
\text { nodular (53) }\end{array}$ & NR \\
\hline $\begin{array}{l}\text { Edwards } 1990 \\
2107219\end{array}$ & $\begin{array}{l}\text { interferon } \\
\text { gamma, } \\
0.05 \\
\end{array}$ & range $(37,69)$ & & NR & & $\begin{array}{l}\text { BCC: superficial (57), } \\
\text { nodular (43) }\end{array}$ & NR \\
\hline $\begin{array}{l}\text { Edwards } 1990 \\
2383027\end{array}$ & $\begin{array}{l}\text { Interferon } \\
\text { alfa-2b, } 30 \\
\text { million IU }\end{array}$ & range $(35,65)$ & & NR & & $\begin{array}{l}\text { BCC: superficial (50), } \\
\text { nodular (50) }\end{array}$ & NR \\
\hline $\begin{array}{l}\text { Edwards } 1990 \\
2383027\end{array}$ & $\begin{array}{l}\text { Interferon } \\
\text { alfa-2b, } 10 \\
\text { million IU }\end{array}$ & range $(35,65)$ & & NR & & $\begin{array}{l}\text { BCC: superficial (50), } \\
\text { nodular }(50)\end{array}$ & NR \\
\hline $\begin{array}{l}\text { Eigentler } 2007 \\
17610993\end{array}$ & $\begin{array}{l}\text { imiquimod } \\
5 \% 8 \text { weeks }\end{array}$ & $\begin{array}{l}\text { median } 65 ; \\
\text { range }(38,88)\end{array}$ & $\begin{array}{l}\text { diameter: } 8.2 \\
\mathrm{~mm} \text {; median8.0 } \\
\mathrm{mm} \text {; range }(4,15)\end{array}$ & 27 & $\begin{array}{l}\text { II 51; III 44.4; } \\
\text { IV } 4.4\end{array}$ & BCC: nodular (100) & $\begin{array}{l}\text { face (24.4), scalp (2.2), } \\
\text { ear (8.9), trunk/neck (4.4), } \\
\text { perioral (4.4), periorbital } \\
(8.9), \text { nose }(42), \\
\text { arm/shoulder (4.4) }\end{array}$ \\
\hline
\end{tabular}




\begin{tabular}{|c|c|c|c|c|c|c|c|}
\hline $\begin{array}{l}\text { Author Year } \\
\text { PMID }\end{array}$ & Arm & $\begin{array}{l}\text { Age, mean } \\
\text { (SD); range }\end{array}$ & $\begin{array}{l}\text { Lesion area, } \\
\text { mean (SD); } \\
\text { range }\end{array}$ & $\begin{array}{l}\text { Female, } \\
\%\end{array}$ & $\begin{array}{l}\text { Fitzpatrick } \\
\text { score } \%\end{array}$ & Lesion type (\%) & Lesion location (\%) \\
\hline $\begin{array}{l}\text { Eigentler } 2007 \\
17610993\end{array}$ & $\begin{array}{l}\text { imiquimod } \\
5 \% 12 \\
\text { weeks }\end{array}$ & $\begin{array}{l}\text { median } 63 ; \\
\text { range }(39,79)\end{array}$ & $\begin{array}{l}\text { diameter: } 9.6 \\
\text { mm; median } 9.0 \text {; } \\
\text { range }(5,15)\end{array}$ & 33 & $\begin{array}{l}1 \text { 4.4;II 52.2; } \\
\text { III 41.3; IV } 2.2\end{array}$ & BCC: nodular (100) & $\begin{array}{l}\text { face (19.6), scalp (2.2), } \\
\text { ear (10.9), trunk/neck } \\
(8.7), \text { perioral }(2.2), \\
\text { periorbital }(6.5), \text { nose (37), } \\
\text { arm/shoulder (4.4), leg/hip } \\
(4.3)\end{array}$ \\
\hline Eimpunth 2014 & Total & range $(29,88)$ & & 33 & & BCC: unspecified (100) & NR \\
\hline $\begin{array}{l}\text { Foley } 2009 \\
20064185\end{array}$ & $\begin{array}{l}\text { methyl- } \\
\text { aminolevulin } \\
\text { atePDT }\end{array}$ & $\begin{array}{l}66(\mathrm{NR}) ; \text { range } \\
(28,88)\end{array}$ & $\begin{array}{l}\text { diameter: } 8.8 \\
\mathrm{~mm} \text {; range }(6,20)\end{array}$ & 28.78 & $\begin{array}{l}\text { I } 41 ; \text { II 39; III- } \\
\text { IV } 20\end{array}$ & BCC: nodular (100) & $\begin{array}{l}\text { face/scalp (25), } \\
\text { extremities (20), Trunk } 32 \\
(43 \%) \\
\text { Neck } 9(12 \%)\end{array}$ \\
\hline $\begin{array}{l}\text { Foley } 2009 \\
20064185\end{array}$ & placebo PDT & $\begin{array}{l}67(\mathrm{NR}) ; \text { range } \\
(39,88)\end{array}$ & $\begin{array}{l}\text { diameter: } 9.0 \\
\mathrm{~mm} \text {; range }(6,22)\end{array}$ & 20 & $\begin{array}{l}\text { I 29; II 43; III- } \\
\text { IV } 28\end{array}$ & BCC: nodular (100) & $\begin{array}{l}\text { face/scalp (31), } \\
\text { extremities (23), Trunk } 34 \\
(45 \%) \\
\text { Neck } 1(1 \%)\end{array}$ \\
\hline $\begin{array}{l}\text { Garcia-Martin } 2011 \\
21242584\end{array}$ & $\begin{array}{l}\text { imiquimod } \\
5 \%\end{array}$ & $\begin{array}{l}73.13(\mathrm{NR}) \\
\text { range }(53,84)\end{array}$ & $\begin{array}{l}\text { diameter: } 7.6 \\
\mathrm{~mm} \text {; range (2-12) }\end{array}$ & 33.3 & & BCC: unspecified (100) & eyelid (100) \\
\hline $\begin{array}{l}\text { Garcia-Martin } 2011 \\
21242584\end{array}$ & radiotherapy & $\begin{array}{l}74.18(\mathrm{NR}) \\
\text { range }(65,83)\end{array}$ & $\begin{array}{l}\text { diameter: } 7.41 \\
\mathrm{~mm} \text {; range }(4-12)\end{array}$ & 41.7 & & BCC: unspecified (100) & eyelid (100) \\
\hline $\begin{array}{l}\text { Geisse } 2002 \\
12196749\end{array}$ & $\begin{array}{l}\text { Imiquimod } \\
\text { 3x/wk }\end{array}$ & $\begin{array}{l}62 \text { (NR); range } \\
(36,85)\end{array}$ & median $1.0 \mathrm{~cm} 2$ & NR & & BCC: superficial (100) & $\begin{array}{l}\text { neck/face/forehead (4), } \\
\text { upper extremity (not hand) } \\
\text { (15), trunk (73), lower } \\
\text { extremity/thigh (not foot) } \\
\text { (8) }\end{array}$ \\
\hline $\begin{array}{l}\text { Geisse } 2002 \\
12196749\end{array}$ & $\begin{array}{l}\text { Imiquimod } \\
5 x / \text { wk }\end{array}$ & $\begin{array}{l}55(\mathrm{NR}) ; \text { range } \\
(38,84)\end{array}$ & median $0.6 \mathrm{~cm} 2$ & NR & & BCC: superficial (100) & $\begin{array}{l}\text { neck/face/forehead (3), } \\
\text { upper extremity (not hand) } \\
\text { (31), trunk (55), lower } \\
\text { extremity/thigh (not foot) } \\
\text { (10) }\end{array}$ \\
\hline $\begin{array}{l}\text { Geisse } 2002 \\
12196749\end{array}$ & $\begin{array}{l}\text { Imiquimod } \\
\text { 1x/day }\end{array}$ & $\begin{array}{l}56(\mathrm{NR}) ; \text { range } \\
(35,85)\end{array}$ & median $0.7 \mathrm{~cm} 2$ & NR & & BCC: superficial (100) & $\begin{array}{l}\text { neck/face/forehead (7), } \\
\text { upper extremity (not hand) } \\
\text { (21), trunk (64), lower } \\
\text { extremity/thigh (not foot) } \\
\text { (7) }\end{array}$ \\
\hline $\begin{array}{l}\text { Geisse } 2002 \\
12196749\end{array}$ & $\begin{array}{l}\text { Imiquimod } \\
2 x / \text { day }\end{array}$ & $\begin{array}{l}69 \text { (NR); range } \\
(51,85)\end{array}$ & median $1.0 \mathrm{~cm} 2$ & NR & & BCC: superficial (100) & $\begin{array}{l}\text { neck/face/forehead (8), } \\
\text { upper extremity (not hand) } \\
\text { (54), trunk (31), lower } \\
\text { extremity/thigh (not foot) } \\
\text { (8) }\end{array}$ \\
\hline $\begin{array}{l}\text { Geisse } 2002 \\
12196749\end{array}$ & $\begin{array}{l}\text { vehicle } \\
\text { (control) }\end{array}$ & $\begin{array}{l}58(\mathrm{NR}) ; \text { range } \\
(38,85)\end{array}$ & median $0.8 \mathrm{~cm} 2$ & NR & & BCC: superficial (100) & $\begin{array}{l}\text { neck/face/forehead (9), } \\
\text { upper extremity (not hand) }\end{array}$ \\
\hline
\end{tabular}




\begin{tabular}{|c|c|c|c|c|c|c|c|}
\hline $\begin{array}{l}\text { Author Year } \\
\text { PMID }\end{array}$ & Arm & $\begin{array}{l}\text { Age, mean } \\
\text { (SD); range }\end{array}$ & $\begin{array}{l}\text { Lesion area, } \\
\text { mean (SD); } \\
\text { range }\end{array}$ & $\begin{array}{l}\text { Female, } \\
\%\end{array}$ & $\begin{array}{l}\text { Fitzpatrick } \\
\text { score \% }\end{array}$ & Lesion type (\%) & Lesion location (\%) \\
\hline & & & & & & & $\begin{array}{l}\text { (34), trunk (47), lower } \\
\text { extremity/thigh (not foot) } \\
\text { (9) }\end{array}$ \\
\hline $\begin{array}{l}\text { Geisse } 2004 \\
15097956\end{array}$ & $\begin{array}{l}\text { Imiquimod } \\
\text { 5x/wk }\end{array}$ & $\begin{array}{l}58.4(13.1) \\
\text { median } 59 ; \\
\text { range }(31,89)\end{array}$ & & 37 & $\begin{array}{l}\text { I 15; II 54; III } \\
26 ; \text { IV } 5\end{array}$ & BCC: unspecified (100) & $\begin{array}{l}\text { neck (4), trunk: anterior } \\
\text { lower (1), trunk: anterior } \\
\text { upper (17), trunk: } \\
\text { posterior lower ( } 7) \text {, trunk: } \\
\text { posterior upper (24), lower } \\
\text { extremity (excluding foot) } \\
\text { (15), upper extremity } \\
\text { (excluding hand) (31), } \\
\text { chin (1), forehead (1) }\end{array}$ \\
\hline $\begin{array}{l}\text { Geisse } 2004 \\
15097956\end{array}$ & $\begin{array}{l}\text { Vehicle } \\
5 x / \text { wk or } \\
7 x / \text { wk }\end{array}$ & $\begin{array}{l}58.7(12.4) ; \\
\text { range }(32,85)\end{array}$ & & 38 & $\begin{array}{l}\text { I 19; II 43; III } \\
32 ; \text { IV } 5\end{array}$ & BCC: unspecified (100) & $\begin{array}{l}\text { neck (1), trunk: anterior } \\
\text { lower (1), trunk: anterior } \\
\text { upper (20), trunk: } \\
\text { posterior lower (6), trunk: } \\
\text { posterior upper (20), lower } \\
\text { extremity (excluding foot) } \\
\text { (10.5), upper extremity } \\
\text { (excluding hand) (39), } \\
\text { cheek (1), chin (1), } \\
\text { forehead (1) }\end{array}$ \\
\hline $\begin{array}{l}\text { Geisse } 2004 \\
15097956\end{array}$ & $\begin{array}{l}\text { Imiquimod } \\
7 x / w k\end{array}$ & $\begin{array}{l}59.4(12.27) ; \\
\text { median } 58 ; \\
\text { range }(29,88)\end{array}$ & & 41 & $\begin{array}{l}\text { I 16; II 46; III } \\
34 ; \text { IV } 4\end{array}$ & BCC: unspecified (100) & $\begin{array}{l}\text { neck (5), trunk: anterior } \\
\text { lower } 3 \text {, trunk: anterior } \\
\text { upper (13), trunk: } \\
\text { posterior lower (8), trunk: } \\
\text { posterior upper (26), lower } \\
\text { extremity (excluding foot) } \\
\text { (11), upper extremity } \\
\text { (excluding hand) (33), } \\
\text { cheek (1), chin (1), } \\
\text { forehead (1) } \\
\text { Face: nose } 1(1 \%)\end{array}$ \\
\hline $\begin{array}{l}\text { Haak } 2015 \\
24903544\end{array}$ & MAL PDT & NR & $\begin{array}{l}\text { diameter: median } \\
8.5 \mathrm{~mm} \text { (IQR 6, } \\
10.5)\end{array}$ & 37.5 & I; II 56; III 44 & BCC: nodular (100) & $\begin{array}{l}\text { nose (37), forehead (31), } \\
\text { cheek (6), oral area (13), } \\
\text { periorbital area (13) }\end{array}$ \\
\hline $\begin{array}{l}\text { Haak } 2015 \\
24903544\end{array}$ & $\begin{array}{l}\text { AFXL MAL } \\
\text { PDT }\end{array}$ & NR & $\begin{array}{l}\text { diameter: } \\
\text { median } 7 \mathrm{~mm} \\
(\mathrm{IQR} 6,8)\end{array}$ & 68.8 & I; II 69; III 31 & BCC: nodular (100) & $\begin{array}{l}\text { nose (56), forehead (19), } \\
\text { cheek (13), oral area (6), } \\
\text { periorbital area (6) }\end{array}$ \\
\hline Hall 19863514075 & $\begin{array}{l}\text { Radiotherap } \\
\mathrm{y}\end{array}$ & & $\begin{array}{l}\text { diameter: } 19<1 \\
\mathrm{~cm}, 251-2 \mathrm{~cm}, 5\end{array}$ & NR & & BCC: unspecified (100) & $\begin{array}{l}\text { face and neck (82), eyelid } \\
(6), \text { trunk (12) }\end{array}$ \\
\hline
\end{tabular}




\begin{tabular}{|c|c|c|c|c|c|c|c|}
\hline $\begin{array}{l}\text { Author Year } \\
\text { PMID }\end{array}$ & Arm & $\begin{array}{l}\text { Age, mean } \\
\text { (SD); range }\end{array}$ & $\begin{array}{l}\text { Lesion area, } \\
\text { mean (SD); } \\
\text { range }\end{array}$ & $\begin{array}{l}\text { Female, } \\
\%\end{array}$ & $\begin{array}{l}\text { Fitzpatrick } \\
\text { score } \%\end{array}$ & Lesion type (\%) & Lesion location (\%) \\
\hline & & & $>2 \mathrm{~cm}$ & & & & \\
\hline Hall 19863514075 & Cryotherapy & & $\begin{array}{l}\text { diameter: } 19<1 \\
\mathrm{~cm}, 231-2 \mathrm{~cm}, 2 \\
>2 \mathrm{~cm}\end{array}$ & NR & & BCC: unspecified (100) & $\begin{array}{l}\text { face and neck (65), eyelid } \\
\text { (17), trunk (17) }\end{array}$ \\
\hline Ko 201424102369 & $\begin{array}{l}\text { Er:YAG AFL } \\
\text { PDT }\end{array}$ & $68.9(13.2)$ & & 52.4 & $\begin{array}{l}\text { III 9.5; IV } \\
71.4 ; \text { V } 19.1\end{array}$ & SCC: Bowen's (100) & extremities (100) \\
\hline Ko 201424102369 & MAL-PDT & $68.9(13.2)$ & & 52.4 & $\begin{array}{l}\text { III 9.5; IV } \\
71.4 ; \text { V } 19.1\end{array}$ & SCC: Bowen's (100) & extremities (100) \\
\hline $\begin{array}{l}\text { Kuijpers } 2006 \\
16865869\end{array}$ & $\begin{array}{l}\text { ALA-PDT } \\
\text { (total) }\end{array}$ & $\begin{array}{l}68.4(N R) ; \\
\text { median } 73 ; \\
\text { range }(39,87)\end{array}$ & $\begin{array}{l}\text { diameter: } 8.1 \mathrm{~mm} \\
(4.12)\end{array}$ & 34.9 & & BCC: nodular (100) & $\begin{array}{l}\text { forehead/temple+nose/par } \\
\text { anasal (36.4), } \\
\text { cheek/chin/lips (9.1), ears } \\
\text { (9.1), extremities (9.1), } \\
\text { trunk/neck (36.4) }\end{array}$ \\
\hline $\begin{array}{l}\text { Kuijpers } 2006 \\
16865869\end{array}$ & $\begin{array}{l}\text { MAL-PDT } \\
\text { (total) }\end{array}$ & $\begin{array}{l}68.4(N R) ; \\
\text { median } 73 ; \\
\text { range }(39,87)\end{array}$ & $\begin{array}{l}\text { diameter: } 8.4 \\
(3.28)\end{array}$ & 34.9 & & BCC: nodular (100) & $\begin{array}{l}\text { forehead/temple+nose/par } \\
\text { anasal (38.1), } \\
\text { cheek/chin/lips (4.8), ears } \\
\text { (14.3), extremities (4.8), } \\
\text { trunk/neck (38.1) }\end{array}$ \\
\hline $\begin{array}{l}\text { Kuijpers } 2006 \\
16865869\end{array}$ & $\begin{array}{l}\text { ALA-PDT } \\
\text { (debulking } \\
\text { subgroup) } \\
\end{array}$ & 68.4 (NR); median & 73 ; range $(39,87)$ & 34.9 & & BCC: nodular (100) & NR \\
\hline $\begin{array}{l}\text { Kuijpers } 2006 \\
16865869\end{array}$ & $\begin{array}{l}\text { ALA-PDT } \\
\text { (no } \\
\text { debulking } \\
\text { subgroup) }\end{array}$ & 68.4 (NR); median & $73 ;$ range $(39,87)$ & 34.9 & & BCC: nodular (100) & NR \\
\hline $\begin{array}{l}\text { Kuijpers } 2006 \\
16865869\end{array}$ & $\begin{array}{l}\text { MAL-PDT } \\
\text { (debulking } \\
\text { subgroup) }\end{array}$ & 68.4 (NR); median & $73 ;$ range $(39,87)$ & 34.9 & & BCC: nodular (100) & NR \\
\hline $\begin{array}{l}\text { Kuijpers } 2006 \\
16865869\end{array}$ & $\begin{array}{l}\text { MAL-PDT } \\
\text { (no } \\
\text { debulking } \\
\text { subgroup) }\end{array}$ & 68.4 (NR); median & 73 ; range $(39,87)$ & 34.9 & & BCC: nodular (100) & NR \\
\hline $\begin{array}{l}\text { Kuijpers } 2007 \\
17451581\end{array}$ & $\begin{array}{l}\text { Curettage }+ \\
\text { Cryosurgery }\end{array}$ & $\begin{array}{l}67(\mathrm{NR}) ; \text { range } \\
(34,92)\end{array}$ & $\begin{array}{l}\text { diameter: } 5.4 \mathrm{~mm} \\
(2.9)\end{array}$ & 43 & & BCC: nodular (100) & $\begin{array}{l}\text { Forehead/temple, } \\
\text { Cheek/chin, Periocular } \\
\text { (80), Lips/mouth (4), } \\
\text { Ears/periauricular (8), } \\
\text { Neck, chest/back (8) }\end{array}$ \\
\hline $\begin{array}{l}\text { Kuijpers } 2007 \\
17451581\end{array}$ & $\begin{array}{l}\text { Surgical } \\
\text { excision }\end{array}$ & $\begin{array}{l}67(\mathrm{NR}) ; \text { range } \\
(34,92)\end{array}$ & $\begin{array}{l}\text { diameter: } 5.3 \mathrm{~mm} \\
(2.6)\end{array}$ & 43 & & $\begin{array}{l}\text { BCC: superficial (8), } \\
\text { nodular (92) }\end{array}$ & $\begin{array}{l}\text { Forehead/temple, } \\
\text { Cheek/chin, Periocular } \\
\text { (76), Lips/mouth (6), }\end{array}$ \\
\hline
\end{tabular}




\begin{tabular}{|c|c|c|c|c|c|c|c|}
\hline $\begin{array}{l}\text { Author Year } \\
\text { PMID }\end{array}$ & Arm & $\begin{array}{l}\text { Age, mean } \\
\text { (SD); range }\end{array}$ & $\begin{array}{l}\text { Lesion area, } \\
\text { mean (SD); } \\
\text { range }\end{array}$ & $\begin{array}{l}\text { Female, } \\
\%\end{array}$ & $\begin{array}{l}\text { Fitzpatrick } \\
\text { score } \%\end{array}$ & Lesion type (\%) & Lesion location (\%) \\
\hline & & & & & & & $\begin{array}{l}\text { Ears/periauricular (6), } \\
\text { Neck, chest/back (12) }\end{array}$ \\
\hline $\begin{array}{l}\text { Marks } 2001 \\
11312429\end{array}$ & Total & $\begin{array}{l}61(\mathrm{NR}) ; \text { range } \\
(23,83)\end{array}$ & $\begin{array}{l}\text { range }(0.5,2 \\
\mathrm{cm} 2)\end{array}$ & 27 & II 46; III 32 & $\begin{array}{l}\text { BCC: superficial (98); } \\
\text { nodular (1); follicular (1) }\end{array}$ & $\begin{array}{l}\text { Upper extremities (32), } \\
\text { upper trunk (28), } \\
\text { head/neck/lower limbs } \\
(40)\end{array}$ \\
\hline $\begin{array}{l}\text { Migden } 2015 \\
25981810 \\
\end{array}$ & $\begin{array}{l}\text { sonidegib } \\
200\end{array}$ & $\begin{array}{l}\text { median } 67 ; \\
\text { range }(25,92) \\
\end{array}$ & & 39 & & $\begin{array}{l}\text { BCC: advanced (91), } \\
\text { metastatic (9) }\end{array}$ & head and neck (100) \\
\hline $\begin{array}{l}\text { Migden } 2015 \\
25981810 \\
\end{array}$ & $\begin{array}{l}\text { sonidegib } \\
800\end{array}$ & $\begin{array}{l}\text { median } 65 ; \\
\text { range }(24,93)\end{array}$ & & 36 & & $\begin{array}{l}\text { BCC: advanced (71), } \\
\text { metastatic (29) }\end{array}$ & head and neck (100) \\
\hline Miller 19978996264 & Total & $\begin{array}{l}61 \text { (NR); range } \\
(29,86)\end{array}$ & $\begin{array}{l}80 \mathrm{~mm} 2 ; \text { range } \\
18,225\end{array}$ & 20 & & $\begin{array}{l}\text { BCC: superficial (31), } \\
\text { nodular (69) }\end{array}$ & $\begin{array}{l}\text { head (7), extremities (40), } \\
\text { trunk/neck (52) }\end{array}$ \\
\hline $\begin{array}{l}\text { Morton } 1996 \\
8977678\end{array}$ & cryotherapy & $\begin{array}{l}76(\mathrm{NR}) ; \text { range } \\
(62,88)\end{array}$ & $\begin{array}{l}82 \mathrm{~mm} 2 ; \text { range } \\
(30,360)\end{array}$ & 84 & & SCC: Bowen's (100) & $\begin{array}{l}\text { hands (5), face (15), legs } \\
(80)\end{array}$ \\
\hline $\begin{array}{l}\text { Morton } 1996 \\
8977678\end{array}$ & $\begin{array}{l}\text { photodynami } \\
\text { c }\end{array}$ & $\begin{array}{l}76(\mathrm{NR}) ; \text { range } \\
(62,88)\end{array}$ & $\begin{array}{l}150 \mathrm{~mm} 2 ; \text { range } \\
(25,441)\end{array}$ & 84 & & SCC: Bowen's (100) & $\begin{array}{l}\text { hands (5), face (10), legs } \\
\text { (85) }\end{array}$ \\
\hline $\begin{array}{l}\text { Morton } 2006 \\
16785375\end{array}$ & MAL PDT & $\begin{array}{l}71.9(\mathrm{NR}) \\
\text { range }(43,89)\end{array}$ & $\begin{array}{l}\text { diameter: } 18.9 \\
\mathrm{~mm} \text {; range (5, } \\
\text { 40mm) }\end{array}$ & 62 & $\begin{array}{l}\text { I 10; II } 47 ; \text { III } \\
38 ; \text { IV } 5\end{array}$ & SCC: Bowen's (100) & $\begin{array}{l}\text { face/scalp (23), } \\
\text { extremities (65), } \\
\text { trunk/neck (12) }\end{array}$ \\
\hline $\begin{array}{l}\text { Morton } 2006 \\
16785375\end{array}$ & PDT placebo & $\begin{array}{l}73.4(\mathrm{NR}) ; \\
\text { range }(53,88)\end{array}$ & $\begin{array}{l}\text { diameter: } 19.3 \\
\mathrm{~mm} \text {; range }(8, \\
40 \mathrm{~mm})\end{array}$ & 65 & $\begin{array}{l}\text { I 24; II 53; III } \\
18 ; \text { IV } 6\end{array}$ & SCC: Bowen's (100) & $\begin{array}{l}\text { face/scalp (25), } \\
\text { extremities (67), } \\
\text { trunk/neck (8) }\end{array}$ \\
\hline $\begin{array}{l}\text { Morton } 2006 \\
16785375\end{array}$ & Cryotherapy & $\begin{array}{l}74.0(N R) ; \\
\text { range }(45,99)\end{array}$ & $\begin{array}{l}\text { diameter: } 19.4 \\
\mathrm{~mm} \text {; range }(6, \\
45 \mathrm{~mm})\end{array}$ & 59 & $\begin{array}{l}\text { I } 4 ; \text { II } 49 ; \text { III } \\
39 ; \text { IV } 9\end{array}$ & SCC: Bowen's (100) & $\begin{array}{l}\text { face/scalp (29), } \\
\text { extremities (57), } \\
\text { trunk/neck (14) }\end{array}$ \\
\hline $\begin{array}{l}\text { Morton } 2006 \\
16785375\end{array}$ & Fluorouracil & $\begin{array}{l}72.5(\mathrm{NR}) ; \\
\text { range }(39,86)\end{array}$ & $\begin{array}{l}\text { diameter: } 20.9 \\
\mathrm{~mm} \text {; range }(9, \\
37 \mathrm{~mm})\end{array}$ & 63 & $\begin{array}{l}\text { I 20; II 37; III } \\
40 ; \text { IV } 3\end{array}$ & SCC: Bowen's (100) & $\begin{array}{l}\text { face/scalp (19), } \\
\text { extremities (69), } \\
\text { trunk/neck (11) }\end{array}$ \\
\hline $\begin{array}{l}\text { Mosterd } 2008 \\
18717680\end{array}$ & ALA-PDT & $\begin{array}{l}64.0(\mathrm{NR}) ; \\
\text { range }(24,83)\end{array}$ & $\begin{array}{l}\text { diameter: } 8.9 \mathrm{~mm} \\
(4.0 \mathrm{~mm}) ; \\
\text { median(IQR ) } \\
\text { range () }\end{array}$ & 48.2 & & BCC: nodular (100) & $\begin{array}{l}\text { face (53); "rest of the } \\
\text { body" }(47 \%)\end{array}$ \\
\hline $\begin{array}{l}\text { Mosterd } 2008 \\
18717680\end{array}$ & $\begin{array}{l}\text { Surgical } \\
\text { excision }\end{array}$ & $\begin{array}{l}65.1(\mathrm{NR}) ; \\
\text { range }(21,91)\end{array}$ & $\begin{array}{l}\text { diameter: } 9.3 \mathrm{~mm} \\
(4.3 \mathrm{~mm}) ; \\
\text { median(IQR ) } \\
\text { range () }\end{array}$ & 50 & & BCC: nodular (100) & $\begin{array}{l}\text { face }(51) ; \text { "rest of the } \\
\text { body" }(49 \%)\end{array}$ \\
\hline $\begin{array}{l}\text { Mosterd } 2008 \\
19010733\end{array}$ & MMS & $67.4(12.7)$ & $\begin{array}{l}1.28 \mathrm{~cm} 2(1.36) \\
\text { diameter: } 13.76 \\
\mathrm{~mm}(6.43)\end{array}$ & 39.7 & & $\begin{array}{l}\text { BCC: unspecified (100), } \\
51.5 \% \text { aggressive }\end{array}$ & $\begin{array}{l}\text { frontal/temporal (26), } \\
\text { cheek/chin (9), (peri)nasal } \\
\text { (34), lips/perioral (7), } \\
\text { periocular (8), ears (4), } \\
\text { periauricular (12) }\end{array}$ \\
\hline
\end{tabular}




\begin{tabular}{|c|c|c|c|c|c|c|c|}
\hline $\begin{array}{l}\text { Author Year } \\
\text { PMID }\end{array}$ & Arm & $\begin{array}{l}\text { Age, mean } \\
\text { (SD); range }\end{array}$ & $\begin{array}{l}\text { Lesion area, } \\
\text { mean (SD); } \\
\text { range }\end{array}$ & $\begin{array}{l}\text { Female, } \\
\%\end{array}$ & $\begin{array}{l}\text { Fitzpatrick } \\
\text { score } \%\end{array}$ & Lesion type (\%) & Lesion location (\%) \\
\hline $\begin{array}{l}\text { Mosterd } 2008 \\
19010733\end{array}$ & $\begin{array}{l}\text { Surgical } \\
\text { excision }\end{array}$ & $68.7(12.2)$ & $\begin{array}{l}1.77 \mathrm{~cm} 2(1.28) \\
\text { diameter: } 15.97 \\
\mathrm{~mm}(8.17)\end{array}$ & 38.2 & & $\begin{array}{l}\text { BCC: unspecified (100), } \\
43.1 \% \text { aggressive }\end{array}$ & $\begin{array}{l}\text { frontal/temporal (32), } \\
\text { cheek/chin (8), (peri)nasal } \\
\text { (30), lips/perioral (4), } \\
\text { periocular (8), ears (8), } \\
\text { periauricular (10) }\end{array}$ \\
\hline $\begin{array}{l}\text { Orenberg } 1992 \\
1430394\end{array}$ & $7.5 \mathrm{mg} 5-\mathrm{FU}$ & $\begin{array}{l}60 \text { (NR); range } \\
(22,78)\end{array}$ & $123.9 \mathrm{~mm} 2$ & 5 & & BCC: nodular (100) & $\begin{array}{l}\text { face }(30), \text { extemities (30), } \\
\text { trunk/neck (40) }\end{array}$ \\
\hline $\begin{array}{l}\text { Orenberg } 1992 \\
1430394\end{array}$ & 15 mg 5-FU & $\begin{array}{l}60 \text { (NR); range } \\
(22,78)\end{array}$ & $76.4 \mathrm{~mm} 2$ & 5 & & BCC: nodular (100) & $\begin{array}{l}\text { face (10), scalp (10), lip } \\
(10), \text { ear (30), extemities } \\
(10), \text { trunk/neck (30) }\end{array}$ \\
\hline $\begin{array}{l}\text { Patel } 2006 \\
16713457\end{array}$ & $\begin{array}{l}\text { imiquimod } \\
5 \%\end{array}$ & $\begin{array}{l}74(8) ; \text { range } \\
(54,83)\end{array}$ & $\begin{array}{l}429 \mathrm{~mm} 2(489) ; \\
\text { range }(23,1776)\end{array}$ & 40 & & SCC: Bowen's (100) & NR \\
\hline $\begin{array}{l}\text { Patel } 2006 \\
16713457 \\
\end{array}$ & vehicle & $\begin{array}{l}74(8) ; \text { range } \\
(60,86)\end{array}$ & $\begin{array}{l}248 \mathrm{~mm} 2(166) ; \\
\text { range }(84,555)\end{array}$ & 87.5 & & SCC: Bowen's (100) & NR \\
\hline $\begin{array}{l}\text { Rhodes } 2004 \\
14732655\end{array}$ & MAL PDT & $\begin{array}{l}69 \text { (NR); range } \\
(40,95)\end{array}$ & & 38 & $\begin{array}{l}\text { I } 8 ; \text { II } 50 ; \text { III } \\
40 ; \text { IV } 2\end{array}$ & BCC: nodular (100) & $\begin{array}{l}\text { face/scalp (40), } \\
\text { extremities (11), } \\
\text { trunk/neck (49) }\end{array}$ \\
\hline $\begin{array}{l}\text { Rhodes } 2004 \\
14732655\end{array}$ & excision & $\begin{array}{l}67(\mathrm{NR}) ; \text { range } \\
(38,82)\end{array}$ & & 41 & $\begin{array}{l}\text { I 8; II 43; III } \\
43 ; \text { IV } 6\end{array}$ & BCC: nodular (100) & $\begin{array}{l}\text { face/scalp (58), } \\
\text { extremities (9), trunk/neck } \\
(29)\end{array}$ \\
\hline $\begin{array}{l}\text { Salim } 2003 \\
12653747\end{array}$ & PDT & $\begin{array}{l}76(\mathrm{NR}) ; \text { range } \\
(65,88)\end{array}$ & & 80 & & SCC: Bowen's (100) & extremities (100) \\
\hline $\begin{array}{l}\text { Salim } 2003 \\
12653747\end{array}$ & $5-\mathrm{FU}$ & $\begin{array}{l}76 \text { (NR); range } \\
(65,88)\end{array}$ & & 80 & & SCC: Bowen's (100) & face (12), extremities (88) \\
\hline Salmanpoor 2012 & $\begin{array}{l}\text { Surgical } \\
\text { excision }\end{array}$ & $\begin{array}{l}57.3(\mathrm{NR}) \\
\text { range }(21,84)\end{array}$ & & 37 & & BCC: unspecified (100) & face and scalp (100) \\
\hline Salmanpoor 2012 & Curettage & $\begin{array}{l}57.3(\mathrm{NR}) ; \\
\text { range }(21,84)\end{array}$ & & 37 & & BCC: unspecified (100) & face and scalp (100) \\
\hline Salmanpoor 2012 & $\begin{array}{l}\text { Electodessic } \\
\text { ation and } \\
\text { curettage }\end{array}$ & $\begin{array}{l}57.3(\mathrm{NR}) ; \\
\text { range }(21,84)\end{array}$ & & 37 & & BCC: unspecified (100) & face and scalp (100) \\
\hline $\begin{array}{l}\text { Schleier } 2007 \\
25047438\end{array}$ & $\begin{array}{l}\text { ALA- } \\
\text { thermogel } \\
\text { PDT }\end{array}$ & $\begin{array}{l}69.9(\mathrm{NR}) ; \\
\text { range }(42,96)\end{array}$ & & 46.15 & & BCC: superficial (100) & $\begin{array}{l}\text { face (54.17), scalp } \\
(20.83), \text { lip (2.78), eyelid } \\
(1.39), \text { extremities (9.72), } \\
\text { trunk/neck (11.11) }\end{array}$ \\
\hline $\begin{array}{l}\text { Schleier } 2007 \\
25047438\end{array}$ & $\begin{array}{l}\text { mALA- } \\
\text { thermogel } \\
\text { PDT }\end{array}$ & $\begin{array}{l}71.8(N R) ; \\
\text { range }(49,88)\end{array}$ & & 36.36 & & BCC: superficial (100) & $\begin{array}{l}\text { face }(52.5), \text { scalp }(30), \\
\text { extremities (5), trunk/neck } \\
(12.5)\end{array}$ \\
\hline $\begin{array}{l}\text { Schulze } 2005 \\
15888150\end{array}$ & $\begin{array}{l}\text { imiquimod } \\
5 \%\end{array}$ & $\begin{array}{l}64.3(13.06) ; \\
\text { median } 67 ; \\
\text { range }(25,83)\end{array}$ & & 39 & $\begin{array}{l}\text { I 5; II 48; III } \\
42 ; \text { IV 5; V } 1\end{array}$ & BCC: superficial (100) & $\begin{array}{l}\text { cheek (1), forehead (0), } \\
\text { extremities (including } \\
\text { hand) }(20), \text { trunk/neck }\end{array}$ \\
\hline
\end{tabular}




\begin{tabular}{|c|c|c|c|c|c|c|c|}
\hline $\begin{array}{l}\text { Author Year } \\
\text { PMID }\end{array}$ & Arm & $\begin{array}{l}\text { Age, mean } \\
\text { (SD); range }\end{array}$ & $\begin{array}{l}\text { Lesion area, } \\
\text { mean (SD); } \\
\text { range }\end{array}$ & $\begin{array}{l}\text { Female, } \\
\%\end{array}$ & $\begin{array}{l}\text { Fitzpatrick } \\
\text { score } \%\end{array}$ & Lesion type (\%) & Lesion location (\%) \\
\hline & & & & & & & (70) \\
\hline $\begin{array}{l}\text { Schulze } 2005 \\
15888150\end{array}$ & vehicle & $\begin{array}{l}64.5(11.43) ; \\
\text { median } 68 ; \\
\text { range }(31,86)\end{array}$ & & 39 & $\begin{array}{l}\text { I 1; II 46; III } \\
\text { 41; IV 10; V } 1\end{array}$ & BCC: superficial (100) & $\begin{array}{l}\text { cheek (1), forehead (5), } \\
\text { scalp (1), extremities } \\
\text { (including hand) (30), } \\
\text { trunk/neck (61) }\end{array}$ \\
\hline $\begin{array}{l}\text { Shumack } 2002 \\
12224978(12 \\
\text { weeks })\end{array}$ & $\begin{array}{l}\text { vehicle } \\
\text { cream }\end{array}$ & NR & median $0.8 \mathrm{~cm} 2$ & 42 & & BCC: nodular (100) & $\begin{array}{l}\text { face (17), trunk/neck } \\
\text { (54.2), upper extremity } \\
\text { (not hand) (25), lower } \\
\text { extremity (not foot) (4) }\end{array}$ \\
\hline $\begin{array}{l}\text { Shumack } 2002 \\
12224978(12 \\
\text { weeks })\end{array}$ & $\begin{array}{l}\text { imiquimod } \\
\text { (IMQ) 5\% } \\
\text { cream - } \\
\text { Twice daily } \\
\text { for } 7 \text { days } \\
\text { per week }\end{array}$ & NR & median $0.8 \mathrm{~cm} 2$ & 75 & & BCC: nodular (100) & face (25), trunk/neck (75) \\
\hline $\begin{array}{l}\text { Shumack } 2002 \\
12224978(12 \\
\text { weeks })\end{array}$ & $\begin{array}{l}\text { imiquimod } \\
\text { (IMQ) } 5 \% \\
\text { cream - } \\
\text { Once daily } \\
\text { for } 7 \text { days } \\
\text { per week }\end{array}$ & NR & median $0.7 \mathrm{~cm} 2$ & 10 & & BCC: nodular (100) & $\begin{array}{l}\text { face (29), trunk/neck (33), } \\
\text { upper extremity (not hand) } \\
\text { (19), lower extremity (not } \\
\text { foot) (10) }\end{array}$ \\
\hline $\begin{array}{l}\text { Shumack } 2002 \\
12224978(12 \\
\text { weeks) }\end{array}$ & $\begin{array}{l}\text { imiquimod } \\
\text { (IMQ) } 5 \% \\
\text { cream - } \\
\text { Once daily } \\
\text { for } 5 \text { days } \\
\text { per week }\end{array}$ & NR & median $0.7 \mathrm{~cm} 2$ & 35 & & BCC: nodular (100) & $\begin{array}{l}\text { face (48), trunk/neck (26), } \\
\text { Upper extremity (not } \\
\text { hand) (17), lower } \\
\text { extremity (not foot) (9) }\end{array}$ \\
\hline $\begin{array}{l}\text { Shumack } 2002 \\
12224978(12 \\
\text { weeks) }\end{array}$ & $\begin{array}{l}\text { imiquimod } \\
\text { (IMQ) } 5 \% \\
\text { cream - } \\
\text { Once daily } \\
\text { for } 3 \text { days } \\
\text { per week }\end{array}$ & NR & median $0.7 \mathrm{~cm} 2$ & 30 & & BCC: nodular (100) & $\begin{array}{l}\text { face (40), trunk/neck (35), } \\
\text { upper extremity (not hand) } \\
\text { (20), lower extremity (not } \\
\text { foot) (5) }\end{array}$ \\
\hline $\begin{array}{l}\text { Shumack } 2002 \\
12224978 \text { (6 weeks) }\end{array}$ & $\begin{array}{l}\text { imiquimod } \\
\text { (IMQ) } 5 \% \\
\text { cream - } \\
\text { Twice daily } \\
\text { for } 7 \text { days } \\
\text { per week }\end{array}$ & NR & median $0.6 \mathrm{~cm} 2$ & 0 & & BCC: nodular (100) & face $(100)$ \\
\hline $\begin{array}{l}\text { Shumack } 2002 \\
12224978 \text { (6 weeks) }\end{array}$ & $\begin{array}{l}\text { imiquimod } \\
\text { (IMQ) } 5 \%\end{array}$ & $63(41.1)$ & median $0.8 \mathrm{~cm} 2$ & 13 & & BCC: nodular (100) & $\begin{array}{l}\text { face (28), trunk/neck } \\
\text { (11.11), Upper extremity }\end{array}$ \\
\hline
\end{tabular}




\begin{tabular}{|c|c|c|c|c|c|c|c|}
\hline $\begin{array}{l}\text { Author Year } \\
\text { PMID }\end{array}$ & Arm & $\begin{array}{l}\text { Age, mean } \\
\text { (SD); range }\end{array}$ & $\begin{array}{l}\text { Lesion area, } \\
\text { mean (SD); } \\
\text { range }\end{array}$ & $\begin{array}{l}\text { Female, } \\
\%\end{array}$ & $\begin{array}{l}\text { Fitzpatrick } \\
\text { score } \%\end{array}$ & Lesion type (\%) & Lesion location (\%) \\
\hline & $\begin{array}{l}\text { cream - } \\
\text { Once daily } \\
\text { for } 3 \text { days } \\
\text { per week }\end{array}$ & & & & & & $\begin{array}{l}\text { (not hand) (25), lower } \\
\text { extremity (not foot) (13) }\end{array}$ \\
\hline $\begin{array}{l}\text { Shumack } 2002 \\
12224978 \text { (6 weeks) }\end{array}$ & $\begin{array}{l}\text { imiquimod } \\
\text { (IMQ) } 5 \% \\
\text { cream - } \\
\text { Twice daily } \\
\text { for } 7 \text { days } \\
\text { per week }\end{array}$ & 69 (11.2) & median $0.8 \mathrm{~cm} 2$ & 13 & & BCC: nodular (100) & $\begin{array}{l}\text { face (32), trunk/neck (39), } \\
\text { Upper extremity (not } \\
\text { hand) (26), lower } \\
\text { extremity (not foot) (3) }\end{array}$ \\
\hline $\begin{array}{l}\text { Shumack } 2002 \\
12224978 \text { (6 weeks) }\end{array}$ & $\begin{array}{l}\text { imiquimod } \\
\text { (IMQ) } 5 \% \\
\text { cream - } \\
\text { Once daily } \\
\text { for } 7 \text { days } \\
\text { per week }\end{array}$ & $66(12.4)$ & median $0.8 \mathrm{~cm} 2$ & 29 & & BCC: nodular (100) & $\begin{array}{l}\text { face (11), trunk/neck (48), } \\
\text { Upper extremity (not } \\
\text { hand) (26), lower } \\
\text { extremity (not foot) (3) }\end{array}$ \\
\hline $\begin{array}{l}\text { Siller } 2010 \\
20546215\end{array}$ & total & $\begin{array}{l}59(\mathrm{NR}) ; \text { range } \\
(34,86)\end{array}$ & $\begin{array}{l}\text { diameter: } 9 \mathrm{~mm} \text {; } \\
\text { range }(4,15 \mathrm{~mm})\end{array}$ & 27 & & BCC: superficial (100) & NR \\
\hline $\begin{array}{l}\text { Spencer } 2006 \\
16393600\end{array}$ & $\begin{array}{l}\text { imiquimod } \\
5 \%\end{array}$ & NR & & 40 & & BCC: nodular (100) & $\begin{array}{l}\text { face (60), ear (10), } \\
\text { unspecified other (30) }\end{array}$ \\
\hline $\begin{array}{l}\text { Spencer } 2006 \\
16393600\end{array}$ & vehicle & NR & & 10 & & BCC: nodular (100) & $\begin{array}{l}\text { face (50), ear (20), } \\
\text { unspecified other (30) }\end{array}$ \\
\hline $\begin{array}{l}\text { Sterry } 2002 \\
12452875 \text { (nodular) }\end{array}$ & $\begin{array}{l}\text { Imiquimod (2 } \\
\text { days/week) } \\
\text { with } \\
\text { occlusion }\end{array}$ & 66 (13.2); NR & median: $0.6 \mathrm{~cm} 2$ & 50 & & BCC: nodular (100) & $\begin{array}{l}\text { Face (10), Scalp (1), } \\
\text { extremities (2), trunk/neck } \\
(9)\end{array}$ \\
\hline $\begin{array}{l}\text { Sterry } 2002 \\
12452875 \text { (nodular) }\end{array}$ & $\begin{array}{l}\text { Imiquimod (3 } \\
\text { days/week) } \\
\text { with } \\
\text { occlusion }\end{array}$ & 66 (14.6); NR & median: $0.7 \mathrm{~cm} 2$ & 30 & & BCC: nodular (100) & $\begin{array}{l}\text { Face (18), extremities (2), } \\
\text { trunk/neck (3) }\end{array}$ \\
\hline $\begin{array}{l}\text { Sterry } 2002 \\
12452875 \text { (nodular) }\end{array}$ & $\begin{array}{l}\text { Imiquimod (2 } \\
\text { days/week) } \\
\text { without } \\
\text { occlusion }\end{array}$ & 67 (8.9); NR & median: $1.0 \mathrm{~cm} 2$ & 24 & & BCC: nodular (100) & $\begin{array}{l}\text { Face (9), extremities (1), } \\
\text { trunk/neck (10) }\end{array}$ \\
\hline $\begin{array}{l}\text { Sterry } 2002 \\
12452875 \text { (nodular) }\end{array}$ & $\begin{array}{l}\text { Imiquimod (3 } \\
\text { days/week) } \\
\text { without } \\
\text { occlusion }\end{array}$ & 66 (13.2); NR & median: $0.6 \mathrm{~cm} 2$ & 46 & & BCC: nodular (100) & $\begin{array}{l}\text { Face (11), extremities (5), } \\
\text { trunk/neck (8) }\end{array}$ \\
\hline $\begin{array}{l}\text { Sterry } 2002 \\
12452875 \\
\text { (superficial) }\end{array}$ & \multicolumn{7}{|c|}{$\begin{array}{l}\text { Imiquimod ( } 2 \text { days/week) with } \\
\text { occlusion }\end{array}$} \\
\hline
\end{tabular}




\begin{tabular}{|c|c|c|c|c|c|c|c|}
\hline $\begin{array}{l}\text { Author Year } \\
\text { PMID }\end{array}$ & Arm & $\begin{array}{l}\text { Age, mean } \\
\text { (SD); range }\end{array}$ & $\begin{array}{l}\text { Lesion area, } \\
\text { mean (SD); } \\
\text { range }\end{array}$ & $\begin{array}{l}\text { Female, } \\
\%\end{array}$ & $\begin{array}{l}\text { Fitzpatrick } \\
\text { score } \%\end{array}$ & Lesion type (\%) & Lesion location (\%) \\
\hline $\begin{array}{l}\text { Sterry } 2002 \\
12452875 \\
\text { (superficial) }\end{array}$ & \multicolumn{2}{|c|}{$\begin{array}{l}\text { Imiquimod ( } 3 \text { days/week) with } \\
\text { occlusion }\end{array}$} & & & & & \\
\hline $\begin{array}{l}\text { Sterry } 2002 \\
12452875 \\
\text { (superficial) }\end{array}$ & \multicolumn{2}{|c|}{$\begin{array}{l}\text { Imiquimod (2 days/week) } \\
\text { without occlusion }\end{array}$} & & & & & \\
\hline $\begin{array}{l}\text { Sterry } 2002 \\
12452875 \\
\text { (superficial) } \\
\end{array}$ & \multicolumn{2}{|c|}{$\begin{array}{l}\text { Imiquimod (3 days/week) } \\
\text { without occlusion }\end{array}$} & & & & & \\
\hline $\begin{array}{l}\text { Szeimies } 2008 \\
18624836\end{array}$ & MAL-PDT & $\begin{array}{l}64.5(12.7) ; \\
\text { range }(33,85)\end{array}$ & $\begin{array}{l}\text { diameter: } 12.5 \\
\mathrm{~mm}(3.7)\end{array}$ & 36.0 & & BCC: superficial (100) & $\begin{array}{l}\text { face/scalp (11.1), } \\
\text { extremities (28.9), } \\
\text { trunk/neck (60) }\end{array}$ \\
\hline $\begin{array}{l}\text { Szeimies } 2008 \\
18624836\end{array}$ & excision & $\begin{array}{l}63.1(13.9) ; \\
\text { range }(31,92)\end{array}$ & $\begin{array}{l}\text { diameter: } 12.6 \\
\mathrm{~mm}(3.7)\end{array}$ & 31.3 & & BCC: superficial (100) & $\begin{array}{l}\text { face/scalp (4.5), } \\
\text { extremities (25.0), } \\
\text { trunk/neck (70.5) }\end{array}$ \\
\hline $\begin{array}{l}\text { Thissen } 2000 \\
10940063\end{array}$ & cryotherapy & NR & & NR & & $\begin{array}{l}\text { BCC: superficial (17), } \\
\text { nodular (83) }\end{array}$ & $\begin{array}{l}\text { face (46), eyelid (4), ear } \\
\text { (4), trunk/neck (6), } \\
\text { forehead/temple (34), } \\
\text { chin/perioral (6) }\end{array}$ \\
\hline $\begin{array}{l}\text { Thissen } 2000 \\
10940063\end{array}$ & $\begin{array}{l}\text { surgical } \\
\text { excision }\end{array}$ & NR & & NR & & $\begin{array}{l}\text { BCC: superficial (12), } \\
\text { nodular (88) }\end{array}$ & $\begin{array}{l}\text { face (43), eyelid (8), } \\
\text { trunk/neck (14), } \\
\text { forehead/temple (25), } \\
\text { chin/perioral (10) }\end{array}$ \\
\hline $\begin{array}{l}\text { Torres } 2004 \\
15606733\end{array}$ & $\begin{array}{l}\text { imiquimod, } 2 \\
\text { weeks }\end{array}$ & NR & $\begin{array}{l}\text { median } 0.9 \mathrm{~cm} 2 \\
(\mathrm{IQR} 0.2,2.0)\end{array}$ & 33.3 & & $\begin{array}{l}\text { BCC: superficial (42), } \\
\text { nodular (58) }\end{array}$ & NR \\
\hline $\begin{array}{l}\text { Torres } 2004 \\
15606733\end{array}$ & $\begin{array}{l}\text { imiquimod, } 4 \\
\text { weeks }\end{array}$ & NR & $\begin{array}{l}\text { median } 0.8 \mathrm{~cm} 2 \\
(\text { IQR } 0.5,1.3)\end{array}$ & 41.7 & & $\begin{array}{l}\text { BCC: superficial (33), } \\
\text { nodular (67) }\end{array}$ & NR \\
\hline $\begin{array}{l}\text { Torres } 2004 \\
15606733\end{array}$ & $\begin{array}{l}\text { imiquimod, } 6 \\
\text { weeks }\end{array}$ & NR & $\begin{array}{l}\text { median } 1.2 \mathrm{~cm} 2 \\
(\text { IQR } 0.5,2.7)\end{array}$ & 33.3 & & $\begin{array}{l}\text { BCC: superficial (17), } \\
\text { nodular (83) }\end{array}$ & NR \\
\hline $\begin{array}{l}\text { Torres } 2004 \\
15606733\end{array}$ & $\begin{array}{l}\text { vehicle } \\
\text { controlled- } \\
\text { pooled }\end{array}$ & NR & $\begin{array}{l}\text { median } 1.2 \mathrm{~cm} 2 \\
\text { (IQR } 0.5,2.7)\end{array}$ & 19.4 & & $\begin{array}{l}\text { BCC: superficial (33), } \\
\text { nodular (67) }\end{array}$ & NR \\
\hline Tran 201222511036 & $\begin{array}{l}\text { S1: PDL } 15 \\
\mathrm{j} / \mathrm{cm} 2\end{array}$ & NR & $\begin{array}{l}88 \mathrm{~mm} 2(\mathrm{SE} \\
12.1)\end{array}$ & 57 & I and II 100\% & $\begin{array}{l}\text { BCC: superficial (12.5), } \\
\text { nodular (62.5), } \\
\text { multifocal (12.5); SCC: } \\
\text { in situ (12.5) }\end{array}$ & $\begin{array}{l}\text { extremities (12), } \\
\text { trunk/neck (88) }\end{array}$ \\
\hline Tran 201222511036 & $\begin{array}{l}\text { S2: PDL } 7.5 \\
\mathrm{j} / \mathrm{cm} 2\end{array}$ & NR & $\begin{array}{l}105 \mathrm{~mm} 2 \text { (SE } \\
23.6)\end{array}$ & 43 & I and II 100\% & $\begin{array}{l}\text { BCC: nodular (50), } \\
\text { multifocal (27.5); SCC: } \\
\text { in situ (12.5) }\end{array}$ & $\begin{array}{l}\text { extremities (50), } \\
\text { trunk/neck (50) }\end{array}$ \\
\hline Tran 201222511036 & No treatment & NR & $\begin{array}{l}94 \mathrm{~mm} 2 \text { (SE } \\
15.2)\end{array}$ & 43 & I and II 100\% & $\begin{array}{l}\text { BCC: nodular (57), } \\
\text { multifocal (29); SCC: in }\end{array}$ & $\begin{array}{l}\text { extremities (43), } \\
\text { trunk/neck (57) }\end{array}$ \\
\hline
\end{tabular}




\begin{tabular}{|c|c|c|c|c|c|c|c|}
\hline $\begin{array}{l}\text { Author Year } \\
\text { PMID }\end{array}$ & Arm & $\begin{array}{l}\text { Age, mean } \\
\text { (SD); range }\end{array}$ & $\begin{array}{l}\text { Lesion area, } \\
\text { mean (SD); } \\
\text { range }\end{array}$ & $\begin{array}{l}\text { Female, } \\
\%\end{array}$ & $\begin{array}{l}\text { Fitzpatrick } \\
\text { score } \%\end{array}$ & Lesion type (\%) & Lesion location (\%) \\
\hline & & & & & & situ (16) & \\
\hline $\begin{array}{l}\text { van der Geer } 2012 \\
22385074\end{array}$ & $\begin{array}{l}\text { Imiquimod + } \\
\text { Mohs }\end{array}$ & $\begin{array}{l}69(\mathrm{NR}) ; \text { range } \\
(95 \% \mathrm{Cl} 65,73)\end{array}$ & & 37 & $129 ; I I 66$ & BCC: nodular (100) & $\begin{array}{l}\text { H-zone (57), nose (23), } \\
\text { ear } 4(11) \text {, scalp + frontal } \\
(23), \text { other regions (cheek, } \\
\text { temporal, chin) (43) }\end{array}$ \\
\hline $\begin{array}{l}\text { van der Geer } 2012 \\
22385074\end{array}$ & $\begin{array}{l}\text { no treatment } \\
+ \text { Mohs }\end{array}$ & $\begin{array}{l}68(\mathrm{NR}) ; \text { range } \\
(95 \% \mathrm{Cl} 64,72)\end{array}$ & $\begin{array}{l}\text { median } 110 \mathrm{~mm} 2 \\
(\mathrm{IQR} 80,160)\end{array}$ & 31 & $126 ; 1166$ & BCC: nodular (100) & $\begin{array}{l}\text { H-zone (66), nose (26), } \\
\text { ear (17), scalp + frontal } \\
(14), \text { other regions (cheek, } \\
\text { temporal, chin) (43) }\end{array}$ \\
\hline $\begin{array}{l}\text { Wang } 2001 \\
11298545\end{array}$ & Total & range $(42,88)$ & & 50 & & $\begin{array}{l}\text { BCC: superficial and } \\
\text { nodular }\end{array}$ & $\begin{array}{l}\text { legs (11), arms (7), trunk } \\
(54), \text { head/neck (28) }\end{array}$ \\
\hline $\begin{array}{l}\text { Wettstein } 2013 \\
23566745\end{array}$ & $\begin{array}{l}\text { Ringer's } \\
\text { lactate } \\
\text { (control } \\
\text { group) }\end{array}$ & $\begin{array}{l}59 \text { (NR); range } \\
(34,86)\end{array}$ & $2.5 \mathrm{~cm} 2(1.72)$ & 26.67 & & BCC: nodular (100) & $\begin{array}{l}\text { nose (46.2), cheek (23.1), } \\
\text { frontal (7.7), ear (23.1) }\end{array}$ \\
\hline $\begin{array}{l}\text { Wettstein } 2013 \\
23566745\end{array}$ & $\begin{array}{l}\text { interferon } \\
\text { alpha-2b }\end{array}$ & $\begin{array}{l}59(\mathrm{NR}) \text {; range } \\
(34,86)\end{array}$ & $3.1 \mathrm{~cm} 2(2.51)$ & 26.67 & & BCC: nodular (100) & $\begin{array}{l}\text { nose (50), cheek (10), } \\
\text { frontal (20), ear (20) }\end{array}$ \\
\hline \multicolumn{8}{|l|}{ NRCS } \\
\hline $\begin{array}{l}\text { Ahmed } 2000 \\
11069453 \\
\end{array}$ & Curettage & $74 ; 46,89$ & $\begin{array}{l}336 \mathrm{~mm} 2 ; 30- \\
1890\end{array}$ & 82 & & SCC: Bowen's (100) & $\begin{array}{l}\text { extremities (38), trunk (2), } \\
\text { head/neck (4) }\end{array}$ \\
\hline $\begin{array}{l}\text { Ahmed } 2000 \\
11069453\end{array}$ & Cryotherapy & $74 ; 46,89$ & $\begin{array}{l}336 \mathrm{~mm} 2 ; 30- \\
1890\end{array}$ & 82 & & SCC: Bowen's (100) & $\begin{array}{l}\text { extremities (29), trunk (4), } \\
\text { head/neck (3) }\end{array}$ \\
\hline $\begin{array}{l}\text { Ballester-Sanchez } \\
201626985197\end{array}$ & $\begin{array}{l}\text { brachythera } \\
\text { py } 36.6 \mathrm{~Gy}\end{array}$ & 70 (3); NR & $\begin{array}{l}\text { diameter: } 11.54 \\
(0.96)\end{array}$ & 50 & $\begin{array}{l}\text { II: } 9(45 \%) \\
\text { III: } 11(55 \%)\end{array}$ & $\begin{array}{l}\text { BCC: } \\
\text { superficial/multicentric } \\
(50 \%), \text { BCC: nodular } \\
(50 \%)\end{array}$ & $\begin{array}{l}\text { head/neck (15), } \\
\text { trunk/extremities (5) }\end{array}$ \\
\hline $\begin{array}{l}\text { Ballester-Sanchez } \\
201626985197\end{array}$ & $\begin{array}{l}\text { brachythera } \\
\text { py } 42 \text { Gy }\end{array}$ & 79 (2); NR & $\begin{array}{l}\text { diameter: } 12.2 \\
(0.68)\end{array}$ & 40 & $\begin{array}{l}\text { II: } 10(50 \%) \\
\text { III: } 10(50 \%)\end{array}$ & $\begin{array}{l}\text { BCC: } \\
\text { superficial/multicentric } \\
(40 \%), \text { BCC: nodular } \\
(60 \%)\end{array}$ & $\begin{array}{l}\text { head/neck (15), } \\
\text { trunk/extremities (5) }\end{array}$ \\
\hline $\begin{array}{l}\text { Chren } 2013 \\
23190903\end{array}$ & $\begin{array}{l}\text { electrodessi } \\
\text { cation and } \\
\text { curettage } \\
\end{array}$ & NR & $\begin{array}{l}\text { diameter: } 9.0 \mathrm{~mm} \\
(5.6)\end{array}$ & 21 & $\begin{array}{l}\text { I or II: } 97 \\
(41.1)\end{array}$ & $\begin{array}{l}\text { BCC: unspecified (83), } \\
\text { SCC: unspecified (17) }\end{array}$ & $\begin{array}{l}\text { H-Zone of face (10.7); } \\
\text { other (unspecified) (89.3) }\end{array}$ \\
\hline $\begin{array}{l}\text { Chren } 2013 \\
23190904 \\
\end{array}$ & excision & NR & $\begin{array}{l}\text { diameter: } 9.5 \mathrm{~mm} \\
(6.1)\end{array}$ & 21 & $\begin{array}{l}\text { I or II: } 180 \\
(38.2)\end{array}$ & $\begin{array}{l}\text { BCC: unspecified (69), } \\
\text { SCC: unspecified (31) }\end{array}$ & $\begin{array}{l}\text { H-Zone of face (25.9); } \\
\text { other (unspecified) (74.1) }\end{array}$ \\
\hline $\begin{array}{l}\text { Chren } 2013 \\
23190905\end{array}$ & Mohs & NR & $\begin{array}{l}\text { diameter: } 7.8 \mathrm{~mm} \\
(4.4)\end{array}$ & 33 & $\begin{array}{l}\text { I or II: } 196 \\
(42)\end{array}$ & $\begin{array}{l}\text { BCC: unspecified (77), } \\
\text { SCC: unspecified (23) }\end{array}$ & $\begin{array}{l}\text { H-Zone of face (64.6); } \\
\text { other (unspecified) (35.4) }\end{array}$ \\
\hline $\begin{array}{l}\text { Cosgarea } 2012 \\
22738399\end{array}$ & ALA PDT & $65 ; 51,85$ & & 47 & $\begin{array}{l}\text { I: 3, II: } 21, \text { III: } \\
12, \text { IV: } 1\end{array}$ & $\begin{array}{l}\text { BCC: } \\
\text { superficial/multicentric } \\
(64.5 \%), \text { BCC: nodular }\end{array}$ & NR \\
\hline
\end{tabular}




\begin{tabular}{|c|c|c|c|c|c|c|c|}
\hline $\begin{array}{l}\text { Author Year } \\
\text { PMID }\end{array}$ & Arm & $\begin{array}{l}\text { Age, mean } \\
\text { (SD); range }\end{array}$ & $\begin{array}{l}\text { Lesion area, } \\
\text { mean (SD); } \\
\text { range }\end{array}$ & $\begin{array}{l}\text { Female, } \\
\%\end{array}$ & $\begin{array}{l}\text { Fitzpatrick } \\
\text { score } \%\end{array}$ & Lesion type (\%) & Lesion location (\%) \\
\hline & & & & & & $(35.5 \%)$ & \\
\hline $\begin{array}{l}\text { Cosgarea } 2012 \\
22738399\end{array}$ & $\begin{array}{l}\text { surgical } \\
\text { excision }\end{array}$ & $66 ; 49,90$ & & 47.5 & $\begin{array}{l}\text { I: } 3, \text { II: } 19, \text { III: } \\
\text { 13, IV: } 1\end{array}$ & $\begin{array}{l}\text { BCC: } \\
\text { superficial/multicentric } \\
(63 \%), \text { BCC: nodular } \\
(37 \%)\end{array}$ & $\begin{array}{l}\text { face/scalp (21), } \\
\text { extremities (3), trunk/neck } \\
(24)\end{array}$ \\
\hline $\begin{array}{l}\text { Graells } 2014 \\
24139468\end{array}$ & Imiquimod & NR & & 50.7 & & $\begin{array}{l}\text { BCC: superficial(60), } \\
\text { nodular (2), } \\
\text { Infiltrative/micronodular/ } \\
\text { morphea } \\
\text { form/scelorosing (38) }\end{array}$ & $\begin{array}{l}\text { exremities (7.14), } \\
\text { trunk/neck (92.86) }\end{array}$ \\
\hline $\begin{array}{l}\text { Graells } 2014 \\
24139468\end{array}$ & Surgery & NR & & 43.27 & & $\begin{array}{l}\text { BCC: superficial(17), } \\
\text { nodular (32), } \\
\text { Infiltrative/micronodular/ } \\
\text { morphea } \\
\text { form/scelorosing (51) }\end{array}$ & $\begin{array}{l}\text { exremities (5.64), } \\
\text { trunk/neck (94.37) }\end{array}$ \\
\hline $\begin{array}{l}\text { Lippert } 2013 \\
23725586\end{array}$ & Total & 61.9 (NR); NR & & 43 & & BCC: nodular (100) & $\begin{array}{l}49 \text { head (not } \mathrm{H} \text {-zone or } \\
\text { adjacent to the eyes or } \\
\text { ears) cheeks, or neck; } 7 \\
\text { other parts of the body }\end{array}$ \\
\hline $\begin{array}{l}\text { Pampena } 2016 \\
26589877\end{array}$ & 3675 cGy & $81.3(8.7)$ & & 45.8 & & $\begin{array}{l}\text { BCC: unspecified (66), } \\
\text { SCC: unspecified (34) }\end{array}$ & $\begin{array}{l}\text { exremities (8.9), trunk } \\
(2.1), \text { head/neck (89) }\end{array}$ \\
\hline $\begin{array}{l}\text { Pampena } 2016 \\
26589878\end{array}$ & 4500 cGy & $73.3(10.2)$ & & 35.6 & & $\begin{array}{l}\text { BCC: unspecified } \\
\text { (80.5), SCC: } \\
\text { unspecified (19.5) }\end{array}$ & $\begin{array}{l}\text { exremities (5.4), trunk } \\
(5.4), \text { head/neck (89.2) }\end{array}$ \\
\hline $\begin{array}{l}\text { Shah } 2009 \\
19588534\end{array}$ & $\begin{array}{l}\text { Pulse dye } \\
\text { laser }\end{array}$ & NR & & NR & & $\begin{array}{l}\text { BCC: } \\
\text { superficial/multicentric } \\
\text { (43\%), BCC: nodular } \\
(47.5 \%), \text { BCC: } \\
\text { infiltrative/micronodular/ } \\
\text { morpheaform/scelorosin } \\
\text { g }(9.5 \%)\end{array}$ & $\begin{array}{l}\text { extremities (2), trunk/neck } \\
\text { (19) }\end{array}$ \\
\hline $\begin{array}{l}\text { Shah } 2009 \\
19588534\end{array}$ & no treatment & NR & & NR & & $\begin{array}{l}\text { BCC: superficial and } \\
\text { nodular }\end{array}$ & NR \\
\hline $\begin{array}{l}\text { Sofen } 2015 \\
25913533\end{array}$ & $\begin{array}{l}\text { vismodegib } \\
12 \text { weeks }\end{array}$ & $\begin{array}{l}60.5(11.2) ; 43, \\
81\end{array}$ & $\begin{array}{l}\text { diameter: } \\
\text { median: } 1.2 \mathrm{~cm} ; \\
\text { range: } 1-3\end{array}$ & 21 & & BCC: nodular (100) & $\begin{array}{l}\text { Scalp/head/neck and } \\
\text { cape area }(100 \%)\end{array}$ \\
\hline $\begin{array}{l}\text { Sofen } 2015 \\
25913533\end{array}$ & $\begin{array}{l}\text { vismodegib } \\
12 \text { weeks + } \\
24 \text { weeks } \\
\text { observation }\end{array}$ & $\begin{array}{l}65.2 \text { (13.3); 40, } \\
86\end{array}$ & $\begin{array}{l}\text { diameter: } \\
\text { median: } 1.5 ; \\
\text { range: } 1-2\end{array}$ & 12 & & BCC: nodular (100) & $\begin{array}{l}\text { Scalp/head/neck and } \\
\text { trunk/limbs }(100 \%)\end{array}$ \\
\hline
\end{tabular}




\begin{tabular}{|c|c|c|c|c|c|c|c|}
\hline $\begin{array}{l}\text { Author Year } \\
\text { PMID }\end{array}$ & Arm & $\begin{array}{l}\text { Age, mean } \\
\text { (SD); range }\end{array}$ & $\begin{array}{l}\text { Lesion area, } \\
\text { mean (SD); } \\
\text { range }\end{array}$ & $\begin{array}{l}\text { Female, } \\
\%\end{array}$ & $\begin{array}{l}\text { Fitzpatrick } \\
\text { score } \%\end{array}$ & Lesion type (\%) & Lesion location (\%) \\
\hline $\begin{array}{l}\text { Sofen } 2015 \\
25913533\end{array}$ & $\begin{array}{l}\text { vismodegib } \\
16 \text { weeks }\end{array}$ & $\begin{array}{l}65.1(11.8) ; 47 \\
89\end{array}$ & $\begin{array}{l}\text { diameter: } \\
\text { median: } 1.2 ; \\
\text { range: } 1-3\end{array}$ & 32 & & BCC: nodular (100) & $\begin{array}{l}\text { Scalp/head/neck and } \\
\text { trunk/limbs (100\%) }\end{array}$ \\
\hline $\begin{array}{l}\text { Sullivan } 2003 \\
14725659\end{array}$ & $\begin{array}{l}\text { imiquimod } \\
5 \%\end{array}$ & $63 ; 57,78$ & diameter: $9.5 \mathrm{~mm}$ & 33 & & BCC: superficial (100) & $\begin{array}{l}\text { Trunk/neck (4), forearm } \\
\text { (2) }\end{array}$ \\
\hline $\begin{array}{l}\text { Sullivan } 2003 \\
14725659 \\
\end{array}$ & vehicle & $59 ; 52,62$ & diameter: $7.5 \mathrm{~mm}$ & 33 & & BCC: superficial (100) & $\begin{array}{l}\text { Trunk/neck (4), forearm } \\
(2)\end{array}$ \\
\hline
\end{tabular}




\section{Appendix E. Arm Details}

Table E-1. Arm details

\begin{tabular}{|c|c|c|c|c|c|c|c|c|c|}
\hline $\begin{array}{l}\text { Author, } \\
\text { year, } \\
\text { PMID }\end{array}$ & Arm & $\begin{array}{l}\text { Surgical } \\
\text { interventions } \\
\text { (margins) }\end{array}$ & $\begin{array}{l}\text { Thermal } \\
\text { interventions } \\
\text { (description) }\end{array}$ & Radiation & $\begin{array}{l}\text { Photodynamic } \\
\text { Therapy dose }\end{array}$ & $\begin{array}{l}\text { Photodynamic } \\
\text { Therapy } \\
\text { description }\end{array}$ & $\begin{array}{l}\text { Medical } \\
\text { interventions } \\
\text { description }\end{array}$ & $\begin{array}{l}\text { Medical } \\
\text { interventions } \\
\text { other }\end{array}$ & $\begin{array}{l}\text { Curettage } \\
\text { number of } \\
\text { passes }\end{array}$ \\
\hline \multicolumn{10}{|l|}{ RCTs } \\
\hline $\begin{array}{l}\text { Abbade } \\
2015\end{array}$ & MAL-PDT & & & & $630 \mathrm{~nm}$ & $\begin{array}{l}2 \text { sessions in } 1 \\
\text { week, MAL, } \\
\text { "previously the } \\
\text { lesions were } \\
\text { shaved" }\end{array}$ & & & \\
\hline $\begin{array}{l}\text { Al-Niaimi } \\
2015 \\
26157307\end{array}$ & PDT + MMS & $\begin{array}{l}\text { Mohs } 2-10 \\
\text { weeks following } \\
\text { PDT treatment }\end{array}$ & & & $\begin{array}{l}\text { non-coherent red } \\
\text { light/average } \\
\text { wavelength } 631 \\
\mathrm{~nm} \text { at } 70-100 \\
\mathrm{~mW} / \mathrm{cm} 2 \text { to } 37 \\
\mathrm{~J} / \mathrm{cm} 2\end{array}$ & $\begin{array}{l}2 \text { sessions } 1 \\
\text { week apart to } 74 \\
\mathrm{~J} / \mathrm{cm} 2,160 \mathrm{mg} / \mathrm{g} \\
\mathrm{MAL}, \text { preparing } \\
\text { the site with } \\
\text { topical acetone } \\
\text { and light abrasion } \\
\text { with curettage }\end{array}$ & & & \\
\hline $\begin{array}{l}\text { Allen } 1979 \\
298425\end{array}$ & cryotherapy & & $\begin{array}{l}\text { Cryotherapy } \\
\text { (liquid nitrogen } \\
\text { spray from the } \\
\text { Brymil } \\
\text { cryospray) }\end{array}$ & & & & & & \\
\hline $\begin{array}{l}\text { Allen } 1979 \\
298425\end{array}$ & radiotherapy & & & $\begin{array}{l}\text { Photons } \\
\text { (gamma or } \\
\text { x), } 9 \text { times } \\
\text { a week for } \\
\text { one month }\end{array}$ & & & & & \\
\hline $\begin{array}{l}\text { Alpsoy } \\
1996 \\
8708151\end{array}$ & IFN alfa-2a & & & & & & $\begin{array}{l}\text { IFN alfa-2a } \\
\text { (Intralesional) } 3 \\
\text { times/weekly } \\
\text { (total } 10 \\
\text { injections) }\end{array}$ & $\begin{array}{l}1.5 \text { megaunits } \\
\text { of intralesional } \\
\text { IFN if the lesion } \\
\text { was less than } 2 \\
\mathrm{~cm} 2,3 \\
\text { megaunits, if }\end{array}$ & \\
\hline
\end{tabular}




\begin{tabular}{|c|c|c|c|c|c|c|c|c|c|}
\hline $\begin{array}{l}\text { Author, } \\
\text { year, } \\
\text { PMID }\end{array}$ & Arm & $\begin{array}{l}\text { Surgical } \\
\text { interventions } \\
\text { (margins) }\end{array}$ & $\begin{array}{l}\text { Thermal } \\
\text { interventions } \\
\text { (description) }\end{array}$ & Radiation & $\begin{array}{l}\text { Photodynamic } \\
\text { Therapy dose }\end{array}$ & $\begin{array}{l}\text { Photodynamic } \\
\text { Therapy } \\
\text { description }\end{array}$ & $\begin{array}{l}\text { Medical } \\
\text { interventions } \\
\text { description }\end{array}$ & $\begin{array}{l}\text { Medical } \\
\text { interventions } \\
\text { other }\end{array}$ & $\begin{array}{l}\text { Curettage } \\
\text { number of } \\
\text { passes }\end{array}$ \\
\hline & & & & & & & & $\begin{array}{l}\text { greater than } 2 \\
\mathrm{~cm} 2\end{array}$ & \\
\hline $\begin{array}{l}\text { Alpsoy } \\
1996 \\
8708151\end{array}$ & IFN alfa-2b & & & & & & $\begin{array}{l}\text { IFN alfa-2b } \\
\text { (Intralesional) } 3 \\
\text { times/weekly } \\
\text { (total } 10 \\
\text { injections) }\end{array}$ & $\begin{array}{l}1.5 \text { megaunits } \\
\text { of intralesional } \\
\text { IFN if the lesion } \\
\text { was less than } 2 \\
\mathrm{~cm} 2,3 \\
\text { megaunits, if } \\
\text { greater than } 2 \\
\mathrm{~cm} 2\end{array}$ & \\
\hline $\begin{array}{l}\text { Alpsoy } \\
1996 \\
8708151\end{array}$ & $\begin{array}{l}\text { IFN alfa-2a + } \\
\text { IFN alfa-2b }\end{array}$ & & & & & & $\begin{array}{l}\text { IFN alfa-2a and } \\
\text { 2b (injected } \\
\text { alternately) } \\
\text { (Intralesional) } 3 \\
\text { times/weekly } \\
\text { (total } 10 \\
\text { injections) }\end{array}$ & $\begin{array}{l}1.5 \text { megaunits } \\
\text { of intralesional } \\
\text { IFN if the lesion } \\
\text { was less than } 2 \\
\mathrm{~cm} 2,3 \\
\text { megaunits, if } \\
\text { greater than } 2 \\
\mathrm{~cm} 2\end{array}$ & \\
\hline $\begin{array}{l}\text { Arits } 2013 \\
23683751\end{array}$ & MAL-PDT & & & & $\begin{array}{l}\text { LED } 630 \mathrm{~nm} \text { for } \\
7 \mathrm{~min} \text { at to } 37 \\
\mathrm{~J} / \mathrm{cm} 2 \text {, total dose } \\
74 \mathrm{~J} / \mathrm{cm} 2\end{array}$ & $\begin{array}{l}2 \text { sessions } 1 \\
\text { week apart, } 16 \% \\
\text { MAL, non- } \\
\text { traumatic surface } \\
\text { preparation }\end{array}$ & & & \\
\hline $\begin{array}{l}\text { Arits } 2013 \\
23683751\end{array}$ & Imiquimod & & & & & & $\begin{array}{l}\text { Imiquimod 5\% } \\
\text { (Topical) daily } 5 \\
\text { days/week for } 6 \\
\text { weeks }\end{array}$ & $\begin{array}{l}\text { apply in a thin } \\
\text { layer to the } \\
\text { tumour } \\
\text { including 5-10 } \\
\text { mm of the } \\
\text { surrounding } \\
\text { skin with no } \\
\text { occlusive } \\
\text { dressing } \\
\text { applied. } \\
\text { Patients were } \\
\text { advised to } \\
\text { apply the cream } \\
\text { at least } 1 \mathrm{~h} \\
\text { before going to } \\
\text { bed and to wipe } \\
\text { it off after } 8 \mathrm{~h} \text {. }\end{array}$ & \\
\hline Arits 2013 & Fluorouacil & & & & & & Fluorouacil 5\% & apply in a thin & \\
\hline
\end{tabular}




\begin{tabular}{|c|c|c|c|c|c|c|c|c|c|}
\hline $\begin{array}{l}\text { Author, } \\
\text { year, } \\
\text { PMID }\end{array}$ & Arm & $\begin{array}{l}\text { Surgical } \\
\text { interventions } \\
\text { (margins) }\end{array}$ & $\begin{array}{l}\text { Thermal } \\
\text { interventions } \\
\text { (description) }\end{array}$ & Radiation & $\begin{array}{l}\text { Photodynamic } \\
\text { Therapy dose }\end{array}$ & $\begin{array}{l}\text { Photodynamic } \\
\text { Therapy } \\
\text { description }\end{array}$ & $\begin{array}{l}\text { Medical } \\
\text { interventions } \\
\text { description }\end{array}$ & $\begin{array}{l}\text { Medical } \\
\text { interventions } \\
\text { other }\end{array}$ & $\begin{array}{l}\text { Curettage } \\
\text { number of } \\
\text { passes }\end{array}$ \\
\hline 23683751 & 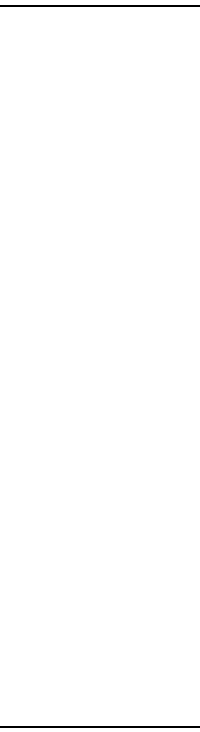 & r & & & & & $\begin{array}{l}\text { (Topical) twice } \\
\text { daily (morning } \\
\text { and evening) for } \\
4 \text { weeks }\end{array}$ & $\begin{array}{l}\text { layer to the } \\
\text { tumour } \\
\text { including 5-10 } \\
\text { mm of the } \\
\text { surrounding } \\
\text { skin, with no } \\
\text { occusive } \\
\text { dressing } \\
\text { applied. } \\
\text { Patients were } \\
\text { advised to wipe } \\
\text { off the } \\
\text { remnants } \\
\text { before applying } \\
\text { a new layer. } \\
\text { There was no } \\
\text { time limit on } \\
\text { how long the } \\
\text { cream was to } \\
\text { remain applied } \\
\end{array}$ & \\
\hline $\begin{array}{l}\text { Avril } 1997 \\
9218740\end{array}$ & surgery & $\begin{array}{l}\text { Frozen section } \\
(2 \mathrm{~mm})\end{array}$ & & & & & & & \\
\hline $\begin{array}{l}\text { Avril } 1997 \\
9218740\end{array}$ & radiotherapy & & & $\begin{array}{l}\text { Photons } \\
\text { (gamma or } \\
\text { x) for } \\
\text { Interstitial } \\
\text { brachythera } \\
\text { py (65-70 } \\
\text { Gy } \\
\text { delivered at } \\
\text { the } \\
\text { reference } \\
\text { isodose, } \\
\text { according } \\
\text { to the Paris } \\
\text { dosimetry } \\
\text { method, } \\
\text { over 5-7 } \\
\text { days) or } \\
\text { Superficial } \\
\text { contacthera }\end{array}$ & & & & & \\
\hline
\end{tabular}




\begin{tabular}{|c|c|c|c|c|c|c|c|c|c|}
\hline $\begin{array}{l}\text { Author, } \\
\text { year, } \\
\text { PMID }\end{array}$ & Arm & $\begin{array}{l}\text { Surgical } \\
\text { interventions } \\
\text { (margins) }\end{array}$ & $\begin{array}{l}\text { Thermal } \\
\text { interventions } \\
\text { (description) }\end{array}$ & Radiation & $\begin{array}{l}\text { Photodynamic } \\
\text { Therapy dose }\end{array}$ & $\begin{array}{l}\text { Photodynamic } \\
\text { Therapy } \\
\text { description }\end{array}$ & $\begin{array}{l}\text { Medical } \\
\text { interventions } \\
\text { description }\end{array}$ & $\begin{array}{l}\text { Medical } \\
\text { interventions } \\
\text { other }\end{array}$ & $\begin{array}{l}\text { Curettage } \\
\text { number of } \\
\text { passes }\end{array}$ \\
\hline & 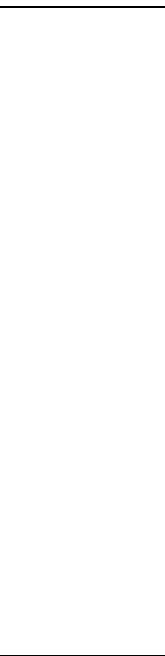 & & & $\begin{array}{l}\text { py (for BCC } \\
<2 \mathrm{~mm}, 2 \\
\text { sessions, } \\
\text { each } \\
\text { delivering } \\
\text { 18-20 Gy } \\
\text { with a 2- } \\
\text { week } \\
\text { interval) or } \\
\text { Convention } \\
\text { al } \\
\text { radiotherap } \\
\text { y (2-4 Gy, } \\
3-4 \text { times } \\
\text { per week, } \\
\text { up to a total } \\
\text { dose of } 60 \\
\text { Gy) }\end{array}$ & & & & & \\
\hline $\begin{array}{l}\text { Basset- } \\
\text { Seguin } \\
2008 \\
18693158\end{array}$ & MAL-PDT & & & & $\begin{array}{l}\text { (Curelight } \AA \text {; } \\
\text { PhotoCure ASA, } \\
\text { Oslo Nor- } \\
\text { way)/570-670 nm } \\
\text { to } 75 \mathrm{~J} / \mathrm{cm}\end{array}$ & $\begin{array}{l}\text { standard was one } \\
\text { session; crust). } \\
\text { Lesions with non- } \\
\text { complete } \\
\text { response were } \\
\text { treated again with } \\
\text { two MAL PDT } \\
\text { sessions } 7 \text { days } \\
\text { apart apart to } \\
\text { *depends on } \\
\text { number of } \\
\text { sessions., } 160 \\
\text { mg/g MAL, the } \\
\text { lesions were } \\
\text { prepared by slight } \\
\text { surface } \\
\text { debridement } \\
\text { using a curette or } \\
\text { scalpel blade to } \\
\text { facilitate access } \\
\text { of the cream and } \\
\text { light. Lesion } \\
\text { preparation was }\end{array}$ & & & \\
\hline
\end{tabular}




\begin{tabular}{|c|c|c|c|c|c|c|c|c|c|}
\hline $\begin{array}{l}\text { Author, } \\
\text { year, } \\
\text { PMID }\end{array}$ & Arm & $\begin{array}{l}\text { Surgical } \\
\text { interventions } \\
\text { (margins) }\end{array}$ & $\begin{array}{l}\text { Thermal } \\
\text { interventions } \\
\text { (description) }\end{array}$ & Radiation & $\begin{array}{l}\text { Photodynamic } \\
\text { Therapy dose }\end{array}$ & $\begin{array}{l}\text { Photodynamic } \\
\text { Therapy } \\
\text { description }\end{array}$ & $\begin{array}{l}\text { Medical } \\
\text { interventions } \\
\text { description }\end{array}$ & $\begin{array}{l}\text { Medical } \\
\text { interventions } \\
\text { other }\end{array}$ & $\begin{array}{l}\text { Curettage } \\
\text { number of } \\
\text { passes }\end{array}$ \\
\hline & & & & & & $\begin{array}{l}\text { always very } \\
\text { superficial and } \\
\text { insufficient to } \\
\text { cause pain.A } 1 \\
\text { mm layer of MAL } \\
\text { cream was } \\
\text { applied to each } \\
\text { lesion and } 5 \mathrm{~mm} \\
\text { of surrounding } \\
\text { tissue, and then } \\
\text { covered with an } \\
\text { adhesive } \\
\text { occlusive } \\
\text { dressing for } 3 \\
\text { hours. The } \\
\text { dressings were } \\
\text { then removed and } \\
\text { the cream } \\
\text { washed off with } \\
0.9 \% \text { saline } \\
\text { solution before } \\
\text { illumination }\end{array}$ & & & \\
\hline $\begin{array}{l}\text { Basset- } \\
\text { Seguin } \\
2008 \\
18693158\end{array}$ & $\begin{array}{l}\text { Cryotherapy } \\
\text { ( } 2 \text { freeze } \\
\text { thaw cycles) }\end{array}$ & & $\begin{array}{l}\text { Cryotherapy } \\
\text { (Cryotherapy } \\
\text { was performed } \\
\text { using a hand- } \\
\text { held liquid } \\
\text { nitrogen spray } \\
\text { and a double } \\
\text { freeze-thaw } \\
\text { cycle. After an } \\
\text { initial ice field } \\
\text { forma- tion with } \\
\text { a } 3 \text { mm rim of } \\
\text { clinically } \\
\text { healthy tissue, } \\
\text { the ice field } \\
\text { was maintained } \\
\text { for up to } 20 \\
\text { seconds. The } \\
\text { procedure was }\end{array}$ & & & & & & \\
\hline
\end{tabular}




\begin{tabular}{|c|c|c|c|c|c|c|c|c|c|}
\hline $\begin{array}{l}\text { Author, } \\
\text { year, } \\
\text { PMID }\end{array}$ & Arm & $\begin{array}{l}\text { Surgical } \\
\text { interventions } \\
\text { (margins) }\end{array}$ & $\begin{array}{l}\text { Thermal } \\
\text { interventions } \\
\text { (description) }\end{array}$ & Radiation & $\begin{array}{l}\text { Photodynamic } \\
\text { Therapy dose }\end{array}$ & $\begin{array}{l}\text { Photodynamic } \\
\text { Therapy } \\
\text { description }\end{array}$ & $\begin{array}{l}\text { Medical } \\
\text { interventions } \\
\text { description }\end{array}$ & $\begin{array}{l}\text { Medical } \\
\text { interventions } \\
\text { other }\end{array}$ & $\begin{array}{l}\text { Curettage } \\
\text { number of } \\
\text { passes }\end{array}$ \\
\hline & & & $\begin{array}{l}\text { repeated after a } \\
\text { thaw of } 2-3 \\
\text { times the freeze } \\
\text { duration. } \\
\text { Lesions with } \\
\text { non-complete } \\
\text { response or } \\
\text { repeat double } \\
\text { freeze-thaw } \\
\text { cryo- therapy } \\
\text { and then } \\
\text { evaluated } 3 \\
\text { months later; } 2 \\
\text { passes) }\end{array}$ & & & & & & \\
\hline $\begin{array}{l}\text { Bath- } \\
\text { Hextall } \\
2014 \\
24332516\end{array}$ & Imiquimod & & & & & & $\begin{array}{l}\text { Imiquimod 5\% } \\
\text { (Topical) once } \\
\text { daily for } 6 \\
\text { (superficial - } \\
\text { clinically } \\
\text { diagnosed) or } \\
12 \text { (nodular) } \\
\text { weeks }\end{array}$ & before bed & \\
\hline $\begin{array}{l}\text { Bath- } \\
\text { Hextall } \\
2014 \\
24332516 \\
\end{array}$ & excision & $\begin{array}{l}\text { excsision (4 } \\
\mathrm{mm})\end{array}$ & & & & & & & \\
\hline $\begin{array}{l}\text { Berroeta } \\
2007 \\
17573890\end{array}$ & PDT & $\begin{array}{l}\text { excision (4-5 } \\
\mathrm{mm})\end{array}$ & & & $\begin{array}{l}630 \mathrm{~nm} \text { to } 125 \\
\mathrm{~mW} \mathrm{~cm} \wedge-2\end{array}$ & $\begin{array}{l}20 \% ;>50 \mathrm{mg} \\
\mathrm{cm}^{\wedge}-2 \text { ALA, gentle } \\
\text { superficial } \\
\text { curettage, } 5- \\
\text { aminolaevulinic } \\
\text { acid fir } 6 \mathrm{~h} \text { under } \\
\text { occlusion }\end{array}$ & & & \\
\hline $\begin{array}{l}\text { Berroeta } \\
2007 \\
17573890 \\
\end{array}$ & excision & & & & & & & & \\
\hline $\begin{array}{l}\text { Beutner } \\
1999 \\
10570388\end{array}$ & $\begin{array}{l}\text { imiquimod } \\
\text { 2x/day }\end{array}$ & & & & & & $\begin{array}{l}\text { imiquimod 5\% } \\
\text { (Topical) twice } \\
\text { daily for } 10 \\
\text { weeks (median) }\end{array}$ & & \\
\hline Beutner & imiquimod & & & & & & imiquimod 5\% & & \\
\hline
\end{tabular}




\begin{tabular}{|c|c|c|c|c|c|c|c|c|c|}
\hline $\begin{array}{l}\text { Author, } \\
\text { year, } \\
\text { PMID }\end{array}$ & Arm & $\begin{array}{l}\text { Surgical } \\
\text { interventions } \\
\text { (margins) }\end{array}$ & $\begin{array}{l}\text { Thermal } \\
\text { interventions } \\
\text { (description) }\end{array}$ & Radiation & $\begin{array}{l}\text { Photodynamic } \\
\text { Therapy dose }\end{array}$ & $\begin{array}{l}\text { Photodynamic } \\
\text { Therapy } \\
\text { description }\end{array}$ & $\begin{array}{l}\text { Medical } \\
\text { interventions } \\
\text { description }\end{array}$ & $\begin{array}{l}\text { Medical } \\
\text { interventions } \\
\text { other }\end{array}$ & $\begin{array}{l}\text { Curettage } \\
\text { number of } \\
\text { passes }\end{array}$ \\
\hline $\begin{array}{l}1999 \\
10570388\end{array}$ & 1x/day & & & & & & $\begin{array}{l}\text { (Topical) once } \\
\text { daily for } 13 \\
\text { weeks (median) }\end{array}$ & & \\
\hline $\begin{array}{l}\text { Beutner } \\
1999 \\
10570388\end{array}$ & $\begin{array}{l}\text { imiquimod } \\
\text { 3x/week }\end{array}$ & & & & & & $\begin{array}{l}\text { imiquimod } 5 \% \\
\text { (Topical) three } \\
\text { times weekly for } \\
14.5 \text { weeks } \\
\text { (median) }\end{array}$ & & \\
\hline $\begin{array}{l}\text { Beutner } \\
1999 \\
10570388\end{array}$ & $\begin{array}{l}\text { imiquimod } \\
\text { 2x/week }\end{array}$ & & & & & & $\begin{array}{l}\text { imiquimod 5\% } \\
\text { (Topical) twice } \\
\text { weekly for } 16 \\
\text { weeks (median) }\end{array}$ & & \\
\hline $\begin{array}{l}\text { Beutner } \\
1999 \\
10570388\end{array}$ & $\begin{array}{l}\text { imiquimod } \\
\text { 1x/week }\end{array}$ & & & & & & $\begin{array}{l}\text { imiquimod } 5 \% \\
\text { (Topical) once } \\
\text { weekly for } 16 \\
\text { weeks (median) }\end{array}$ & & \\
\hline $\begin{array}{l}\text { Beutner } \\
1999 \\
10570388\end{array}$ & $\begin{array}{l}\text { vehicle (3 } \\
\text { 2x/day, } 2 \\
\text { 1x/day, } 2 \\
\text { 3x/week, } 2 \\
\text { 2x/week, } 2 \\
\text { 1x/week) }\end{array}$ & & & & & & $\begin{array}{l}\text { vehicle cream } \\
5 \% \text { (Topical) } 3 \\
2 x / \text { day, } 2 \\
\text { 1x/day, } 2 \\
\text { 3x/week, } 2 \\
\text { 2x/week, } 2 \\
\text { 1x/week for } 16 \\
\text { weeks (median) }\end{array}$ & & \\
\hline $\begin{array}{l}\text { Brinkhuize } \\
\text { n } 2016 \\
27067393\end{array}$ & Diclofenac & & & & & & $\begin{array}{l}\text { diclofenac } \\
\text { sodium } 3 \% \text { gel } \\
\text { in hyaluronic } \\
\text { acid } 2.5 \% \\
\text { (Topical) twice } \\
\text { daily for } 8 \text { weeks }\end{array}$ & & \\
\hline $\begin{array}{l}\text { Brinkhuize } \\
\text { n } 2016 \\
27067393 \\
\end{array}$ & Calcitriol & & & & & & $\begin{array}{l}\text { Calcitriol } 3 \mathrm{u} ? \mathrm{~g} / \mathrm{g} \\
\text { (Topical) twice } \\
\text { daily for } 8 \text { weeks }\end{array}$ & & \\
\hline $\begin{array}{l}\text { Brinkhuize } \\
\text { n } 2016 \\
27067393\end{array}$ & $\begin{array}{l}\text { Diclofenac + } \\
\text { Calcitriol }\end{array}$ & & & & & & $\begin{array}{l}\text { diclofenac } \\
\text { sodium }+ \\
\text { Calcitriol } 3 \% \text { gel } \\
\text { in hyaluronic } \\
\text { acid } 2.5 \%+3 \\
\text { ?ug/g (Topical) } \\
\text { twice daily for } 8 \\
\text { weeks }\end{array}$ & $\begin{array}{l}\text { diclofenac gel } \\
\text { application was } \\
\text { followed by } \\
\text { calcitriol } \\
\text { ointment with a } \\
\text { 2-minute } \\
\text { interval }\end{array}$ & \\
\hline
\end{tabular}




\begin{tabular}{|c|c|c|c|c|c|c|c|c|c|}
\hline $\begin{array}{l}\text { Author, } \\
\text { year, } \\
\text { PMID }\end{array}$ & Arm & $\begin{array}{l}\text { Surgical } \\
\text { interventions } \\
\text { (margins) }\end{array}$ & $\begin{array}{l}\text { Thermal } \\
\text { interventions } \\
\text { (description) }\end{array}$ & Radiation & $\begin{array}{l}\text { Photodynamic } \\
\text { Therapy dose }\end{array}$ & $\begin{array}{l}\text { Photodynamic } \\
\text { Therapy } \\
\text { description }\end{array}$ & $\begin{array}{l}\text { Medical } \\
\text { interventions } \\
\text { description }\end{array}$ & $\begin{array}{l}\text { Medical } \\
\text { interventions } \\
\text { other }\end{array}$ & $\begin{array}{l}\text { Curettage } \\
\text { number of } \\
\text { passes }\end{array}$ \\
\hline $\begin{array}{l}\text { Brinkhuize } \\
\text { n } 2016 \\
27067393 \\
\end{array}$ & No treatment & & & & & & & & \\
\hline $\begin{array}{l}\text { Butler } \\
2009 \\
19018814 \\
\end{array}$ & $\begin{array}{l}\text { Vehicle } \\
\text { group+MOHs }\end{array}$ & Mohs & & & & & $\begin{array}{l}\text { Vehicle } \\
\text { (Topical) nightly } \\
\text { for } 6 \text { weeks }\end{array}$ & & \\
\hline $\begin{array}{l}\text { Butler } \\
2009 \\
19018814 \\
\end{array}$ & $\begin{array}{l}\text { imiquimod } 5 \% \\
\text { Cream } \\
\text { group+MOHs }\end{array}$ & Mohs & & & & & $\begin{array}{l}\text { Imiquimod } \\
\text { (Topical) nightly } \\
\text { for } 6 \text { weeks }\end{array}$ & & \\
\hline Cai 2015 & ALA-PDT + & & CO2 Laser & & red light from & $1-3$ treatment & & & \\
\hline 25899562 & CO2 Laser & & $\begin{array}{l}\text { Therapy without } \\
\text { curettage } \\
\text { (vaporization } \\
\text { under local } \\
\text { anesthesia, } \\
\text { power ranging } \\
\text { between } 2 \text { and } \\
3 \mathrm{~W} \text {. lesions } \\
\text { vaporized to the } \\
\text { leveled of the } \\
\text { papillary dermis } \\
\text { in nonhairy } \\
\text { areas and to } \\
\text { the level fo the } \\
\text { midreticular } \\
\text { dermis in hairy } \\
\text { areas. during } \\
\text { process } \\
\text { vaporized } \\
\text { tissues were } \\
\text { erased with a } \\
\text { cotton swab } \\
\text { soaked with } \\
\text { broogeramine } \\
\text { to expose the } \\
\text { fresh wound.) }\end{array}$ & & $\begin{array}{l}\text { laser radiation } \\
\text { source (qishi } \\
\text { Laser } \\
\text { institute) } / 630 \mathrm{~nm} \\
\text { to } 180 \mathrm{j} / \mathrm{cm}^{\wedge} 2\end{array}$ & $\begin{array}{l}\text { sessions one } \\
\text { week intervals } \\
\text { apart, } 20 \% \text { ALA, } \\
\text { vaporization } \\
\text { under local } \\
\text { anesthesia, power } \\
\text { ranging between } \\
2 \text { and } 3 \text { W. } \\
\text { lesions vaporized } \\
\text { to the leveled of } \\
\text { the papillary } \\
\text { dermis in } \\
\text { nonhairy areas } \\
\text { and to the level fo } \\
\text { the midreticular } \\
\text { dermis in hairy } \\
\text { areas. during } \\
\text { process vaporized } \\
\text { tissues were } \\
\text { erased with a } \\
\text { cotton swab } \\
\text { soaked with } \\
\text { broogeramine to } \\
\text { expose the fresh } \\
\text { wound; ala } \\
\text { hydrochloride } \\
\text { applied to lesion } \\
\text { and surrounding } \\
\text { area (0 to } 4 \text { mm } \\
\text { away from } \\
\text { margin). lesion }\end{array}$ & & & \\
\hline
\end{tabular}




\begin{tabular}{|c|c|c|c|c|c|c|c|c|c|}
\hline $\begin{array}{l}\text { Author, } \\
\text { year, } \\
\text { PMID }\end{array}$ & Arm & $\begin{array}{l}\text { Surgical } \\
\text { interventions } \\
\text { (margins) }\end{array}$ & $\begin{array}{l}\text { Thermal } \\
\text { interventions } \\
\text { (description) }\end{array}$ & Radiation & $\begin{array}{l}\text { Photodynamic } \\
\text { Therapy dose }\end{array}$ & $\begin{array}{l}\text { Photodynamic } \\
\text { Therapy } \\
\text { description }\end{array}$ & $\begin{array}{l}\text { Medical } \\
\text { interventions } \\
\text { description }\end{array}$ & $\begin{array}{l}\text { Medical } \\
\text { interventions } \\
\text { other }\end{array}$ & $\begin{array}{l}\text { Curettage } \\
\text { number of } \\
\text { passes }\end{array}$ \\
\hline & ( & & & & & $\begin{array}{l}\text { sites maintained } \\
\text { under occlusion } \\
\text { for } 5 \mathrm{~h} \text { using and } \\
\text { occlusive and } \\
\text { light shielding } \\
\text { dressing. After } \\
\text { occlusion, } \\
\text { dressing removed } \\
\text { and ALA washed } \\
\text { of with } 0.9 \% \\
\text { saline solution. } \\
\text { depending on } \\
\text { response of pts, } \\
\text { treatments done } \\
1,2,3 \text { times } \\
\text { (separated by } \\
\text { weekly intervals) }\end{array}$ & & & \\
\hline $\begin{array}{l}\text { Cai } 2015 \\
25899562\end{array}$ & CO2 Laser & & $\begin{array}{l}\text { CO2 Laser } \\
\text { Therapy without } \\
\text { curettage } \\
\text { (vaporization } \\
\text { under local } \\
\text { anesthesia, } \\
\text { power ranging } \\
\text { between } 2 \text { and } \\
3 \mathrm{~W} \text {. lesions } \\
\text { vaporized to the } \\
\text { leveled of the } \\
\text { papillary dermis } \\
\text { in nonhairy } \\
\text { areas and to } \\
\text { the level fo the } \\
\text { midreticular } \\
\text { dermis in hairy } \\
\text { areas. during } \\
\text { process } \\
\text { vaporized } \\
\text { tissues were } \\
\text { erased with a } \\
\text { cotton swab } \\
\text { soaked with }\end{array}$ & & & & & & \\
\hline
\end{tabular}




\begin{tabular}{|c|c|c|c|c|c|c|c|c|c|}
\hline $\begin{array}{l}\text { Author, } \\
\text { year, } \\
\text { PMID }\end{array}$ & Arm & $\begin{array}{l}\text { Surgical } \\
\text { interventions } \\
\text { (margins) }\end{array}$ & $\begin{array}{l}\text { Thermal } \\
\text { interventions } \\
\text { (description) }\end{array}$ & Radiation & $\begin{array}{l}\text { Photodynamic } \\
\text { Therapy dose }\end{array}$ & $\begin{array}{l}\text { Photodynamic } \\
\text { Therapy } \\
\text { description }\end{array}$ & $\begin{array}{l}\text { Medical } \\
\text { interventions } \\
\text { description }\end{array}$ & $\begin{array}{l}\text { Medical } \\
\text { interventions } \\
\text { other }\end{array}$ & $\begin{array}{l}\text { Curettage } \\
\text { number of } \\
\text { passes }\end{array}$ \\
\hline & & & $\begin{array}{l}\text { broogeramine } \\
\text { to expose the } \\
\text { fresh wound.) } \\
\text { depending on } \\
\text { response of pts, } \\
\text { treatments } \\
\text { done } 1,2,3 \\
\text { times } \\
\text { (separated by } \\
\text { weekly } \\
\text { intervals) }\end{array}$ & & & & & & \\
\hline $\begin{array}{l}\text { Carija } \\
2016 \\
27516420\end{array}$ & PDT-ALA & & & & $150 \mathrm{~J} / \mathrm{cm} 2$ & $\begin{array}{l}1 \text { session } 3-5 \\
\text { minutes, } 20 \% \\
\text { ALA, surface } \\
\text { crusts removed } \\
\text { and abraded }\end{array}$ & & & \\
\hline $\begin{array}{l}\text { Carija } \\
2016 \\
27516420\end{array}$ & $\begin{array}{l}\text { PDT-ALA + } \\
\text { PDL }\end{array}$ & & & & $150 \mathrm{~J} / \mathrm{cm} 2$ & $\begin{array}{l}1 \text { session } 3-5 \\
\text { minutes, } 20 \% \\
\text { ALA, surface } \\
\text { crusts removed } \\
\text { and abraded; } \\
\text { Pulsed dye laser, } \\
\text { three passes }\end{array}$ & & & \\
\hline $\begin{array}{l}\text { Choi } 2016 \\
26551044\end{array}$ & $\begin{array}{l}\text { Er:YAG } \\
\text { ablative } \\
\text { fractional } \\
\text { laser-primed } \\
\text { MAL- PDT }\end{array}$ & & & & $\begin{array}{l}\text { Aktilite CL128/ } \\
632 \mathrm{~nm} \text { to } 37 \\
\mathrm{~J} / \mathrm{m}^{\wedge} 2\end{array}$ & $\begin{array}{l}1 \text { session, 16\% } \\
\text { MAL, lesions } \\
\text { were then } \\
\text { cleansed with } \\
\text { saline gauze, and } \\
\text { a lidocaine- } \\
\text { prilocaine 5\% } \\
\text { cream (EMLA; } \\
\text { Astra } \\
\text { Pharmaceuticals, } \\
\text { LP, Westborough, } \\
\text { MA, USA) was } \\
\text { applied to the } \\
\text { treatment area for } \\
30 \text { min under } \\
\text { occlusion. After } \\
\text { the anaesthetic } \\
\text { cream was }\end{array}$ & & & \\
\hline
\end{tabular}




\begin{tabular}{|c|c|c|c|c|c|c|c|c|c|}
\hline $\begin{array}{l}\text { Author, } \\
\text { year, } \\
\text { PMID }\end{array}$ & Arm & $\begin{array}{l}\text { Surgical } \\
\text { interventions } \\
\text { (margins) }\end{array}$ & $\begin{array}{l}\text { Thermal } \\
\text { interventions } \\
\text { (description) }\end{array}$ & Radiation & $\begin{array}{l}\text { Photodynamic } \\
\text { Therapy dose }\end{array}$ & $\begin{array}{l}\text { Photodynamic } \\
\text { Therapy } \\
\text { description }\end{array}$ & $\begin{array}{l}\text { Medical } \\
\text { interventions } \\
\text { description }\end{array}$ & $\begin{array}{l}\text { Medical } \\
\text { interventions } \\
\text { other }\end{array}$ & $\begin{array}{l}\text { Curettage } \\
\text { number of } \\
\text { passes }\end{array}$ \\
\hline & & & & & & $\begin{array}{l}\text { removed, AFL } \\
\text { was performed } \\
\text { using a } 2940 \text { nm } \\
\text { Er:YAG AFL } \\
\text { (Joule; Sciton } \\
\text { Inc., Palo Alto, } \\
\text { CA, USA) with a } \\
550 \text { Im ablation } \\
\text { depth, level one } \\
\text { coagulation, 22\% } \\
\text { treatment density } \\
\text { and a single } \\
\text { pulse. Immedi- } \\
\text { ately after AFL, a } \\
1 \text { mm thick layer } \\
\text { of methyl } \\
\text { aminolevulinate } \\
\text { (16\% Metvix } \\
\text { cream; PhotoCure } \\
\text { ASA, Oslo, } \\
\text { Norway) was } \\
\text { applied to the } \\
\text { lesion and to } 5 \\
\text { mm of the } \\
\text { surrounding } \\
\text { healthy tissue. } \\
\text { The area was } \\
\text { covered with an } \\
\text { occlusive } \\
\text { dressing (Tega- } \\
\text { derm; } 3 M \text { Co., } \\
\text { Saint Paul, MN, } \\
\text { USA) for } 3 \text { h, after } \\
\text { which the } \\
\text { remaining cream } \\
\text { was removed with } \\
\text { saline gauze. } \\
\text { molne }\end{array}$ & & & \\
\hline $\begin{array}{l}\text { Choi } 2016 \\
26551044\end{array}$ & MAL-PDT & & & & $\begin{array}{l}\text { Aktilite CL128/ } \\
632 \mathrm{~nm} \text { to } 37 \\
\mathrm{~J} / \mathrm{cm}^{\wedge} 2\end{array}$ & $\begin{array}{l}2 \text { sessions } 7 \text { days } \\
\text { apart, } 16 \% \text { MAL, } \\
1 \text { mm thick layer } \\
\text { of methyl }\end{array}$ & & & \\
\hline
\end{tabular}




\begin{tabular}{|c|c|c|c|c|c|c|c|c|c|}
\hline $\begin{array}{l}\text { Author, } \\
\text { year, } \\
\text { PMID }\end{array}$ & Arm & $\begin{array}{l}\text { Surgical } \\
\text { interventions } \\
\text { (margins) }\end{array}$ & $\begin{array}{l}\text { Thermal } \\
\text { interventions } \\
\text { (description) }\end{array}$ & Radiation & $\begin{array}{l}\text { Photodynamic } \\
\text { Therapy dose }\end{array}$ & $\begin{array}{l}\text { Photodynamic } \\
\text { Therapy } \\
\text { description }\end{array}$ & $\begin{array}{l}\text { Medical } \\
\text { interventions } \\
\text { description }\end{array}$ & $\begin{array}{l}\text { Medical } \\
\text { interventions } \\
\text { other }\end{array}$ & $\begin{array}{l}\text { Curettage } \\
\text { number of } \\
\text { passes }\end{array}$ \\
\hline & & & & & & $\begin{array}{l}\text { aminolevulinate } \\
\text { (16\% Metvix } \\
\text { cream; PhotoCure } \\
\text { ASA, Oslo, } \\
\text { Norway) was } \\
\text { applied to the } \\
\text { lesion and to } 5 \\
\text { mm of the } \\
\text { surrounding } \\
\text { healthy tissue. } \\
\text { The area was } \\
\text { covered with an } \\
\text { occlusive } \\
\text { dressing (Tega- } \\
\text { derm; } 3 M \text { Co., } \\
\text { Saint Paul, MN, } \\
\text { USA) for } 3 \text { h, after } \\
\text { which the } \\
\text { remaining cream } \\
\text { was removed with } \\
\text { saline gauze. }\end{array}$ & & & \\
\hline $\begin{array}{l}\text { Choi } 2017 \\
28199463\end{array}$ & $\begin{array}{l}\text { Er:YAG } \\
\text { ablative } \\
\text { fractional } \\
\text { laser-primed } \\
\text { MAL- PDT }\end{array}$ & & & & $37 \mathrm{~J} / \mathrm{cm} 2$ & $\begin{array}{l}1 \text { session, 17\% } \\
\text { MAL; Aktilite } \\
\text { CL128; 632nm; } \\
\text { pretreatment with } \\
\text { 2940-nm Er:YAG } \\
\text { AFL }\end{array}$ & & & \\
\hline $\begin{array}{l}\text { Choi } 2017 \\
28199463\end{array}$ & MAL-PDT & & & & $74 \mathrm{~J} / \mathrm{cm} 2$ & $\begin{array}{l}2 \text { sessions } 7 \text { days } \\
\text { apart, } 17 \% \text { MAL; } \\
\text { Aktilite CL128; } \\
632 \mathrm{~nm}\end{array}$ & & & \\
\hline $\begin{array}{l}\text { Cornell } \\
1990 \\
2229497\end{array}$ & interferon & & & & & & $\begin{array}{l}\text { interferon alfa- } \\
2 \text { b } 1.5 \text { million IU } \\
\text { (Intralesional) } 3 \\
\text { times per week } \\
\text { for } 3 \text { weeks }\end{array}$ & $\begin{array}{l}\text { Each test site } \\
\text { was cleansed } \\
\text { with alcohol, } \\
\text { and the area } \\
\text { underlying } \\
\text { visible skin } \\
\text { changes by the } \\
\text { tumor and the } \\
\text { substance of } \\
\text { each lesion was }\end{array}$ & \\
\hline
\end{tabular}




\begin{tabular}{|c|c|c|c|c|c|c|c|c|c|}
\hline $\begin{array}{l}\text { Author, } \\
\text { year, } \\
\text { PMID }\end{array}$ & Arm & $\begin{array}{l}\text { Surgical } \\
\text { interventions } \\
\text { (margins) }\end{array}$ & $\begin{array}{l}\text { Thermal } \\
\text { interventions } \\
\text { (description) }\end{array}$ & Radiation & $\begin{array}{l}\text { Photodynamic } \\
\text { Therapy dose }\end{array}$ & $\begin{array}{l}\text { Photodynamic } \\
\text { Therapy } \\
\text { description }\end{array}$ & $\begin{array}{l}\text { Medical } \\
\text { interventions } \\
\text { description }\end{array}$ & $\begin{array}{l}\text { Medical } \\
\text { interventions } \\
\text { other }\end{array}$ & $\begin{array}{l}\text { Curettage } \\
\text { number of } \\
\text { passes }\end{array}$ \\
\hline & & & & & & & & $\begin{array}{l}\text { injected with } \\
0.15 \mathrm{ml} \text { of the } \\
\text { test solution }\end{array}$ & \\
\hline $\begin{array}{l}\text { Cornell } \\
1990 \\
2229497\end{array}$ & placebo & & & & & & $\begin{array}{l}\text { phosphate } \\
\text { buffers, } \\
\text { humanalbumin,a } \\
\text { nd glycine. } \\
\text { (Intralesional) } 3 \\
\text { times per week } \\
\text { for } 3 \text { weeks }\end{array}$ & $\begin{array}{l}\text { Each test site } \\
\text { was cleansed } \\
\text { with alcohol, } \\
\text { and the area } \\
\text { underlying } \\
\text { visible skin } \\
\text { changes by the } \\
\text { tumor and the } \\
\text { substance of } \\
\text { each lesion was } \\
\text { injected with } \\
0.15 \text { ml of the } \\
\text { test solution }\end{array}$ & \\
\hline $\begin{array}{l}\text { Edwards } \\
1990 \\
2107219\end{array}$ & $\begin{array}{l}\text { interferon } \\
\text { gamma, } 0.01\end{array}$ & & & & & & $\begin{array}{l}\text { interferon } \\
\text { gamma } 0.01 \\
\text { (Intralesional) } 3 \\
\text { times/week on } \\
\text { alternate days } \\
\text { for } 3 \text { weeks }\end{array}$ & & \\
\hline $\begin{array}{l}\text { Edwards } \\
1990 \\
2107219\end{array}$ & $\begin{array}{l}\text { interferon } \\
\text { gamma, } 0.05\end{array}$ & & & & & & $\begin{array}{l}\text { interferon } \\
\text { gamma } 0.05 \\
\text { (Intralesional) } 3 \\
\text { times/week on } \\
\text { alternate days } \\
\text { for } 3 \text { weeks }\end{array}$ & & \\
\hline $\begin{array}{l}\text { Edwards } \\
1990 \\
2383027\end{array}$ & $\begin{array}{l}\text { Interferon } \\
\text { alfa-2b, } 30 \\
\text { million IU }\end{array}$ & & & & & & $\begin{array}{l}\text { Interferon alfa- } \\
\text { 2b } 30 \text { million IU } \\
\text { (Intralesional) } \\
\text { weekly for } 3 \\
\text { weeks }\end{array}$ & $\begin{array}{l}\text { Patients were } \\
\text { given } 650 \text { mg of } \\
\text { acetaminophen } \\
\text { orally at the } \\
\text { time of injection }\end{array}$ & \\
\hline $\begin{array}{l}\text { Edwards } \\
1990 \\
2383027\end{array}$ & $\begin{array}{l}\text { Interferon } \\
\text { alfa-2b, } 10 \\
\text { million IU }\end{array}$ & & & & & & $\begin{array}{l}\text { Interferon alfa- } \\
\text { 2b } 10 \text { million IU } \\
\text { (Intralesional) } \\
\text { once }\end{array}$ & $\begin{array}{l}\text { Patients were } \\
\text { given } 650 \mathrm{mg} \text { of } \\
\text { acetaminophen } \\
\text { orally at the } \\
\text { time of injection }\end{array}$ & \\
\hline $\begin{array}{l}\text { Eigentler } \\
2007 \\
17610993\end{array}$ & $\begin{array}{l}\text { imiquimod } 5 \% \\
\text { ( } 8 \text { weeks) }\end{array}$ & & & & & & $\begin{array}{l}\text { imiquimod 5\% } \\
\text { (Topical) thrice } \\
\text { weekly for } 8\end{array}$ & & \\
\hline
\end{tabular}




\begin{tabular}{|c|c|c|c|c|c|c|c|c|c|}
\hline $\begin{array}{l}\text { Author, } \\
\text { year, } \\
\text { PMID }\end{array}$ & Arm & $\begin{array}{l}\text { Surgical } \\
\text { interventions } \\
\text { (margins) }\end{array}$ & $\begin{array}{l}\text { Thermal } \\
\text { interventions } \\
\text { (description) }\end{array}$ & Radiation & $\begin{array}{l}\text { Photodynamic } \\
\text { Therapy dose }\end{array}$ & $\begin{array}{l}\text { Photodynamic } \\
\text { Therapy } \\
\text { description }\end{array}$ & $\begin{array}{l}\text { Medical } \\
\text { interventions } \\
\text { description }\end{array}$ & $\begin{array}{l}\text { Medical } \\
\text { interventions } \\
\text { other }\end{array}$ & $\begin{array}{l}\text { Curettage } \\
\text { number of } \\
\text { passes }\end{array}$ \\
\hline & & & & & & & weeks & & \\
\hline $\begin{array}{l}\text { Eigentler } \\
2007 \\
17610993\end{array}$ & $\begin{array}{l}\text { imiquimod } 5 \% \\
\text { (12 weeks) }\end{array}$ & & & & & & $\begin{array}{l}\text { imiquimod 5\% } \\
\text { (Topical) thrice } \\
\text { weekly for } 12 \\
\text { weeks }\end{array}$ & & \\
\hline $\begin{array}{l}\text { Eimpunth } \\
2014\end{array}$ & $\begin{array}{l}\text { pulsed dye } \\
\text { laser }\end{array}$ & & & & $\begin{array}{l}\text { fluence of } 7.5 \\
\mathrm{~J} / \mathrm{cm} 2,3-\mathrm{ms} \\
\text { pulse width }\end{array}$ & \multicolumn{2}{|c|}{$\begin{array}{l}\text { one session of double stacked-pulses } \\
\text { of PDL treatment using } 10 \mathrm{~mm} \text { spot } \\
\text { size at the office visit. Lesions were } \\
\text { treated with } 1 \text { single session and } \\
\text { included a } 6-\mathrm{mm} \text { margin of normal } \\
\text { skin around the clinically apparent } \\
\text { tumor }\end{array}$} & & \\
\hline $\begin{array}{l}\text { Eimpunth } \\
2014\end{array}$ & no treatment & & & & & & & & \\
\hline $\begin{array}{l}\text { Foley } \\
2009 \\
20064185\end{array}$ & $\begin{array}{l}\text { methyl- } \\
\text { aminolevulina } \\
\text { tePDT }\end{array}$ & & & & $\begin{array}{l}\text { CureLight/570- } \\
670 \mathrm{~nm} \text { at } 50- \\
200 \mathrm{~mW}^{\prime} / \mathrm{cm}^{\wedge} 2 \text { to } \\
75 \mathrm{j} / \mathrm{cm}^{\wedge} 2\end{array}$ & $\begin{array}{l}\text { 1-2 treatment } \\
\text { cycles (assessed } \\
3-6 \text { months) } 1 \\
\text { week apart, } 160 \\
\text { mg/g MAL, the } \\
\text { surfaceof the } \\
\text { lesion was } \\
\text { prepared by } \\
\text { gentle } \\
\text { tumorsurface } \\
\text { debridement } \\
\text { using a curette, } \\
\text { which removedthe } \\
\text { stratumcorneuma } \\
\text { nd surfaceof the } \\
\text { friable tumor } \\
\text { tissue. A layer of } \\
\text { cream(MAL or } \\
\text { placebo), } 1 \text { mm } \\
\text { thick, was } \\
\text { appliedto each } \\
\text { lesion and } 5 \text { mm } \\
\text { of } \\
\text { surroundingtissue } \\
\text { and covered with } \\
\text { an adhesive } \\
\text { occlusivedressing }\end{array}$ & & & \\
\hline
\end{tabular}




\begin{tabular}{|c|c|c|c|c|c|c|c|c|c|}
\hline $\begin{array}{l}\text { Author, } \\
\text { year, } \\
\text { PMID }\end{array}$ & Arm & $\begin{array}{l}\text { Surgical } \\
\text { interventions } \\
\text { (margins) }\end{array}$ & $\begin{array}{l}\text { Thermal } \\
\text { interventions } \\
\text { (description) }\end{array}$ & Radiation & $\begin{array}{l}\text { Photodynamic } \\
\text { Therapy dose }\end{array}$ & $\begin{array}{l}\text { Photodynamic } \\
\text { Therapy } \\
\text { description }\end{array}$ & $\begin{array}{l}\text { Medical } \\
\text { interventions } \\
\text { description }\end{array}$ & $\begin{array}{l}\text { Medical } \\
\text { interventions } \\
\text { other }\end{array}$ & $\begin{array}{l}\text { Curettage } \\
\text { number of } \\
\text { passes }\end{array}$ \\
\hline & & & & & & $\begin{array}{l}\text { (e.g. Tegaderm, } \\
\text { 3M, St Paul, MN, } \\
\text { USA). After } 3 \mathrm{~h} \text {, } \\
\text { the dressings } \\
\text { were removed } \\
\text { and the } \\
\text { creamwas } \\
\text { washedoff with } \\
0.9 \% \text { saline } \\
\text { solution, } \\
\text { immediately } \\
\text { followed by } \\
\text { illumination }\end{array}$ & & & \\
\hline $\begin{array}{l}\text { Foley } \\
2009 \\
20064185\end{array}$ & placebo PDT & & & & $\begin{array}{l}\text { CureLight/570- } \\
670 \mathrm{~nm} \text { at } 50- \\
200 \mathrm{~mW}^{\circ} \mathrm{cm}^{\wedge} 2 \text { to } \\
75 \mathrm{j} / \mathrm{cm}^{\wedge} 2\end{array}$ & $\begin{array}{l}\text { 1-2 treatment } \\
\text { cycles (assessed } \\
\text { 3- } 6 \text { months) } 1 \\
\text { week apart, } \\
\text { placebo, the } \\
\text { surface of the } \\
\text { lesion was } \\
\text { prepared by } \\
\text { gentle tumor } \\
\text { surface } \\
\text { debridement } \\
\text { using a curette, } \\
\text { which removed } \\
\text { the stratum } \\
\text { corneum and } \\
\text { surface of the } \\
\text { friable tumor } \\
\text { tissue, to facilitate } \\
\text { access of the } \\
\text { cream and light to } \\
\text { the tissue. The } \\
\text { purpose of this } \\
\text { debridement was } \\
\text { to debulk rather } \\
\text { than remove the } \\
\text { tumor. placebo),1 } \\
\text { mm thick, was } \\
\text { appliedto each }\end{array}$ & & & \\
\hline
\end{tabular}




\begin{tabular}{|c|c|c|c|c|c|c|c|c|c|}
\hline $\begin{array}{l}\text { Author, } \\
\text { year, } \\
\text { PMID }\end{array}$ & Arm & $\begin{array}{l}\text { Surgical } \\
\text { interventions } \\
\text { (margins) }\end{array}$ & $\begin{array}{l}\text { Thermal } \\
\text { interventions } \\
\text { (description) }\end{array}$ & Radiation & $\begin{array}{l}\text { Photodynamic } \\
\text { Therapy dose }\end{array}$ & $\begin{array}{l}\text { Photodynamic } \\
\text { Therapy } \\
\text { description }\end{array}$ & $\begin{array}{l}\text { Medical } \\
\text { interventions } \\
\text { description }\end{array}$ & $\begin{array}{l}\text { Medical } \\
\text { interventions } \\
\text { other }\end{array}$ & $\begin{array}{l}\text { Curettage } \\
\text { number of } \\
\text { passes }\end{array}$ \\
\hline & & & & & & $\begin{array}{l}\text { lesion and } 5 \mathrm{~mm} \\
\text { of } \\
\text { surroundingtissue } \\
\text { and covered with } \\
\text { an adhesive } \\
\text { occlusivedressing } \\
\text { (e.g. Tegaderm, } \\
\text { 3M, St Paul, MN, } \\
\text { USA). After } 3 \mathrm{~h} \text {, } \\
\text { the dressings } \\
\text { were removed } \\
\text { and the } \\
\text { creamwas } \\
\text { washedoff with } \\
\text { 0.9\% saline } \\
\text { solution, } \\
\text { immediately } \\
\text { followed by } \\
\text { illumination }\end{array}$ & & & \\
\hline $\begin{array}{l}\text { Garcia- } \\
\text { Martin } \\
2011 \\
21242584\end{array}$ & imiquimod 5\% & & & & & & $\begin{array}{l}\text { Imiquimod } 5 \% \\
\text { (Topical) } 5 \text { times } \\
\text { per week for } 6 \\
\text { weeks }\end{array}$ & $\begin{array}{l}\text { plus carbomer } \\
0.2 \% \text { cream }\end{array}$ & \\
\hline $\begin{array}{l}\text { Garcia- } \\
\text { Martin } \\
2011 \\
21242584\end{array}$ & radiotherapy & & & $\begin{array}{l}\text { Photons } \\
\text { (gamma or } \\
\text { x) to } 4000- \\
7000 \mathrm{cGy} \text {, } \\
10-15 \\
\text { sessions, 2- } \\
3 \text { times per } \\
\text { week for } 5 \\
\text { weeks }\end{array}$ & & & & & \\
\hline $\begin{array}{l}\text { Geisse } \\
2002 \\
12196749\end{array}$ & $\begin{array}{l}\text { Imiquimod } \\
\text { 3x/wk }\end{array}$ & & & & & & $\begin{array}{l}\text { Imiquimod 5\% } \\
\text { (Topical) } \\
\text { 3x/week for } 12 \\
\text { weeks }\end{array}$ & $\begin{array}{l}\text { mean total } \\
\text { dose: } 43 \mathrm{mg}\end{array}$ & \\
\hline $\begin{array}{l}\text { Geisse } \\
2002 \\
12196749\end{array}$ & $\begin{array}{l}\text { Imiquimod } \\
5 x / w k\end{array}$ & & & & & & $\begin{array}{l}\text { Imiquimod 5\% } \\
\text { (Topical) } \\
5 x / \text { week for } 12 \\
\text { weeks }\end{array}$ & $\begin{array}{l}\text { mean total } \\
\text { dose: } 43 \mathrm{mg}\end{array}$ & \\
\hline Geisse & Imiquimod & & & & & & Imiquimod 5\% & mean total & \\
\hline
\end{tabular}




\begin{tabular}{|c|c|c|c|c|c|c|c|c|c|}
\hline $\begin{array}{l}\text { Author, } \\
\text { year, } \\
\text { PMID }\end{array}$ & Arm & $\begin{array}{l}\text { Surgical } \\
\text { interventions } \\
\text { (margins) }\end{array}$ & $\begin{array}{l}\text { Thermal } \\
\text { interventions } \\
\text { (description) }\end{array}$ & Radiation & $\begin{array}{l}\text { Photodynamic } \\
\text { Therapy dose }\end{array}$ & $\begin{array}{l}\text { Photodynamic } \\
\text { Therapy } \\
\text { description }\end{array}$ & $\begin{array}{l}\text { Medical } \\
\text { interventions } \\
\text { description }\end{array}$ & $\begin{array}{l}\text { Medical } \\
\text { interventions } \\
\text { other }\end{array}$ & $\begin{array}{l}\text { Curettage } \\
\text { number of } \\
\text { passes }\end{array}$ \\
\hline $\begin{array}{l}2002 \\
12196749 \\
\end{array}$ & 1x/day & & & & & & $\begin{array}{l}\text { (Topical) daily } \\
\text { for } 12 \text { weeks }\end{array}$ & dose: 69mg & \\
\hline $\begin{array}{l}\text { Geisse } \\
2002 \\
12196749\end{array}$ & $\begin{array}{l}\text { Imiquimod } \\
\text { 2x/day }\end{array}$ & & & & & & $\begin{array}{l}\text { Imiquimod 5\% } \\
\text { (Topical) twice } \\
\text { daily for } 12 \\
\text { weeks }\end{array}$ & $\begin{array}{l}\text { mean total } \\
\text { dose: } 146 \mathrm{mg}\end{array}$ & \\
\hline $\begin{array}{l}\text { Geisse } \\
2004 \\
15097956\end{array}$ & $\begin{array}{l}\text { Imiquimod } \\
5 x / w k\end{array}$ & & & & & & $\begin{array}{l}\text { Imiquimod } 5 \% \\
\text { (Topical) } 5 \\
\text { times/week for } 6 \\
\text { weeks }\end{array}$ & & \\
\hline $\begin{array}{l}\text { Geisse } \\
2004 \\
15097956\end{array}$ & $\begin{array}{l}\text { Imiquimod } \\
7 x / \text { wk }\end{array}$ & & & & & & $\begin{array}{l}\text { Imiquimod } 5 \% \\
\text { (Topical) } 7 \\
\text { times/week for } 6 \\
\text { weeks }\end{array}$ & & \\
\hline $\begin{array}{l}\text { Geisse } \\
2004 \\
15097956\end{array}$ & $\begin{array}{l}\text { Vehicle } 5 x / w k \\
\text { or } 7 x / \text { wk }\end{array}$ & & & & & & $\begin{array}{l}\text { vehicle } 5 \% \\
\text { (Topical) } 5 \text { or } 7 \\
\text { times/week for } 6 \\
\text { weeks }\end{array}$ & & \\
\hline $\begin{array}{l}\text { Haak } \\
2015 \\
24903544\end{array}$ & MAL PDT & & & & $\begin{array}{l}\text { LED for } 8 \text { min to } \\
37 \mathrm{~J} / \mathrm{cm} 2\end{array}$ & $\begin{array}{l}2 \text { sessions } 7-10 \\
\text { days apart to } 74 \\
\mathrm{~J} / \mathrm{cm} 2,16 \% \mathrm{MAL} \text {, } \\
\text { partial debulking } \\
\text { was performed } \\
\text { with a ring curette } \\
\text { (M.H.) and areas } \\
\text { compressed until } \\
\text { bleeding stopped }\end{array}$ & & & \\
\hline $\begin{array}{l}\text { Haak } \\
2015 \\
24903544\end{array}$ & $\begin{array}{l}\text { AFXL MAL } \\
\text { PDT }\end{array}$ & & & & $\begin{array}{l}\text { LED for } 8 \text { min to } \\
37 \mathrm{~J} / \mathrm{cm} 2\end{array}$ & $\begin{array}{l}2 \text { sessions } 7-10 \\
\text { days apart to } 74 \\
\mathrm{~J} / \mathrm{cm} 2,16 \% \mathrm{MAL} \text {, } \\
\text { partial debulking } \\
\text { was performed } \\
\text { with a ring curette } \\
\text { (M.H.) and areas } \\
\text { compressed until } \\
\text { bleeding stopped. } \\
\text { UltraPulse? } \\
\text { fractional CO2 }\end{array}$ & & & \\
\hline
\end{tabular}




\begin{tabular}{|c|c|c|c|c|c|c|c|c|c|}
\hline $\begin{array}{l}\text { Author, } \\
\text { year, } \\
\text { PMID }\end{array}$ & Arm & $\begin{array}{l}\text { Surgical } \\
\text { interventions } \\
\text { (margins) }\end{array}$ & $\begin{array}{l}\text { Thermal } \\
\text { interventions } \\
\text { (description) }\end{array}$ & Radiation & $\begin{array}{l}\text { Photodynamic } \\
\text { Therapy dose }\end{array}$ & $\begin{array}{l}\text { Photodynamic } \\
\text { Therapy } \\
\text { description }\end{array}$ & $\begin{array}{l}\text { Medical } \\
\text { interventions } \\
\text { description }\end{array}$ & $\begin{array}{l}\text { Medical } \\
\text { interventions } \\
\text { other }\end{array}$ & $\begin{array}{l}\text { Curettage } \\
\text { number of } \\
\text { passes }\end{array}$ \\
\hline & & & & & & $\begin{array}{l}\text { laser system } \\
\text { using a DeepFx } \\
\text { handpiece to } \\
\text { deliver two } \\
\text { stacked pulses of } \\
40 \mathrm{~mJ} \text { per pulse } \\
\text { at a density of } 5 \%\end{array}$ & & & \\
\hline $\begin{array}{l}\text { Hall } 1986 \\
3514075\end{array}$ & Radiotherapy & & & $\begin{array}{l}\text { For lesions } \\
>1 \mathrm{~cm}, \\
\text { External } \\
\text { Photons } \\
\text { (gamma or } \\
\text { x) to } 3750 \\
\text { Gy, } 10 \\
\text { treatments } \\
\text { over } 12 \\
\text { days. NR } \\
<1 \mathrm{~cm}\end{array}$ & & & & & \\
\hline $\begin{array}{l}\text { Hall } 1986 \\
3514075\end{array}$ & Cryotherapy & & $\begin{array}{l}\text { Cryotherapy } \\
\text { without } \\
\text { curettage (Cry- } \\
\text { Owen spray } \\
\text { gun for face } \\
\text { and trunk; } \\
\text { Brymil } \\
\text { cryospray near } \\
\text { the eye) }\end{array}$ & & & & & & \\
\hline $\begin{array}{l}\text { Ko } 2014 \\
24102369\end{array}$ & $\begin{array}{l}\text { Er:YAG AFL } \\
\text { PDT }\end{array}$ & & & & $\begin{array}{l}\text { Aktilite/632 to } 37 \\
\mathrm{~J} \mathrm{~cm}^{\wedge}-2\end{array}$ & $\begin{array}{l}1 \text { session, 16\% } \\
\text { MAL, AFL } \\
\text { therapy was } \\
\text { performed using a } \\
\text { 2940-nm Er: YAG } \\
\text { AFL (Joule?; } \\
\text { Sciton Inc., Palo } \\
\text { Alto, CA, U.S.A.) } \\
\text { at 550- } 600 \text { Im } \\
\text { ablation depth, } \\
\text { level } 1 \\
\text { coagulation, 22\% } \\
\text { treatment density } \\
\text { and a single }\end{array}$ & & & \\
\hline
\end{tabular}




\begin{tabular}{|c|c|c|c|c|c|c|c|c|c|}
\hline $\begin{array}{l}\text { Author, } \\
\text { year, } \\
\text { PMID }\end{array}$ & Arm & $\begin{array}{l}\text { Surgical } \\
\text { interventions } \\
\text { (margins) }\end{array}$ & $\begin{array}{l}\text { Thermal } \\
\text { interventions } \\
\text { (description) }\end{array}$ & Radiation & $\begin{array}{l}\text { Photodynamic } \\
\text { Therapy dose }\end{array}$ & $\begin{array}{l}\text { Photodynamic } \\
\text { Therapy } \\
\text { description }\end{array}$ & $\begin{array}{l}\text { Medical } \\
\text { interventions } \\
\text { description }\end{array}$ & $\begin{array}{l}\text { Medical } \\
\text { interventions } \\
\text { other }\end{array}$ & $\begin{array}{l}\text { Curettage } \\
\text { number of } \\
\text { passes }\end{array}$ \\
\hline & & & & & & $\begin{array}{l}\text { pulse. } \\
\text { Immediately } \\
\text { afterwards, a 1- } \\
\text { mm thick layer of } \\
\text { MAL (16\% } \\
\text { Metvix? cream; } \\
\text { PhotoCure ASA, } \\
\text { Oslo, Norway) } \\
\text { was applied to the } \\
\text { lesion and to } 5 \\
\text { mm of } \\
\text { surrounding } \\
\text { healthy tissue. } \\
\text { The area was } \\
\text { covered with an } \\
\text { occlusive } \\
\text { dressing } \\
\text { (Tegaderm?; } 3 \mathrm{M} \text {, } \\
\text { Saint Paul, MN, } \\
\text { U.S.A.) for } 3 \mathrm{~h} \text {, } \\
\text { after which the } \\
\text { remaining cream } \\
\text { was removed with } \\
\text { saline gauze }\end{array}$ & & & \\
\hline $\begin{array}{l}\text { Ko } 2014 \\
24102369\end{array}$ & MAL-PDT & & & & $\begin{array}{l}\text { Aktilite/632 to } 37 \\
\mathrm{~J} \mathrm{~cm}^{\wedge}-2\end{array}$ & $\begin{array}{l}2 \text { sessions } 7 \text { days } \\
\text { apart to } 37 \mathrm{~J} \mathrm{~cm}^{\wedge} \\
-2(\times 2), 16 \% \mathrm{MAL} \text {, } \\
\text { a 1-mm thick } \\
\text { layer of MAL } \\
\text { (16\% Metvix? } \\
\text { cream; PhotoCure } \\
\text { ASA, Oslo, } \\
\text { Norway) was } \\
\text { applied to the } \\
\text { lesion and to } 5 \\
\text { mm of } \\
\text { surrounding } \\
\text { healthy tissue. } \\
\text { The area was } \\
\text { covered with an } \\
\text { occlusive }\end{array}$ & & & \\
\hline
\end{tabular}




\begin{tabular}{|c|c|c|c|c|c|c|c|c|c|}
\hline $\begin{array}{l}\text { Author, } \\
\text { year, } \\
\text { PMID }\end{array}$ & Arm & $\begin{array}{l}\text { Surgical } \\
\text { interventions } \\
\text { (margins) }\end{array}$ & $\begin{array}{l}\text { Thermal } \\
\text { interventions } \\
\text { (description) }\end{array}$ & Radiation & $\begin{array}{l}\text { Photodynamic } \\
\text { Therapy dose }\end{array}$ & $\begin{array}{l}\text { Photodynamic } \\
\text { Therapy } \\
\text { description }\end{array}$ & $\begin{array}{l}\text { Medical } \\
\text { interventions } \\
\text { description }\end{array}$ & $\begin{array}{l}\text { Medical } \\
\text { interventions } \\
\text { other }\end{array}$ & $\begin{array}{l}\text { Curettage } \\
\text { number of } \\
\text { passes }\end{array}$ \\
\hline & & & & & & $\begin{array}{l}\text { dressing } \\
\text { (Tegaderm?; 3M, } \\
\text { Saint Paul, MN, } \\
\text { U.S.A.) for } 3 \mathrm{~h} \text {, } \\
\text { after which the } \\
\text { remaining cream } \\
\text { was removed with } \\
\text { saline gauze }\end{array}$ & & & \\
\hline $\begin{array}{l}\text { Kuijpers } \\
2006 \\
16865869\end{array}$ & $\begin{array}{l}\text { ALA-PDT } \\
\text { (total) (no } \\
\text { subgroup } \\
\text { stratification, } \\
\text { combination } \\
\text { of arms } 2 \text { and } \\
\text { 3) }\end{array}$ & $3 \mathrm{~mm}$ & & & $\begin{array}{l}\text { broadband, metal } \\
\text { halogen light; } \\
600-730 \mathrm{~nm} \text { at } \\
100 \mathrm{mwatt} / \mathrm{cm}^{\wedge} 2 \text {, } \\
\text { total dose } 75 \\
\mathrm{~J} / \mathrm{cm}^{\wedge} 2\end{array}$ & $\begin{array}{l}2 \text { sessions } 7 \text { days } \\
\text { apart, 20\% ALA, } \\
20 \% 5-A L A \text { on } \\
\text { tumor + 5mm } \\
\text { margin in non- } \\
\text { transparent layer } \\
2 \mathrm{~mm} \text { thick, } \\
\text { covered with } \\
\text { polyurethane and } \\
\text { opaque dressing } \\
\text { for } 3 \text { hours then } \\
\text { cleaned with } \\
\text { saline }\end{array}$ & & & 0 \\
\hline $\begin{array}{l}\text { Kuijpers } \\
2006 \\
16865869\end{array}$ & $\begin{array}{l}\text { ALA-PDT } \\
\text { (debulking } \\
\text { subgroup) } \\
\text { (ALA-PDT + } \\
\text { allocation to } \\
\text { debulking } \\
\text { group) }\end{array}$ & $3 \mathrm{~mm}$ & & & $\begin{array}{l}\text { broadband, metal } \\
\text { halogen light; } \\
600-730 \mathrm{~nm} \text { at } \\
100 \mathrm{mwatt} / \mathrm{cm}^{\wedge} 2 \text {, } \\
\text { total dose } 75 \\
\mathrm{~J} / \mathrm{cm}^{\wedge} 2\end{array}$ & $\begin{array}{l}2 \text { sessions } 7 \text { days } \\
\text { apart, } 20 \% \text { ALA, } \\
20 \% 5-A L A \text { on } \\
\text { tumor + } 5 \mathrm{~mm} \\
\text { margin in non- } \\
\text { transparent layer } \\
2 \mathrm{~mm} \text { thick, } \\
\text { covered with } \\
\text { polyurethane and } \\
\text { opaque dressing } \\
\text { for } 3 \text { hours then } \\
\text { cleaned with } \\
\text { saline; debulking } \\
\text { group: all tumor } \\
\text { tissue above skin } \\
\text { level with Stiefel } \\
\text { sharp curette nr. } 4 \\
\text { after topical } \\
\text { anesthesia }\end{array}$ & & & 1 \\
\hline Kuijpers & ALA-PDT (no & $3 \mathrm{~mm}$ & & & broadband, metal & 2 sessions 7 days & & & 0 \\
\hline
\end{tabular}




\begin{tabular}{|c|c|c|c|c|c|c|c|c|c|}
\hline $\begin{array}{l}\text { Author, } \\
\text { year, } \\
\text { PMID }\end{array}$ & Arm & $\begin{array}{l}\text { Surgical } \\
\text { interventions } \\
\text { (margins) }\end{array}$ & $\begin{array}{l}\text { Thermal } \\
\text { interventions } \\
\text { (description) }\end{array}$ & Radiation & $\begin{array}{l}\text { Photodynamic } \\
\text { Therapy dose }\end{array}$ & $\begin{array}{l}\text { Photodynamic } \\
\text { Therapy } \\
\text { description }\end{array}$ & $\begin{array}{l}\text { Medical } \\
\text { interventions } \\
\text { description }\end{array}$ & $\begin{array}{l}\text { Medical } \\
\text { interventions } \\
\text { other }\end{array}$ & $\begin{array}{l}\text { Curettage } \\
\text { number of } \\
\text { passes }\end{array}$ \\
\hline $\begin{array}{l}2006 \\
16865869\end{array}$ & $\begin{array}{l}\text { debulking } \\
\text { subgroup) } \\
\text { (ALA-PDT + } \\
\text { no allocation } \\
\text { to debulking } \\
\text { group) }\end{array}$ & & & & $\begin{array}{l}\text { halogen light; } \\
600-730 \mathrm{~nm} \text { at } \\
100 \mathrm{mwatt} / \mathrm{cm}^{\wedge} 2 \\
\text { total dose } 75 \\
\mathrm{~J} / \mathrm{cm}^{\wedge} 2\end{array}$ & $\begin{array}{l}\text { apart, 20\% ALA, } \\
20 \% 5-A L A \text { on } \\
\text { tumor + 5mm } \\
\text { margin in non- } \\
\text { transparent layer } \\
2 \mathrm{~mm} \text { thick, } \\
\text { covered with } \\
\text { polyurethane and } \\
\text { opaque dressing } \\
\text { for } 3 \text { hours then } \\
\text { cleaned with } \\
\text { saline }\end{array}$ & & & \\
\hline $\begin{array}{l}\text { Kuijpers } \\
2006 \\
16865869\end{array}$ & $\begin{array}{l}\text { MAL-PDT } \\
\text { (total) (MAL- } \\
\text { PDT + no } \\
\text { stratification } \\
\text { by subgroup, } \\
\text { combination } \\
\text { of arms 5+6) }\end{array}$ & $3 \mathrm{~mm}$ & & & $\begin{array}{l}\text { broadband, metal } \\
\text { halogen light; } \\
600-730 \mathrm{~nm} \text { at } \\
100 \mathrm{mwatt} / \mathrm{cm}^{\wedge} 2 \text {, } \\
\text { total dose } 75 \\
\mathrm{~J} / \mathrm{cm}^{\wedge} 2\end{array}$ & $\begin{array}{l}7 \text { days apart, } 16 \% \\
\text { MAL, } 16 \% \text { MAL } \\
\text { on tumor }+5 \mathrm{~mm} \\
\text { margin in non- } \\
\text { transparent layer } \\
2 \mathrm{~mm} \text { thick, } \\
\text { covered with } \\
\text { polyurethane and } \\
\text { opaque dressing } \\
\text { for } 3 \text { hours then } \\
\text { cleaned with } \\
\text { saline }\end{array}$ & & & 0 \\
\hline $\begin{array}{l}\text { Kuijpers } \\
2006 \\
16865869\end{array}$ & $\begin{array}{l}\text { MAL-PDT } \\
\text { (debulking } \\
\text { subgroup) } \\
\text { (MAL-PDT + } \\
\text { allocation to } \\
\text { debulking } \\
\text { group) }\end{array}$ & $3 \mathrm{~mm}$ & & & $\begin{array}{l}\text { broadband, metal } \\
\text { halogen light; } \\
600-730 \mathrm{~nm} \text { at } \\
100 \mathrm{mwatt} / \mathrm{cm}^{\wedge} 2 \text {, } \\
\text { total dose } 75 \\
\mathrm{~J} / \mathrm{cm}^{\wedge} 2\end{array}$ & $\begin{array}{l}7 \text { days apart, 16\% } \\
\text { MAL, } 16 \% \text { MAL } \\
\text { on tumor }+5 \mathrm{~mm} \\
\text { margin in non- } \\
\text { transparent layer } \\
2 \mathrm{~mm} \text { thick, } \\
\text { covered with } \\
\text { polyurethane and } \\
\text { opaque dressing } \\
\text { for } 3 \text { hours then } \\
\text { cleaned with } \\
\text { saline; debulking } \\
\text { group: all tumor } \\
\text { tissue above skin } \\
\text { level with Stiefel } \\
\text { sharp curette nr. } 4 \\
\text { after topical }\end{array}$ & & & 1 \\
\hline
\end{tabular}




\begin{tabular}{|c|c|c|c|c|c|c|c|c|c|}
\hline $\begin{array}{l}\text { Author, } \\
\text { year, } \\
\text { PMID }\end{array}$ & Arm & $\begin{array}{l}\text { Surgical } \\
\text { interventions } \\
\text { (margins) }\end{array}$ & $\begin{array}{l}\text { Thermal } \\
\text { interventions } \\
\text { (description) }\end{array}$ & Radiation & $\begin{array}{l}\text { Photodynamic } \\
\text { Therapy dose }\end{array}$ & $\begin{array}{l}\text { Photodynamic } \\
\text { Therapy } \\
\text { description }\end{array}$ & $\begin{array}{l}\text { Medical } \\
\text { interventions } \\
\text { description }\end{array}$ & $\begin{array}{l}\text { Medical } \\
\text { interventions } \\
\text { other }\end{array}$ & $\begin{array}{l}\text { Curettage } \\
\text { number of } \\
\text { passes }\end{array}$ \\
\hline & & & & & & anesthesia & & & \\
\hline $\begin{array}{l}\text { Kuijpers } \\
2006 \\
16865869\end{array}$ & $\begin{array}{l}\text { MAL-PDT (no } \\
\text { debulking } \\
\text { subgroup) } \\
\text { (MAL-PDT + } \\
\text { no allocation } \\
\text { to debulking } \\
\text { group) }\end{array}$ & $3 \mathrm{~mm}$ & & & $\begin{array}{l}\text { broadband, metal } \\
\text { halogen light; } \\
600-730 \mathrm{~nm} \text { at } \\
100 \mathrm{mwatt}^{\mathrm{c}} \mathrm{cm}^{\wedge} 2 \text {, } \\
\text { total dose } 75 \\
\mathrm{~J} / \mathrm{cm}^{\wedge} 2\end{array}$ & $\begin{array}{l}7 \text { days apart, } 16 \% \\
\text { MAL, } 16 \% \mathrm{MAL} \\
\text { on tumor }+5 \mathrm{~mm} \\
\text { margin in non- } \\
\text { transparent layer } \\
2 \mathrm{~mm} \text { thick, } \\
\text { covered with } \\
\text { polyurethane and } \\
\text { opaque dressing } \\
\text { for } 3 \text { hours then } \\
\text { cleaned with } \\
\text { saline }\end{array}$ & & & 0 \\
\hline $\begin{array}{l}\text { Kuijpers } \\
2007 \\
17451581\end{array}$ & $\begin{array}{l}\text { Curettage }+ \\
\text { Cryosurgery }\end{array}$ & & $\begin{array}{l}\text { Cryotherapy } \\
\text { (Curettage with } \\
\text { sharp curette. } \\
\text { Cryo with liquid } \\
\text { nitrogen with } \\
\text { neoprene open } \\
\text { cone) }\end{array}$ & & & & & & \\
\hline $\begin{array}{l}\text { Kuijpers } \\
2007 \\
17451581 \\
\end{array}$ & $\begin{array}{l}\text { Surgical } \\
\text { excision }\end{array}$ & excision $(3 \mathrm{~mm})$ & & & & & & & \\
\hline $\begin{array}{l}\text { Marks } \\
2001 \\
11312429 \\
\end{array}$ & $\begin{array}{l}\text { Imiquimod } \\
\text { BID }\end{array}$ & & & & & & $\begin{array}{l}\text { Imiquimod 5\% } \\
\text { (Topical) BID for } \\
6 \text { weeks }\end{array}$ & & \\
\hline $\begin{array}{l}\text { Marks } \\
2001 \\
11312429 \\
\end{array}$ & $\begin{array}{l}\text { Imiquimod } \\
\text { OD }\end{array}$ & & & & & & $\begin{array}{l}\text { Imiquimod 5\% } \\
\text { (Topical) OD for } \\
6 \text { weeks }\end{array}$ & & \\
\hline $\begin{array}{l}\text { Marks } \\
2001 \\
11312429\end{array}$ & $\begin{array}{l}\text { Imiquimod } \\
\text { BID 3/week }\end{array}$ & & & & & & $\begin{array}{l}\text { Imiquimod 5\% } \\
\text { (Topical) BID } 3 \\
\text { times per week } \\
\text { for } 6 \text { weeks }\end{array}$ & & \\
\hline $\begin{array}{l}\text { Marks } \\
2001 \\
11312429\end{array}$ & $\begin{array}{l}\text { Imiquimod } \\
\text { OD 3/week }\end{array}$ & & & & & & $\begin{array}{l}\text { Imiquimod 5\% } \\
\text { (Topical) OD } 3 \\
\text { times per week } \\
\text { for } 6 \text { weeks }\end{array}$ & & \\
\hline
\end{tabular}




\begin{tabular}{|c|c|c|c|c|c|c|c|c|c|}
\hline $\begin{array}{l}\text { Author, } \\
\text { year, } \\
\text { PMID }\end{array}$ & Arm & $\begin{array}{l}\text { Surgical } \\
\text { interventions } \\
\text { (margins) }\end{array}$ & $\begin{array}{l}\text { Thermal } \\
\text { interventions } \\
\text { (description) }\end{array}$ & Radiation & $\begin{array}{l}\text { Photodynamic } \\
\text { Therapy dose }\end{array}$ & $\begin{array}{l}\text { Photodynamic } \\
\text { Therapy } \\
\text { description }\end{array}$ & $\begin{array}{l}\text { Medical } \\
\text { interventions } \\
\text { description }\end{array}$ & $\begin{array}{l}\text { Medical } \\
\text { interventions } \\
\text { other }\end{array}$ & $\begin{array}{l}\text { Curettage } \\
\text { number of } \\
\text { passes }\end{array}$ \\
\hline & & & & & & & $\begin{array}{l}\text { disease } \\
\text { progression (as } \\
\text { confi rmed by } \\
\text { independent } \\
\text { central review), } \\
\text { intolerable toxic } \\
\text { eff ects, } \\
\text { withdrawal of } \\
\text { consent, death, } \\
\text { discontinuation } \\
\text { at an } \\
\text { investigator's } \\
\text { discretion, dose } \\
\text { interruption } \\
\text { lasting longer } \\
\text { than } 21 \text { days } \\
\text { (unless the } \\
\text { patient was } \\
\text { responding to } \\
\text { study treatment } \\
\text { and had not } \\
\text { progressed, in } \\
\text { which case } \\
\text { resumption of } \\
\text { treatment was } \\
\text { permitted at the } \\
\text { investigator's } \\
\text { discretion), use } \\
\text { of a prohibited } \\
\text { medication, start } \\
\text { of another } \\
\text { antineoplastic } \\
\text { therapy, or study } \\
\text { termination. }\end{array}$ & $\begin{array}{l}\text { reductions were } \\
\text { permitted for } \\
\text { toxic eff ects } \\
\text { deemed to be } \\
\text { related to study } \\
\text { treatment }\end{array}$ & \\
\hline $\begin{array}{l}\text { Migden } \\
2015 \\
25981810\end{array}$ & sonidegib 800 & & & & & & $\begin{array}{l}\text { sonidegib } 800 \\
\text { mg (Oral) once } \\
\text { daily for until } \\
\text { documented } \\
\text { disease } \\
\text { progression (as } \\
\text { confi rmed by }\end{array}$ & $\begin{array}{l}\text { Dose } \\
\text { interruptions of } \\
21 \text { days or } \\
\text { fewer, or dose } \\
\text { reductions were } \\
\text { permitted for } \\
\text { toxic eff ects }\end{array}$ & \\
\hline
\end{tabular}




\begin{tabular}{|c|c|c|c|c|c|c|c|c|c|}
\hline $\begin{array}{l}\text { Author, } \\
\text { year, } \\
\text { PMID }\end{array}$ & Arm & $\begin{array}{l}\text { Surgical } \\
\text { interventions } \\
\text { (margins) }\end{array}$ & $\begin{array}{l}\text { Thermal } \\
\text { interventions } \\
\text { (description) }\end{array}$ & Radiation & $\begin{array}{l}\text { Photodynamic } \\
\text { Therapy dose }\end{array}$ & $\begin{array}{l}\text { Photodynamic } \\
\text { Therapy } \\
\text { description }\end{array}$ & $\begin{array}{l}\text { Medical } \\
\text { interventions } \\
\text { description }\end{array}$ & $\begin{array}{l}\text { Medical } \\
\text { interventions } \\
\text { other }\end{array}$ & $\begin{array}{l}\text { Curettage } \\
\text { number of } \\
\text { passes }\end{array}$ \\
\hline & & & & & & & $\begin{array}{l}\text { independent } \\
\text { central review), } \\
\text { intolerable toxic } \\
\text { eff ects, } \\
\text { withdrawal of } \\
\text { consent, death, } \\
\text { discontinuation } \\
\text { at an } \\
\text { investigator's } \\
\text { discretion, dose } \\
\text { interruption } \\
\text { lasting longer } \\
\text { than } 21 \text { days } \\
\text { (unless the } \\
\text { patient was } \\
\text { responding to } \\
\text { study treatment } \\
\text { and had not } \\
\text { progressed, in } \\
\text { which case } \\
\text { resumption of } \\
\text { treatment was } \\
\text { permitted at the } \\
\text { investigator's } \\
\text { discretion), use } \\
\text { of a prohibited } \\
\text { medication, start } \\
\text { of another } \\
\text { antineoplastic } \\
\text { therapy, or study } \\
\text { termination. }\end{array}$ & $\begin{array}{l}\text { deemed to be } \\
\text { related to study } \\
\text { treatment }\end{array}$ & \\
\hline $\begin{array}{l}\text { Miller } \\
1997 \\
8996264\end{array}$ & $\begin{array}{l}1.0 \mathrm{~mL} 5-\mathrm{FU} \\
\text { weekly/6 } \\
\text { weeks }\end{array}$ & & & & & & $\begin{array}{l}\text { 5-FU/epi } 1.0 \mathrm{~mL} \\
\text { (Intralesional) } \\
\text { once weekly for } \\
6 \text { weeks }\end{array}$ & & \\
\hline $\begin{array}{l}\text { Miller } \\
1997 \\
8996264\end{array}$ & $\begin{array}{l}0.5 \mathrm{~mL} 5-\mathrm{FU} \\
\text { weekly/6 } \\
\text { weeks }\end{array}$ & & & & & & $\begin{array}{l}5 \text {-FU/epi } 0.5 \mathrm{~mL} \\
\text { (Intralesional) } \\
\text { once weekly for } \\
6 \text { weeks }\end{array}$ & & \\
\hline $\begin{array}{l}\text { Miller } \\
1997\end{array}$ & $\begin{array}{l}1.0 \mathrm{~mL} 5-\mathrm{FU} \\
2 \mathrm{x} \text { weekly/3 }\end{array}$ & & & & & & $\begin{array}{l}\text { 5-FU/epi } 1.0 \mathrm{~mL} \\
\text { (Intralesional) }\end{array}$ & & \\
\hline
\end{tabular}




\begin{tabular}{|c|c|c|c|c|c|c|c|c|c|}
\hline $\begin{array}{l}\text { Author, } \\
\text { year, } \\
\text { PMID }\end{array}$ & Arm & $\begin{array}{l}\text { Surgical } \\
\text { interventions } \\
\text { (margins) }\end{array}$ & $\begin{array}{l}\text { Thermal } \\
\text { interventions } \\
\text { (description) }\end{array}$ & Radiation & $\begin{array}{l}\text { Photodynamic } \\
\text { Therapy dose }\end{array}$ & $\begin{array}{l}\text { Photodynamic } \\
\text { Therapy } \\
\text { description }\end{array}$ & $\begin{array}{l}\text { Medical } \\
\text { interventions } \\
\text { description }\end{array}$ & $\begin{array}{l}\text { Medical } \\
\text { interventions } \\
\text { other }\end{array}$ & $\begin{array}{l}\text { Curettage } \\
\text { number of } \\
\text { passes }\end{array}$ \\
\hline 8996264 & weeks & & & & & & $\begin{array}{l}\text { twice weekly for } \\
3 \text { weeks }\end{array}$ & & \\
\hline $\begin{array}{l}\text { Miller } \\
1997 \\
8996264\end{array}$ & $\begin{array}{l}1.0 \mathrm{~mL} 5-\mathrm{FU} \\
\text { twi } 2 \mathrm{x} \\
\text { weekly/3 } \\
\text { weeks }\end{array}$ & & & & & & $\begin{array}{l}\text { 5-FU/epi } 0.5 \mathrm{~mL} \\
\text { (Intralesional) } \\
\text { twice weekly for } \\
3 \text { weeks }\end{array}$ & & \\
\hline $\begin{array}{l}\text { Miller } \\
1997 \\
8996264\end{array}$ & $\begin{array}{l}0.5 \mathrm{~mL} 5-\mathrm{FU} \\
3 x \text { weekly/2 } \\
\text { weeks }\end{array}$ & & & & & & $\begin{array}{l}\text { 5-FU/epi } 0.5 \mathrm{~mL} \\
\text { (Intralesional) } \\
\text { three times } \\
\text { weekly for } 2 \\
\text { weeks }\end{array}$ & & \\
\hline $\begin{array}{l}\text { Morton } \\
1996 \\
8977678\end{array}$ & cryotherapy & & $\begin{array}{l}\text { Cryotherapy } \\
\text { (Liquid nitrogen } \\
\text { was applied to } \\
\text { lesions via a } \\
\text { hand-held } \\
\text { 'Cryac' spray. } \\
\text { After initial } \\
\text { iceiicld } \\
\text { formation, the } \\
\text { freeze was } \\
\text { maintained for } \\
20 \text { SH A single } \\
\text { freeze-thaw } \\
\text { cycle technique } \\
\text { was employed } \\
\text { with a } 2-3 \text { mm } \\
\text { rim of clinically } \\
\text { healthy tissue } \\
\text { included in the } \\
\text { treatment field.) }\end{array}$ & & & & & & \\
\hline $\begin{array}{l}\text { Morton } \\
1996 \\
8977678\end{array}$ & photodynamic & & & & $\begin{array}{l}300 \mathrm{~W} \text { xenon } \\
\text { short arc plasma } \\
\text { discharge for } 30 \\
\text { min to } 125 \mathrm{~J} / \mathrm{cm} 2\end{array}$ & $\begin{array}{l}1 \text { session, } 20 \% \\
\text { ALA }\end{array}$ & & & \\
\hline $\begin{array}{l}\text { Morton } \\
2006 \\
\end{array}$ & MAL PDT & & & & $\begin{array}{l}\text { noncoherent red } \\
\text { light wavelength, }\end{array}$ & $\begin{array}{l}2 \text { sessions } 1 \\
\text { week apart, } 160\end{array}$ & & & \\
\hline
\end{tabular}




\begin{tabular}{|c|c|c|c|c|c|c|c|c|c|}
\hline $\begin{array}{l}\text { Author, } \\
\text { year, } \\
\text { PMID }\end{array}$ & Arm & $\begin{array}{l}\text { Surgical } \\
\text { interventions } \\
\text { (margins) }\end{array}$ & $\begin{array}{l}\text { Thermal } \\
\text { interventions } \\
\text { (description) }\end{array}$ & Radiation & $\begin{array}{l}\text { Photodynamic } \\
\text { Therapy dose }\end{array}$ & $\begin{array}{l}\text { Photodynamic } \\
\text { Therapy } \\
\text { description }\end{array}$ & $\begin{array}{l}\text { Medical } \\
\text { interventions } \\
\text { description }\end{array}$ & $\begin{array}{l}\text { Medical } \\
\text { interventions } \\
\text { other }\end{array}$ & $\begin{array}{l}\text { Curettage } \\
\text { number of } \\
\text { passes }\end{array}$ \\
\hline 16785375 & & & & & $\begin{array}{l}570-670 \mathrm{~nm} \text { to } \\
75 \mathrm{~J} / \mathrm{cm} 2\end{array}$ & $\begin{array}{l}\text { mg/g MAL, gentle } \\
\text { surface } \\
\text { debridement with } \\
\text { a curette. }\end{array}$ & & & \\
\hline $\begin{array}{l}\text { Morton } \\
2006 \\
16785375\end{array}$ & PDT placebo & & & & $\begin{array}{l}\text { noncoherent red } \\
\text { light wavelength, } \\
570-670 \mathrm{~nm} \text { to } \\
75 \mathrm{~J} / \mathrm{cm} 2\end{array}$ & $\begin{array}{l}2 \text { sessions } 1 \\
\text { week apart, } \\
\text { placebo cream, } \\
\text { gentle surface } \\
\text { debridement with } \\
\text { a curette. } \\
\text { Retreated at } 12 \\
\text { weeks if partial } \\
\text { response }\end{array}$ & & & \\
\hline $\begin{array}{l}\text { Morton } \\
2006 \\
16785375\end{array}$ & $\begin{array}{l}\text { Cryotherapy } \\
\text { or } \\
\text { Fluorouracil }\end{array}$ & & $\begin{array}{l}\text { Cryotherapy } \\
\text { without } \\
\text { curettage } \\
\text { (Cryotherapy } \\
\text { was performed } \\
\text { with a handheld } \\
\text { liquid nitrogen } \\
\text { spray, using a } \\
\text { single } \\
\text { freeze/thaw } \\
\text { cycle. After an } \\
\text { initial ice field } \\
\text { formation with a } \\
\text { 2-mm rim of } \\
\text { clinically } \\
\text { healthy tissue, } \\
\text { the ice field was } \\
\text { maintained for a } \\
\text { minimum of } 20 \\
\text { seconds; } 1 \\
\text { pass) }\end{array}$ & & & & $\begin{array}{l}\text { Fluorouracil 5\% } \\
\text { (Topical) once } \\
\text { daily during the } \\
\text { first week and } \\
\text { twice daily } \\
\text { weeks } 2-4 \text { for } 4 \\
\text { weeks }\end{array}$ & $\begin{array}{l}\text { Retreated at } 12 \\
\text { weeks if partial } \\
\text { response }\end{array}$ & \\
\hline $\begin{array}{l}\text { Mosterd } \\
2008 \\
18717680\end{array}$ & ALA-PDT & & & & $\begin{array}{l}\text { broadband } \\
\text { metal-halogen } \\
\text { light source/585- } \\
720 \mathrm{~nm} \text { for } 15 \\
\mathrm{~min} \text { at } 100 \mathrm{~mW} \\
\mathrm{~cm}-2 \text { to } 75 \mathrm{~J} \mathrm{~cm}- \\
2 \text {, total dose } 150\end{array}$ & $\begin{array}{l}2 \text { sessions } 60 \\
\text { minutes apart, } \\
20 \% \text { ALA, } \\
\text { debulking } 3 \\
\text { weeks before } \\
\text { procedure }\end{array}$ & & & \\
\hline
\end{tabular}




\begin{tabular}{|c|c|c|c|c|c|c|c|c|c|}
\hline $\begin{array}{l}\text { Author, } \\
\text { year, } \\
\text { PMID }\end{array}$ & Arm & $\begin{array}{l}\text { Surgical } \\
\text { interventions } \\
\text { (margins) }\end{array}$ & $\begin{array}{l}\text { Thermal } \\
\text { interventions } \\
\text { (description) }\end{array}$ & Radiation & $\begin{array}{l}\text { Photodynamic } \\
\text { Therapy dose }\end{array}$ & $\begin{array}{l}\text { Photodynamic } \\
\text { Therapy } \\
\text { description }\end{array}$ & $\begin{array}{l}\text { Medical } \\
\text { interventions } \\
\text { description }\end{array}$ & $\begin{array}{l}\text { Medical } \\
\text { interventions } \\
\text { other }\end{array}$ & $\begin{array}{l}\text { Curettage } \\
\text { number of } \\
\text { passes }\end{array}$ \\
\hline & & & & & $\mathrm{J} \mathrm{cm}-2$ & & & & \\
\hline $\begin{array}{l}\text { Mosterd } \\
2008 \\
18717680 \\
\end{array}$ & $\begin{array}{l}\text { Surgical } \\
\text { excision }\end{array}$ & excision (3 mm) & & & & & & & \\
\hline $\begin{array}{l}\text { Mosterd } \\
2008 \\
19010733 \\
\end{array}$ & MMS & Mohs (3 mm) & & & & & & & \\
\hline $\begin{array}{l}\text { Mosterd } \\
2008 \\
19010733\end{array}$ & $\begin{array}{l}\text { Surgical } \\
\text { excision }\end{array}$ & excision $(3 \mathrm{~mm})$ & & & & & & & \\
\hline $\begin{array}{l}\text { Orenberg } \\
1992 \\
1430394\end{array}$ & $\begin{array}{l}7.5 \mathrm{mg} 5-\mathrm{FU} \\
(0.25 \mathrm{ml} \text { of } \\
\text { MPI } 5003 \text { ( } 7.5 \\
\text { mg 5-FU) } \\
\text { intralesionally } \\
\text { ) }\end{array}$ & & & & & & $\begin{array}{l}5 \text {-FU } 30 \mathrm{mg} / \mathrm{ml} \\
\text { (Intralesional) } \\
\text { weekly for up to } \\
6 \text { weeks }\end{array}$ & & \\
\hline $\begin{array}{l}\text { Orenberg } \\
1992 \\
1430394\end{array}$ & $\begin{array}{l}15 \mathrm{mg} 5-\mathrm{FU} \\
(0.5 \mathrm{ml} \text { of } \mathrm{MPI} \\
5003(15 \mathrm{mg} \\
5-\mathrm{FU}) \\
\text { intralesionally } \\
)\end{array}$ & & & & & & $\begin{array}{l}5-\mathrm{FU} 30 \mathrm{mg} / \mathrm{ml} \\
\text { (Intralesional) } \\
\text { weekly for up to } \\
6 \text { weeks }\end{array}$ & & \\
\hline $\begin{array}{l}\text { Patel } 2006 \\
16713457\end{array}$ & imiquimod 5\% & & & & & & $\begin{array}{l}\text { imiquimod } 5 \% \\
\text { (Topical) daily } \\
\text { for } 16 \text { weeks }\end{array}$ & $\begin{array}{l}\text { wash with tap } \\
\text { water and pat } \\
\text { dry before } \\
\text { applying nightly }\end{array}$ & \\
\hline $\begin{array}{l}\text { Patel } 2006 \\
16713457\end{array}$ & vehicle & & & & & & $\begin{array}{l}\text { vehicle } \\
\text { (Topical) daily } \\
\text { for } 16 \text { weeks }\end{array}$ & $\begin{array}{l}\text { wash with tap } \\
\text { water and pat } \\
\text { dry before } \\
\text { applying nightly }\end{array}$ & \\
\hline $\begin{array}{l}\text { Rhodes } \\
2004 \\
14732655\end{array}$ & MAL PDT & & & & $\begin{array}{l}\text { red light, } 570-670 \\
\mathrm{~nm} \text { at } 50-200 \\
\mathrm{~mW} \text {, total dose } \\
75 \mathrm{~J} / \mathrm{cm} 2\end{array}$ & $\begin{array}{l}2 \text { sessions } 1 \\
\text { week apart, MAL, } \\
\text { surface crust or } \\
\text { scale was } \\
\text { removed with a } \\
\text { scalpel blade }\end{array}$ & & & \\
\hline $\begin{array}{l}\text { Rhodes } \\
2004 \\
14732655 \\
\end{array}$ & excision & excision (5 mm) & & & & & & & \\
\hline $\begin{array}{l}\text { Salim } \\
2003\end{array}$ & $\begin{array}{l}\text { PDT (ALA } \\
\text { PDT) }\end{array}$ & & & & $\begin{array}{l}\text { Xenon lamp } 630 \\
\pm 15 \mathrm{~nm} \text { for } 12-\end{array}$ & $\begin{array}{l}1 \text { session, } 20 \% \\
\text { ALA }\end{array}$ & & & \\
\hline
\end{tabular}




\begin{tabular}{|c|c|c|c|c|c|c|c|c|c|}
\hline $\begin{array}{l}\text { Author, } \\
\text { year, } \\
\text { PMID }\end{array}$ & Arm & $\begin{array}{l}\text { Surgical } \\
\text { interventions } \\
\text { (margins) }\end{array}$ & $\begin{array}{l}\text { Thermal } \\
\text { interventions } \\
\text { (description) }\end{array}$ & Radiation & $\begin{array}{l}\text { Photodynamic } \\
\text { Therapy dose }\end{array}$ & $\begin{array}{l}\text { Photodynamic } \\
\text { Therapy } \\
\text { description }\end{array}$ & $\begin{array}{l}\text { Medical } \\
\text { interventions } \\
\text { description }\end{array}$ & $\begin{array}{l}\text { Medical } \\
\text { interventions } \\
\text { other }\end{array}$ & $\begin{array}{l}\text { Curettage } \\
\text { number of } \\
\text { passes }\end{array}$ \\
\hline 12653747 & & & & & $\begin{array}{l}40 \mathrm{~min} \text { at } 50-90 \\
\mathrm{~mW} / \mathrm{cm} 2 \text { to } 100 \\
\mathrm{~J} / \mathrm{cm} 2\end{array}$ & & & & \\
\hline $\begin{array}{l}\text { Salim } \\
2003 \\
12653747\end{array}$ & 5-FU (Efudix) & & & & & & $\begin{array}{l}\text { 5-FU (Topical) } \\
\text { Daily for } 1 \text { week } \\
\text { then BID for } 3 \\
\text { weeks for } 4 \\
\text { weeks total } \\
\text { treatment }\end{array}$ & & \\
\hline $\begin{array}{l}\text { Salmanpo } \\
\text { or } 2012\end{array}$ & $\begin{array}{l}\text { Surgical } \\
\text { excision }\end{array}$ & excision (4 mm) & & & & & & & \\
\hline $\begin{array}{l}\text { Salmanpo } \\
\text { or } 2012\end{array}$ & Curettage & & & & & & & & NR \\
\hline $\begin{array}{l}\text { Salmanpo } \\
\text { or } 2012\end{array}$ & $\begin{array}{l}\text { Electodessica } \\
\text { tion and } \\
\text { curettage }\end{array}$ & & $\begin{array}{l}\text { Diathermy ( } 2 \\
\text { mm cauttery } \\
\text { margins after } \\
\text { curettage; } \\
\text { dessication) } \\
\end{array}$ & & & & & & \\
\hline $\begin{array}{l}\text { Schleier } \\
2007 \\
25047438\end{array}$ & $\begin{array}{l}\text { ALA- } \\
\text { thermogel } \\
\text { PDT }\end{array}$ & & & & $\begin{array}{l}\text { diode laser, } \\
\text { Ceralas } 635 \text { PDT } \\
\text { at } 0.1 \mathrm{~W} / \mathrm{cm}^{\wedge} 2 \text { to } \\
120 \mathrm{~J} / \mathrm{cm}^{\wedge} 2\end{array}$ & $\begin{array}{l}\text { 1-3 times based } \\
\text { on response at } \\
\text { follow up, 10\% } \\
\text { ALA, ALA } \\
\text { dissolved in } \\
\text { thermogel } 1 \text { hour } \\
\text { before treatment. } \\
\text { combination gel } \\
\text { applied } 3 \text { mm } \\
\text { beyond visible } \\
\text { margin of tumor. } \\
\text { the gel layer was } \\
5 \text { mm thick. the } \\
\text { area was covered } \\
\text { with plaster and } \\
\text { protected from } \\
\text { light. three hours } \\
\text { after application, } \\
\text { residue was } \\
\text { removed. }\end{array}$ & & & \\
\hline $\begin{array}{l}\text { Schleier } \\
2007 \\
25047438 \\
\end{array}$ & $\begin{array}{l}\text { mALA- } \\
\text { thermogel } \\
\text { PDT } \\
\end{array}$ & & & & $\begin{array}{l}\text { diode laser, } \\
\text { Ceralas } 635 \text { PDT } \\
\text { at } 0.1 \mathrm{~W} / \mathrm{cm}^{\wedge} 2 \text { to }\end{array}$ & $\begin{array}{l}1-3 \text { times based } \\
\text { on response at } \\
\text { follow up, } 10 \%\end{array}$ & & & \\
\hline
\end{tabular}




\begin{tabular}{|c|c|c|c|c|c|c|c|c|c|}
\hline $\begin{array}{l}\text { Author, } \\
\text { year, } \\
\text { PMID }\end{array}$ & Arm & $\begin{array}{l}\text { Surgical } \\
\text { interventions } \\
\text { (margins) }\end{array}$ & $\begin{array}{l}\text { Thermal } \\
\text { interventions } \\
\text { (description) }\end{array}$ & Radiation & $\begin{array}{l}\text { Photodynamic } \\
\text { Therapy dose }\end{array}$ & $\begin{array}{l}\text { Photodynamic } \\
\text { Therapy } \\
\text { description }\end{array}$ & $\begin{array}{l}\text { Medical } \\
\text { interventions } \\
\text { description }\end{array}$ & $\begin{array}{l}\text { Medical } \\
\text { interventions } \\
\text { other }\end{array}$ & $\begin{array}{l}\text { Curettage } \\
\text { number of } \\
\text { passes }\end{array}$ \\
\hline & & & & & $120 \mathrm{~J} / \mathrm{cm}^{\wedge} 2$ & $\begin{array}{l}\text { methyl-ALA, } \\
\text { methyl-ALA } \\
\text { dissolved in } \\
\text { thermogel } 1 \text { hour } \\
\text { before treatment. } \\
\text { combination gel } \\
\text { applied } 3 \text { mm } \\
\text { beyond visible } \\
\text { margin of tumor. } \\
\text { the gel layer was } \\
5 \text { mm thick. the } \\
\text { area was covered } \\
\text { with plaster and } \\
\text { protected from } \\
\text { light. three hours } \\
\text { after application, } \\
\text { residue was } \\
\text { removed. }\end{array}$ & & & \\
\hline $\begin{array}{l}\text { Schulze } \\
2005 \\
15888150 \\
\end{array}$ & imiquimod 5\% & & & & & & $\begin{array}{l}\text { imiquimod 5\% } \\
\text { (Topical) daily } \\
\text { for } 6 \text { weeks }\end{array}$ & & \\
\hline $\begin{array}{l}\text { Schulze } \\
2005 \\
15888150 \\
\end{array}$ & vehicle & & & & & & $\begin{array}{l}\text { vehicle cream } \\
\text { (Topical) daily } \\
\text { for } 6 \text { weeks }\end{array}$ & & \\
\hline $\begin{array}{l}\text { Shumack } \\
2002 \\
12224978 \\
(12 \\
\text { weeks) }\end{array}$ & vehicle cream & & & & & & $\begin{array}{l}\text { vehicle Placebo } \\
\text { (Topical) twice } \\
\text { daily for } 7 \text { days } \\
\text { per week or } \\
\text { once daily for } 7 \\
\text { days per week } \\
\text { or once daily for } \\
5 \text { days per week } \\
\text { for } 12 \text { weeks }\end{array}$ & & \\
\hline $\begin{array}{l}\text { Shumack } \\
2002 \\
12224978 \\
(12 \\
\text { weeks) }\end{array}$ & $\begin{array}{l}\text { imiquimod } \\
\text { (IMQ) 5\% } \\
\text { cream - Twice } \\
\text { daily for } 7 \\
\text { days per } \\
\text { week }\end{array}$ & & & & & & $\begin{array}{l}\text { imiquimod 5\% } \\
\text { (Topical) Twice } \\
\text { daily for } 7 \text { days } \\
\text { per week for } 12 \\
\text { weeks }\end{array}$ & $\begin{array}{l}\text { Patients applied } \\
\text { topical } 5 \% \\
\text { imiquimod } \\
\text { cream to } 1 \\
\text { target tu- mor } \\
\text { just prior to } \\
\text { normal sleeping } \\
\text { hours according }\end{array}$ & \\
\hline
\end{tabular}




\begin{tabular}{|c|c|c|c|c|c|c|c|c|c|}
\hline $\begin{array}{l}\text { Author, } \\
\text { year, } \\
\text { PMID }\end{array}$ & Arm & $\begin{array}{l}\text { Surgical } \\
\text { interventions } \\
\text { (margins) }\end{array}$ & $\begin{array}{l}\text { Thermal } \\
\text { interventions } \\
\text { (description) }\end{array}$ & Radiation & $\begin{array}{l}\text { Photodynamic } \\
\text { Therapy dose }\end{array}$ & $\begin{array}{l}\text { Photodynamic } \\
\text { Therapy } \\
\text { description }\end{array}$ & $\begin{array}{l}\text { Medical } \\
\text { interventions } \\
\text { description }\end{array}$ & $\begin{array}{l}\text { Medical } \\
\text { interventions } \\
\text { other }\end{array}$ & $\begin{array}{l}\text { Curettage } \\
\text { number of } \\
\text { passes }\end{array}$ \\
\hline & & & & & & & & $\begin{array}{l}\text { to the dosing } \\
\text { regimen to } \\
\text { which they } \\
\text { were assigned. } \\
\text { The target } \\
\text { tumor was } \\
\text { washed with } \\
\text { mild soap just } \\
\text { prior to cream } \\
\text { application, and } \\
\text { the cream was } \\
\text { rubbed into and } \\
\text { around } \\
\text { (approximately } \\
\text { up to } 1 \mathrm{~cm} \text { ) the } \\
\text { tumor. The } \\
\text { cream } \\
\text { remained in } \\
\text { place for at } \\
\text { least } 8 \text { hours } \\
\text { without } \\
\text { occlusion. }\end{array}$ & \\
\hline $\begin{array}{l}\text { Shumack } \\
2002 \\
12224978 \\
(12 \\
\text { weeks) }\end{array}$ & $\begin{array}{l}\text { imiquimod } \\
\text { (IMQ) 5\% } \\
\text { cream - Once } \\
\text { daily for } 7 \\
\text { days per } \\
\text { week }\end{array}$ & & & & & & $\begin{array}{l}\text { imiquimod } 5 \% \\
\text { (Topical) Once } \\
\text { daily for } 7 \text { days } \\
\text { per week for } 12 \\
\text { weeks }\end{array}$ & $\begin{array}{l}\text { Patients applied } \\
\text { topical 5\% } \\
\text { imiquimod } \\
\text { cream to } 1 \\
\text { target tu- mor } \\
\text { just prior to } \\
\text { normal sleeping } \\
\text { hours according } \\
\text { to the dosing } \\
\text { regimen to } \\
\text { which they } \\
\text { were assigned. } \\
\text { The target } \\
\text { tumor was } \\
\text { washed with } \\
\text { mild soap just } \\
\text { prior to cream } \\
\text { application, and } \\
\text { the cream was }\end{array}$ & \\
\hline
\end{tabular}




\begin{tabular}{|c|c|c|c|c|c|c|c|c|c|}
\hline $\begin{array}{l}\text { Author, } \\
\text { year, } \\
\text { PMID }\end{array}$ & Arm & $\begin{array}{l}\text { Surgical } \\
\text { interventions } \\
\text { (margins) }\end{array}$ & $\begin{array}{l}\text { Thermal } \\
\text { interventions } \\
\text { (description) }\end{array}$ & Radiation & $\begin{array}{l}\text { Photodynamic } \\
\text { Therapy dose }\end{array}$ & $\begin{array}{l}\text { Photodynamic } \\
\text { Therapy } \\
\text { description }\end{array}$ & $\begin{array}{l}\text { Medical } \\
\text { interventions } \\
\text { description }\end{array}$ & $\begin{array}{l}\text { Medical } \\
\text { interventions } \\
\text { other }\end{array}$ & $\begin{array}{l}\text { Curettage } \\
\text { number of } \\
\text { passes }\end{array}$ \\
\hline & & & & & & & & $\begin{array}{l}\text { rubbed into and } \\
\text { around } \\
\text { (approximately } \\
\text { up to } 1 \mathrm{~cm} \text { ) the } \\
\text { tumor. The } \\
\text { cream } \\
\text { remained in } \\
\text { place for at } \\
\text { least } 8 \text { hours } \\
\text { without } \\
\text { occlusion. }\end{array}$ & \\
\hline $\begin{array}{l}\text { Shumack } \\
2002 \\
12224978 \\
(12 \\
\text { weeks) }\end{array}$ & $\begin{array}{l}\text { imiquimod } \\
\text { (IMQ) } 5 \% \\
\text { cream - Once } \\
\text { daily for } 5 \\
\text { days per } \\
\text { week }\end{array}$ & & & & & & $\begin{array}{l}\text { imiquimod } 5 \% \\
\text { (Topical) Once } \\
\text { daily for } 5 \text { days } \\
\text { per week for } 12 \\
\text { weeks }\end{array}$ & $\begin{array}{l}\text { Patients applied } \\
\text { topical 5\% } \\
\text { imiquimod } \\
\text { cream to } 1 \\
\text { target tu- mor } \\
\text { just prior to } \\
\text { normal sleeping } \\
\text { hours according } \\
\text { to the dosing } \\
\text { regimen to } \\
\text { which they } \\
\text { were assigned. } \\
\text { The target } \\
\text { tumor was } \\
\text { washed with } \\
\text { mild soap just } \\
\text { prior to cream } \\
\text { application, and } \\
\text { the cream was } \\
\text { rubbed into and } \\
\text { around } \\
\text { (approximately } \\
\text { up to } 1 \text { cm) the } \\
\text { tumor. The } \\
\text { cream } \\
\text { remained in } \\
\text { place for at } \\
\text { least } 8 \text { hours } \\
\text { without } \\
\text { occlusion. }\end{array}$ & \\
\hline
\end{tabular}




\begin{tabular}{|c|c|c|c|c|c|c|c|c|c|}
\hline $\begin{array}{l}\text { Author, } \\
\text { year, } \\
\text { PMID }\end{array}$ & Arm & $\begin{array}{l}\text { Surgical } \\
\text { interventions } \\
\text { (margins) }\end{array}$ & $\begin{array}{l}\text { Thermal } \\
\text { interventions } \\
\text { (description) }\end{array}$ & Radiation & $\begin{array}{l}\text { Photodynamic } \\
\text { Therapy dose }\end{array}$ & $\begin{array}{l}\text { Photodynamic } \\
\text { Therapy } \\
\text { description }\end{array}$ & $\begin{array}{l}\text { Medical } \\
\text { interventions } \\
\text { description }\end{array}$ & $\begin{array}{l}\text { Medical } \\
\text { interventions } \\
\text { other }\end{array}$ & $\begin{array}{l}\text { Curettage } \\
\text { number of } \\
\text { passes }\end{array}$ \\
\hline $\begin{array}{l}\text { Shumack } \\
2002 \\
12224978 \\
(12 \\
\text { weeks) }\end{array}$ & $\begin{array}{l}\text { imiquimod } \\
\text { (IMQ) 5\% } \\
\text { cream - Once } \\
\text { daily for } 3 \\
\text { days per } \\
\text { week }\end{array}$ & & & & & & $\begin{array}{l}\text { imiquimod } 5 \% \\
\text { (Topical) Once } \\
\text { daily for } 3 \text { days } \\
\text { per week for } 12 \\
\text { weeks }\end{array}$ & $\begin{array}{l}\text { Patients applied } \\
\text { topical 5\% } \\
\text { imiquimod } \\
\text { cream to } 1 \\
\text { target tu- mor } \\
\text { just prior to } \\
\text { normal sleeping } \\
\text { hours according } \\
\text { to the dosing } \\
\text { regimen to } \\
\text { which they } \\
\text { were assigned. } \\
\text { The target } \\
\text { tumor was } \\
\text { washed with } \\
\text { mild soap just } \\
\text { prior to cream } \\
\text { application, and } \\
\text { the cream was } \\
\text { rubbed into and } \\
\text { around } \\
\text { (approximately } \\
\text { up to } 1 \text { cm) the } \\
\text { tumor. The } \\
\text { cream } \\
\text { remained in } \\
\text { place for at } \\
\text { least } 8 \text { hours } \\
\text { without } \\
\text { occlusion. }\end{array}$ & \\
\hline $\begin{array}{l}\text { Shumack } \\
2002 \\
12224978 \\
(6 \text { weeks) }\end{array}$ & $\begin{array}{l}\text { imiquimod } \\
\text { (IMQ) 5\% } \\
\text { cream - Twice } \\
\text { daily for } 7 \\
\text { days per } \\
\text { week }\end{array}$ & & & & & & $\begin{array}{l}\text { Imiquimod 5\% } \\
\text { (Topical) Twice } \\
\text { daily for } 7 \text { days } \\
\text { per week for } 6 \\
\text { weeks }\end{array}$ & $\begin{array}{l}\text { Patients applied } \\
\text { topical 5\% } \\
\text { imiquimod } \\
\text { cream to } 1 \\
\text { target tu- mor } \\
\text { just prior to } \\
\text { normal sleeping } \\
\text { hours according } \\
\text { to the dosing } \\
\text { regimentowhich } \\
\text { theywereassign }\end{array}$ & \\
\hline
\end{tabular}




\begin{tabular}{|c|c|c|c|c|c|c|c|c|c|}
\hline $\begin{array}{l}\text { Author, } \\
\text { year, } \\
\text { PMID }\end{array}$ & Arm & $\begin{array}{l}\text { Surgical } \\
\text { interventions } \\
\text { (margins) }\end{array}$ & $\begin{array}{l}\text { Thermal } \\
\text { interventions } \\
\text { (description) }\end{array}$ & Radiation & $\begin{array}{l}\text { Photodynamic } \\
\text { Therapy dose }\end{array}$ & $\begin{array}{l}\text { Photodynamic } \\
\text { Therapy } \\
\text { description }\end{array}$ & $\begin{array}{l}\text { Medical } \\
\text { interventions } \\
\text { description }\end{array}$ & $\begin{array}{l}\text { Medical } \\
\text { interventions } \\
\text { other }\end{array}$ & $\begin{array}{l}\text { Curettage } \\
\text { number of } \\
\text { passes }\end{array}$ \\
\hline & & & & & & & & $\begin{array}{l}\text { ed.Thetargettu } \\
\text { morwaswashed } \\
\text { with mild soap } \\
\text { just prior to } \\
\text { cream } \\
\text { application, and } \\
\text { the cream was } \\
\text { rubbed into and } \\
\text { around } \\
\text { (approximately } \\
\text { up to } 1 \mathrm{~cm} \text { ) the } \\
\text { tumor. The } \\
\text { cream } \\
\text { remained in } \\
\text { place for at } \\
\text { least } 8 \text { hours } \\
\text { without } \\
\text { occlusion. }\end{array}$ & \\
\hline $\begin{array}{l}\text { Shumack } \\
2002 \\
12224978 \\
(6 \text { weeks) }\end{array}$ & $\begin{array}{l}\text { imiquimod } \\
\text { (IMQ) } 5 \% \\
\text { cream - Once } \\
\text { daily for } 3 \\
\text { days per } \\
\text { week }\end{array}$ & & & & & & $\begin{array}{l}\text { Imiquimod 5\% } \\
\text { (Topical) Once } \\
\text { daily for } 3 \text { days } \\
\text { per week for } 6 \\
\text { weeks }\end{array}$ & $\begin{array}{l}\text { Patients applied } \\
\text { topical 5\% } \\
\text { imiquimod } \\
\text { cream to } 1 \\
\text { target tu- mor } \\
\text { just prior to } \\
\text { normal sleeping } \\
\text { hours according } \\
\text { to the dosing } \\
\text { regimentowhich } \\
\text { theywereassign } \\
\text { ed.Thetargettu } \\
\text { morwaswashed } \\
\text { with mild soap } \\
\text { just prior to } \\
\text { cream } \\
\text { application, and } \\
\text { the cream was } \\
\text { rubbed into and } \\
\text { around } \\
\text { (approximately } \\
\text { up to } 1 \mathrm{~cm} \text { ) the } \\
\text { tumor. The }\end{array}$ & \\
\hline
\end{tabular}




\begin{tabular}{|c|c|c|c|c|c|c|c|c|c|}
\hline $\begin{array}{l}\text { Author, } \\
\text { year, } \\
\text { PMID }\end{array}$ & Arm & $\begin{array}{l}\text { Surgical } \\
\text { interventions } \\
\text { (margins) }\end{array}$ & $\begin{array}{l}\text { Thermal } \\
\text { interventions } \\
\text { (description) }\end{array}$ & Radiation & $\begin{array}{l}\text { Photodynamic } \\
\text { Therapy dose }\end{array}$ & $\begin{array}{l}\text { Photodynamic } \\
\text { Therapy } \\
\text { description }\end{array}$ & $\begin{array}{l}\text { Medical } \\
\text { interventions } \\
\text { description }\end{array}$ & $\begin{array}{l}\text { Medical } \\
\text { interventions } \\
\text { other }\end{array}$ & $\begin{array}{l}\text { Curettage } \\
\text { number of } \\
\text { passes }\end{array}$ \\
\hline & & & & & & & & $\begin{array}{l}\text { cream } \\
\text { remained in } \\
\text { place for at } \\
\text { least } 8 \text { hours } \\
\text { without } \\
\text { occlusion. }\end{array}$ & \\
\hline $\begin{array}{l}\text { Shumack } \\
2002 \\
12224978 \\
(6 \text { weeks) }\end{array}$ & $\begin{array}{l}\text { imiquimod } \\
\text { (IMQ) 5\% } \\
\text { cream - Twice } \\
\text { daily for } 3 \\
\text { days per } \\
\text { week }\end{array}$ & & & & & & $\begin{array}{l}\text { Imiquimod 5\% } \\
\text { (Topical) Twice } \\
\text { daily for } 3 \text { days } \\
\text { per week for } 6 \\
\text { weeks }\end{array}$ & $\begin{array}{l}\text { Patients applied } \\
\text { topical 5\% } \\
\text { imiquimod } \\
\text { cream to } 1 \\
\text { target tu- mor } \\
\text { just prior to } \\
\text { normal sleeping } \\
\text { hours according } \\
\text { to the dosing } \\
\text { regimentowhich } \\
\text { theywereassign } \\
\text { ed. Thetargettu } \\
\text { morwaswashed } \\
\text { with mild soap } \\
\text { just prior to } \\
\text { cream } \\
\text { application, and } \\
\text { the cream was } \\
\text { rubbed into and } \\
\text { around } \\
\text { (approximately } \\
\text { up to } 1 \text { cm) the } \\
\text { tumor. The } \\
\text { cream } \\
\text { remained in } \\
\text { place for at } \\
\text { least } 8 \text { hours } \\
\text { without } \\
\text { occlusion. }\end{array}$ & \\
\hline $\begin{array}{l}\text { Shumack } \\
2002 \\
12224978 \\
(6 \text { weeks) }\end{array}$ & $\begin{array}{l}\text { imiquimod } \\
\text { (IMQ) 5\% } \\
\text { cream - Once } \\
\text { daily for } 7 \\
\text { days per } \\
\text { week }\end{array}$ & & & & & & $\begin{array}{l}\text { Imiquimod 5\% } \\
\text { (Topical) Once } \\
\text { daily for } 7 \text { days } \\
\text { per week for } 6 \\
\text { weeks }\end{array}$ & $\begin{array}{l}\text { Patients applied } \\
\text { topical } 5 \% \\
\text { imiquimod } \\
\text { cream to } 1 \\
\text { target tu- mor } \\
\text { just prior to }\end{array}$ & \\
\hline
\end{tabular}




\begin{tabular}{|c|c|c|c|c|c|c|c|c|c|}
\hline $\begin{array}{l}\text { Author, } \\
\text { year, } \\
\text { PMID }\end{array}$ & Arm & $\begin{array}{l}\text { Surgical } \\
\text { interventions } \\
\text { (margins) }\end{array}$ & $\begin{array}{l}\text { Thermal } \\
\text { interventions } \\
\text { (description) }\end{array}$ & Radiation & $\begin{array}{l}\text { Photodynamic } \\
\text { Therapy dose }\end{array}$ & $\begin{array}{l}\text { Photodynamic } \\
\text { Therapy } \\
\text { description }\end{array}$ & $\begin{array}{l}\text { Medical } \\
\text { interventions } \\
\text { description }\end{array}$ & $\begin{array}{l}\text { Medical } \\
\text { interventions } \\
\text { other }\end{array}$ & $\begin{array}{l}\text { Curettage } \\
\text { number of } \\
\text { passes }\end{array}$ \\
\hline & & & & & & & & $\begin{array}{l}\text { normal sleeping } \\
\text { hours according } \\
\text { to the dosing } \\
\text { regimentowhich } \\
\text { theywereassign } \\
\text { ed.Thetargettu } \\
\text { morwaswashed } \\
\text { with mild soap } \\
\text { just prior to } \\
\text { cream } \\
\text { application, and } \\
\text { the cream was } \\
\text { rubbed into and } \\
\text { around } \\
\text { (approximately } \\
\text { up to } 1 \text { cm) the } \\
\text { tumor. The } \\
\text { cream } \\
\text { remained in } \\
\text { place for at } \\
\text { least } 8 \text { hours } \\
\text { without } \\
\text { occlusion. }\end{array}$ & \\
\hline $\begin{array}{l}\text { Siller } 2010 \\
20546215\end{array}$ & $\begin{array}{l}\text { vehicle gel, } \\
\text { treatment arm } \\
\text { A; day } 1 \text { and } \\
2 \text { (subjects } \\
\text { randomized } \\
\text { to apply } \\
\text { vehicle cream } \\
\text { (control) on } \\
\text { day } 1 \text { and } 2 . \text { ) }\end{array}$ & & & & & & $\begin{array}{l}\text { vehicle cream } \\
\text { (Topical) } 2 X \text { for } \\
\text { N/A }\end{array}$ & $\begin{array}{l}\text { Day } 1 \text { and Dat } \\
2 ; \text { investigator } \\
\text { applied gel } \\
\text { directly to } \\
\text { sBCC using a } \\
\text { micropipette } \\
\text { and a circular } \\
\text { template. } \\
\text { volume of gel } \\
\text { based on } \\
\text { longest post- } \\
\text { biopsy lesion } \\
\text { diameter } \\
\text { (ranged btw } \\
0.25-5.20 \\
\text { micrograms } / \mathrm{cm} \\
\text { 2) }\end{array}$ & \\
\hline Siller 2010 & ingenol & & & & & & ingenol & Day 1 and Day & \\
\hline
\end{tabular}




\begin{tabular}{|c|c|c|c|c|c|c|c|c|c|}
\hline $\begin{array}{l}\text { Author, } \\
\text { year, } \\
\text { PMID }\end{array}$ & Arm & $\begin{array}{l}\text { Surgical } \\
\text { interventions } \\
\text { (margins) }\end{array}$ & $\begin{array}{l}\text { Thermal } \\
\text { interventions } \\
\text { (description) }\end{array}$ & Radiation & $\begin{array}{l}\text { Photodynamic } \\
\text { Therapy dose }\end{array}$ & $\begin{array}{l}\text { Photodynamic } \\
\text { Therapy } \\
\text { description }\end{array}$ & $\begin{array}{l}\text { Medical } \\
\text { interventions } \\
\text { description }\end{array}$ & $\begin{array}{l}\text { Medical } \\
\text { interventions } \\
\text { other }\end{array}$ & $\begin{array}{l}\text { Curettage } \\
\text { number of } \\
\text { passes }\end{array}$ \\
\hline 20546215 & $\begin{array}{l}\text { mebutate gel, } \\
0.0025 \%, \\
\text { treatment arm } \\
\text { A-days } 1 \text { and } \\
2 \text { (subjects } \\
\text { randomized } \\
\text { to apply } \\
0.0025 \% \\
\text { ingenol } \\
\text { mebutate on } \\
\text { days } 1 \text { and } 2 \text {. } \\
\text { ) }\end{array}$ & & & & & & $\begin{array}{l}\text { mebutate } \\
0.0025 \% \\
\text { (Topical) 2x for } \\
\text { N/A }\end{array}$ & $\begin{array}{l}\text { 2; investigator } \\
\text { applied gel } \\
\text { directly to } \\
\text { sBCC using a } \\
\text { micropipette } \\
\text { and a circular } \\
\text { template. } \\
\text { volume of gel } \\
\text { based on } \\
\text { longest post- } \\
\text { biopsy lesion } \\
\text { diameter } \\
\text { (ranged btw } \\
0.25-5.20 \\
\text { micrograms/cm } \\
2 \text { ) } \\
\end{array}$ & \\
\hline $\begin{array}{l}\text { Siller } 2010 \\
20546215\end{array}$ & $\begin{array}{l}\text { ingenol } \\
\text { mebutate gel, } \\
0.01 \%, \\
\text { treatment arm } \\
\text { A- day } 1 \text { and } \\
2 \text { (subjects } \\
\text { randomized } \\
\text { to apply } \\
0.01 \% \\
\text { ingenol } \\
\text { mebutate on } \\
\text { days } 1 \text { and } 2 \text {. } \\
\text { ) }\end{array}$ & & & & & & $\begin{array}{l}\text { ingenol } \\
\text { mebutate } 0.01 \% \\
\text { (Topical) } 2 x \text { for } \\
\text { N/A }\end{array}$ & $\begin{array}{l}\text { Day } 1 \text { and Day } \\
\text { 2; investigator } \\
\text { applied gel } \\
\text { directly to } \\
\text { sBCC using a } \\
\text { micropipette } \\
\text { and a circular } \\
\text { template. } \\
\text { volume of gel } \\
\text { based on } \\
\text { longest post- } \\
\text { biopsy lesion } \\
\text { diameter } \\
\text { (ranged btw } \\
0.25-5.20 \\
\text { micrograms/cm } \\
\text { 2) }\end{array}$ & \\
\hline $\begin{array}{l}\text { Siller } 2010 \\
20546215\end{array}$ & $\begin{array}{l}\text { ingenol } \\
\text { mebutate gel, } \\
0.05 \%, \\
\text { treatment arm } \\
\text { A-day } 1 \text { and } 2 \\
\text { (subjects } \\
\text { randomized } \\
\text { to apply }\end{array}$ & & & & & & $\begin{array}{l}\text { ingenol } \\
\text { mebutate } 0.05 \% \\
\text { (Topical) } 2 x \text { for } \\
\text { N/A }\end{array}$ & $\begin{array}{l}\text { Day } 1 \text { and Day } \\
2 ; \text { investigator } \\
\text { applied gel } \\
\text { directly to } \\
\text { sBCC using a } \\
\text { micropipette } \\
\text { and a circular } \\
\text { template. }\end{array}$ & \\
\hline
\end{tabular}




\begin{tabular}{|c|c|c|c|c|c|c|c|c|c|}
\hline $\begin{array}{l}\text { Author, } \\
\text { year, } \\
\text { PMID }\end{array}$ & Arm & $\begin{array}{l}\text { Surgical } \\
\text { interventions } \\
\text { (margins) }\end{array}$ & $\begin{array}{l}\text { Thermal } \\
\text { interventions } \\
\text { (description) }\end{array}$ & Radiation & $\begin{array}{l}\text { Photodynamic } \\
\text { Therapy dose }\end{array}$ & $\begin{array}{l}\text { Photodynamic } \\
\text { Therapy } \\
\text { description }\end{array}$ & $\begin{array}{l}\text { Medical } \\
\text { interventions } \\
\text { description }\end{array}$ & $\begin{array}{l}\text { Medical } \\
\text { interventions } \\
\text { other }\end{array}$ & $\begin{array}{l}\text { Curettage } \\
\text { number of } \\
\text { passes }\end{array}$ \\
\hline & $\begin{array}{l}0.05 \% \\
\text { ingenol } \\
\text { mebutate on } \\
\text { days } 1 \text { and } 2 . \\
\text { ) }\end{array}$ & & & & & & & $\begin{array}{l}\text { volume of gel } \\
\text { based on } \\
\text { longest post- } \\
\text { biopsy lesion } \\
\text { diameter } \\
\text { (ranged btw } \\
0.25-5.20 \\
\text { micrograms/cm } \\
2 \text { ) }\end{array}$ & \\
\hline $\begin{array}{l}\text { Siller } 2010 \\
20546215\end{array}$ & $\begin{array}{l}\text { vehicle gel, } \\
\text { treatment arm } \\
\text { B- day } 1 \text { and } \\
8 \text { (subjects } \\
\text { randomized } \\
\text { to apply } \\
\text { vehicle cream } \\
\text { (control) on } \\
\text { day } 1 \text { and } 8 . \text { ) }\end{array}$ & & & & & & $\begin{array}{l}\text { vehicle (Topical) } \\
2 x \text { for N/A }\end{array}$ & $\begin{array}{l}\text { Day } 1 \text { and Day } \\
\text { 8; investigator } \\
\text { applied gel } \\
\text { directly to } \\
\text { sBCC using a } \\
\text { micropipette } \\
\text { and a circular } \\
\text { template. } \\
\text { volume of gel } \\
\text { based on } \\
\text { longest post- } \\
\text { biopsy lesion } \\
\text { diameter } \\
\text { (ranged btw } \\
0.25-5.20 \\
\text { micrograms/cm } \\
2 \text { ) }\end{array}$ & \\
\hline $\begin{array}{l}\text { Siller } 2010 \\
20546215\end{array}$ & $\begin{array}{l}\text { ingenol } \\
\text { mebutate gel, } \\
0.0025 \%, \\
\text { treatment arm } \\
\text { B-days } 1 \text { and } \\
8 \text { (subjects } \\
\text { randomized } \\
\text { to apply } \\
0.0025 \% \\
\text { ingenol } \\
\text { mebutate on } \\
\text { days } 1 \text { and } 8 . \\
\text { ) }\end{array}$ & & & & & & $\begin{array}{l}\text { ingenol } \\
\text { mebutate } \\
0.0025 \% \\
\text { (Topical) 2x for } \\
\text { N/A }\end{array}$ & $\begin{array}{l}\text { Day } 1 \text { and Day } \\
\text { 8; investigator } \\
\text { applied gel } \\
\text { directly to } \\
\text { sBCC using a } \\
\text { micropipette } \\
\text { and a circular } \\
\text { template. } \\
\text { volume of gel } \\
\text { based on } \\
\text { longest post- } \\
\text { biopsy lesion } \\
\text { diameter } \\
\text { (ranged btw } \\
0.25-5.20\end{array}$ & \\
\hline
\end{tabular}




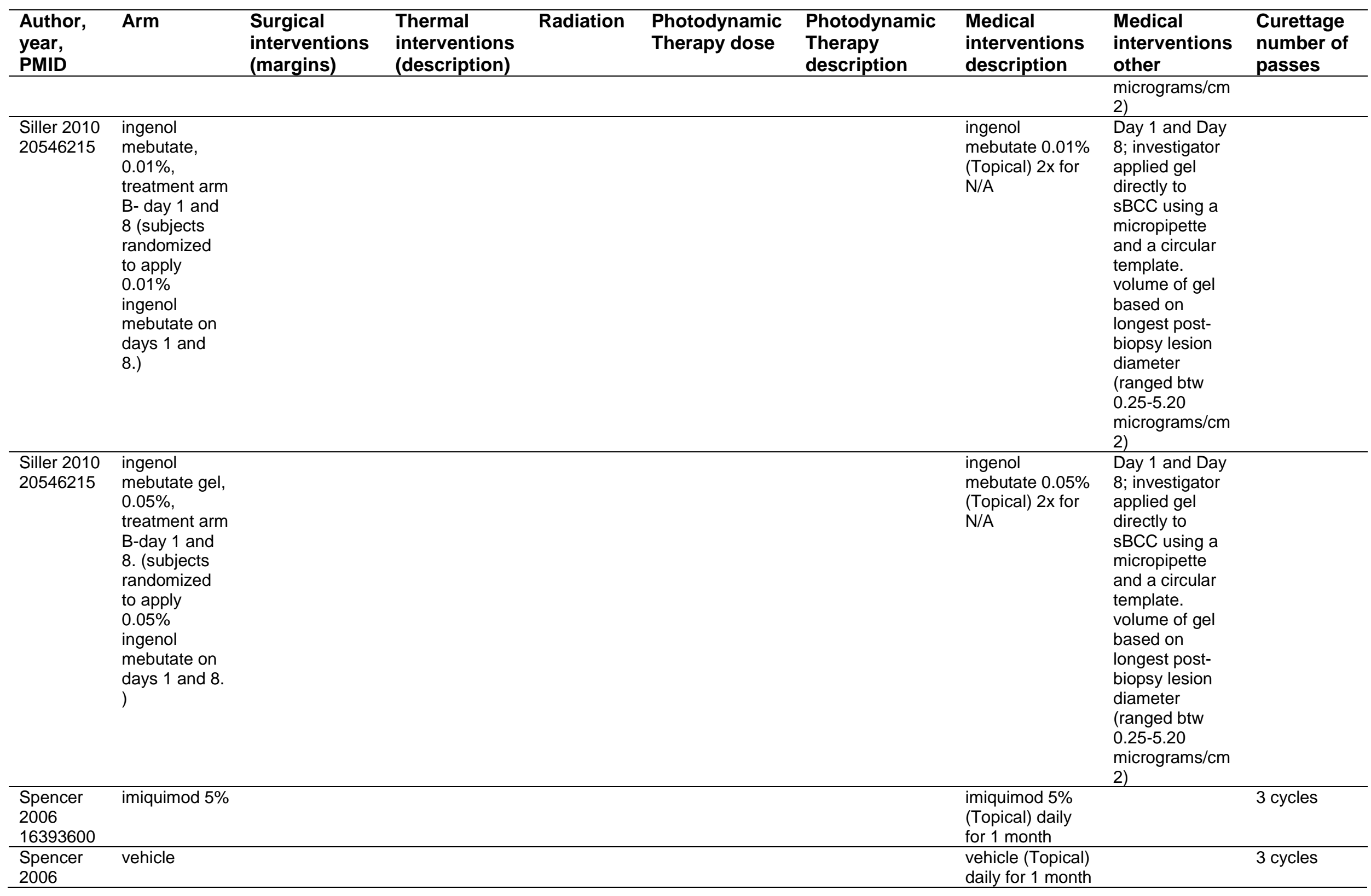




\begin{tabular}{|c|c|c|c|c|c|c|c|c|c|}
\hline $\begin{array}{l}\text { Author, } \\
\text { year, } \\
\text { PMID } \\
\end{array}$ & Arm & $\begin{array}{l}\text { Surgical } \\
\text { interventions } \\
\text { (margins) }\end{array}$ & $\begin{array}{l}\text { Thermal } \\
\text { interventions } \\
\text { (description) }\end{array}$ & Radiation & $\begin{array}{l}\text { Photodynamic } \\
\text { Therapy dose }\end{array}$ & $\begin{array}{l}\text { Photodynamic } \\
\text { Therapy } \\
\text { description }\end{array}$ & $\begin{array}{l}\text { Medical } \\
\text { interventions } \\
\text { description }\end{array}$ & $\begin{array}{l}\text { Medical } \\
\text { interventions } \\
\text { other }\end{array}$ & $\begin{array}{l}\text { Curettage } \\
\text { number of } \\
\text { passes }\end{array}$ \\
\hline \multicolumn{10}{|l|}{16393600} \\
\hline $\begin{array}{l}\text { Sterry } \\
2002 \\
12452875\end{array}$ & $\begin{array}{l}\text { Imiquimod (3 } \\
\text { days/week) } \\
\text { with occlusion }\end{array}$ & & & & & & $\begin{array}{l}\text { Imiquimod 5\% } \\
\text { (Topical) } 3 \\
\text { days/week for } 6 \\
\text { weeks }\end{array}$ & $\begin{array}{l}\text { bedtime; left on } \\
\text { for } 8 \text { hours; with } \\
\text { occlusive } \\
\text { dressing }\end{array}$ & \\
\hline $\begin{array}{l}\text { Sterry } \\
2002 \\
12452875\end{array}$ & $\begin{array}{l}\text { Imiquimod (2 } \\
\text { days/week) } \\
\text { without } \\
\text { occlusion }\end{array}$ & & & & & & $\begin{array}{l}\text { Imiquimod 5\% } \\
\text { (Topical) } 2 \\
\text { days/week for } 6 \\
\text { weeks }\end{array}$ & $\begin{array}{l}\text { bedtime; left on } \\
\text { for } 8 \text { hours; } \\
\text { without } \\
\text { occlusive } \\
\text { dressing }\end{array}$ & \\
\hline $\begin{array}{l}\text { Sterry } \\
2002 \\
12452875 \\
\end{array}$ & $\begin{array}{l}\text { Imiquimod (3 } \\
\text { days/week) } \\
\text { without } \\
\text { occlusion }\end{array}$ & & & & & & $\begin{array}{l}\text { Imiquimod 5\% } \\
\text { (Topical) } 3 \\
\text { days/week for } 6 \\
\text { weeks }\end{array}$ & $\begin{array}{l}\text { bedtime; left on } \\
\text { for } 8 \text { hours; } \\
\text { without } \\
\text { occlusive } \\
\text { dressing }\end{array}$ & \\
\hline $\begin{array}{l}\text { Sterry } \\
2002 \\
12452875 \\
\text { (nodular) } \\
\end{array}$ & $\begin{array}{l}\text { Imiquimod (2 } \\
\text { days/week) } \\
\text { with occlusion }\end{array}$ & & & & & & $\begin{array}{l}\text { Imiquimod } 5 \% \\
\text { (Topical) } 2 \\
\text { days/week for } 6 \\
\text { weeks }\end{array}$ & $\begin{array}{l}\text { bedtime; left on } \\
\text { for } 8 \text { hours; with } \\
\text { occlusive } \\
\text { dressing }\end{array}$ & \\
\hline $\begin{array}{l}\text { Sterry } \\
2002 \\
12452875 \\
\text { (nodular) } \\
\end{array}$ & $\begin{array}{l}\text { Imiquimod (3 } \\
\text { days/week) } \\
\text { with occlusion }\end{array}$ & & & & & & $\begin{array}{l}\text { Imiquimod } 5 \% \\
\text { (Topical) } 3 \\
\text { days/week for } 6 \\
\text { weeks }\end{array}$ & $\begin{array}{l}\text { bedtime; left on } \\
\text { for } 8 \text { hours; with } \\
\text { occlusive } \\
\text { dressing }\end{array}$ & \\
\hline $\begin{array}{l}\text { Sterry } \\
2002 \\
12452875 \\
\text { (nodular) }\end{array}$ & $\begin{array}{l}\text { Imiquimod (2 } \\
\text { days/week) } \\
\text { without } \\
\text { occlusion }\end{array}$ & & & & & & $\begin{array}{l}\text { Imiquimod } 5 \% \\
\text { (Topical) } 2 \\
\text { days/week for } 6 \\
\text { weeks }\end{array}$ & $\begin{array}{l}\text { bedtime; left on } \\
\text { for } 8 \text { hours; } \\
\text { without } \\
\text { occlusive } \\
\text { dressing }\end{array}$ & \\
\hline $\begin{array}{l}\text { Sterry } \\
2002 \\
12452875 \\
\text { (nodular) }\end{array}$ & $\begin{array}{l}\text { Imiquimod (3 } \\
\text { days/week) } \\
\text { without } \\
\text { occlusion }\end{array}$ & & & & & & $\begin{array}{l}\text { Imiquimod 5\% } \\
\text { (Topical) } 3 \\
\text { days/week for } 6 \\
\text { weeks }\end{array}$ & $\begin{array}{l}\text { bedtime; left on } \\
\text { for } 8 \text { hours; } \\
\text { without } \\
\text { occlusive } \\
\text { dressing }\end{array}$ & \\
\hline
\end{tabular}




\begin{tabular}{|c|c|c|c|c|c|c|c|c|c|}
\hline $\begin{array}{l}\text { Author, } \\
\text { year, } \\
\text { PMID }\end{array}$ & Arm & $\begin{array}{l}\text { Surgical } \\
\text { interventions } \\
\text { (margins) }\end{array}$ & $\begin{array}{l}\text { Thermal } \\
\text { interventions } \\
\text { (description) }\end{array}$ & Radiation & $\begin{array}{l}\text { Photodynamic } \\
\text { Therapy dose }\end{array}$ & $\begin{array}{l}\text { Photodynamic } \\
\text { Therapy } \\
\text { description }\end{array}$ & $\begin{array}{l}\text { Medical } \\
\text { interventions } \\
\text { description }\end{array}$ & $\begin{array}{l}\text { Medical } \\
\text { interventions } \\
\text { other }\end{array}$ & $\begin{array}{l}\text { Curettage } \\
\text { number of } \\
\text { passes }\end{array}$ \\
\hline & & & & & & & & developed & \\
\hline $\begin{array}{l}\text { Sullivan } \\
2003 \\
14725659\end{array}$ & vehicle & & & & & & $\begin{array}{l}\text { vehicle cream } \\
\text { NR (Topical) } \\
\text { nightly on } \\
\text { weekdays for } 10 \\
+-3 \text { applications }\end{array}$ & & \\
\hline $\begin{array}{l}\text { Szeimies } \\
2008 \\
18624836\end{array}$ & MAL-PDT & & & & $\begin{array}{l}\text { large-field LED } \\
\text { for } 7-10 \text { min to } \\
37 \mathrm{~J} / \mathrm{cm}^{\wedge} 2\end{array}$ & $\begin{array}{l}2 \text { sessions, } 160 \\
\text { mg/g MAL, } \\
\text { without bleeding } \\
\text { or pain, to remove } \\
\text { scales and crusts } \\
\text { and roughen } \\
\text { legion surface, } \\
\text { followed by layer } \\
\text { of } 1 \text { mm thick } \\
\text { MAL to lesion and } \\
\text { surrounding 5-10 } \\
\text { mm area }\end{array}$ & & & \\
\hline $\begin{array}{l}\text { Szeimies } \\
2008 \\
18624836 \\
\end{array}$ & excision & $\begin{array}{l}\text { excsision (3 } \\
\mathrm{mm})\end{array}$ & & & & & & & \\
\hline $\begin{array}{l}\text { Thissen } \\
2000 \\
10940063\end{array}$ & cryotherapy & & $\begin{array}{l}\text { Cryotherapy } \\
\text { with curettage } \\
\text { (treated with } \\
\text { liquid nitrogen) } \\
\end{array}$ & & & & & & \\
\hline $\begin{array}{l}\text { Thissen } \\
2000 \\
10940063 \\
\end{array}$ & $\begin{array}{l}\text { surgical } \\
\text { excision }\end{array}$ & excision $(3 \mathrm{~mm})$ & & & & & & & \\
\hline $\begin{array}{l}\text { Torres } \\
2004 \\
15606733\end{array}$ & $\begin{array}{l}\text { imiquimod, } 2 \\
\text { weeks (pt } \\
\text { applied } \\
\text { imiquimod } \\
5 \times / \text { week x } 2 \\
\text { weeks prior to } \\
\text { MOHs. ) } \\
\end{array}$ & Mohs & & & & & $\begin{array}{l}\text { imiquimod 5\% } \\
\text { (Topical) } \\
\text { 5x/week for } 2 \\
\text { weeks }\end{array}$ & $\begin{array}{l}\text { apply cream to } \\
\text { target tumor } \\
\text { area and } 1 \mathrm{~cm} \\
\text { of skin } \\
\text { surrounding } \\
\text { tumor }\end{array}$ & \\
\hline $\begin{array}{l}\text { Torres } \\
2004 \\
15606733\end{array}$ & $\begin{array}{l}\text { imiquimod, } 4 \\
\text { weeks (pt } \\
\text { applied } \\
\text { imiquimod } 5 \\
\text { x/week x } 4 \\
\text { weeks prior to }\end{array}$ & Mohs & & & & & $\begin{array}{l}\text { imiquimod 5\% } \\
\text { (Topical) } \\
\text { 5x/week for } 4 \\
\text { weeks }\end{array}$ & $\begin{array}{l}\text { apply cream to } \\
\text { target tumor } \\
\text { area and } 1 \mathrm{~cm} \\
\text { of skin } \\
\text { surrounding } \\
\text { tumor }\end{array}$ & \\
\hline
\end{tabular}




\begin{tabular}{|c|c|c|c|c|c|c|c|c|c|}
\hline $\begin{array}{l}\text { Author, } \\
\text { year, } \\
\text { PMID }\end{array}$ & Arm & $\begin{array}{l}\text { Surgical } \\
\text { interventions } \\
\text { (margins) }\end{array}$ & $\begin{array}{l}\text { Thermal } \\
\text { interventions } \\
\text { (description) }\end{array}$ & Radiation & $\begin{array}{l}\text { Photodynamic } \\
\text { Therapy dose }\end{array}$ & $\begin{array}{l}\text { Photodynamic } \\
\text { Therapy } \\
\text { description }\end{array}$ & $\begin{array}{l}\text { Medical } \\
\text { interventions } \\
\text { description }\end{array}$ & $\begin{array}{l}\text { Medical } \\
\text { interventions } \\
\text { other }\end{array}$ & $\begin{array}{l}\text { Curettage } \\
\text { number of } \\
\text { passes }\end{array}$ \\
\hline & $\mathrm{MOHs})$ & & & & & & & & \\
\hline $\begin{array}{l}\text { Torres } \\
2004 \\
15606733\end{array}$ & $\begin{array}{l}\text { imiquimod, } 6 \\
\text { weeks (pt } \\
\text { applied } \\
\text { imiquimod } \\
5 \times / \text { week x } 6 \\
\text { weeks prior to } \\
\text { MOHs) }\end{array}$ & Mohs & & & & & $\begin{array}{l}\text { imiquimod 5\% } \\
\text { (Topical) } \\
5 x / \text { week for } 6 \\
\text { weeks }\end{array}$ & $\begin{array}{l}\text { apply cream to } \\
\text { target tumor } \\
\text { area and } 1 \mathrm{~cm} \\
\text { of skin } \\
\text { surrounding } \\
\text { tumor }\end{array}$ & \\
\hline $\begin{array}{l}\text { Torres } \\
2004 \\
15606733\end{array}$ & $\begin{array}{l}\text { vehicle } \\
\text { controlled- } \\
\text { pooled } \\
\text { (applied study } \\
\text { cream } \\
5 x / \text { week for } 2, \\
4, \text { or } 6 \text { weeks } \\
\text { prior to } \\
\text { MOHs. ) }\end{array}$ & Mohs & & & & & $\begin{array}{l}\text { vehicle cream } \\
\text { (Topical) } \\
5 x / \text { week for } 2,4 \text {, } \\
\text { and } 6 \text { weeks }\end{array}$ & $\begin{array}{l}\text { apply cream to } \\
\text { target tumor } \\
\text { area and } 1 \mathrm{~cm} \\
\text { of skin } \\
\text { surrounding } \\
\text { tumor }\end{array}$ & \\
\hline $\begin{array}{l}\text { Tran } 2012 \\
22511036\end{array}$ & $\begin{array}{l}\text { S1: PDL } 15 \\
\mathrm{j} / \mathrm{cm} 2\end{array}$ & & & & $\begin{array}{l}595 \mathrm{nM} \text { pulsed- } \\
\text { dye laser: pulse } \\
\text { energy of } 15 \\
\mathrm{~J} / \mathrm{cm} 2,3- \\
\text { millisecond pulse } \\
\text { length }\end{array}$ & $\begin{array}{l}\text { no dynamic } \\
\text { cooling, using a 7- } \\
\text { mm spot size with } \\
10 \% \text { overlap of } \\
\text { pulses and two } \\
\text { passes; } 4 \mathrm{~mm} \\
\text { margin }\end{array}$ & & & \\
\hline $\begin{array}{l}\text { Tran } 2012 \\
22511036\end{array}$ & $\begin{array}{l}\text { S2: PDL } 7.5 \\
\mathrm{j} / \mathrm{cm} 2\end{array}$ & & & & $\begin{array}{l}595 \text { nM pulsed- } \\
\text { dye laser: } 7.5 \\
\text { J/cm2, 3- } \\
\text { millisecond pulse } \\
\text { length }\end{array}$ & $\begin{array}{l}\text { no dynamic } \\
\text { cooling, using a } \\
10-\mathrm{mm} \text { spot size } \\
\text { with } 10 \% \text { overlap } \\
\text { of pulses and } \\
\text { double-stacked } \\
\text { pulses with a } \\
\text { repetitive pulse } \\
\text { rate of } 1.5 \mathrm{~Hz} ; 4 \\
\text { mm margin }\end{array}$ & & & \\
\hline $\begin{array}{l}\text { Tran } 2012 \\
22511036\end{array}$ & No treatment & & & & & & & & \\
\hline $\begin{array}{l}\text { van der } \\
\text { Geer } 2012 \\
22385074 \\
\end{array}$ & $\begin{array}{l}\text { Imiquimod + } \\
\text { Mohs }\end{array}$ & Mohs & & & & & \multicolumn{3}{|c|}{$\begin{array}{l}\text { Imiquimod } 5 \% \text { (Topical) daily/5 } \\
\text { days per week for } 4 \text { weeks }\end{array}$} \\
\hline $\begin{array}{l}\text { van der } \\
\text { Geer } 2012\end{array}$ & $\begin{array}{l}\text { no treatment } \\
+ \text { Mohs }\end{array}$ & Mohs & & & & & & & \\
\hline
\end{tabular}




\begin{tabular}{|c|c|c|c|c|c|c|c|c|c|}
\hline $\begin{array}{l}\text { Author, } \\
\text { year, } \\
\text { PMID } \\
\end{array}$ & Arm & $\begin{array}{l}\text { Surgical } \\
\text { interventions } \\
\text { (margins) }\end{array}$ & $\begin{array}{l}\text { Thermal } \\
\text { interventions } \\
\text { (description) }\end{array}$ & Radiation & $\begin{array}{l}\text { Photodynamic } \\
\text { Therapy dose }\end{array}$ & $\begin{array}{l}\text { Photodynamic } \\
\text { Therapy } \\
\text { description }\end{array}$ & $\begin{array}{l}\text { Medical } \\
\text { interventions } \\
\text { description }\end{array}$ & $\begin{array}{l}\text { Medical } \\
\text { interventions } \\
\text { other }\end{array}$ & $\begin{array}{l}\text { Curettage } \\
\text { number of } \\
\text { passes }\end{array}$ \\
\hline \multicolumn{10}{|l|}{22385074} \\
\hline $\begin{array}{l}\text { Wang } \\
2001 \\
11298545\end{array}$ & ALA-PDT & & & & $\begin{array}{l}635 \mathrm{~nm} \text { at } 80+/- \\
20 \mathrm{~mW} / \mathrm{cm} 2 \text { to } \\
60 \mathrm{~J} / \mathrm{cm} 2\end{array}$ & $\begin{array}{l}1 \text { session, } 20 \% \\
\text { ALA. When the } \\
\text { stratum corneum } \\
\text { was intact, it was } \\
\text { carefully scraped } \\
\text { off with a scalpel. } \\
\text { Lipids were } \\
\text { removed using } \\
96 \% \text { ethanol. } \\
\text { Crusts were } \\
\text { softened with } \\
\text { isotonic saline } \\
\text { and then lifted off. }\end{array}$ & & & \\
\hline $\begin{array}{l}\text { Wang } \\
2001 \\
11298545\end{array}$ & cryosurgery & & $\begin{array}{l}\text { Cryotherapy } \\
\text { without } \\
\text { curettage } \\
\text { (CRY-AC } \\
\text { spray) }\end{array}$ & & & & & & \\
\hline $\begin{array}{l}\text { Wettstein } \\
2013 \\
23566745\end{array}$ & $\begin{array}{l}\text { Mohs + } \\
\text { Ringer's } \\
\text { lactate } \\
\text { (control } \\
\text { group) }\end{array}$ & excision (NR) & & & & & $\begin{array}{l}\text { Ringer } 1 \times 10^{\wedge} 6 \\
\text { IU (Intralesional) } \\
\text { once for N/A }\end{array}$ & $\begin{array}{l}\text { immediately } \\
\text { after surgical } \\
\text { excision }\end{array}$ & \\
\hline $\begin{array}{l}\text { Wettstein } \\
2013 \\
23566745\end{array}$ & $\begin{array}{l}\text { Mohs + } \\
\text { interferon } \\
\text { alpha-2b }\end{array}$ & excision (NR) & & & & & $\begin{array}{l}\text { inf alpha-2b } \\
1 \times 10^{\wedge} 6 \mathrm{IU} \\
\text { (Intralesional) } \\
\text { once for N/A } \\
\end{array}$ & $\begin{array}{l}\text { immediately } \\
\text { after surgical } \\
\text { excision }\end{array}$ & \\
\hline \multicolumn{10}{|l|}{ NRCS } \\
\hline $\begin{array}{l}\text { Ahmed } \\
2000 \\
11069453 \\
\end{array}$ & Curettage & excsision (3 mm) & & & & & & & \\
\hline $\begin{array}{l}\text { Ahmed } \\
2000 \\
11069453\end{array}$ & Cryotherapy & & $\begin{array}{l}\text { Cryotherapy } \\
\text { without } \\
\text { curettage } \\
\text { (Liquid nitrogen } \\
\text { with a } 3 \mathrm{~mm} \\
\text { margin; the } \\
\text { freeze was then } \\
\text { maintained for }\end{array}$ & & & & & & \\
\hline
\end{tabular}




\begin{tabular}{|c|c|c|c|c|c|c|c|c|c|}
\hline $\begin{array}{l}\text { Author, } \\
\text { year, } \\
\text { PMID }\end{array}$ & Arm & $\begin{array}{l}\text { Surgical } \\
\text { interventions } \\
\text { (margins) }\end{array}$ & $\begin{array}{l}\text { Thermal } \\
\text { interventions } \\
\text { (description) }\end{array}$ & Radiation & $\begin{array}{l}\text { Photodynamic } \\
\text { Therapy dose }\end{array}$ & $\begin{array}{l}\text { Photodynamic } \\
\text { Therapy } \\
\text { description }\end{array}$ & $\begin{array}{l}\text { Medical } \\
\text { interventions } \\
\text { description }\end{array}$ & $\begin{array}{l}\text { Medical } \\
\text { interventions } \\
\text { other }\end{array}$ & $\begin{array}{l}\text { Curettage } \\
\text { number of } \\
\text { passes }\end{array}$ \\
\hline & & & $\begin{array}{l}5 \pm 10 \mathrm{~s} \text {. The } \\
\text { lesion was } \\
\text { allowed to thaw } \\
\text { fully and the } \\
\text { freeze was } \\
\text { repeated; ) }\end{array}$ & & & & & & \\
\hline $\begin{array}{l}\text { Ballester- } \\
\text { Sanchez } \\
2016 \\
26985197 \\
\end{array}$ & $\begin{array}{l}\text { brachytherap } \\
\text { y } 36.6 \text { Gy }\end{array}$ & & & $\begin{array}{l}\text { Photons } \\
\text { (gamma or } \\
\text { x) to } 36.6 \\
\text { Gy, } \\
\text { Brachythera } \\
\text { py/Plesioth } \\
\text { erapy, } 6 \\
\text { sessions, } \\
2 x / \text { week for } \\
3 \text { weeks }\end{array}$ & & & & & \\
\hline $\begin{array}{l}\text { Ballester- } \\
\text { Sanchez } \\
2016 \\
26985197 \\
\end{array}$ & $\begin{array}{l}\text { brachytherap } \\
\text { y } 42 \text { Gy }\end{array}$ & & & $\begin{array}{l}\text { Photons } \\
\text { (gamma or } \\
\text { x) to } 42 \text { Gy, } \\
\text { Brachythera } \\
\text { py/Plesioth } \\
\text { erapy, } 6 \\
\text { sessions, } \\
2 x / \text { week for } \\
3 \text { weeks }\end{array}$ & & & & & \\
\hline $\begin{array}{l}\text { Chren } \\
2013 \\
23190903\end{array}$ & $\begin{array}{l}\text { electrodessic } \\
\text { ation and } \\
\text { curettage }\end{array}$ & Mohs & $\begin{array}{l}\text { Diathermy } \\
\text { (electrodessicat } \\
\text { ion and } \\
\text { curettage; } 3 \\
\text { passes) }\end{array}$ & & & & & & \\
\hline $\begin{array}{l}\text { Chren } \\
2013 \\
23190904 \\
\end{array}$ & excision & $\begin{array}{l}\text { excsision } \\
\text { (median } 3.0 \\
\mathrm{~mm} \text { ) }\end{array}$ & & & & & & & \\
\hline $\begin{array}{l}\text { Chren } \\
2013 \\
23190905 \\
\end{array}$ & Mohs & & & & & & & & \\
\hline $\begin{array}{l}\text { Cosgarea } \\
2012 \\
22738399 \\
\end{array}$ & ALA PDT & & & & $\begin{array}{l}\text { red led to } 37 \\
\mathrm{~J} / \mathrm{cm} 2\end{array}$ & $\begin{array}{l}2 \text { sessions } 1 \\
\text { month apart to } 74 \\
\mathrm{~J} / \mathrm{cm} 2,20 \% \text { ALA }\end{array}$ & & & \\
\hline $\begin{array}{l}\text { Cosgarea } \\
2012\end{array}$ & $\begin{array}{l}\text { surgical } \\
\text { excision }\end{array}$ & excsision $(3 \mathrm{~mm})$ & & & & & & & \\
\hline
\end{tabular}




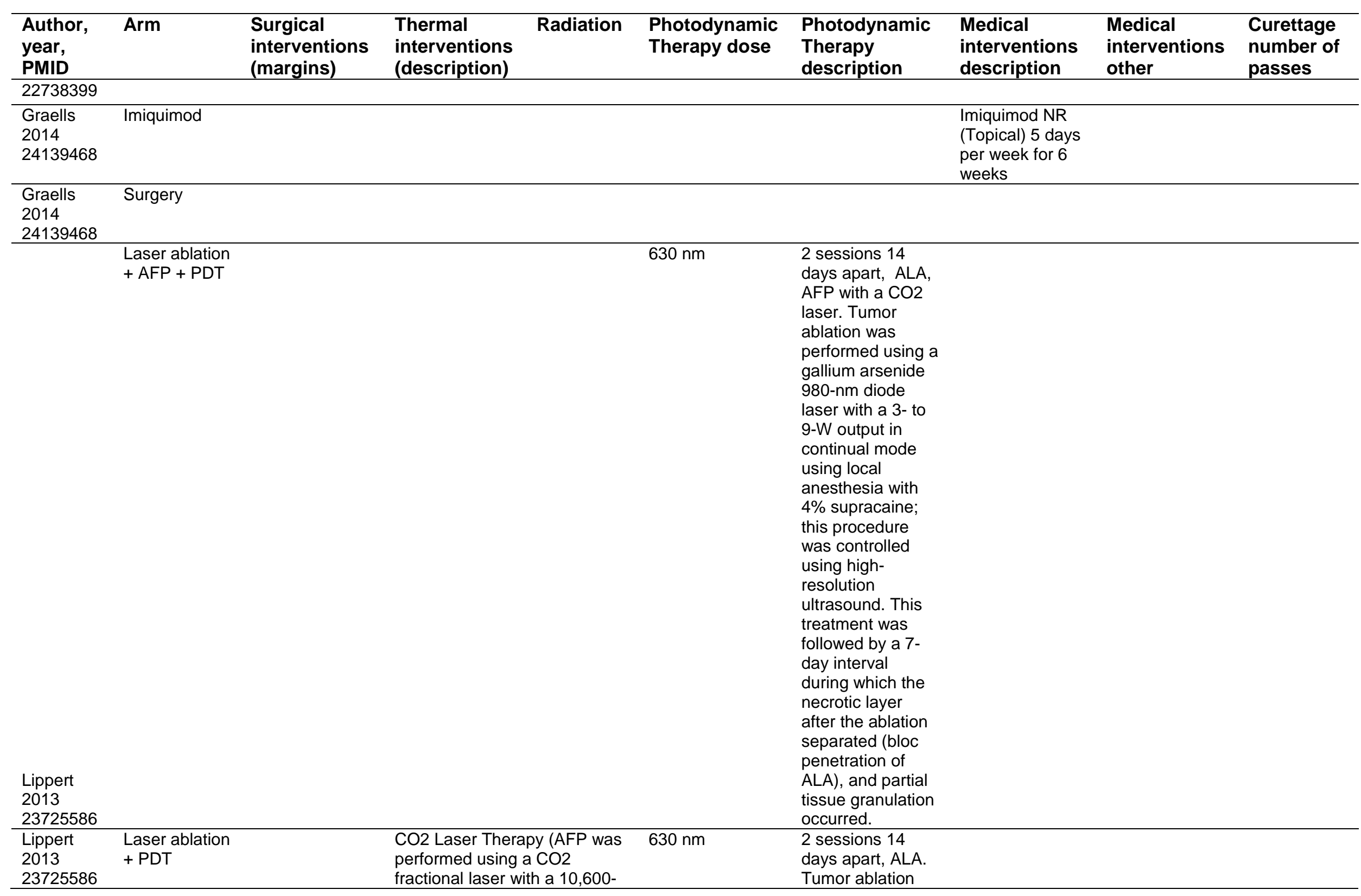




\begin{tabular}{|c|c|c|c|c|c|c|c|c|c|}
\hline $\begin{array}{l}\text { Author, } \\
\text { year, } \\
\text { PMID }\end{array}$ & Arm & $\begin{array}{l}\text { Surgical } \\
\text { interventions } \\
\text { (margins) }\end{array}$ & $\begin{array}{l}\text { Thermal } \\
\text { interventions } \\
\text { (description) }\end{array}$ & Radiation & $\begin{array}{l}\text { Photodynamic } \\
\text { Therapy dose }\end{array}$ & $\begin{array}{l}\text { Photodynamic } \\
\text { Therapy } \\
\text { description }\end{array}$ & $\begin{array}{l}\text { Medical } \\
\text { interventions } \\
\text { description }\end{array}$ & $\begin{array}{l}\text { Medical } \\
\text { interventions } \\
\text { other }\end{array}$ & $\begin{array}{l}\text { Curettage } \\
\text { number of } \\
\text { passes }\end{array}$ \\
\hline & & & $\begin{array}{l}\mathrm{nm} \text { wavelength ( } \\
\text { index, 8; density, } \\
15 \mathrm{~W}) \text { ) }\end{array}$ & $\begin{array}{l}\text { רode, SX; SX } \\
\text { 15\%; power, }\end{array}$ & & $\begin{array}{l}\text { was performed } \\
\text { using a gallium } \\
\text { arsenide 980-nm } \\
\text { diode laser with a } \\
\text { 3- to 9-W output } \\
\text { in continual mode } \\
\text { using local } \\
\text { anesthesia with } \\
4 \% \text { supracaine; } \\
\text { this procedure } \\
\text { was controlled } \\
\text { using high- } \\
\text { resolution } \\
\text { ultrasound. This } \\
\text { treatment was } \\
\text { followed by a 7- } \\
\text { day interval } \\
\text { during which the } \\
\text { necrotic layer } \\
\text { after the ablation } \\
\text { separated (bloc } \\
\text { penetration of } \\
\text { ALA), and partial } \\
\text { tissue granulation } \\
\text { occurred }\end{array}$ & & & \\
\hline $\begin{array}{l}\text { Pampena } \\
2016 \\
26589877\end{array}$ & 3675 cGy & & & $\begin{array}{l}\text { External } \\
\text { Photons } \\
\text { (gamma or } \\
\text { x) to } 3675 \\
\text { cGy, } 7 \\
\text { sessions } \\
\text { weekly }\end{array}$ & & & & & \\
\hline $\begin{array}{l}\text { Pampena } \\
2016 \\
26589878\end{array}$ & 4500 cGy & & & $\begin{array}{l}\text { External } \\
\text { Photons } \\
\text { (gamma or } \\
\text { x) to } 4500 \\
\text { cGy, } 15 \\
\text { sessions } \\
\text { daily }\end{array}$ & & & & & \\
\hline $\begin{array}{l}\text { Shah } \\
2009\end{array}$ & $\begin{array}{l}\text { Pulse dye } \\
\text { laser }\end{array}$ & & $\begin{array}{l}\text { CO2 Laser } \\
\text { Therapy ( } 595\end{array}$ & & & & & & \\
\hline
\end{tabular}




\begin{tabular}{|c|c|c|c|c|c|c|c|c|c|}
\hline $\begin{array}{l}\text { Author, } \\
\text { year, } \\
\text { PMID }\end{array}$ & Arm & $\begin{array}{l}\text { Surgical } \\
\text { interventions } \\
\text { (margins) }\end{array}$ & $\begin{array}{l}\text { Thermal } \\
\text { interventions } \\
\text { (description) }\end{array}$ & Radiation & $\begin{array}{l}\text { Photodynamic } \\
\text { Therapy dose }\end{array}$ & $\begin{array}{l}\text { Photodynamic } \\
\text { Therapy } \\
\text { description }\end{array}$ & $\begin{array}{l}\text { Medical } \\
\text { interventions } \\
\text { description }\end{array}$ & $\begin{array}{l}\text { Medical } \\
\text { interventions } \\
\text { other }\end{array}$ & $\begin{array}{l}\text { Curettage } \\
\text { number of } \\
\text { passes }\end{array}$ \\
\hline 19588534 & & & $\begin{array}{l}\text { nm Pulse dye } \\
\text { lasereach pass } \\
\text { at } 15 \mathrm{~J} / \mathrm{cm} 2 \\
\text { pulse length of } \\
3 \mathrm{~ms} ; 4 \text { passes } \\
\text { at } 2 \text { week } \\
\text { intervals) }\end{array}$ & & & & & & \\
\hline $\begin{array}{l}\text { Shah } \\
2009 \\
19588534\end{array}$ & no treatment & & & & & & & & \\
\hline $\begin{array}{l}\text { Sofen } \\
2015 \\
25913533 \\
\end{array}$ & $\begin{array}{l}\text { vismodegib } \\
12 \text { weeks }\end{array}$ & & & & & & $\begin{array}{l}\text { vismodegib } 150 \\
\text { mg/d (Oral) for } \\
12 \text { weeks }\end{array}$ & & \\
\hline $\begin{array}{l}\text { Sofen } \\
2015 \\
25913533 \\
\end{array}$ & $\begin{array}{l}\text { vismodegib } \\
12 \text { weeks + } \\
24 \text { weeks } \\
\text { observation }\end{array}$ & & & & & & $\begin{array}{l}\text { vismodegib } 150 \\
\mathrm{mg} / \mathrm{d} \text { (Oral) for } \\
12 \text { weeks }\end{array}$ & $\begin{array}{l}+24 \text { weeks } \\
\text { observation } \\
\text { period }\end{array}$ & \\
\hline $\begin{array}{l}\text { Sofen } \\
2015 \\
25913533 \\
\end{array}$ & $\begin{array}{l}\text { vismodegib } \\
16 \text { weeks }\end{array}$ & & 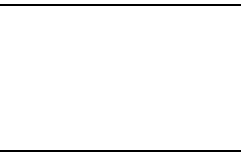 & & & & $\begin{array}{l}\text { vismodegib } 150 \\
\mathrm{mg} / \mathrm{d} \text { (Oral) for } \\
16 \text { weeks }\end{array}$ & $\begin{array}{l}8 \text { weeks }+4 \\
\text { weeks } \\
\text { observation }+8 \\
\text { weeks }\end{array}$ & \\
\hline $\begin{array}{l}\text { Sullivan } \\
2003 \\
14725659\end{array}$ & imiquimod 5\% & & & & & & $\begin{array}{l}\text { imiquimod } 5 \% \\
\text { (Topical) nightly } \\
\text { on weekdays for } \\
10 \text { applications }\end{array}$ & $\begin{array}{l}\text { schedule } \\
\text { immediate } \\
\text { exicision if } \\
\text { irritation } \\
\text { developed }\end{array}$ & \\
\hline $\begin{array}{l}\text { Sullivan } \\
2003 \\
14725659\end{array}$ & vehicle & & & & & & $\begin{array}{l}\text { vehicle cream } \\
\text { NR (Topical) } \\
\text { nightly on } \\
\text { weekdays for } 10 \\
+-3 \text { applications }\end{array}$ & & \\
\hline
\end{tabular}




\section{Appendix F. Risk of Bias}

Table F-1. Risk of bias in RCTs

\begin{tabular}{|c|c|c|c|c|c|c|c|c|c|c|c|c|}
\hline Study & $\begin{array}{l}\text { Adequate } \\
\text { generation } \\
\text { of a } \\
\text { randomized } \\
\text { sequence } \\
\text { reported }\end{array}$ & $\begin{array}{l}\text { Adequate } \\
\text { allocation } \\
\text { concealment } \\
\text { reported }\end{array}$ & $\begin{array}{l}\text { Group } \\
\text { similarity } \\
\text { at } \\
\text { baseline }\end{array}$ & $\begin{array}{l}\text { Adequate } \\
\text { blinding of } \\
\text { PATIENTS } \\
\text { reported }\end{array}$ & $\begin{array}{l}\text { Adequate } \\
\text { blinding of } \\
\text { PROVIDERS } \\
\text { reported }\end{array}$ & $\begin{array}{l}\text { Adequate } \\
\text { blinding of } \\
\text { OUTCOME } \\
\text { ASSESSORS } \\
\text { reported }\end{array}$ & $\begin{array}{l}\text { Intention } \\
\text { to treat } \\
\text { analysis } \\
?\end{array}$ & $\begin{array}{l}\text { Incom- } \\
\text { plete } \\
\text { results } \\
\text { data }\end{array}$ & $\begin{array}{l}\text { Incomplete } \\
\text { results data: } \\
\text { Differential } \\
\text { missingness }\end{array}$ & $\begin{array}{l}\text { Adverse } \\
\text { events (of } \\
\text { interest) } \\
\text { precisely } \\
\text { defined }\end{array}$ & $\begin{array}{l}\text { Selective } \\
\text { Reporting }\end{array}$ & $\begin{array}{l}\text { Overall, } \\
\text { by } \\
\text { outcome }\end{array}$ \\
\hline $\begin{array}{l}\text { Abbade } 2015 \\
\text { (Conference } \\
\text { abstract) } \\
\text { (Brazil) }\end{array}$ & No Data & No Data & Yes & No & No & No Data & Yes & No & No & No Data & No & Moderate \\
\hline $\begin{array}{l}\text { Al-Niaimi } \\
2015 \\
26157307 \\
\text { (UK) }\end{array}$ & Unsure & Yes & Yes & No & No & Yes & No & Yes & Yes & No & $\begin{array}{l}\text { (12 month } \\
\text { results } \\
\text { mentioned } \\
\text { in the } \\
\text { protocol not } \\
\text { given; } \\
\text { recurrence } \\
\text { rates not } \\
\text { given by } \\
\text { arm; only } 1 \\
\text { AE given) }\end{array}$ & $\begin{array}{l}\text { cosmetic } \\
\text { outcomes } \\
: \text { low } \\
\text { recurrenc } \\
\text { e: } \\
\text { moderate } \\
\text { to high }\end{array}$ \\
\hline $\begin{array}{l}\text { Allen } 1979 \\
298425 \text { (UK) }\end{array}$ & $\begin{array}{l}\text { Yes } \\
\text { ("subjects } \\
\text { randomly } \\
\text { assigned in } \\
\text { a coded, } \\
\text { controlled } \\
\text { trial.") }\end{array}$ & $\begin{array}{l}\text { Yes } \\
\text { ("randomly } \\
\text { coded } \\
\text { allocation of } \\
\text { treatment") }\end{array}$ & $\begin{array}{l}\text { No Data } \\
\text { (No Table } \\
1 \text { / patient } \\
\text { characteri } \\
\text { stics } \\
\text { reported.) }\end{array}$ & $\begin{array}{l}\text { Yes } \\
\text { (Subjects } \\
\text { could not } \\
\text { be blinded } \\
\text { to } \\
\text { treatment } \\
\text { allocation } \\
\text { (Cryotherap } \\
\text { y vs. } \\
\text { Radiothera } \\
\text { py)) }\end{array}$ & $\begin{array}{l}\text { No Data (No } \\
\text { mention is } \\
\text { made of } \\
\text { blinding } \\
\text { providers; } \\
\text { Review } \\
\text { Authors do } \\
\text { not discuss } \\
\text { whether this } \\
\text { would impact } \\
\text { the outcome.) }\end{array}$ & $\begin{array}{l}\text { No Data (No } \\
\text { mention is } \\
\text { made of } \\
\text { blinding } \\
\text { outcome } \\
\text { assessors; } \\
\text { Review } \\
\text { Authors do } \\
\text { not discuss } \\
\text { whether this } \\
\text { would impact } \\
\text { the outcome.) }\end{array}$ & $\begin{array}{l}\text { No Data } \\
\text { (No } \\
\text { dropouts } \\
\text { reported. } \\
\text { ) }\end{array}$ & $\begin{array}{l}\text { No } \\
\text { Data } \\
\text { (Only } \\
\text { Recurr } \\
\text { ence } \\
\text { was } \\
\text { reporte } \\
d \text {, but it } \\
\text { was } \\
\text { reporte } \\
d \\
\text { complet } \\
\text { ely for } \\
\text { both } \\
\text { arms of } \\
\text { the } \\
\text { trial.) } \\
\end{array}$ & $\begin{array}{l}\text { No Data (See } \\
\text { above) }\end{array}$ & $\begin{array}{l}\text { No Data } \\
\text { (No } \\
\text { Adverse } \\
\text { Events } \\
\text { were } \\
\text { reported) }\end{array}$ & $\begin{array}{l}\text { Low RoB } \\
\text { (Outcome } \\
\text { of interest, } \\
\text { recurrence, } \\
\text { was } \\
\text { reported by } \\
\text { arm.) }\end{array}$ & High \\
\hline $\begin{array}{l}\text { Alpsoy } 1996 \\
8708151\end{array}$ & Unsure & Unsure & Yes & Unsure & Unsure & Unsure & Yes & No & No & Yes & & High \\
\hline
\end{tabular}




\begin{tabular}{|c|c|c|c|c|c|c|c|c|c|c|c|c|}
\hline Study & $\begin{array}{l}\text { Adequate } \\
\text { generation } \\
\text { of a } \\
\text { randomized } \\
\text { sequence } \\
\text { reported }\end{array}$ & $\begin{array}{l}\text { Adequate } \\
\text { allocation } \\
\text { concealment } \\
\text { reported }\end{array}$ & $\begin{array}{l}\text { Group } \\
\text { similarity } \\
\text { at } \\
\text { baseline }\end{array}$ & $\begin{array}{l}\text { Adequate } \\
\text { blinding of } \\
\text { PATIENTS } \\
\text { reported }\end{array}$ & $\begin{array}{l}\text { Adequate } \\
\text { blinding of } \\
\text { PROVIDERS } \\
\text { reported }\end{array}$ & $\begin{array}{l}\text { Adequate } \\
\text { blinding of } \\
\text { OUTCOME } \\
\text { ASSESSORS } \\
\text { reported }\end{array}$ & $\begin{array}{l}\text { Intention } \\
\text { to treat } \\
\text { analysis } \\
?\end{array}$ & $\begin{array}{l}\text { Incom- } \\
\text { plete } \\
\text { results } \\
\text { data }\end{array}$ & $\begin{array}{l}\text { Incomplete } \\
\text { results data: } \\
\text { Differential } \\
\text { missingness }\end{array}$ & $\begin{array}{l}\text { Adverse } \\
\text { events (of } \\
\text { interest) } \\
\text { precisely } \\
\text { defined }\end{array}$ & $\begin{array}{l}\text { Selective } \\
\text { Reporting }\end{array}$ & $\begin{array}{l}\text { Overall, } \\
\text { by } \\
\text { outcome }\end{array}$ \\
\hline \multicolumn{13}{|l|}{ (Turkey) } \\
\hline $\begin{array}{l}\text { Arits } 2013 \\
23683751 \\
\text { (Netherlands) }\end{array}$ & Yes & Yes & Yes & $\begin{array}{l}\text { No } \\
\text { (patients } \\
\text { were not } \\
\text { blinded) }\end{array}$ & $\begin{array}{l}\text { No } \\
\text { (caregivers } \\
\text { were not } \\
\text { blinded) }\end{array}$ & $\begin{array}{l}\text { Yes (all } \\
\text { outcome } \\
\text { assessors } \\
\text { (except for } \\
\text { AEs, which } \\
\text { were } \\
\text { assessed by } \\
\text { patients) were } \\
\text { blinded) }\end{array}$ & Yes & No & No & Yes & No & Low \\
\hline $\begin{array}{l}\text { Avril } 1997 \\
9218740 \\
\text { (France) }\end{array}$ & $\begin{array}{l}\text { Unsure } \\
\text { (method of } \\
\text { randomizatio } \\
\mathrm{n} \text { not } \\
\text { reported) }\end{array}$ & Yes & Yes & $\begin{array}{l}\text { No (The } \\
\text { lack of } \\
\text { blinding is } \\
\text { concerning } \\
\text { for patient } \\
\text { and } \\
\text { physician } \\
\text { reported } \\
\text { cosmetic } \\
\text { outcomes, } \\
\text { but they } \\
\text { also report } \\
\text { outcomes } \\
\text { from third- } \\
\text { party } \\
\text { blinded } \\
\text { assessors) }\end{array}$ & $\begin{array}{l}\text { No (The lack } \\
\text { of blinding is } \\
\text { concerning } \\
\text { for patient } \\
\text { and physician } \\
\text { reported } \\
\text { cosmetic } \\
\text { outcomes, but } \\
\text { they also } \\
\text { report } \\
\text { outcomes } \\
\text { from third- } \\
\text { party blinded } \\
\text { assessors) }\end{array}$ & $\begin{array}{l}\text { No (The lack } \\
\text { of blinding is } \\
\text { concerning } \\
\text { for patient } \\
\text { and physician } \\
\text { reported } \\
\text { cosmetic } \\
\text { outcomes, but } \\
\text { they also } \\
\text { report } \\
\text { outcomes } \\
\text { from third- } \\
\text { party blinded } \\
\text { assessors) }\end{array}$ & $\begin{array}{l}\text { Unsure } \\
\text { (ITT not } \\
\text { reported, } \\
\text { low } \\
\text { number } \\
\text { of } \\
\text { dropouts) }\end{array}$ & $\begin{array}{l}\text { Yes } \\
(23 \% \\
\text { and } \\
27 \% \\
\text { lost to } \\
\text { followu } \\
\mathrm{p} \text { by } \\
\text { mean } \\
\text { followu } \\
\mathrm{p} \text { time } \\
\text { of } 41 \\
\text { months } \\
\text { ) }\end{array}$ & $\begin{array}{l}\text { No (similar } \\
\text { rates between } \\
\text { arms) }\end{array}$ & $\begin{array}{l}\text { No (they } \\
\text { were } \\
\text { reported, } \\
\text { but not } \\
\text { well } \\
\text { defined) }\end{array}$ & $\begin{array}{l}\text { (Neither } \\
\text { paper } \\
\text { reported } \\
\text { AEs } \\
\text { adequately) }\end{array}$ & High \\
\hline $\begin{array}{l}\text { Basset- } \\
\text { Seguin } 2008 \\
18693158 \text { (13 } \\
\text { centers in } 7 \\
\text { european } \\
\text { countries) }\end{array}$ & Unsure & Yes & Yes & No & No & Unsure & No & No & No & Yes & & $\begin{array}{l}\text { Low to } \\
\text { moderate } \\
\text { for all } \\
\text { outcomes }\end{array}$ \\
\hline $\begin{array}{l}\text { Bath-Hextall } \\
2014 \\
24332516 \\
\text { (UK) }\end{array}$ & Yes & Yes & Yes & No & No & Yes & $\begin{array}{l}\text { Yes } \\
\text { (Modified } \\
\text { ITT: all } \\
\text { randomiz } \\
\text { ed }\end{array}$ & Yes & No & Yes & No & Low \\
\hline
\end{tabular}




\begin{tabular}{|c|c|c|c|c|c|c|c|c|c|c|c|c|}
\hline Study & $\begin{array}{l}\text { Adequate } \\
\text { generation } \\
\text { of a } \\
\text { randomized } \\
\text { sequence } \\
\text { reported }\end{array}$ & $\begin{array}{l}\text { Adequate } \\
\text { allocation } \\
\text { concealment } \\
\text { reported }\end{array}$ & $\begin{array}{l}\text { Group } \\
\text { similarity } \\
\text { at } \\
\text { baseline }\end{array}$ & $\begin{array}{l}\text { Adequate } \\
\text { blinding of } \\
\text { PATIENTS } \\
\text { reported }\end{array}$ & $\begin{array}{l}\text { Adequate } \\
\text { blinding of } \\
\text { PROVIDERS } \\
\text { reported }\end{array}$ & $\begin{array}{l}\text { Adequate } \\
\text { blinding of } \\
\text { OUTCOME } \\
\text { ASSESSORS } \\
\text { reported }\end{array}$ & $\begin{array}{l}\text { Intention } \\
\text { to treat } \\
\text { analysis } \\
?\end{array}$ & $\begin{array}{l}\text { Incom- } \\
\text { plete } \\
\text { results } \\
\text { data }\end{array}$ & $\begin{array}{l}\text { Incomplete } \\
\text { results data: } \\
\text { Differential } \\
\text { missingness }\end{array}$ & $\begin{array}{l}\text { Adverse } \\
\text { events (of } \\
\text { interest) } \\
\text { precisely } \\
\text { defined }\end{array}$ & $\begin{array}{l}\text { Selective } \\
\text { Reporting }\end{array}$ & $\begin{array}{l}\text { Overall, } \\
\text { by } \\
\text { outcome }\end{array}$ \\
\hline & & & & & & & $\begin{array}{l}\text { patients } \\
\text { who } \\
\text { received } \\
\text { at least } 1 \\
\text { applicatio } \\
\mathrm{n} \text { of } \\
\text { imiquimo } \\
\mathrm{d} \text { or } \\
\text { surgery } \\
\text { and for } \\
\text { whom } \\
\text { the } \\
\text { outcome } \\
\text { was } \\
\text { available) }\end{array}$ & & & & & \\
\hline $\begin{array}{l}\text { Berroeta } \\
2007 \\
17573890 \\
\text { (United } \\
\text { Kingdom) }\end{array}$ & Yes & Yes & No Data & No & No & Yes & Yes & No & No & Yes & $\begin{array}{l}\text { Yes (Said } \\
\text { they } \\
\text { measured } \\
\text { at multiple } \\
\text { timepoints } \\
\text { but only } \\
\text { reported } 1 \\
\text { year) }\end{array}$ & Moderate \\
\hline $\begin{array}{l}\text { Beutner } 1999 \\
10570388 \\
\text { (USA) }\end{array}$ & No Data & No Data & $\begin{array}{l}\text { No (Group } \\
\text { sizes are } \\
\text { very } \\
\text { small) }\end{array}$ & Unsure & Yes & No Data & $\begin{array}{l}\text { Yes (no } \\
\text { dropouts } \\
\text { or } \\
\text { crossove } \\
\text { r) }\end{array}$ & No & No & Yes & No & $\begin{array}{l}\text { Moderate } \\
\text { to high } \\
\text { due to } \\
\text { small } \\
\text { sample } \\
\text { size and } \\
\text { baseline } \\
\text { difference } \\
\text { s }\end{array}$ \\
\hline $\begin{array}{l}\text { Brinkhuizen } \\
2016 \\
27067393 \\
\text { (Netherlands) }\end{array}$ & Yes & Yes & $\begin{array}{l}\text { No } \\
\text { (superficia } \\
\text { I not } \\
\text { similar, } \\
\text { nodular } \\
\text { similar } \\
\end{array}$ & No & No & Yes & Yes & No & No & Yes & $\begin{array}{l}\text { None } \\
\text { immediatel } \\
\text { y apparent }\end{array}$ & $\begin{array}{l}\text { Low to } \\
\text { moderate }\end{array}$ \\
\hline
\end{tabular}




\begin{tabular}{|c|c|c|c|c|c|c|c|c|c|c|c|c|}
\hline Study & $\begin{array}{l}\text { Adequate } \\
\text { generation } \\
\text { of a } \\
\text { randomized } \\
\text { sequence } \\
\text { reported }\end{array}$ & $\begin{array}{l}\text { Adequate } \\
\text { allocation } \\
\text { concealment } \\
\text { reported }\end{array}$ & $\begin{array}{l}\text { Group } \\
\text { similarity } \\
\text { at } \\
\text { baseline }\end{array}$ & $\begin{array}{l}\text { Adequate } \\
\text { blinding of } \\
\text { PATIENTS } \\
\text { reported }\end{array}$ & $\begin{array}{l}\text { Adequate } \\
\text { blinding of } \\
\text { PROVIDERS } \\
\text { reported }\end{array}$ & $\begin{array}{l}\text { Adequate } \\
\text { blinding of } \\
\text { OUTCOME } \\
\text { ASSESSORS } \\
\text { reported }\end{array}$ & $\begin{array}{l}\text { Intention } \\
\text { to treat } \\
\text { analysis } \\
?\end{array}$ & $\begin{array}{l}\text { Incom- } \\
\text { plete } \\
\text { results } \\
\text { data }\end{array}$ & $\begin{array}{l}\text { Incomplete } \\
\text { results data: } \\
\text { Differential } \\
\text { missingness }\end{array}$ & $\begin{array}{l}\text { Adverse } \\
\text { events (of } \\
\text { interest) } \\
\text { precisely } \\
\text { defined }\end{array}$ & $\begin{array}{l}\text { Selective } \\
\text { Reporting }\end{array}$ & $\begin{array}{l}\text { Overall, } \\
\text { by } \\
\text { outcome }\end{array}$ \\
\hline & & & enough) & & & & & & & & & \\
\hline $\begin{array}{l}\text { Butler } 2009 \\
19018814 \\
\text { (texas, usa) }\end{array}$ & Yes & Yes & Yes & Yes & Yes & Yes & $\begin{array}{l}\text { No (3 } \\
\text { patients } \\
\text { who } \\
\text { failed to } \\
\text { complete } \\
\text { the study } \\
\text { were } \\
\text { included } \\
\text { as } \\
\text { treatment } \\
\text { failures. } \\
\text { this is not } \\
\text { ITT.) }\end{array}$ & No & $\begin{array}{l}\text { No (3 patients } \\
\text { in imiquimod } \\
\text { group and } 0 \\
\text { patients in } \\
\text { vehicle } \\
\text { groupp) }\end{array}$ & Yes & No & $\begin{array}{l}\text { Low for } \\
\text { all } \\
\text { outcomes }\end{array}$ \\
\hline $\begin{array}{l}\text { Cai } 2015 \\
25899562 \\
\text { (china) }\end{array}$ & Unsure & Yes & Yes & Unsure & Yes & Yes & $\begin{array}{l}\text { Unsure } \\
\text { (study } \\
\text { states: } \\
\text { "patients } \\
\text { randomly } \\
\text { assigned } \\
\text { to two } \\
\text { groups } \\
\text { accordin } \\
\text { g to their } \\
\text { hospital } \\
\text { identificat } \\
\text { ion } \\
\text { number" } \\
\text { did not } \\
\text { mention } \\
\text { a specific } \\
\text { computer } \\
\text { generator } \\
\text { ) }\end{array}$ & No & No & $\begin{array}{l}\text { No (no } \\
\text { table for } \\
\text { adverse } \\
\text { events; } \\
\text { study } \\
\text { loosely } \\
\text { describes } \\
\text { ae in the } \\
\text { body of } \\
\text { the text for } \\
\text { study arm) }\end{array}$ & & $\begin{array}{l}\text { Low for } \\
\text { efficacy; } \\
\text { high for } \\
\text { AEs }\end{array}$ \\
\hline $\begin{array}{l}\text { Carija } 2016 \\
27516420 \\
\text { (Croatia) }\end{array}$ & No & No & Yes & unsure & Yes & Yes & No & No & No & Yes & Yes & $\begin{array}{l}\text { Moderate } \\
\text { for all } \\
\text { outcomes }\end{array}$ \\
\hline
\end{tabular}




\begin{tabular}{|c|c|c|c|c|c|c|c|c|c|c|c|c|}
\hline Study & $\begin{array}{l}\text { Adequate } \\
\text { generation } \\
\text { of a } \\
\text { randomized } \\
\text { sequence } \\
\text { reported }\end{array}$ & $\begin{array}{l}\text { Adequate } \\
\text { allocation } \\
\text { concealment } \\
\text { reported }\end{array}$ & $\begin{array}{l}\text { Group } \\
\text { similarity } \\
\text { at } \\
\text { baseline }\end{array}$ & $\begin{array}{l}\text { Adequate } \\
\text { blinding of } \\
\text { PATIENTS } \\
\text { reported }\end{array}$ & $\begin{array}{l}\text { Adequate } \\
\text { blinding of } \\
\text { PROVIDERS } \\
\text { reported }\end{array}$ & $\begin{array}{l}\text { Adequate } \\
\text { blinding of } \\
\text { OUTCOME } \\
\text { ASSESSORS } \\
\text { reported }\end{array}$ & $\begin{array}{l}\text { Intention } \\
\text { to treat } \\
\text { analysis } \\
?\end{array}$ & $\begin{array}{l}\text { Incom- } \\
\text { plete } \\
\text { results } \\
\text { data }\end{array}$ & $\begin{array}{l}\text { Incomplete } \\
\text { results data: } \\
\text { Differential } \\
\text { missingness }\end{array}$ & $\begin{array}{l}\text { Adverse } \\
\text { events (of } \\
\text { interest) } \\
\text { precisely } \\
\text { defined }\end{array}$ & $\begin{array}{l}\text { Selective } \\
\text { Reporting }\end{array}$ & $\begin{array}{l}\text { Overall, } \\
\text { by } \\
\text { outcome }\end{array}$ \\
\hline $\begin{array}{l}\text { Choi } 2016 \\
26551044 \\
\text { (Korea) }\end{array}$ & $\begin{array}{l}\text { Unsure (did } \\
\text { not } \\
\text { elaborate on } \\
\text { how subjects } \\
\text { were } \\
\text { randomized) }\end{array}$ & Yes & Yes & Yes & Yes & Yes & $\begin{array}{l}\text { No (five } \\
\text { subjects } \\
\text { dropped } \\
\text { out } \\
\text { prematur } \\
\text { ely for } \\
\text { unrelated } \\
\text { reasons } \\
\text { to study } \\
\text { and were } \\
\text { analyzed } \\
\text { as } \\
\text { treatment } \\
\text { failures. } \\
\text { discusse } \\
\text { d with } \\
\text { gaelen } \\
\text { who did } \\
\text { not think } \\
\text { it } \\
\text { effected } \\
\text { outcome } \\
\text { s or data } \\
\text { based on } \\
\text { bounding } \\
\text { analysis.) }\end{array}$ & No & No & Yes & No & $\begin{array}{l}\text { Low for } \\
\text { all } \\
\text { outcomes }\end{array}$ \\
\hline $\begin{array}{l}\text { Choi } 2017 \\
28199463 \\
\text { (Korea) }\end{array}$ & Yes & No & Yes & No & No & Yes & Yes & No & No & Yes & No & $\begin{array}{l}\text { Low for } \\
\text { all } \\
\text { outcomes }\end{array}$ \\
\hline $\begin{array}{l}\text { Cornell } 1990 \\
2229497 \\
\text { (U.S.) }\end{array}$ & Yes & No Data & $\begin{array}{l}\text { Yes } \\
\text { (Location } \\
\text { might be } \\
\text { slightly } \\
\text { different, } \\
\text { disadvant } \\
\text { ages the } \\
\text { treatment } \\
\text { group) }\end{array}$ & Yes & No & Yes & Yes & No & No & Yes & No & $\begin{array}{l}\text { Low for } \\
\text { all } \\
\text { outcomes }\end{array}$ \\
\hline
\end{tabular}




\begin{tabular}{|c|c|c|c|c|c|c|c|c|c|c|c|c|}
\hline Study & $\begin{array}{l}\text { Adequate } \\
\text { generation } \\
\text { of a } \\
\text { randomized } \\
\text { sequence } \\
\text { reported }\end{array}$ & $\begin{array}{l}\text { Adequate } \\
\text { allocation } \\
\text { concealment } \\
\text { reported }\end{array}$ & $\begin{array}{l}\text { Group } \\
\text { similarity } \\
\text { at } \\
\text { baseline }\end{array}$ & $\begin{array}{l}\text { Adequate } \\
\text { blinding of } \\
\text { PATIENTS } \\
\text { reported }\end{array}$ & $\begin{array}{l}\text { Adequate } \\
\text { blinding of } \\
\text { PROVIDERS } \\
\text { reported }\end{array}$ & $\begin{array}{l}\text { Adequate } \\
\text { blinding of } \\
\text { OUTCOME } \\
\text { ASSESSORS } \\
\text { reported }\end{array}$ & $\begin{array}{l}\text { Intention } \\
\text { to treat } \\
\text { analysis } \\
?\end{array}$ & $\begin{array}{l}\text { Incom- } \\
\text { plete } \\
\text { results } \\
\text { data }\end{array}$ & $\begin{array}{l}\text { Incomplete } \\
\text { results data: } \\
\text { Differential } \\
\text { missingness }\end{array}$ & $\begin{array}{l}\text { Adverse } \\
\text { events (of } \\
\text { interest) } \\
\text { precisely } \\
\text { defined }\end{array}$ & $\begin{array}{l}\text { Selective } \\
\text { Reporting }\end{array}$ & $\begin{array}{l}\text { Overall, } \\
\text { by } \\
\text { outcome }\end{array}$ \\
\hline $\begin{array}{l}\text { Edwards } \\
1990 \\
2107219 \\
\text { (U.S.) }\end{array}$ & $\begin{array}{l}\text { Unsure (not } \\
\text { reported; } \\
\text { randomizatio } \\
\mathrm{n} \text { done in } \\
\text { blocks by } \\
\text { lesion type } \\
\text { (superficial } \\
\text { or nodular)) }\end{array}$ & $\begin{array}{l}\text { Unsure (not } \\
\text { reported) }\end{array}$ & $\begin{array}{l}\text { Unsure } \\
\text { (baseline } \\
\text { data not } \\
\text { reported) }\end{array}$ & $\begin{array}{l}\text { Unsure (not } \\
\text { reported) }\end{array}$ & $\begin{array}{l}\text { Unsure (not } \\
\text { reported) }\end{array}$ & $\begin{array}{l}\text { Unsure (not } \\
\text { reported) }\end{array}$ & Yes & No & No & Yes & $\begin{array}{l}\text { (Adverse } \\
\text { events and } \\
\text { cosmetic } \\
\text { outcomes } \\
\text { were not } \\
\text { presented } \\
\text { by arm.) }\end{array}$ & $\begin{array}{l}\text { This } \\
\text { paper } \\
\text { lacks } \\
\text { detail on } \\
\text { study } \\
\text { design, } \\
\text { so it is } \\
\text { unclear } \\
\text { whether it } \\
\text { was } \\
\text { properly } \\
\text { conducte } \\
\text { d } \\
\text { Moderate } \\
\text { to high }\end{array}$ \\
\hline $\begin{array}{l}\text { Edwards } \\
1990 \\
2383027 \\
\text { (U.S.) }\end{array}$ & $\begin{array}{l}\text { Unsure (not } \\
\text { reported; } \\
\text { subjects } \\
\text { randomized } \\
\text { in blocks } \\
\text { based on } \\
\text { lesion type) }\end{array}$ & $\begin{array}{l}\text { Unsure (not } \\
\text { reported) }\end{array}$ & $\begin{array}{l}\text { Unsure } \\
\text { (no } \\
\text { baseline } \\
\text { details } \\
\text { given) }\end{array}$ & Yes & Yes & Yes & $\begin{array}{l}\text { Yes (no } \\
\text { drop } \\
\text { outs, no } \\
\text { crossove } \\
\text { rs) }\end{array}$ & No & No & $\begin{array}{l}\text { No } \\
\text { (Adverse } \\
\text { events } \\
\text { were not } \\
\text { defined } \\
\text { and were } \\
\text { not given } \\
\text { by arm) }\end{array}$ & $\begin{array}{l}\text { (There } \\
\text { appears to } \\
\text { be some } \\
\text { selective } \\
\text { reporting: } \\
\text { cosmetic } \\
\text { outcomes } \\
\text { were only } \\
\text { reported in } \\
\text { a subset of } \\
\text { patients } \\
\text { and not by } \\
\text { arm, } \\
\text { adverse } \\
\text { events } \\
\text { were not } \\
\text { reported by } \\
\text { arm. <- } \\
\text { seems to } \\
\text { be true in } \\
\text { all studies) }\end{array}$ & $\begin{array}{l}\text { This is an } \\
\text { older } \\
\text { study and } \\
\text { a very } \\
\text { short } \\
\text { report, so } \\
\text { things } \\
\text { may have } \\
\text { been } \\
\text { done } \\
\text { right but } \\
\text { not } \\
\text { adequatel } \\
\text { y } \\
\text { reported } \\
\text { Moderate } \\
\text { to high }\end{array}$ \\
\hline $\begin{array}{l}\text { Eigentler } \\
2007\end{array}$ & No Data & No Data & Unsure & No & No Data & No Data & No & No & No & $\begin{array}{l}\text { Unsure } \\
\text { (partial }\end{array}$ & & $\begin{array}{l}\text { Moderate } \\
\text { to low }\end{array}$ \\
\hline
\end{tabular}




\begin{tabular}{|c|c|c|c|c|c|c|c|c|c|c|c|c|}
\hline Study & $\begin{array}{l}\text { Adequate } \\
\text { generation } \\
\text { of a } \\
\text { randomized } \\
\text { sequence } \\
\text { reported }\end{array}$ & $\begin{array}{l}\text { Adequate } \\
\text { allocation } \\
\text { concealment } \\
\text { reported }\end{array}$ & $\begin{array}{l}\text { Group } \\
\text { similarity } \\
\text { at } \\
\text { baseline }\end{array}$ & $\begin{array}{l}\text { Adequate } \\
\text { blinding of } \\
\text { PATIENTS } \\
\text { reported }\end{array}$ & $\begin{array}{l}\text { Adequate } \\
\text { blinding of } \\
\text { PROVIDERS } \\
\text { reported }\end{array}$ & $\begin{array}{l}\text { Adequate } \\
\text { blinding of } \\
\text { OUTCOME } \\
\text { ASSESSORS } \\
\text { reported }\end{array}$ & $\begin{array}{l}\text { Intention } \\
\text { to treat } \\
\text { analysis } \\
?\end{array}$ & $\begin{array}{l}\text { Incom- } \\
\text { plete } \\
\text { results } \\
\text { data }\end{array}$ & $\begin{array}{l}\text { Incomplete } \\
\text { results data: } \\
\text { Differential } \\
\text { missingness }\end{array}$ & $\begin{array}{l}\text { Adverse } \\
\text { events (of } \\
\text { interest) } \\
\text { precisely } \\
\text { defined }\end{array}$ & $\begin{array}{l}\text { Selective } \\
\text { Reporting }\end{array}$ & $\begin{array}{l}\text { Overall, } \\
\text { by } \\
\text { outcome }\end{array}$ \\
\hline $\begin{array}{l}17610993 \\
\text { (Germany) }\end{array}$ & & & & & & & & & & $\begin{array}{l}\text { reporting, } \\
\text { but they } \\
\text { say } \\
\text { there's no } \\
\text { difference } \\
\text { between } \\
\text { arms) } \\
\end{array}$ & & \\
\hline $\begin{array}{l}\text { Eimpunth } \\
2014 \\
\text { (Conference } \\
\text { abstract) } \\
\text { (unclear) }\end{array}$ & No Data & No Data & No Data & No & No Data & No Data & Yes & No & No & No & (probably) & $\begin{array}{l}\text { It is very } \\
\text { difficult to } \\
\text { assess } \\
\text { quality } \\
\text { based on } \\
\text { the } \\
\text { abstract } \\
\text { alone }\end{array}$ \\
\hline $\begin{array}{l}\text { Foley } 2009 \\
20064185 \\
\text { (U.S. and } \\
\text { australia) }\end{array}$ & Yes & Yes & $\begin{array}{l}\text { Unsure } \\
\text { (They did } \\
\text { note a } \\
\text { significant } \\
\text { difference } \\
\text { btw } \\
\text { groups in } \\
\text { the } \\
\text { distributio } \\
n \text { of } \\
\text { Fitzpatrick } \\
\text { skin } \\
\text { phototype } \\
\text { (p<0.05), } \\
\text { largely } \\
\text { caused by } \\
\text { greater } \\
\text { proportion } \\
\text { of patients } \\
\text { with skin } \\
\text { type } 1 \text { in } \\
\text { the MAL } \\
\text { group) }\end{array}$ & Yes & Yes & Yes & $\begin{array}{l}\text { No (3 } \\
\text { dropouts } \\
\text { (2 in MAL } \\
\text { and } 1 \text { in } \\
\text { placebo) } \\
\text { inconsist } \\
\text { ent and } \\
\text { unclearly } \\
\text { presente } \\
\text { d.) }\end{array}$ & No & No & Yes & No & $\begin{array}{l}\text { Low for } \\
\text { all } \\
\text { outcomes }\end{array}$ \\
\hline
\end{tabular}




\begin{tabular}{|c|c|c|c|c|c|c|c|c|c|c|c|c|}
\hline Study & $\begin{array}{l}\text { Adequate } \\
\text { generation } \\
\text { of a } \\
\text { randomized } \\
\text { sequence } \\
\text { reported }\end{array}$ & $\begin{array}{l}\text { Adequate } \\
\text { allocation } \\
\text { concealment } \\
\text { reported }\end{array}$ & $\begin{array}{l}\text { Group } \\
\text { similarity } \\
\text { at } \\
\text { baseline }\end{array}$ & $\begin{array}{l}\text { Adequate } \\
\text { blinding of } \\
\text { PATIENTS } \\
\text { reported }\end{array}$ & $\begin{array}{l}\text { Adequate } \\
\text { blinding of } \\
\text { PROVIDERS } \\
\text { reported }\end{array}$ & $\begin{array}{l}\text { Adequate } \\
\text { blinding of } \\
\text { OUTCOME } \\
\text { ASSESSORS } \\
\text { reported }\end{array}$ & $\begin{array}{l}\text { Intention } \\
\text { to treat } \\
\text { analysis } \\
?\end{array}$ & $\begin{array}{l}\text { Incom- } \\
\text { plete } \\
\text { results } \\
\text { data }\end{array}$ & $\begin{array}{l}\text { Incomplete } \\
\text { results data: } \\
\text { Differential } \\
\text { missingness }\end{array}$ & $\begin{array}{l}\text { Adverse } \\
\text { events (of } \\
\text { interest) } \\
\text { precisely } \\
\text { defined }\end{array}$ & $\begin{array}{l}\text { Selective } \\
\text { Reporting }\end{array}$ & $\begin{array}{l}\text { Overall, } \\
\text { by } \\
\text { outcome }\end{array}$ \\
\hline $\begin{array}{l}\text { Garcia-Martin } \\
2011 \\
21242584 \\
\text { (Spain) }\end{array}$ & Unclear RoB & Unclear RoB & Low RoB & High RoB & $\begin{array}{l}\text { Moderate } \\
\text { RoB }\end{array}$ & Unclear RoB & Low RoB & $\begin{array}{l}\text { Low } \\
\text { RoB }\end{array}$ & & Low RoB & $\begin{array}{l}\text { (opthomolo } \\
\text { gist rated } \\
\text { cosmetic } \\
\text { outcome } \\
\text { prespecifie } \\
\text { d in the } \\
\text { methods } \\
\text { but not } \\
\text { reported in } \\
\text { the results) }\end{array}$ & $\begin{array}{l}\text { Low to } \\
\text { moderate } \\
\text { due to } \\
\text { lack of } \\
\text { blinding }\end{array}$ \\
\hline $\begin{array}{l}\text { Geisse } 2002 \\
12196749 \\
\text { (U.S.) }\end{array}$ & Yes & Yes & Yes & Yes & Yes & Yes & Yes & No & No & $\begin{array}{l}\text { Yes } \\
\text { (Some } \\
\text { AEs were } \\
\text { not } \\
\text { reported } \\
\text { for vehicle } \\
\text { groups) }\end{array}$ & $\begin{array}{l}\text { (not } \\
\text { immediatel } \\
\text { y apparent) }\end{array}$ & $\begin{array}{l}\text { Low for } \\
\text { all } \\
\text { outcomes }\end{array}$ \\
\hline $\begin{array}{l}\text { Geisse } 2004 \\
15097956 \\
\text { (U.S.) }\end{array}$ & Yes & Yes & $\begin{array}{l}\text { No (ages } \\
\text { and } \\
\text { locations } \\
\text { of tumors } \\
\text { differ) }\end{array}$ & Yes & Yes & Yes & Yes & No & No & $\begin{array}{l}\text { No (AE } \\
\text { reporting } \\
\text { was there, } \\
\text { but } \\
\text { inconsiste } \\
\text { nt } \\
\text { (sometime } \\
\text { s by arm, } \\
\text { sometime } \\
\text { s with } \\
\text { numbers, } \\
\text { etc)) }\end{array}$ & $\begin{array}{l}\text { (I don't see } \\
\text { any sign of } \\
\text { overt } \\
\text { selective } \\
\text { reporting) }\end{array}$ & $\begin{array}{l}\text { Low; } \\
\text { moderate } \\
\text { for AEs }\end{array}$ \\
\hline $\begin{array}{l}\text { Haak } 2015 \\
24903544 \\
\text { (Denmark) }\end{array}$ & Yes & Yes & Yes & No & No & $\begin{array}{l}\text { Yes (except } \\
\text { patient } \\
\text { cosmetic } \\
\text { outcomes) }\end{array}$ & Yes & No & No & Yes & $\begin{array}{l}\text { (none } \\
\text { immediatel } \\
\text { y obvious) }\end{array}$ & $\begin{array}{l}\text { Low for } \\
\text { all } \\
\text { outcomes }\end{array}$ \\
\hline $\begin{array}{l}\text { Hall } 1986 \\
3514075 \text { (UK) }\end{array}$ & $\begin{array}{l}\text { No (Not } \\
\text { mentioned } \\
\text { how } \\
\text { randomized) }\end{array}$ & $\begin{array}{l}\text { No (Not } \\
\text { mentioned) }\end{array}$ & $\begin{array}{l}\text { No } \\
\text { (Differenc } \\
\text { e in size } \\
\text { and }\end{array}$ & $\begin{array}{l}\text { No (Not } \\
\text { possible to } \\
\text { blind) }\end{array}$ & $\begin{array}{l}\text { No (Not } \\
\text { possible to } \\
\text { blind) }\end{array}$ & $\begin{array}{l}\text { No (Not } \\
\text { mentioned) }\end{array}$ & $\begin{array}{l}\text { No (Only } \\
\text { analyzed } \\
\text { patients } \\
\text { with }\end{array}$ & $\begin{array}{l}\text { Unsure } \\
\text { (Only } \\
\text { gives } \\
\text { dropout }\end{array}$ & $\begin{array}{l}\text { No Data (Only } \\
\text { gives dropouts } \\
\text { for whole } \\
\text { study not per }\end{array}$ & No & No & $\begin{array}{l}\text { Unsure } \\
\text { Differenti } \\
\text { al } \\
\text { missingn }\end{array}$ \\
\hline
\end{tabular}




\begin{tabular}{|c|c|c|c|c|c|c|c|c|c|c|c|c|}
\hline Study & $\begin{array}{l}\text { Adequate } \\
\text { generation } \\
\text { of a } \\
\text { randomized } \\
\text { sequence } \\
\text { reported }\end{array}$ & $\begin{array}{l}\text { Adequate } \\
\text { allocation } \\
\text { concealment } \\
\text { reported }\end{array}$ & $\begin{array}{l}\text { Group } \\
\text { similarity } \\
\text { at } \\
\text { baseline }\end{array}$ & $\begin{array}{l}\text { Adequate } \\
\text { blinding of } \\
\text { PATIENTS } \\
\text { reported }\end{array}$ & $\begin{array}{l}\text { Adequate } \\
\text { blinding of } \\
\text { PROVIDERS } \\
\text { reported }\end{array}$ & $\begin{array}{l}\text { Adequate } \\
\text { blinding of } \\
\text { OUTCOME } \\
\text { ASSESSORS } \\
\text { reported }\end{array}$ & $\begin{array}{l}\text { Intention } \\
\text { to treat } \\
\text { analysis } \\
?\end{array}$ & $\begin{array}{l}\text { Incom- } \\
\text { plete } \\
\text { results } \\
\text { data }\end{array}$ & $\begin{array}{l}\text { Incomplete } \\
\text { results data: } \\
\text { Differential } \\
\text { missingness }\end{array}$ & $\begin{array}{l}\text { Adverse } \\
\text { events (of } \\
\text { interest) } \\
\text { precisely } \\
\text { defined }\end{array}$ & $\begin{array}{l}\text { Selective } \\
\text { Reporting }\end{array}$ & \begin{tabular}{|l|} 
Overall, \\
by \\
outcome
\end{tabular} \\
\hline & & & location) & & & & $\begin{array}{l}\text { follow-up } \\
\text { data) }\end{array}$ & $\begin{array}{l}\text { s for } \\
\text { whole } \\
\text { study } \\
\text { not per } \\
\text { group) }\end{array}$ & group) & & & $\begin{array}{l}\text { ess not } \\
\text { reported }\end{array}$ \\
\hline $\begin{array}{l}\text { Ko } 2014 \\
24102369 \\
\text { (Korea) }\end{array}$ & Unsure & No & Yes & No & Unsure & Yes & $\begin{array}{l}\text { Unsure } \\
\text { (ITT } \\
\text { populatio } \\
\text { n was 19. } \\
\text { they had } \\
\text { one } \\
\text { dropout } \\
\text { (unclear } \\
\text { how } \\
\text { many } \\
\text { lesions) } \\
\text { who } \\
\text { violated } \\
\text { protocol } \\
\text { and } \\
\text { counted } \\
\text { as } \\
\text { treatment } \\
\text { failure. } \\
\text { bc the } \\
\text { exact } \\
\text { number } \\
\text { of lesions } \\
\text { randomiz } \\
\text { ed for the } \\
19 \text { pts } \\
\text { was not } \\
\text { abailable } \\
\text { for ITT } \\
\text { eval, pp } \\
\text { was used } \\
\text { for data } \\
\text { extractio } \\
\text { n.) }\end{array}$ & No & No & Yes & $\begin{array}{l}\text { (not } \\
\text { immediatel } \\
\text { y apparent) }\end{array}$ & Low \\
\hline
\end{tabular}




\begin{tabular}{|c|c|c|c|c|c|c|c|c|c|c|c|c|}
\hline Study & $\begin{array}{l}\text { Adequate } \\
\text { generation } \\
\text { of a } \\
\text { randomized } \\
\text { sequence } \\
\text { reported }\end{array}$ & $\begin{array}{l}\text { Adequate } \\
\text { allocation } \\
\text { concealment } \\
\text { reported }\end{array}$ & $\begin{array}{l}\text { Group } \\
\text { similarity } \\
\text { at } \\
\text { baseline }\end{array}$ & $\begin{array}{l}\text { Adequate } \\
\text { blinding of } \\
\text { PATIENTS } \\
\text { reported }\end{array}$ & $\begin{array}{l}\text { Adequate } \\
\text { blinding of } \\
\text { PROVIDERS } \\
\text { reported }\end{array}$ & $\begin{array}{l}\text { Adequate } \\
\text { blinding of } \\
\text { OUTCOME } \\
\text { ASSESSORS } \\
\text { reported }\end{array}$ & $\begin{array}{l}\text { Intention } \\
\text { to treat } \\
\text { analysis } \\
?\end{array}$ & $\begin{array}{l}\text { Incom- } \\
\text { plete } \\
\text { results } \\
\text { data }\end{array}$ & $\begin{array}{l}\text { Incomplete } \\
\text { results data: } \\
\text { Differential } \\
\text { missingness }\end{array}$ & $\begin{array}{l}\text { Adverse } \\
\text { events (of } \\
\text { interest) } \\
\text { precisely } \\
\text { defined }\end{array}$ & $\begin{array}{l}\text { Selective } \\
\text { Reporting }\end{array}$ & $\begin{array}{l}\text { Overall, } \\
\text { by } \\
\text { outcome }\end{array}$ \\
\hline $\begin{array}{l}\text { Kuijpers } 2006 \\
16865869 \\
\text { (Netherlands) }\end{array}$ & Yes & Yes & $\begin{array}{l}\text { Maybe (4 } \\
\text { superficial } \\
\text { BCC in } \\
\text { surgery } \\
\text { arm. All } \\
\text { others } \\
\text { nodular.) }\end{array}$ & $\begin{array}{l}\text { No (Not } \\
\text { possible) }\end{array}$ & $\begin{array}{l}\text { No (Not } \\
\text { possible) }\end{array}$ & $\begin{array}{l}\text { Unsure (3rd } \\
\text { blinded party } \\
\text { did } \\
\text { assessments } \\
\text { "where } \\
\text { possible") }\end{array}$ & $\begin{array}{l}\text { No (Not } \\
\text { true } \\
\text { intention } \\
\text { to treat, } \\
\text { complete } \\
\text { r } \\
\text { analysis) }\end{array}$ & $\begin{array}{l}\text { Yes } \\
\text { (13/51 } \\
\text { tumors } \\
\text { in the } \\
\text { cryo } \\
\text { group } \\
\text { lost to } \\
\text { follow- } \\
\text { up } \\
\text { 2/49 in } \\
\text { surgical } \\
\text { group) }\end{array}$ & $\begin{array}{l}\text { Yes (Cryo } \\
\text { group had } 13 \\
\text { missing at } 5 \\
\text { years vs. } 2 \text { in } \\
\text { excision } \\
\text { group) }\end{array}$ & No & $\begin{array}{l}\text { (Missing } \\
\text { systematic } \\
\text { reporting of } \\
\text { AEs) }\end{array}$ & $\begin{array}{l}\text { Moderate } \\
\text { to high } \\
\text { due to } \\
\text { missingn } \\
\text { ess }\end{array}$ \\
\hline $\begin{array}{l}\text { Kuijpers } 2007 \\
17451581 \\
\text { (Netherlands) }\end{array}$ & $\begin{array}{l}\text { No Data } \\
\text { ("randomly } \\
\text { assigned" is } \\
\text { only } \\
\text { mention) }\end{array}$ & Unsure & $\begin{array}{l}\text { Yes (seem } \\
\text { similar } \\
\text { enough) }\end{array}$ & Unsure & Unsure & $\begin{array}{l}\text { Yes } \\
\text { (pathologist } \\
\text { was blinded) }\end{array}$ & $\begin{array}{l}\text { Yes (no } \\
\text { dropouts) }\end{array}$ & No & No & No & $\begin{array}{l}\text { No (no } \\
\text { reporting of } \\
\text { adverse } \\
\text { events } \\
\text { other than } \\
\text { pain) }\end{array}$ & $\begin{array}{l}\text { Low for } \\
\text { effectiven } \\
\text { ess } \\
\text { outcomes } \\
\text { and } \\
\text { moderate } \\
\text { for AEs }\end{array}$ \\
\hline $\begin{array}{l}\text { Marks } 2001 \\
11312429 \\
\text { (Australia and } \\
\text { New Zealand) }\end{array}$ & $\begin{array}{l}\text { No (Not } \\
\text { reported) }\end{array}$ & $\begin{array}{l}\text { No (Not } \\
\text { reported) }\end{array}$ & $\begin{array}{l}\text { Unsure } \\
\text { (Minimal } \\
\text { data given } \\
\text { in table 1) }\end{array}$ & $\begin{array}{l}\text { No (Open- } \\
\text { label) }\end{array}$ & $\begin{array}{l}\text { Unsure } \\
\text { (Open-label) }\end{array}$ & $\begin{array}{l}\text { Unsure } \\
\text { (Open-label) }\end{array}$ & $\begin{array}{l}\text { No (Not } \\
\text { true ITT } \\
\text { but } \\
\text { number } \\
\text { of } \\
\text { dropouts } \\
\text { is low) }\end{array}$ & No & No & Yes & $\begin{array}{l}\text { (Unclear - } \\
\text { no protocol } \\
\text { available } \\
\text { but all } \\
\text { outcomes } \\
\text { of interest } \\
\text { available) }\end{array}$ & $\begin{array}{l}\text { Moderate } \\
\text { to high }\end{array}$ \\
\hline $\begin{array}{l}\text { Migden } 2015 \\
25981810 \\
\text { (worldwide) }\end{array}$ & Yes & Yes & Yes & Yes & Yes & Yes & $\begin{array}{l}\text { Yes (both } \\
\text { ITT and } \\
\text { as } \\
\text { treated } \\
\text { results } \\
\text { reported) }\end{array}$ & $\begin{array}{l}\text { No } \\
\text { (Very } \\
\text { high } \\
\text { dropout } \\
\text { rate; } \\
\text { most } \\
\text { due to } \\
\text { advers } \\
\text { e } \\
\text { events. } \\
\text { Boundi } \\
\text { ng }\end{array}$ & $\begin{array}{l}\text { No (dropout } \\
\text { rates and } \\
\text { reasons were } \\
\text { similar across } \\
\text { arms) }\end{array}$ & Yes & $\begin{array}{l}\text { (Possible; } \\
\text { only a small } \\
\text { number (7) } \\
\text { of QOL } \\
\text { results } \\
\text { reported; } \\
\text { NCT record } \\
\text { does not } \\
\text { call for any } \\
\text { QOL } \\
\text { results.) }\end{array}$ & $\begin{array}{l}\text { Moderate } \\
\text { due to } \\
\text { dropouts }\end{array}$ \\
\hline
\end{tabular}




\begin{tabular}{|c|c|c|c|c|c|c|c|c|c|c|c|c|}
\hline Study & $\begin{array}{l}\text { Adequate } \\
\text { generation } \\
\text { of a } \\
\text { randomized } \\
\text { sequence } \\
\text { reported }\end{array}$ & $\begin{array}{l}\text { Adequate } \\
\text { allocation } \\
\text { concealment } \\
\text { reported }\end{array}$ & $\begin{array}{l}\text { Group } \\
\text { similarity } \\
\text { at } \\
\text { baseline }\end{array}$ & $\begin{array}{l}\text { Adequate } \\
\text { blinding of } \\
\text { PATIENTS } \\
\text { reported }\end{array}$ & $\begin{array}{l}\text { Adequate } \\
\text { blinding of } \\
\text { PROVIDERS } \\
\text { reported }\end{array}$ & $\begin{array}{l}\text { Adequate } \\
\text { blinding of } \\
\text { OUTCOME } \\
\text { ASSESSORS } \\
\text { reported }\end{array}$ & $\begin{array}{l}\text { Intention } \\
\text { to treat } \\
\text { analysis } \\
?\end{array}$ & $\begin{array}{l}\text { Incom- } \\
\text { plete } \\
\text { results } \\
\text { data }\end{array}$ & $\begin{array}{l}\text { Incomplete } \\
\text { results data: } \\
\text { Differential } \\
\text { missingness }\end{array}$ & $\begin{array}{l}\text { Adverse } \\
\text { events (of } \\
\text { interest) } \\
\text { precisely } \\
\text { defined }\end{array}$ & $\begin{array}{l}\text { Selective } \\
\text { Reporting }\end{array}$ & $\begin{array}{l}\text { Overall, } \\
\text { by } \\
\text { outcome }\end{array}$ \\
\hline & & & & & & & & $\begin{array}{l}\text { analysi } \\
\text { s } \\
\text { suggest } \\
\text { there is } \\
\text { high } \\
\text { risk of } \\
\text { bias } \\
\text { due to } \\
\text { dropout } \\
\text { s) }\end{array}$ & & & & \\
\hline $\begin{array}{l}\text { Miller } 1997 \\
8996264 \\
\text { (USA) }\end{array}$ & $\begin{array}{l}\text { Unclear RoB } \\
\text { (randomizati } \\
\text { on } \\
\text { procedure } \\
\text { undefined) }\end{array}$ & $\begin{array}{l}\text { Unclear RoB } \\
\text { (randomizatio } \\
\text { n procedure } \\
\text { undefined) }\end{array}$ & No Data & $\begin{array}{l}\text { Low RoB } \\
\text { (open label } \\
\text { but } \\
\text { outcomes } \\
\text { aren't likely } \\
\text { influenced) }\end{array}$ & $\begin{array}{l}\text { Low RoB } \\
\text { (open label } \\
\text { but outcomes } \\
\text { aren't likely } \\
\text { influenced) }\end{array}$ & $\begin{array}{l}\text { Low RoB } \\
\text { (open label } \\
\text { but outcomes } \\
\text { aren't likely } \\
\text { influenced) }\end{array}$ & $\begin{array}{l}\text { Unsure } \\
\text { (FLAG } \\
\text { some } \\
\text { drop outs } \\
\text { related to } \\
\text { treatment } \\
\text { ) }\end{array}$ & $\begin{array}{l}\begin{array}{l}\text { Yes } \\
\text { (drops }\end{array} \\
\text { outs } \\
\text { occurre } \\
\text { d either } \\
\text { prior to } \\
\text { complet } \\
\text { ion or } \\
\text { were } \\
\text { unrelat } \\
\text { ed to } \\
\text { treatme } \\
\text { nt) }\end{array}$ & Yes & Low RoB & $\begin{array}{l}\begin{array}{l}\text { No } \\
\text { (adverse }\end{array} \\
\text { events } \\
\text { selectively } \\
\text { reported or } \\
\text { not } \\
\text { stratified, } \\
\text { cosmetic } \\
\text { outcome } \\
\text { not fully } \\
\text { reported, } \\
\text { histologic } \\
\text { clearance } \\
\text { is reported } \\
\text { fully) }\end{array}$ & $\begin{array}{l}\text { Moderate } \\
\text { for } \\
\text { clearance } \\
\text {, high for } \\
\text { other } \\
\text { outcomes }\end{array}$ \\
\hline $\begin{array}{l}\text { Morton } 1996 \\
8977678 \\
\text { (Scotland) }\end{array}$ & $\begin{array}{l}\text { Unsure (not } \\
\text { fully } \\
\text { randomized) }\end{array}$ & Unsure & Yes & No & No & $\begin{array}{l}\text { No (only one } \\
\text { outcome } \\
\text { assessor was } \\
\text { reported to be } \\
\text { blinded and } \\
\text { that outcome } \\
\text { was given at } \\
\text { the fewest } \\
\text { timepoints) }\end{array}$ & $\begin{array}{l}\text { No (per } \\
\text { protocol, } \\
\text { not too } \\
\text { many } \\
\text { dropouts } \\
\text { for } 1 \\
\text { year, } \\
\text { unclear } \\
\text { for } 2 \\
\text { years) }\end{array}$ & $\begin{array}{l}\text { Yes } \\
\text { (possibl } \\
\text { y for } \\
\text { long- } \\
\text { term) }\end{array}$ & $\begin{array}{l}\text { Yes (possibly } \\
\text { for long-term) }\end{array}$ & Yes & $\begin{array}{l}\text { (It feels } \\
\text { like there } \\
\text { may be } \\
\text { some } \\
\text { selective } \\
\text { reporting in } \\
\text { the } \\
\text { aesthetic } \\
\text { outcomes) }\end{array}$ & $\begin{array}{l}\text { Low to } \\
\text { moderate } \\
\text { due to } \\
\text { lack of } \\
\text { blinding } \\
\text { and long } \\
\text { term } \\
\text { dropouts }\end{array}$ \\
\hline $\begin{array}{l}\text { Morton } 2006 \\
16785375 \\
\text { (Europe) }\end{array}$ & $\begin{array}{l}\text { No Data } \\
\text { (Not } \\
\text { reported) }\end{array}$ & $\begin{array}{l}\text { No Data (Not } \\
\text { reported) }\end{array}$ & $\begin{array}{l}\text { Unsure } \\
\text { (Lesions } \\
\text { size was } \\
\end{array}$ & $\begin{array}{l}\text { No } \\
\text { (unblinded) }\end{array}$ & $\begin{array}{l}\text { No } \\
\text { (unblinded) }\end{array}$ & $\begin{array}{l}\text { No } \\
\text { (unblinded) }\end{array}$ & $\begin{array}{l}\text { Yes (no } \\
\text { dropouts) }\end{array}$ & No & No & Yes & $\begin{array}{l}\text { (Does not } \\
\text { appear to } \\
\text { be any) }\end{array}$ & $\begin{array}{l}\text { Older } \\
\text { study } \\
\text { with poor }\end{array}$ \\
\hline
\end{tabular}




\begin{tabular}{|c|c|c|c|c|c|c|c|c|c|c|c|c|}
\hline Study & $\begin{array}{l}\text { Adequate } \\
\text { generation } \\
\text { of a } \\
\text { randomized } \\
\text { sequence } \\
\text { reported }\end{array}$ & $\begin{array}{l}\text { Adequate } \\
\text { allocation } \\
\text { concealment } \\
\text { reported }\end{array}$ & $\begin{array}{l}\text { Group } \\
\text { similarity } \\
\text { at } \\
\text { baseline }\end{array}$ & $\begin{array}{l}\text { Adequate } \\
\text { blinding of } \\
\text { PATIENTS } \\
\text { reported }\end{array}$ & $\begin{array}{l}\text { Adequate } \\
\text { blinding of } \\
\text { PROVIDERS } \\
\text { reported }\end{array}$ & $\begin{array}{l}\text { Adequate } \\
\text { blinding of } \\
\text { OUTCOME } \\
\text { ASSESSORS } \\
\text { reported }\end{array}$ & $\begin{array}{l}\text { Intention } \\
\text { to treat } \\
\text { analysis } \\
?\end{array}$ & $\begin{array}{l}\text { Incom- } \\
\text { plete } \\
\text { results } \\
\text { data }\end{array}$ & $\begin{array}{l}\text { Incomplete } \\
\text { results data: } \\
\text { Differential } \\
\text { missingness }\end{array}$ & $\begin{array}{l}\text { Adverse } \\
\text { events (of } \\
\text { interest) } \\
\text { precisely } \\
\text { defined }\end{array}$ & $\begin{array}{l}\text { Selective } \\
\text { Reporting }\end{array}$ & $\begin{array}{l}\text { Overall, } \\
\text { by } \\
\text { outcome }\end{array}$ \\
\hline & & & $\begin{array}{l}\text { different; } \\
\text { this was } \\
\text { accounted } \\
\text { for in a } \\
\text { regression } \\
\text {.) }\end{array}$ & & & & & & & & & $\begin{array}{l}\text { reporting. } \\
\text { Lack of } \\
\text { blinding } \\
\text { may } \\
\text { affect AE } \\
\text { reporting, } \\
\text { but } \\
\text { unlikely } \\
\text { to affect } \\
\text { clearance } \\
\text { or } \\
\text { recurrenc } \\
\text { e } \\
\text { high due } \\
\text { to poor } \\
\text { reporting }\end{array}$ \\
\hline $\begin{array}{l}\text { Mosterd } 2008 \\
18717680 \\
\text { (Netherlands) }\end{array}$ & Yes & Yes & Yes & Yes & No & No & Yes & Yes & No & Yes & $\begin{array}{l}\text { (Aesthetic } \\
\text { outcomes } \\
\text { only } \\
\text { reported in } \\
\text { combined } \\
\text { recurrent/pr } \\
\text { imary arm. } \\
\text { Subgroup } \\
\text { analysis for } \\
\text { more } \\
\text { severe } \\
\text { cancers } \\
\text { missing } \\
\text { followup } \\
\text { Ns;) }\end{array}$ & $\begin{array}{l}\text { Low to } \\
\text { maybe } \\
\text { moderate } \\
\text { because } \\
\text { of loss to } \\
\text { followup. }\end{array}$ \\
\hline $\begin{array}{l}\text { Mosterd } 2008 \\
19010733 \\
\text { (Netherlands) }\end{array}$ & Yes & Yes & $\begin{array}{l}\text { Unsure } \\
\text { (Very few } \\
\text { baseline } \\
\text { details } \\
\text { were } \\
\text { given. } \\
\text { Those that }\end{array}$ & $\begin{array}{l}\text { No (No } \\
\text { blinding) }\end{array}$ & $\begin{array}{l}\text { No (No } \\
\text { blinding) }\end{array}$ & $\begin{array}{l}\text { No (No } \\
\text { blinding) }\end{array}$ & Yes & $\begin{array}{l}\text { Yes (48 } \\
\text { months } \\
>30 \% \\
\text { missing } \\
\text { ness) }\end{array}$ & $\begin{array}{l}\text { Yes (48 } \\
\text { months } \\
\text { differential) }\end{array}$ & $\begin{array}{l}\text { No (AEs } \\
\text { were not } \\
\text { defined) }\end{array}$ & $\begin{array}{l}\text { (The lack } \\
\text { of } \\
\text { specificity } \\
\text { in } A E \text { and } \\
\text { baseline } \\
\text { data } \\
\text { reporting }\end{array}$ & $\begin{array}{l}\text { Moderate } \\
\text { for early } \\
\text { followup } \\
\text { and high } \\
\text { for later } \\
\text { followup }\end{array}$ \\
\hline
\end{tabular}




\begin{tabular}{|c|c|c|c|c|c|c|c|c|c|c|c|c|}
\hline Study & $\begin{array}{l}\text { Adequate } \\
\text { generation } \\
\text { of a } \\
\text { randomized } \\
\text { sequence } \\
\text { reported }\end{array}$ & $\begin{array}{l}\text { Adequate } \\
\text { allocation } \\
\text { concealment } \\
\text { reported }\end{array}$ & $\begin{array}{l}\text { Group } \\
\text { similarity } \\
\text { at } \\
\text { baseline }\end{array}$ & $\begin{array}{l}\text { Adequate } \\
\text { blinding of } \\
\text { PATIENTS } \\
\text { reported }\end{array}$ & $\begin{array}{l}\text { Adequate } \\
\text { blinding of } \\
\text { PROVIDERS } \\
\text { reported }\end{array}$ & $\begin{array}{l}\text { Adequate } \\
\text { blinding of } \\
\text { OUTCOME } \\
\text { ASSESSORS } \\
\text { reported }\end{array}$ & $\begin{array}{l}\text { Intention } \\
\text { to treat } \\
\text { analysis } \\
?\end{array}$ & $\begin{array}{l}\text { Incom- } \\
\text { plete } \\
\text { results } \\
\text { data }\end{array}$ & $\begin{array}{l}\text { Incomplete } \\
\text { results data: } \\
\text { Differential } \\
\text { missingness }\end{array}$ & $\begin{array}{l}\text { Adverse } \\
\text { events (of } \\
\text { interest) } \\
\text { precisely } \\
\text { defined }\end{array}$ & $\begin{array}{l}\text { Selective } \\
\text { Reporting }\end{array}$ & $\begin{array}{l}\text { Overall, } \\
\text { by } \\
\text { outcome }\end{array}$ \\
\hline & & & $\begin{array}{l}\text { are given } \\
\text { are } \\
\text { similar.) }\end{array}$ & & & & & & & & $\begin{array}{l}\text { may } \\
\text { suggest } \\
\text { selective } \\
\text { reporting) }\end{array}$ & \\
\hline $\begin{array}{l}\text { Orenberg } \\
1992 \\
1430394 \\
(\text { USA) }\end{array}$ & Unclear RoB & Unclear RoB & No & Low RoB & Low RoB & Low RoB & 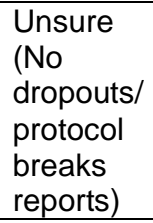 & No & No & High RoB & Yes & $\begin{array}{l}\text { High, } \\
\text { Lots of } \\
\text { uncertaint } \\
\text { y, very } \\
\text { small } \\
\text { study } \\
\end{array}$ \\
\hline $\begin{array}{l}\text { Patel } 2006 \\
16713457 \\
\text { (United } \\
\text { Kingdom) }\end{array}$ & Yes & Yes & $\begin{array}{l}\text { No (legion } \\
\text { size } \\
\text { different } \\
\text { between } \\
\text { groups) }\end{array}$ & Yes & Yes & Yes & No & $\begin{array}{l}\text { Yes } \\
(3 / 15)\end{array}$ & $\begin{array}{l}\text { Yes }(20 \% \text { in } \\
\text { one arm, no } \\
\text { dropout in } \\
\text { other arm) }\end{array}$ & $\begin{array}{l}\text { No (not } \\
\text { well- } \\
\text { defined, } \\
\text { not } \\
\text { reported } \\
\text { by arm) }\end{array}$ & & $\begin{array}{l}\text { High, } \\
\text { blinding } \\
\text { is good } \\
\text { but } \\
\text { groups } \\
\text { are not } \\
\text { similar, } \\
\text { there is } \\
\text { differentia } \\
\text { I } \\
\text { missingn } \\
\text { ess, and } \\
\text { outcomes } \\
\text { are not } \\
\text { reported } \\
\text { by arm }\end{array}$ \\
\hline $\begin{array}{l}\text { Rhodes } 2004 \\
14732655 \\
\text { (Europe) }\end{array}$ & Yes & Yes & $\begin{array}{l}\text { No } \\
\text { (location } \\
\text { of lesions } \\
\text { differed } \\
\text { significantl } \\
\text { y; this } \\
\text { matters } \\
\text { because a } \\
\text { subgroup } \\
\text { analysis } \\
\text { by location } \\
\text { of lesion }\end{array}$ & No & No & $\begin{array}{l}\text { No (could } \\
\text { lead to bias } \\
\text { as lack of } \\
\text { cure was } \\
\text { established } \\
\text { clinically and } \\
\text { both } \\
\text { investigators } \\
\text { and patients } \\
\text { assessed } \\
\text { cosmetic } \\
\text { outcomes) }\end{array}$ & $\begin{array}{l}\text { No (per } \\
\text { protocol } \\
\text { analysis } \\
\text { was } \\
\text { done. } \\
\text { The } \\
\text { authors } \\
\text { state that } \\
\text { an ITT } \\
\text { analysis } \\
\text { was } \\
\text { nearly }\end{array}$ & $\begin{array}{l}\text { Yes } \\
\text { (No for } \\
\text { the } \\
\text { early } \\
\text { followu } \\
\text { p; yes } \\
\text { for } \\
\text { followu } \\
p \\
\text { beyond } \\
1 \text { year) }\end{array}$ & No & Yes & $\begin{array}{l}\text { (hard to } \\
\text { tell) }\end{array}$ & $\begin{array}{l}\text { High, } \\
\text { especially } \\
\text { given that } \\
\text { the } \\
\text { funding } \\
\text { came } \\
\text { from a } \\
\text { PDT } \\
\text { source }\end{array}$ \\
\hline
\end{tabular}




\begin{tabular}{|c|c|c|c|c|c|c|c|c|c|c|c|c|}
\hline Study & $\begin{array}{l}\text { Adequate } \\
\text { generation } \\
\text { of a } \\
\text { randomized } \\
\text { sequence } \\
\text { reported }\end{array}$ & $\begin{array}{l}\text { Adequate } \\
\text { allocation } \\
\text { concealment } \\
\text { reported }\end{array}$ & $\begin{array}{l}\text { Group } \\
\text { similarity } \\
\text { at } \\
\text { baseline }\end{array}$ & $\begin{array}{l}\text { Adequate } \\
\text { blinding of } \\
\text { PATIENTS } \\
\text { reported }\end{array}$ & $\begin{array}{l}\text { Adequate } \\
\text { blinding of } \\
\text { PROVIDERS } \\
\text { reported }\end{array}$ & $\begin{array}{l}\text { Adequate } \\
\text { blinding of } \\
\text { OUTCOME } \\
\text { ASSESSORS } \\
\text { reported }\end{array}$ & $\begin{array}{l}\text { Intention } \\
\text { to treat } \\
\text { analysis } \\
?\end{array}$ & $\begin{array}{l}\text { Incom- } \\
\text { plete } \\
\text { results } \\
\text { data }\end{array}$ & $\begin{array}{l}\text { Incomplete } \\
\text { results data: } \\
\text { Differential } \\
\text { missingness }\end{array}$ & $\begin{array}{l}\text { Adverse } \\
\text { events (of } \\
\text { interest) } \\
\text { precisely } \\
\text { defined }\end{array}$ & $\begin{array}{l}\text { Selective } \\
\text { Reporting }\end{array}$ & $\begin{array}{l}\text { Overall, } \\
\text { by } \\
\text { outcome }\end{array}$ \\
\hline & & & was done) & & & & identical) & & & & & \\
\hline $\begin{array}{l}\text { Salim } 2003 \\
12653747 \\
(\mathrm{UK})\end{array}$ & $\begin{array}{l}\text { No (Not } \\
\text { reported) }\end{array}$ & $\begin{array}{l}\text { No (Not } \\
\text { reported) }\end{array}$ & $\begin{array}{l}\text { No } \\
\text { (Lesion } \\
\text { location } \\
\text { not } \\
\text { similar. } \\
\text { Other } \\
\text { characteri } \\
\text { stics not } \\
\text { provided) }\end{array}$ & $\begin{array}{l}\text { No (Not } \\
\text { reported) }\end{array}$ & $\begin{array}{l}\text { No (Not } \\
\text { reported) }\end{array}$ & $\begin{array}{l}\text { No (Not } \\
\text { reported) }\end{array}$ & Yes & No & $\begin{array}{l}\text { Yes (Dropouts } \\
\text { occurred only } \\
\text { in the 5-FU } \\
\text { group) }\end{array}$ & No & $\begin{array}{l}\text { (Did not } \\
\text { report } A E \\
\text { assessmen } \\
\text { ts from } \\
\text { each visit) }\end{array}$ & $\begin{array}{l}\text { High risk } \\
\text { of bias } \\
\text { due to } \\
\text { between- } \\
\text { group } \\
\text { difference } \\
\text { slocation } \\
\text { and } \\
\text { selective } \\
\text { reporting } \\
\text { of AEs }\end{array}$ \\
\hline $\begin{array}{l}\text { Salmanpoor } \\
2012 \text { (Iran) }\end{array}$ & $\begin{array}{l}\text { No (Not } \\
\text { reported) }\end{array}$ & $\begin{array}{l}\text { No (Not } \\
\text { reported) }\end{array}$ & $\begin{array}{l}\text { No Data } \\
\text { (No Table } \\
1 \text { or other } \\
\text { compariso } \\
\text { n) }\end{array}$ & $\begin{array}{l}\text { Unsure } \\
\text { (Not } \\
\text { reported) }\end{array}$ & $\begin{array}{l}\text { Unsure (Not } \\
\text { reported) }\end{array}$ & $\begin{array}{l}\text { Unsure (Not } \\
\text { reported) }\end{array}$ & $\begin{array}{l}\text { Yes (No } \\
\text { dropouts } \\
\text { reported) }\end{array}$ & $\begin{array}{l}\text { No (No } \\
\text { missing } \\
\text { data } \\
\text { reporte } \\
\text { d) }\end{array}$ & $\begin{array}{l}\text { No (No } \\
\text { missing data } \\
\text { reported) }\end{array}$ & $\begin{array}{l}\text { Not } \\
\text { Applicable } \\
\text { (No AEs } \\
\text { discussed) }\end{array}$ & $\begin{array}{l}\text { (No AEs } \\
\text { reported) }\end{array}$ & High \\
\hline $\begin{array}{l}\text { Schleier } 2007 \\
25047438 \\
\text { (Germany } \\
\text { (Friedrich- } \\
\text { Schiller } \\
\text { University } \\
\text { Jena)) }\end{array}$ & Yes & No & Yes & Yes & Yes & Yes & Unsure & No & & $\begin{array}{l}\text { Yes (pain } \\
\text { specifics } \\
\text { unavailabl } \\
\text { e) }\end{array}$ & & $\begin{array}{l}\text { Moderate } \\
\text { for all } \\
\text { outcomes }\end{array}$ \\
\hline $\begin{array}{l}\text { Schulze } 2005 \\
15888150 \\
\text { (Europe) }\end{array}$ & $\begin{array}{l}\text { Yes } \\
\text { (randomized } \\
\text { to imiquimod } \\
\text { or vehicle in } \\
\text { a } 1: 1 \text { ratio } \\
\text { according to } \\
\text { a computer- } \\
\text { generated } \\
\text { randomizatio } \\
\text { n schedule) }\end{array}$ & $\begin{array}{l}\text { Yes (Study } \\
\text { personnel } \\
\text { remained } \\
\text { blinded to the } \\
\text { randomization } \\
\text { until the } \\
\text { database was } \\
\text { complete and } \\
\text { locked.) }\end{array}$ & Yes & $\begin{array}{l}\text { Yes } \\
\text { (Subjects, } \\
\text { study } \\
\text { personnel } \\
\text { and the } \\
\text { sponsor's } \\
\text { clinical } \\
\text { research } \\
\text { team were } \\
\text { blinded to } \\
\text { study } \\
\text { cream } \\
\text { identity and } \\
\text { treatment } \\
\end{array}$ & No & Unsure & Yes & Yes & Yes & No & Yes & Low \\
\hline
\end{tabular}




\begin{tabular}{|c|c|c|c|c|c|c|c|c|c|c|c|c|}
\hline Study & $\begin{array}{l}\text { Adequate } \\
\text { generation } \\
\text { of a } \\
\text { randomized } \\
\text { sequence } \\
\text { reported }\end{array}$ & $\begin{array}{l}\text { Adequate } \\
\text { allocation } \\
\text { concealment } \\
\text { reported }\end{array}$ & $\begin{array}{l}\text { Group } \\
\text { similarity } \\
\text { at } \\
\text { baseline }\end{array}$ & $\begin{array}{l}\text { Adequate } \\
\text { blinding of } \\
\text { PATIENTS } \\
\text { reported }\end{array}$ & $\begin{array}{l}\text { Adequate } \\
\text { blinding of } \\
\text { PROVIDERS } \\
\text { reported }\end{array}$ & $\begin{array}{l}\text { Adequate } \\
\text { blinding of } \\
\text { OUTCOME } \\
\text { ASSESSORS } \\
\text { reported }\end{array}$ & $\begin{array}{l}\text { Intention } \\
\text { to treat } \\
\text { analysis } \\
?\end{array}$ & $\begin{array}{l}\text { Incom- } \\
\text { plete } \\
\text { results } \\
\text { data }\end{array}$ & $\begin{array}{l}\text { Incomplete } \\
\text { results data: } \\
\text { Differential } \\
\text { missingness }\end{array}$ & $\begin{array}{l}\text { Adverse } \\
\text { events (of } \\
\text { interest) } \\
\text { precisely } \\
\text { defined }\end{array}$ & $\begin{array}{l}\text { Selective } \\
\text { Reporting }\end{array}$ & $\begin{array}{l}\text { Overall, } \\
\text { by } \\
\text { outcome }\end{array}$ \\
\hline & & & & $\begin{array}{l}\text { assignment } \\
\text { ) }\end{array}$ & & & & & & & & \\
\hline $\begin{array}{l}\text { Shumack } \\
2002 \\
12224977 \text { (12 } \\
\text { weeks) } \\
\text { (Australia and } \\
\text { New Zealand; } \\
\text { And United } \\
\text { States) }\end{array}$ & $\begin{array}{l}\text { No (92 } \\
\text { patients } \\
\text { randomized } \\
\text { to Imiquimod } \\
\text { and placebo } \\
\text { according to } \\
\text { the dosing } \\
\text { scheme: } \\
\text { - once daily } \\
\text { for } 3 \text { days } \\
\text { per week (20 } \\
\text { Active, } 8 \\
\text { Vehicle) } \\
\text {-once daily } \\
\text { for } 5 \text { days } \\
\text { per week (23 } \\
\text { A, } 6 \text { V) } \\
\text { - once daily } \\
\text { for } 7 \text { days } \\
\text { per week ( } 21 \\
\text { A, } 10 \text { V)) }\end{array}$ & $\begin{array}{l}\text { No Data } \\
\text { (method of } \\
\text { allocation } \\
\text { concealment } \\
\text { was not } \\
\text { reported) }\end{array}$ & $\begin{array}{l}\text { No (Twice } \\
\text { daily for } 7 \\
\text { days per } \\
\text { week } \\
\text { group (4 } \\
\text { active, } 0 \\
\text { control) } \\
\text { Mean age } \\
\text { is different } \\
\text { from } \\
\text { range of } \\
\text { age in } \\
\text { other } \\
\text { groups } \\
\text { and } \\
\text { combined } \\
\text { vehicle) }\end{array}$ & Yes & $\begin{array}{l}\text { No Data } \\
\text { ("double } \\
\text { blind") }\end{array}$ & $\begin{array}{l}\text { No Data } \\
\text { ("double } \\
\text { blind") }\end{array}$ & $\begin{array}{l}\text { Yes (15 } \\
\text { were } \\
\text { discontin } \\
\text { ued from } \\
\text { the } \\
\text { study. } \\
\text { Post } \\
\text { treatment } \\
\text { excision } \\
\text { results } \\
\text { were } \\
\text { obtained } \\
\text { for } 11 \text { of } \\
\text { these. } \\
\text { Intention } \\
\text { to Treat } \\
\text { was } \\
\text { reported. } \\
\text { ) }\end{array}$ & $\begin{array}{l}\text { No } \\
\text { (Cleara } \\
\text { nce } \\
\text { outcom } \\
\text { e was } \\
\text { partially } \\
\text { reporte } \\
\text { d. } \\
\text { Report } \\
\text { ed for } \\
\text { combin } \\
\text { ed } \\
\text { vehicle } \\
\text { separat } \\
\text { e from } \\
\text { dosing } \\
\text { regime } \\
\text { n } \\
\text { groups, } \\
\text { where } \\
\text { only } \\
\text { results } \\
\text { of } \\
\text { imiquim } \\
\text { od } \\
\text { patients } \\
\text { were } \\
\text { reporte } \\
\text { d.) }\end{array}$ & No & $\begin{array}{l}\text { No (AE } \\
\text { were } \\
\text { defined } \\
\text { but \# of } \\
\text { counts } \\
\text { within } \\
\text { each arm } \\
\text { was not } \\
\text { completely } \\
\text { reported.) }\end{array}$ & $\begin{array}{l}\text { Yes (AE } \\
\text { were } \\
\text { defined but } \\
\text { \# of counts } \\
\text { within each } \\
\text { arm was } \\
\text { not } \\
\text { completely } \\
\text { reported.) }\end{array}$ & $\begin{array}{l}\text { Low for } \\
\text { clearance } \\
\text { outcomes } \\
\text {, unclear } \\
\text { for AEs }\end{array}$ \\
\hline $\begin{array}{l}\text { Shumack } \\
2002 \\
12224977 \text { (6 } \\
\text { weeks) } \\
\text { (Australia and } \\
\text { New Zealand; } \\
\text { And United } \\
\end{array}$ & $\begin{array}{l}\text { Yes (99 } \\
\text { patients } \\
\text { randomized } \\
\text { to Imiquimod } \\
\text { and placebo } \\
\text { according to } \\
\text { the dosing }\end{array}$ & No Data & $\begin{array}{l}\text { No } \\
\text { (Noticeabl } \\
\text { e } \\
\text { difference } \\
\text { in age for } \\
\text { Twice } \\
\text { daily for } 7 \\
\end{array}$ & Yes & No Data & No Data & $\begin{array}{l}\text { Yes }(9 \\
\text { patients } \\
\text { were } \\
\text { discontin } \\
\text { ued from } \\
\text { the } \\
\text { study, }\end{array}$ & No & No & Yes & No & $\begin{array}{l}\text { Low } \\
\text { Adverse } \\
\text { events } \\
\text { reported } \\
\text { but not } \\
\text { for every } \\
\text { arm } \\
\end{array}$ \\
\hline
\end{tabular}




\begin{tabular}{|c|c|c|c|c|c|c|c|c|c|c|c|c|}
\hline Study & $\begin{array}{l}\text { Adequate } \\
\text { generation } \\
\text { of a } \\
\text { randomized } \\
\text { sequence } \\
\text { reported }\end{array}$ & $\begin{array}{l}\text { Adequate } \\
\text { allocation } \\
\text { concealment } \\
\text { reported }\end{array}$ & $\begin{array}{l}\text { Group } \\
\text { similarity } \\
\text { at } \\
\text { baseline }\end{array}$ & $\begin{array}{l}\text { Adequate } \\
\text { blinding of } \\
\text { PATIENTS } \\
\text { reported }\end{array}$ & $\begin{array}{l}\text { Adequate } \\
\text { blinding of } \\
\text { PROVIDERS } \\
\text { reported }\end{array}$ & $\begin{array}{l}\text { Adequate } \\
\text { blinding of } \\
\text { OUTCOME } \\
\text { ASSESSORS } \\
\text { reported }\end{array}$ & $\begin{array}{l}\text { Intention } \\
\text { to treat } \\
\text { analysis } \\
?\end{array}$ & $\begin{array}{l}\text { Incom- } \\
\text { plete } \\
\text { results } \\
\text { data }\end{array}$ & $\begin{array}{l}\text { Incomplete } \\
\text { results data: } \\
\text { Differential } \\
\text { missingness }\end{array}$ & $\begin{array}{l}\text { Adverse } \\
\text { events (of } \\
\text { interest) } \\
\text { precisely } \\
\text { defined }\end{array}$ & $\begin{array}{l}\text { Selective } \\
\text { Reporting }\end{array}$ & $\begin{array}{l}\text { Overall, } \\
\text { by } \\
\text { outcome }\end{array}$ \\
\hline States) & $\begin{array}{l}\text { scheme: } \\
\text { - once daily } \\
\text { for } 3 \text { days } \\
\text { per week } \\
\text { (32) } \\
\text {-twice daily } \\
\text { for } 3 \text { days } \\
\text { per week } \\
\text { (31) } \\
\text { - once daily } \\
\text { for } 7 \text { days } \\
\text { per week } \\
\text { (35) } \\
\text { - twice daily } \\
\text { for } 7 \text { days } \\
\text { per week } \\
\text { (1)) }\end{array}$ & & $\begin{array}{l}\text { days/ } \\
\text { week arm } \\
(n=1))\end{array}$ & & & & $\begin{array}{l}\text { but only } \\
4 \text { did not } \\
\text { undergo } \\
\text { post- } \\
\text { treatment } \\
\text { excision } \\
5 \text { of } 99 \\
\text { enrolled } \\
\text { did not } \\
\text { undergo } \\
\text { post } \\
\text { treatment } \\
\text { excision. } \\
\text { ITT not } \\
\text { reported. } \\
\text { ) }\end{array}$ & & & & & \\
\hline $\begin{array}{l}\text { Siller } 2010 \\
20546215 \text { (8 } \\
\text { private } \\
\text { dermatology } \\
\text { clinics } \\
\text { Australia) }\end{array}$ & Yes & Yes & Yes & Yes & Yes & Yes & Yes & Unsure & No & Yes & & $\begin{array}{l}\text { Low for } \\
\text { all } \\
\text { outcomes }\end{array}$ \\
\hline $\begin{array}{l}\text { Spencer } 2006 \\
16393600 \\
\text { (United } \\
\text { States) }\end{array}$ & $\begin{array}{l}\text { No Data } \\
\text { (randomizati } \\
\text { on not } \\
\text { reported) }\end{array}$ & No Data & $\begin{array}{l}\text { Unsure } \\
\text { (very low } \\
\text { n) }\end{array}$ & No Data & No Data & $\begin{array}{l}\text { No (blinding } \\
\text { not reported) }\end{array}$ & $\begin{array}{l}\text { Yes (no } \\
\text { dropouts) }\end{array}$ & No & No & No & $\begin{array}{l}\text { No (not all } \\
\text { time points } \\
\text { reported.) }\end{array}$ & $\begin{array}{l}\text { High risk } \\
\text { of bias }\end{array}$ \\
\hline $\begin{array}{l}\text { Sterry } 2002 \\
12452875 \\
\text { (nodular) } \\
\text { (Europe) }\end{array}$ & Yes & Yes & Yes & No & No & Unsure & No & No & Yes & $\begin{array}{l}\text { No (Few } \\
\text { AEs } \\
\text { reported } \\
\text { by arm; in } \\
\text { general } \\
\text { unclear } \\
\text { AE } \\
\text { reporting) }\end{array}$ & $\begin{array}{l}\text { (Not } \\
\text { immediatel } \\
\text { y evident) }\end{array}$ & $\begin{array}{l}\text { Low for } \\
\text { efficacy } \\
\text { and } \\
\text { moderate } \\
\text { to high } \\
\text { for AEs }\end{array}$ \\
\hline $\begin{array}{l}\text { Sterry } 2002 \\
12452875 \\
\text { (superficial) }\end{array}$ & Yes & Yes & No & No & No & Unsure & No & No & Yes & $\begin{array}{l}\text { No (Few } \\
\text { AEs } \\
\text { reported }\end{array}$ & & $\begin{array}{l}\text { Low to } \\
\text { moderate } \\
\text { for }\end{array}$ \\
\hline
\end{tabular}




\begin{tabular}{|c|c|c|c|c|c|c|c|c|c|c|c|c|}
\hline Study & $\begin{array}{l}\text { Adequate } \\
\text { generation } \\
\text { of a } \\
\text { randomized } \\
\text { sequence } \\
\text { reported }\end{array}$ & $\begin{array}{l}\text { Adequate } \\
\text { allocation } \\
\text { concealment } \\
\text { reported }\end{array}$ & $\begin{array}{l}\text { Group } \\
\text { similarity } \\
\text { at } \\
\text { baseline }\end{array}$ & $\begin{array}{l}\text { Adequate } \\
\text { blinding of } \\
\text { PATIENTS } \\
\text { reported }\end{array}$ & $\begin{array}{l}\text { Adequate } \\
\text { blinding of } \\
\text { PROVIDERS } \\
\text { reported }\end{array}$ & $\begin{array}{l}\text { Adequate } \\
\text { blinding of } \\
\text { OUTCOME } \\
\text { ASSESSORS } \\
\text { reported }\end{array}$ & $\begin{array}{l}\text { Intention } \\
\text { to treat } \\
\text { analysis } \\
?\end{array}$ & $\begin{array}{l}\text { Incom- } \\
\text { plete } \\
\text { results } \\
\text { data }\end{array}$ & $\begin{array}{l}\text { Incomplete } \\
\text { results data: } \\
\text { Differential } \\
\text { missingness }\end{array}$ & $\begin{array}{l}\text { Adverse } \\
\text { events (of } \\
\text { interest) } \\
\text { precisely } \\
\text { defined }\end{array}$ & $\begin{array}{l}\text { Selective } \\
\text { Reporting }\end{array}$ & $\begin{array}{l}\text { Overall, } \\
\text { by } \\
\text { outcome }\end{array}$ \\
\hline (Europe) & & & & & & & & & & $\begin{array}{l}\text { by arm; in } \\
\text { general } \\
\text { unclear } \\
\mathrm{AE} \\
\text { reporting) }\end{array}$ & & $\begin{array}{l}\text { efficacy } \\
\text { and } \\
\text { moderate } \\
\text { to high } \\
\text { for AEs }\end{array}$ \\
\hline $\begin{array}{l}\text { Szeimies } \\
2008 \\
18624836 \\
\text { (United } \\
\text { Kingdom/Ger } \\
\text { many/Switzerl } \\
\text { and/Australia) }\end{array}$ & Yes & Yes & Yes & No & No & No & $\begin{array}{l}\text { Unsure } \\
\text { (per } \\
\text { protocol } \\
\text { analysis) }\end{array}$ & No & $\begin{array}{l}\text { Yes (some } \\
\text { outcomes) }\end{array}$ & No & & $\begin{array}{l}\text { Low to } \\
\text { moderate }\end{array}$ \\
\hline $\begin{array}{l}\text { Thissen } 2000 \\
10940063 \\
\text { (Netherlands) }\end{array}$ & No Data & No Data & Yes & $\begin{array}{l}\text { No (Not } \\
\text { possible to } \\
\text { blind } \\
\text { patients to } \\
\text { treatment } \\
\text { allocation } \\
\text { (cryosurger } \\
\text { y vs. } \\
\text { surgical } \\
\text { excision)) }\end{array}$ & $\begin{array}{l}\text { No Data (It is } \\
\text { not reported if } \\
\text { providers } \\
\text { were blinded, } \\
\text { might be high } \\
\text { RoB for } \\
\text { clinical } \\
\text { recurrence } \\
\text { outcome) }\end{array}$ & 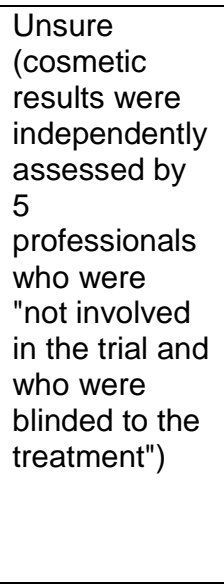 & $\begin{array}{l}\text { Yes (few } \\
\text { drop-outs } \\
\text { not } \\
\text { reported } \\
\text { by arm (3 } \\
\text { did not } \\
\text { appear } \\
\text { for } \\
\text { control } \\
\text { visits and } \\
1 \text { died), } \\
\text { not } \\
\text { related to } \\
\text { treatment } \\
\text { or } \\
\text { outcome) }\end{array}$ & $\begin{array}{l}\text { No } \\
\text { (Cleara } \\
\text { nce is } \\
\text { fully } \\
\text { reporte } \\
\text { d by } \\
\text { arm.) }\end{array}$ & No & $\begin{array}{l}\text { Yes (AEs: } \\
\text { secondary } \\
\text { wound } \\
\text { infections; } \\
\text { moderate } \\
\text { to severe } \\
\text { swelling of } \\
\text { treated } \\
\text { area. } \\
\text { (Reported } \\
\text { by Arm)) }\end{array}$ & No & $\begin{array}{l}\text { Moderate } \\
\text { to high } \\
\text { because } \\
\text { of } \\
\text { blinding } \\
\text { only }\end{array}$ \\
\hline $\begin{array}{l}\text { Torres } 2004 \\
15606733 \\
\text { (loma linda, } \\
\text { CA; boston, } \\
\text { MA) }\end{array}$ & $\begin{array}{l}\text { Yes } \\
\text { (computer- } \\
\text { generated } \\
\text { schedule) }\end{array}$ & No & Yes & Yes & Yes & $\begin{array}{l}\text { Unsure } \\
\text { (histologist) }\end{array}$ & Yes & No & & $\begin{array}{l}\text { No (Not } \\
\text { well } \\
\text { reported) }\end{array}$ & $\begin{array}{l}\text { No } \\
\text { (probably } \\
\text { not) }\end{array}$ & $\begin{array}{l}\text { Low for } \\
\text { all } \\
\text { outcomes }\end{array}$ \\
\hline $\begin{array}{l}\text { Tran } 2012 \\
22511036 \\
\text { (US) }\end{array}$ & No Data & No Data & $\begin{array}{l}\text { No } \\
\text { (groups } \\
\text { were not } \\
\text { similar at } \\
\text { baseline, }\end{array}$ & Yes & No & No & Yes & No & No & Yes & (unclear) & $\begin{array}{l}\text { Moderate } \\
\text { to high } \\
\text { due to } \\
\text { nonsimila } \\
r\end{array}$ \\
\hline
\end{tabular}




\begin{tabular}{|c|c|c|c|c|c|c|c|c|c|c|c|c|}
\hline Study & $\begin{array}{l}\text { Adequate } \\
\text { generation } \\
\text { of a } \\
\text { randomized } \\
\text { sequence } \\
\text { reported }\end{array}$ & $\begin{array}{l}\text { Adequate } \\
\text { allocation } \\
\text { concealment } \\
\text { reported }\end{array}$ & $\begin{array}{l}\text { Group } \\
\text { similarity } \\
\text { at } \\
\text { baseline }\end{array}$ & $\begin{array}{l}\text { Adequate } \\
\text { blinding of } \\
\text { PATIENTS } \\
\text { reported }\end{array}$ & $\begin{array}{l}\text { Adequate } \\
\text { blinding of } \\
\text { PROVIDERS } \\
\text { reported }\end{array}$ & $\begin{array}{l}\text { Adequate } \\
\text { blinding of } \\
\text { OUTCOME } \\
\text { ASSESSORS } \\
\text { reported }\end{array}$ & $\begin{array}{l}\text { Intention } \\
\text { to treat } \\
\text { analysis } \\
?\end{array}$ & $\begin{array}{l}\text { Incom- } \\
\text { plete } \\
\text { results } \\
\text { data }\end{array}$ & $\begin{array}{l}\text { Incomplete } \\
\text { results data: } \\
\text { Differential } \\
\text { missingness }\end{array}$ & $\begin{array}{l}\text { Adverse } \\
\text { events (of } \\
\text { interest) } \\
\text { precisely } \\
\text { defined }\end{array}$ & $\begin{array}{l}\text { Selective } \\
\text { Reporting }\end{array}$ & $\begin{array}{l}\text { Overall, } \\
\text { by } \\
\text { outcome }\end{array}$ \\
\hline & & & $\begin{array}{l}\text { though the } \\
\text { difference } \\
\text { s were not } \\
\text { statisticall } \\
y \\
\text { significant } \\
\text { (probably } \\
\text { because } \\
\text { of the } \\
\text { small } \\
\text { sample } \\
\text { size)) }\end{array}$ & & & & & & & & & baselines \\
\hline $\begin{array}{l}\text { van der Geer } \\
2012 \\
22385074 \\
\text { (Netherlands) }\end{array}$ & Yes & Yes & Yes & $\begin{array}{l}\text { No (no } \\
\text { mention of } \\
\text { blinding) }\end{array}$ & $\begin{array}{l}\text { No (no } \\
\text { mention of } \\
\text { blinding) }\end{array}$ & $\begin{array}{l}\text { No (High } \\
\text { RoB, no } \\
\text { mention of } \\
\text { blinding, and } \\
\text { only clinical } \\
\text { clearance } \\
\text { outcome) }\end{array}$ & Yes & No & No & Yes & $\begin{array}{l}\text { (none that i } \\
\text { could spot } \\
\text { easily) }\end{array}$ & Moderate \\
\hline $\begin{array}{l}\text { Wang } 2001 \\
11298545 \\
\text { (England) }\end{array}$ & No Data & Unsure & $\begin{array}{l}\text { Unsure } \\
\text { (The two } \\
\text { treatment } \\
\text { groups } \\
\text { were } \\
\text { comparabl } \\
\text { e } \\
\text { concernin } \\
\text { g medical } \\
\text { history of } \\
\text { the } \\
\text { patients } \\
\text { and status } \\
\text { at the } \\
\text { medical } \\
\text { examinati } \\
\text { on.) }\end{array}$ & $\begin{array}{l}\text { No (No } \\
\text { blinding } \\
\text { regimen } \\
\text { was } \\
\text { possible } \\
\text { due to the } \\
\text { nature of } \\
\text { the } \\
\text { treatment } \\
\text { procedures. } \\
\text { ) }\end{array}$ & $\begin{array}{l}\text { No (No } \\
\text { blinding } \\
\text { regimen was } \\
\text { possible due } \\
\text { to the nature } \\
\text { of the } \\
\text { treatment } \\
\text { procedures.) }\end{array}$ & No Data & Yes & No & No & Yes & & $\begin{array}{l}\text { Low to } \\
\text { moderate } \\
\text { due to } \\
\text { poor } \\
\text { reporting }\end{array}$ \\
\hline $\begin{array}{l}\text { Wettstein } \\
2013\end{array}$ & Yes & No Data & Yes & Yes & Yes & No Data & Yes & No & No & Yes & (Low) & Low \\
\hline
\end{tabular}




\begin{tabular}{|c|c|c|c|c|c|c|c|c|c|c|c|c|}
\hline Study & $\begin{array}{l}\text { Adequate } \\
\text { generation } \\
\text { of a } \\
\text { randomized } \\
\text { sequence } \\
\text { reported }\end{array}$ & $\begin{array}{l}\text { Adequate } \\
\text { allocation } \\
\text { concealment } \\
\text { reported }\end{array}$ & $\begin{array}{l}\text { Group } \\
\text { similarity } \\
\text { at } \\
\text { baseline }\end{array}$ & $\begin{array}{l}\text { Adequate } \\
\text { blinding of } \\
\text { PATIENTS } \\
\text { reported }\end{array}$ & $\begin{array}{l}\text { Adequate } \\
\text { blinding of } \\
\text { PROVIDERS } \\
\text { reported }\end{array}$ & $\begin{array}{l}\text { Adequate } \\
\text { blinding of } \\
\text { OUTCOME } \\
\text { ASSESSORS } \\
\text { reported }\end{array}$ & $\begin{array}{l}\text { Intention } \\
\text { to treat } \\
\text { analysis } \\
?\end{array}$ & $\begin{array}{l}\text { Incom- } \\
\text { plete } \\
\text { results } \\
\text { data }\end{array}$ & $\begin{array}{l}\text { Incomplete } \\
\text { results data: } \\
\text { Differential } \\
\text { missingness }\end{array}$ & $\begin{array}{l}\text { Adverse } \\
\text { events (of } \\
\text { interest) } \\
\text { precisely } \\
\text { defined }\end{array}$ & $\begin{array}{l}\text { Selective } \\
\text { Reporting }\end{array}$ & $\begin{array}{l}\text { Overall, } \\
\text { by } \\
\text { outcome }\end{array}$ \\
\hline $\begin{array}{l}23566745 \\
\text { (Switzerland) }\end{array}$ & & & & & & & & & & & & \\
\hline
\end{tabular}

Table F-2. NRCS

\begin{tabular}{|c|c|c|c|c|c|c|c|c|}
\hline Study & $\begin{array}{l}\text { Group } \\
\text { similarity at } \\
\text { baseline }\end{array}$ & $\begin{array}{l}\text { Adequate } \\
\text { blinding of } \\
\text { outcome } \\
\text { assessors }\end{array}$ & $\begin{array}{l}\text { Incomplete } \\
\text { results data }\end{array}$ & $\begin{array}{l}\text { Differential } \\
\text { missingness }\end{array}$ & $\begin{array}{l}\text { Adverse events (of } \\
\text { interest) precisely } \\
\text { defined }\end{array}$ & & $\begin{array}{l}\text { Selective } \\
\text { Reporting }\end{array}$ & $\begin{array}{l}\text { Overall, by } \\
\text { outcome }\end{array}$ \\
\hline $\begin{array}{l}\text { Ahmed } 2000 \\
11069453 \\
(\text { UK) }\end{array}$ & $\begin{array}{l}\text { Unsure } \\
\text { (Baseline data } \\
\text { not given by } \\
\text { arm except } \\
\text { lesion location, } \\
\text { which was } \\
\text { balanced) }\end{array}$ & No & & $\begin{array}{l}\text { Unsure (okay for } \\
\text { clinical clearance } \\
\text { and pain; } \\
\text { problematic for } \\
\text { recurrence) }\end{array}$ & $\begin{array}{l}\text { Unsure (dropouts not } \\
\text { given by arm) }\end{array}$ & & & $\begin{array}{l}\text { High (primarily } \\
\text { because of } \\
\text { unclear } \\
\text { reporting) }\end{array}$ \\
\hline $\begin{array}{l}\text { Ballester- } \\
\text { Sanchez } \\
2016 \\
26985197 \\
\text { (Spain) }\end{array}$ & $\begin{array}{l}\text { Unsure (ages } \\
\text { differ } \\
\text { significantly; } \\
\text { exact location of } \\
\text { tumors not } \\
\text { given) }\end{array}$ & No & No & No & $\begin{array}{l}\text { Unsure (only } 2 \text { AEs } \\
\text { reported, but those } \\
\text { were reported well) }\end{array}$ & $\begin{array}{l}\text { Unsure } \\
\text { (Unclear } \\
\text { results } \\
\text { reporting) }\end{array}$ & & Moderate \\
\hline $\begin{array}{l}\text { Chren } 2013 \\
23190903 \\
\text { (U.S.) }\end{array}$ & $\begin{array}{l}\text { No (Patients, } \\
\text { tumors, and } \\
\text { care differed in } \\
\text { the treatment } \\
\text { groups (Table } \\
\text { 1). For example, } \\
\text { tumors treated } \\
\text { with destruction } \\
\text { were much less } \\
\text { likely to be } \\
\text { located in the H- } \\
\text { zone of the } \\
\text { face, and much } \\
\text { less likely to } \\
\text { have }\end{array}$ & $\begin{array}{l}\text { Yes (primary } \\
\text { source of data on } \\
\text { recurrence was } \\
\text { the medical } \\
\text { record. patients } \\
\text { who consented } \\
\text { were examined a } \\
\text { median of } 8.6 \\
\text { years after } \\
\text { treatment by a } \\
\text { dermatologist } \\
\text { (MMC) blinded to } \\
\text { treatment type.) }\end{array}$ & $\begin{array}{l}\text { No (Patients } \\
\text { lost to follow-up } \\
\text { were similar to } \\
\text { those with } \\
\text { follow-up in } \\
\text { most features } \\
\text { but were more } \\
\text { likely to be } \\
\text { female (38\% } \\
\text { vs. } 26 \% \text { ), to } \\
\text { have worse } \\
\text { mental health } \\
\text { status (median } \\
\text { SF-12 Mental } \\
\text { Component }\end{array}$ & No & $\begin{array}{l}\text { No (Adverse events } \\
\text { were not reported in } \\
\text { any of the } 4 \text { papers } \\
\text { from this study) }\end{array}$ & $\begin{array}{l}\text { (Consecutive } \\
\text { patients) }\end{array}$ & & Low \\
\hline
\end{tabular}




\begin{tabular}{|c|c|c|c|c|c|c|c|c|}
\hline Study & $\begin{array}{l}\text { Group } \\
\text { similarity at } \\
\text { baseline }\end{array}$ & $\begin{array}{l}\text { Adequate } \\
\text { blinding of } \\
\text { outcome } \\
\text { assessors }\end{array}$ & $\begin{array}{l}\text { Incomplete } \\
\text { results data }\end{array}$ & $\begin{array}{l}\text { Differential } \\
\text { missingness }\end{array}$ & $\begin{array}{l}\text { Adverse events (of } \\
\text { interest) precisely } \\
\text { defined }\end{array}$ & & $\begin{array}{l}\text { Selective } \\
\text { Reporting }\end{array}$ & $\begin{array}{l}\text { Overall, by } \\
\text { outcome }\end{array}$ \\
\hline & $\begin{array}{l}\text { histological risk } \\
\text { factors for } \\
\text { recurrence.) }\end{array}$ & & $\begin{array}{l}\text { Score } 41.2 \text { vs } \\
51.5), \text { and to } \\
\text { have BCC } \\
\text { rather than } \\
\text { SCC (89\% vs } \\
75 \%) .)\end{array}$ & & & & & \\
\hline $\begin{array}{l}\text { Cosgarea } \\
2012 \\
22738399 \\
\text { (Romania) }\end{array}$ & Yes & No & No & No & No & $\begin{array}{l}\text { Yes (Baseline } \\
\text { numbers for } \\
\text { skin type and } \\
\text { \# lesions per } \\
\text { patient do not } \\
\text { add up) }\end{array}$ & No & Moderate \\
\hline $\begin{array}{l}\text { Graells } 2014 \\
24139468 \\
\text { (Spain) }\end{array}$ & $\begin{array}{l}\text { No (The } \\
\text { imiquimod and } \\
\text { surgery groups } \\
\text { differed: higher } \\
\text { frequency of } \\
\text { superficial } \\
\text { BCCs in } \\
\text { patients treated } \\
\text { with imiquimod } \\
\text { vs. surgery, the } \\
\text { proportion of } \\
\text { patients with a } \\
\text { history of } \\
\text { multiple BCCs } \\
\text { and current } \\
\text { multiple BCCs } \\
\text { was higher in } \\
\text { the group of } \\
\text { patients who } \\
\text { received } \\
\text { imiquimod.) }\end{array}$ & No & $\begin{array}{l}\text { Unsure (For } \\
\text { "subsequent } \\
\text { BCC" outcome, } \\
67 \text { subjects } \\
\text { were lost to } \\
\text { follow-up } \\
\text { ( } 10.7 \% \text { of } \\
\text { whole group), } \\
\text { but distribution } \\
\text { of missing by } \\
\text { arm is not } \\
\text { reported.) }\end{array}$ & No & $\begin{array}{l}\text { Unsure (AE was only } \\
\text { defined for Imiquimod. } \\
\text { Imiquimod-induced } \\
\text { inflammation was } \\
\text { classified as mild (not } \\
\text { requiring any change } \\
\text { in treatment), } \\
\text { moderate (requir- ing } \\
\text { the addition of a } \\
\text { corticosteroid- } \\
\text { antibiotic cream but no } \\
\text { change in imiquimod } \\
\text { treatment), or intense } \\
\text { (requiring the } \\
\text { temporary or } \\
\text { permanent withdrawal } \\
\text { of treatment).) }\end{array}$ & $\begin{array}{l}\text { Yes } \\
\text { (Multivariate } \\
\text { analysis did } \\
\text { not include } \\
\text { variables } \\
\text { such as: size, } \\
\text { histology, or } \\
\text { location of } \\
\text { BCC), all of } \\
\text { which may } \\
\text { explain the } \\
\text { difference in } \\
\text { risk between } \\
\text { imiquimod } \\
\text { and surgery.) }\end{array}$ & $\begin{array}{l}\text { No (Lack of } \\
\text { clinical } \\
\text { clearance } \\
\text { was not } \\
\text { reported for } \\
\text { surgery arm. } \\
\text { Adverse } \\
\text { Events were } \\
\text { only defined } \\
\text { for } \\
\text { Imiquimod } \\
\text { arm. No AEs } \\
\text { reported for } \\
\text { surgery arm.) }\end{array}$ & High \\
\hline $\begin{array}{l}\text { Lippert } 2013 \\
23725586 \\
\text { (Czech } \\
\text { Republic) }\end{array}$ & Yes & No & No & No & $\begin{array}{l}\text { Unsure (AEs not } \\
\text { reported in depth) }\end{array}$ & & $\begin{array}{l}\text { Unsure } \\
\text { (Cosmetic } \\
\text { outcomes } \\
\text { not reported } \\
\text { by arm) }\end{array}$ & $\begin{array}{l}\text { Moderate to low } \\
\text { (outcome } \\
\text { assessors not } \\
\text { blinded; AEs } \\
\text { and cosmetic } \\
\text { outcomes given } \\
\text { very short shrift) }\end{array}$ \\
\hline
\end{tabular}




\begin{tabular}{|c|c|c|c|c|c|c|c|c|}
\hline Study & $\begin{array}{l}\text { Group } \\
\text { similarity at } \\
\text { baseline }\end{array}$ & $\begin{array}{l}\text { Adequate } \\
\text { blinding of } \\
\text { outcome } \\
\text { assessors }\end{array}$ & $\begin{array}{l}\text { Incomplete } \\
\text { results data }\end{array}$ & $\begin{array}{l}\text { Differential } \\
\text { missingness }\end{array}$ & $\begin{array}{l}\text { Adverse events (of } \\
\text { interest) precisely } \\
\text { defined }\end{array}$ & & \begin{tabular}{|l|} 
Selective \\
Reporting
\end{tabular} & $\begin{array}{l}\text { Overall, by } \\
\text { outcome }\end{array}$ \\
\hline $\begin{array}{l}\text { Pampena } \\
2016 \\
26589877 \\
\text { (Italy) }\end{array}$ & $\begin{array}{l}\text { Yes (nothing > } \\
20 \% \text { differential, } \\
\text { mean age may } \\
\text { be of concern) }\end{array}$ & $\begin{array}{l}\text { Yes (OS and } \\
\text { DFS likely not } \\
\text { affected by } \\
\text { blinding. } \\
\text { Cosmetic } \\
\text { outcome } \\
\text { assessor } \\
\text { blinded) }\end{array}$ & No (no missing) & No (no missing) & No Data (no AEs) & & $\begin{array}{l}\text { No (all } 3 \\
\text { outcomes } \\
\text { reported) }\end{array}$ & $\begin{array}{l}\text { Low (NRCS, no } \\
\text { Aes) }\end{array}$ \\
\hline $\begin{array}{l}\text { Shah } 2009 \\
19588534 \\
\text { (U.S.) }\end{array}$ & $\begin{array}{l}\text { Unsure (stated, } \\
\text { but baselines } \\
\text { not given for } \\
\text { controls) }\end{array}$ & No & No & No & No (AEs not reported) & & & Moderate \\
\hline $\begin{array}{l}\text { Sofen } 2015 \\
25913533 \\
\text { (U.S.) }\end{array}$ & $\begin{array}{l}\text { Unsure (From } \\
\text { the baseline } \\
\text { table, yes, but } \\
\text { the number in } \\
\text { each region for } \\
\text { each arm is not } \\
\text { given.) }\end{array}$ & No & Yes & Yes & Yes & & No & $\begin{array}{l}\text { Moderate } \\
\text { (NRCS, no Aes) }\end{array}$ \\
\hline $\begin{array}{l}\text { Sullivan } \\
2003 \\
14725659 \\
\text { (US) }\end{array}$ & $\begin{array}{l}\text { (some } \\
\text { differences, but } \\
\text { most likely due } \\
\text { to small sample } \\
\text { size) }\end{array}$ & $\begin{array}{l}\text { Yes } \\
\text { (dermatologist } \\
\text { and pathologist } \\
\text { blinded) }\end{array}$ & No (no missing) & No (no missing) & $\begin{array}{l}\text { No Data (none } \\
\text { reported) }\end{array}$ & $\begin{array}{l}\text { (small } \\
\text { sample size } \\
(6 \text { per arm)) }\end{array}$ & Yes (no AEs) & Moderate \\
\hline $\begin{array}{l}\text { Wilson } 2012 \\
22145798 \\
\text { (U.S.) }\end{array}$ & $\begin{array}{l}\text { No (Statistically } \\
\text { significant } \\
\text { difference } \\
\text { between } \\
\text { patients at } \\
\text { private site and } \\
\text { VA site in terms } \\
\text { of Age (private } \\
\text { patients are } \\
\text { younger), } \\
\text { gender (private } \\
\text { patients are } \\
\text { more female), } \\
\text { annual income } \\
\text { (private patients } \\
\text { are less likely to }\end{array}$ & $\begin{array}{l}\text { No Data } \\
\text { (Blinding of } \\
\text { outcome } \\
\text { assessors not } \\
\text { reported.Exposur } \\
\text { e of interest was } \\
\text { treatment center: } \\
\text { private treatment } \\
\text { center or VA } \\
\text { center.) }\end{array}$ & $\begin{array}{l}\text { Low Risk (No } \\
\text { loss of follow- } \\
\text { up reported.) }\end{array}$ & Low Risk & $\begin{array}{l}\text { No Data (Authors do } \\
\text { not mention AEs at } \\
\text { all.) }\end{array}$ & No & No & $\begin{array}{l}\text { Low } \\
\text { (Differences at } \\
\text { Baseline were } \\
\text { controlled for in } \\
\text { multivariate } \\
\text { analysis. It is } \\
\text { reported that it } \\
\text { is unlikely that } \\
\text { Clinical } \\
\text { differences of } \\
\text { patients } \\
\text { accounted for } \\
\text { all the variation } \\
\text { in care between } \\
\text { the treatment } \\
\text { centers) }\end{array}$ \\
\hline
\end{tabular}




\begin{tabular}{|l|l|l|l|l|l|l|l|}
\hline Study & $\begin{array}{l}\text { Group } \\
\text { similarity at } \\
\text { baseline }\end{array}$ & $\begin{array}{l}\text { Adequate } \\
\text { blinding of } \\
\text { outcome } \\
\text { assessors }\end{array}$ & $\begin{array}{l}\text { Incomplete } \\
\text { results data }\end{array}$ & $\begin{array}{l}\text { Differential } \\
\text { missingness }\end{array}$ & $\begin{array}{l}\text { Adverse events (of } \\
\text { interest) precisely } \\
\text { defined }\end{array}$ & $\begin{array}{l}\text { Selective } \\
\text { Reporting }\end{array}$ & $\begin{array}{l}\text { Overall, by } \\
\text { outcome }\end{array}$ \\
\hline $\begin{array}{l}\text { be poor), tumor } \\
\text { size (private } \\
\text { tumors are } \\
\text { smaller in } \\
\text { diameter), } \\
\text { histologic type } \\
\text { (private tumors } \\
\text { are less likely to } \\
\text { be SCC), } \\
\text { location (private } \\
\text { tumors are less } \\
\text { likely to be on } \\
\text { head and neck), } \\
\text { and H-zone } \\
\text { (private tumors } \\
\text { are less likely to } \\
\text { be in the h-zone } \\
\text { of the face).) }\end{array}$ & & & & & & \\
\hline
\end{tabular}




\section{Appendix G. Summary Results From Unadjusted NRCS}

Table G-1. Summary results from unadjusted NRCS

\begin{tabular}{|c|c|c|c|c|c|c|c|c|c|c|}
\hline $\begin{array}{l}\text { Author } \\
\text { year PMID }\end{array}$ & $\begin{array}{l}\text { Total N } \\
\text { (patients) }\end{array}$ & $\begin{array}{l}\text { Lesion } \\
\text { type } \\
\text { (location) }\end{array}$ & Treatment & $\begin{array}{l}\text { Treatment } \\
\text { notes }\end{array}$ & $\begin{array}{l}\text { Outcome } \\
\text { (lack of } \\
\text { clearancel } \\
\text { recurrence) }\end{array}$ & $\begin{array}{l}\text { n patients } \\
\text { (in arm)/N } \\
\text { patients } \\
\text { (in arm) } \\
\text { (\% } \\
\text { patients) }\end{array}$ & $\begin{array}{l}\text { n lesions } \\
\text { (arm)/N } \\
\text { lesions } \\
\text { (in arm) } \\
\text { (\% } \\
\text { lesions) }\end{array}$ & $\begin{array}{l}\text { Odds } \\
\text { Ratio (95\% } \\
\text { Cl) }\end{array}$ & $\begin{array}{l}\text { Adverse } \\
\text { events } \\
\text { reported } \\
(y / n)\end{array}$ & $\begin{array}{l}\text { Aditional } \\
\text { outcomes } \\
\text { reported }\end{array}$ \\
\hline $\begin{array}{l}\text { Rank } 1973 \\
4700671\end{array}$ & 1942 & $\mathrm{BCC}$ & $\begin{array}{l}\text { A. Surgical } \\
\text { Excision without } \\
\text { interoperative } \\
\text { evaluation }\end{array}$ & $\begin{array}{l}\text { unclear } \\
\text { whether the } \mathrm{n} \\
\text { here is } \\
\text { lesions or } \\
\text { patients }\end{array}$ & Recurrence & $3 / 566(0.5)$ & & $\begin{array}{l}\text { A vs. D1: } \\
0.14(0.04 \\
0.47)\end{array}$ & $\mathrm{n}$ & \\
\hline $\begin{array}{l}\text { Rank } 1973 \\
4700671\end{array}$ & 1942 & $\mathrm{BCC}$ & $\begin{array}{l}\text { D1. External } \\
\text { beam radiation }\end{array}$ & $\begin{array}{l}\text { unclear } \\
\text { whether the } \mathrm{n} \\
\text { here is } \\
\text { lesions or } \\
\text { patients }\end{array}$ & Recurrence & $\begin{array}{l}31 / 857 \\
(3.6)\end{array}$ & & & $\mathrm{n}$ & \\
\hline $\begin{array}{l}\text { Rank } 1973 \\
4700671\end{array}$ & 1942 & SCC & $\begin{array}{l}\text { A. Surgical } \\
\text { Excision without } \\
\text { interoperative } \\
\text { evaluation }\end{array}$ & $\begin{array}{l}\text { unclear } \\
\text { whether the } \mathrm{n} \\
\text { here is } \\
\text { lesions or } \\
\text { patients }\end{array}$ & Recurrence & $4 / 288(1.4)$ & & $\begin{array}{l}\text { A vs. D1: } \\
0.45(0.13 \\
1.56)\end{array}$ & $\mathrm{n}$ & \\
\hline $\begin{array}{l}\text { Rank } 1973 \\
4700671\end{array}$ & 1942 & SCC & $\begin{array}{l}\text { D1. External } \\
\text { beam radiation }\end{array}$ & $\begin{array}{l}\text { unclear } \\
\text { whether the } \mathrm{n} \\
\text { here is } \\
\text { lesions or } \\
\text { patients }\end{array}$ & Recurrence & $7 / 231(3)$ & & & $\mathrm{n}$ & \\
\hline $\begin{array}{l}\text { Chernosky } \\
1978 \\
663726\end{array}$ & 1898 & $\begin{array}{l}\mathrm{BCC}+ \\
\mathrm{SCC}\end{array}$ & $\begin{array}{l}\text { A. Surgical } \\
\text { Excision without } \\
\text { interoperative } \\
\text { evaluation }\end{array}$ & & Recurrence & & $\begin{array}{l}6 / 494 \\
(1.21)\end{array}$ & $\begin{array}{l}\text { A vs. D1: } \\
0.47(0.17 \\
1.31)\end{array}$ & $\mathrm{n}$ & \\
\hline $\begin{array}{l}\text { Chernosky } \\
1978 \\
663726 \\
\end{array}$ & 1898 & $\begin{array}{l}\mathrm{BCC}+ \\
\mathrm{SCC}\end{array}$ & $\begin{array}{l}\text { D1. External } \\
\text { beam radiation }\end{array}$ & & Recurrence & & $\begin{array}{l}10 / 395 \\
(2.53)\end{array}$ & $\begin{array}{l}\text { D1 vs. C2: } \\
1.53(0.77, \\
3.07) \\
\end{array}$ & $\mathrm{n}$ & \\
\hline $\begin{array}{l}\text { Chernosky } \\
1978 \\
663726 \\
\end{array}$ & 1898 & $\begin{array}{l}\text { BCC + } \\
\text { SCC }\end{array}$ & $\begin{array}{l}\text { C2. } \\
\text { Diathermy/electr } \\
\text { odessication }\end{array}$ & & Recurrence & & $\begin{array}{l}46 / 2763 \\
(1.66)\end{array}$ & $\begin{array}{l}\text { A vs. C2: } \\
0.73(0.31 \\
1.71) \\
\end{array}$ & $\mathrm{n}$ & \\
\hline $\begin{array}{l}\text { Mazeron } \\
1988 \\
3146781 \\
\end{array}$ & 1326 & $\begin{array}{l}\text { BCC + } \\
\text { SCC (nose) }\end{array}$ & $\begin{array}{l}\text { D2. } \\
\text { Brachytherapy/PI } \\
\text { eisiotherapy }\end{array}$ & $\begin{array}{l}\text { interstitial } \\
\text { implantation }\end{array}$ & Recurrence & & $\begin{array}{l}19 / 578 \\
(3.3)\end{array}$ & $\begin{array}{l}\text { D2 vs. D1 } \\
\text { (ortho): } 0.68 \\
(0.38,1.21)\end{array}$ & $y$ & $\begin{array}{l}\text { cosmetic } \\
\text { outcomes }\end{array}$ \\
\hline
\end{tabular}




\begin{tabular}{|c|c|c|c|c|c|c|c|c|c|c|}
\hline $\begin{array}{l}\text { Author } \\
\text { year PMID }\end{array}$ & $\begin{array}{l}\text { Total N } \\
\text { (patients) }\end{array}$ & $\begin{array}{l}\text { Lesion } \\
\text { type } \\
\text { (location) }\end{array}$ & Treatment & $\begin{array}{l}\text { Treatment } \\
\text { notes }\end{array}$ & $\begin{array}{l}\text { Outcome } \\
\text { (lack of } \\
\text { clearancel } \\
\text { recurrence) }\end{array}$ & $\begin{array}{l}\text { n patients } \\
\text { (in arm)/N } \\
\text { patients } \\
\text { (in arm) } \\
\text { (\% } \\
\text { patients) }\end{array}$ & $\begin{array}{l}\text { n lesions } \\
\text { (arm)/N } \\
\text { lesions } \\
\text { (in arm) } \\
\text { (\% } \\
\text { lesions) }\end{array}$ & $\begin{array}{l}\text { Odds } \\
\text { Ratio (95\% } \\
\text { Cl) }\end{array}$ & $\begin{array}{l}\text { Adverse } \\
\text { events } \\
\text { reported } \\
(y / n)\end{array}$ & $\begin{array}{l}\text { Aditional } \\
\text { outcomes } \\
\text { reported }\end{array}$ \\
\hline $\begin{array}{l}\text { Mazeron } \\
1988 \\
3146781\end{array}$ & 1326 & $\begin{array}{l}\text { BCC + } \\
\text { SCC (nose) }\end{array}$ & $\begin{array}{l}\text { D1. External } \\
\text { beam radiation }\end{array}$ & orthovoltage & Recurrence & & $\begin{array}{l}31 / 648 \\
(4.7)\end{array}$ & $\begin{array}{l}\text { D1 (ortho) } \\
\text { vs. D1 } \\
\text { (mega): } 0.21 \\
(0.12,0.4)\end{array}$ & $y$ & $\begin{array}{l}\text { cosmetic } \\
\text { outcomes }\end{array}$ \\
\hline $\begin{array}{l}\text { Mazeron } \\
1988 \\
3146781 \\
\end{array}$ & 1326 & $\begin{array}{l}\text { BCC + } \\
\text { SCC (nose) }\end{array}$ & $\begin{array}{l}\text { D1. External } \\
\text { beam radiation }\end{array}$ & megavoltage & Recurrence & & 19/100 (19) & $\begin{array}{l}D 2 \text { vs. } D 1 \\
\text { (mega): } 0.14 \\
(0.07,0.29)\end{array}$ & $y$ & $\begin{array}{l}\text { cosmetic } \\
\text { outcomes }\end{array}$ \\
\hline $\begin{array}{l}\text { Knox } 1967 \\
6020491\end{array}$ & 1417 & BCC & $\begin{array}{l}\text { D1. External } \\
\text { beam radiation }\end{array}$ & xray & Lack of cure & & $7 / 144$ (4.8) & $\begin{array}{l}\text { A vs. D1: } \\
0.66(0.25 \\
1.73)\end{array}$ & $\mathrm{n}$ & \\
\hline $\begin{array}{l}\text { Knox } 1967 \\
6020491\end{array}$ & 1417 & $\mathrm{BCC}$ & $\begin{array}{l}\text { A. Surgical } \\
\text { Excision without } \\
\text { interoperative } \\
\text { evaluation }\end{array}$ & & Lack of cure & & $\begin{array}{l}11 / 339 \\
(3.2)\end{array}$ & $\begin{array}{l}\text { A vs. C2: } \\
1.84(0.85 \\
3.96)\end{array}$ & $\mathrm{n}$ & \\
\hline $\begin{array}{l}\text { Knox } 1967 \\
6020491\end{array}$ & 1417 & $\mathrm{BCC}$ & $\begin{array}{l}\text { C2. } \\
\text { Diathermy/electr } \\
\text { odessication }\end{array}$ & & Lack of cure & & $\begin{array}{l}17 / 948 \\
(1.8)\end{array}$ & $\begin{array}{l}\text { D1 vs. C2: } \\
2.8(1.14 \\
6.87)\end{array}$ & $\mathrm{n}$ & \\
\hline $\begin{array}{l}\text { Knox } 1967 \\
6020491\end{array}$ & 1417 & SCC & $\begin{array}{l}\text { D1. External } \\
\text { beam radiation }\end{array}$ & xray & Lack of cure & & $8 / 101(7.9)$ & $\begin{array}{l}\text { A vs. D1: } \\
0.52(0.19 \\
1.38)\end{array}$ & $\mathrm{n}$ & \\
\hline $\begin{array}{l}\text { Knox } 1967 \\
6020491\end{array}$ & 1417 & SCC & $\begin{array}{l}\text { A. Surgical } \\
\text { Excision without } \\
\text { interoperative } \\
\text { evaluation }\end{array}$ & & Lack of cure & & 9/211 (4.3) & $\begin{array}{l}\text { A vS. C2: } \\
3.42 \text { (1.26, } \\
9.32)\end{array}$ & $\mathrm{n}$ & \\
\hline $\begin{array}{l}\text { Knox } 1967 \\
6020491\end{array}$ & 1417 & SCC & $\begin{array}{l}\mathrm{C} 2 . \\
\text { Diathermy/electr } \\
\text { odessication }\end{array}$ & & Lack of cure & & $7 / 545$ (1.3) & $\begin{array}{l}\text { D1 vS. C2: } \\
6.61 \text { (2.34, } \\
18.67)\end{array}$ & $\mathrm{n}$ & \\
\hline $\begin{array}{l}\text { Tourli } 2016 \\
26870972\end{array}$ & 1380 & $\begin{array}{l}\text { BCC (head } \\
\text { and neck } \\
\text { region) }\end{array}$ & $\begin{array}{l}\text { A. Surgical } \\
\text { Excision without } \\
\text { interoperative } \\
\text { evaluation }\end{array}$ & wide excision & Recurrence & & $5 / 380$ (1.4) & $\begin{array}{l}A \text { vs } B: 5.65 \\
(1.34,23.75)\end{array}$ & $\mathrm{n}$ & \\
\hline $\begin{array}{l}\text { Tourli } 2016 \\
26870972\end{array}$ & 1380 & $\begin{array}{l}\text { BCC (head } \\
\text { and neck } \\
\text { region) }\end{array}$ & $\begin{array}{l}\text { B. Surgical } \\
\text { Excision with } \\
\text { interoperative } \\
\text { evaluation }\end{array}$ & delayed Mohs & Recurrence & & $\begin{array}{l}3 / 1274 \\
(0.23)\end{array}$ & & $\mathrm{n}$ & \\
\hline $\begin{array}{l}\text { Ashby } 1989 \\
2702595\end{array}$ & 1154 & $\begin{array}{l}\mathrm{BCC}+ \\
\mathrm{SCC}\end{array}$ & $\begin{array}{l}\text { A. Surgical } \\
\text { Excision without }\end{array}$ & & Lack of cure & $\begin{array}{l}18 / 614 \\
(2.9) \\
\end{array}$ & & $\begin{array}{l}\text { A vs. D1: } \\
0.46(0.25,\end{array}$ & $\mathrm{n}$ & \\
\hline
\end{tabular}




\begin{tabular}{|c|c|c|c|c|c|c|c|c|c|c|}
\hline $\begin{array}{l}\text { Author } \\
\text { year PMID }\end{array}$ & $\begin{array}{l}\text { Total N } \\
\text { (patients) }\end{array}$ & $\begin{array}{l}\text { Lesion } \\
\text { type } \\
\text { (location) }\end{array}$ & Treatment & $\begin{array}{l}\text { Treatment } \\
\text { notes }\end{array}$ & $\begin{array}{l}\text { Outcome } \\
\text { (lack of } \\
\text { clearancel } \\
\text { recurrence) }\end{array}$ & $\begin{array}{l}\text { n patients } \\
\text { (in arm)/N } \\
\text { patients } \\
\text { (in arm) } \\
\text { (\% } \\
\text { patients) }\end{array}$ & $\begin{array}{l}\text { n lesions } \\
\text { (arm)/N } \\
\text { lesions } \\
\text { (in arm) } \\
\text { (\% } \\
\text { lesions) }\end{array}$ & $\begin{array}{l}\text { Odds } \\
\text { Ratio (95\% } \\
\text { Cl) }\end{array}$ & $\begin{array}{l}\text { Adverse } \\
\text { events } \\
\text { reported } \\
(y / n)\end{array}$ & $\begin{array}{l}\text { Aditional } \\
\text { outcomes } \\
\text { reported }\end{array}$ \\
\hline & & & $\begin{array}{l}\text { interoperative } \\
\text { evaluation }\end{array}$ & & & & & $0.83)$ & & \\
\hline $\begin{array}{l}\text { Ashby } 1989 \\
2702595\end{array}$ & 1154 & $\begin{array}{l}\mathrm{BCC}+ \\
\mathrm{SCC}\end{array}$ & $\begin{array}{l}\text { D1. External } \\
\text { beam radiation }\end{array}$ & & Lack of cure & $\begin{array}{l}30 / 482 \\
(6.2)\end{array}$ & & $\begin{array}{l}\text { D1 vs. C1: } \\
0.73(0.09 \\
5.85)\end{array}$ & $\mathrm{n}$ & \\
\hline $\begin{array}{l}\text { Ashby } 1989 \\
2702595\end{array}$ & 1154 & $\begin{array}{l}\mathrm{BCC}+ \\
\mathrm{SCC}\end{array}$ & C1. Cryotherapy & & Lack of cure & $1 / 12(8.3)$ & & $\begin{array}{l}\text { A vs. C1: } \\
0.33(0.04 \\
2.71)\end{array}$ & $\mathrm{n}$ & \\
\hline $\begin{array}{l}\text { Futoryan } \\
1995 \\
7773598\end{array}$ & 1047 & $\begin{array}{l}\mathrm{BCC}+ \\
\mathrm{SCC}\end{array}$ & $\begin{array}{l}\text { B. Surgical } \\
\text { Excision with } \\
\text { interoperative } \\
\text { evaluation }\end{array}$ & & infection & & $\begin{array}{l}13 / 530 \\
(2.5)\end{array}$ & $\begin{array}{l}\text { A vs. B: } 0.86 \\
(0.38,1.95)\end{array}$ & $y$ & \\
\hline $\begin{array}{l}\text { Futoryan } \\
1995 \\
7773598\end{array}$ & 1047 & $\begin{array}{l}\mathrm{BCC}+ \\
\mathrm{SCC}\end{array}$ & $\begin{array}{l}\text { A. Surgical } \\
\text { Excision without } \\
\text { interoperative } \\
\text { evaluation }\end{array}$ & & infection & & $\begin{array}{l}11 / 517 \\
(2.1)\end{array}$ & & $y$ & \\
\hline $\begin{array}{l}\text { Honeycutt } \\
1973 \\
4750203 \\
\end{array}$ & 484 & SCC & $\begin{array}{l}\text { C2. } \\
\text { Diathermy/electr } \\
\text { odessication }\end{array}$ & & Recurrence & & $3 / 281$ (1.1) & & $\mathrm{n}$ & \\
\hline $\begin{array}{l}\text { Honeycutt } \\
1973 \\
4750203 \\
\end{array}$ & 484 & SCC & $\begin{array}{l}\text { D1. External } \\
\text { beam radiation }\end{array}$ & & Recurrence & & $0 / 18(0)$ & & $\mathrm{n}$ & \\
\hline $\begin{array}{l}\text { Honeycutt } \\
1973 \\
4750203 \\
\end{array}$ & 484 & SCC lip & $\begin{array}{l}\text { C2. } \\
\text { Diathermy/electr } \\
\text { odessication }\end{array}$ & & Recurrence & & $3 / 29(10.3)$ & $\begin{array}{l}\text { A vs. C2: } \\
1.24(0.18, \\
8.31)\end{array}$ & $\mathrm{n}$ & \\
\hline $\begin{array}{l}\text { Honeycutt } \\
1973 \\
4750203\end{array}$ & 484 & SCC lip & $\begin{array}{l}\text { A. Surgical } \\
\text { Excision without } \\
\text { interoperative } \\
\text { evaluation }\end{array}$ & & Recurrence & & 2/16 (12.5) & & $\mathrm{n}$ & \\
\hline $\begin{array}{l}\text { Jebodhsingh } \\
2012 \\
22560426\end{array}$ & 385 & $\begin{array}{l}\text { BCC } \\
\text { (periocular) }\end{array}$ & $\begin{array}{l}\text { A. Surgical } \\
\text { Excision without } \\
\text { interoperative } \\
\text { evaluation }\end{array}$ & & Recurrence & $51 / 346(15)$ & & $\begin{array}{l}\text { A vs. B: } 2.31 \\
(0.69,7.73)\end{array}$ & $\mathrm{n}$ & \\
\hline $\begin{array}{l}\text { Jebodhsingh } \\
2012 \\
22560426\end{array}$ & 385 & $\begin{array}{l}\text { BCC } \\
\text { (periocular) }\end{array}$ & $\begin{array}{l}\text { B. Surgical } \\
\text { Excision with } \\
\text { interoperative } \\
\text { evaluation }\end{array}$ & Mohs & Recurrence & $3 / 43(8)$ & & & $\mathrm{n}$ & \\
\hline
\end{tabular}




\begin{tabular}{|c|c|c|c|c|c|c|c|c|c|c|}
\hline $\begin{array}{l}\text { Author } \\
\text { year PMID }\end{array}$ & $\begin{array}{l}\text { Total N } \\
\text { (patients) }\end{array}$ & $\begin{array}{l}\text { Lesion } \\
\text { type } \\
\text { (location) }\end{array}$ & Treatment & $\begin{array}{l}\text { Treatment } \\
\text { notes }\end{array}$ & $\begin{array}{l}\text { Outcome } \\
\text { (lack of } \\
\text { clearancel } \\
\text { recurrence) }\end{array}$ & $\begin{array}{l}\text { n patients } \\
\text { (in arm)/N } \\
\text { patients } \\
\text { (in arm) } \\
\text { (\% } \\
\text { patients) }\end{array}$ & $\begin{array}{l}\text { n lesions } \\
\text { (arm)/N } \\
\text { lesions } \\
\text { (in arm) } \\
\text { (\% } \\
\text { lesions) }\end{array}$ & $\begin{array}{l}\text { Odds } \\
\text { Ratio (95\% } \\
\text { Cl) }\end{array}$ & $\begin{array}{l}\text { Adverse } \\
\text { events } \\
\text { reported } \\
(y / n)\end{array}$ & $\begin{array}{l}\text { Aditional } \\
\text { outcomes } \\
\text { reported }\end{array}$ \\
\hline $\begin{array}{l}\text { Van } \\
\text { Hezewijk } \\
2010\end{array}$ & 333 & $\begin{array}{l}\text { BCC + } \\
\text { SCC }\end{array}$ & $\begin{array}{l}\text { D1. External } \\
\text { beam radiation }\end{array}$ & 54 gy & Recurrence & & $5 / 159(3.1)$ & $\begin{array}{l}\text { D1 (high } \\
\text { dose) vs. D1 } \\
\text { (low dose): } \\
0.86(0.29, \\
2.56)\end{array}$ & $\mathrm{n}$ & $\begin{array}{l}\text { cosmetic } \\
\text { outcomes }\end{array}$ \\
\hline $\begin{array}{l}\text { Van } \\
\text { Hezewijk } \\
2010 \\
\end{array}$ & 333 & $\begin{array}{l}\mathrm{BCC}+ \\
\mathrm{SCC}\end{array}$ & $\begin{array}{l}\text { D1. External } \\
\text { beam radiation }\end{array}$ & 44 gy & Recurrence & & $\begin{array}{l}10 / 275 \\
(3.6)\end{array}$ & & $\mathrm{n}$ & $\begin{array}{l}\text { cosmetic } \\
\text { outcomes }\end{array}$ \\
\hline $\begin{array}{l}\text { Hansen } \\
2008 \\
18363722\end{array}$ & 298 & Bowen's & $\begin{array}{l}\text { A. Surgical } \\
\text { Excision without } \\
\text { interoperative } \\
\text { evaluation }\end{array}$ & $\begin{array}{l}\text { elliptical or } \\
\text { shave } \\
\text { excision }\end{array}$ & Recurrence & & $8 / 188(4.3)$ & & $\mathrm{n}$ & \\
\hline $\begin{array}{l}\text { Hansen } \\
2008 \\
18363722 \\
\end{array}$ & 298 & Bowen's & C1. Cryotherapy & & Recurrence & & $2 / 24(8.3)$ & & $\mathrm{n}$ & \\
\hline $\begin{array}{l}\text { Hansen } \\
2008 \\
18363722 \\
\end{array}$ & 298 & Bowen's & $\begin{array}{l}\text { C3. Curettage + } \\
\text { diathermy }\end{array}$ & $\begin{array}{l}\text { Curettage } \\
\text { and } \\
\text { fulgaration }\end{array}$ & Recurrence & & $2 / 46(4.3)$ & & $\mathrm{n}$ & \\
\hline $\begin{array}{l}\text { Hansen } \\
2008 \\
18363722\end{array}$ & 298 & Bowen's & $\begin{array}{l}\text { F1. Topical or } \\
\text { intralesional 5- } \\
\text { FU...Define: }\end{array}$ & topical & Recurrence & & $1 / 24(4.2)$ & & $\mathrm{n}$ & \\
\hline $\begin{array}{l}\text { Hansen } \\
2008 \\
18363722\end{array}$ & 298 & Bowen's & $\begin{array}{l}\text { B. Surgical } \\
\text { Excision with } \\
\text { interoperative } \\
\text { evaluation }\end{array}$ & Mohs & Recurrence & & $2 / 83(2.4)$ & & $\mathrm{n}$ & \\
\hline $\begin{array}{l}\text { Hansen } \\
2008 \\
18363722 \\
\end{array}$ & 298 & Bowen's & $\begin{array}{l}\text { C2. } \\
\text { Diathermy/electr } \\
\text { odessication }\end{array}$ & & Recurrence & & $0 / 16(0)$ & & $\mathrm{n}$ & \\
\hline $\begin{array}{l}\text { Hansen } \\
2008 \\
18363722\end{array}$ & 298 & Bowen's & $\begin{array}{l}\text { F2. Topical or } \\
\text { intralesional } \\
\text { Imiquimod...Defin } \\
\text { e: }\end{array}$ & topical & Recurrence & & $0 / 7(0)$ & & $\mathrm{n}$ & \\
\hline $\begin{array}{l}\text { Pereira } 2013 \\
23486132\end{array}$ & 289 & NMSC & $\begin{array}{l}\text { A. Surgical } \\
\text { Excision without } \\
\text { interoperative } \\
\text { evaluation }\end{array}$ & & Recurrence & 29/289 (10) & & $\begin{array}{l}\text { A vs. B: } 3.61 \\
(1.47,8.86)\end{array}$ & $\mathrm{n}$ & \\
\hline Pereira 2013 & 289 & NMSC & B. Surgical & Mohs & Recurrence & $6 / 200(3)$ & & & $\mathrm{n}$ & \\
\hline
\end{tabular}




\begin{tabular}{|c|c|c|c|c|c|c|c|c|c|c|}
\hline $\begin{array}{l}\text { Author } \\
\text { year PMID }\end{array}$ & $\begin{array}{l}\text { Total N } \\
\text { (patients) }\end{array}$ & $\begin{array}{l}\text { Lesion } \\
\text { type } \\
\text { (location) }\end{array}$ & Treatment & $\begin{array}{l}\text { Treatment } \\
\text { notes }\end{array}$ & $\begin{array}{l}\text { Outcome } \\
\text { (lack of } \\
\text { clearancel } \\
\text { recurrence) }\end{array}$ & $\begin{array}{l}\text { n patients } \\
\text { (in arm)/N } \\
\text { patients } \\
\text { (in arm) } \\
\text { (\% } \\
\text { patients) }\end{array}$ & $\begin{array}{l}\text { n lesions } \\
\text { (arm)/N } \\
\text { lesions } \\
\text { (in arm) } \\
\text { (\% } \\
\text { lesions) }\end{array}$ & $\begin{array}{l}\text { Odds } \\
\text { Ratio (95\% } \\
\text { Cl) }\end{array}$ & $\begin{array}{l}\text { Adverse } \\
\text { events } \\
\text { reported } \\
\text { (y/n) }\end{array}$ & $\begin{array}{l}\text { Aditional } \\
\text { outcomes } \\
\text { reported }\end{array}$ \\
\hline 23486132 & & & $\begin{array}{l}\text { Excision with } \\
\text { interoperative } \\
\text { evaluation }\end{array}$ & & & & & & & \\
\hline $\begin{array}{l}\text { Nevrkla } \\
1974 \\
4425623\end{array}$ & 200 & $\mathrm{BCC}$ & $\begin{array}{l}\text { A. Surgical } \\
\text { Excision without } \\
\text { interoperative } \\
\text { evaluation }\end{array}$ & & Recurrence & $1 / 35(2.9)$ & & $\begin{array}{l}\text { A vs. D1: } \\
0.44(0.05 \\
3.68)\end{array}$ & $y$ & \\
\hline $\begin{array}{l}\text { Nevrkla } \\
1974 \\
4425623 \\
\end{array}$ & 200 & $\mathrm{BCC}$ & $\begin{array}{l}\text { D1. External } \\
\text { beam radiation }\end{array}$ & $\begin{array}{l}\text { low-voltage } x- \\
\text { rays }\end{array}$ & Recurrence & $8 / 129(6.2)$ & & $\begin{array}{l}\text { D1 vs D2: } \\
0.73(0.18 \\
2.9)\end{array}$ & $y$ & \\
\hline $\begin{array}{l}\text { Nevrkla } \\
1974 \\
4425623\end{array}$ & 200 & $\mathrm{BCC}$ & $\begin{array}{l}\text { D2. } \\
\text { Brachytherapy/PI } \\
\text { eisiotherapy }\end{array}$ & $\begin{array}{l}\text { implant of } \\
\text { radium } \\
\text { needles or } \\
\text { radon seeds }\end{array}$ & Recurrence & $3 / 36(8.3)$ & & $\begin{array}{l}\text { A vs. D2: } \\
0.32(0.03 \\
3.27)\end{array}$ & $y$ & \\
\hline $\begin{array}{l}\text { Werlinger } \\
2002 \\
12472494\end{array}$ & 191 & SCC & $\begin{array}{l}\text { A. Surgical } \\
\text { Excision without } \\
\text { interoperative } \\
\text { evaluation }\end{array}$ & & Recurrence & & $0 / 20(0)$ & & $\mathrm{n}$ & \\
\hline $\begin{array}{l}\text { Werlinger } \\
2002 \\
12472494 \\
\end{array}$ & 191 & SCC & $\begin{array}{l}\text { C3. Curettage + } \\
\text { diathermy }\end{array}$ & $\begin{array}{l}\text { Curettage \& } \\
\text { Desiccation }\end{array}$ & Recurrence & & $2 / 56(3.6)$ & & $\mathrm{n}$ & \\
\hline $\begin{array}{l}\text { Werlinger } \\
2002 \\
12472494\end{array}$ & 191 & $\mathrm{BCC}$ & $\begin{array}{l}\text { A. Surgical } \\
\text { Excision without } \\
\text { interoperative } \\
\text { evaluation }\end{array}$ & & Recurrence & & $1 / 90(1.1)$ & $\begin{array}{l}\text { A vs. C3: } \\
0.37(0.04, \\
3.63)\end{array}$ & $\mathrm{n}$ & \\
\hline $\begin{array}{l}\text { Werlinger } \\
2002 \\
12472494 \\
\end{array}$ & 191 & $\mathrm{BCC}$ & $\begin{array}{l}\text { C3. Curettage + } \\
\text { diathermy }\end{array}$ & $\begin{array}{l}\text { Curettage \& } \\
\text { Desiccation }\end{array}$ & Recurrence & & $3 / 102(2.9)$ & & $\mathrm{n}$ & \\
\hline $\begin{array}{l}\text { Mclntosh } \\
1983 \\
6647186\end{array}$ & 186 & $\mathrm{BCC}$ & $\begin{array}{l}\text { A. Surgical } \\
\text { Excision without } \\
\text { interoperative } \\
\text { evaluation }\end{array}$ & & Recurrence & & $5 / 62(8.1)$ & $\begin{array}{l}\text { A vs. D1: } \\
4.25(0.8 \\
22.65)\end{array}$ & $\mathrm{n}$ & \\
\hline $\begin{array}{l}\text { McIntosh } \\
1983 \\
6647186 \\
\end{array}$ & 186 & $\mathrm{BCC}$ & $\begin{array}{l}\text { D1. External } \\
\text { beam radiation }\end{array}$ & & Recurrence & & 2/99 (2) & $\begin{array}{l}\text { D1 vs. C1: } \\
0.33(0.04 \\
2.44)\end{array}$ & $\mathrm{n}$ & \\
\hline $\begin{array}{l}\text { McIntosh } \\
1983\end{array}$ & 186 & $\mathrm{BCC}$ & C1. Cryotherapy & & Recurrence & & $2 / 34(5.9)$ & $\begin{array}{l}\text { A vs. C1: } 1.4 \\
(0.26,7.65)\end{array}$ & $\mathrm{n}$ & \\
\hline
\end{tabular}




\begin{tabular}{|c|c|c|c|c|c|c|c|c|c|c|}
\hline $\begin{array}{l}\text { Author } \\
\text { year PMID }\end{array}$ & $\begin{array}{l}\text { Total N } \\
\text { (patients) }\end{array}$ & $\begin{array}{l}\text { Lesion } \\
\text { type } \\
\text { (location) }\end{array}$ & Treatment & $\begin{array}{l}\text { Treatment } \\
\text { notes }\end{array}$ & $\begin{array}{l}\text { Outcome } \\
\text { (lack of } \\
\text { clearancel } \\
\text { recurrence) }\end{array}$ & $\begin{array}{l}\text { n patients } \\
\text { (in arm)/N } \\
\text { patients } \\
\text { (in arm) } \\
\text { (\% } \\
\text { patients) }\end{array}$ & $\begin{array}{l}\text { n lesions } \\
\text { (arm)/N } \\
\text { lesions } \\
\text { (in arm) } \\
\text { (\% } \\
\text { lesions) }\end{array}$ & $\begin{array}{l}\text { Odds } \\
\text { Ratio (95\% } \\
\text { Cl) }\end{array}$ & $\begin{array}{l}\text { Adverse } \\
\text { events } \\
\text { reported } \\
\text { (y/n) }\end{array}$ & $\begin{array}{l}\text { Aditional } \\
\text { outcomes } \\
\text { reported }\end{array}$ \\
\hline \multicolumn{11}{|l|}{6647186} \\
\hline $\begin{array}{l}\text { Harrison } \\
1987 \\
3676083\end{array}$ & 123 & $\mathrm{BCC}$ & $\begin{array}{l}\text { A. Surgical } \\
\text { Excision without } \\
\text { interoperative } \\
\text { evaluation }\end{array}$ & & Recurrence & $2 / 15(13.3)$ & & $\begin{array}{l}\text { A vs. C3: } \\
1.85(0.15 \\
23.07)\end{array}$ & $y$ & \\
\hline $\begin{array}{l}\text { Harrison } \\
1987 \\
3676083 \\
\end{array}$ & 123 & $\mathrm{BCC}$ & $\begin{array}{l}\text { C3. Curettage + } \\
\text { diathermy }\end{array}$ & $\begin{array}{l}\text { Curettage } \\
\text { and cautery }\end{array}$ & Recurrence & $1 / 13(7.7)$ & & & $y$ & \\
\hline $\begin{array}{l}\text { Mebed } 2010 \\
21503006\end{array}$ & 120 & $\mathrm{BCC}+\mathrm{SCC}$ & $\begin{array}{l}\text { A. Surgical } \\
\text { Excision without } \\
\text { interoperative } \\
\text { evaluation }\end{array}$ & $\begin{array}{l}\text { with and } \\
\text { without } \\
\text { adjuvant } \\
\text { radiotherapy }\end{array}$ & Recurrence & 2/103 (1.9) & & & $\mathrm{y}$ & \\
\hline $\begin{array}{l}\text { Mebed } 2010 \\
21503006\end{array}$ & 120 & $\mathrm{BCC}+\mathrm{SCC}$ & $\begin{array}{l}\text { F3. Topical or } \\
\text { intralesional } \\
\text { Interferon } \\
\text { (INF)...Define: }\end{array}$ & intralesional & Recurrence & $3 / 7(42.9)$ & & & $y$ & \\
\hline $\begin{array}{l}\text { Mebed } 2010 \\
21503006 \\
\end{array}$ & 120 & $\mathrm{BCC}+\mathrm{SCC}$ & $\begin{array}{l}\text { D1. External } \\
\text { beam radiation }\end{array}$ & & Recurrence & $0 / 8(0)$ & & & $y$ & \\
\hline $\begin{array}{l}\text { Tarstedt } \\
2016 \\
26841041 \\
\end{array}$ & 116 & Bowen's & $\begin{array}{l}\text { E1. PDT: MAL + } \\
\text { red } \\
\text { light...Define: }\end{array}$ & & $\begin{array}{l}\text { Lack of clinical } \\
\text { clearance }\end{array}$ & & $4 / 18(22.2)$ & $\begin{array}{l}\text { E1 vs. E2: } \\
2.29(0.22, \\
24.14)\end{array}$ & $\mathrm{n}$ & \\
\hline $\begin{array}{l}\text { Tarstedt } \\
2016 \\
26841041 \\
\end{array}$ & 116 & Bowen's & $\begin{array}{l}\text { E2. PDT: ALA + } \\
\text { blue } \\
\text { light...Define: }\end{array}$ & & $\begin{array}{l}\text { Lack of clinical } \\
\text { clearance }\end{array}$ & & $1 / 9(11.1)$ & & $\mathrm{n}$ & \\
\hline $\begin{array}{l}\text { Tarstedt } \\
2016 \\
26841041 \\
\end{array}$ & 116 & nodal BCC & $\begin{array}{l}\text { E1. PDT: MAL + } \\
\text { red } \\
\text { light...Define: }\end{array}$ & & $\begin{array}{l}\text { Lack of clinical } \\
\text { clearance }\end{array}$ & & $4 / 25(16)$ & $\begin{array}{l}\text { E1 vs. E2: } \\
1.02(0.2, \\
5.2)\end{array}$ & $\mathrm{n}$ & \\
\hline $\begin{array}{l}\text { Tarstedt } \\
2016 \\
26841041 \\
\end{array}$ & 116 & nodal BCC & $\begin{array}{l}\text { E2. PDT: ALA + } \\
\text { blue } \\
\text { light...Define: }\end{array}$ & & $\begin{array}{l}\text { Lack of clinical } \\
\text { clearance }\end{array}$ & & $3 / 19$ (15.8) & & $\mathrm{n}$ & \\
\hline $\begin{array}{l}\text { Tarstedt } \\
2016 \\
26841041 \\
\end{array}$ & 116 & $\begin{array}{l}\text { superficial } \\
\text { BCC }\end{array}$ & $\begin{array}{l}\text { E1. PDT: MAL + } \\
\text { red } \\
\text { light...Define: }\end{array}$ & & $\begin{array}{l}\text { Lack of clinical } \\
\text { clearance }\end{array}$ & & $5 / 39(12.8)$ & $\begin{array}{l}\text { E1 vs. E2: } \\
1.08(0.23 \\
4.97)\end{array}$ & $n$ & \\
\hline $\begin{array}{l}\text { Tarstedt } \\
2016 \\
26841041 \\
\end{array}$ & 116 & $\begin{array}{l}\text { superficial } \\
\text { BCC }\end{array}$ & $\begin{array}{l}\text { E2. PDT: ALA + } \\
\text { blue } \\
\text { light...Define: }\end{array}$ & & $\begin{array}{l}\text { Lack of clinical } \\
\text { clearance }\end{array}$ & & $3 / 25$ (12) & & $n$ & \\
\hline
\end{tabular}




\begin{tabular}{|c|c|c|c|c|c|c|c|c|c|c|}
\hline $\begin{array}{l}\text { Author } \\
\text { year PMID }\end{array}$ & $\begin{array}{l}\text { Total N } \\
\text { (patients) }\end{array}$ & $\begin{array}{l}\text { Lesion } \\
\text { type } \\
\text { (location) }\end{array}$ & Treatment & $\begin{array}{l}\text { Treatment } \\
\text { notes }\end{array}$ & $\begin{array}{l}\text { Outcome } \\
\text { (lack of } \\
\text { clearancel } \\
\text { recurrence) }\end{array}$ & $\begin{array}{l}\text { n patients } \\
\text { (in arm)/N } \\
\text { patients } \\
\text { (in arm) } \\
\text { (\% } \\
\text { patients) }\end{array}$ & $\begin{array}{l}\text { n lesions } \\
\text { (arm)/N } \\
\text { lesions } \\
\text { (in arm) } \\
\text { (\% } \\
\text { lesions) }\end{array}$ & $\begin{array}{l}\text { Odds } \\
\text { Ratio (95\% } \\
\text { Cl) }\end{array}$ & $\begin{array}{l}\text { Adverse } \\
\text { events } \\
\text { reported } \\
(y / n)\end{array}$ & $\begin{array}{l}\text { Aditional } \\
\text { outcomes } \\
\text { reported }\end{array}$ \\
\hline $\begin{array}{l}\text { Avila } 1977 \\
589557\end{array}$ & 97 & $\begin{array}{l}\mathrm{BCC}+\mathrm{SCC} \\
\text { (pinna) }\end{array}$ & $\begin{array}{l}\text { A. Surgical } \\
\text { Excision without } \\
\text { interoperative } \\
\text { evaluation }\end{array}$ & & Recurrence & $2 / 50(4)$ & & $\begin{array}{l}\text { A vs. D1: } \\
0.27(0.05 \\
1.42)\end{array}$ & $y$ & \\
\hline $\begin{array}{l}\text { Avila } 1977 \\
589557\end{array}$ & 97 & $\begin{array}{l}\mathrm{BCC}+\mathrm{SCC} \\
\text { (pinna) }\end{array}$ & $\begin{array}{l}\text { D1. External } \\
\text { beam radiation }\end{array}$ & x-rays & Recurrence & $6 / 45(13.3)$ & & & $y$ & \\
\hline Wang 2016 & 95 & BCC & $\begin{array}{l}\text { E3. PDT other } \\
\text { (specify)...Define: }\end{array}$ & $\begin{array}{l}\text { PDT } \\
\text { combined } \\
\text { with the } \\
\text { application of } \\
\text { the topical } \\
\text { photosensitiz } \\
\text { er ALA and } \\
\text { systemic } \\
\text { light-sensitive } \\
\text { drua HPD }\end{array}$ & $\begin{array}{l}\text { Lack of clinical } \\
\text { clearance }\end{array}$ & $0 / 9(0)$ & $0 / 14(0)$ & & $\mathrm{n}$ & \\
\hline Wang 2016 & 95 & $\mathrm{BCC}$ & $\begin{array}{l}\text { E3. PDT other } \\
\text { (specify)...Define: }\end{array}$ & HPD-PDT & $\begin{array}{l}\text { Lack of clinical } \\
\text { clearance }\end{array}$ & $2 / 13(15.4)$ & $2 / 13(15.4)$ & & $\mathrm{n}$ & \\
\hline Wang 2016 & 95 & $\mathrm{BCC}$ & $\begin{array}{l}\text { E2. PDT: ALA + } \\
\text { blue } \\
\text { light...Define: }\end{array}$ & $\begin{array}{l}\text { ALA-PDT } \\
\text { following CO2 } \\
\text { laser } \\
\text { vaporization }\end{array}$ & $\begin{array}{l}\text { Lack of clinical } \\
\text { clearance }\end{array}$ & $0 / 14(0)$ & $0 / 14(0)$ & & $\mathrm{n}$ & \\
\hline Wang 2016 & 95 & SCC & $\begin{array}{l}\text { E3. PDT other } \\
\text { (specify)...Define: }\end{array}$ & $\begin{array}{l}\text { PDT } \\
\text { combined } \\
\text { with the } \\
\text { application of } \\
\text { the topical } \\
\text { photosensitiz } \\
\text { er ALA and } \\
\text { systemic } \\
\text { light-sensitive } \\
\text { drug HPD }\end{array}$ & $\begin{array}{l}\text { Lack of clinical } \\
\text { clearance }\end{array}$ & & $1 / 26(3.8)$ & & $\mathrm{n}$ & \\
\hline Wang 2016 & 95 & SCC & $\begin{array}{l}\text { E3. PDT other } \\
\text { (specify)...Define: }\end{array}$ & HPD-PDT & $\begin{array}{l}\text { Lack of clinical } \\
\text { clearance }\end{array}$ & $0 / 10(0)$ & $0 / 10(0)$ & & $\mathrm{n}$ & \\
\hline Wang 2016 & 95 & SCC & $\begin{array}{l}\text { E2. PDT: ALA + } \\
\text { blue }\end{array}$ & $\begin{array}{l}\text { ALA-PDT } \\
\text { following } \mathrm{CO} 2\end{array}$ & $\begin{array}{l}\text { Lack of clinical } \\
\text { clearance }\end{array}$ & & $2 / 18$ (11.1) & & $\mathrm{n}$ & \\
\hline
\end{tabular}




\begin{tabular}{|c|c|c|c|c|c|c|c|c|c|c|}
\hline $\begin{array}{l}\text { Author } \\
\text { year PMID }\end{array}$ & $\begin{array}{l}\text { Total N } \\
\text { (patients) }\end{array}$ & $\begin{array}{l}\text { Lesion } \\
\text { type } \\
\text { (location) }\end{array}$ & Treatment & $\begin{array}{l}\text { Treatment } \\
\text { notes }\end{array}$ & $\begin{array}{l}\text { Outcome } \\
\text { (lack of } \\
\text { clearancel } \\
\text { recurrence) }\end{array}$ & $\begin{array}{l}\text { n patients } \\
\text { (in arm)/N } \\
\text { patients } \\
\text { (in arm) } \\
\text { (\% } \\
\text { patients) }\end{array}$ & $\begin{array}{l}\text { n lesions } \\
\text { (arm)/N } \\
\text { lesions } \\
\text { (in arm) } \\
\text { (\% } \\
\text { lesions) }\end{array}$ & $\begin{array}{l}\text { Odds } \\
\text { Ratio (95\% } \\
\text { Cl) }\end{array}$ & $\begin{array}{l}\text { Adverse } \\
\text { events } \\
\text { reported } \\
\text { (y/n) }\end{array}$ & $\begin{array}{l}\text { Aditional } \\
\text { outcomes } \\
\text { reported }\end{array}$ \\
\hline & & & light...Define: & $\begin{array}{l}\text { laser } \\
\text { vaporization }\end{array}$ & & & & & & \\
\hline $\begin{array}{l}\text { Cox } 1995 \\
7669642 \\
\end{array}$ & 91 & Bowen's & C1. Cryotherapy & & Recurrence & & $6 / 82(7.3)$ & & $y$ & \\
\hline $\begin{array}{l}\text { Cox } 1995 \\
7669642\end{array}$ & 91 & Bowen's & $\begin{array}{l}\text { D1. External } \\
\text { beam radiation }\end{array}$ & & Recurrence & & $0 / 59(0)$ & & $y$ & \\
\hline $\begin{array}{l}\text { Kowalzick } \\
1994\end{array}$ & 87 & $\mathrm{BCC}$ & $\begin{array}{l}\text { F3. Topical or } \\
\text { intralesional } \\
\text { Interferon } \\
\text { (INF)...Define: }\end{array}$ & $\begin{array}{l}\text { intralesional } 1 \\
\text { MU/weekly } \\
\text { for } 3 \text { weeks }\end{array}$ & $\begin{array}{l}\text { Lack of clinical } \\
\text { clearance }\end{array}$ & $4 / 7(57.1)$ & & & $y$ & \\
\hline $\begin{array}{l}\text { Kowalzick } \\
1994\end{array}$ & 87 & $\mathrm{BCC}$ & $\begin{array}{l}\text { F3. Topical or } \\
\text { intralesional } \\
\text { Interferon } \\
\text { (INF)...Define: }\end{array}$ & $\begin{array}{l}\text { intralesional } 3 \\
\text { MU/weekly } \\
\text { for } 3 \text { weeks }\end{array}$ & $\begin{array}{l}\text { Lack of clinical } \\
\text { clearance }\end{array}$ & $6 / 7(85.7)$ & & & $y$ & \\
\hline $\begin{array}{l}\text { Kowalzick } \\
1994\end{array}$ & 87 & $\mathrm{BCC}$ & $\begin{array}{l}\text { F3. Topical or } \\
\text { intralesional } \\
\text { Interferon } \\
\text { (INF)...Define: }\end{array}$ & $\begin{array}{l}\text { intralesional } \\
0.5 \mathrm{MU} / \text { twice } \\
\text { weekly for } 3 \\
\text { weeks }\end{array}$ & $\begin{array}{l}\text { Lack of clinical } \\
\text { clearance }\end{array}$ & $6 / 7(85.7)$ & & & $y$ & \\
\hline $\begin{array}{l}\text { Kowalzick } \\
1994\end{array}$ & 87 & $\mathrm{BCC}$ & $\begin{array}{l}\text { F3. Topical or } \\
\text { intralesional } \\
\text { Interferon } \\
\text { (INF)...Define: }\end{array}$ & $\begin{array}{l}\text { intralesional } 1 \\
\text { MU/twice } \\
\text { weekly for } 3 \\
\text { weeks }\end{array}$ & $\begin{array}{l}\text { Lack of clinical } \\
\text { clearance }\end{array}$ & $6 / 10(60)$ & & & $y$ & \\
\hline $\begin{array}{l}\text { Kowalzick } \\
1994\end{array}$ & 87 & $\mathrm{BCC}$ & $\begin{array}{l}\text { F3. Topical or } \\
\text { intralesional } \\
\text { Interferon } \\
\text { (INF)...Define: }\end{array}$ & $\begin{array}{l}\text { intralesional } 3 \\
\text { MU/twice } \\
\text { weekly for } 3 \\
\text { weeks }\end{array}$ & $\begin{array}{l}\text { Lack of clinical } \\
\text { clearance }\end{array}$ & $5 / 10(50)$ & & & $y$ & \\
\hline $\begin{array}{l}\text { Kowalzick } \\
1994\end{array}$ & 87 & $\mathrm{BCC}$ & $\begin{array}{l}\text { F3. Topical or } \\
\text { intralesional } \\
\text { Interferon } \\
\text { (INF)...Define: }\end{array}$ & $\begin{array}{l}\text { intralesional } \\
0.5 \mathrm{MU} / \text { three } \\
\text { times weekly } \\
\text { for } 3 \text { weeks }\end{array}$ & $\begin{array}{l}\text { Lack of clinical } \\
\text { clearance }\end{array}$ & $5 / 14(35.7)$ & & & $y$ & \\
\hline $\begin{array}{l}\text { Kowalzick } \\
1994\end{array}$ & 87 & $\mathrm{BCC}$ & $\begin{array}{l}\text { F3. Topical or } \\
\text { intralesional } \\
\text { Interferon } \\
\text { (INF)...Define: }\end{array}$ & $\begin{array}{l}\text { intralesional } 1 \\
\text { MU/three } \\
\text { times weekly } \\
\text { for } 3 \text { weeks }\end{array}$ & $\begin{array}{l}\text { Lack of clinical } \\
\text { clearance }\end{array}$ & 2/14 (14.3) & & & $y$ & \\
\hline $\begin{array}{l}\text { Reschly } \\
2010 \\
20677531 \\
\end{array}$ & 75 & $\begin{array}{l}\text { SCC } \\
\text { (males over } \\
\text { age 60) }\end{array}$ & $\begin{array}{l}\text { C3. Curettage + } \\
\text { diathermy }\end{array}$ & $\begin{array}{l}\text { Curettage \& } \\
\text { Electrodesicc } \\
\text { ation }\end{array}$ & Recurrence & & $0 / 14(0)$ & & $\mathrm{n}$ & \\
\hline
\end{tabular}




\begin{tabular}{|c|c|c|c|c|c|c|c|c|c|c|}
\hline $\begin{array}{l}\text { Author } \\
\text { year PMID }\end{array}$ & $\begin{array}{l}\text { Total N } \\
\text { (patients) }\end{array}$ & $\begin{array}{l}\text { Lesion } \\
\text { type } \\
\text { (location) }\end{array}$ & Treatment & $\begin{array}{l}\text { Treatment } \\
\text { notes }\end{array}$ & $\begin{array}{l}\text { Outcome } \\
\text { (lack of } \\
\text { clearancel } \\
\text { recurrence) }\end{array}$ & $\begin{array}{l}\text { n patients } \\
\text { (in arm)/N } \\
\text { patients } \\
\text { (in arm) } \\
\text { (\% } \\
\text { patients) }\end{array}$ & $\begin{array}{l}\text { n lesions } \\
\text { (arm)/N } \\
\text { lesions } \\
\text { (in arm) } \\
\text { (\% } \\
\text { lesions) }\end{array}$ & $\begin{array}{l}\text { Odds } \\
\text { Ratio (95\% } \\
\text { Cl) }\end{array}$ & $\begin{array}{l}\text { Adverse } \\
\text { events } \\
\text { reported } \\
(y / n)\end{array}$ & $\begin{array}{l}\text { Aditional } \\
\text { outcomes } \\
\text { reported }\end{array}$ \\
\hline $\begin{array}{l}\text { Reschly } \\
2010 \\
20677531\end{array}$ & 75 & $\begin{array}{l}\text { SCC } \\
\text { (males over } \\
\text { age 60) }\end{array}$ & $\begin{array}{l}\text { A. Surgical } \\
\text { Excision without } \\
\text { interoperative } \\
\text { evaluation }\end{array}$ & & Recurrence & & $1 / 16(6)$ & & $\mathrm{n}$ & \\
\hline $\begin{array}{l}\text { Bean } 1984 \\
6463702\end{array}$ & 70 & $\begin{array}{l}\text { BCC+SCC } \\
\text { (hand) }\end{array}$ & $\begin{array}{l}\text { A. Surgical } \\
\text { Excision without } \\
\text { interoperative } \\
\text { evaluation }\end{array}$ & & Recurrence & & $2 / 67(3)$ & & $\mathrm{n}$ & metastasis \\
\hline $\begin{array}{l}\text { Bean } 1984 \\
6463702 \\
\end{array}$ & 70 & $\begin{array}{l}\text { BCC+SCC } \\
\text { (hand) }\end{array}$ & $\begin{array}{l}\text { D1. External } \\
\text { beam radiation }\end{array}$ & & Recurrence & & $1 / 3(33.3)$ & & $\mathrm{n}$ & metastasis \\
\hline $\begin{array}{l}\text { Bean } 1984 \\
6463702\end{array}$ & 70 & $\begin{array}{l}\text { BCC+SCC } \\
\text { (hand) }\end{array}$ & $\begin{array}{l}\text { F1. Topical or } \\
\text { intralesional 5- } \\
\text { FU...Define: }\end{array}$ & & Recurrence & & $3 / 3(100)$ & & $\mathrm{n}$ & metastasis \\
\hline $\begin{array}{l}\text { Bean } 1984 \\
6463702\end{array}$ & 70 & $\begin{array}{l}\mathrm{BCC}+\mathrm{SCC} \\
\text { (hand) }\end{array}$ & $\begin{array}{l}\text { C2. } \\
\text { Diathermy/electr } \\
\text { odessication }\end{array}$ & & Recurrence & & $1 / 3(33.3)$ & & $\mathrm{n}$ & metastasis \\
\hline $\begin{array}{l}\text { Bean } 1984 \\
6463702 \\
\end{array}$ & 70 & $\begin{array}{l}\text { BCC+SCC } \\
\text { (hand) }\end{array}$ & C1. Cryotherapy & & Recurrence & & $1 / 3(33.3)$ & & $\mathrm{n}$ & metastasis \\
\hline $\begin{array}{l}\text { Cham } 1991 \\
1913614\end{array}$ & 41 & BCC & $\begin{array}{l}\text { F5. Medical } \\
\text { other...Define: }\end{array}$ & $\begin{array}{l}\text { BEC } \\
\text { (Curaderm) }\end{array}$ & $\begin{array}{l}\text { Lack of clinical } \\
\text { clearance }\end{array}$ & & $0 / 39(0)$ & & $y$ & \\
\hline $\begin{array}{l}\text { Cham } 1991 \\
1913614 \\
\end{array}$ & 41 & $\mathrm{BCC}$ & $\begin{array}{l}\text { F5. Medical } \\
\text { other...Define: }\end{array}$ & placebo & $\begin{array}{l}\text { Lack of clinical } \\
\text { clearance }\end{array}$ & & $2 / 2(100)$ & & $y$ & \\
\hline $\begin{array}{l}\text { Aguilar } 2010 \\
20456549\end{array}$ & 67 & $\mathrm{BCC}+\mathrm{SCC}$ & $\begin{array}{l}\text { A. Surgical } \\
\text { Excision without } \\
\text { interoperative } \\
\text { evaluation }\end{array}$ & & $\begin{array}{l}\text { Lack of clinical } \\
\text { clearance (1- } \\
\text { efficacy) }\end{array}$ & & $1 / 34(2.5)$ & $\begin{array}{l}\text { A vs. E1: } \\
0.25(0.02 \\
2.58)\end{array}$ & $\mathrm{n}$ & costs \\
\hline $\begin{array}{l}\text { Aguilar } 2010 \\
20456549\end{array}$ & 67 & $\mathrm{BCC}+\mathrm{SCC}$ & $\begin{array}{l}\text { E1. PDT: MAL + } \\
\text { red } \\
\text { light...Define: }\end{array}$ & & $\begin{array}{l}\text { Lack of clinical } \\
\text { clearance (1- } \\
\text { efficacy) }\end{array}$ & & $3 / 28(10.5)$ & $\begin{array}{l}\text { E1 vs. F2: } \\
0.84(0.15, \\
4.61) \\
\end{array}$ & $\mathrm{n}$ & costs \\
\hline $\begin{array}{l}\text { Aguilar } 2010 \\
20456549\end{array}$ & 67 & $\mathrm{BCC}+\mathrm{SCC}$ & $\begin{array}{l}\text { F2. Topical or } \\
\text { intralesional } \\
\text { Imiquimod...Defin } \\
\text { e: }\end{array}$ & topical & $\begin{array}{l}\text { Lack of clinical } \\
\text { clearance (1- } \\
\text { efficacy) }\end{array}$ & & $3 / 24(12.5)$ & $\begin{array}{l}\text { A vs. F2: } \\
0.21(0.02 \\
2.18)\end{array}$ & $\mathrm{n}$ & costs \\
\hline Marks 2004 & 67 & $\mathrm{BCC}$ & $\begin{array}{l}\text { F2. Topical or } \\
\text { intralesional } \\
\text { Imiquimod...Defin } \\
\text { e: }\end{array}$ & $\begin{array}{l}\text { topical } 5 \\
\text { times/week }\end{array}$ & $\begin{array}{l}\text { Lack of clinical } \\
\text { clearance }\end{array}$ & $0 / 36(0)$ & & & $y$ & \\
\hline
\end{tabular}




\begin{tabular}{|c|c|c|c|c|c|c|c|c|c|c|}
\hline $\begin{array}{l}\text { Author } \\
\text { year PMID }\end{array}$ & $\begin{array}{l}\text { Total N } \\
\text { (patients) }\end{array}$ & $\begin{array}{l}\text { Lesion } \\
\text { type } \\
\text { (location) }\end{array}$ & Treatment & $\begin{array}{l}\text { Treatment } \\
\text { notes }\end{array}$ & $\begin{array}{l}\text { Outcome } \\
\text { (lack of } \\
\text { clearancel } \\
\text { recurrence) }\end{array}$ & $\begin{array}{l}\text { n patients } \\
\text { (in arm)/N } \\
\text { patients } \\
\text { (in arm) } \\
\text { (\% } \\
\text { patients) }\end{array}$ & $\begin{array}{l}\text { n lesions } \\
\text { (arm)/N } \\
\text { lesions } \\
\text { (in arm) } \\
\text { (\% } \\
\text { lesions) }\end{array}$ & $\begin{array}{l}\text { Odds } \\
\text { Ratio (95\% } \\
\text { Cl) }\end{array}$ & $\begin{array}{l}\text { Adverse } \\
\text { events } \\
\text { reported } \\
(y / n)\end{array}$ & $\begin{array}{l}\text { Aditional } \\
\text { outcomes } \\
\text { reported }\end{array}$ \\
\hline Marks 2004 & 67 & $\mathrm{BCC}$ & $\begin{array}{l}\text { F2. Topical or } \\
\text { intralesional } \\
\text { Imiquimod...Defin } \\
\text { e: }\end{array}$ & $\begin{array}{l}\text { topical } 7 \\
\text { times/week }\end{array}$ & $\begin{array}{l}\text { Lack of clinical } \\
\text { clearance }\end{array}$ & $2 / 30(7)$ & & & $y$ & \\
\hline $\begin{array}{l}\text { Kadakia } \\
2016 \\
26780196\end{array}$ & 53 & $\begin{array}{l}\text { SCC } \\
\text { (scalp) } \\
\text { immunoco } \\
\text { mpromised }\end{array}$ & $\begin{array}{l}\text { A. Surgical } \\
\text { Excision without } \\
\text { interoperative } \\
\text { evaluation }\end{array}$ & $\begin{array}{l}\text { Surgical } \\
\text { excision or } \\
\text { Mohs with } \\
\text { post- } \\
\text { operative } \\
\text { radiation }\end{array}$ & Recurrence & $8 / 45$ (17.8) & & $\begin{array}{l}\text { A + D1 vs A: } \\
0.36(0.07 \\
1.83)\end{array}$ & $y$ & $\begin{array}{l}\text { metastasis/ } \\
\text { death }\end{array}$ \\
\hline $\begin{array}{l}\text { Kadakia } \\
2016 \\
26780196\end{array}$ & 53 & $\begin{array}{l}\text { SCC } \\
\text { (scalp) } \\
\text { immunoco } \\
\text { mpromised }\end{array}$ & $\begin{array}{l}\text { A. Surgical } \\
\text { Excision without } \\
\text { interoperative } \\
\text { evaluation }\end{array}$ & $\begin{array}{l}\text { Surgical } \\
\text { excision or } \\
\text { Mohs }\end{array}$ & Recurrence & $3 / 8(37.5)$ & & & $y$ & $\begin{array}{l}\text { metastasis/ } \\
\text { death }\end{array}$ \\
\hline $\begin{array}{l}\text { Shiffman } \\
1975 \\
1125865\end{array}$ & 52 & $\begin{array}{l}\text { SCC } \\
\text { (pinna) }\end{array}$ & $\begin{array}{l}\text { A. Surgical } \\
\text { Excision without } \\
\text { interoperative } \\
\text { evaluation }\end{array}$ & & Recurrence & $2 / 31(6.5)$ & & $\begin{array}{l}\text { A vs. C3: } \\
0.19(0.03 \\
1.19)\end{array}$ & $\mathrm{n}$ & metastasis \\
\hline $\begin{array}{l}\text { Shiffman } \\
1975 \\
1125865\end{array}$ & 52 & $\begin{array}{l}\text { SCC } \\
\text { (pinna) }\end{array}$ & $\begin{array}{l}\text { C3. Curettage + } \\
\text { diathermy }\end{array}$ & $\begin{array}{l}\text { curettage }+ \\
\text { electrodessic } \\
\text { ation or } \\
\text { surgery }\end{array}$ & Recurrence & $4 / 15(26.7)$ & & & $\mathrm{n}$ & metastasis \\
\hline $\begin{array}{l}\text { Ibbotson } \\
2012 \\
22971196 \\
\end{array}$ & 40 & $\mathrm{BCC}$ & $\begin{array}{l}\text { E1. PDT: MAL + } \\
\text { red } \\
\text { light...Define: }\end{array}$ & & $\begin{array}{l}\text { Lack of clinical } \\
\text { clearance }\end{array}$ & $8 / 20(40)$ & & $\begin{array}{l}\text { E1 vs. E2: } \\
1.24(0.34, \\
4.46)\end{array}$ & $y$ & \\
\hline $\begin{array}{l}\text { Ibbotson } \\
2012 \\
22971196 \\
\end{array}$ & 40 & $\mathrm{BCC}$ & $\begin{array}{l}\text { E2. PDT: ALA + } \\
\text { blue } \\
\text { light...Define: }\end{array}$ & & $\begin{array}{l}\text { Lack of clinical } \\
\text { clearance }\end{array}$ & $7 / 20(35)$ & & & $y$ & \\
\hline $\begin{array}{l}\text { Yoon } 1992 \\
1463102\end{array}$ & 40 & SCC & $\begin{array}{l}\text { A. Surgical } \\
\text { Excision without } \\
\text { interoperative } \\
\text { evaluation }\end{array}$ & excision only & Recurrence & $8 / 13(62)$ & & $\begin{array}{l}\text { A vs. B: } 3.52 \\
(0.76,16.39)\end{array}$ & $\mathrm{n}$ & $\begin{array}{l}\text { metastasis/ } \\
\text { death }\end{array}$ \\
\hline $\begin{array}{l}\text { Yoon } 1992 \\
1463102\end{array}$ & 40 & SCC & $\begin{array}{l}\text { B. Surgical } \\
\text { Excision with } \\
\text { interoperative } \\
\text { evaluation }\end{array}$ & Mohs & Recurrence & $5 / 16(31)$ & & & $\mathrm{n}$ & $\begin{array}{l}\text { metastasis/ } \\
\text { death }\end{array}$ \\
\hline Glass 1974 & 24 & epidermoid & A. Surgical & & Recurrence & $2 / 19$ (10.5) & & A vs. D1: & $\mathrm{n}$ & \\
\hline
\end{tabular}




\begin{tabular}{|c|c|c|c|c|c|c|c|c|c|c|}
\hline $\begin{array}{l}\text { Author } \\
\text { year PMID }\end{array}$ & $\begin{array}{l}\text { Total N } \\
\text { (patients) }\end{array}$ & $\begin{array}{l}\text { Lesion } \\
\text { type } \\
\text { (location) }\end{array}$ & Treatment & $\begin{array}{l}\text { Treatment } \\
\text { notes }\end{array}$ & $\begin{array}{l}\text { Outcome } \\
\text { (lack of } \\
\text { clearancel } \\
\text { recurrence) }\end{array}$ & $\begin{array}{l}\text { n patients } \\
\text { (in arm)/N } \\
\text { patients } \\
\text { (in arm) } \\
\text { (\% } \\
\text { patients) }\end{array}$ & $\begin{array}{l}\text { n lesions } \\
\text { (arm)/N } \\
\text { lesions } \\
\text { (in arm) } \\
\text { (\% } \\
\text { lesions) }\end{array}$ & $\begin{array}{l}\text { Odds } \\
\text { Ratio (95\% } \\
\text { Cl) }\end{array}$ & $\begin{array}{l}\text { Adverse } \\
\text { events } \\
\text { reported } \\
\text { (y/n) }\end{array}$ & $\begin{array}{l}\text { Aditional } \\
\text { outcomes } \\
\text { reported }\end{array}$ \\
\hline 4808574 & & $\begin{array}{l}\text { carcinoma; } \\
\text { incompletel } \\
\text { y excised }\end{array}$ & $\begin{array}{l}\text { Excision without } \\
\text { interoperative } \\
\text { evaluation }\end{array}$ & & & & & $\begin{array}{l}0.47(0.03 \\
6.57)\end{array}$ & & \\
\hline $\begin{array}{l}\text { Glass } 1974 \\
4808574\end{array}$ & 24 & $\begin{array}{l}\text { epidermoid } \\
\text { carcinoma; } \\
\text { incompletel } \\
\text { y excised }\end{array}$ & $\begin{array}{l}\text { D1. External } \\
\text { beam radiation }\end{array}$ & radiotherapy & Recurrence & $1 / 5(20)$ & & & $\mathrm{n}$ & \\
\hline $\begin{array}{l}\text { Valentine } \\
2011 \\
21077899\end{array}$ & 40 & $\mathrm{BCC}$ & $\begin{array}{l}\text { E1. PDT: MAL + } \\
\text { red } \\
\text { light...Define: }\end{array}$ & $10 \mathrm{gp} 2$ & & & & & $y$ & \\
\hline $\begin{array}{l}\text { Valentine } \\
2011 \\
21077899\end{array}$ & 40 & $\mathrm{BCC}$ & $\begin{array}{l}\text { E2. PDT: ALA + } \\
\text { blue } \\
\text { light...Define: }\end{array}$ & 10 gp1 & & & & & $y$ & \\
\hline $\begin{array}{l}\text { Valentine } \\
2011 \\
21077899\end{array}$ & 40 & Bowen's & $\begin{array}{l}\text { E1. PDT: MAL + } \\
\text { red } \\
\text { light...Define: }\end{array}$ & 10 gp 4 & & & & & $y$ & \\
\hline $\begin{array}{l}\text { Valentine } \\
2011 \\
21077899 \\
\end{array}$ & 40 & Bowen's & $\begin{array}{l}\text { E2. PDT: ALA + } \\
\text { blue } \\
\text { light...Define: }\end{array}$ & 10 gp 3 & & & & & $y$ & \\
\hline $\begin{array}{l}\text { Halnan } 1968 \\
5710508\end{array}$ & 104 & $\begin{array}{l}\mathrm{BCC}+ \\
\mathrm{SCC}\end{array}$ & $\begin{array}{l}\text { A. Surgical } \\
\text { Excision without } \\
\text { interoperative } \\
\text { evaluation }\end{array}$ & 10 lesions & & & & & $y$ & $\begin{array}{l}\text { cosmetic } \\
\text { outcomes }\end{array}$ \\
\hline $\begin{array}{l}\text { Halnan } 1968 \\
5710508\end{array}$ & 104 & $\begin{array}{l}\mathrm{BCC}+ \\
\mathrm{SCC}\end{array}$ & $\begin{array}{l}\text { D1. External } \\
\text { beam radiation }\end{array}$ & $\begin{array}{l}\text { x-ray therapy } \\
=58 \text { lesions }\end{array}$ & & & & & $y$ & $\begin{array}{l}\text { cosmetic } \\
\text { outcomes }\end{array}$ \\
\hline $\begin{array}{l}\text { Halnan } 1968 \\
5710508\end{array}$ & 104 & $\begin{array}{l}\mathrm{BCC}+ \\
\mathrm{SCC}\end{array}$ & $\begin{array}{l}\text { D2. } \\
\text { Brachytherapy/PI } \\
\text { eisiotherapy }\end{array}$ & $\begin{array}{l}\text { radon gold } \\
\text { see implant }= \\
38 \text { lesions }\end{array}$ & & & & & $y$ & $\begin{array}{l}\text { cosmetic } \\
\text { outcomes }\end{array}$ \\
\hline $\begin{array}{l}\text { Bu } 2016 \\
27888160\end{array}$ & 20 & $\mathrm{BCC}$ & $\begin{array}{l}\text { E2. PDT: ALA + } \\
\text { blue } \\
\text { light...Define: }\end{array}$ & $\begin{array}{l}\text { Excision }+ \\
\text { ALA PDT }\end{array}$ & Recurrence & $0 / 10(0)$ & & & & \\
\hline $\begin{array}{l}\text { Bu } 2016 \\
27888160\end{array}$ & 20 & $\mathrm{BCC}$ & $\begin{array}{l}\text { A. Surgical } \\
\text { Excision without } \\
\text { interoperative } \\
\text { evaluation }\end{array}$ & $\begin{array}{l}\text { Excision + } \\
\text { excision }\end{array}$ & recurrence & $0 / 10(0)$ & & & & \\
\hline $\begin{array}{l}\text { Haseltine } \\
2016\end{array}$ & 61 & $\begin{array}{l}\mathrm{BCC}+ \\
\mathrm{SCC}\end{array}$ & $\begin{array}{l}\text { D2. } \\
\text { Brachytherapy/PI }\end{array}$ & & $\begin{array}{l}\text { Lack of clinical } \\
\text { clearance }\end{array}$ & $0 / 8(0)$ & & $\begin{array}{l}\text { D2 vs D1: } \\
0.04(0,\end{array}$ & $y$ & $\begin{array}{l}\text { cosmetic } \\
\text { outcomes }\end{array}$ \\
\hline
\end{tabular}




\begin{tabular}{|c|c|c|c|c|c|c|c|c|c|c|}
\hline $\begin{array}{l}\text { Author } \\
\text { year PMID }\end{array}$ & $\begin{array}{l}\text { Total N } \\
\text { (patients) }\end{array}$ & $\begin{array}{l}\text { Lesion } \\
\text { type } \\
\text { (location) }\end{array}$ & Treatment & $\begin{array}{l}\text { Treatment } \\
\text { notes }\end{array}$ & $\begin{array}{l}\text { Outcome } \\
\text { (lack of } \\
\text { clearancel } \\
\text { recurrence) }\end{array}$ & $\begin{array}{l}\text { n patients } \\
\text { (in arm)/N } \\
\text { patients } \\
\text { (in arm) } \\
\text { (\% } \\
\text { patients) }\end{array}$ & $\begin{array}{l}\text { n lesions } \\
\text { (arm)/N } \\
\text { lesions } \\
\text { (in arm) } \\
\text { (\% } \\
\text { lesions) }\end{array}$ & $\begin{array}{l}\text { Odds } \\
\text { Ratio (95\% } \\
\text { Cl) }\end{array}$ & $\begin{array}{l}\text { Adverse } \\
\text { events } \\
\text { reported } \\
\text { (y/n) }\end{array}$ & $\begin{array}{l}\text { Aditional } \\
\text { outcomes } \\
\text { reported }\end{array}$ \\
\hline 27504127 & & & eisiotherapy & & & & & 21.14) & & \\
\hline $\begin{array}{l}\text { Haseltine } \\
2016 \\
27504127 \\
\end{array}$ & 61 & $\begin{array}{l}\mathrm{BCC}+ \\
\mathrm{SCC}\end{array}$ & $\begin{array}{l}\text { D1. External } \\
\text { beam radiation }\end{array}$ & $\begin{array}{l}\text { hypofractionat } \\
\text { ion }\end{array}$ & $\begin{array}{l}\text { Lack of clinical } \\
\text { clearance }\end{array}$ & $7 / 29(24)$ & & $\begin{array}{l}\text { D2 vs D1: } \\
0.05(0, \\
27.38)\end{array}$ & $y$ & $\begin{array}{l}\text { cosmetic } \\
\text { outcomes }\end{array}$ \\
\hline $\begin{array}{l}\text { Haseltine } \\
2016 \\
27504127 \\
\end{array}$ & 61 & $\begin{array}{l}\mathrm{BCC}+ \\
\mathrm{SCC}\end{array}$ & $\begin{array}{l}\text { D1. External } \\
\text { beam radiation }\end{array}$ & $\begin{array}{l}\text { standard } \\
\text { fractionation }\end{array}$ & $\begin{array}{l}\text { Lack of clinical } \\
\text { clearance }\end{array}$ & $4 / 20(20)$ & & $\begin{array}{l}\text { D1 vs } \\
\text { D1:1.27 } \\
(0.32,5.09)\end{array}$ & $y$ & $\begin{array}{l}\text { cosmetic } \\
\text { outcomes }\end{array}$ \\
\hline $\begin{array}{l}\text { Salido- } \\
\text { Vallejo } 2016 \\
26369617\end{array}$ & 86 & $\begin{array}{l}\text { SCC } \\
\text { (infiltrating) }\end{array}$ & $\begin{array}{l}\text { F5. Medical } \\
\text { other...Define: }\end{array}$ & $\begin{array}{l}\text { Methotrexate } \\
+ \text { excision }\end{array}$ & $\begin{array}{l}\text { tumor area } \\
\text { reduction }\end{array}$ & 43 & $\begin{array}{l}\text { neg } 0.52 \\
\text { cm2 (0.85) }\end{array}$ & $\begin{array}{l}\text { mean } \\
\text { difference - } \\
1.01(-1.38,- \\
0.64) \\
P<0.001\end{array}$ & $y$ & \\
\hline $\begin{array}{l}\text { Salido- } \\
\text { Vallejo } 2016 \\
26369617\end{array}$ & 86 & $\begin{array}{l}\text { SCC } \\
\text { (infiltrating) }\end{array}$ & $\begin{array}{l}\text { A. Surgical } \\
\text { Excision without } \\
\text { interoperative } \\
\text { evaluation }\end{array}$ & excision only & $\begin{array}{l}\text { tumor area } \\
\text { reduction }\end{array}$ & 43 & $\begin{array}{l}0.49 \mathrm{~cm} 2 \\
(0 . .88)\end{array}$ & & $y$ & \\
\hline $\begin{array}{l}\text { Overmark } \\
2016 \\
26073523\end{array}$ & 239 & $\begin{array}{l}\text { SCC (in } \\
\text { situ) }\end{array}$ & $\begin{array}{l}\text { A. Surgical } \\
\text { Excision without } \\
\text { interoperative } \\
\text { evaluation }\end{array}$ & & recurrence & & $1 / 125(0.8)$ & $\begin{array}{l}\text { A vs. C1: } \\
0.16(0.02, \\
1.61)\end{array}$ & & \\
\hline $\begin{array}{l}\text { Overmark } \\
2016 \\
26073523\end{array}$ & 239 & $\begin{array}{l}\text { SCC (in } \\
\text { situ) }\end{array}$ & C1. Cryotherapy & & recurrence & & $3 / 64(4.7)$ & $\begin{array}{l}\text { C1 vs E1: } \\
0.23(0.06 \\
0.85)\end{array}$ & & \\
\hline $\begin{array}{l}\text { Overmark } \\
2016 \\
26073523 \\
\end{array}$ & 239 & $\begin{array}{l}\text { SCC (in } \\
\text { situ) }\end{array}$ & $\begin{array}{l}\text { E1. PDT: MAL + } \\
\text { red } \\
\text { light...Define: }\end{array}$ & & recurrence & & $13 / 74(18)$ & $\begin{array}{l}\text { A vs E1: } \\
0.04(0,0.3)\end{array}$ & & \\
\hline $\begin{array}{l}\text { Nassiripour } \\
2016 \_2816 \overline{3} \\
737\end{array}$ & 630 & $\begin{array}{l}\mathrm{BCC}+ \\
\mathrm{SCC}\end{array}$ & $\begin{array}{l}\text { A. Surgical } \\
\text { Excision without } \\
\text { interoperative } \\
\text { evaluation }\end{array}$ & & recurrence & $\begin{array}{l}28 / 354 \\
(7.9)\end{array}$ & & $\begin{array}{l}\text { A vs B: } 0.9 \\
(0.51,1.59)\end{array}$ & & \\
\hline $\begin{array}{l}\text { Nassiripour } \\
2016 \_2816 \overline{3} \\
737\end{array}$ & 630 & $\begin{array}{l}\mathrm{BCC}+ \\
\mathrm{SCC}\end{array}$ & $\begin{array}{l}\text { B. Surgical } \\
\text { Excision with } \\
\text { interoperative } \\
\text { evaluation }\end{array}$ & & recurrence & $\begin{array}{l}24 / 276 \\
(8.7)\end{array}$ & & & & \\
\hline $\begin{array}{l}\text { Marconi } \\
2016\end{array}$ & 597 & $\begin{array}{l}\text { BCC + } \\
\text { SCC }\end{array}$ & $\begin{array}{l}\text { D1. External } \\
\text { beam radiation }\end{array}$ & $2 \mathrm{~Gy}$ & $\begin{array}{l}\text { lack of } \\
\text { histological } \\
\text { clearance }\end{array}$ & & $\begin{array}{l}32 / 500 \\
(6.4)\end{array}$ & $\begin{array}{l}\text { D1 (high } \\
\text { dose) vs. D1 } \\
\text { (low dose): }\end{array}$ & $y$ & \\
\hline
\end{tabular}




\begin{tabular}{|c|c|c|c|c|c|c|c|c|c|c|}
\hline $\begin{array}{l}\text { Author } \\
\text { year PMID }\end{array}$ & $\begin{array}{l}\text { Total N } \\
\text { (patients) }\end{array}$ & $\begin{array}{l}\text { Lesion } \\
\text { type } \\
\text { (location) }\end{array}$ & Treatment & $\begin{array}{l}\text { Treatment } \\
\text { notes }\end{array}$ & $\begin{array}{l}\text { Outcome } \\
\text { (lack of } \\
\text { clearancel } \\
\text { recurrence) }\end{array}$ & $\begin{array}{l}\text { n patients } \\
\text { (in arm)/N } \\
\text { patients } \\
\text { (in arm) } \\
\text { (\% } \\
\text { patients) }\end{array}$ & $\begin{array}{l}\text { n lesions } \\
\text { (arm)/N } \\
\text { lesions } \\
\text { (in arm) } \\
\text { (\% } \\
\text { lesions) }\end{array}$ & $\begin{array}{l}\text { Odds } \\
\text { Ratio (95\% } \\
\text { Cl) }\end{array}$ & $\begin{array}{l}\text { Adverse } \\
\text { events } \\
\text { reported } \\
(y / n)\end{array}$ & $\begin{array}{l}\text { Aditional } \\
\text { outcomes } \\
\text { reported }\end{array}$ \\
\hline & & & & & & & & $\begin{array}{l}0.26(0.12, \\
0.54)\end{array}$ & & \\
\hline $\begin{array}{l}\text { Marconi } \\
2016\end{array}$ & 597 & $\begin{array}{l}\mathrm{BCC}+ \\
\mathrm{SCC}\end{array}$ & $\begin{array}{l}\text { D1. External } \\
\text { beam radiation }\end{array}$ & $>2$ Gy & $\begin{array}{l}\text { lack of } \\
\text { histological } \\
\text { clearance }\end{array}$ & & $9 / 521(1.7)$ & & $y$ & \\
\hline
\end{tabular}




\section{Appendix H. Adverse Events Reported}

\begin{tabular}{|c|c|c|}
\hline Arm type & Outcome Description & \# studies reporting outcome \\
\hline cryotherapy & Blistering & 3 \\
\hline cryotherapy & wound infection & 3 \\
\hline cryotherapy & Necrosis & 4 \\
\hline cryotherapy & Ulceration & 4 \\
\hline cryotherapy & inflammation/swelling & 5 \\
\hline cryotherapy & scarring & 5 \\
\hline cryotherapy & pain & 24 \\
\hline excision & bleeding & 5 \\
\hline excision & cataract and lachrymal duct stenosis & 1 \\
\hline excision & crusting & 10 \\
\hline excision & dyspigmentations and telangiectasia & 2 \\
\hline excision & Ectropion & 1 \\
\hline excision & edema/oedema & 5 \\
\hline excision & erosion & 6 \\
\hline excision & Erythema & 14 \\
\hline excision & headache & 1 \\
\hline excision & inflammation/swelling & 5 \\
\hline excision & itching & 10 \\
\hline excision & malaise & 1 \\
\hline excision & necrosis & 4 \\
\hline excision & pain & 25 \\
\hline excision & photosensitivity reaction & 1 \\
\hline excision & Radiodystrophy & 1 \\
\hline excision & scabbing & 1 \\
\hline excision & scaling & 4 \\
\hline excision & soreness & 1 \\
\hline excision & spots or pimples & 1 \\
\hline excision & skin infection & 1 \\
\hline excision & skin irritation & 9 \\
\hline excision & swelling & 1 \\
\hline excision & weeping & 1 \\
\hline excision & wound dehiscence & 2 \\
\hline excision & wound infection & 3 \\
\hline laser & dyspigmentation & 2 \\
\hline laser & hypopigmentation & 2 \\
\hline laser & Purpura & 2 \\
\hline laser & blistering & 3 \\
\hline laser & bullae & 3 \\
\hline laser & scarring & 5 \\
\hline
\end{tabular}




\begin{tabular}{|c|c|c|}
\hline Arm type & Outcome Description & \# studies reporting outcome \\
\hline laser & crusting & 10 \\
\hline laser & Erythema & 13 \\
\hline medical & Alanine aminotransferase elevation & 1 \\
\hline medical & alkaline phosphatase elevation & 2 \\
\hline medical & application site reaction & 5 \\
\hline medical & arthralgia & 1 \\
\hline medical & back pain & 1 \\
\hline medical & bleeding & 5 \\
\hline medical & Blink discomfort and dry eye & 1 \\
\hline medical & Burning & 9 \\
\hline medical & crusting & 10 \\
\hline medical & Desquamation & 1 \\
\hline medical & diarrhea & 2 \\
\hline medical & discharge & 1 \\
\hline medical & drainage & 1 \\
\hline medical & Ectropion & 1 \\
\hline medical & edema/oedema & 5 \\
\hline medical & erosion & 6 \\
\hline medical & Erythema & 14 \\
\hline medical & excoriation/flaking & 2 \\
\hline medical & fatigue & 2 \\
\hline medical & fever & 1 \\
\hline medical & headache & 4 \\
\hline medical & hypopigmentation & 2 \\
\hline medical & induration & 1 \\
\hline medical & inflammation/swelling & 5 \\
\hline medical & Intense conjunctival irritation & 1 \\
\hline medical & itching & 10 \\
\hline medical & lesions at remote site & 1 \\
\hline medical & leukopenia & 1 \\
\hline medical & loss of eyelashes & 1 \\
\hline medical & malaise/cold or flu like symptoms & 3 \\
\hline medical & nausea & 3 \\
\hline medical & necrosis & 4 \\
\hline medical & pain & 25 \\
\hline medical & Paresthesia & 1 \\
\hline medical & Pruritus & 2 \\
\hline medical & pustules & 1 \\
\hline medical & rash & 3 \\
\hline medical & redness & 2 \\
\hline medical & scabbing & 4 \\
\hline
\end{tabular}




\section{Arm type}

medical

medical

medical

medical

medical

medical

medical

medical

medical

medical

medical

medical

medical

medical

medical

medical

\section{Outcome Description}

Scaling

sensitivity

sinusitis

skin irritation

soreness

spots or pimples

swelling

Telangiectasia

tenderness

thrombocytopenia

Ulceration

upper respiritory tract infection

Vesicles

weeping

Wound dehiscence

Wounds
\# studies reporting outcome

4

1

1

9

1

1

1

1

2

1

4

1

3

2

2

1

$\begin{array}{llr}\text { PDT } & \text { oozing } & 1 \\ \text { PDT } & \text { photosensitivity reaction } & 1 \\ \text { PDT } & \text { skin infection } & 1 \\ \text { PDT } & \text { squamae } & 1 \\ \text { PDT } & \text { tingling } & 1 \\ \text { PDT } & \text { warmth } & 1 \\ \text { PDT } & \text { hypopigmentation } & 1 \\ \text { PDT } & \text { hyperpigmentation } & 3 \\ \text { PDT } & \text { Infection } & 2 \\ \text { PDT } & \text { redness } & 2 \\ \text { PDT } & \text { wound dehiscence } & 2 \\ \text { PDT } & \text { Blistering } & 3 \\ \text { PDT } & \text { bullae } & 3 \\ \text { PDT } & \text { stinging } & 3 \\ \text { PDT } & \text { vesicles } & 3 \\ \text { PDT } & \text { wound infection } & 3 \\ \text { PDT } & \text { bleeding } & 4 \\ \text { PDT } & \text { scaling } & 4 \\ \text { PDT } & \text { Ulceration } & 4 \\ \text { PDT } & \text { necrosis } & 4 \\ \text { PDT } & \text { edema/oedema } & 5 \\ \text { PDT } & \text { erosion } & 6 \\ \text { PDT } & \text { scarring } & 6 \\ \text { PDT } & \text { inflamation/swelling } & 5 \\ \text { PDT } & \text { burning } & 9\end{array}$




\begin{tabular}{llc} 
Arm type & Outcome Description & \# \\
PDT & skin irritation & 10 \\
PDT & itching & 9 \\
PDT & crusting & 10 \\
PDT & Erythema & 13 \\
PDT & pain & 25 \\
\hline radiotherapy & Blink discomfort and dry eye & 1 \\
radiotherapy & cataract and lachrymal duct stenosis & 1 \\
radiotherapy & Intense conjunctival irritation & 1 \\
radiotherapy & loss of eyelashes & 1 \\
radiotherapy & Radiodystrophy & 1 \\
radiotherapy & slight pain in lower eyelid & 1 \\
radiotherapy & dyspigmentations and telangiectasia & 2 \\
radiotherapy & Ectropion & 2 \\
radiotherapy & Necrosis & 4 \\
radiotherapy & scarring & 5
\end{tabular}




\section{Appendix I. Study-Level Results}

Table I-1. Recurrence, all BCC

\begin{tabular}{|c|c|c|c|c|}
\hline Study & Arm & Lesion Location & $n / N$ & Result \\
\hline 11298545 Wang & (E) cryosurgery & NR & $6 / 39$ & OR $0.7(0.4,1.22)$ \\
\hline 11298545 Wang & (C) ALA-PDT & NR & $11 / 44$ & OR $0.7(0.4,1.22)$ \\
\hline 14732655 Rhodes & $(A, B)$ excision & $\begin{array}{l}\text { face/scalp (58), extremities (9), } \\
\text { trunk/neck (29) }\end{array}$ & $0 / 35$ & OR $0.08(0.01,0.52)$ \\
\hline 14732655 Rhodes & (E) MAL PDT & $\begin{array}{l}\text { face/scalp (40), extremities (11), } \\
\text { trunk/neck (49) }\end{array}$ & $0 / 31$ & OR $0.08(0.01,0.52)$ \\
\hline 17451581 Kuijpers & $\begin{array}{l}\text { (A,B) Surgical } \\
\text { excision }\end{array}$ & $\begin{array}{l}\text { Forehead/temple, Cheek/chin, } \\
\text { Periocular (76), Lips/mouth (6), } \\
\text { Ears/periauricular (6), Neck, } \\
\text { chest/back (12) }\end{array}$ & $4 / 47$ & OR $0.42(0.14,1.25)$ \\
\hline 17451581 Kuijpers & $\begin{array}{l}\text { (C) Curettage + } \\
\text { Cryosurgery }\end{array}$ & $\begin{array}{l}\text { Forehead/temple, Cheek/chin, } \\
\text { Periocular (80), Lips/mouth (4), } \\
\text { Ears/periauricular (8), Neck, } \\
\text { chest/back (8) }\end{array}$ & $9 / 38$ & OR $0.42(0.14,1.25)$ \\
\hline $\begin{array}{l}18693158 \text { Basset- } \\
\text { Seguin }\end{array}$ & (E) Cryotherapy & $\begin{array}{l}\text { face/scalp (4), extremities (20), } \\
\text { trunk/neck (76) }\end{array}$ & $19 / 93$ & OR $0.7(0.4,1.22)$ \\
\hline $\begin{array}{l}18693158 \text { Basset- } \\
\text { Seguin }\end{array}$ & (C) MAL-PDT & $\begin{array}{l}\text { face/scalp (6), extremities (22), } \\
\text { trunk/neck (72) }\end{array}$ & $22 / 100$ & OR $0.7(0.4,1.22)$ \\
\hline 18717680 Mosterd & $\begin{array}{l}(A, B) \text { Surgical } \\
\text { excision }\end{array}$ & face (51); Irest of the body (49\%) & $0 / 88$ & OR $0.08(0.01,0.52)$ \\
\hline 18717680 Mosterd & (E) ALA-PDT & face (53); Irest of the body (47\%) & $25 / 83$ & OR $0.08(0.01,0.52)$ \\
\hline $\begin{array}{l}21242584 \text { Garcia- } \\
\text { Martin }\end{array}$ & (D) radiotherapy & eyelid (100) & $0 / 12$ & OR $1.24(0.02,67.04)$ \\
\hline $\begin{array}{l}21242584 \text { Garcia- } \\
\text { Martin }\end{array}$ & $(F)$ imiquimod $5 \%$ & eyelid (100) & $0 / 15$ & OR $1.24(0.02,67.04)$ \\
\hline 24903544 Haak & (E) AFXL MAL PDT & $\begin{array}{l}\text { nose (56), forehead (19), cheek (13), } \\
\text { oral area (6), periorbital area (6) }\end{array}$ & $3 / 16$ & OR $0.7(0.4,1.22)$ \\
\hline 24903544 Haak & (C) MAL PDT & $\begin{array}{l}\text { nose (37), forehead (31), cheek (6), } \\
\text { oral area (13), periorbital area (13) }\end{array}$ & $7 / 16$ & OR $0.7(0.4,1.22)$ \\
\hline 3514075 Hall & (D) Cryotherapy & $\begin{array}{l}\text { face and neck (65), eyelid (17), trunk } \\
\text { (17) }\end{array}$ & $17 / 44$ & OR $14.8(3.17,69)$ \\
\hline 3514075 Hall & (C) Radiotherapy & $\begin{array}{l}\text { face and neck (82), eyelid (6), trunk } \\
\text { (12) }\end{array}$ & $2 / 49$ & OR $14.8(3.17,69)$ \\
\hline 9218740 Avril & $(A, B)$ surgery & $\begin{array}{l}\text { nose (53), cheek, pre- and } \\
\text { retroauricular areas (21), eyelids, } \\
\text { internal and external eye angles (19), } \\
\text { forehead, temple, between eyebrows } \\
36 \text { (21), chin, cutaneous superior lip } \\
10 \text { (6), ear (3) }\end{array}$ & $1 / 174$ & OR $0.12(0.01,0.96)$ \\
\hline 9218740 Avril & (D) radiotherapy & $\begin{array}{l}\text { nose (28), cheek, pre- and } \\
\text { retroauricular areas (24), eyelids, } \\
\text { internal and external eye angles (20), } \\
\text { forehead, temple, between eyebrows } \\
\text { (17), chin, cutaneous superior lip ( } 7 \text { ), } \\
\text { ear (3) }\end{array}$ & $8 / 173$ & OR $0.12(0.01,0.96)$ \\
\hline Abbade & $\begin{array}{l}\text { (A,B) Surgical } \\
\text { excision }\end{array}$ & head and neck (100) & $0 / 35$ & OR $0.08(0.01,0.52)$ \\
\hline Abbade & (E) MAL-PDT & head and neck (100) & $2 / 33$ & OR $0.08(0.01,0.52)$ \\
\hline Salmanpoor & $\begin{array}{l}(A, B) \text { Surgical } \\
\text { excision }\end{array}$ & face and scalp (100) & $2 / 24$ & OR $0.42(0.14,1.25)$ \\
\hline Salmanpoor & $\begin{array}{l}\text { (A,B) Surgical } \\
\text { excision }\end{array}$ & face and scalp (100) & $2 / 24$ & OR $0.36(0.06,2.23)$ \\
\hline
\end{tabular}




\begin{tabular}{|c|c|c|c|c|}
\hline Study & Arm & Lesion Location & $\mathrm{n} / \mathrm{N}$ & Result \\
\hline Salmanpoor & $\begin{array}{l}\text { (C) Electodessication } \\
\text { and curettage }\end{array}$ & face and scalp (100) & $2 / 25$ & OR $0.35(0.06,2.13)$ \\
\hline Salmanpoor & $\begin{array}{l}\text { (C) Electodessication } \\
\text { and curettage }\end{array}$ & face and scalp (100) & $2 / 25$ & OR $0.42(0.14,1.25)$ \\
\hline Salmanpoor & (H) Curettage & face and scalp (100) & $4 / 20$ & OR $0.36(0.06,2.23)$ \\
\hline Salmanpoor & $(\mathrm{H})$ Curettage & face and scalp (100) & $4 / 20$ & OR $0.35(0.06,2.13)$ \\
\hline Carija & (E) ALA PDT + PDL & extremities (23.5), trunk/neck (76.5) & $5 / 25$ & OR $0.79(0.46,1.34)$ \\
\hline Carija & (C) ALA PDT & extremities (3.6), trunk/neck (96.4) & $1 / 22$ & OR $0.79(0.46,1.34)$ \\
\hline Bath-Hextall & (F2) Imiquimod & $\begin{array}{l}\text { Face (37), Trunk (38), Neck (6), Arm } \\
\text { (6), Leg (10), Other (3) }\end{array}$ & $11 / 206$ & OR $4.94(1.08,22.58)$ \\
\hline Bath-Hextall & (A) Excision & $\begin{array}{l}\text { Face (33), Trunk (39), Neck (9) Arm } \\
\text { (7), Leg (9), Other (3) }\end{array}$ & $2 / 177$ & OR $4.94(1.08,22.58)$ \\
\hline
\end{tabular}

Table I-2. Lack of histological clearance, all BCC

\begin{tabular}{|c|c|c|c|c|}
\hline Study & Arm & Lesion Location & $n / N$ & Result \\
\hline $\begin{array}{l}10570388 \\
\text { Beutner }\end{array}$ & (F) imiquimod 5\% & NR & $20 / 24$ & OR $0.16(0.02,1.56)$ \\
\hline $\begin{array}{l}10570388 \\
\text { Beutner }\end{array}$ & $\begin{array}{l}(\mathrm{I}, \mathrm{J}) \text { vehicle (3 2x/day, } 2 \\
1 \mathrm{x} / \text { day, } 23 \mathrm{x} / \text { week, } 2 \\
2 \mathrm{x} / \text { week, } 2 \text { 1x/week) }\end{array}$ & $\begin{array}{l}\text { face }(9) \text {, upper extremity }(46), \\
\text { anterior upper trunk (9), neck (9), } \\
\text { posterior lower trunk (27) }\end{array}$ & $1 / 11$ & OR $0.16(0.02,1.56)$ \\
\hline $\begin{array}{l}10940063 \\
\text { Thissen }\end{array}$ & $(A, B)$ surgical excision & $\begin{array}{l}\text { face (43), eyelid (8), trunk/neck (14), } \\
\text { forehead/temple (25), chin/perioral } \\
(10)\end{array}$ & $0 / 48$ & OR $0.13(0.01,2.67)$ \\
\hline $\begin{array}{l}10940063 \\
\text { Thissen }\end{array}$ & (C) cryotherapy & $\begin{array}{l}\text { face (46), eyelid (4), ear (4), } \\
\text { trunk/neck (6), forehead/temple } \\
\text { (34), chin/perioral (6) }\end{array}$ & $3 / 48$ & OR $0.13(0.01,2.67)$ \\
\hline $\begin{array}{l}11298545 \\
\text { Wang }\end{array}$ & (C) cryosurgery & NR & $6 / 39$ & OR $0.62(0.26,1.49)$ \\
\hline $\begin{array}{l}11298545 \\
\text { Wang }\end{array}$ & (E) ALA-PDT & NR & $11 / 44$ & OR $0.62(0.26,1.49)$ \\
\hline $\begin{array}{l}12196749 \\
\text { Geisse }\end{array}$ & (F) Imiquimod 5\% & NR & $23 / 94$ & OR $0.16(0.02,1.56)$ \\
\hline $\begin{array}{l}12196749 \\
\text { Geisse }\end{array}$ & $(\mathrm{I}, \mathrm{J})$ vehicle (control) & $\begin{array}{l}\text { neck/face/forehead (9), upper } \\
\text { extremity (not hand) (34), trunk (47), } \\
\text { lower extremity/thigh (not foot) (9) }\end{array}$ & $26 / 31$ & OR $0.16(0.02,1.56)$ \\
\hline $\begin{array}{l}12224977- \\
12 \text { week } \\
\text { Shumack }\end{array}$ & (F) Imiquimod 5\% & $\mathrm{NR}$ & $21 / 68$ & OR $0.16(0.02,1.56)$ \\
\hline $\begin{array}{l}12224977- \\
12 \text { week } \\
\text { Shumack }\end{array}$ & $(\mathrm{I}, \mathrm{J})$ vehicle cream & $\begin{array}{l}\text { face (17), trunk/neck (54.2), upper } \\
\text { extremity (not hand) ( } 25), \text { lower } \\
\text { extremity (not foot) (4) }\end{array}$ & $21 / 24$ & OR $0.16(0.02,1.56)$ \\
\hline $\begin{array}{l}15097956 \\
\text { Geisse }\end{array}$ & (F) Imiquimod 5\% & NR & $49 / 346$ & OR $0.16(0.02,1.56)$ \\
\hline $\begin{array}{l}15097956 \\
\text { Geisse }\end{array}$ & $\begin{array}{l}(\mathrm{I}, \mathrm{J}) \text { Vehicle } 5 \mathrm{x} / \mathrm{wk} \text { or } \\
7 \mathrm{x} / \mathrm{wk}\end{array}$ & $\begin{array}{l}\text { neck (1), trunk: anterior lower (1), } \\
\text { trunk: anterior upper (20), trunk: } \\
\text { posterior lower (6), trunk: posterior } \\
\text { upper (20), lower extremity } \\
\text { (excluding foot) (10.5), upper } \\
\text { extremity (excluding hand) (39), } \\
\text { cheek (1), chin (1), forehead (1) }\end{array}$ & $335 / 346$ & OR $0.16(0.02,1.56)$ \\
\hline $\begin{array}{l}15888150 \\
\text { Schulze }\end{array}$ & (F) imiquimod 5\% & $\begin{array}{l}\text { cheek (1), forehead (0), extremities } \\
\text { (including hand) (20), trunk/neck } \\
\text { (70) }\end{array}$ & $17 / 84$ & OR $0.16(0.02,1.56)$ \\
\hline $\begin{array}{l}15888150 \\
\text { Schulze }\end{array}$ & $(\mathrm{I}, \mathrm{J})$ vehicle & $\begin{array}{l}\text { cheek (1), forehead (5), scalp (1), } \\
\text { extremities (including hand) (30), } \\
\text { trunk/neck (61) }\end{array}$ & $77 / 82$ & OR $0.16(0.02,1.56)$ \\
\hline
\end{tabular}




\begin{tabular}{|c|c|c|c|c|}
\hline Study & Arm & Lesion Location & $n / N$ & Result \\
\hline $\begin{array}{l}20064185 \\
\text { Foley }\end{array}$ & $\begin{array}{l}\text { (I,J) methyl- } \\
\text { aminolevulinatePDT }\end{array}$ & $\begin{array}{l}\text { face/scalp (25), extremities (20), } \\
\text { Trunk (32), Neck (9) }\end{array}$ & $20 / 75$ & OR $0.13(0.06,0.27)$ \\
\hline $\begin{array}{l}20064185 \\
\text { Foley }\end{array}$ & (E) placebo PDT & $\begin{array}{l}\text { face/scalp (31), extremities (23), } \\
\text { Trunk (34), Neck (1) }\end{array}$ & $55 / 75$ & OR $0.13(0.06,0.27)$ \\
\hline $\begin{array}{l}20546215 \\
\text { Siller }\end{array}$ & (F) ingenol mebutate gel & NR & $37 / 48$ & OR $0.16(0.02,1.56)$ \\
\hline $\begin{array}{l}20546215 \\
\text { Siller }\end{array}$ & $\begin{array}{l}(\mathrm{I}, \mathrm{J}) \text { vehicle gel, } \\
\text { treatment arm B- day } 1 \\
\text { and } 8\end{array}$ & NR & $5 / 6$ & OR $0.16(0.02,1.56)$ \\
\hline $\begin{array}{l}22511036 \\
\text { Tran }\end{array}$ & $(\mathrm{I}, \mathrm{J}) \mathrm{PDL}$ & NR & $8 / 14$ & OR $0.25(0.06,1.01)$ \\
\hline $\begin{array}{l}22511036 \\
\text { Tran }\end{array}$ & (C) No treatment & extremities (43), trunk/neck (57) & $4 / 6$ & OR $0.25(0.06,1.01)$ \\
\hline $\begin{array}{l}23683751 \\
\text { Arits }\end{array}$ & (F) MAL-PDT & $\begin{array}{l}\text { head/neck excluding H-zone (12), } \\
\text { extremities (29), trunk (59), upper } \\
\text { extremities (16), lower extremities } \\
\text { (13) }\end{array}$ & $10 / 126$ & OR $6.16(1.32,28.69)$ \\
\hline $\begin{array}{l}23683751 \\
\text { Arits }\end{array}$ & (E) Imiquimod & $\begin{array}{l}\text { head/neck excluding } \mathrm{H} \text {-zone (12), } \\
\text { extremities (27), trunk (61), upper } \\
\text { extremities (13), lower extremities } \\
(14)\end{array}$ & $2 / 145$ & OR $6.16(1.32,28.69)$ \\
\hline $\begin{array}{l}24903544 \\
\text { Haak }\end{array}$ & (C) AFXL MAL PDT & $\begin{array}{l}\text { nose (56), forehead (19), cheek } \\
\text { (13), oral area (6), periorbital area } \\
\text { (6) }\end{array}$ & $6 / 16$ & OR $0.62(0.26,1.49)$ \\
\hline $\begin{array}{l}24903544 \\
\text { Haak }\end{array}$ & (E) MAL PDT & $\begin{array}{l}\text { nose (37), forehead (31), cheek (6), } \\
\text { oral area (13), periorbital area (13) }\end{array}$ & $7 / 16$ & OR $0.62(0.26,1.49)$ \\
\hline $\begin{array}{l}27067393 \\
\text { Brinkhuizen }\end{array}$ & (F) Calcitriol & trunk/neck (59), genetalia (41) & $16 / 16$ & OR $0.16(0.02,1.56)$ \\
\hline $\begin{array}{l}27067393 \\
\text { Brinkhuizen }\end{array}$ & $(\mathrm{I}, \mathrm{J})$ No treatment & extremities (53), trunk/neck (47) & $16 / 16$ & OR $0.16(0.02,1.56)$ \\
\hline Abbade & (A,B) Surgical excision & head and neck (100) & $0 / 35$ & OR $0.12(0.01,2.47)$ \\
\hline Abbade & (E) MAL-PDT & head and neck (100) & $3 / 33$ & OR $0.12(0.01,2.47)$ \\
\hline Eimpunth & $(\mathrm{I}, \mathrm{J})$ pulsed dye laser & NR & $4 / 14$ & OR $0.25(0.06,1.01)$ \\
\hline Eimpunth & (C) no treatment & NR & $8 / 10$ & OR $0.25(0.06,1.01)$ \\
\hline
\end{tabular}

Table I-3. Lack of clinical clearance, all BCC

\begin{tabular}{|l|l|l|l|l|}
\hline Study & Arm & Lesion Location & $\mathbf{n} / \mathbf{N}$ & Result \\
\hline $\begin{array}{l}11298545 \\
\text { Wang }\end{array}$ & (C) cryosurgery & NR & $5 / 39$ & OR $0.61(0.1,3.56)$ \\
\hline $\begin{array}{l}11298545 \\
\text { Wang }\end{array}$ & (E) ALA-PDT & NR & $2 / 44$ & OR 0.61 (0.1, 3.56) \\
\hline $\begin{array}{l}14732655 \\
\text { Rhodes }\end{array}$ & (A,B) excision & $\begin{array}{l}\text { face/scalp (58), extremities (9), } \\
\text { trunk/neck (29) }\end{array}$ & $1 / 52$ & OR $0.25(0.08,0.74)$ \\
\hline $\begin{array}{l}14732655 \\
\text { Rhodes }\end{array}$ & (E) MAL PDT & $\begin{array}{l}\text { face/scalp (40), extremities (11), } \\
\text { trunk/neck (49) }\end{array}$ & $1 / 53$ & OR 0.25 (0.08, 0.74) \\
\hline $\begin{array}{l}15888150 \\
\text { Schulze }\end{array}$ & (F) imiquimod 5\% & $\begin{array}{l}\text { cheek (1), forehead (0), extremities } \\
\text { (including hand) (20), trunk/neck } \\
\text { (70) }\end{array}$ & $19 / 84$ & OR 0.04 (0.02, 0.07) \\
\hline $\begin{array}{l}\text { 15888150 } \\
\text { Schulze } \\
\text { extremities (including hand) (30), } \\
\text { trunk/neck (61) }\end{array}$ & (I,J) vehicle & NR & $77 / 82$ & OR 0.04 (0.02, 0.07) \\
\hline $\begin{array}{l}17573890 \\
\text { Berroeta }\end{array}$ & (A,B) excision & NR & $4 / 19$ & OR 0.25 (0.08, 0.74) \\
\hline $\begin{array}{l}17573890 \\
\text { Berroeta }\end{array}$ & (E) PDT & $8 / 21$ & OR 0.25 (0.08, 0.74) \\
\hline
\end{tabular}




\begin{tabular}{|c|c|c|c|c|}
\hline Study & Arm & Lesion Location & $n / N$ & Result \\
\hline $\begin{array}{l}18624836 \\
\text { Szeimies }\end{array}$ & $(\mathrm{A}, \mathrm{B})$ excision & $\begin{array}{l}\text { face/scalp (4.5) , extremities (25.0), } \\
\text { trunk/neck (70.5) }\end{array}$ & $0 / 117$ & OR $0.25(0.08,0.74)$ \\
\hline $\begin{array}{l}18624836 \\
\text { Szeimies }\end{array}$ & (E) MAL-PDT & $\begin{array}{l}\text { face/scalp (11.1), extremities (28.9), } \\
\text { trunk/neck (60) }\end{array}$ & $11 / 118$ & OR $0.25(0.08,0.74)$ \\
\hline $\begin{array}{l}18693158 \\
\text { Basset- } \\
\text { Seguin }\end{array}$ & (C) Cryotherapy & $\begin{array}{l}\text { face/scalp (4), extremities (20), } \\
\text { trunk/neck (76) }\end{array}$ & $5 / 98$ & OR $0.61(0.1,3.56)$ \\
\hline $\begin{array}{l}18693158 \\
\text { Basset- } \\
\text { Seguin }\end{array}$ & (E) MAL-PDT & $\begin{array}{l}\text { face/scalp (6), extremities (22), } \\
\text { trunk/neck (72) }\end{array}$ & $3 / 103$ & OR $0.61(0.1,3.56)$ \\
\hline $\begin{array}{l}20546215 \\
\text { Siller }\end{array}$ & $\begin{array}{l}\text { (F) ingenol mebutate } \\
\text { gel }\end{array}$ & NR & $36 / 48$ & OR $0.04(0.02,0.07)$ \\
\hline $\begin{array}{l}20546215 \\
\text { Siller }\end{array}$ & $\begin{array}{l}(\mathrm{I}, \mathrm{J}) \text { vehicle gel, } \\
\text { treatment arm } \mathrm{B} \text { - day } 1 \\
\text { and } 8\end{array}$ & NR & $6 / 6$ & OR $0.04(0.02,0.07)$ \\
\hline $\begin{array}{l}21242584 \\
\text { Garcia- } \\
\text { Martin }\end{array}$ & (D) radiotherapy & eyelid (100) & $0 / 12$ & OR $1.24(0.02,67.04)$ \\
\hline $\begin{array}{l}21242584 \\
\text { Garcia- } \\
\text { Martin }\end{array}$ & (F) imiquimod 5\% & eyelid (100) & $0 / 15$ & OR $1.24(0.02,67.04)$ \\
\hline $\begin{array}{l}2229497 \\
\text { Cornell }\end{array}$ & (F) interferon & $\begin{array}{l}\text { head and face (25), extermities } \\
\text { (12), trunk/neck (63) }\end{array}$ & $22 / 118$ & OR $0.04(0.02,0.07)$ \\
\hline $\begin{array}{l}2229497 \\
\text { Cornell }\end{array}$ & $(\mathrm{I}, \mathrm{J})$ placebo & $\begin{array}{l}\text { head and face (17), extermities } \\
(14), \text { trunk/neck (59) }\end{array}$ & $33 / 41$ & OR $0.04(0.02,0.07)$ \\
\hline $\begin{array}{l}24332516 \\
\text { Bath-Hextall }\end{array}$ & (F) excision & $\begin{array}{l}\text { face (33), trunk (39), neck (9), arm } \\
\text { (7), leg (9), other (3) }\end{array}$ & $1 / 98$ & OR $0.58(0.05,6.47)$ \\
\hline $\begin{array}{l}24332516 \\
\text { Bath-Hextall }\end{array}$ & $(A, B)$ Imiquimod & $\begin{array}{l}\text { face (37), trunk (38), neck (6), arm } \\
(6), \text { leg (10), other (3) }\end{array}$ & $2 / 114$ & OR $0.58(0.05,6.47)$ \\
\hline $\begin{array}{l}24903544 \\
\text { Haak }\end{array}$ & (C) AFXL MAL PDT & $\begin{array}{l}\text { nose (56), forehead (19), cheek } \\
\text { (13), oral area (6), periorbital area } \\
(6)\end{array}$ & $0 / 16$ & OR $0.61(0.1,3.56)$ \\
\hline $\begin{array}{l}24903544 \\
\text { Haak }\end{array}$ & (E) MAL PDT & $\begin{array}{l}\text { nose (37), forehead (31), cheek (6), } \\
\text { oral area (13), periorbital area (13) }\end{array}$ & $2 / 16$ & OR $0.61(0.1,3.56)$ \\
\hline $\begin{array}{l}26551044 \\
\text { Choi }\end{array}$ & $\begin{array}{l}\text { (C) Er:YAG ablative } \\
\text { fractional laser-primed } \\
\text { MAL- PDT }\end{array}$ & NR & $6 / 21$ & OR $0.61(0.1,3.56)$ \\
\hline $\begin{array}{l}26551044 \\
\text { Choi }\end{array}$ & (E) MAL-PDT & NR & $17 / 21$ & OR $0.61(0.1,3.56)$ \\
\hline $\begin{array}{l}298425 \\
\text { Allen }\end{array}$ & (D) cryotherapy & NR & $1 / 15$ & OR $3.41(0.13,90.49)$ \\
\hline $\begin{array}{l}298425 \\
\text { Allen }\end{array}$ & (C) radiotherapy & NR & $0 / 16$ & OR $3.41(0.13,90.49)$ \\
\hline $\begin{array}{l}9218740 \\
\text { Avril }\end{array}$ & (D) surgery & $\begin{array}{l}\text { nose (53), cheek, pre- and } \\
\text { retroauricular areas ( } 21) \text {, eyelids, } \\
\text { internal and external eye angles } \\
\text { (19), forehead, temple, between } \\
\text { eyebrows } 36(21) \text {, chin, cutaneous } \\
\text { superior lip } 10(6) \text {, ear (3) }\end{array}$ & $0 / 174$ & OR $0.14(0.01,2.72)$ \\
\hline $\begin{array}{l}9218740 \\
\text { Avril }\end{array}$ & $(A, B)$ radiotherapy & $\begin{array}{l}\text { nose (28), cheek, pre- and } \\
\text { retroauricular areas (24), eyelids, } \\
\text { internal and external eye angles } \\
\text { (20), forehead, temple, between } \\
\text { eyebrows (17), chin, cutaneous } \\
\text { superior lip (7), ear (3) }\end{array}$ & $3 / 173$ & OR $0.14(0.01,2.72)$ \\
\hline
\end{tabular}


Table I-4. Recurrence, ScCIS

\begin{tabular}{|l|l|l|l|l|}
\hline Study & Arm & Lesion Location & n/N & Result \\
\hline $\begin{array}{l}12653747 \\
\text { Salim }\end{array}$ & (E) PDT & extremities (100) & $6 / 33$ & OR 0.21 (0.07, 0.64) \\
\hline $\begin{array}{l}12653747 \\
\text { Salim }\end{array}$ & (F) 5-FU & face (12), extremities (88) & $17 / 33$ & OR 0.21 (0.07, 0.64) \\
\hline $\begin{array}{l}16785375 \\
\text { Morton }\end{array}$ & $\begin{array}{l}\text { (C) Cryotherapy or } \\
\text { Fluorouracil }\end{array}$ & NR & $19 / 97$ & OR 1.21 (0.61, 2.4) \\
\hline $\begin{array}{l}16785375 \\
\text { Morton }\end{array}$ & $\begin{array}{l}\text { (C) Cryotherapy or } \\
\text { Fluorouracil }\end{array}$ & NR & $19 / 97$ & OR 0.24 (0.03, 1.84) \\
\hline $\begin{array}{l}\text { Morton } \\
\text { 16785375 } \\
\text { Morton }\end{array}$ & (E) MAL PDT & $\begin{array}{l}\text { face/scalp (23), extremities (65), } \\
\text { trunk/neck (12) }\end{array}$ & $15 / 103$ & OR 0.17 (0.02, 1.3) \\
\hline $\begin{array}{l}16785375 \\
\text { Morton }\end{array}$ & (I,J) PDT placebo & $\begin{array}{l}\text { face/scalp (23), extremities (65), } \\
\text { trunk/neck (12) } \\
\text { trunk/neck (8) }\end{array}$ & $15 / 103$ & OR 1.21 (0.61, 2.4) \\
\hline $\begin{array}{l}16785375 \\
\text { Morton }\end{array}$ & (I,J) PDT placebo & $\begin{array}{l}\text { face/scalp (25), extremities (67), } \\
\text { trunk/neck (8) }\end{array}$ & $2 / 4$ & OR 0.17 (0.02, 1.3) \\
\hline 24102369 Ko & (C) Er:YAG AFL PDT & extremities (100) & $1 / 19$ & OR 1.21 (0.61, 2.4) \\
\hline $\begin{array}{l}24102369 \text { Ko } \\
\text { (E) MAL-PDT }\end{array}$ & extremities (100) & $1 / 19$ & OR 1.21 (0.61, 2.4) \\
\hline $\begin{array}{l}\text { 8977678 } \\
\text { 89rton }\end{array}$ & (C) cryotherapy & hands (5), face (15), legs (80) & $2 / 20$ & OR 1.21 (0.61, 2.4) \\
\hline \begin{tabular}{l} 
Morton \\
\hline
\end{tabular} & (E) photodynamic & hands (5), face (10), legs (85) & $0 / 20$ & OR 1.21 (0.61, 2.4) \\
\hline
\end{tabular}

Table 1-5. Lack of histological clearance, SCCIS

\begin{tabular}{|l|l|l|l|l|}
\hline Study & Arm & Lesion Location & n/N & Result \\
\hline 16713457 Patel & $(\mathrm{F})$ imiquimod 5\% & NR & $12-$ Mar & OR 0.01 (0, 0.24) \\
\hline 16713457 Patel & $(\mathrm{I}, \mathrm{J})$ vehicle & NR & $16 / 16$ & OR 0.01 (0, 0.24) \\
\hline
\end{tabular}

Table I-6. Lack of clinial clearance, SCCIS

\begin{tabular}{|l|l|l|l|l|}
\hline Author & Arm & Lesion Location & $\mathbf{n} / \mathbf{N}$ & Result \\
\hline 12653747 Salim & (E) PDT & extremities (100) & $4 / 33$ & OR 0.28 (0.08, 0.98) \\
\hline 12653747 Salim & (F) 5-FU & face (12), extremities (88) & $11 / 33$ & OR 0.28 (0.08, 0.98) \\
\hline 16713457 Patel & (F) imiquimod 5\% & NR & $3 / 12$ & OR 0.01 (0, 0.24) \\
\hline 16713457 Patel & (I,J) vehicle & NR & $16 / 16$ & OR 0.01 (0, 0.24) \\
\hline 16785375 Morton & $\begin{array}{l}\text { (C) Cryotherapy or } \\
\text { Fluorouracil }\end{array}$ & NR & $17 / 114$ & OR 0.94 (0.46, 1.94) \\
\hline 16785375 Morton & $\begin{array}{l}\text { (C) Cryotherapy or } \\
\text { Fluorouracil }\end{array}$ & NR & $17 / 114$ & OR 0.05 (0.01, 0.16) \\
\hline 16785375 Morton & (E) MAL PDT & $\begin{array}{l}\text { face/scalp (23), extremities (65), } \\
\text { trunk/neck (12) }\end{array}$ & $8 / 111$ & OR 0.02 (0.01, 0.08) \\
\hline 16785375 Morton & (E) MAL PDT & $\begin{array}{l}\text { face/scalp (23), extremities (65), } \\
\text { trunk/neck (12) }\end{array}$ & $8 / 111$ & OR 0.94 (0.46, 1.94) \\
\hline 16785375 Morton & (I,J) PDT placebo & $\begin{array}{l}\text { face/scalp (25), extremities (67), } \\
\text { trunk/neck (8) }\end{array}$ & $15 / 19$ & OR 0.05 (0.01, 0.16) \\
\hline 16785375 Morton & (I,J) PDT placebo & $\begin{array}{l}\text { face/scalp (25), extremities (67), } \\
\text { trunk/neck (8) }\end{array}$ & $15 / 19$ & OR 0.02 (0.01, 0.08) \\
\hline 24102369 Ko & (C) Er:YAG AFL PDT & extremities (100) & $4 / 32$ & OR 0.94 (0.46, 1.94) \\
\hline 24102369 Ko & (E) MAL-PDT & extremities (100) & $13 / 26$ & OR 0.94 (0.46, 1.94) \\
\hline 8977678 Morton & (C) cryotherapy & hands (5), face (15), legs (80) & $0 / 20$ & OR 0.94 (0.46, 1.94) \\
\hline 8977678 Morton & (E) photodynamic & hands (5), face (10), legs (85) & $0 / 20$ & OR 0.94 (0.46, 1.94) \\
\hline
\end{tabular}

